






\section{LE ISOLE PELAGIE E LA LORO FLORA}





\title{
STEFANO SOMMIER
}

\section{LE ISOLE PELAGIE}

\section{LAMPEDUSA, LINOSA, LAMPIONE}

\author{
E LA LORO FLORA
}

CON UN ELENCO COMPLETO DELLE PIANTE

\section{PAN'TELLERIA}
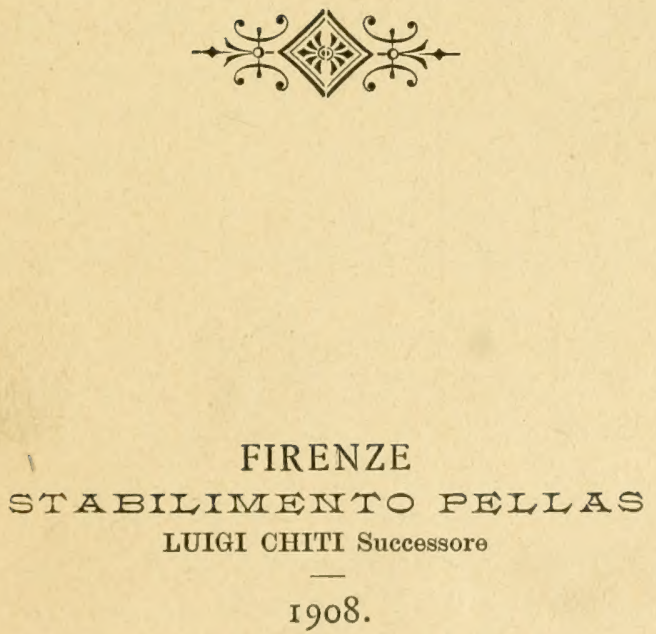



\section{PREFAZIONE}

Nel 1873 il prof. Parlatore, specialmente in vista di raccogliere materiali per la sua Flor'c Itcliance alla quale allora lavorava assiduamente, mandava in Sicilia il sig. Luigi Ajuti, giardiniere botanico del R. Istituto Superiore, per una campagna botanica primaverile, che doveva estendersi fino alle isolette di Linosa e di Lampedusa, ed ebbe caro che io mi unissi all'Ajuti in quel viaggio.

Oggri ancora il portarsi alle isole Pelagie non è cosa molto comola; ma ben piu disagevole era il viaggrio in quel tempo. Unico mezzo di comunicazione era il piccolo veliero che portara da Porto Empedocle a Lampedusa la posta ed i condannati a domicilio coatto. Col cap̧itano di quella meschina imbarcazione, che in Sicilia porta il nome arabo di sciabecco, contrattammo il nostro passaggio.

Quanto staremo in mare? - ghli chiedemmo.

Forse ventiquattr' ore se il vento è buono; ma portino con sè provviste per otto giorni perchè non si puó sapere; - fư la . suggestiva risposta.

Il 15 aprile c' imbarcammo a Porto Empedocle, in compagnia di una squadra di condannati con i relativi carabinieri. Lo sciabecco aveva una sola più che modesta cabina, quella del capi- 
tano, che questi gentilmente ci cedette. Condannati e carabinieri furono allogati nelia stiva. Comincio il viagrio sotto buoni auspici: 11 a dopo poco, cambiato e rinfrescato il rento, doremmo cambiar corso e metter la prua sopra Pantelleria che arvistammo il giorno dopo la nostra partenza. Mutatosi da capo il vento in furioso libeccio, fummo respinti fino a rivedere le coste di Sicilia, quando un nuovo mutamento ci permise di rimettere la prua su Lampedusa dove arrivammo il 18 aprile. Eravamo rimasti cosi il trastullo delle onde e dei venti per tre giorni e tre notti, durante i quali, è inutile dirlo, le nostre provviste rimasero intatte !

Il nostro ritorno fu piú fortunato. Partiti da Lampedusa il $1^{\circ}$ maggio con buon vento di mezzogiorno, giungemmo a Porto Empedocle in poco più di ventiquattr' ore.

Ma se non era facile arrivare a Lampedusa, meno facile ancora era il giungere a Linosa. Per andarvi dovemmo noleggiare a Lampedusa una barchetta scoperta. Con questa, scelto un giorno di buon vento, il 21 aprile giungemmo in sei ore a Linosa, con qualche peripezia soltanto all' arrivo, lo sbarco essendo difficile col vento fresco su quella costa rocciosa. Il 25 tornammo colla nostra barca a Lampedusa.

Sarebbe stato mio desiderio andare anche a Lampione. Ma allora, come ai tempi di Calcara, lo vietarano le leggi sanitarie; ossia non avremmo potuto sbarcare in questo isolotto disabitato senza suttoporci poi ad una lunga quarantena.

$$
* *
$$

Quando presentai questo scritto al Congresso di Palermo, erano 'trascorsi quasi trent' anni dall' epoca di quella gita; eppure rivedendo le mie raccolte mi ero persuaso che meritarano ancora di esser pubblicate. Buon numero delle nostre piante figuravano nella Flora ilaliana di Parlatore e nella sua continuazione per Caruel, e precisamente quelle delle famiglie pubblicate dopo il 1873. Molte delle specie che quando le raccogliemmo non erano note delle isole Pelagie, erano state poi trorate e pubbli- 
cate dai signori Lojacono, Ross e Solla. Tuttavia ve ne erano non poche la cui presenza in quelle isole non era stata ancora segnalata. E del resto, anche astrazion fatta dalle nuoviti contenute nelle mie due florule, mi era sembrato prezzo dell'opera il riunire in un corpo quanto era stato fin allora pubblicato frammentariamente ed in opuscoli non facili a procurarsi, e presentare cosi il quadro esatto della flora delle due isole quale allora la conosceramo. Questa pubblicazione mi pareva poi che avesse un interesse speciale non soltanto perchè le nostre due isolette sono gli ultimi lembi meridionali di terra appartenenti all' Italia, ma anche perchè le loro florule permettono di fare il confronto fra due isole vicine e quindi sottoposte a condizioni climatiche uguali, ma assolutamente diverse per origine e per natura di suolo.

La pubblicazione di questo mio laroro ha subito un lungo ritardo, perché era destinato agli Atti del Congresso di Palermo, la cui stampa dopo essere stata rimandata fu poi definitiramente abbandonata. Nel frattempo il dott. Zodda ha fatto una escursione botanica nelle Pelagie, ed io stesso ho potuto tornare a risitarle in compagnia del giardiniere botanico Antonino Riccobono. Cosi la nostra conoscenza di queste isole ha fatto un altro passo, e posso presentarne le florule con notevoli aggiunte. ì dunque il caso di dire che ogni male non vien per nuocere. 



\section{LE ISOLE PELAGIE}

Il nome di Pelagie, adoperato come nome proprio per designare un gruppo di isole, non è molto antico. Ha evidentemente avuto origine dall'appellativo di $\pi s \lambda a y \iota s$, pelagie o di alto mare, dato da Tolomeo e da Strabone ad alcune isole del Mediterraneo fra la Sicilia e l'Africa.

Difatti rediamo che Mattioli, nella versione italiana di Tolomeo

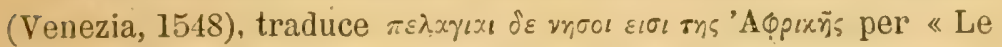
Isole d'Aphrica chiamate Pelagie sono queste » dando all'appellatiro di pelagie il valore di nome proprio. Il termine di pelagie però non fu sempre applicato nello stesso modo, d'onde sono nate non poche confusioni. Tolomeo per esempio (Geograph., lib. IV, cap. I, 13) dice che le isole d'alto mare ( $\left.\pi \varepsilon \lambda \times \gamma_{t} \times x_{l}\right)$ appartenenti all'Africa sono Cossyra (Pantelleria), Gaulus (Gozo) e Melite (Malta), e pone Lopadusa ed Ethusa (Linosa) fra le isole adiacenti all'Africa. Strabone invece applica il termine di pelagia soltanto all' isola di Lampedusa ( $\eta \pi \sigma 0 \sigma \cdot \pi \varepsilon \lambda x \gamma \iota x \quad \lambda 0 \pi x \delta \sigma \tilde{v} \sigma \sigma x$. Geograph., lib. XVII, cap. III).

Nelle carte nautiche dal principio del secolo XIV alla fine del XVI ${ }^{1}$ Lampedusa, Linosa e Lampione non si trovano mai designate con un nome collettivo.

Il nome di Pelagie, adoperato come nome proprio per designare in modo ben definito come oggi le due isole di Lampedusa e Linosa e l' isolotto di Lampione, si trova in Fazello nel 1558."

1 Vedasi la splendida edizione di facsimili pubblicati da Nordenskjöld col nome di Periplus.

2 Vedi bibliografia. 
Peró anche più tardi troviamo chi seguita a adoprarlo in altro senso. Ortelio, per esempio, nella seconda metá del secolo XVI scrive nel suo "Thesaurus geographicus » che le isole Pelagie sono cinque, e quello che ne dice prova quale idea confusa avesse intorno ad esse. Baudrand (Geographia, 1682) e Baudrand e Ferrari (Novum lexicon geograph., 1738) ripetono che le Pelagie sono cinque e sono le «Tarichiae rel Phoenicorum insulae» di Strabone, le Conigliere di Nigro. ${ }^{1} \mathrm{E}$ si che da tre secoli i portolani indicavano con esattezza mirabile per quei tempi le isole di quella parte del Mediterraneo, quel mare era solcato da naviglio di commercio e di guerra, e Malta già da quasi un secolo era in possesso dei Cavalieri Ospitalieri. Ciò prova quanto la geografia scolastica, che si basava sugli antichi testi spesso male interpretati, fosse rimasta arretrata rispetto. alle conoscenze dei nariganti.

\section{BOTANICI CHE HANNO VISTTATO LE ISOLE PELAGIE}

Avanti noi pare che tre o quattro soli botanici avessero visitato Lampedusa e due soli Linosa.

Nel 1787 La Billardiére si fermò brevemente a Lampedusa mentre era in viaggio per la Siria. ${ }^{2}$ Vi raccolse due piante non ancora conosciute, la Periploca angustifolia ${ }^{3}$ che egli stesso descrisse e figuró nel suo «Icones plantarum Syriae rariorum »

1 Bruzen la Martinière, nel 1736, nel suo Gran Dizionario geo-, grafico, mentre rileva l'errore di Ortelio, ritiene che siano da intendersi per isole Pelagie quelle indicate da Tolomeo. Perfino Reclus nella sua Géographie Universelle dice che le Pelagie comprendono Lampedusa, Linosa ed altre minori(!).

${ }^{2}$ Gussone, Notizie sulle isole Linosa, Lampione e Lampedusa, p. 71, e Calcara, Descrizione dell'isola di Lampedusa, p. 28 in nota.

3 Peró quando la descrisse nel 1791 era già stato preceduto da Aiton, il quale, nel Hortus Kewensis del 1789 ( $1^{\text {a }}$ edizione, vol. I, p. 301), aveva descritto la stessa pianta proveniente dalle isole $\mathrm{Ca}-$ nario col nome di Periploca laevigaia. 
Decas secunda, tab. VII, pag. 13-14, ove è detto: «Hab. juxta Ladoiceam et in insula Lampelosa », e la Diplotaxis scaposa che renne descritta da De Candolle. Non sono state pubblicate, che io sappia, altre piante raccolte da La Billardière in Lampedusa, ${ }^{2}$ e Gussone dice espressamente che egli non ha lasciato alcun cenno su di questa isola. L'erbario dell' illustre botanico francese conservasi a Firenze intercalato nell'erbario Webb, ${ }^{2}$ e forse vi si trovano altre piante provenienti da Lampedusa; ma è impossibile rintracciarle, mancando in generale l'indicazione della provenienza sulle etichette di La Blllardière.

Un altro botanico parrebbe che visitasse Lampedusa al principio del secolo passato; ma chi egli fosse, ed in quale anno vi andasse non ho potuto sapere. Il fatto si rileva da un catalogo di piante delle Isole Maltesi e di Lampedusa pubblicato dal padre Giacinto ${ }^{3}$ nel 1825, nel quale, al dire del Grech Delicata, ${ }^{4}$ sono citate 117 piante di Lampedusa. Siano forse state raccolte in quest' isola da La Billardière e venute alla conoscenza del Giácinto? Siano state raccolte dal Giacinto stesso o da uno dei suoi collaboratori Naudi o Zerafa? Avevo pensato che queste piante si troverebbero forse ancora negli erbarî della Universitá di Valetta; ma la speranza che l'esame di quegli erbarî potesse gettare qualche luce sull'argomento è pressochè svanita dopo la mia visita a Malta in quest'anno, poichè ho dovuto constatare che della magrgior parte delle piante non è indicata la provenienza, e quando questa è segnata, manca di solito la data e il nome del raccoglitore. Nei pacchi esaminati trovai una sola pianta di Lampedusa, la Periploca angustifolia, con eti-

1 Parlatore, Fl. It., vol. V, p. 550, cita ancora, ma dubitativamente, Hypericum Egyptiacum come raccolto in Lampedusa da La Billardière. Difatti trovansi due esemplari di questa specie nell'erbario Webb provenienti dall'erbario di La Billardière, ma senza indicazione di località (uno nell' inserto di Triadenia microphylla, l'altro nell' inserto di Triadenia Webbii).

2 Parlatore, Les collections botaniques du Musée royal de Physique et d'Histoire naturelle de Florense au printemps de 1874. Firenze, 1874, p. 15-16.

3 Vedi in bibliografia a Hyacintturs.

4 J. C. Grech Delicata, Flora Melitensis etc. Melitae, 1853 (Praefatio, p. xi). 
chetta che non diceva altro se non "Asclepias della Lampedusa ». Certo si è che Gussone non sapera nè dello scritto di Giacinto nè di quelle piante state raccolte in Lampedusa avanti l'epoca sua.

Nel 1828 Gussone, a bordo di un brigantino della Marina Reale messo a sua disposizione dal Re Francesco di Napoli per un viaggio circuminsulare, ${ }^{2}$ si reco in Lampedusa, dove soggiornó dal 2 al 31 di agosto, come risulta dalla tabella meteorologica pubblicata nelle sue « Notizie ecc. ». Dalla stessa tabella si deduce che a Linosa fu soltanto il 31 agosto da mattina a sera; ed a Lampione pure da mattina a sera il 15 agosto. Nel 1832 comunicó alla R. Accademia delle Scienze di Napoli i risultati di questa sua visita alle isole Pelagie in una Memoria che contiene una descrizione e notizie geologiche, botaniche, meteorologiche e storiche di quelle isole, insieme alla descrizione della Stapelia Europaea. Nel «Supplementum ad Flor. Sic. Prodr. » egli incominciò a pubblicare le piante raccolte nelle Pelagie, le quali poi furono riportate tutte nella sua «Florae Siculae Synopsis ». Le piante stesse sono conservate nel suo Erbario Siculo alla Università di Napoli. L' Erbario centrale di Firenze ne possiede alcuni esemplari.

Nella biografia di Gussone scritta da Pasquale è detto che il barone Porcari, viaggiatore e collettore, fu a Lampedusa con Gussone, e che lo stesso Porcari mandó a Gussone delle piante di quell' isola. Ma delle visite del Porcari a Lampedusa non ho potuto sapere altro.

Gussone è il solo botanico che abbia visitato l" isolotto di Lampione.

Nel 1846 Calcara, professore interino di Storia naturale nella R. Universitá di Palermo, visitava le nostre isole (oltre a quella di Pantelleria). Giungeva in Lampedusa il 18 maggio. L' 8 giugno si recava a Linosa da dove, dopo un soggiorno di due o tre giorni, ritornava in Lampedusa trattenendovisi fino al 15 giugno. Durante questo viaggio era accompagnato da Vincenzo Messina, giardiniere dell' Orto botanico di Palermo, incaricato

1 Pasquale, Dooumenti biografici di G. Gussone, p. 114. 
ta Tineo di coadiuvare Calcara nella raccolta delle piante. ${ }^{1}$ In un rapporto preliminare ci ha lasciato un cenno del suo viaggio, e notizie succinte sulle due isole. In due opuscoli separati poi, parla di Lamperlusa e di Linosa, descrivendole e dando intorno ad esse variate notizie, in parte desunte dalla memoria di Gussone.

In questi opuscoli, per ognuna delle due isole, sotto i titoli di «Florula Lopadusana» e «Florula di Linosa d dà l'elenco delle piante raccolte da Gussone e da lui. stesso. Le piante riportate da esso e dal giardiniere Messina furono studiate da Vincenzo Tineo allora direttore dell'Orto botanico di Palermo, e si conservano nell' Erbario dell' Universitá di Palermo, dal quale l'Erbario di Firenze ba ricevuto non pochi doppî. Tineo pubblicó nelle sue "Plant. rar. Siciliae », alcune novitá trovate fra le piante di Calcara.

Calcara, come egli stesso ci informa ("Rapporto»p. 15 e «Descrizione di Lampedusa » p. 19 in nota), non potè visitare Lampione, dore leggi sanitarie vietarano l'approdo.

Nel 1884 pare che si risvegliasse ad un tratto l' interesse per la flora di queste isole, forse stimolato dal fatto che era stata stabilita una comunicazione regolare colla Sicilia per mezzo di piroscafi. Difatti il 6 aprile di quell'anno sbarcarano in Lampedusa tre botanici, cioé i signori M. Lojacono, assistente del Giardino botanico di Palermo, il dott. H. Ross e il barone v. Zwierlein, e vi si trattenevano fino al 16. I due ultimi si recarono poi a Linosa dove si trattennero dal 16 al 20 aprile, mentre Lojacono tornava a Porto Empedocle. Il 13 aprile poi dello stesso anno giungeva in Lampedusa un quarto botanico, il dott. R. Solla, e vi si tratteneva fino al 20. Egli soggiornava poi a Linosa dal 21 al 30 aprile.

Il dott. Ross, il 25 luglio 18s4, presentara alla Sociełá botanica di Berlino un brevissimo rapporto sul suo viaggio intitolato "Eine botanische Excursion nach den Inseln Lampedusa und Linosa", con un elenco delle piante interessanti raccolte da esso nelle due isole. Le piante critiche furono determinate

1 Ció rilevasi da Tiseo, Plantar. rar. Sic., fasc. II, p. 19-20 in nota. 
dal prof. Ascherson, le Medicago dal dott. Urban, i licheni dal prot. Magnus.

Nel settembre del 1884 il sig. Lojacono dava alle stampe col titolo «Una escursione botanica in Lampedusa, », una breve relazione del suo viaggio con una succinta descrizione di Lampedusa ed un "Catalogo delle piante vascolari di Linosa e Lampedusa » nel quale sono annoverate le piante raccolte dal barone v. Zwierlein e da lui in Lampedusa, ed alcune piante di Linosa che gli furono comunicate dal barone $\mathrm{v}$. Zwierlein, ed inoltre sono riportate, ma in parte soltanto, le piante della « Florula Lopadusana » di Calcara da esso non trorate. Le piante raccolte da. Lojacono sono riportate pure in parte nella sua « Flora Sicula ».

Il barone v. Zwierlein non ha pubblicato nulla. Le piante da esso raccolte figurano nell' elenco di Lojacono. Esse sono in parte conservate negli Erbarî dell' Universitá di Messina.

Il dott. Solla scrisse una lettera datata da Messina 5 maggio 1884 e pubblicata nella Oesterr. bot. Zeitschr., nella quale è descritto il paesaggio botanico di Lampedusa e di Linosa. Presentó poi, il 5 novembre 1884, alla Societá zonlogico-botanica di Vienna una breve memoria intitolata « Phytobiologische Beobachtungen auf einer Excursion nach Lampedusa und Linosa » (datata dalla fine di agosto) che contiene delle osservazioni sulla flora di queste isole, ed un elenco delle piante da esso racco!te in ognuma. I licheni furono determinati dal dott. A. Jatta. 11 dott. Solla pubblicava inoltre nella Oesterr: bot. Zeitschr., anno 1885, col titolo "Auf einer Excursion nach den pelagischen Inseln, April 1884, gesammelte Meeresalgen ", un elenco di alghe marine raccolte in questo viaggio (2:) di Lampedusa e 18 di Linosa). Le piante da esso raccolte nelle Pelagie si trovano negli Erbarî di Messina e di Berlino. La buona descrizione dell'aspetto floristico delle Pelagie dataci dal Solla è tanto più interessante in quanto che d'allora in poi quell' aspetto è assai mutato.

L'anno dopo la nostra visita a Lampedusa il sig. Conti Dini, ufliciale postale in quell'isola, inandó al prof. Parlatore ed a me alcune poche piante raccolte in autunno ed inverno.

Anche il D.r Ross e il sig. Lojacono ricevettero (e distribuirono nelle loro exsiccala) alcune piante di Lampedusa raccolte dopo la loro escursione da persone di conoscenza residenti in quell' isola. 
Finalmente nel 1905 le Pelagie furono visitate, per incarico del Conte Martelli, dal dott. Zodda, accompagnato dal dott. Sturniolo. Questi botanici si trattennero a Lampedusa dal 31 marzo al 9 aprile, el a Linosa dal 9 al 16 aprile, riportando ricea messe di piante che dal Conte Martelli e dal dott. Zutda furorio gentilmente messe a mia disposizione onde io ne potessi tener conto nelle mie florule.

Trovandomi obbligato, per queste ultime raccolte posteriori alla presentazione del mio lavoro al Congresso di Palermo, di modificare il mio manoscritto e di rifarne la parte statistica, mi decisi di completare possibilmente la nostra conoscenza di quelle flore segnatamente nella loro parte briologica, visitando Lampedusa e Linosa in epoca più precoce di quella in cui fino allora erano state esplorate. Feci dunque in questa primavera, dopo 33 anni, una seconda visita alle Pelagrie, soggrornando una settimana a Linosa (dal $1^{\circ}$ all' 8 marzo) ed un'altra a Lampedusa (dall' 8 al 15 marzo) e visitando poi Pantelleria e Malta pel meglio poterne confrontare la flora con quella delle Pelagie. In questa gita a Linosa, Lampedusa e Pantelleria ebbi a compagno e validissimo coadiutore nella raccolta il giardiniere botanico dell'Orto di Palermo, Antonino Riccobono, al quale il Direttore di quell' Istituto botanico, prof. Borzi, con squisita cortesia di cui sono lieto esprimere qui la mia gratitudine, aveva a tal uopo concesso un lungo congedo. In oggi il recarsi in Lampedusa ed in Linosa non presenta piủ le difficoltá di una volta, poichè due volte ogni settimana vi approda un piroscafo, una volta andando da Porto Empedocle a Pantelleria, ed una volta tornando. Con tempo buono il tragitto da Porto Empedocle a Linosa dura circa dieci ore, e da Linosa a Lampedusa poco più di tre ore. In Linosa peró può succedere che a causa del tempo cattivo lo sbarco sia impossibile.

Neppure questa volta mi fu dato, per le condizioni del mars, di recarmi nell' isolotto di Lampione dove bisogna andare in barca, come non avevano potuto recarvisi l'anno avanti i signori Zodda e Sturniolo.

Ancora recentissimamente ho ricevuto alcune piante raccolte, dopo la mia ultima visita a Lampedusa, dalla guardia campestre Martorana, che mi era stata guida in tutte le mie gite. Anche 
queste piante hanno portato un piccolo contributo alla conoscenza di quella flora.

Nelle Flore italiane di Bertoloni, Parlatore e Fiori si trovano qualche rolta citati Tineo, Todaro e Pasruale per Lampedusa, Linosa e Lampione come se essi vi avessero raccolto piante." Nessuno di questi tre botanici visitó le Pelagie. L' errore proviene dal fatto che Tineo e Todaro distribuirono piante raccolte da Calcara e che Pasquale distribui piante di Gussone, senza citare sempre sulle etichette da chi erano state raccolte.

1 Auche dallo specchio degli erbarî fiorentini, nello scritto di Parlatoro intitolato Collections holaniques du Mrusée de Florence ecc. Firenze, 1871, 1). 35, 36, si potrebbe credere che Lampedusa e Linosa fossero state visitate da Todaro. Ivi perú dev intendersi «distribuite » e non « raccolte» da Todaro.

2 Todaro è giunto a tal punto di trascuratezza da lasciare stampare su di una etichetta (N.0275 della sua «Flora sicula exsiccata»): "Statice albida - Maio - Lampione legit Todaro », mentre è ben noto che l'unico botanico che visito Lampione è Gussone, il quale vi fu nel mese di agosto! 


\section{APPUNTI BIBLI0GRAFICI}

Questi appunti non hanno la pretesa di essere una bibliografia completa per le isole Pelagie. Ho tralasciato di proposito gli antichi scrittori che ne menzionano solo il nome o poco più, ed i lavori piú recenti nei quali poco o nulla di originale si contiene su di esse. Ma certo mi saranno sfuggiti làvori meritevoli di essere citati. Ho peró cercato, per quanto mi è stato possibile, di dare completa la bibliografia botanica.

Fazello. E. Thomae Fazelli Siculi Or. Praedicatorum. - De Rebus Siculis decades duae nunc primum in lucem editae. Ex offic. Joan.' Matth. Maydae. Panormi, 1558 mense Maio.

In questa opera dedicata «D. Philippo Hispania, Angliae, Siciliae ctc. regi potentissimo », nella prima decade, libro primo « $\mathrm{De}$ situ Siciliae et insularum adiacentium descriptione caput prinum », a p. 9, il frate Fazello dice: "In medio mari inter Cercinam (oggi Kerkena), et Siciliam, sunt Pelagiae insulae desertre tres, Lampedusa, Lalenusa et Scola, non multum ab invicem refugientes. Lampedusa earum est maxima, p. m. 12 ambitus, quae vetustum nomen a coruscationibus, quas crebro emittit adeptum, adhuc servat: ubi olim ejusdem appellationis erat arx, et oppidulum, cujus monumenta. Specus est in en, Sacellum D. Marine sacrum. Qua Occidentem respicit, rupibus altis et immensis scatet. Qua vero Orientem, Gerbarumque insulam spectat, supina instar fluctum procumbit». Descrizione eccellente, che prova come Fazello avesse le sue informazioni da chi conosceva Lampedusa de visu.

Descrive poi il naufragio di 8 delle 15 galere di Carlo $V$, con più di 1000 uomini, comandate da Antonio Doria (Aurea) genovese, avvenuto ai tempi suoi (nel 1551) sulle coste di Lampedusa in seguito ad una terribile tempesta.

Di questa opera del Fazello esistono vario edizioni e traduzioni. In una edizione dedicata a Carlo $\mathrm{V}$ ed a suo figlio Filippo II, con 
titolo alquanto diverso (Fazellus frat. Thomas ordinis praedicatorum. Rerum Sicularum scriptores ex recentioribus praecipui, in unum corpus nunc primum congesti, diligentique recognitione plurimis in locis emendati. Francofurti ad Moenum apud And. Wechelum 1579) ed in una antica traduzione « in lingua Toscana 》 del P. M. Remigio fiorentino stampata a Venezia nel 1574, si trovano leggiere varianti nella scrittura dei nomi.

Gli scrittori posteriori hanno in generale ripetuto quanto ha scritto il Fazello.

Anania Gio. Lorenzo (d'). - L'Universale fabrica del mondo, ovvero Cosmografia. Appresso Andrea Muschio. Venetia, 1596.

A p. 316 è rammentata Lampidosa dove « arde continuamente una lampa avanti l' imagine di nostra Donna, ove s'afferma da molti non haverle mai mancato l'olio, rifondendovene sempre i nocchieri, che v'arrivano, o siano Christiani, o Mahomettani ». E più oltre dice: «poi segue Limosa, prima Ethusa».

Goltzius Hubertus. - Graeciae universae Asiaeque Minoris et insularum nomismata veterum. Antverpiae, 1618-1620.

A tav. 28 dellə isole, è figurata una moneta con testa di Giove da un lato e dall'altro un pesce coll' iscrizione $\Lambda$ OII $\triangle \triangle O \Upsilon \Sigma \Sigma A I \Omega N$; ed a p. 289 è detto $\Lambda$ OIA $\Delta O \Upsilon \Sigma \wedge I S N$. Lopadusa. In Africo mari non procul a Thapso Lopadusa jacet ignobilis insula quam Plinius longam dicit.

Goltzius Hubertus, - Siciliae ot Magnae Grasciae historia. Lugduni Batavorum et Francofurti, 1629.

Nella «Siciliae historia posterior» a p. 68 parla del naufragio descritto da Fazello delle 8 triremi di Carlo V, avvenuto nel 1551 sulle coste di Lopadusa, mentre l'ammiraglio Doria inseguiva il famigerato Dragù per cacciarlo dai mari, secondo l'ordine ricevuto dall' imperatore.

Astolfi Don Felice Canonico del Salvatore. - Historia universale delle immagini miracolose della Gran Madre di Dio riverite in tutte le parti del Mondo ecc. Venotia, 1623.

A p. 313 leggesi: "Trovasi vicino a Sicilia un Isoletta dishabi. tata, nomata Lampadusa, et appresso il mare in uno scoglio evvi alzata una cappelletta, dentro la qual' è riposta l' Imagine» (della Madonna di Trapani), e ripete poi quanto ne ha detto Lorenzo d'Anania. 
Abela Commendatore Fra Gio. Francesco. - Desviwione di Mall con le sue antichiti, ed altre notitic. P. Bonacota. Malta, 1617.

A p. 247, pairlando della venuta dei Goti in Malta, Abela, commendatore e vicacancelliere dell'Ordine dei Maltesi, descrive e dà il facsimile di una iscrizione in caratteri gotici, alti un palmo, che egli trovo «nell'Isola Lapedosa » nel 1610 mentre era in crociera per quei mari sulla Capitana delle galere maltesi. Ai due lati dell'iscrizione si vedevano « due scudi d'arme di basso riliovo nella pietra, ch'haveano scolpiti nel campo cinque monti con una dentatura pel orlo dello scudo ». L'iscrizione suona cosi:

Bartolomeus de Marsara dictu ian Crassu

Capitaniu me feci fare Ani prima indictio.

Questa iscrizione scorgevasi «nella parte più sublime della facciata, che rimaneva in piedi d'un antico Castello, o Torre, se pur affatto non s'è rovinata».

E probabile che negli Archivi dei Cavalieri di Malta si trovino altre notizie interessanti per la storia di Lampedusa che doveva continuamente essere visitata dai bastimenti dell'Ordine.

Pagnozzi F. Francesco Maria da Pistoia prodicatore cappuccino. Maric trionfante con la pompa di una triplicata corona di stelle risplendenti ecc. ecc. P. A. Fortunati, Pistoia, 1655.

A p. 612 dice: "In un'Isola disabitata presso la Sicilia, degna di gran venerazione è la Madonna di Lampedosa, dağli stessi Turchi onorata, e riverita: come dicon le storie, e ne son assicurato da uno dei Caralieri di Malta i quali soli levar possone le limosine, che ivi ritrovano, portaudole alla S. S. Madonna di Trapani : a cui molto simile e la Madonna di Lampedosa, essendo pur questa, come quella, in forma di Statua, e d'alabastro, col bambino G. in braccio. Dore più volto si è veduto questo mixacolo, che se alcuno dell'oblazioni di questa benedetta Madonna rubba cos'alcuna, non può mai quindi allontanarsi il Naviglio, finche non sia fatta la restituzione: levandosi tempesta in mare, o simil' altro fortunio accadendo, ancorche un solo d'una squadra di galee fosse stato il ladro. » Ripete poi prass' a poco quanto aveva scritto l'Anania 59 anni prima.

Maggio P. D. Francesco Maria Cherico Regolare. - Vita e morte del venerabile P. F. Alipio di S. Giuseppe ecc. Ignatio de' Laznri. Roma, 1657.

Nel capitolo IX, narrando come le ossa del Padre Alipio furono trasportate da 'Tripoli a Malta e all' isola di Lampedusa, a p. 128-134: ha occasione di parlare di questa isola alla quale dir il nome che porta ancora oggi. Dopo avere citato lo versioni di questo nome presso alcuni scrittori antichi, riforisce in parte quanto no dissero Fazello, 
Anania, Astolfi e Pagnozzi. Riporta lo parole del Padre Pierantonio Spinello (De Festis ac 'Templis) il quale dice press' a poco lo stesso del Pagnozzi. Cita ancora il Padre Giovanni Rhó ne: Sabati (Esempio 94), Fra Giovanni Manno (Tstoria), Fra Giovanbattista Lezana (Annali), i quali con altri autori dicono che " la bellissima Statua della « Madonna di Trapani che fu scolpita in Cipro l'anno 730 e da Geru«salemme fu trasferita da alcuni Cavalieri Templarij della Citti di "Pisa, correndo tempesta il Navilio, che la portava, si salvò in «Lampedusa, e che di essere ivi'stata la Statua di Nostra Signora, «ne serba la memoria infino a oggi una piccola Chiesetta, cui anche «i Barbari sogliono venerare..... F. Finalmente riporta la iscrizione del 1653 che rammenta questo salvamento della Statua, iscrizione che trovasi su di un «bel quadro grande d'argento, con la detta isola » conservato nella Chiesa di Trapani. Questa iscrizione è interessante anche perchè ricorda la donazione di Lampedusa a un Caro. Vi si legge difatti che quel bel quadro d'argento è un ex voto offerto alla Madonna da «D. Giulio di Tomasi e Caro, Cava"lier di San Giacomo, Duca di Palma, e Signore della medesima "Lampedusa, essendo detta Isola, celebre per diverse Istorie e fa«vole di Poeti, stata conceduta dal Re Alfonso alla sua Fami"glia..... Il Maggio riporta inoltre $\mathrm{i}$ versi nei quali Ariosto parla di Lampedusa.

Secondo il Maggio, il nome di questa isola potrebbe derivare dall'esservi continuamonte accesa una lampada davanti l'immagine della Madonna (!).

Labbe Phil. et Cossorti Gabr. - Sacro sancta Concilia... Tom. VII ab anno 787 ad annum 847. Latetiae Parisiorum, 1671.

Nella lettera quinta di Leone III a Carlomagno (colonna 1116) il papa riferisce all' imperatore d'occidente le notizie avute dai Greci intorno alle loro guerre coi Saraceni, e fra le altre cose narra come i Saraceni con tredici navi approdassero all'isola detta Lampcdusa. Essendo sopraggiunte in esplorazione sette navi dei Greci (i quali avevano ottenuto il concorso di quelli di Gaeta e d'Amalfi) i Mauri odiati da Dio ne fecero prigionieri gli equipaggi e li uccisero. L'armata dei Greci non vedendo tornare quei loro esploratori si mosse tutta e coll'aiuto di Cristo i Greci trucidarono tutti quegli iniqui Mauri cosicchè non ne lasciarono vivo neppure uno. L'armata greca era comandata da un patrizio (o governatore) di Sicilia nominato dall' imperatore d'Oriente Michele, il quale regnó dall'811 all' 813. Amari (Storia dei Mussulmani di Sicilia vol. I p. 227-228) riforisce questo avvenimento all's13, e la lettera di Leone III allo stesso anno. 
Pacichelli abate Gio. Battista. - . Temorie de? Yingli per l' Europu

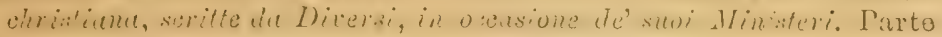

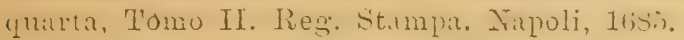

A p. 117 cita Limosa deserta, e di Lampadusa dice che ha una cappella in rucca consacrata alla Beata Vergime, o clio vi si trova tutto quanto abbisogna "al rinfresco di un vascello, como sarebbo a dire del biscotto, corde, ancore, legname, ferri, vele, seta, olio, vestiti ed altra robba di questo taglio, senza veruna guardia, le quali costumsi prendere, anche da' Turchi o qualsiasi Natione, giusta l'occorrenza, rimettendo nel sito l'equivalente, siccome ho lotto, e udite narrar nelle vicinanze, da più persone degne di f'ede ».

(Massa). - La Sicilia in prospsttiva. Parte seconda, cioè le Citlà, Castella, Terre, e Luaghii esistenti, e non esistenti in Sicilia, la Toporgrafia Littorale, li Siogli, Isole, e Penisole intorno ad essa. Esposti in veduta da un religioso dellu compagnia di Giesu. Decticutc all' illustrissimo Senato Palermitano. Stamp. F. Cichè. Palerno, 1709.

Nella parte intitolata : "Le Penisole, gli Scogli e l'Isole intorno ad essa con l'Etimologie, ed Historie più segnalate, e co' suoi nomi in uso appresso l'Autori Latini, e Greci, Antichi e Moderni ", « p. $4 \mathbf{i}$, dice cho le Isole Pelagie sono tre, Limpertusa, Lalenusa e Scola. Cita di nuovo Lampione a p. 489, sotto il nomo di Scuola (latimo Schala) cumo una delle tre Peligir. - Di Linosa si vede cho aveva una idea assai confusa. Infatti a p. 148 la cita sotto il nome di Labenusa (latino Labenusa o Lalenusa) e la cita di nuovo a p. 456 sotto il nome di Limosa (latino Ethusa) imbrogliandosi colle sinonimie di Ethusa, Egusa e Larunesia e facendo una confusione generale fra Favignaua (Egusa), Linosa (Ethusa) e Pantelleria. A p. 437 pexó avova rimproverato a Plinio di confondere Egrusa con Athusa dicendo correttamente che il primo nome si applicava a Favignana ed il secondo a Limosa (ossia Linosa). Che la sua Limosa sia proprio Linosa si rileva dai gradi sotto i quali la pone.

A Lampedusa dedica un lungo articolo da p. 448 a p. 455, in buona parto copiato dal Naggio. Rammenta prima $i$ varî nomi che gli sono stati dati dagli scrittori antichi e le etimologie proposto. Parla poi del naufragio delle galere di Antonio Doria nel 1551, dell' iscrizione trovata sul Castello d'Orlando dall'Abela che dice scritta in carattere gotico, ma con idioma latino, e siciliano corrotto, o cita, come il Maggio, i versi dell'Ariosto. Dice che l'isola è deserta, e ne di una breve descrizione. Fra lo sue calo ne rammenta una che era detta di Dragut perchè vi esistono pozzi scavati da quel famigerato corsaro. Descrive la grotta dove era la statua della Vergine, rispettata anche dai Maomettani, e si dilunga nell'enumerarne i 
miracoli. Da quella grotta si passava, egli dice, in un'altra dedicata al culto di Maometto. Racconta, come il Maggio, che vi si salvó la. famosissima statua della Madonna di Trapani, mentre veniva trasportata da Gerusalemme. Afferma, sull'autorità del Ventimiglia, che il danaro ofterto alla Madonna in Lampedusa ascendeva talvolta a 600 scudi, e che vi si trovavano anche monete turchesche, il che provava che anche quei Barbari tributavano offerte a Maria. Si ribella contro l'erudito Coronelli perchè questi ritiene essere ridicola superstizione dei Turchi che niuno potesse lasciare l'isola se avesse portato via qualche oggetto o denaro lasciato come oblazione alla Madonna, citando naufragi che ne furono la conseguenza anche col mare calmo. Nega pure che i Cavalieri di Malta andassero periodicamente a raccogliervi il danaro offerto alla Madonna per impiegarlo a pro degli infermi del loro spedale in Malta, come rorrebbe il Coronelli, ma dice che quel danaro i Cavalieri Maltesi 1o portavano a Trapani al convento dove si venera la Madonna di Trapani. Cita varî autori, specialmente ecclesiastici, in appoggio al suo dire. Nega infine quanto afferma il Coronelli, che riescisse " assai molesto il soggiorno in quell' isola per le spaventevoli visioni di fantasmi $\theta$ di spettri che si diano in tempo di notte a vedere».

Amico Vito M. - Lexicon topographicum Siculum. In quo Siciliae Urbes, Opida, cum diruta, tum extantia, Montes, Flumina, Portus, adjacentes Insulae, ae singula Loca describuntur, illustrantur. Tomus secundus, Pars prima. Catanae, 1759.

Linosa vi è semplicemente citata a p. 201 come una delle Pelagie sotto il nome di Lalenusa: A Lampedusa sono dedicate tre pagine (201-204). La dice deserta al tempo in cui scriveva (deserta omnino squallet) se pure non vi era un eremita, come per il passato, a custodia della Cappella della Vergine, cosa che egli confessa di non sapere. Parla dei ruderi del Castello, che egli dice chiamato «Torre d'Orlando », e costruito, secondo l'antica iscrizione trovatavi dall'Abela, da Bartolommeo di Marsala.

Descrive la cappella scavata nella roccia e consacrata alla Vergine, dalla quale si passa in altra cripta attigua dove i Turchi venerano il loro pseudo profeta. Il buon frate benedettino ci mostra. che non era diminuito il culto per la Madonna di Lampedusa, anche due secoli dopo l'epoca in cui per il primo ce ne parla Fazello. Esso difatti afferma ancora che questa Vergine fa innumerevoli miracoli anche pei Turchi, e che $j$ naviganti di tutte le nazioni, per assicurarsi un buon viaggio, le fanno delle ricche offerte in natura $e$ in danaro. Di quando in quando, egli dice, i Maltesi vengono a prendere questo danaro e lo portano religiosamente nel tempio della Vergine a Trapani. Nessun altro ardirebbe portar via da quel luogo nò danaro nò altro per timore della vendetta divina. - Parla del- 
l'occupazione di Lampedusa per parte dei Saraceni nell'anno $813 \theta$ della loro espulsione da quest' isola per il talasiarca crreco Gregorio.

Racconta poi di un combattimento navale fira Carlo Magno ed i Saraceni presso Lampedusa. La fiaba di questa battaglia navalo di Carlo Magno, di cui poi hinno parlato ancho altri, indotti in errore dall'Amico, ha avuto probabilmente origino dilla falsa inter. pretazione della lettera di Leone III a Carlomagno (vedi a Labbo). L'Amico rammenta poi il disastro marittimo dell'ammiraglio Antonio Doria sulle coste di Lampedusa nel 1551; e dopo avere parlato della donazione dell' isola a Giovanni Caro, enumera tutti i discendenti di questo cortigiano di Re Alfonso, che ebbero il dominio, almeno nominale, di Lampedusa fino al tempo in cui scriveva.

Si vede che l'Amico ha tratto una parte delle sue informazioni dal Massa, come questi trasse parte delle sue dal Maggio.

Torremuzza (Gabrial L. Castellus). - Siciliae veteres nummi. Typis regis. Palermo, 1781.

Sulla tavola 95 è riprodotta la moneta con testa di Giove da un lato e figura di pesce con $\Lambda O \Pi A \Delta O T \Sigma \Sigma A I \Omega N$ dall'altra, già figurata da Golzio. L'autore che dapprima si mostra alquanto scettico intorno alle affermazioni del Golzio, si ricrede nell'A uctarium del 1789 a p. 19, avendo egli stesso potuto acquistare una moneta somigliante a quella di Golzio su cui leggesi ancora chiaramente il principio dell'iscrizione $\Lambda$ OП . Questa seconda moneta è riprodotta a tavola VIII.

Hyacinthus Pater F. Carolus Carmelita excalcoatus, Botanicae Professor ecc. adjuvantibus Augustino Naudi, et Stephano Ze= rafa. - Plantae insularum Melitae, Gaulos, et Lampedosae. 1825. Sei pagine in $-8^{\circ}$.

La mia attenzione fu richiamata su di questo lavoro da quanto no scrive il Grech Delicata nella prefazione a p. xI della sua «Flora Melitensis $\%$. Dopo lunghe ed infruttuose ricerche finalmente, grazie alla somma cortesia dell'avv. conte Caruana Gatto botanico maltese, ho potuto vedere questo raro opuscoletto. Esso fu pubblicato dal Hyacinthus in appendice al suo lavoro intitolato: « Mezzo stabile di prosperiti per le isole di. Malta e Gozo », e le sue pagine portano la numerazinne da 37 a 42 . E un semplice clenco altubtico di 851 specie, fanerogame e crittogame, indigene ed esotiche, senza indicazione di localita, per cui è impossibile rilevare da esso quali siano le piante spontance e coltivate delle isole Maltesi, e quali quelle di Lampedusa. E noppure nel lavoro in cui quest'elenco figura come appendice, è data alcuna informazione in proposito. Solamente dal Grech Delicata, 1. c., approndiamo che in questo catalogo sono contenute 
117 specio di Lampedusa di cui 15 particolari a quest' isola o 102 comuni ad essa o alle isole Maltesi. Come Grech Delicata abbia fatto a saperlo non è detto. Nè l'autore del catalogo, nè Grech Delicata dicono da chi fossero raccolte quelle piante. - Hyacinthus, in italiano Giacinto, era genovese, e fu professore di botanica all'Universiti di Malta. ${ }^{1}$ Uno dei snoi colluboratori, Zurata o Zursha, sli successe nella cattedra di botanica a Malta.

Smith cap. William Henry. - Memoir desiriptive of the resources, inhabitants and hydrography of Sicily and its istands, interspersed with antiquarian and other notices. J. Murray. London, 1824. (Pag. 281-289 e xxiv-xxxv).

L'Autore, che come comandante della Hottiglia inglese nelle acque della Sicilia, ebbe occasione di visitare più d'una volta Linosa, Lampedusa o Lampione, nel libro destinato ad illustrare il suo atlanto della Sicilia pubblicato dall' Ammiragliato inglese, descrive queste isolo e dà alcune notizie storiche su di esse.

Colucci Salvatore. - Delle isole Pelagis ed in parlicolare di quella nominata Lampechusa, relativamente al progetto di convertivla per luogo di deportazione. Manoscritto, 1823?

Questa memoria venne adoperata da Calcara il quale ne parla nella «Descrizione di Lampedusa» a p. 3, e ci fa sapere come il Colucci visitasse Lampedusa nel Luglio 1828, $\theta$ la sua memoria manoscritta venisse conservata nell'Archivio della gran Corte dei conti.

Gussone cav. D. Giovanni. - Nutizie sulle isole Linosa, Lampione e Lampedusa, e descrizione di una nuova spesie di Stapelia che trovasi in questa ultima; lette nell'anno 1Sš2. Atti della Reale Accademia delle Scienze, Sezione della Societí Reale Borbonica, Vol. IV. Sezione Botanica. Napoli, 1839. (Pag. 74-97 con una tavola).

In questa classica memoria, oltre kd alcune notizie storiche, ad una tabella di osservazioni meteorologiche fatte in Lampedusa in Agosto 1828, e alla descrizione della nuova Stapelia, troviamo le prime informazioni un poco dettagliate sopra queste isole, e specialmente sulla loro geologia e la loro flora. E uno scritto magistrale di cui si può dire multum in parvo, ed è fonte alla quale hanno attinto molti che dopo di lui scrissero di Lampedusa e di Linosa.

\footnotetext{
' Saccardo, La botanica in Ilalia.
} 
Gussone Joannes. - Supplementum ad Florde Siculae Prodromum, quod et speximen florae insularum Siciliae ulteriori adjacentium. Regia Typographia. Neapoli. Fase. I 18:32; finc. II 1431. (Dalla Momandria-monogynia alla diadelphia-decandria). In-So di 203 pagine. '

Quando Gussone incomincio il suo « Florae Siculae Prodromus 》, non arera ameota visitato le isolette appartenenti allia Sicilia, né sapera di altru botanico, salvo La Billardiere, che vi fosso stato, e quindi non ne fia alcuna menzione in quel lavoro. Per questo, dopo compiuto il suo viaggio marittimo, si accinse a pubblicare il «Supplementum " lestinato precipuamente, como egli avvorte nella prefazione, ad illustrare la flora di quelle isolette, fra cui sono comprese Linosa, Lampedusa e Lampione. Egli non ultimò quel. Supplemento probabilmente perchè si era deciso a pubblicare la «Synopsis » nella quale sono date le indicazioni di tutte le località della Sicilia e dello isolette ad essa appartenenti. Lo indicazioni riguardanti le isole Pelagie si trovano riportate nella «Synopsis», il cho rende inutile la consultazione del «Supplementum».

Amari Emerico. - Calcara, nella «Descrizione di Lampedusa » a p. 45, cita l'Amari, segretario di una Commissione governativa, come autore di «Un progetto di colonizzazione che meglio potrebbe convenire nella isola di Lampedusa e sue adiacenze », pubblicato nel fascicolo 16 del $1^{\circ}$ quadrimestre del $18 \pm 1$ del giornale di Statistica. Non ho potuto vedere questo progetto.

Gussone J. - Florae Siculae S'ynopsis exhibens plantas vasculares in Sicilia insu'isque adjacentibus huc usque detectas secundum systema linneanum dispositas. Typ. Tramater. Neapoli. Vol. I 1842 ; Vol. II 1843.

Sono citate tutte le specie da Gussone stesso raccolte a Lampedusa, Linosa e Lampione.

Tinéo Vincenzo. - Plantarum rariorum Siciliae minus cognitarum. Fasciculi 2 e 3. Palermo, 1846.

Vi sono descritte tre piante di Lampedusa $\theta$ di Linosa raccolte da Calcara, due como nuove per la scienza: Daucus Lopadusamus (Lampedusa), Castellia tuberculata (Linosa), ed una come nuova per l' Italia: Échinops spinosus L. (Lampedusa). A p. 19-20 (fasc. II) in nota sono date alcune notizie del viaggio di Calcara alle Pelagie.

\footnotetext{
'Ilo potuto consultaro questo lavoro assai raro grazie alla cortesia del prof. Delpino che mi ha mandato l'esemplare della biblioteca universitaria di Napoli.
} 
Calcara Pietro. - Rapporto del viaggio scientifico eseguito nelle isole di Lampedusa, Linosa e Pantellaria ed in altri punti della Sicilia. Stamp. di R. Pagano. Palermo, 1846. In-160 di 32 pagine. ${ }^{1}$

In questo breve Rapporto alla Commissione di pubblica istruzione ed educazione di Sicilia, le prime 16 pagine sono dedicate a Lampedusa ed a Linosa. A p. 10 dice che « Lampedusa offre poche piante spontanee e che per conseguenza la sua flora deve reputarsi la più povera di quante isole trovansi presso la Sicilia, il che è manifesto indizio della steriliti di quel suolo ». Del resto tutto quanto vi dice è poi ripetuto nelle due monografie dedicate a quelle isole.

Calcara Pietro. - Descrizione dell' isola di Lampedusa. Stamp. R. Pagano. Palermo, 1847.

In questo opuscolo di 45 pagine, accompagnato da una carta dell'isole a grande scala, trovasi, da p. 22 a p. 30, la Florula di Lampedusa. Ti sono contenute inoltre variate notizie sull' isola, come lo indicano i titoli dei 15 capitoli in cui quel lavoro è diviso: I Geografia fisica; II Meteorologia; III Idrologia; IV Orittognosia; V Paleontologia; VI Geognosia; VII Geogonia; VIII Florula Lopadusana; IX Geografia botanica; X Boschi; XI Agricoltura; XII Animali invertebrati; XIII Animali vertebrati; XIV Malattie dominanti; XV Storia civile. - 亡 il lavoro che in compendio fornisce il maggior numero di notizio sopra Lampedusa, e ad esso hanno attinto tutti quelli che ne scrissero dopo Calcarr.

Parlatore Filippo. - Flora Italiana ossia descrizione delle piante che crescono spontanee o vegetano come tali in Italia $e$ nelle isole ad essa aggiacenti disposte secondo il metodo naturale. Firenze. Vol. I-V, 1848-1872. Continuata da Teodoro Caruel con varî collaboratori. Vol. VI-X, 1883-1893.

Sono citate tutte le piante di Lampedusa e di Linosa note all'epoca della stampa dei singoli volumi.

Sanvisente B. - L' isola di Lampedusa eretta a colonia dal munificentissimo nostro Sovrano Ferdinando II, descritta dal cav. B. Sanvisente capitano di fregata e governatore della medesima con un cenno sulle minori isole Linosa e Lampione. R. Tipografia Militaro. Napoli, 1849. In-40 di pagine 124.

L'Autore, capitano di fregata della marina napoletana, fu mandato nel 1843 a Lampedusa come Governatore dell' isola per installarvi una colonia. La dedica a Ferdinando II è datata del Dicem-

\footnotetext{
- Opuscolo assai raro che ho potuto leggere grazio alla cortesia del prof. Delpino che me lo ba inviato dalla biblioteca della Universita di Napoli.
} 
bre 1847. Il lavoro è diviso in 10 capitoli: I Geografia fisica; II Met9orologia; III Geologia; IV Botanica; V Agricoltura; VI Zoologia; TII Stato politico; VIII Immegliamenti; IX Storia civile; $\mathrm{X}$ Conclusioni. Una breve appendice è dedicata a Linosa e Lampione. È accompagnato da una cartina per mostrare la posizione delle Pelagie rispetto alla Sicilia, all'Africa e a Malta, e da una carta di Lampedusa all' $1: 24,000$ con una pianta particolare del porto a $1: 10,800$.

La divisione del lavoro è quasi identica a quella della memoria di Calcara, come molto simile è il contenuto dei singoli capitoli, in modo da far credere che l'uno abbia avuto sott'occhio il lavoro dell'altro. Stando alla data della dedica, i due lavori sarebbero scritti lo stesso anno. Quello di Sanvisente peró fu stampato due anni dopo quello di Calcara. Sembrerebbe che per le notizie topografiche, politiche - e storiche Calcara avesse parafrasato il Sanvisente, mentre per la storia naturale è chiaro come questi, che non era affatto naturalista, si sia giovato (malamente) dello scritto di Calcara, o abbia attinto alla medesima fonte. L' elenco delle piante, salvo lievissime differenze, non solo è uguale, ma si trovano spesso nell'uno e nell'altro gli stessi errori nella scrittura dei nomi. Quello di Sanvisente peró contiene maggior numero d'indicazioni di localiti, e vi sono citate alcune piante coltivate che mancano nella florula di Calcara. Tutto ciò rende difficile a capire la genesi di questi due lavori i cui autori non si citano mai l'un l'altro. Non sarei alieno perciò dal credere che la florula non fosse scritta vè da Calcara nè da Sanvisente, ma da Tineo sui materiali raccolti da Calcara, e che il manoscritto di Tineo fosse adoperato quasi contemporaneamente dai due che figurano come suoi autori. Se cosi fosse, Calcara avrebbe soppresso una parte delle località ed alcune specie coltivate. Sanvisente ha poi aggiunto a molte pianto il nome volgare e gli usi, tratti da opere generali, o quindi di nessun interesse per Lampedusa.

Sanvisente dà delle tabelle meteorologiche per gli anni 1846 e 1847. Quelle termometriche, che vanno dal 20 Giugno al 31 Dicembre 1847, darebbero una massima di $23^{\circ}, 4 \mathrm{R}$. in Agosto, e una minima di $8^{\circ} \mathrm{R}$. in Dicembre. Senonchè queste osservazioni sembrano affatto inattendibili, poichè indicano una uniformità di temperatura nelle varie ore del giorno e nei varî giorni d'ogni mese quali in nessun luogo si riscontrano. Le poche osservazioni fatte da Gussone nel mese di Agosto 1828 e da Calcara nei mesi di Maggio e Giugno del 1846 indicano delle escursioni diurne ben maggiori, a maggiori difierenze fra le temperature dei varî giorni.

Calcara Pietro. - Desorizione dell'isola di Linosa. Stamp. P. Marvillo. Palermo, 1851.

Questo opuscolo di 30 pagine è diviso in capitoli press' a poco come la descrizione di Lampedusa. La « Florula di Linosa» cho ne 
i il cap. IIII, occup le pagine 20-23. Il litoro é accompaguato ta una carta geologica di Linosa.

Amari Michele. - Storia dei Mrusutmani di Sicilia. Tolume primo. Firenze, 1854.

A p. 128, parlando delle scorrorie dei AIusulmani, dice che questi nell'813, approdarono a Lampedusa con tredici legni; oppressero sette legui sottili mandativi dal patrizio di Sicilia ad esplorare, e uccisero le ciurme; so non che venuto il grosso dell'armata bizantina, furono a lor volta sopraffatti i Musulmąni, $\theta$ passati a fil di spada. (Tolto dalla lettera di Leone III a Carlo Magno, del 7 Settembre. - Vedi a Labbe).

Pasquale Giuseppe Antonio. - Documenti biografici di Giovanni Guszons Botanico napolitano tratti dalle sue opere e specialmente dal suo erbario. (Memoria letta all Accademia nella tornata del 15 Gennaio 1871). Atti dell'Accademia Poutaniana, vol. X. Na. poli, 1876. Pagine 99.152.

A p. 113-114 si parla più specialmente delle visite di Gussone alle isole di Lampedusa, Lampione e Linosa, $\theta$ vi è detto che talvolta si vede nell'erbario di Gussone l'antografo del Porcari, viaggiatore e collettore, che spediva a Gussone piante di Lampedusa.

A p. 134-35 è detto che dalle etichette del suo erbario si rileva che Gussone fu nel Luglio 1828 a Lampedusa col Porcari e nell'Agrosto a Lampione. Pare che sulle etichetto non siano mai riportate date piu precise. Dal 13 al 18 Maggio 1829, Gussone fu in Pantelleria.

D'Albertis Enrico. - Crociera del "Violante 》 durante l'anno 1876. Aunali del Museo Civico di Storia nat. di Gonova, vol. XI, 1877. Estratto. Genova, 1878.

A proposito di una fermata di poco più di un'ora in Linosa (14 Settembre 1876) e di poco più di un giorno in Lampedusa (1516 Settembre), l'autore dà una buona descrizione di queste isole ed alcuni cenni sulla loro storia (p. 225 a 241). A p. 233 sono citate alcune alghe raccolte in Lampedusia (redi Piccone). A p. 234 vi è una veduta del porto di Lampedusa.

Avogadro di Vigliano E. - Lampeduse. Napoli, 1880.

Non ho potuto procurarmi questo lavoro, che conosco solo per averlo visto citato da Lo Re, e nel Boll. della Società Geogr. ital. anno XV 1881 a p. 390, ove è detto: «Brere descrizione geografica, economica, storica ed etrografic $r$ dell' isola ». 
Lo Re A. - Le condizioni economico-agrarie delle isole di LamñedusaLinosa, relazione a $S$. E. il Ministro di Ayn. Ind. e Comm. Sicilia agricola, anuo III n. 6 e seg. Palermo, 1885.

Questa relazions è datata da Girgenti 27 Marzo 188t. Le prime 26 pagine sono dedicate a Larnpedusa. Vi sono acerbamente criticate le relazioni di altri sopra lo stesso argomento. L'autore dà il buon consiglio di mandare come coatti a Lampedusa dei contadini e specialmente dei viticultori, ritenendo che la vite sia li pianta piu rimuneratrice in quei terreni. Raccomanda di mettere un freno al diboscamento e dì altri consigli e suggerimenti per il bene economico dell' isola.

Le 9 pagine seguenti sono dedicate a Linosa. Da esse si rileva che ancora nel $183 \pm$ una parte dei coloni abitava nelle grotte scavate nel tufo. In fondo a questa memoria vi è un catalogo di piante intitolato «Flora di Lampedusa e Linosa il quale non è altro che una trascrizione della florula di Lampedusa (e non di Linosa) del Calcara.

Piccone Ant. - Tisultati algologici delle crociere del « Violante 》. Annali del Museo civico di St. Nat. di Genova, vol. XX, 1881.

Sono citate le 18 specie di alghe marine raccolte dal cap. D'Albortis a Lampedusa nel 1876. - (Erano già stato citato dallo stesso autore in altre memorie pubblicate negli stessi «Annali » e nelle Memorie Sc. fis. mat. nat. della R. Accademia dei Lincei, ser. $3^{a}$, rol. IV, p. 34).

Speciale S. - Ise isole Pelagie, nota preliminare (con una tavola). Bollettino del R. Comitato Geologico d'Italia, Serie II vol. V, p. 161 -166. Roma, 1884.

Sono cenni geologici sulle isole di Lampedusa e di Linosa visitate dall'Autore. Questi non potè recarsi a Lampione. Sono citati alcuni larori in cui parlasi delle Pelagie.

Solla Rüdiger Felix. - Esterraichische botanische Zeitschrift 1884, Juni, p. 232-31.

Una lettera datata da Messina 5 Maggio, nella quale descrive il paesaggio botanico di Lampedusa e di Linosa.

Ross Hermann. - Eins botanische Excursion nach den Inseln Lampedusa und Linosa (eingegangen am 25 Juli 1884). Berichte der doutschen botanischen Gesellschaft; anno 1884, p. 344-19.

Brovi osservazioni sulle due isole ed elenco delle piante più interessanti raccolte dall'autoro. 
Solla Ruidiger Felix. - Plyytolyyologische Beobachtungen auf einer Eixsur. sion nach Lampedusa und Linosa (vorgelegt in der Versammlung am 5 November 1884). Verhandlungen der K. K. Zoologischbotanischen Gesellschaft in. Wien, Jahrgang 1884, p. 465-180.

Le prime 7 pagine sono dedicate alla descrizione di Lampedusa e di Linosa specialmente dal punto di vista botanico. Nelle 9 pagine seguenti sono enumerate le piante raccolte nelle due isole messe in prospetto, ed è indicato se trovate in fiore, frutto o con sole foglie. Alle fanerogame seguono alcuni licheni che furono determinati dal dott. Jatta.

Lojacono M. - Una escursione botanica in Lampedusa, datato $\mathrm{Pa}$ lermo Settembre 1884. Naturalista Siciliano, anno III 1883-84, p. 339-343 e anno IV 1881-85, p. 40-44, 92-96, 105-109, 133-139.

Nelle 14 prime pagine l'Autore descrive il suo viaggio e dà notizie generali sulle due isole e sulla loro flora. Le 14 seguenti contengono il "Catalogo delle piante vascolari di Linosa e Lampedusa ».

Solla Rüdiger Felix. - Auf einer Excursion nach den pelagischen Inseln, April 1884, gesammelte Meeresalgen. Esterreichische botanische Zeitschrift, Jahrgang 1885, n. 2.

L'Autore dà in 7 pagine una breve descrizione della costiera delle due isole, ed un elenco delle alghe marine che vi ha raccolte e che sono state determinate dal dott. Ferd. Hauck.

Trabucco G. - Quadro dei terreni ed elenco delle rosce della provincia di Girgenti. Como, 1889.

Contiene notizie geologiche sulle isolo di Linosa e di Lampedusa.

Trabucco Giacomo. - L' isola di Lampedusa. Studio geo-paleontologico. Roma, 1890 (opuscolo di 38 pagine estratto dal Bollettino della Società geologica italiana, vol. IX, 1890, fasc. $3^{\circ}$, p. 209 e seg.).

È un accurata studio geo-paleontologico di Lampedusa che l'Autore visitò nel Maggio del 1888, preceduto da indicazioni bibliografiche, notizio storiche, descrizione fisica, e qualche conno sulla flora, sulla fauna, sull'agricoltura e sul commercio dell' isola. La memoria è accompagnata da una tavola con figure di conchiglio fossili, da una cartina del Mediterraneo fra la T'unisia e la Sicilia, con una sezione indicante le profondità del mare fra la Tunisia, Lampedusa, Malta e la Sicilia, e da una carta geologica di Lampedusa all'1 per 50,000 . 
Nicotra L. - Solhedule speciografiche riferentisi alla flora siciliana. Il Naturalista siciliano, X. Palermo; 1890.

A p. 67 l'Autore dice di avere ricevuto piante di Linosa e Lampedusa dal barone Zwierlein di cui non dà l'elenco perchè trovansi citate nei lavori di Solla e di Ross, e perchè molte delle piante dello $Z$ wierlein avendo perduto $\mathrm{l}^{\prime}$ indicazione di localita non si sa se debbano riferirsi alle dette isole o alla Sicilia meridionale. Fra queste piante d'incerta provenienza evvi una nuova varieti Zwier. leinii della Plantago Psyllium L. di cui è data la descrizione (p. 67-68).

Nicotra Leopoldo. - Elementi statistici della Flora siciliana. \$10. Fatti salienti che riguardano l'abitazione. N. Giorn. bot. it., vol. XXII. Firenze, 1890.

A p. 520-21 l'Autore dì un prospetto delle specie esistenti nelle isolette appartenenti alla Sicilia e mancanti sull' isola stessa. Secondo quel prospetto Lampedusa possederebbe 19 di queste specie, di cui 5 sole si trovano anche in altre di quelle isolette, rimanendo 14 specie che figurano nella Flora siciliana unicamente per la loro presenza in Lampedusa. - A Linosa sono attribuite 7 specio mancanti al continente siciliano, di cui 4 si trovano anche in altre isolette appartenenti alla Sicilia. - A p. 522 osserva che Lampedusa è l' isoletta appartenente alla Sicilia la più ricca di specie non rappresentate sul continente siciliano.

Lojacono Pojero M. - Flora Sicula o descrizione delle piante vascolari sponianee o indigenate in Sicilia. Palermo. Vol. I parte $1^{\mathrm{a}}$ 1888, vol. I parte $2^{a} 1891$, vol. II parte $1^{a} 1902$.

$\mathrm{Vi}$ sono citate, in parte, le piante raccolte in Lampedusa $\theta$ in Linosa dallo stesso Lojacono e da altri.

Trabucco Giacomo. - L' isola di Linosa. Studio geologico. Nota preliminare. Rassegna delle Scienze geologiche in Italia, anno I, fasc. 1-2. Roma, 1891.

Due pagine di descrizione e notizie geologiche.

Tellini. - Trabusco, L'Iso'a di Lxmpedusa. Rassegna delle Scienze geologicho in Italia, anno I, p. 127-28. Roma, 1891.

È una rassegna della memoria del prof. Trabucco sull' isola di Lampedusa. Sono rilevate specialmente le conclusioni cui giunge il Trabucco, cioè che Lampedusa e Lampione appartengono geologicamente e geograficamente all'Africa, che verso la fine del Miocene l'Africa si estendeva a settentrione verso la Sicilia assai più di adesso, che l'emersione delle due isolette coincido con quella della costa africana vicina, ed avvenne alla fine del Pliocene. 
Trabucco G. - Risposta ad alcune osservazioni alla nota « $L$ ' Isolu di Isampedusa ecc.». Boll. Soc. geol. it. Roma, 1892.

L'Autore, rispondendo al prof. De Stefani il quale aveva espresso l'opinione che il calcare di Lampedusa appartenesse al Miocene anzichè al Pliocene, insiste nella sua opiniono. Rileva nuovamente la concordanza stratigrafica fra Lamperlusa e la vicina costa d'Africa, e dice che tale concordanza non esiste con le isole Maltesi le quali appartengono al Miocene modio.

Tellini. - Trabucso, Risposta al aleune osservazioni ecc. Rassegna delle Scienze geol. in Italia, anno II p. 291-95. Roma, 1892.

In questo articolo polemico Tellini ribadisce gli appunti già mossi al lavoro del Trabucco sull' isola di Lampedusa. Sono indicate le profondita marine di quella parte del Mediterraneo secondo le migliori e più recenti fonti, che assegnano al mare fra Mehodîa $\theta$ Lampione una profondità massima di $55 \mathrm{~m}$. e fra Lampedusa e Malta di $962 \mathrm{~m}$.

Trabucco G. - L'isola di Linosa. Stritio geo-fisico. Atti del terzo Congresso geografico italiano tənuto in Firenze dal 12 al 17 Aprile 1898, vol. II, p. 148-162. Firenze, 1899. Ristampato in Rivista geografica italiana, anno VI, fasc. 2-3. Firenze, 1899.

I'Autore che visitó Linosa nel Maggio del 1888, ne dà una breve descrizione, nccenna alla sua storia, al clima, alla popolazione, alla flora, alla fauna, all'agricoltura, e si dilunga sulla sua costituzione e storia geologica. Cita cinque fossili potuti determinare mei tufi fossiliferi dell' isola. La memoria è accompagnata da due vignette, una carta grografica con sezioni indicanti le profondità del mare circostante, ed una carta geologica di Linosa all' 1 per 25,000; contiene pure indicazioni bibliografiche.

Il paragrafo dedicato alla botanica disgraziatamente non è attendibile, essendo errate tanto le citazioni delle piante quanto la loro statistica, per avere l'autore fatto confusione fra le florule di Lampedusa e di Linosa.

Ross Hermann. - Beitrüge zur Flora von Sizilien. Erlüuterungen und kritische Bemerkingen sum Herbarium Siculum, in Bull. de l'Herb. Boissier. Geṅ̀ve, I Teil, vol. VII n. 4 Avril 1899; II Teil, sec. série, n. 12, Décembre 1901 .

Sono note critiche o illustrative alle piante distribuite nel $\mathrm{Her}$ barium Siculum fra le quali trovansene alcune delle isole Pelagie. Nella prefazione (Teil I p. 266) vi sono alcune osservazioni generali sopra Lampedusa. 
Sommier S. - I'iunte indite di Intmpetusat e di Linosu. Istill. Soc. bot. it. 1!10) 1). 215. Firenze, 1!50\%.

Sono enumerate 20 specio li Linosa e 2) di Lampelusa racenlte da Ajuti e Sommier nel 1S:i, che non erano state ancorib publicate per queste isule.

Lanza D. - Note sulla Flora di Sicilia. Boll. R. Orto bot. di Palermo, anno IV. Palermo, 1905.

A p. 31 parla delli Silene apetala Willd. raccolta a Linosa da Calcara fiu dal 1816, ma rimasta fin' ora indetorminata.

Nicotra L. - Altri ragguagli sulie Fumarie italiane. Atti e Rendic. Acc. Dafnica di scienze ecc. Acireals, 1905.

Contiene alcume osservazioni sopra Fumarie di Lampedusa e di Linosa.

Lojacono Pojero. - Addenta et emendanda ad Floram Siculam. Malpighia XX p. 37. Genova, 1906.

Contiene qualche osservazione su piante di Lampedusa.

INDICE DEGLI AUTORI CITATI.

\begin{tabular}{|c|c|}
\hline & \\
\hline F.... & gio P. D. F. M. . . . \\
\hline$\ldots \ldots$ & sa ..... \\
\hline$\ldots \ldots$ & A. (v. Hyacinthus). \\
\hline м. . . . . . . . & ra L. . . . . 27, \\
\hline a G. L. $\left(d^{\prime}\right) \ldots$ & ab. G. B. \\
\hline 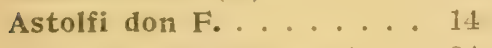 & I F. F. M. . . \\
\hline di Vigliano E. . . & 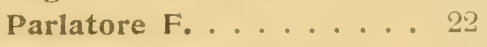 \\
\hline ...... 22 & e G. A......... \\
\hline$\ldots \ldots$ & A. .......... \\
\hline v. Labbe). & \\
\hline T. ....... & . . \\
\hline$\ldots \ldots$ & . W. H. ... \\
\hline Hyacinthus). & \\
\hline$\ldots \ldots$ & ;. . . . \\
\hline G... :20, & S.... \\
\hline$\ldots \ldots$ & 27 , \\
\hline t Cossorti G. . & $\ldots \ldots$ \\
\hline$\cdots \cdot$ & muzza ... \\
\hline jero M. 26,27 , & \\
\hline . . . & v. Hyacinthus). \\
\hline
\end{tabular}




\section{LAMPEDUSA}

NOME E STORIA.

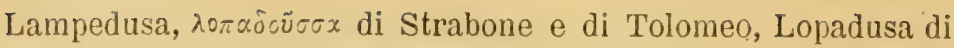
Plinio, è la maggiore delle tre Pelagie. Essa ha mutato assai poco il suo nome attraverso ai secoli, poichẻ tutti quelli coi quali la troviamo menzionata da allora in poi, non sono altro che variazioni piủ o meno libere su di un medesimo tema, o semplici errori di trascrizione. ${ }^{\prime}$

Qualche ingegnoso ricercatore di etimologie ha preteso che quel nome venisse dalla frequenza dei lampi che vi si osservano. Altri lo fece derivare da $\lambda \varepsilon \pi x \varsigma$ scoglio o $\lambda \varepsilon \pi \alpha \iota$ s roccioso. Altri ancora credette che i nomi di Lampedusa e di Lampione derivassero da $\lambda x \mu \pi x$, fiaccola, per essere stati collocati su quelle isole dei fanali come segnali ai naviganti. Kiepert ne trova l'origine in una parola fenicia che significa ardere, origine che egli giustifica dicendo che Lampedusa è essenzialmente vulcanica. ${ }^{2}$ A tale interpretazione vi è una sola difficoltá, ed è che di vulcani a Lampedusa non vi è traccia! Lojacono, dimenticando che l'etimologia di Lopadusa nón va ricercata nel latino poichè i Romani non fecero altro che modificare leggermente il norne

1 Sulle carte antiche e nei varî autori, spэcialmente nei commentatori degli antichi, troviamo queste ed altre varianti: Lopadosa, Lapadusa, Lapedosa, Lipidusa, Lipadusa, Lampedosa, Lampidusa, Lanbedusa, Leopadusa, Lepadosa ecc. Le variazioni più aberranti sono: Lampedola, Lepadula, Lampido e Lampas. I dizionarî geografici francesi la chiamano Lampadouse.

${ }^{2}$ Kiepert, Lehrbush der alten Geographie, Berlin, 1875. Lo stesso ripete EaLI, Nomina geographica. Leipzig, 1893. 
greco, lo fa derivare da lapidosa, aggettivo che in vero le si converrebbe assai !

Secondu Màssa, è stata proposta ancora l'etimologia dal nome greco di un pesce $\lambda \in \pi \times \delta \varepsilon \xi$ che vi si pescava in abbondanza. Questa etimologia fu forse suggerita dalle monete attribuite a Lampedusa, e sulle quali è rappresentato un pesce. " Finalmente ricorderó che $\lambda \varepsilon \pi x \varsigma \lambda \varepsilon \pi x \hat{0} 0 ;$ era anche il nome dato dai Greci alle patelle, quel piccolo mollusco univalve che si trova abbondante sugli scogli marini di Lampedusa come in generale su tutti gli scogli dei nostri mari, per cui forse qualcuno ha sup-

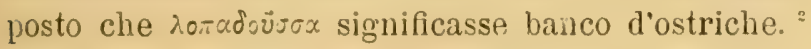

Gli autori che hanno scritto di Lampedusa rilevano che essa in antico fu abitata da colonie greche, romane, cartaginesi ed arabe. Lo provano anfore, lucerne, lacrimatoj e vasi di vetro, cripte sepolcrali, grotte ridotte ad abitazioni, cisterne, pozzi, avanzi di fabbriche con mosaici e monete di varie provenienze e di varî tempi dalle consolari romane alle arabo-turche. ${ }^{3}$ Le due monete con iscrizione greca illustrate dal Goltzius e dal Torremuzza, sembrano dimostrare il conto in cui quest' isola era tenuta. Vi sono vicino al porto delle spaziose catacombe romane, in parte adoprate ora come magazzini, nelle quali anche quest'anno ho trovato delle ossa umane, ed ho visto che vi erano tuttora delle sepolture intatte; ed ancora oggi quando si lavora la terra si trovano frequentemente avanzi, specialmente dell'epoca romana.

È probabile che Lampedusa, a causa della sua posizione, aresse una particolare importanza all'epoca delle guerre puniche, quantunque nessuna memoria storica di quel tempo ne sia rimasta. Di essa del resto tace la storia fino al principio del secolo Ix. Strabone dice soltanto che è situata in alto mare di

1 Vedi Golzio, in billiografia.

= J. J. EGLI, Nomina geograplica. Nigro (D. M. Nigri Veneti Geogr. Comm. Basileae, 1557), p. 363 dice « Lepadusa ...... insula a patellis dicta ostreorum genere ».

${ }^{3}$ SAnvisente (p. 105) dice di avere raccolto in Lampedusa o spedito al Duca di Cumia delle monete Siracusane, Agrigantine, Romane Consolari ed Impэriali, Arabe, Turche, Veneziane, Francesi o Maltesi. 
faccia alla citti africana di Thapsos. Plinio, Hist. Nat., la cita nel libro III e nel libro V; ma tutto quanto ne sa è che dista circa 50 miglia da Cercina e Cercinitis (oggi isole Kerkena) ed è lunga 6 miglia. In Tolomeo si trova semplicemente citata fra le isole dell'Africa. Da una lettera del Papa Leone III a Carlomagno" sappiamo che nell' 813 in Lampedusa sbarcarono i Saraceni e vi trucidarono gli equipaggni di sette galere delI'Impuatore d'Oriente, erl alla loro rolta furono poi passati a fil di spada dal grosso dell'al'mata greca. ${ }^{2}$

Questo brano di storia illumina di una fosca luce il passato di Lampedusa. Chi sa quante rolte simili scene di sangue si salanno ripetute nei molti secoli durante i. cuali i Saraceni infestarono il Mediterraneo. Eppure è probabile che per l'estensione discreta dell'isola, la coltivabilitá del suo suolo e la ricchezza in pesci delle sue acque, vi si siano stabilite, forse anche piu volte, nei periodi di tregua, delle colonie di pacifici agricoltori e pescatori venuti dalla Sicilia, da Malta o dalla vicina costa d'Africa. Na se cosi fu, queste colonie certamente saranno state soggette alle razzie, e forse a distruzione completa da parte dei pirati, cosicchè Lampedusa puỏ essere stata a varie riprese abitata e deserta. Le grotte che vi si vedono scavate nella roccia devono rimontare a tempo molto antico, ma hanno probabilmente servito di ricovero agli uomini in varie epoche. Vi sorsero poi altre abitazioni, poiché Fazello nel 1558 dice che vi si trovava un oppicuulum abbandonato.

Dell'approdo in Lampedusa a diverse epoche di bastimenti di varie provenienze fanno fede le monete raccolte dal Sanvisente di cui ho fatto menzione sopra.

Nel 1436 pare si consilerasse Lampedusa coine appartenente al Regno d'Aragrona e di Sicilia, poichè in quell'anno Alfonso il

1 V. in bibliogr. a Labbe e Amari.

: Probabilmente é questa lettera scritta del Papa per informare Carlomagno delle sorti dei Cristiani d'Oriente che, male interpretata, ha fatto dire all'A mico (seguito poi da Gussone e da Calcara) che Carlomagno areva battuto i Saraceni a Lampedusa e si era impadronito di quest'isola. - E forse quel ricordo storico che ha dato all'Ariosto l'idea di scegliere Lampedisa come teatro della tenzone fra Agramante e Orlando. 
Magnanimo la concesse con tutti i poteri baronali, e il diritto di costruirvi una torre, ad un suo fido, Giovanni Caro di Montechiaro, dai cui discendenti passó alla famiglia Tommasi che oggi ancora conserva fra i suoi titoli quello di principi di Lampedusa. ${ }^{1}$

Che effettivamente vi fu costruita una torre o fortezza, lo sappiamo da Fazello, il quale la dice gii abbandonata nel 1558; e da Fra Giovanni Francesco Abela, Commendatore dell' Ordine Maltese, sappiamo che quella torre venne eretta per ordine del Capitano Bartolomeo de Mar'sara detto Jan Crassu. Di questa fortezza, forse sari stato più volte contrastato il possesso dai mussulmani e) dai criatiani, specialmente dopo l'insediamento a Malta, nel 1530, dell' Ordine degli Ospitalieri di Gerusalemme, d'allora in poi detto di Malta. E questa probabilmente la torre che fu poi detta d'Orlando, e che è rammentata da varî scrittori. Delle frequenti visite, anche pacifiche, a Lampedusa dei Cavalieri di Malta, come dei Saraceni, fanno fede del resto molti scrittori che dal XVI al XVIII secolo parlano della Madonna miracolosa di Lampedusa di cui dirò in appresso.

Triste celebriti si acquistò più tardi Lampedusa per un memorabile naufragio avrenuto il 4 luglio 1551 sulle sue coste. Raccontano varî autori come, essendo allora Andrea Doria comandante in capo delle forze navali di Carlo Quinto, con incarico speciale di liberare il mare dal famigerato ammiraglio turco Dragut, una parte delie galere imperiali al comando di Antonio Doria ${ }^{2}$ fu sorpresa da una furiosa tempesta nelle acque di Lampedusa, ed otto triremi gettate a riva vi si infransero, venendo a perdere la vita più di mille uomini.

Il frate Fazello che narrava questo avvenimento nel 1558, dice di Lampedusa che, al pari di Linosa, era allora deserta.

1 Il Maggio riporta una iscrizione del 1653 conservata nella chiesa di Trapani, in cui Don Giulio di Tomasi e Caro rammenta quella donazione del $R e$ Alfonso alla sua famiglia.

${ }^{2}$ Alcuni degli scrittori che parlano di questo naufragio confondono i due Genovesi Antonio e Andrea Doria. Antonio Doria, quantunque meno celebre di Andrea, si distinse esso pure in vario guerre e fu anche Vicerè di Sicilia. Nel 1551 era al servizio di Carlo V, sotto gli ordini di Andrea Doria. 
Appare dunque fantastica la notizia data da Sanvisente (senza citare la sua fonte), che cioé il famigerato Dragut traesse, nel 1553, dalla popolazione allora vivente in Lampedusa, mille schiavi, poichè Fazello non avrebbe mancato di narrare un fatto cosi grave avvenuto a tempo suo. Anche Ariosto doveva avere sentito dire che Lampedusa era deserta, poichè tale la dice, descrivendola come arena della tenzone fra tre Saraceni ed altrettanti Cristiani. Deserta ancora la dicono il Pacichelli nel 1685, il Massa nel 1709, il Torremuzza nel 1781.

Tuttavia l'affermazione del Pacichelli, che vi si trovasse ogni genere di merce per i bastimenti, senza alcuno a custodirla, e che onestamente venisse pagata da chi approdando all' isola ne prendeva quanto gli abbisognava, appare immaginaria, poiché un tale scambio di merci senza contrattanti o commercio muto, sembra impossibile in una isola esposta alle continue visite di pirati e di naviglio belligerante.

Notizie certe sulla popolazione dell'isola abbiamo soltanto dal 1776 in poi, quando Ferdinando IV ne tentò la colonizzazione." Quei primi coloni pare che fossero decimati dalla peste pochi anni dopo. Gussone e Calcara parlano di lapidi trovate presso il porto su cui leggesi: "Qui ritrovasi un cadavere morto di peste in giugno 1784. »Due di queste lapidi con identica iscrizione ho ancora vedute quest'anno, una delle quali trovata recentemente nel fare uno scavo. Nel 1800 vi stabili una piccola colonia di agricoltori il Maltese Salvatore Gatt. Per concessione di questo, pochi anni dopo vi fondó uno stabilimento agricolo più importante l'Inglese Fernandez, portandovi tre o quattrocento persone. Allora, nel 1810, fu cominciato a costruire l'attuale rozzo castello, nel posto dove eranvi quattro antiche torri in cattivo stato. Ma questo tentativo di colonizzazione al pari degli altri falli, e quando il capitano Smith visito Lampedusa verso il 1820, vi trovó 12 o 14 contadini Maltesi sparsi per l'isola, ove dimoravano entro grotte, e la famiglia del sig. Fernandez, che abitava in una casetta, senza alcuna protezione contro i predoni o i bastimenti infetti che suolevano farvi scalo. Quando Gussone visitó

${ }^{1}$ Secondo Calcara e Sanvisente, anche avanti, nel 1760, vi si sarebbero stabiliti sei francesi con un prete. 
Lampedusa nel 18:S, la trovo abitata soltanto da 24 Maltesi che vi conducerano, vita assai stentata.

La colonizzazione in regola di Lampedusa per parte del Governo delle due Sicilie rimonta solo al 1843 , quando ne prese possesso il capitano di fregata Sanvisente in nome del Governo, previo accordo coi principi di Lampedusa e colla famiglia Gatt. All' epoca in cui Sanvisente scrisse il suo lavoro sopra Lampedusa, cioè nel 1847, l'isola contava 700 abitanti, tutti oriundi di diverse parti della Sicilia ed isole adiacenti, segnatamente di Pantelleria.

Nel 1847 Ferdinando II visitó Lampedusa, facendo sperimentare la sua munificenza a tutti, come ce ne informa Sanvisente « e vantaggiando a preferenza la classe delle nubili onde incoraggiare e favorire i matrimonî ».

Nel 1872 vi fu dal Governo italiano impiantata una colonia di condannati a domicilio coatto, che esiste tuttora. All'epoca in cui visitai Lampedusa per la prima rolta, cioè un anno dopo, ri erano circa 900 abilanti liberi, 200 coatti ed un piccolo presidio. In oggi la popolazione libera è di circa 2000 abitanti, ed i coatti sono quasi 500. Il presidio conta 70 uomini.

Il posșesso di Lampedusa, per ragioni strategiche, pare che venisse ambito dalla Russia sotto Caterina II, e durante le guerre Napoleoniche dall' Inghilter'a.

Del dominio del folkilore è la storia, o leggenda, dell'Eremita di Lampedusa. Secondo essa abitava in antico in quest'isola un eremita opportunista, il quale aveva arredato in modo diverso due tempietti contigui, consacrati l'uno a Cristo e l'altro a Maometto. Quando scorgeva una vela all'orizzonte, la seguiva con occhio vigile ed appena si era accertato se portasse la mezzaluna o la croce, chiudeva uno dei suoi luoghi di culto, e si preparava ad ufliziare nell'altro per ingraziarsi l'equipaggio nel caso che il bastimento aresse approdato in Lampedusa. Questo ha dato origine al detto comune in Sicilia « fare come il romito di Lampedusa », che vale quanto sapersi adattare alle circostanze.

Più conosciuti peró di questa leggenda sono oggi la Madonna di Lampedusa ed i miracoli da essa fatti. Tutti gli isolani samo raccontare, e leggesi stampato su di immagini e preghiere illustrate, come un tale Andrea Anfossi di Castellaro in diocesi 
di Ventimiglia, tratto in schiavitả dai Turchi, fuggrisse da Lampedusa su di un tronco dalbero scavato, tenendo in mano come vela l'immagine della Madonna. Coll'aiuto di questa giunse salvo al suo paese in Liguria, dove la Madonna di Lampedusa è ancora oggi oggetto di un culto speciale e da dove probabilmente è venuta questa leggenda alla popolazione recente di Lampedusa.

Il fatto sta che, fin da tempo antico, esisteva in Lampedusa una cappella con una statua della Madonna. Molti sono gli autori, in specie ecclesiastici, cominciando dal frate Fazello nel 1558, che ne parlano, esaltandone le virtù miracolose, tanto che si puó dire che per due secoli Lampedusa fu conosciuta nella letteratura, più che per altro, per la sua Madonna miracolosa. Pare che quella statua fosse fatta ad immagine di quella di Trapani, perchè in Lampedusa si salvò da una tempesta il bastimento che trasportava questa statua della Mradonna da Gerusalemme a Trapani. Tutti quegli autori attestano che era tenuta in gran conto non solo dai Cristiani, ma anche dai Mảomettani, i quali spesso approdavano in Lampedusa. Secondo il Coronelli i Turchi, «acciecati dalla superstizione », deponevano sempre dei doni in quella Cappella, ed i Caralieri di Malta, «illuminati dalla rera fede », li portavano via a profitto del loro spedale, il che proverebbe che i Cavalieri di Malta erano meno scrupolosi dei Turchi e prendevano forse al tempo stesso le offerte fatte a Maometto e quelle fatte alla Madonna. Peró il buon religioso Massa scagiona i Cavalieri di Malta dall'accusa di avere adoprato i danari offerti alla Madonna per il loro spedale, affermando che li portavano invece scrupolosamente alla Madonna in Trapani. Pare che la superstizione impedisse ad altri di toccare quei doni, poichè varî autori attestano che chiunque, all'infuori dei Cavalieri Maltesi, avesse ardito involare cosa alcuna offerta alla Madonna, non avrebbe potuto lasciar l' isola senza fare tosto naufragio.

La chiesetta dedicata alla Madonna trovasi in Lampedusa nel Vallone da essa detto della Madonna, ed è in parte scavata nella roccia. Li presso vi sono altre grotte, anch'esse opera dell'uomo.

Secondo una leggenda, pure riferita da varî autori, di notte tempo si vedevano in Lampedusa spaventevoli apparizioni, per cui i bastimenti evitarano di passarvi la notte. 
A titolo di curiositả poi, riporto qui i versi coi quali l'Ariosto descrive Lampedusa: ${ }^{1}$
"Consenti il re Agramante, e di periglio
Useì, pigliando la spiaggia mancina,
Che per salute de' nocchieri giace
Tra gli Afri, o di Vulcan l'alta fornace."
". . . . . . . . Lipadusa
Una isoletta è questa, che dal maré
Medesmo che la cinge è circonfusa,
D' abitazioni è l'isoletta vuota,
Piena d' umil mortelle $\theta$ di ginepri;
Gioconda solitudine e remota
A cervi, a daini, a caprioli, a lepri:
E, fuor ch'a pescatori, ò poco nota,
Ove sovente a rimondati vepri
Sospendon, per seccar, l'umide reti :
Dormono intanto i pesci in mar quïeti, .

Se in questa descrizione vi è piủ poesia che veritá, ancora meno verita evvi nelle obiezioni che Ariosto dice essergli mosse da Fulgoso:

". . . . . . . l' isola si fiera,

Montuosa o inegual ritrovò tanto,

Che non è, dice, in tutto il luogo strano

Ove un sol piè si possa metter piano: ${ }^{2}$

poiché invece tutta l'isola è pianeggiante.

I ruderi che in Lampedusa portarono il nome, oggi dimenticato, di Torre di Orlando, secondo qualche commentatore hanno dato all'Ariosto l'idea di scegliere quest'isola come scena della pugna fra Agramante e Orlando. Ma se pure vi è una connessione fra quel nome e il poema dell'Ariosto, è piú probabile che il nome della torre sia conseguenza e non origine della finzione del poeta ferrarese. ${ }^{3}$ Difatti, nelle prime menzioni di quella torre o fortezza, quelle cioè del Fazello (1558) e dell'Abela (1647), non è detto che si chiamasse torre d'Orlando, e la trovo per la prima volta designata con tal nome dal Massa nel 1709.

1 Orlando furioso, XL, 44, 55 e 45.

2 Idem, XLII, 20.

3 Pro Rajia, Le fontidell' Orlando furioso, $2^{a}$ ediz., p. 557. G. Sansoni, Firenze. 
Ancora a titolo di curiosità rammenteró che il poeta Wieland, alla fine del secolo.XVIII, scrisse un poema nel quale narra come in Lampedusa si salvassero da un naufragio due signorine e due giovanotti di Palermo (Rosina e Clelia, Guido e Sinibaldo) con due loro ancelle, ed ivi trovassero due eremiti, e come da questi naufraghi e da quegli eremiti avesse origine la popolazione di Lampedusa. ${ }^{1}$

\section{DESCRIZIONE, PRODOTTI, CLIMA.}

Lampedusa è situata fra $35^{\circ} 29^{\prime}, 4$ e $35^{\circ} 31^{\prime}, 6$ Lat. N., e fra $0^{\circ} 3^{\prime}, 8$ e $0^{\circ} 10^{\prime}, 8$ Long. E. di Roma. È distante $205 \mathrm{Km}$. dalla Marina di Palma che è il punto più prossimo della Sicilia, e $113 \mathrm{Km}$. dal Capo Mehediah, punto più prossimo della costa d'Africa. Da Lampione dista $18 \mathrm{Km}$., da Linosa $42 \mathrm{Km}$., da Pantelleria $141 \mathrm{Km}$., dalle isole Maltesi (Gozo) $150 \mathrm{Km}$.

Ha forma quasi di triangolo isoscele, coi due lati uguali molto più lunghi del ter'zo. Il lato più corto è a Levante, da dove l'isola va gradatamente assottigliandosi verso Ponente. Il suo asse maggiore, che è quindi press' a poco in direzione Est-Ovest, è lungo $11 \mathrm{Km}$. La sua massima larghezza, che è verso l'estremitả orientale, è di $\mathrm{Km}$. 3,700. Il suo perimetro, tenendo conto delle sinuositá della costa, è di Km. 40. La massima altezza, misurata nel punto detto «Albero del Sole» sulla costa Nord-Ovest, è

1 Winland, Clelia und Sinibald oder die Bevöllerung von Lampeduse, in Kleinere Schriften. L'autore tedesco dice di trarre il suo racconto da un antico scritto siciliano. Lo Smith dal canto suo, senza rammentare Wieland, parla di una leggenda siciliana quasi identica. Non saprei dire quindi se sia Wieland che ha creato la leggenda, o se questa esistesse prima di Iui. Calcara e Sanvisente, i quali evidentemente non conoscevano il poemetto tedesco, dichiarano di non conoscere alcuna leggenda come quella di cui parla lo Smith. Rajna (1. cit.) trova l'ispirazione del Wieland nel naufragio di Ruggero nell' Orlando furioso. 
di $133^{\mathrm{m}}$. La sua superficie é di Kmq. 20,1974. ${ }^{\mathrm{1}}$ Ne esiste la carta all' 1 a 25000 dell' Istituto Geografico militare. La costa Nord, che presenta poche sinuositi, s' innalza scoscesa e generalmente inaccessibile, specialmente verso Ponente dove sono le altezze maggiori. É costituita da rocce stratificate orizzontalmente el è tanto a picco, che da molti punti, ed anche dal piu alto (l'Albero del Sole, $133^{\mathrm{m}}$ ), si puó buttare un sasso nel mare. Da quel lato non vi sono vere cale e si puó discendere al mare solo in pochi punti. Dal suo orlo Nord l' isola va gradatamente abbassandosi verso Levante e Mezzogiorno dove la costa è frastagliata da varie piccole insenature, la maggiore delle quali, che s'addentra per circa $800-900$ metri, forma il porto naturale dell' isola. Questo porto é aperto solo ai venti di Mezzogiorno e di Libeccio, ed offre un buon ancoraggio a bastimenti non superiori alle 500 tonnellate. La superficie dell' isola è ondulata, non presentando forti pendenze se non in vicinanza immediata deI mare e sui fianchi delle vallate o burroni che mettono capo alle insenature della costa Sud. Il punto più basso della costa, dove il piano dell' isola si abbassa fino al mare, è la Punta Sottile all' estremo Sud-Est.

Dal lato di Mezzogionno, separato dalla costa da poche diecine di metri di mare cosi poco profondo che si può attraversare a guado, evvi un isolotto detto Isola o Scoglio dei Conigli, che ha circa 300 metri di lungo per 150 di largo. Questo isolotto all' epoca romana era probabilmente congiunto coll'isola, altrimenti non mi spiegherei la presenza di una cisterna rivestita di cemento; evidentemente romana, che vi osservai.

I terreni coltivati sono principalmente nel centro dell' isola, nella sua parte orientale ed a Sud, nel basso di alcuni valloni meno lontani dal porto. Adesso circa un terzo dell' isola è ridotto a coltura. I campi sono tutti circondati da muri a secco, fatti non meno per liberare il terreno dai sassi, che per dividere le proprietá, e per difesa contro gli animali. Dalle carte che ac-

1 Attilio Mori, L'area delle minori isole italiane in Rivista geogr. ital., anno III, fasc. X, Dicembre 1896. Il prof. A. Mori dell' Istituto geografico militare ha gentilmente controllato le indicazioni geografiche che ho qui date. 
compagnano i lavori di Sanvisente e di Calcara si rileva che le terre coltivate sono assai cresciute dal 1846 in poi. Alcune peró delle terre che furono una volta coltivate, non lo sono più adesso, come lo provano delle traccie di muri a secco intorno a campi abbandonati, che rimontano forse all' epoca dei Maltesi Gatt. Le abitazioni sono quasi tutte intorno al porto, pochissime essendo le case coloniche sparse per l'isola. Verso la punta Nord-Est sorge un fanale di $3^{a}$ classe, che funziona dal 1890 in poi.

Veicoli non vi sono in Lampedusa, e quindi neppure strade carrozzabili. Il Governatore Sanvisente, è vero, dalle idee grandiose, abbozzò due strade che si partivano dal porto; ma se mai furono in uso, sono adesso abbandonate.

Nei campi si coltivano principalmente il grano, l'orzo (in parte per foraggio), l'avena, le patate, le fave, i piselli, le lenti, i fagioli. Ma il raccolto viene spesso danneggiato dalle cavallette, quasi sempre dalla mancanza di pioggia e sempre dal vento. La vite, che ai tempi di Calcara non esisteva a Lampedusa, è adesso coltivata su larga scala e dá buoni prodotti. " I fichi d’India, piantati irregolarmente o a filari, nei campi o come siepi intorno ad essi, abbondano e sono una grande risorsa per l'isola, non solo, perchè gli abitanti si cibano dei loro frutti, ma anche perchè le loro pale sono un ottimo alimento per il bestiame. E stata tentata pure, ma senza successo, la coltura del sommacco, e, secondo Sanvisente, anche del tè (?). Quella del Mesembryanthemum crystallinum per estrårne la soda, che una volta si faceva, adesso è cessata. Negli orticelli, oltre a pochi ortaggi, si coltivano aranci e limoni, fichi ed alcuni altri alberi da frutto, ma in piccola quantitá e con esito ben poco felice. Sole a sfidare i venti sono alcune belle palme da dattero, che rallegrano un poco il monotono paesaggio nella vicinanza del paese.

Gli abitanti tengono dei somari come animali da basto, poche vaccine, mancando per queste il pascolo, un discreto numero di pecore e capre, ${ }^{2}$ maiali e galline. Ma più remunerativa del-

1 Mi fu detto che si poteva valutare a 300,000 il numero di viti ora esistenti in Lampedusa.

${ }^{2} \mathrm{Vi}$ erano 110 vaccine, 615 capre e 1340 pecore nell'isola all'epoca della mia ultima visita. 
l'agricoltura e della pastorizia è la pesca delle spugne e delle sardine, che richiama a certe opoche nel porto di Lampedusa anche barche e marinari d'altre parti. ${ }^{1}$ Le acque di quest'isola godono meritata fama per l'abbondanza e l'ottima qualita dei loro pesci. Mentre ero in Lampedusa quest'anno si vendeva il pesce buonissimo a 20 centesimi il chilo, e Lo Re scriveva, nel 1884, che allora valeva da 7 a 10 centesimi il chilo. Non poco movimento commerciale, e quindi guadagno per gli isolani, porta poi con sé anche la colonia penale, col relativo presidio.

\section{$\therefore$}

Non vi è nell' isola acqua corrente, salvo quando piove, e manca pure quasi del tutto l'acqua stagnante. In qualche punto si trova acqua sorgiva, ma in quantitá affatto trascurabile. Scavando dei pozzi s' incontra, al livello del mare, dell' acqua, che però è in generale più o meno salmastra, per cui fin dai tempi antichi si era provveduto all'acqua da bere con cisterne di cui l' isola, secondo Sanvisente, contava 153.

La pioggia, salvo in inverno, è molto scarsa, talvolta nulla per mesi interi. Ho visto i contadini a sospirarla gia nel mese di Marzo, e spesso succede che, per mancanza d'acqua, i raccolti siano seriamente danneggiati. I venti sono gagliardi, cosicchè gli alberi da frutto e gli agrumi possono vivere soltanto al riparo di muri, rimanendo le loro punte bruciate appena oltrepassano l'altezza della cinta. I piccoli giardini, circondati da muri a secco alti 2-3 m., sono poco numerosi, e situati specialmente nei valloni, dove al riparo artificiale si aggiunge quello naturale. Peró anche dentro a tali recinti ho visto quest' anno molte piante di agrumi seccate dall'alidore e dal vento.

1 Alla pesca delle spugne, che dura da Marzo a Novembre, prendono parte una cinquantina di barche di Lampedusa (dette sacch'o leva) con cinque uomini ognuna, ed una trentina di barche che vengono di fuori, specialmente dalla Grecia. Le sardine si pescano dalla metà di Marzo a tutto Giugno, ed occupano una ottantina di barche, montate ognuna da sei marinari. Quest'anno la pesca delle sardine è stata cosi abbondante che, a quanto mi scrivono, alcune barche hanno fatto 2000 lire di gruadagno netto. 
I dati meteorologici esatti per Lampedusa sono assai scarsi, e. per averne alcuni attendibili dobbiamo rimontare al tempo di Gussone e di Calcara. Da una tabella di quest'ultimo si rileva che i giorni in cui piovve più o meno furono:

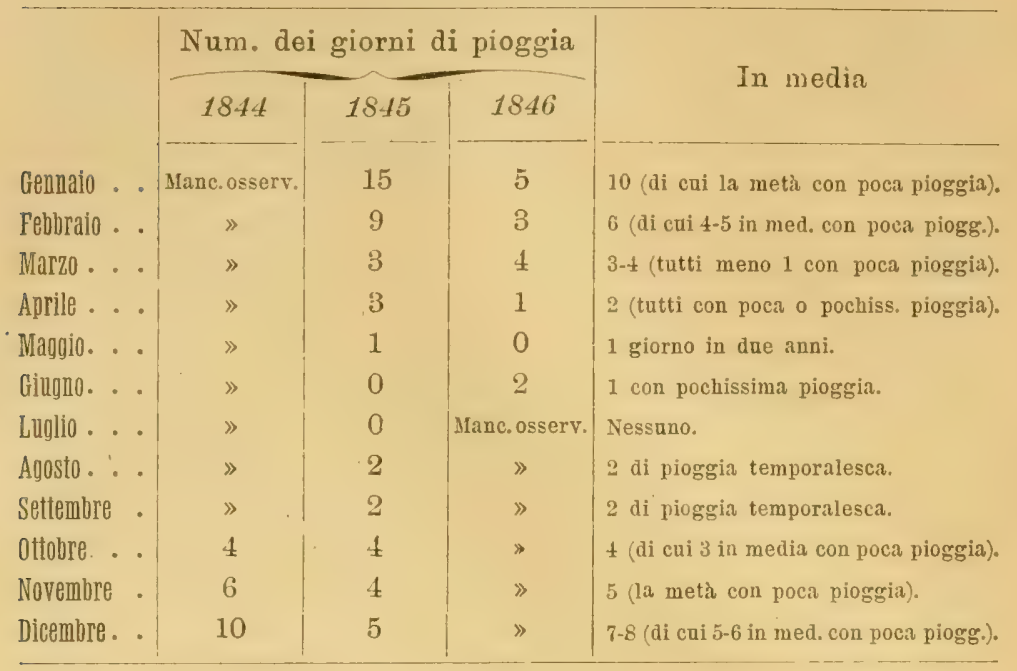

Dalla tabella di Gussone vediamo che nel mese di Agosto 1828 non vi fu affatto pioggia, che il cielo fu prevalentemente sereno, che i venti predominanti furono di Nord e di Nord-Ovest, che vi fu in generale rugiada abbondante. Al dire degli isolani in inverno sono frequenti le nebbie.

Per la temperatura abbiamo una tabella di Gussone che va dal 2 al 31 Agosto 1828, dalla quale si rileva che la massima delle temperature osservate alle 12 e alle $13 \mathrm{fu}$ in Centigradi di 33. 3, e la minima delle temperature osservate alle 6 fu di 22. 7. La media delle 30 temperature prese alle 6 fu di $25^{\circ} 4$; la media delle 30 temperature prese alle $19 \mathrm{fu}$ di $26^{\circ} 4$ e quella delle 29 temperature prese alle $12 \mathrm{fu}$ di $29^{\circ} 5$. - Calcara dá pure (in Farenheit) le temperature osservate da esso dal 26 Maggio all' \& Giugno 1846. Esse, ridotte in Centigradi, mostrano per quei giorni una massima di $26^{\circ}$ (alle 12 del 4 Giugno) ed una minima di $19^{\circ} 5$ (alle 20 del 2 Giugno). La media delle temperature osservate durante quei 14 giorni fu alle ore 6 di 21.6 , alle 12 di 24. 2 , ed alle 20 di 21.3. - Alle temperature notate da 
Sanvisente non si può prestar fede come ho detto nella bibliografia. ${ }^{1}$

Quello che si sa dalla gente dell' isola è che neve non si vede mai, e ghiaccio neppure, ma quasi ogni anno un po' di grandine, e che vi sono piuttosto frequenti le nebbie, ed abbondante la rugiada. In generale il clima deve dirsi mite, presentando minime alte e massime poco elevate.

\section{GEOLOGIA.}

Primi a scrivere della geologia di Lampedusa furono Gussone e Calcara. Il prof. Trabucco che vi dedico uno studio spẹciale, oltre a riferire le proprie osservazioni, riassume quanto ne $\mathrm{fu}$ detto anche incidentalmente da varî scrittori. Riporto qui soltanto quel poco che interessa più particolarmente il botanico.

L'isola è interamente composta di terreni sedimentarî calcarei, alternanti in qualche luogo con strati di marna. Quei terreni furono depositati dai mari del miocene superiore o del pliocene inferiore. Terreni analoghi, della medesima epoca, trovansi in Sicilia. Secondo Gussone sono assimilabili ai calcari di Malta e di Siracusa. Il calcare più o meno magnesifero, passante talora a vero calcare dolomitico, talvolta marnoso, è in parte compatto e duro, in parte friabile. L'emer'sione di Lampedusa e di Lampione coincide con quella della vicina costa d'Africa, ed è avvenuta sul finire del pliocene. Lampedusa è stata probabilmente congiunta con Lampione, dal quale la separa un braccio di mare poco profondo ( 85 metri al massimo), e forse anche con la vicina costa d'Africa che ha identica formazione (la profondita massima fra il punto più vicino dell' Africa e Lampedusa è di 117 metri). ${ }^{2}$ Certo non è mai stata unita nè alla Sicilia nè a

${ }^{1}$ Lo $R \theta$, in fondo alla sua memoria, indica come temperatura massima $29^{\circ} 5 \mathrm{Cgr}$. per il 24 Agosto, e come minima $10^{\circ} \mathrm{Cgr}$. per il 31 Dicembre. Ma non solo non dice in quale anno, ma neppure in quale delle due isole Lampedusa e Linosa alle quali è dedicato il suo scritto!

2 Teluini, Rassegna Se. geol. it., 1892, p. 293. 
Nalta, dalle quali es separata da profonditi marine multo maggiori. La superficie di Lampedusa adesso pare che vada diminuendo per la corrosione dei flutti. Una cisterna che ho trovata nell'isolotto dei Conigli prova che all' epoca romana questo isolotto era congiunto con Lampedusa e dimostra quindi che anche in tempi storici quest'isola ha subito una sensibile diminuzione. Le vallate prodotte dalla erosione di acque correnti stanno del resto a testimoniare di una estensione assai maggiore, in antico, di terre emerse.

\section{FAUNA.}

Della fauna di Lampedusa ha dato per il primo alcune notizie Calcara. Egli osserva che gli invertebrati appartengono in generale a specie comuni in Sicilia. Tuttavia, fra i molluschi dei quali più specialmente si occupa, e di cui cita varie specie terrestri e marine, descrive come nuove due conchiglie terrestri, la Helix Cumiae e la Clarsilia Lopadusae. Sanvisente pure dá alcuni elenchi di animali. Egli evidentemente, come per le piante, ha attinto alle medesime fonti che Calcara, mostrando peró troppo chiaramente di non sapersene servire." Anche Trabucco cita un certo numero di animali, per la maggior parte tratti dagli elenchi di Calcara e di Failla-Tedaidi. Il prof. E. H. Giglioli, che visito Lampedusa direrse volte, facendovi raccolte zoologiche, mi ha fornito le seguenti informazioni sui vertebrati.

Ai tempi di Sanvisente e di Calcara esistevano nell' isola ancora alcuni cervi (Cervus corsicanus Erxl.), ${ }^{2}$ forse introdotti dagli antichi Signori dell' isola; ma erano giả estinti all'epoca della mia prima visita. Le capre selvatiche, che pure vi si trovavano, furono distrutte per ordine di Sanvisente, come egli stesso

1 Per darne un esempio dirò che fra gli anfib̂̂ mutanti cita la Murena anguille (sic) accanto alla Phoca vitulina!

* Calcara lo chiama Cervus elaphus; ma il prof. Giglioli che ne ha trovato ancora un cranio in Lampedusa, ha potuto accertarsi che apparteneva alla specie di Corsica e Sardegna. 
racconta. Il mammifero più grosso che oggi si trovi a Lampedusa è il Pelagizes monachus, la nostra foca comune, che frequenta le grotte della costa. I conigli (Lepus cuniculus L.), i ratti (Mus rathus L.) ed i topolini (Mus musculus L.), che Calcara dice essere tanto numerosi da danneggiare seriamente le campagne, vi sono oggi ancora frequenti, specialmente gli ultimi. Quegli autori parlano pure del gatto selvatico, ma si deve certo intendere gatto domestico rinselvatichito, come ve n' è qualcuno anche oggi. Una specie almeno di pipistrello, il Vesperugo $I$ ruhli (Natt.) var. albo-limbata, vi è abbondante.

Gli uccelli sedentarî, veduti o presi dal prof. Giglioli, sono i seguenti :

\begin{tabular}{|c|c|}
\hline $\begin{array}{l}\text { Passer hispaniolensis (Temm.) } \\
\text { ? Sturnus unicolor La Marm. } \\
\text { ?Emberiza Cirlus L. } \\
\text { Melanocorypha Calundra (L.) } \\
\text { Monticola Cyanus (L.) } \\
\text { Sylvia conspicillata La Marm. } \\
\text { Hirundo rustica L., estiva } \\
\text { Cypselus Apus (L.), estivo } \\
\text { Cypselus Melba (L.), estivo } \\
\text { Alcedo Ispicla L. } \\
\text { Athene Nochua (Scop.) }\end{array}$ & $\begin{array}{l}\text { Hypotriorchis Eleonorae } \\
\quad \text { (Gené) } \\
\text { Phalacrocorax Graculus (L.) } \\
\text { Columba livia Bonnat. } \\
\text { Tuntur communis Selby } \\
\text { Tringoüdes Hypoleucus (L.) } \\
\text { Chroocephalus ridibundus (L.) } \\
\text { Lamis fuscus L. } \\
\text { Pulfinus anglorum (Temm.) } \\
\text { Puffinus Iruhli (Boie) } \\
\text { Procellaria pelagica. L. }\end{array}$ \\
\hline
\end{tabular}

Molte poi sono le specie che si riposano in Lampedusa nelle loro migrazioni fra l'Africa e l' Europa, e queste certo hanno avuto e seguitano ad avere importanza per la disseminazione delle piante. Gussone, Calcara e Sanvisente lamentano i damni gravissimi che le gru (Grus communis Bechst.) recano alle messi nella sosta che fanno in Lampedusa sul finire di Maggio e al principio di Giugno. Oggri peró mi fu detto che se ne vedevano bensi di passo, ma che non facevano danuo.

Non rara, nel mare di Lampedusa, è la grande tartaruga marina (Talassochelys corticata Rondel.) mentre in terra trovasi frequente la testuggine comune (Teshudo graeca L.), non piu tanto abbondante peró quanto la descrivono Gussone e Calcara. In gran numero si trovano i Gongylues ocellatus (Forsk.), detti Tiri, e non rari gli Hemidactylus verruculatas Cuv. ed i 
Platyclactylus mareritanicus (Laur.), detti Salamiri (quest'ultimi presi da me anche nell' isolotto dei Conigli). La volgare lucertola invece, che è tanto abbondante a Linosa, e che trovasi anche nell'isolotto di Lampione e sul piccolo scoglio dei Conigli, manca assolutamente a Lampedusa, il che è abbastanza strano. Due sono le specie di serpenti che si trorano in Lampedusa, innocue entrambe, la Coelopeltis lacertina (WVagl.) ed il Macroprotodon (Coronella) cucullatus (Is. Geoffr.). "

Questi rettili, che vi furono raccolti tanto dal prof. Giglioli quanto da me, pare che siano i soli esistenti in Lampedusa. ${ }^{2}$

Le rane mancano; vi si trova invece frequente un rospo, il Bufo variabilis Pall.

Il mare intorno a Lampedusa è molto ricco di pesci. Calcara dả un piccolo elenco di quelli più comuni. Di maggiore interesse per il commercio sono le sardine (o sarde) e le acciughe (dette alici o alaci), che vengono salate ed esportate. Vi abbondano pure i cefalopodi, e meritata celebritá vi godono i calamari ed i totani.

Calcara dà il nome di alcune specie di crostacei da esso osservati. L'arigusta e il lupacanto, che esso non menziona, si trovano nelle acque di Lampedusa, ma in poca quantità.

Agli insetti di Lampedusa ha dedicato uno studio speciale il Failla-Tedaldi ${ }^{3}$ che vi fece un soggiorno di cinque giorni, oc-

1 Questa specie Nord-Africana che io scopersi, per la prima volta in Europa, a Lampedusa nel 1873, ha dato argomento ad una nota del prof. Giglioli intitolata «Beiträge zur Kenntniss der Wirbelthiere Italiens 》 in Archiv f. Naturgesch. 1877.

2 Oltre alle tartarughe, Calcara cita 3 soli rettili: Hemidactylus triedrus (=H. verruculatus) Cuv., Podarcis muralis Wagl., Coluber elaphis Shaw. - Certamente invece di Podarcis muralis deve leggersi Gongylus ocellatus, poichè dalla gente del paese mi fu affermato, e lo stesso mi conferma il prof. Giglioli, che mancava la lucertola, mentre abbonda il Gongylus che Calcara non menziona. Ed invece di Coluber elaplis deve, secondo ogni probabilita, leggersi Macroprotodon cucullatus o Coelopeltis lacertina, le due sole specie di serpenti di cui il prof. Giglioli ed io abbiamo potuto constatare l'esistenza in Lampedusa.

${ }^{3}$ L. Failla-Thdaldi, Escursione entomologica all'isola di Lampedusa. «Il Naturalista Siciliano », anno VI, 18S6-87, pagg. 53, 69, 102, 157. Questo scritto contiene, oltre alla parte entomologica, alcune osservazioni di vario genere sopra Lampedusa. 
cupandosi piủ specialmente dei coleotteri. Egli osserva che la fauna entomologica di Lampedusa è relativamente ricca. Pochi perỏ sono i lepidotteri, specialmente i diurni. Numerosi i carabidi che mancano del tutto in Pantelleria. Vi ha trovato quatche specie africana non ancora conosciuta di Europa. Attribuisce la grande quantitá dei carabidi carnivori alla abbondanza in Lampedusa di conchiglie terrestri; e dice che essi difettano in Pantelleria, perchè in quest' isola, a causa della mancanza di calcare, scarseggiano le conchiglie. Numerose sono le specie di ortotteri; che numerosi ne sono gli individui lo sanno pur troppo i contadini, che tanto spesso vedono le loro mèssi divorate dalle cavallette. Oltre agli insetti raccolti, il Tedaldi enumera alcuni aracnidi.

Il prof. Emery, al quale ho sottoposto le formiche da me raccolte in Lampedusa, vi ha riconosciuto queste specie: Messor barbarus barbarus L. var. nigra Andrè, Camponotus rufoglaucus micans Nyl. e Monomorium Salomonis L., una varietà non descritta.

Devo ancora fare speciale menzione delle patelle che abbondano sugli scogli (e di cui Calcara cita tre specie distinte), a causa della gratitudine che devo loro per l'eccellente antipasto che mi fornivano, e perchè ad esse, da alcuni, è attribuita l'origine del nome di Lampedusa.

Non manca pur troppo a Lampedusa neanche la fauna molesta, e mosche, zanzare e pulci, al dire degli abitanti, vi sono in estate una vera piaga.

\section{FLORA.}

Il paesaggio botanico è profondamente modificato dal tempo di Gussone in poi, ed è cambiato anche dopo la mia prima visita a Lampedusa nel 1873. Gussone difatti ci descrive l'isola come coperta di verdi fruticeti, di dense boscaglie, in alcuni luoghi di cespugli densissimi e quasi impenetrabili, e ci parla di alberi di discreta altezza. Il capitano Smith nel 1824 scriveva che le 
legna da ardere vi abbondavano e venivano portate di lì a Malta ed a Tripoli. Nel 1873 non vidi più densi fruticeti, ma trovai ancora buona parte dell'isola coperta di bassa macchia, e vidi qualche pino isolato; ed ancora nel 1884 Ross, Solla, Lojacono, Lo Re parlano di macchia lamentandone peró la già progredita distruzione.

Oggi macchia non vi è più, ed ancora meno vi si vedono alberi. Il Corbezzolo, che dava il suo nome (Imbriacola) ad una estesa vallata che n'era rivestita al tempo di Calcara, è interamente scomparso di lå, ed appena se ne sono salvate alcune piante all' estremo Ponente, per l'inaccessibilitá dei luoghi dove crescono. L'isola é diventata squallida e il suo carattere predo. minante è l'aridità. Il suo scheletro di bianca roccia, messo a nudo, rifrange i raggi del sole in modo da offendere gli occhi. I suoi alberi sono caduti sotto l'accetta del boscaiolo, ed i suoi frutici a poco alla volta sono stati consumati dai forni del paese. Pochi avanzi di macchia rimangono ancora verso Capo Ponente nella parte dell'isola più lontana dal porto ed in qualche burrone, cioé nei luoghi di più difficile accesso; ma scompariranno ben presto anche questi, poichè vedevo ancora quest' anno i coatti venire in paese con fastelli di Pistacia Lentiscus e d'altri pochi superstiti che erano andati a svellere ad ore di distanzi. Le capre e le pecore poi, hanno compiuto l'opera di distruzione, divorando i germogli dovunque spuntavano. Perfino i Cistus, che avevo visti ancora abbondanti nel 1872, si trovano ora soltanto in pochi luoghi, meschini e radi. La Pistacia Lentiscus, la Phillyrea, l' Erica multiflora, l' Hypericum Egyptiacum, il Teucrinm frulicans, la Periploca, il Prasium, il Thymus capitalus, il Lycium Europaeum, l'oleastro, che rimangono, per la maggior parte non si possono più chiamare frutici. Essi si sono rimpiattati nelle fessure delle roccie e fra i sassi dei muri a secco, o, se sono in campo aperto, presentano una forma pulvinata ed erinacea, lasciando esposti alla superficie soltanto un intreccio di rami nudi sotto i quali se ne stanno nascoste le foglie. Non è il vento, ma sono i denti tosatori degli ovini che li hanno foggiati in quel modo. Solo le foglie degli Asfodeli e della Scilla maritima, acri e caustiche fin tanto che sono fresche, sfidano la roracitá degli erbivori. 
Qualche mutamento nella fhora es arrenuto pure per effetto (iell' estensione delle colture. Cosi nel piano detto "la Salina», spesso rammentato da Gussone e da Calcara per varie piante alofile, non si trorano ora che campi di grano e vigne.

Se sono spariti del tutto gli alberi, e dalla maggior parte dell'isola i frutici, e con essi probabilmente alcune piante più umili che virevano al riparo della macchia, è stata introdotta invece qualche pianta che al tempo di Gussone non vi esisteva. La Oxalis cernua che Gussone non menziona, è al giorno d'oggi la peste dell'isola, che ha invasa in tutte le sue parti, coltivate ed incolte, imprimendo profondamente il suo stampo, colle sue vistose infiorescenze gialle, nella fisionomia delia flora primaverile. Ed i Gladiolıs, non visti da Gussone nè da Calcara, sono un ornamento dei campi, poco gradito ai coloni. La moltiplicizione del fico d'India, che Calcara indicava di due soli punti, e che adesso è un tratto saliente del paesaggio botanico nelle parti coltivate, deve pure aver'e molto modificato l'aspetto dell' isola.

A causa della unifol'mita del suolo, della sua denudazione, della sua natura generalmente pianeggiante, e quindi della poca diversita delle esposizioni e per la mancanza d'acqua, vi è poca varieta nelle stazioni e molte sono le piante che si trovano indifferentemente in tutta l'isola.

La maggior parte del suolo, lá dore non è coltivato, consiste in rupi bianche e nude o incrostate da licheni, ed in terra calcareo-argillosa, di colore ocraceo, risultante dallo sgretolalamento di quella pietra calcarea leggermente marnosa, che si è accumulata negli intervalli della roccia, nei suoi crepacci e nei suoi incavi. Questa terra, che si raccoglie in aree abbastanza estese lá dove il suolo è pianegrgiante, è compatta, e prosciugando diventa assai dura e si screpola. E probabile che nella stagione delle pioggie, a causa della sua natura compatta e della impermeabiliti del sottosuolo, si mantenga umida e favorisca così lo sviluppo della microflora precoce nella quale vi sono alcune piante annanti dell'umidita come il Juncus bufonius e le epatiche in genere. Ma cessate le pioggie indurisce presto, diventa arida ed arresta l'accrescimento delle piante annue (l'ho gia trovata dura e screpolata in Marzo). Difatti questi terreni, dove prima 
cresceva la macchia, sono ora il dominio delle piante nane di loro natura o ridotte nane per la stazione in cui crescono, che presto seccano e scompaiono, e di altre che hanno la loro riserva in bulbi o tuberi sotterranei. Li abbondano: Plantago Coronopus, Catapodium loliaceum, Juncus bufonius var. pumilio, Diplotaxis scaposa, Hyoseris scabra, Euphorbia retusa e E. peploides, Trigonella maritima e.T. Monspeliaca, Linaria reflexa, Sideritis Romana, Sagina maritima, Evax pygmaea, Filago Gussonei, Tillaea muscosa, Colula aurea, Senecio leucanthemifolius, Asteriscus aquaticus, Seriola Elnensis, Brachypodium distachyum, Hippocrepis unisiliquosa, Bupleurum glaucum, Eryngium dichotomum, Convolvulus lineatus, Ranunculus bullatus, Thrincia tuberosa, Romulea Columnae, Iris Sisyrinchium, Scilla autumnalis, Colchicum Bentolonii, varie Riccia ed altre epatiche. Li pure crescono la Stapelia, la Jasonia, la Cynara horrida ed in abbondanza l'Asphodelus ramosus e la Scilla maritima, due piante che ora costituiscono il tratto più saliente del paesaggio botanico di Lampedusa.

In alcuni incavi della roccia l'acqua piovana si mantiene più a lungo, formando dei piccoli bacini di pochi metri, e talvolta șolo di pochi decimetri di superficie, nei quali si rinvengono fitti tappeti di Elatine Gussonei in mezzo ai quali cresce. la Butliardn Vaillantii, due piantine che si trovano intrecciate fra loro come un feltro, e spesso sono ricoperte da uno strato compatto di un'alga verde. Li si trovano pure il Juncus bufonius normalmente sviluppato ed i Lythrum.

Dai lati di Mezzogiono e di Levante, nel fondo di alcune cale, la rena, calcarea rigettata dal mare ha formato delle spiaggie sulle quali si accumulano le foglie della Posidonia rigettate dal mare. Queste spiaggrie peró sono poco estese ed in generale nude. Soltanto in una Cala vicino al porto, nel luogo detto Arena Bianca, si trovano l'Euphorbia Paralios, il Pancratium maritimum e la Cakile maritima, le sole piante esclu. sivamente psammofile che io abbia trovate nell' isola.

Sta\%ione ben caratterizzata è quella delle rupi e terre in immediata vicinanza del mare. Ma poche sono le piante ad essa limitate, come le Statice, la silene sedoides, la Passerina hir. suta e l'Inula crithmoides. Condizioni quasi uguali si trovano 
altrove, sicchè anche più lontano dal mare s'incontrano piante generalmente considerate come marine, quali le Frantienia e

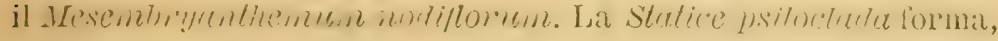

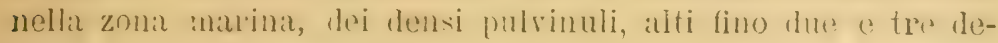
cimetri, tanto compatti che vi si puó camminare supra senza che cedano sotto il peso del corpo.

Come piante piu caratteristiche della stazione rupestre si possono citare Cmucianclla rupestris, Hypericum . Egyptiacum, Sedum dasyphlyllum, S. Nicaeense, Umbilicus liorizontalis, Capparis rupestris, Satureja fasciculata; ma in generale sui dorsi denudati dell'isola quella stazione è poco distinta, poichè si confonde con quella delle terre aride di cui ho parlato, colle quali le rupi e i sassi sono cosi intimamente consociati. Peró, nei burroni più stretti e lai tianchi più ripidi che scendono alle Cale di Mezzogiono, si trova fra le rupi e su di esse, una vegetazione che ha un aspetto diverso da quello delle parti pianegrianti lell'isola. Li, riparate dai venti el un poco anche dai raggi cocenti del sole, mono esposte ad essere mutilate dall'uomo e dalle bestie, crescono più rigogliose e meno deformate fra le rupi, la Rula, la Pislaciu ed altre piante di quelle che prima costituivano la macchia, e si trova ancora il Carubbo in forma di 'arbusto, l'Euphorbia a'cnił’oides e fin'ariche qualche rara pianta di Juniperus Phoenicea o qualche, piú rara ancora, Lonicera implexa; l'Hypericum Egyptiacum vi forma degli eleganti cespuglietti abbondantemente rivestiti di fiori in Mar\%o. Fra i frutici crescono la Succowia Balearica e la Melica minula, e più abbondanti che altrove siunalzano gli Asparagus delle cui griorani messe è facile qui in breve ora fare ampia raccolta. Alcune rupi, che qui conser'rano un po' d'umidita, sono rivestite di Vaillantia e di Caliipellis, di muschi e di epatiche, e nelle loro fessure nascono piu rigugliose alcune delle piante rupicole citate sopra.

Poche sono le piante spontanee che, come i Gladiolus e ia Bellevalia conosa, crescono fra le méssi ripulite con cura dai contadiui; ma nei campi in maggese o abbandonati, vi ì una flora arrense discretanente ricca, e generalmente meno stentata che nelle altre parti dell' isola. Li abbondano alcune delle piante arrensi le piü comuni lella regione mediterranea insieme ad altre piú particolari al Mezzogionno. Vi si trovano le Vicia, 
le Medicago, le Fumaria, le Calendula, i Papaver, i Bromus, i Lotus edulis e ornilhopodioines, Slellaria media, Anagallis arvensis, Sherardia arvensis, Scandix, Tordylium, Avena barbata, insieme a Oxalis cernua, Pinardia, Silene ruhella, dionis microcarpus, Carrichtera vellae, Solanum Sodomaeum, Glau. cium phoeniceum, Sénecio leucanthemifolius, Malva microcarpa, Euphorbia pinea, Echium confusum ecc., varie delle quali specie sono, del resto, comuni in tutta l'isola, come l' Oxalis, l'Anagallis, il Senecio, l'Euphorbia, la Slellaria, la Scandix e la Sherardia.

Sui muri a secco e fra i fichi d'India crescono di preferenza Bryonir acuta, Clematis cirrlosa, Rubia peregrina, Convolvulus althaeoides.

Il terreno sotto i fichi d'India e al piede dei muriccioli dal lato di tramontana, dove si mantiene più Iungamente l'umiditá, è, insieme alle rupi ombrose nel fondo dei valloni più stretti, la stazione preferita dei muschi e delle epatiche. E sotto alle Opunzie che ho trovato il Petalophyllum Ralfsii.

Stillicidî appena esistono in Lampedusa. In un solo punto della costa meridionale, non lungi dall' isola dei Conigli, ho trovata una rupe dalla quale sgocciolava un po' d'acqua, e dove, insieme ad alghe di acqua dolce, cresceva il Capel Venere.

Le stazioni « ad vias» $\mathrm{e}$ 《in ruderatis » meritano appena di essere distinte in Lampedusa, poichè non vi si trovano piante che non siano comuni altrove, tranne forse qualche Carducs, il Silybum, l'Hyosciamus e l'Ecballion.

Dove la vegetazione è più bella, e dí una idea di quello che potrebbe essere Lampedusa se non fossero stati inconsultamente permessi il diboscamento ed il libero pascolo, è fra le protettrici opuntia ed in certi giardinetti, o recinti ben difesi dagli animali. Ivi, come nel fondo di qualche vallone, vi sono ancora alcuni frutici, e fra essi rigogliosi s'innalzano la Ferula, il Foeniculum, la Magydaris e specialmente lo Smymium; ed ivi più grandi che altrove crescono Borago officinalis, Urospermum picroides, Sonchus levis, Hyoseris radiata, 'Thrincia tuberosa, Cerastium glomeratum e Torilis nodosa. Gli Asparagus, i Convolvulus, le Fumaria si innalzano appoggiati ai frutici, i Galim intrecciano i loro fusti, più laıghe si fanno le foglie 
dell'Arisarmom e s'incontra qualche pianta sciafila come Geranim Robertianum e Scrofularia peregrina.

Ma dove ancora meglio ci si rende conto di quello che sarebbe la vegetazione se fosse sottratta all'azione dell'nomo e degli ovini, è sull isolotto dei Conigli. Ivi, quantunque sia poco più che uno scoglio di quattro o cinque ettari di superficie, battuto dai venti e spluzzato dalie onde, si trova una rigogliosa vegetazione, e, considerando l'area ristretta, una grande varietá nelle specie. In meno d' un' ora vi raccogliemmo, in Marzo, 75 fanerogame. Il terreno, meno le rupi in vicinanza immediata del mare, vi è tutto rivestito di vegetazione. La macchia vi è folta, composta di Salsola fruticosa, Atriplex Halimus, Euphorbia dendroides, Piriploca angustifolia, Pistacia Lentiscus, Prasium majus, e Salsola longifolia. La Capparis rupestris vi allunga i stoi rami, alti vi crescono il Cardueus argyroa, la Ferula nodifora, la Thapsia Garganica, e le piante erbacee all'ombra della macchia ed in un terreno piu ricco di humus, vi acquistano un insolito sviluppo. Splendido v'innalza i suoi grossi fusti fistolosi il Sonchus glaucescens rivaleggiando coi suoi grandi capolini dorati col Picridium Tingitcmum e colle ricche infiorescenze del Senecio leucanthemifolius, ed il Lotus cylisoides vi forma dei grandi e densi pulvinuli, in Marto tutti coperti di fiori, che facevano un bel contrasto colle corolle azzurre dell' Anagallis e dell' Echium confusum. Una visita a quell' isolotto è un rero riposo dopo lo squallore abbagliante delle nude roccie di Lampedusa.

Se sul finire dell'inverno ed in primavera è poco ridente l'aspetto floristico di Lampedusa, ben più triste deve essere in estate. Anche avanti che la macchia venisse distrutta, Gussone, che vi fu in Agosto, dice che in quell'epoca non si trovava più traccia di pascolo, ed i bovi e le capre erano ridotti a nutrirsi dei rami teneri e delle foglie degli oleastri, delle Phillyrea e perfino della Periploca angustifolia, quantunque questa causi al bestiame infiammazione el esulcerazione della bocca; le pecore allora mangiavano le foglie appassite della Scilla maritima e dell'Asphodelus ramosus, i conigli si cibavano di corteccie di Euforbie e si vederano le testugrgini, che allora erano più frequenti di adesso, a rodere i bulbi della scilla nonostante la loro causticitá 


\section{LE PIANTE RACCOLTE IN LAMPEDUSA.}

Gussone visitó Lampedusa nel mese di Agosto, cioè in una delle peggiori stagioni per la flora, quando, per i calori estivi, in quell'aridissima isola molte piante annue della flora primaverile sono interamente scomparse, ed anche molte delle perenni derono essere in uno stato appena riconoscibile. Ciò non ostante egli ci dice (Notizie p. 87) che vi ha raccolto 274 fanerogame. ${ }^{1}$

Calcara vi fu alla fine di Maggio ed al principio di Giugno, epoca assai più farorevole alle raccolte, benchè non sia neppure essa la migliore per quel suolo arido, in latitudine cosi bassa. Ció non ostante il suo contributo alla conoscenza della flora di quest'isola non fu quale avrebbe potuto essere, specialmente se si considera che egli era accompagnato da un ciardiniere dell' Orto botanico di Palermo, allo scopo espresso di aiutarlo nella raccolta delle piante. Calcara avverte, a p. 22, come tutte le specie che egli contrassegna con un solo * sono

1 Questa cifra differisce poco da quella che risulta dallo spoglio della Synopsis (266 fanerogame ed una felce salvo errore). A p. 32 delle «Notizio» si trova una tabella evidentemente errata, poichè mentre secondo il titolo dovrebbe contenere, distribuite per famiglie, le piante fanerogame delle isole Lampedusa, Linosa e Lampione, giunge a in totalts di sole 265 specie, inferiore alla cifra indicata da Gussone per Lampedusa sola. Per una strana coincidenza, che non può essere altro che fortuita, questa cifra di 265 è il numero preciso di piante attribuite a Gussone per Lampedusa nella Florula di Calcara.

Gussone non dice mai di aver ricevuto da altri, piante di Lampedusa. Però fra le piante indicate per quest' isola nella Synopsis, ve ne sono alcune precoci e fugaci (come Bellis annua, Asterolinum, Bulliarda, 'Tillcea, Callitriche, Allium Chamaemoly), che mal si comprende come potessero essere riconosciute nel mese di Agosto. Questo, unito alla notizia, dataci dal suo biografo Pasquale, che Gussone ricevette piante di Lampedusa da un barone Porcari, fa supporre che un certo numero delle piante indicate nella Synopsis fossero state raccolte non da lui stesso, ma da questo Porcari. 
state trovate da Gussone solo; quelle senza * da Calcara solo; quelle con due ** da Gussone e da Calcara. Risulterebbe quindi che delle 288 piante vascolari enumerate nella sua Florula Loparlusana, egli stesso non ne raccogliesse più di 114, di cui sole 23 non erano state prima trovate da Gussone; e questo numero gia esiguo di piante nuove andrebbe forse ancora un poco diminuito, poichè Calcara, per alcune piante, ha dimenticato di indicare con un asterisco che erano state gia raccolte da Gussone, come risulta dalla Synopsis. Dico forse, perché inversamente Calcara ha attribuito a Gussone alcune specie che questi non indica, il che potrebbe compensare quelle dimenticate. Ad ogni modo é evidente che le raccolte di Calcara non furono quello che arrebbero potuto essere.

In epoca per la flora ancora migliore di Calcara, dal 6 al 20 Aprile, visitarono Lampedusa i signori Ross, Lojacono, Zwierlein e Solla.

Ross nella sua memoria cita 57 fanerogame da esso raccolte in Lampedusa; ma, come ci dice, egli ha trascurato le specie piủ comuni.

Le fanerogame raccolte da Solla ed enumerate nel suo primo laroro, ammontano a 192, i licheni a 6 ; le alghe, nel secondo lavoro, a 23.

Lojacono, nel suo catalogo complessivo di Lampedusa e Linosa, cita 341 specie vàscolari. Egli avverte a p. 15 che tutte quelle che non fa precedere dal segno $O$ sono state raccolte da lui stesso. Detraendo dal totale di 341 le 29 specie che sembrano indicate per Linosa sola, ne rimangono 312 per Lampedusa. ${ }^{2}$ Di queste, 18 sono precedute da un $\bigcirc$, cioè sarebbero state trovate da Gussone o Calcara e non da lui, per cui sarebbero 291 le specie raccolte da Lojacono in quell' isola. Però si notano dimenticanze ed errori frequenti nell' apposizione dei segni (anche l'* che dovrebbe significare non essere la pianta stata antecedentemente indicata da Gussone o Calcara, è spesșo messo indebitamente), contradizioni fra queste indicazioni e quelle

- Secondo la Florula di Calcara le piante raccolte da Gussone sareubero 265, compresa la Diplotaxis scaposa.

${ }^{2}$ A p. 28 del suo opuscolo, Lojacono dì cinque righe di statistica, nella quale però tutte le cifre sono sbagliate. 
della Flora Sicula dello stesso Lojacono, e confusioni fra Linosa e Lampedusa; per cui si puó supporre (ed in varî casi dimostrare) che il segno $\bigcirc$ è stato assai spesso dimenticato da Lojacono.' Non si puó dunque indicare con esattezza il numero di specie da esso raccolte.

Il capitano Enrico D'Albertis, durante un breve soggiorno in Lampedusa, nel Settembre 1876, vi raccolse 14 alghe marine che vennero studiate dal prof. A. Piccone. Il loro elenco trovasi nella «Crociera del Violante» a p. 233, e in Piccone ( $r$. bibliogr.).

Zodda e Sturniolo raccolsero 231 fanerogame (da me redute), oltre a 5 briofite e ad un certo numero di licheni.

L'Ajuti ed io facemmo in comune la raccolta di piante, e queste, dopo il nostro ritorno, furono divise in due parti uguali, di cui il Museo botanico fiorentino conservo una. ed io l'altra. Disgraziatamente gli esemplari unici furono essi pure di $\mathbf{x}$ isi fra me e il Museo, e, dopo la morte di Parlatore, quelli del Museo vennero intercalati negli erbarî di Firenze senza che ne fosse stata fatta una nota particolareggiata; per cui, distribuiti nei 1700 e più pacchi dell'Erbario Centrale e nei 1500 pacchi dell'Erbario Webb, sono adesso quasi irreperibili. Per questo puo darsi che qualcuna delle specie da noi raccolta in un solo esemplare non figuri nella mia Florulá, e venga segnalata soltanto quando sará fatto lo studio accurato di qualche genere o di qualche famiglia, come è avvenuto per le famiglie della Flora Italiana di Parlatore pubblicate dono il nostro viaggio.

Il numero delle specie vascolari raccolte dall'Ajuti e da me nel 1873 fu di 246. In quest'anno, con Riccobono, ne abbiamo raccolte 259 , oltre a 38 briofite e ad un certo numero di licheni e di alghe.

Come si vede dalla seguente Florula, il numero di piante vascolari ora conosciute di Lampedusa é di 458. Questo numero peró andrebbe alquanto diminuito, essendo certo che in non pochi casi una medesima specie figura nella mia Florula sotto due

- Non si può fare a meno di essere colpiti dal numero rilevante delle piante di Gussone che risulterebbero ritrovate da Lojacono solo, in tutt'altra stagione, e non ci si può difendere dal sospetto che in molti di questi casi Lojacono abbia dimenticato il segno $\bigcirc$. 
nomi diversi dati da differenti raccoglitori. Nella magrgior parte dei casi non.mi sono creshto autorizzato a finde queste riunioni dietro a semplici congetture, per quanto plausibili potessero essere, non avendo avuto modo di vedere le piante raccolte da altri, salvo quelle di Zodda. Ma d'altra parte si puó essere certi che quella cifra sara notevolmente aumentata da ulteriori ricerche. Basta considerare che Calcara, il solo che abbia raccolto piante in Lampedusa nella seconda meti di Maggio e in Giugno, non lo ha fatto in modo esauriente, che nessuno vi ha erborizzato nella prima metá di Magrio, e che neppure vi è stato alcun botanico in autunno ed in inverno. Induce a crederlo anche il fatto che fra le poche piante mandatemi dalla guardia Martorana nel Giugno passato, ne ho trovate quattro non ancora note di Lampedusa.

Nella seguente Florula ho citato, dopo il nome di ogni pianta, i lavori di Gussone (Notizie e Synopsis), Calcara, Ross, Solla e Lojacono, con la pagina in cui trovasi menzionata Lampedusa. Ho pure citato sempre la Flora Italiana di Parlatore e la Flora analitica d'Italia di Fiori, Paoletti e Bẻguinot quando vi è indicata una specie per Lampedusa. Sollanto eccezionalmente, nei casi in cui mi sembrava opportuno per la sinonimia o per osservazioni speciali, ho citato altri lavori che menzionano piante di Lampedusa. Di Gussone ho citato il Supplemento al Prodromo soltanto in quei rari casi nei quali le indicazioni di questo lavoro non combinano con quelle della Synopsis, oppure quando nel Supplemento una specie è citata con nome diverso da quello che porta nella Synopsis. La Florula di Sanvisente, come ho detto a p. 23, è quasi una ripetizione di quella del Calcara. Per questo non ho creduto necessario citarla altro che nei pochi casi in cui le due Florule non combinano.

A tutte le piante raccolte da me in compagnia dell' Ajuti e di Riccobono ho messo il segno! Ho poi citato gli altri raccoglitori secondo le indicazioni rilevate nei loro scritti."

2 In quanto a Gussone, si può essere certi cho le piante da esso citate nella Synopsis per Lampedusa, vi furono raccolte da lui stesso, tranno la Diplotaxis scaposa e forse alcune specie mandato dal Porcari. Nelle indicazioni di Ross e di Solla non vi è nulla di 
Le note sulla frequenza, localiti e stazione, sono tutte state prese da me sul posto. Percio non riporto le osservazioni di altri se non per le piante rare o per quelle da me non trovate, o quando presentano un interesse speciale.

Rammento che i miei giorni di erborazione con Riccobono, indicati nella Florula con Mrrz., furono dall' 8 al 15 Marzo, e quelli con l'Ajuti indicati con Apr., dal 18 al 21 Aprile e dal 26 Aprile al $1^{\circ}$ Maggrio; quelli di Gussone dal 2 al 31 Agosto; quelli di Calcara dal 18 Maggio al 15 Giugno; quelli di Ross e di Lojacono dal 6 al i6 Aprile; quelli di Solla dal 13 al 20 Aprile e quelli di Zodda dal 31 Marzo al 9 Aprile.

dubbio, salvo qualche determinazione. Ugualmente certa è l'indicazione di Zodda, di cui ho trovato tutti gli esemplari accuratamente etichettati. Invece, per le ragioni esposte a p. 55-56, sono meno certe le indicazioni di Calcara e di Lojacono. Tuttavia, benchè persuaso di farlo spesso indebitamente, li ho segnati come raccoglitori d'una specie, ogni volta che cosi si rileva dai loro scritti, salvo nei casi in cuil'errore si puó dimostrare. 


\section{FLORULA DI LAMPEDUSA}

DICOTYLEDONEAE.

\section{I. - Ranunculacear.}

1. - Clematis cirrhosa L.; Guss. Notizie p. 87, et Fl. Sic. Syn. Ir p. 36; Solla Phyt. Beob. p. 47\%. C. cirrhos a et C. polymorpha b. Calcara Descr. Lamp. p. 27 et 31 ; Lojac. Una Esc. p. 15. C. cirrhosa var. polymorpha Lojac. FI. Sic. I p. 28. C. Balearica Lojac. Nalpighia XX p. 38.

Qua e la sui muri a secco, nelle siepi e fra i residui della macchia. Ver'so Capo Ponente, a Sanguedolce e nel Vallone dell'Imbriacola; Marzo e Aprile foglie! - Raccolta pure da Gussone, Calcara, Solla, Zodda (foglie).

Calcara dice di avere trovato in Lampedisa la C. polymorpha b. Viv. Gussone invece (Notizie e Synopsis) cita per Lampedusa la $C$. cirrhosa. Solla pure indica la $C$. cirrhosa, ma con dubbio, probabilmente perchè sara rimasto incerto se dovesse riferire i suoi esemplari alla C. cirrhosa o alla C. polymorpha Vir. Le piante di Lampedusa hanno le foglie giovani, verso l'estremitá dei rami, ternate, con foglioline, talvolta anche le laterali, picciolettate, mentre le foglie cresciute più in basso sui rami sono indivise o semplicemente lobate; tali foglie non sono affatto coriacee. Appartengono dunque a forme intermedie fra la C. cirrhosa e la C. polymorpha, avvalorando l'opinione the queste non si possano considerare come specie distinte. - Secondo Gussone questa specie a Lampedusa perde le foglie in estate.

2. - Anemone hortensis L.; Guss. Syn. II p. 33; Calcara p. 27; Lojac. Fl. Sic. I p. 29.

Raccolta soltanto da Gussone. 
3. - Adonis microcarpus DC.; Ross Eine bot. Excurs. p. 345. A. microcarpa Lojac. Esc. p. 15. A. microcarmes var. psendodenlatues f". purpureus et f. citrines Lanza Gli Adonis di Sic. e di Sard., in Malpighia V p. 248. A. acstivalis Guss. Suppl, Prodr. p. 184. A. Cupaniana a. et b. citrina Guss. Sym. II p. 38: Calc. p. 27; Solla p. 472. A. dentata Ar'c. Comp. Fl. It. $1^{\text {an }}$ ed. p. 7 ; Lojac. Fl. Sic. I p. 32 et Malpighia XX p. 42. A. ammus L. $\beta$ microcarpus Fiori e Paol. Fl. an. I p. 499.

Comunissima, specialmente nei coltivati, la forma con fiori rossi. Molto meno frequente, ma pure abbondante in alcuni luoghi, la forma con fiori citrini; Mrz. fi. e fr., Apr. fi. e fr.! - Cussone, Ross, Solla (fi. e fr.), Lojacono, Zodda (la forma con fiori rossi ubique in arvis, la forma citrinus all' Imbriacola, fi. e fr.).

4. - Ranunculus bullatus L.; Guss. Syn. II p. 41 ; Calc. p. 27 ; Lojac. Esc. p. 15 et Fl. Sic. I p. 38; Fiori e Paol. FJ. an. I p. 507.

Mi è stato mandato dal sig. $A$. Conti, ufficiale postale in Lampedusa, raccolto in fiore il 3 Novembre 1873. In Marzo ne ho trovato molto comuni le foglie quasi dovunque, meno che nella parte coltivata dell' isola! - Raccolto pure da Gussone e Lojacono.

5. - Ranunculus muricatus L.; Guss. Syn. II p. 50; Calcara p. 27; Lojac. Esc. p. 15 et Fl. Sic. I p. 48.

Raccolto da Gussone e Lojacono.

6. - Nigella Damascena L.; Guss. Syn. II p. 30; Calc. p. 27; Solla p. 472; Lojac. Esc. p. 15. N. Damascena c. Africana Brand in Richter et Gürke Plantae Eur. Tom. II fasc. III p. 418.

Frequente specialmente nei coltivati, ma trovasi anche lontana da questi; Mrz. fi., Apr. fi. e fr.! - Gussone, Solla (fi.), Lojacono, Zodda (fi.).

I miei esemplari di Aprile, come quelli di Zodda, sono per la maggior parte stentati e piccoli in tutte le loro parti (spesso non più alti di $4-5 \mathrm{~cm}$.). È questa la forma descritta da Brand (Monogir. d. Gattung Nigella p. 37, Berlin 1895) col nome di Africana, e indicata dall'Autore per' la costa settentrionale d'Affrica, e per Teneriffa, e da Richter e Gürle per Lampedusa. ì un caso tipico di nanismo prodotto dalla sterilita del suolo.

7. - Delphinium longipes Moris; Guss. Syn. II p. 27; Calc. p. 27; Lojac. Esc. p. 15. D. junceum Guss. Suppl. Prodr. p. 181. D. peregrinum $\delta$ longipes Fiori e Paol. Fl. an. I p. 523. 
Capo Rupestre e Capo Ponente, 28 e 30 Apr., ti. - Gussone e Lojacono.

I numbrosi esemplari che ho racenlti sono tuti plecoli, stentati e pauciflori.

\section{II. - Papaveraceae.}

8. - Papaver hybridum L.; Guss. Syn. II p. 6; Calc. p. 27; Solla p. 472; Lojac. Lisc. p. 15.

Nei luoghi coltivati, molto frequente; Mrz. fi. e fr. giov., A pr., fi. e fr.! - Gussone, Solla (fi.), Lojacono, Zodda (fi. e fr.).

Oltre al tipo, Ajuti ed io abbiamo raccolto in due soli esemplari fra le méssi presso il P.orto la var. Siculem (Guss.). Si distingue nettamente dal tipo per avere i peduncoli come il caule in alto densamente ispidi per peli patenti, e per la capsula più globosa. Le lacinie delle foglie sono strette e acute, ma ció incontrasi pure spesso nel tipo. In frutto il $20 \mathrm{Apr}$ !!

\section{9. - Papaver dubium L.}

Frequente nel coltivato, ma meno del P. hybridum; Mrz. fi. e fr. giov.! - Zodda (fi. e fr.).

Tanto le piante raccolte da me, quanto quelle di Zodda, appartengono allar varieti pinualifinem (Moris). Se, ne trovano spesso esemplari nani.

10. - Papaver Rhoeas. L.; Guss. Syn. II p. 8; Calc. p. 27; Solla p. 472; Lojac. p. 15.

Nel coltivato comune; Mrz. fi., Apr. fi. e fr.! - Gussone, Calcara, Solla (fi.), Lojacono, Zodda (meno comune del 'P. hiybridum fi. e fr.).

Uno dei miei esemplari del 1873 ha i peli dei peduncoli ascendenti-appressati, e corrisponde quindi alla var. d. della Synopsis di Gussone (P. strigosum Boenning?), Anche il dott. Zodda ha trovato questa varieta.

11. - Papaver setigerum DC.; Guss. Syn. II p. 8; Calc. p. 27; Solla p. 472; Lojac. Esc. p. 15 et Fil. Sic. I p. 59.

Nel coltivato frequente; Mrz. fi., Apr. fi. e fr.! - Gussone, Solla (fi.), Lojacono, Zodda (fi. è fr.).

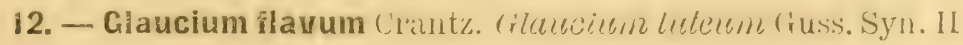
p. 5; Calc. p. 27; Lojac. Esc. p. 15. 
In varî luoghi anche distanti dal mare, ma non comune; Mrz. foglie, 30 Apr. fi.! - Gussone, Calcara (Cala Pisana), Lojacono.

13. - Glaucium corniculatum (I.) Curt.; Ross. p. 345; Solla p. 472; Fiori e Paol. Fl. an. I p. 484. G. phoenicerm Guss. syn. II p. 5; Calc. p. 27; I Iojac. Esc. p. 15 et Fl. Sic. I p. 57. G. corniculalum $\approx$ phoeniceum Solla p. 472.

Frequente nei campi incolti; Mrz. fi. e fr. giov., Apr. fi. e fr.! - Gussone, Calcara, Ross, Solla (fi. e fr.), Lojacono, Zodda (fi. e fir.).

Solla cita il $G$. corniculatum e la var. phoeniceum, d'onde si dovrebbe concludere che ha trovato due forme con colori diversi. Io l'ho visto soltanto con fiori rossi.

14. - Hypecoun procumbens I.; Ross p. 345 ; Lojac. Esc. p. 15 et Fl. Sic. I p. 56; Nicotra Le Fumariacee Italiane p. 22.

In vicinanza del Porto, Cala Pisuna e Cala Francese; 9 e 14 Mrz. fi. e fr. giov., 19 Apr. fi. e fr.! - Ross (margine dei campi presso il Porto), Lojacono (raro).

\section{III. - FuMARIACEAE.}

15. - Fumaria densiflora DC.; Ross p. 346 ; Lojac. Fl. Sic. I p. 64. F. micrantha $\beta$ Parlatoreana Lojac. Esc. p. 15. F. micrantha Nicotra Le Fumar. It. p. 73. F. olficinalis $\gamma$ densiflora Fiori e Paol. Fl. an. I p. 480.

Nel coltivato presso il Porto, non comune; $8 \mathrm{Mr}^{2} \mathrm{z}$. fi. e fr., 19 Apr. fi. e fr.! - Ross, Lojacono (il quale nella Flora Sicula non parla più della var. Parlatoreana).

16. - Fumaria parviflora Lam.; Ross p. 345; Lojac Esc. p. 16; Nicotra Le Fum. It. 1) 71.

Comune e talvolta abbondante nel coltivato; Mrz. fi. e fr., Apr. fi. e fr.! - Ross, Zwierlein (sec. Nicotra loc. cit.), Lojacono, Zodda (Cala Croce, floribus albis, fi. e fr.).

Ve ne sono esemplari ramosi diflusi, lunghi fino a $30 \mathrm{~cm}$., con lacinie foliari lineari-allungate, ed altri che si possono riferire alla var. glauca (Jord.) per essere piccoli, eretti, poco ramificati, con lacinie foliari più brevi e conniventi.

17. - Fumaria flabellata Gasparr.; Nicotra Le Fum. It. p. 60 et Altri ragg. sulle Fum. It. p. 8 . 
Frequente nel coltivato e qua e lá anche nei luoghi incolti; isola dei Conigli; Mrz. fi. e fr., Apr. fi. e fr.! - Zodda (Cala Galera, ad sepes, fi. e fr.).

- Fumaria capreolata L.; Ross p. 346; Solla p. 472; Nicotra Le Fum. It. p. 45 et Altri ragg. sulle Fum. It. p. 3. F. speciosa Lojac. Esc. p. 15.

Ross, Solla (fi. e fr.), Lojacono. Questi, in «Una Esc. », riteneva che i suoi esemplari fossero da riferirsi alla $F$. speciosa Jord. anzichè alla rera capreolata; dalla sua Flora Sic., nella quale non anmette la specie di Jordan per la Sicilia, non si rileva con certezza a quale specie li abbia poi riportati, poichẻ per la $F$. ambigua, alla quale di per sinonimo $F$. speciosa Lojac. non Jord., non cita Lampedusa. Nicotra (Le Fum. It. p. 45) cita di Lampedusa la $F$. capreolata var. speciosa soltanto sulla fede di Lojacono. D'altra parte peró (p. 60) riferisce la $F$. ambigua di Lojacono alla $F$. flabellata Gasp.

Nessuno dei tre botanici che indicano la $F$. capreolata per Lampedusa cita la F. Aabellata, che pure vi è comune. Ció permette di supporre che le loro indicazioni si riferiscano a quest'ultima specie. Escludo quindi per ora la $F$. capreolata dalla florula di Lampedusa.

18. - Fumaria media Lois.; Solla p. 472. F. Gussonei Ross p. 346. F. muralis et F. Gussonii Nicotra Le Fum. It. p. 49 et 53.

Comune nei coltivati, specialmente presso il Porto; Mrz. fi.e fr., Apr. fi. e fr.! - Solla (fi. e fr.), Ross, Zodda (fi. e fr.).

Ross riferisce i suoi esemplari alla var. Gussonei (Boiss.). Fra i miei, alcuni, specialmente fra quelli di Marzo, appartengono a questa varieta, mentre gli altri, come pure quelli di Zodda, sono da ascriversi piuttosto alla var. confusa (Jord.).

19. - Fumaria agraria Lag.; Nicotra Altri ragguagli p. 9. F. major Ross p. 346. F. microsepala Lojac. Esc. p. 15 (ex Nicotra Le Fum. It. p. 57 et Lojac. Fl. Sic. p. 62). F. capreoluta $\gamma$ agraria Fiori e Paol. Fl. an. I p. 479.

Comune nel coltirato; Mrz. fi. e fr.! - Ross, Zodda (fi. e fro.).

Delle due forme spectabilis (Bisch.) e major (Bad.), la pianta di Lampedusa, almeno gli esemplari miei e quelli di Zodda, va riferita piuttosto alla seconda. Difatti ha le brattee uguali o più lunghe dei pedicelli fruttiferi, i'sepali larghi quanto la corolla 
e profondamente dentati, il frutto ottuso appena apicolato, le foglie a lobi generalmente poco discosti.

Lojacono in "Una Escurs, 》 dice della sua $F$. microsepala, che è particolarmente lussureggiante in Lampedusa. Nelia sua Flora Sicula riferisce questa specie alla $F$. agraria, ma non ripete l' indicazione di Lampedusa. Nicotra in «Le Fum. It. 》 p. 58 aveva riferito erroneamente la citazione di Lojacono a Linosa anzichè a Lampedusa e si è corretto in «Altri ragguagli » p. 9.

\section{IV. - Cruciferae.}

20. - Cardamine hirsuta L.; Lojac. Esc. p. 16.

Trovata soltanto da Lojacono.

21. - Sisymbrium officinale L.; Lojac. Esc. p. 16 ; Car. in Parl. Fl. It. IX p. 930.

Trovato soltanto da Lojacono.

22. - Brassica fruticulosa Cyr.; Lojac. Esc. p. 16; Cal. in Parl. Fl. It. IX p. 995; Fiori e Paol. Fl. an. I p. 444.

Alle case di Terranuova, nel coltivato; 14 Mrz. fi. e fr.! Lojacono.

23. - Brassica Tournefortii Gouan: Ross p. 316; Lojac. Esc.

p. 16; Car. in Parl. Fl. It. IX p. 990 ; Fiori e Paol. Fl. an. I p. 447.

Ross (margine dei campi a ponente della colonia), Lojacono (nelle sabbie marittime del Porto, rarissima).

24. - Brassica adpressa (Moench) Bois..; Car. in Parl. Fl. It. IX p. 1008. Sinapis incana Guss. Syn. II p. 203; Calc. p. 28; Lojac. Esc. p. 16.

Gussone, Lojacono.

25. - Brassica Sinapistrum Boiss. Sinapis arvensis Guss. Syn. II p. 202; Calc. p. 28; Lojac. Esc. p. 16 et Fl. Sic. I p. 113. Gussone, Lojacono.

- Emuce sativa Lam.

Sanvisente (L'Isola di Lampedusa p. 63) cita un Cavolo Brassica che chiama anche Ruca, col qual nome suppongo intenda l'Eruca sativa, forse coltivata.

26. - Diplotaxis erucoides (L.) DC.; Gussone II p. 192; Calc. 
p. 27; Lojac. Esc. p. 16; Car. in Parl. Fl. It. IX p. 970; Fiori e Paol. Fl. an. I p. 448.

Gussone, Lojacono.

27. - Diplotaxis scaposa DC. ; Guss. Syn. II p. 193; Calc. p. 28 et 31 ; Nyman Consp. Fl. Eur. p. 49; Ross p. 346 ; Solla p. 472 et 468 ; Lojac. Esc. p. 16 et Fl. Sic. I p. 117; Arc. Comp. Fl. It. $1^{a}$ ed. p. 47 et $2^{a}$ ed. p. 270; Car. in Parl. Fl. It. IX p. 967; Fiori e Paol. Fl. an. I p. 448.

Una delle piante più comuni in Lampedusa, tanto nel coltivato quanto nella parte incolta dell'isola; Mrz. fi. e fr., Apr. fi. e fr.! - La Billardière (1787), Ross (il quale l'ha distribuita nella $V^{a}$ Centuria del suo Herb. Siculum n. 407, Apr. 1904 fi. e fr.). Solla ( $\mathrm{rr}^{\mathrm{r}}$ ), Lojacono (il quale pure l' ha distribuita nelle sue Plantae Ital. sel. n. 59), Zorda (fi. e fr.). Gussone, a causa della stagione inoltrata in cui visito Lampedusa, non ve la vide.

Ho trovato in gran numero, in quest' anno come nel 1873 , gli esemplari nani, alti 2-3 pollici come dice Gussone (2-4 pollici come dice De Candolle nella descrizione originale « Regni Veget. Syst. nat. II p. 635 ») ed anche più piccoli, non più alti di $1-2 \mathrm{~cm}$. Ma sono pure comuni gli esemplari rigogliosi, alti oltre $30 \mathrm{~cm}$., ampiamente ramificati fin dalla base in modo da non poterne distendere uno intero su di un foglio d'erbario. Questa differenza di sviluppo era giá stara notata da Solla p. 468. Il nanismo, che osservasi anche in altre Diplotaxis, non è dunque una caratteristica della. specie, ma un prodotto delle circostanze che danno origine alla microflora, e sará forse ancora più frequente in stagione più precoce. I petali sono lunghi il doppio del calice $(6-S$ mm.) e grandi come quelli della $D$. muralis. Ha dunque torto Gussone che li dice poco più lunghi del calice. Questo errore è ripetuto da Parlatore Fl. It. nella descrizione italiana, quantunque nella descrizione latina (di Caruel) sia detto giustamente «floribus grandibus ». Lo stesso errore è ripetuto anche nella Flora anal. di Fiori e Paol. Nella figura di Delessert (Icon. sel. II tab. 90) i petali sono rappresentati di una lunghezza doppia del calice, il che corrisponde al vero. Lojacono Fl. Sic. dice con ragione «floribus e maximis».

Come notansi grandi differenze nella statura, cosi variano molto i diversi esemplari per la lunghezza dei peduncoli e dolle 
silique e per la foliazione dei fusti. I più grandi, con fusti foliosi nel loro quarto inferiore, con foglie pennatofesse, con peduncoli lunghi fino a $3-4$ ed anche $7 \mathrm{~cm}$, presentano grandissima somiglianza con la D.muralis, da cui sembrano differire soltanto per le silique generalmente meno lunghe. Come si è spesso imbarazzati per distinguere la $D$. tenuifolia dalla $D$. muralis, cosi si rimarrebbe imbarazzati a distinguere da quest'ultima specie cer'ti esemplari della $D$. scaposa, se non fosse per la localitá classica da cui provengono.

Per molto tempo la $D$. scaposa era stata indicata soltanto di Lampedusa. Più tardi fu citata di Sicilia nei Compendî, ma erroneamente come osserva Caruel in Parl. Fl. It. Caruel stesso peró (seguito da Fiori e Paol. Fl. an.) sbağlia indicandola di Malta, poichè le piante maltesi distribuite sotto questo nome da Duthie sono di Diplotaxis viminea. Peró pare che esista sulla costa opposta d'Africa, poichè posseggo degli esemplari di una Diplotaxis raccolti dal M. ${ }^{\text {sc }}$ G. Doria a Tunisi (nelle colline di Belvedere in fiore l' 11 Marzo 1888), che mi sembrano doversi riferire alla D. scaposa.

28. - Carrichtera annua (L.) Prantl; Fiori e Paol. Fl. an. I p. 442. C. Vellae Ross p. 346 ; Lojac. Esc. p. 16 et Fl. Sic. I p. 91 ; Car. in Parl. Fl. It. IX p. 1013. Vella annea Solla p. 472.

In diversi luoghi, specialmente abbondante nei campi, ma trovasi anche lontano dal coltivato, fino verso Capo Ponente e nell'isolotto dei Conigli; Mrz. fi. e fr., Apr. fi. e fr.! - Ross, Solla (fr.), Lojacono, Zodda (fi. e fr.).

29. - Succowia Balearica (L.) Medic.; Ross p. 346; Solla p. 472 ; Lojac. Esc. p. 11, 16 et Fl. Sic. I p. 91; Car. in Parl. IX p. 1015; Fiori e Paol. Fl. an. I p. 441.

Cala Madonna e Cala Galera, tra i frutici nei luoghi più freschi, 11 e 13 Mrz.! - Ross (soltanto in una vallata della Costa Sud ed ivi rara), Solla (fi.), Lojacono (Cala Galera nelle localitá ombrose dei fruticeti, copiosissima, mancante negli altri luoghi; nella. Fl. Sic. dice Cala Madonna), Zodda (Cala Galera ad sepes fi. e fr.).

- Raphanus sativus L.; Lojac. Esc. p. 16.

Lojacono in "Una Esc. » dice: nelle arene marittime del Porto rarissimo. Nella Fl. Sic. non ne riparla. Ad ogni modo sarebbe 
pianta subspontanea, e quindi non l'ammetto fi'a le inquiline di Lampedusa.

30. - Rapistrum rugosum (L.) Berg. : Guss. II i). 141 ; Calc. p. 27; Lojac. Esc. p. 16 et Fl. Sic. I p. 75.

Nel coltivato qua e lá; Mrz. foglie, Apr. fi. e fr.! - Gussone, Calcara, Zodda (fi. e fr. giov.). Stando ad «Una Esc.» si dovrebbe credere che l'avesse raccolto anche Lojacono; ma nella Flora Sic. dice che vi fu raccolto solo da Gussone (dimenticando Calcara).

Tutti i miei esemplari appartengono alla forma lejocarpum Guss. Per i peduncoli lunghetti e piuttosto sottili, e per l'articolo inferiore poco ingrossato, apparterrebbero alla var. Hispanicum (Boiss. e Reut.) b. glabrum Fiori e Paol. Fi. an. Gli esemplari di zodda appartengono alla medesima forma dei miei.

\section{1. - Cakile maritima Scop.}

In una spiagria arenosa presso il Porto, $11 \mathrm{Mrz}$. fi. e fr. giov.! Zodda (prope Portum in arenosis maritimis, fi. e fr.).

Tanto i miei esemplari quanto quelli di Zodda appartengono alla forma a foglie pennatifide.

32. - Alyssum maritimum (L.) Lam.; Guss. Syn. II p. 165; Calc. p. 27; Lojac. Esc. p. 16. Lobularia maritima Solla p. 472. Koniga maritima Car. in Parl. Fl. It. IX p. 755.

Dovunque abbondante, ma specialmente sulle rupi e nei luoghi sassosi ed anche nell' isola dei Conigli; Mrz. fi. e fr., Apr. fi. e fr.! - Gussone, Calcara, Solla (fr.), Lojacono, Zodda (fi. e fr.). -

33. - Capsella Bursa-pastoris (L.) Moench; Guss. Syn. II p. 155; Calc. p. 27; Lojac. Esc. p. 16. Thlaspi Bursa-pastoris Guss. Suppl. Prodr. p. 211.

Nel coltivato presso il Porto; Mrz. fi. e fr., Apr. fr.! - Gussone, Lojacono, Zodda (Cala Galera in cultis, fi. e frro).

34. - Hutchinsia procumbens (L.) Desv.

Luoghi rupestri vicini al mare, a Cala Francese e nell' isola dei Conigli; 8 e $10 \mathrm{Mr}$. fi. e fr.! - Zodda (presso la Lanterna del Porto, in saxosis maritimis, $\mathrm{f} r$.).

Fusti eretti o prostrati, lunghi da meno di $1 \mathrm{~cm}$. a $8-9 \mathrm{~cm}$., coperti, specialmente iu alto, di una pubescenza densa e brevissima; foglie un po' carnose, per la maggior parte intere; fiori piccolissimi. 
35. - Biscutella lyrata L.; Lojac. Esc. p. 16. B. Apula $\beta$ lyrata Car. in Parl. Fl. It. IX p. 650.

'Trovata soltanto da Lojacono.

\section{V. - CAPPARIDACEAE.}

36. - Capparis rupestris Smith; Guss. Notiz. 1) 86 et Syn. II p. 3; Calc. p. 26; Lojac. Esc. p. 16. C. spinosa $\gamma$ inermis Car. in Parl. Fl. It. IX p. 1055.

Fessure delle rupi, Imbriacola, Capo Rupestre, isola dei Conigli; Mrz. e Apr. sole foglie! - Gussone, Calcara (Cala Malucco). Non si capisce se Lojacono abbia egli pure raccolta questa pianta a Lampedusa. Si direbbe di si perchè non la fa precedere dal segno $\bigcirc$; ma si direbbe di no perchè cita Calcara.

Ho visto come questa pianta, presa dagli abitanti di Lampedusa sulle rupi marine, venisse trasportata negli orti ed ivi coltivata.

37. - Capparis spinosa L.; Solla p. 472.

Indicata soltanto da Solla, il quale forse sotto questo nome Linneano intendeva la specie precedente.

\section{VI. - RESEdACEAE.}

38. - Reseda lutea L.; Ross p. 346; Solla p. 472; Lojac. Esc. p. 16; Terracc. in Parl. Fl. It. X p. 176 et 178; Fiori e Paol. Fl. an. I p. 411.

In varî luoghi, ma particolarmente presso il Porto e nel coltivato; Mrz. fi., Apr. fi. e fr. giov.! - Ross, Solla (fi.), Lojacono, Zodda (Cala Galera e Vigna del Sindaco, fi.).

Terracciano 1. c., seguito da Fiori Fl. an., dice che la $R$. lutea di Lampedusa appartiene in parte alla var. Reyeri (Porta e Rigo). Peró nè i miei esemplari nè quelli di Zodda presentano i caratteri di questa varietá, la quale del resto mi sembra assai male distinta.

39. - Reseda alba L.; Solla p. 472. R. fruticulosn Guss. Syn. I P. 530; Caic. p. 26; Lojac. Esc. p. 16; Terracc. in Parl. Fl. It. X p. 183 et 184 .

In varî luoghi; Mrz. fi., Apr. fi. e fr.! - Gussone, Calcara, Solla (fi.), Lojacono, Zodda (a Cavallo Bianco e altrove, in incultis, fi.). 


$$
\text { LAMPEDUSA - RESEDACEAE, CISTACEAE }
$$

Gussone ha distinto una var. glaucescens alla quale lui e Calcara riferiscono le piante di Lampedusa. 'T'erracciano I. c. riferisce gli esemplari di Lampedusa a due forme diverse, la tipica e la Tenoriz.

40. - Reseda luteola L.; Ross p. 346.

Ross (lungo le vie e nei campi), Zodda (Cala Galera in arvis, fi.).

\section{VII. - Cistaceae.}

41. -- Cistus complicatus Lam.; Guss. Syn. II p. 11; Calc. p. 27 et 32 ; Ross. p. 346; Solla p. 472; Lojac. Esc. p. 16 et Fl. Sic. I p. 125; Parl. Fl. It. V p. 583. C. parviforus Guss. Notiz. p. 86; Nyman Consp. p. 70 ; Fiori e Paol. Fl. an. I p. 399.

Questa pianta che era ancora comune in molte parti dell' isola nel 1873, si puo adesso dire quasi rara. L' ho raccolta quest'anno nel Vallone Pollicino, nel Vallone della Madonna e all' Imbriacola, luoghi dove non si può piủ dire, dei suoi cespuglietti bassi e radi, che formino macchia; Mrz. fi. e capsule secche dell' anno avanti, Apr. fi. e fr.! - Gussone (colli aridi calcarei, comunissimo e gregario), Calcara, Ross (frequente nella macchia), 'Solla (fi.), Lojacono (molto meno comune del C. Skanbergi), Zodda (Cala Madonna e all' Imbriacola, fi. e fr.).

Nella Flora Sic. Lojacono dice che cresce in consorzio coi C. salvifolius e C. Monspeliensis. In ciò vi deve essere errore poichè nè lo stesso Lojacono nè altri hanno indicato il $C$. salvifolizes di Lampedusa. - Lojacono l'ha distribuito nelle Pl. It. sel. n. 41 ; e Ross. nel Herb. Sicul. n. $410 \mathrm{col}$ nome di C. parviflomes Lam.

42. - Cistus Monspeliensis L.; (iuss. Notiz. 11. 86 et Sy11. II p. 13; Calc. p. 27 et 32 ; Ross p. 346; Solla p. 472; Lojac. Esc. p. 16; Parl. Fl. It. V [1. 589.

Nel 1873 era ancora uno dei principali elementi costituenti la macchia bassa. Oggi si trova sparso per l' isola, un po' meno scarso del C. complicatus; Mrz. fi. e capsule dell'anno avanti, Apr. fi. e fr. giov. ! - Gussone (ove mancano gli alti frutici, i Cistu. Monspeliensis e parviflorus, l' Euphorbiu dendroides ecc.... li rimpiazzano), Calcara, Ross (molto frequente nella macchia), Lojacono, Solla (fi.), Zodda (fi.). 
Ross indica la var. affinis (Bert. pro specie) come meno frequente del tipo e della specie precedente. Bertoloni stesso peró, nella Flora Italica V p. 339, riferisce il suo C. affinis al C. Monspeliensis come semplice sinonimo.

43. - C. Skanbergi Lojacono Esc. p. 16. C. Skanbergii Fl. Sic. I p. 127-8. C. incanus b. Guss. sec. Lojac. C. Monspeliensis $\beta$ Skanbergi Arc. Comp. Fl. It. 2a ed. p. 291. C. parvifloro $X$ Monspeliensis Fiori e Paol. Fl. an. I p. 400.

Lojacono dice che questa specie è comunissima, specialmente nella parte orientale dell' isola. Zodda, il solo il quale l'abbia raccolta oltre a Lojacono, invece la dice rarissima (Cala Madonna, in aridis dumetis, fi.). - Lojacono l' ha distribuita nelle Pl. It. sel. col n. 42.

Lojacono si meraviglia che una specie tanto distinta e tanto abbondante non sia stata osservata dagli altri botanici che visitarono Lampedusa. Ció si spiega colla supposizione che non è mai stato abbondante come è rimasto nella memoria del suo autore, il che è tanto più probabile inquantochè, al giudizio dello stesso Lojacono, sembra essere un ibrido fra il C. complicatues e il C. Monspeliensis, «delle quali specie mirabilmente divide $\mathrm{i}$ caratteri ».

Gli esemplari raccolti da Zodda corrispondono alla descrizione di Lojacono. Essi sono effettivamente intermedî fra i C. Monspeliensis e complicalus, avvicinandosi di più, per la forma delle foglie, al Monspeliensis, di cui però non hanno affatto la viscusità, ed al complicatus per il colore roseo dei fiori.

- Cistus incanus b. Guss. Syn. II p. 12.

Non considero questa specie come appartenente alla flora di Lampedusa, quantunque sia citata da Gussone, perchè secondo Lojacono Esc., e Fl. Sic. I p. 125 e 127, la citazione di Gussone si riferisce al C. Skanbergi. Calcara non l'ha riportato, ma non si sa se intenzionalmente o per dimenticanza.

44. - Helianthemum glutinosum (L.) Pers.; Parl. Fl. It, V p. 649 ; Solla p. 465 (in nota). Fumana gletinosa Ross p. 346. Frmana viscida Solla p. 472, Lojac. Esc. p. 17.

Raro, in luoghi aridi all'Imbriacola; 12 Mrz. fi., Cala Grande e Capo Rupestre, 29 e $30 \mathrm{Apr}$. fr. $!$ - Ross (nella macchia a Nord della Colonia), Solla (fi. e fr.), Lojacono. 
Ross riferisce i suoi esemplari alla var. viride (Ten.). I miei hanno l'infiorescenza glandolosio-pubescente, e sono intermedî fra il glutinosum tipico e la var. viride.

45. - Helianthemum levipes (L.) Per's.; Guss. Syn. II p. 15 ; Calc. p. 27; Parl. Fl. It. V p. 652. Fumana laevipes Lojac. Esc. p. 17 et FI. Sic. I p. 137.

Raro nei luoghi aridi della costa meridionale; 28 Apr. fi. e fr.! Gussone, Lojacono, Zodda (Cala Galera ad rupes).

46. - Helianthemum guttatum (L.) Mill.; Guss. Suppl. Prodr. p. 180.

Gussone nella Synopsis non fà menzione particolare di Lampedusa per questa pianta, che dice comune nelle isole adiacenti alla Sicilia, ma nel supplemento al Prodromo l'aveva indicata di Lampedusa. É strano pero che nessun altro l'abbia ritrovata, per cui è da ritenersi dubbia la sua presenza in quest' isola.

\section{Vili. - Frankeniaceae.}

47. - Frankenia levis L.; Guss. Syn. I p. 428; Calc. p. 25 ; Lojac. Esc. p. 16 et Fl. Sic. I p. 146; Car. in Parl. Fl. It. IX p. 238 (pro parte).

Gussone, Calcara, Lojacono.

Gussone indica per Lampedusa e Linosa soltanto la var. b. "caulibus villosiusculis subvelutinis calycibus ad angulos sparse strigoso-pilosis», oltre alla $F$. pulverulenta. Lojacono Fl. Sic. riporta la $F$. levis di Lampedusa soltanto sulla fede di Gussone, benchè in «Una Esc. » sembri indicare di averla raccolta egrli pure. Calcara è il solo che dica di avervi trovato tanto la $f$. levis quanto la $F$. hispida, ma non dice della levis se fosse la var. b. o il tipo. E dunque permesso sospettare che la $F$. levis tipica non si trovi in Lampedusa; ed in quanto alla var. b., sarebbe forse meglio unirla alla specie seguente.

48. - Frankenia intermedia IC.; Solla p. 473; Loiac. Esc. p. 16. F. hispida Calc. p. 25. F. levis $\beta$ cinerascens Car. in Pari. FI. It. IX p. 238.

Comune nell' isola, lungo la costa da Punta Sottile a Capo Ponente e fino nel punto più alto dell'isola, all'Albero del Sole; Mrz. in boccio, Apr. fi.! - Calcara, Solla (fi.), Lojacono, Zodda 
(ubique in saxosis maritimis, le forme meno pubescenti; a Cala Grande in aridis, una forma più pubescente, fi. e fr:.).

Tutte le piante che ho osservate e raccolte variano alquanto per la maggiore o minore pubescenza, ma mai tanto da essere riferite nè alla $F$. levis tipica, nè alla vera $F$. hirsuta $L$. Nella citazione delle localita di $F$. levis, nella Flora Italiana, Caruel non dice se si riferiscono al tipo o alla var. cinerascens; in quanto agli esemplari dell' Ajuti, essi, come i miei, appartengono a questa ultima. Sono del resto specie assai male distinte.

49. - Frankenia pulverulenta L.; Guss. Syn. I p. 429; Calc. p. 25; Bert. Fl. It. IV p. 230 ; Lojac. Esc. p. 16; Car. in Parl. Fl. It. IX p. 235.

Frequente nell' isola, ma meno della precedente, sulle rupi marittime e nei luoghi aridi; Mrz. foglie, Apr. fi! - Gussone, Lojacono, Zodda (fi.). - Ross l' ha distribuita nell' Herb. Sic. n. 414, raccolta da un corrispondente fra Maggio e Luglio del 1903, fi. e fr.

\section{iX. - Caryophyllaceae.}

50. - Dianthus rupicola Biv.; Lojac. Esc. p. 18 et Fl. Sic. I p. 163; Tanf. in Parl. Fl. It. IX p. 292. D. Bisignani Guss. Notiz. p. 86 et Syn. I p. 478; Calc. p. 26 et 32 ; Bert. Fl. It. IV p. 552 ; Solla p. 465 (in nota). D. mepicolus Fiori e Paol. Fl. an. I p. 377.

Gussone, Calcara. L' ho ricevuto in fiore da Martorana nei primi giorni di Agosto. Lojacono nella Flora Sicula, col segno! indica di arerlo raccolto in Lampedusa, mentre in «Una Esc.» lo fa precedere da un $\bigcirc$ a significare che non ve lo ha raccolto.

Secondo Gussone se ne trovano a Lampedusa due forme, corrispondenti alle sue varietá a. e c. L'esemplare che ho ricevuto fresco aveva i fiori rosei, e per la strettezza delle foglie sarebbe piuttosto la forma $\mathrm{c}$.

51. - Silene Gallica L.; Guss. Syn. I p. 481; Calc. p. 26; Solla p. 473 ; Lojac. Esc. p. 18; Tanf. in Parl. Fl. It. IX p. 357.

Gussone, Solla (fr.), Lojacono.

52. - Silene nocturna L.; Guss. Syn. I p. 482; Calc. p. 26; Ross p. 346; Lojac. Esc. p. 17 ; Tanf. in Parl. Fl. It. IX p. 365.

Molto frequente nei luoghi coltivati ed incolti; $\mathrm{Mr}$. fi. e fr., Apr. fi. e fr.! - Gussone, Ross, Lojacono, Zodda (fi. e fr.). 
Ross riferisce i suoi esemplari alla var. permixta (Jord.) che Tanfani Fl. It. riferisce alla sua volta alla val'. brachypetala (Rob. et Cast.). I miei numerosi esemplari, come quelli di Zodda, hanno tutti i petali exserti. Alcuni di quelli che ho raccolto in Marzo hanno brattee larghe, quasi cuoriformi alla base, e meriterebbero di essere distinti come forma latibracleata. Altri sono alti pochi centimetri, pauciflori e anche uniflori, ed appartengono alla microflora.

- Silene neglecta Ten. S. reflexa Tanf. in Parl. Fl. It. IX p. 362; Fiori e Paol. Fl. an. I p. 36\%.

Tanfani l. c. dice a torto che questa specie fu raccolta a Lampedusa da Lojacono; questi la cita solo di Linosa. Fiori e Paoletti ripetono l'indicazione errata di 'Tanfani. Non mi consta che alcuno abbia raccolto la S. neglecta a Lampedusa, per cui non la includo fra le piante di quest' isola.

53. - Silene rubella $L_{\text {.. }}$; Lojac. Essc. p. 18 et Fl. Sic. I p. 156 ; Arc. Comp. Fl. It., $2^{a}$ ed. p. 310; Tanf. in Parl. Fl. It. IX p. 399; Fiori e Paol. Fl. an. I p. 365.

L' ho trovata piuttosto frequente nel coltivato; Mrz. fi. e fr., 19 Apr. fi. e fr.! - Lojacono (molto rara; distribuila poi nelle Pl. It. sel. col n. 30), Zodda (Cavallo Bianco in incultis abunde, fi. e fr.).

54. - Silene muscipula L.; Ross p. 346 ; Lojac. Esc. p. 18 et Fl. Sic. I p. 155; Tant. in Parl. Fl. It. IX p. 406; Fiori e Paol. Fl. an. I p. 363.

Nei campi in diverse parti dell'isola, abbondante. L' ho raccolta a Capo Ponente, a Cala Francese, e presso il Porto; Apr. fi. e fr.! - Ross (il quale l'ha poi distribuita nel Herb. Sic.n.416), Lojacono (nei seminati verso Ponente; distribuita nelle PI. It. sel. n. 29).

55. - Silene sedoides Poir.; Ross p. 346; Lojac. Esc. p. 18; Tanf. in Parl. Fl. It. IX p. 401 ; Fiori e Paol. Fl. an. I p. 364.

Abbondante qua e lá sulle rupi in vicinanza del mare, raccolta a Punta Sottile, Cala Croce, Cala Francese e Capo Ponente; Mrz. primi fi., Apr. fi. e fr.! - Ross, Lojacono (Capo Grecale), Zodda (Cala Malucco, fi. e fr.).

56. - Silene inflata Smith; Guss. Syn. I p. 486 ; Lojac. Esc. p. 18. S. Belien b. angustifolia Guss. Suppl. Prodr. p. 124. S. Cucubalus Tanf. in Parl Fl. It. IX p. 345. Silene sp. Solla p. 473? 
In molti luoghi coltivati e incolti; Mrz. fi., Apr. fi. e fr.! Gussone, Lojacono, Zodda (fi. e fr.). Calcara ha dimenticato di citare Gussone. Solla indica di Lampedusa una Silene sp. (in fi.), che dice afline alla $S$. marilima, e che quindi suppongo fosse una forma della $S$. inflata.

Gussone nel Suppl. al Prodr. e nella Synopsis nota che in estate le foglie di questa specie si disseccano qui come a Linosa.

57. - Silene Behen L.; Solla p. 473; Lojac. Esc. p. 17 et Fl. Sic. I p. 154; Ar'c. Comp. Fl. it. $2^{a}$ ed. p. 314 ; Tanf. in Pall. Fl. It. IX p. 408; Fiori e Paol. Fl. an. I p. 363.

Nei campi verso Cala Francese piuttosto abbondante; $30 \mathrm{Apr}$. fi. e fr.! - Solla (fi.), Lojacono (nei luoghi coltivati verso Ponente), Zodda (Cavallo Bianco in incultis, fi. e fr.). Distribuita da Ross nel Herb. Sic. n. 415 raccolta da un corrispondente, fi. e fr. Apr. 1904, e da Lojacono Pl. It. sel. 11. 31.

58. - Cerastium glomeratum Thuill.; Guss. Syn. I p. 506; Calc. p. 26 ; Lojac. Esc. p. 18.

Frequente nei luoghi coltivati ed incolti; Mr\%. fi. e fr.! - Lojacono. Calcara cita questa pianta con un * il che significa trovata solo da Gussone. Questi peró dice soltanto genericamente che la var. b. del C. glomeratum trovasi in tutte le isole adiacenti alla Sicilia.

La pianta di Lampedusa per altro non corrisponde alla var. b. di Gussone, poichè è glandoloso-vischiosa in alto. Trovasi del resto tanto la forma normale con petali piủ lunghi del calice, quanto la forma apetalim (Dum.).

59. - Stellaria media (L.) Cyr.; Guss. Syn. p. 494 (var. a: et b.); Calc. p. 26 ; Lojac. Esc. p. 18; Tanf. in Parl. Fl. It. IX p. 525.

Molto comune in tutta l'isola, nelle sue parti incolte come nel coltivato; Mrz. fi. e fr., Apr. fr.! - Gussone, Lojacono, Zodda (fi. e fr.).

Comunissima è la forma apetala (Ucria), ma trovasi qua e lá anche la forma con petali uguaglianti il calice.

- drenarie serpyllifolia L.; Tanf. in Parl. Fl. It. IX p. 549.

Nella Flora Italiana questa specie è citata a torto per Lampedusa, come trovata da me: Io come l'Aiuti l'abbiamo raccolta soltanto a Linosa. 
60. - Sagina apetala L.; Guss. Suppl. Prodr. p. 49.

Nei luoghi muschiosi e fra le epatiche, non comune; 8 Mrz. fi. e fr.! - Gussone, il quale cita Lampedusa soltanto nel Supplemento al Prodromo, mentre nella Synopsis dice genericamente in Sicilia e nelle isole adiacenti. Calcara ha dimenticato di riportarla.

I miei esemplari sono eccessivamente ridotti, i più non oltrepassando $/ 2 \mathrm{~cm}$. Tutti hanno pochi peli alla base delle foglie e del resto sono glabri; hanno i sepali applicati sulla capsula.

61. - Sagina maritima Don; Lojacono Esc. p. 18; Tanf. in Parl. Fl. It. IX p. 568.

Molto frequente, nana fra le piante della microflora, ed anche sull' isola dei Conigli; Mrz. fi. e fr., Apr. fi. e fr.! - Lojacono, Zodda (fi. e fr'o). Distribuita da Ross Herb. Sic. n. 417 raccolta da un suo corrispondente in fi. e fr: Apr. 1903.

Ho veduto soltanto la forma senza petali.

- Spergula arvensis L.; Tanf. in Parl. Fl. It. IX p. 612.

Citata a torto da Tanfani per Lampedusa come raccolta da Ajuti. Nessuna Spergula abbiamo trovato, Ajuti ed io, in questa isola; solo a Linosa abbiamo raccolto una Spergula, ed era la pentandra.

62. - Spergularia rubra (L.) Pers.; Tanf. in Parl. Fl. It. IX p. 617. Alsine rubra Guss. Syn. I p. 501 ; Calc. p. 26. Arenaria rubra solla p. 473.

Luoghi incolti, aridi, lungo i viottoli ecc.; Mrz. fi., Apr. fi. e fr. ! - Gussone, Calcara, Solla (fi.), Zodda (fi. e fr.). Gussone non aveva citato questa specie per Lampedusa nel Supplemento al Prodromo.

63. - Spergularia diandra (Guss.) Boiss.; Solla p. 473; S. m,bra $\gamma$ diandra Tanf. in Parl. Fl. It. IX p. 617.

In varî luoghi e nell' isola dei Conigli; Mrz. fi., Apr. fi. e fr. ! - Solla (fi.).

64. - Spergularia media (L.) l'er's. S. marim Solla p. 473. S. salina Lojac. Fl. Sic. I p. 184; Tanf. in Parl. Fl. It. IX p. 621. Arenaria helerosperma Guss. Suppl. Prodr. p. 130. Alsine heterosperma Guss. Syn. I p. 501; Calc. p. 26. Lepigonum heterospermum Lojac. Esc. p. 18.

Frequente specialmente dal lato del Porto; Mrz. (i. e fr., Apr. fi. e fr. ! - Gussone, Solla (fi.), Lojacono, Zodda (fr.). 
Le piante raccolte da me e quelle di Zodda hanno la gran magrorionza dei semi apteri, trovandosi soltanto eccezionalmente qualche capsula con parte dei semi cinti da larga ala. I peduncoli sono ben tre volte più lunghi dei fiori, e le capsule oltrepassano di poco il calice. E dunque la forma chiamata nella FI. anal. S. rubra o marina e in Rouy et Foucaud S. Dillenii $\check{\zeta}$ pedicellaris.

\section{X. - Paronychiachae.}

65. - Herniaria cinerea DC.; Guss. Syn. I p. 292; Calc. p. 24; Solla p. 475 ; Lojac. Esc. p. 18. H. glabra $\beta$ hirsuta b. cinerea Fiori e Paol. Fl. an. I p. 335.

Comune nei luoghi aridi fra le microfite; Mrz. fi., Apr. fi. e fr. ! - Gussone, Solla (fi.), Lojacono, Zodda (fi. e fr.).

- Loeflingia Hispanica L.; Fiori e Paol. Fl. an. I p. 336.

Non trovando questa specie citata per Lampedusa da alcun raccoglitore, e dubitando quindi di un errore della Flora analitica, ho pregrato il dott. Terracciano di verificare se nell'erbario di Palermo se ne trovassero esemplari di Lampedusa. Eghli mi ha risposto che si trova effettivamente un esemplare di Loeflingia Hispanica coll' indicazione di Lampedusa, ma però aggiunta posteriormente da Todaro, mentre il nome della specie è scritto da altra mano ignota. Ritengo quindi dubbia la presenza di questa specie in Lampedusa e per questo la escludo dalla numerazione.

66. - Polycarpon tetraphyllum (L.) L.; Guss. Syn. I p. 166 ; Calc. p. 23 ; Solla p. 475 ; Tanf. in Parl. Fl. It. IX p. 625.

Frequente nei luoghi aridi, lungo le vie ecc., ed anche nelisola dei Conigli; Mrz. fi. e fr', Apr. fi. e fr.! - Gussone, Solla (fi.), Zodda (fi. e fr.).

Tanfani l. c. cita per Lampedusa la var. alsinefolizm come raccolta dall'Aiuti. Difatti parte dei miei esemplari si arvicinano a questa rarietá, altri sono nani.

Xi. - Elatinaceae.

67. - Elatine Hydropiper I. var. Gussonei mihi. E. macropoda Solla p. 473; Lojac. Esc. p. 11, 18 et Fl. Sic. I p. 185, non 
Guss. E. campylosperma Ross p. 346, non Seubert. E. Hydropiper \& macropoda Fiori e Paol. Fl. an. I p. 384.

Foliis oblongo-ellipticis vel subspathulatis, inferioribus longe petiolatis, floribus tetrameris, octandris, superioribus subsessilibus, inferioribus pedunculatis, pedunculis folio brevioribus vel parum longioribus, petalis late ellipticis, pulchre roseis, calycem aequantibus, seminibus instar ferri equini vel uncini curvatis.

Ab $E$. Hydropipere praesertim floribus pedunculatis differt; ab E. macropoda magis recedit seminibus valde incurvis, magnitudine et colore petalorum (petala a Gussone in specie sua alba et calyce duplo breviora dicuntur), " nec non pedunculis brevioribus, crassioribus. E. Hydropiper var. pedunculata Moris (=E. campylosperma Seub.) quae nostrae magis affinis est, tamen peduncula longiora, et petala alba calyce breviora habet.

Qua e là nelle piccole pozzanghere che si formano negli incavi della roccia; Marzo fiori! - Ross (luoghi umidi o che furono inondati), Solla (ancora in fiore, ma per la maggior parte disseccata), Lojacono (in tutte le pozzanghere negli incavi del calcare in tutta Lampedusa copiosissima; distribuita nelle Pl. It. sel. col n. 26), Zodda (Cala Croce e Cala Madonna, frutti e ancora qualche fiore). Caruel pubblicando nel vol. IX della Flora It. la famiglia delle Elatinee lasciata manoscritta da Parlatore, ha dimenticato di aggiungere Lampedusa per l' Elatine ivi raccolta e già pubblicata da Ross, Solla e Lojacono.

Nella prima meta di Marzo questa graziosa pianticella era in pieno fiore, e formava, unita di solito alla Bulliarda, dei fitti tappeti verdi alti pochi millimetri, coperti di fiorellini rosa che giungevano appunto al livello del tappeto verde.

Non ho potuto conformarmi all'opinione di chi ha veduto nella Elatine di Lampedusa la. E. macropoda di Gussone. La credenza di Parlatore (Fl. It. IX p. 228), che Gussone attribuisse alla sua specie dei semi poco curvati perchè ne areva visto soltanto esemplari non maturi, non mi pare giustificata. I semi currati devono esserlo fin dalla loro gioventù. La membrana semilunare

"Gli autori in generale dicono della E. macropoda che ha i fiori bianchi. Fa eccezione Lojacono il quale (Fl. Sic.) li dice rosoi, appunto perchè egli aveva veduto la pianta di Lampedusa. 
che trovasi nella curvatura dei semi di E. Hydropiper, membrana menzionata e ligurata da Seubert ${ }^{\prime}$ e che trovasi ben manifesta anche nei semi della Elatine di Lampedusa (che ho visti in grande quantilả negli esemplari di Zodda), prova ad evidenza, mi pare, che la curvatura del seme non avviene all'ultimo stadio del suo sviluppo.

Del resto la $E$. macropoda Guss. ha dato luogo a delle interpretazioni molto diverse, da quelle di Parlatore, Seubert, Grenier et Godron fino a quella di Rouy et Foucaud. Di queste interpretazioni, e delle affinità dell' $E$. macropoda con la E. Fabri Gren. non mi occupo. A me basta di avere rilevato che la pianta di Lampedusa non si puó confondere con la specie di Gussone.

Mi sembra probabile che la Elaline Hydropiper di luoghi più settentrionali, sia rappresentata in regioni più meridionali, e seginatamente nelle nostre isole, da varietà o specie vicarianti che più o meno si scostano dal tipo, ma sono tutte caratterizzate dall' avere i fiori peduncolati. Quella che meno si scosta dal tipo è la Gussonei, quella che più se ne allontana è la macropoda, e fra le due sta la pedunculata di Moris.

La pianta di Lampedusa è una forma terrestre, prostrata e radicante ai nodi, con internodi raccorciati; per il portamento, ma non per i dettagli, somiglia alla figura della E. macropoda. * genuina di Seubert (op. cit. tab. III fig. 9), ed è assai diversa dalla figura della E. Hydropiper var. pedunculata di Moris (Fl. Sard. tab. XX fig. 2).

XiI. - Hypericaceae.

68. - Hypericum Fgyptiacum L.; Guss. Notiz. p. 86, 87 et Syn. II p. 379 ; Calc. p. 28, 31, 32 ; Ross Eine bot. Excurs. p. 346 et Bull. Herb. Boiss. sec. sér. tom. I p. 1210 ; Solla p. 473 ; Lojac. Esc. p. 18 ; Fiori e Paol. Fl. an. I p. 385. H. Egypticum Bert. Fl. It. VIII p. 312. H. heteroslylum Parl. Fl. It. V p. 550. Triadenia Agypliaca Nyman Consp. Fl. Eur. p. 134 ; Lojac. Fl. Sic. I p. 189.

${ }^{1}$ Seubert, Elatinarum Monographia. Verhandl. d. K. Leopuld. Carol. Ak. d. Naturf. XXI, 1845, p. 48 e tab. III, fig. 8 . 
Questo elegante suffrutice è ancora molto abbondante nelle parti non coltivate dell' isola, dove cresce specialmente uelle buche e nei crepacci delle roccie; ma nei luoghi più frequentati dagli ovini è ridotto alla sua base legnosa, con pochi rami mutilati. L'ho raccolto anche sull'isolotto dei Conigli. Era in pieno fiore in Marzo ed ancora fiorito in Aprile! - Gussone, Calcara, Ross, Solla (fi.), Lojacono, Zodda (fi. e fr. giov.). Parlatore, nella Flora Italiana, cita anche come raccoglitori Tineo (che non fu mai a Lampedusa) e dubitativamente Labillardière, per un esemplare dell' erbario Webb (sub Triadenia microphylla Spach.). Distribuito da Ross Herb. Sic. n. 117, e da Lojacono Pl. It. sel. n. 165.

\section{XIII. - Malvaceae.}

69. - Malva Cretica Cav.; Solla p. 473; Lojac. Esc. p. 18. Solla (fi.), Lojacono.

70. - Malva sylvestris L. M. erecta Lojac. Esc. p. 18. M. sylvestris var. erecta (Presl) Fiori Fl. an. II p. 267.

Rara; trovata solo all'Imbriacola; $12 \mathrm{Mrz}$. fi. e fr.! - Lojacono.

Lojacono, in "Una Esc. », dice di avere trovata la var. erecta (Presl); peró nella Flora Sicula cita questa varietà di pochi luoghi in Sicilia e non di Lampedusa. I miei esemplari hanno i carpelli perfettamente glabri ed appartengono al tipo e non a questa varietá.

71. - Malva microcarpa Desf. M. flexuosa b. intermedia Guss. Syn. II p. 223 ; Calc. p. 28; Lojac. Esc. p. 18 et FI. Sic. I p. 195. M. parviflora Ross p. 346 ; Solla p. 473. M. parviflora typica Fiori e Paol. Fl. an. II p. 268.

Molto comune nelle parti coltivate dell' isola, lungo le vie ecc. ma qua e là anche lontano dalle colture, e nell' isolotto dei Conigli; Mrz. fi. e fr., Apr.fi. e fr.! - Gussone, Calcara, Ross, Solla (fi. e fr.), Lojacono, Zodda (fi. e fr.).

Parlatore (Flora Ital. V p. 61) dice che non ha veduto d'Italia alcun esemplare di Malva parviflora L., e ritiene che tutto quanto fu cosi chiamato sia M. microcarpa Desf. Nella Flora analitica invece sono indicate le due specie come frequenti in Italia, e per Lampedusa è indicata la parviflora. Le differenze 
fra queste due specie sono del resto espresse in modo molto diverso dai varî Autori. I miei esemplari di Lampedusa, come quelli di Zodda, sono annui, di solito diffuso-prostrati o ascendenti, e quando sono allungati sono anche flessuosi; hanno da 2 a 5 e fino 7 fiori all'ascella d'una foglia, subsessili, talvolta misti ad altri con peduncoli lunghi fin $2 \% \mathrm{~cm}$, calici accrescenti, non colorati, che arrivano a maturitá ad avere, distesi, un po' più di $1 \mathrm{~cm}$. di diametro, ora patenti ed ora quasi avvolgenti i carpidî, ed i quali sono grandetti ( $2 \mathrm{~mm}$. di diametro) e glabri. I peli del fusto e delle foglie sono a fascetti. Il tubo staminale è glabro. Come si vede, partecipano ai caratteri attribuiti alla parviflora, alla flexuosa e alla microcarpa.

72. - Lavatera Cretica L.; Somm. Piante ined. L. sylvestris Lojac. Fl. Sic. I p. 198.

Presso il Porto e Punta Sottile, abbondante; 27 e 30 Apr. fi. e fr.! - Zodda (Cavallo Bianco, in herbosis, fi. e fr.). Lojacono l'avrebbe raccolta egli pure, secondo che scrive nella Flora Sic., ma in «Una Esc.» non ne areva fatto alcuna menzione.

\section{3. - Lavatera arborea L.}

Rara; ne ho visto solo poche piante non lontano dal Porto e all' Imbriacola, ove mi fu assicurato che era spontanea; Mrz. fi. e fr. giov. :

Gussone areva indicato questa specie soltanto per l' isolotto di Lampione (Notiz. p. 87 et Syn. II p. 229).

\section{XiV. - Geraniaceae.}

74. - Geranium molle L.; Guss. Syn. II p. 216; Calc. p. 28 ; Solla p. 473 ; Lojac. Esc. p. 18 et Fl. Sic. I p. 204 ; Parl. Fl. It. V p. 183.

Comunissimo in tutta l' isola; Mrz. fi. e fr., Apr. fi. e fr.! Gussone, Solla (fi.), Lojacono, Zodda (fi. e fr.).

75. - Geranium rotundifolium L.; Somm. Piante ined.

Verso Capo Rupestre; 30 Apr. fr.!

76. - Geranium Robertianum L.; Calc. p. 28; Solla p. 473; Lojac. Esc. p. 11, 18 et FI. Sic. I p. 207 ; Parl. Fl. It. V p. 201.

Nei luoghi più freschi, non comune, Cala Galera e Sanguedolce; 10 e 13 Mrz. fi. e fr. giov. ! - Galcara, Solla (fi.), Loja- 
cono (la var. b. eriocarpum, nei luoghi ombrosi, rara a Cala Galera), Zodda (Cala Galera in saxosis, fi. e fr.).

Le piante che ho raccolte, come quelle di Zodda, hanno i carpelli perfettamente glabri e non appartengono quindi alla var. eriocaipum.

\section{7. - Erodium cicutarium (L.) L'Hẻrit.}

Non comune; visto soltanto presso il Porto in direzione della Lanterna e della Cala Croce: Mrr. fi. e fr.!

Gli esemplari generalmente piccoli che ho visti, sono da riferirsi alla forma pimpinellifolium (Sibth.) con passaggi alla forma dissection Rouy.

78. - Erodium moschatum (Burm.) L'Hérit.; Somm. Piante ined.

Piuttosto frequente lungo ' $\mathrm{i}$ viottoli e nei campi incolti in varie parti dell'isola; Mr'. fi. e fr., Apr. fi. e fr.! - Zodda (fi. e fr.).

79. - Erodium Chium (Burm.) Willd. E. Chium var. cunea. tum Lojac. Fl. Sic. I p. 209.

Lojacono, nella Flora Sicula, cita questa pianta da lui trovata in Lampedusa, quantunque in «Una Esc. »: non ne faccia menzione.

80. - Erodium malacoides (L.) Willd.; Ross p. 3.16; Solla p. 473.

Comune piủ o meno in tutta l'isola e sull'isolotto dei Conigli; Mrz. fi. e frr, Apr. fi. e fr.! - Ross, Solla (ii. e fr.), Zodda (fi. e fr.).

Oltre alla forma a foglie lobate o inciso-lobate, che è assai comune, trovasi rara la var. subtrilobum (Jord.) forma microphyllum Rouy et Fouc., con foglie sub-pennatilobe piccole, e col becco del frutto non più lungo di $20 \mathrm{~mm}$. Del resto trovasi tanto nana quanto bene- sviluppata.

\section{XV. - LINACEAE.}

81. - Linum Gallicum L.; Lojac. Esc. p. 18.

Cala Croce, Cala Madonna, Capo Ponente e Capo Rupestre, nei luoghi aridi non comune; Mrz. fi., Apr. fi. e fr.! - Lojacono (luoghi erbosi dei fruticeti), Zodda (ver'so Capo l'onente, in aridis et in dumetis, fi. e fr.). 
Sono piú frequenti le forme nane, ridotte fino a 3 ed anche $2 \mathrm{~cm}$.

82. - Linum strictum L.; Solla p. 473. L. inaequale Lojac. Esc. p. 18.

Molto comune in tutta l' isola; Mrz. foglie, Apr. fi. e fr.! Solla (fi. e fr.), Lojacono, Zodda (fi. e fr.).

E molto più frequente la var. axillare Gr. Godr. (= spicatum Lam.), ben caratterizzata; incontrasi peró anche la var. cymosum Gr. Godr. Sono frequenti gli esemplari nani.

\section{XVI. - Oxalidaceae.}

83. - Oxalis cernua Thunberg; Ross p. 346 ; Solla p. 473.

Dovunque; Mrz. fi., Apr. fi.! - Ross (nei campi, talvolta con fiori doppî), Solla (in parte con fiori doppî), Zodda (fiori in parte doppî).

Questa pianta, che al tempo di Gussone pare non esistesse neppure in Sicilia, ma che oramai si puó dire inquilina di gran parte della regione mediterranea meridionale, all'epoca della mia prima visita era già giunta fino a Lampedusa, e quest'anno ho trovato che aveva invaso tutta l'isola, non soltanto nei luoghi coltivati, dove è una vera peste, ma anche lontano dalle colture. É detta "agrodolce» e se ne mangiano gli steli cbe sono piacevolmente aciduli.

Ho notato che le piante a fiore scempio erano rare in confronto di quelle a fiore doppio. Del resto neanche quelle abboniscono i semi. Come si sa, da noi l'oxalis cernua si riproduce sempre agamicamente.

\section{- Oxalis stricta L.}

È citata come inquilina di Lampedusa da Solla Phytob. Beob. p. 465 in nota, probabilmente per errore, come mi scrive il prof. Solla stesso.

XVII. - ZyGOPHYLLACEAE.

84. - Tribulus terrestris L.; Guss. Syn. I p. 462 ; Calc. p. 26 ; Lojac. Esc. p. 18; Parl. Fl. It. V p. 334.

Gussone, Lojacono. 


\section{XVIII. - Rutaceae.}

85. - Ruta bracteosa DC. ; Guss. Syn. I p. 463; Calc. p. 26, 32 ; Solla p. 473 ; Pal'l. Fl. It. V p. 356 ; Lojac. Fl. Sic. I p. 222.

Comune in tutta l' isola; Mrz. fi., Apr. fi. e fr.! - Gussone, Calcara, Solla (fr.), Zodda (fi. e fr.).

\section{XIX. - ANACARDIACEAE.}

86. - Pistacia Lentiscus L.; Guss. Notiz. p. 83,86 et Syn. II p. 627 ; Calc. p. 30,31 , 32 et 34 ; Solla p. 473 ; Lojac. Esc. p. 18 et FI. Sic. I, pars II p. 15.

Questa pianta che al tempo di Gussone era uno dei principali elementi costituenti la macchia, trovasi ancora sparsa per tutta l'isola, ed anche in discreta quantità nei luoghi piú riparati; cresce pure nell' isola dei Conigli; Mrz. fi., Apr. fi.! - Gussone, Calcara, Solla (fi. e fr.), Lojacono, Zodda (fi.).

Gussone dice che i frutti della Pistacia Lentiscus sono mangiati avidamente dai majali e dalle galline; e dice pure che da quei frutti i Maltesi, allora abitanti di Lampedusa, estraevano un olio cattivo col quale facevano candele e sapone (?). Adesso non si fa uso dei frutti del Lentischio, forse perchè non trovasi più in quantitả sufficiente.

\section{XX. - Rhamnaceae.}

87. - Rhamnus Alaternus I., Solla p. 473 ; I.njac. Fl. Sic. I, pars II p. 11.

Solla (fr.). Lojacono nella Flora Sic. l'indica come trovato da lui stesso in Lampedusa, ma in «Una Esc. » non l'aveva citato.

Non l'ho trovato neppure nella mia prima gita. Se ancora esiste deve essere rarissimo ed in qualche lungo di difficile accesso.

\section{XXI. - Papilionaceae.}

88. - Anagyris foetida L.; Guss. Syn. I p. 460 ; Calc. p. 26; Lojac. Esc. p. 18; Tanf. in Parl. Fl. It. X p. 106.

Punta Vilgia; 28 Apr. fr.. - Gussone, Calcara, Lojacono (rarissima nel Vallone dell' Imbriacola). 
89. - Spartium junceum L.; Lojac. Esc. p. 18.

Raro, all' Imbriacola e verso Punta Sottile; 12 Mrz. foglie, 30 A pr. fi.! - Lojacono (rarissimo).

90. - Ononis Sieberi Bess.; Ross p. 346 ; Solla p. 474 ; Lojac. Esc. p. 18 et Fl. Sic. I, pars II p. 38 ; Fiori e Paol. Fl. an. II p. 29.

Frequente nelle parti non coltivate dell' isola; Mrz. foglie, Apr. fi. e fr.: - Ross, Lojacono, Solla (fi.), Zodda (in aridis, fi. e fr.).

91. - Ononis ornithopodioides L.; Ross p. 316 ; Lojac. Esc. p. 18 et Fl. Sic. I, pars II p. 38.

Cala Francese e Sanguedolce; Mrz. foglie! - Ross (sotto i frutici della macchia), Lojacono (rara nei luoghi ombrosi dei fruticeti verso Ponente).

92. - Ononis mollis Savi; Guss. Syn. II p. 259 ; Calc. p. 28 , Solla p. 474; Lojac. Esc. p. 18 et Fl. Sic. I, pars II p. 40.

Comune; Apr. fr. e pochi ultimi fi.! - Gussone, Solla (fi.), Lojacono, Zodda (fi. e fr.).

Sono frequenti gli esemplari nani.

93. - Trigonella Monspeliaca L.; Ross p. 3.17 ; Lojac. Esc. p. 18 et Fl. Sic. I, pars II p. 71.

Frequente fra le microfite nei luoghi aridi; Mrz. fi. e fr. Apr. fr.! - Ross, Lojacono, Zodda (fi. e fr.).

94. - Trigonella maritima Delile; Ross p. 347; Solla p. 47t; Lojac. Esc. p. 18 ; Fiori e Paol. Fl. an. II p. 46.

Frequente nella zona più vicina al mare, ma trovasi anche altrove nei luoghi aridi; cresce pure sull' isolotto dei Conigli; Mrz. fi., Apr. fi. e fr.! - Ross, Solla (fi.e fr.), Lojacono, Zodda (fi. e fr.).

95. - Medicago orbicularis (L.) All.; Solla p. 474.

Nei seminati presso il Porto; $20 \mathrm{Apr}$. fr. ! - Solla (fr.), Zodda (Pozzo del Monaco, in arvis, fr.).

96. - Medicago Helix Willd.; Lojac. Esc. p. 18. M. obscura $\gamma$ Helix Fiori e Paol. Fl. an. II p. 35.

Lojacono l' indica in «Una Esc. » ma non ne fa più menzione nella Flora Sicula, di modo che la sua esistenza in Lampedusa rimane dubbia.

97. - Miedicago tribuloides Desi, ; Solla p. 174 ; Lojac. Esc. p. 18. 
Comunissima, specialmente nei luoghi erbosi ; Mrz. fi. e fr., Apr. fi. e fr.! - Solla (fi. e fr.), Lojacono, Zodda (fi. e fr.).

I numerosi esemplari che ho raccolto, come pure quelli di Zodda, hanno aculei di varia lunghezza. I più si arvicinano molto alla rar. breviaculeata Moris (II. truncatula Gaertı.), presentando, a perfetta maturita, aculei grossi, conici, applicati contro le spire ed appena più lunghi dello spessore di una spira; sono in parte destror'si, in parte sinistrorsi. I frutti giovani presentano le forme crassispina (Vis.) e uncinata (IV.) Fiori FI. an. II p. 36.

98. - Medicago litoralis Rhode; Ross p. 347 ; Solla p. 474. M. Bramii Solla ibid. M. tornata? Lojac. Esc. p. 19.

Isola dei Conigli; Mr\%. fi.! - Ross (anche nella macchia), Solla (fr.), Lojacono, Zodda (alla Salina, in arenosis maritimis, fi. e fr.).

Nella incertezza sul significato dato da Solla e da Lojacono alle $M$. Braunii e $M$. tomala, riferisco le loro piante senz'altro alla $M$. litoralis. Gli esemplari di Zodda come i miei (per quanto si può giudicare senza frutti) appartengono piuttosto alla var. cylindracaea (DC.).

99. - Medicago denticulata Willi.; Solla p. 174. M. hispider. Ross p. 347. M. lappacea Lojac. Esc. p. 18. M. pentacycla Guss. Suppl. Prodr. p. 237. M. Hystrix Guss. Syn. II p. 367 ; Calc. p. 28 ; Lojac. Esc. p. 18.

Molto frequente nei luoghi erbosi delle parti coltivate dell' isola; Mrz. fi. e fr., Apr. fi. e fr.! - Gussone, Ross, Solla (fi.). Lojacono, Zodda (fi. e fr.).

Ho trovato molto piu frequente la var. Impmecea (Dess.) Mroris et De Not. Però ho raccolto pure delle forme che molto si avvicinano alla var. macrocarpa Moris et De Not. $(=$ M. Hystrix Ten.). Gussone cita soltanto quest'ultima, Lojacono l'una e l'altra. Alla prima appartengono gli esemplari di Zodda.

100. - Melilotus parviflorus Desf.; (iuss. Sin. II 1) 3? I; ( Calc. p. 28; Solla p. 474; Lojac. Esc. p. 19.

Nel coltivato presso il Porto; 27 Apr. fi. e fr.! - Gussone, Solla (fi. e fr.), Lojacono, Zodda (alla Salina, in arenusis marjtimis, (i. e fr. giov.).

Gli esemplari di Zodda appartengono alla microflora, essendo ridotti fino a $1 \frac{1}{2}-2 \mathrm{~cm}$. di altezza. 
101. - Melilotus elegans Salzm.; Solla p. 474.

Indicato soltanto da Solla (fr.).

102. - Melilotus sulcatus Desf.; Somm. Piante ined.

Abbondante nel coltivato, e frequente anche lontano dalle colture, come pure nell'isola dei Conigli; Mrz. fi. e fr. giov., Apr. fi. e fi.! - Zodda (fi. e fr.).

La pianta di Lampedusa ha il peduncolo comune assai breve, tutto il racemo piuttosto lasso, che oltrepassa di poco la foglia o piú brevè di essa, e frutti piuttosto grandi. Questa forma pare frequente in Sicilia.

103. - Trifolium suffocatum L.; Guss. Syn. II p. 325; Calc. p. 28 .

Trovato solo da Gussone.

104. - Trifolium glomeratum L.; Guss. Syn. II 1). 326; Calc. p. 28 .

Gussone, Zodda (all' Imbriacola, in herbosis, fi. e fr.).

105. - Trifolium scabrum L.; Guss. Syn. II p. 327 ; Calc. p. 28 ; Solla p. 474.

Gussone, Solla (fi. e fr.).

106. - Trifolium Cherleri I. ; Guss. Syn. II p. 332; Calc. p. 28. Gussone, Zodda (il quale scrive di avere raccolto l'unico esemplare da esso visto all'Aria Rossa, fi. e fro.).

107. - Trifolium stellatum L. ; Fuss. Syn. II p. 334 ; Calc. p. 28.

Solo Gussone.

108. - Trifolium resupinatum L.; Iojac. Fl. Sic. I, par's I[ p. 86. T. suaveolens Guss. Syn. II p. 344 ; Calc. p. 28.

Solo Gussone.

109. - Trifolium tomentosum L.

Zodda solo (Cavallo Bianco in incultis, fi. e fr.).

110. - Trifolium nigrescens Viv.; Guss. Syn. II p. 339 ; Calc. p. 28; Lojac. Fil. Sic. I, pars II p. 81 .

Solo Gussone.

111. - Anthyllis tetraphylla I. Pinysanthyllis Solla 1. 474.

Sanguedolce, Aria Rossa, Capo Ponente; 10, 12 Mrz. fi., 28 Apr. fr. ! - Solla (fi. e fr ), Zodda (Cala Croce e verso Capo Ponente, in arvis, fi. e fr. giov.).

112. - Lotus cytisoides I.: Guss. Suppl. Prodr. L. cytisoides et $L$. coronillaefolius Guss. Syn. II p. 356 ; Calc. p. 28 ; Lojac. 
Esc. p. 19. L. cytisoides var. coronillaefolius Solla p. 474; Lojac. Fl. Sic. I, pars II p. 114.

Comunissimo e formante spesso, vicino al mare, dei densi ed alti pulvinuli, ed anche all' isola dei Conigli; Mrz. fi., Apr. fi. e fr.! Gussone, Solla (fi. e fr.), Lojacono (L. cytisoides), Zodda (fi. e fr.).

Nel Supplemento al Prodromo Gussone indicava solamente il L. cytisoides. I miei esemplari e quelli di Zodda presentano forme molto diverse fra le quali se ne potrebbero trovare talune corrispondenti alla var. coronillaefolius; ma fra queste forme esistono passagri cosi graduati che riesce impossibile separarle in modo soddisfacente.

113. - Lotus edulis L.; Guss. Syn. II p. 350 ; Calc. p. 28 ; Solla p. 474 ; Lojac. Esc. p. 19.

Comunissimo nella maggior parte dell' isola; Mrz. fi., Apr. fi. e fr.! - Gussone, Solla (fr.), Lojacono, Zodda (fi. e fr.).

114. - Lotus ornithopodioides L.; Solla p. 474.

Presso il coltivato, frequente; Mrz. fi. e fr., Apr. fi. e fr.! Solla (fi. e fr.).

115. - Astragalus hamosus L. ; Guss. Syn. II p. 313 ; Calc. p. 28; Solla p. 474; Lojac. Esc. p. 19.

In varî luoghi, specialmente nelle parti coltivate; Mrz. fi., Apr. fi. e fr.! - Gussone, Solla (fr.), Lojacono.

116. - Astragalus Boeticus L.; Guss. Syn. II p. 313; Calc. p. 28; Lojac. Fl. Sic. I, pars II p. 117.

Solo Gussone.

117. - Biserrula Pelecinus L.; Lojac. Esc. p. 19.

Presso il Porto; $28 \mathrm{Apr}$. in fr.! - Lojacono.

118. - Scorpiurus subvillosus L.; Guss. Syı. II p. 307; Calc. p. 28; Solla p. 474; Lojac. Esc. p. 19.

Frequente, specialmente nelle parti coltivate; Mrz. fi., Apr. fr.! - Gussone, Solla (fr.), Lojacono, Zodda (fi. e fr.).

119. - Coronilla scorpioides (I.) Koch; Guss. Syu. II p. 303; Calc. p. 28. Ornithopus scorpioides Guss. Suppl. Prodr. p. 229. Arthrolobizm scorpioides Lojac. Esc. p. 19.

Nel coltivato, frequente; Mrz. fi., Apr. fi. e fr.! - Gussone, Lojacono, Zodda (fi. e fr.).

120. - Hippocrepis multisiliquosa L.; Lojac. Esc. p. 19; Fiori e Faol. Fl. an. II p. 93. 
Lojacono è il solo che in «Una Escurs. citi questa specie di Lampedusa, poichè Fiori Flora an. non l'a altro che ripetere la sua citazione; però nella Flora Sicula non ne parla più e quindi non mi sembra improbabile che l' indicazione in «Una Escurs. » invece che a Lampedusa dovesse riferirsi a Linosa, dove questa pianta è comune.

121. - Hippocrepis ciliata Willd.; Ross p. 347; Solla p. 474; Lojac. Esc. p. 19.

Presso il Porto, a Cala Francese, verso Terranova e a Capo Ponente; Mrz. fi: e fr., A pr. fr.! - Ross (a S.-E. del Porto fra i sassi), Solla (fr.), Lojacono, Zodda (Cavallo Bianco, in saxosis haud communis, fi. e fr.).

122. - Hippocrepis unisiliquosa L.; Lojac. Esc. p. 19.

Molto frequente, specialmente nei luoghi aridi; Mrz. fi. e fr., Apr. fr.! - Lojacono, Zodda (fi. e fr.).

123. - Hedysarum capitatum Desf.; Somm. Piante ined.

Cala Pisana; 30 Apr. fi. e fr.!

\section{- Pisum sativum L.}

Questa specie trovasi qua e lá subspontaneà vicino al coltivato; $19 \mathrm{Apr}$. fi.!

124. - Lathyrus Ochrus (¿.) DC.; Lojac. Esc. p. 19.

Lojacono solo.

125. - Lathyrus Clymenum L. L. tenuifolines Guss. Syn. II p. 278; Calc. p. 28; Lojac. Esc. p. 19. L. Clymenum a tenuifoliues Solla p. 474.

Qua e lá, luoghi coltivati e incolti; Mrz. fi., Apr. fi. e fr.: Gussone, Solla (fi. e fr.), Lojacono, Zodda (fi.).

Le piante che ho viste di Lampedusa hanno per lo più foglie strette e lineari, ed appartengono quindi alla forma tenuifolizs (Desf.) indicata da Gussone, da Solla e da Lojacono.

126. - Lathyrus Cicera L.

Qua e lá nelle parti coltivate; Mr\%. fi. e fr. giov. !

127. - Vicia sativa L.

Frequente specialmente nelle parti coltivate; Mrz. fi. e fr. giov., Apr. fr.! - Zodda (fi. e fr.).

I miei esemplari e quelli di Zodda per la forma delle foglie si avvicinano alquanto alla forma cordata (Wulf.). 
128. - Vicia maculata Presl; Lojac. Esc. p. 19. V. sativa $n m \alpha$ culata Fiori e Paol. Fl. ari. II p. 112.

Lojacollo solo.

129. - Vicia peregrina L.; Lojac. Esc. p. 19.

Solo Lojacono (luoghi arenosi coltivati del Vallone Imbriacola).

130. - Vicia atropurpurea Desl.; Solla p. 474 et flij in nota ).

Indicata solamente da Solla (fi.).

131. - Vicia pseudocracca Bert.; Guss. Syn. II p. 295 ; Calc.

p. 28; Lojac. Esc. p. 19.

Gussone e Lojacono.

\section{2. - Vicia triflora Ten.}

Qua e lá nei campi in riposo, ed in taluni abbondante; Marz. fi. e fr. giov. : - Me ne sono stati mandati dei baccelli e dei semi maturi in Giugno dalla guardia campestre Martorana.

La pianta di lampedusa è perfettamente conforme agli esemplari autentici di Gussone e di Tenore delle Puglie, di Lucania e di Calabria, conservati nell'Erbario Centrale di Firenze.

Moris (Flora Sardoa) credette di dovere identificare questa specie con la Vicia calcarata Desf., e d'allora in poi la V.trifora 'Ten. fu generalmente passata in sinonimia. 'I'uttavia Bertoloni (Fl. It. VII p. 505-6) e Gussone (Syn. II p. 291-2) non accettano questa riunione, ed a quanto mi sembra, con ragione. Difatti nella descrizione originale della $T^{\%}$. calcarata nella Flora Atlantica, è detto: peluncolo uni-, raramente bifloro, due a tre volte piú corto della foglia, calice glabro, corolla celeste pallido, foglioline mucronate, legume lungo $2 \mathrm{~cm}$.; ed alla descrizione corrisponde l'esemplare di Desfontaines conservato nell'Erbario Webb. Nella Vicia Iriflora autoptica invece, ' come in quella di Lampedusa, vediamo il peduncolo 3-5floro, poco più corto della foglia, il calice pubescente-irto, le foglioline emarginate mutiche. La corolla poi sul vivo, negli esemplari di Lampedusa, è di un bel rosso (che peró seccando diventa violaceo), ed i legumi maturi sono lunghi $3 \frac{1}{2}-4 \%$ cm. Inoltre Desfontaines dice della sua $V$. calcarata "affinis Viciae sativae»,

1 Mi riferisco alle piante autoptiche anzichè alla descrizione di Tenore, perché questa è insufficiente. La figura della Fl. Nap. è discreta. 
mentre nessuna affinitá con questa presenta la Vicia triflara. Tenore difatti dice della sua specie che hil l'habitus della V. sylvalica.

Peró le descrizioni della $V$. calcarata posteriori a quella di Desfontaines (p. es. quelle di Moris Fl. Sard., di Battandier et Trabut Fl. d'Algérie, ed anche di Boissier Fl. Or.), sono tali che vi si potrebbe fare entrare la $V$. triflora, è cio perché appunto è stato allargato il senso originario di $V$. calcarata per includervi altre specie. Specie affini alla $V$. calcarala (che peró non si arricinano magoriormente alla $V$. triflora), e da alcuni riunite ad essa, sono pure $T$. biflora Desf. e $V$. cinerea Marsh. Bieb. Se si vuole allargare talmente l'ambito della $V$. calcarata da includervi le $V$. triflora Ten., biflora Desf. e cinerea M. B., se ne fa una specie complessiva che non corrisponde più al concetto del suo autore. Per questo ho creduto di dovere ripristinare il nome di Tenore e ridividere quello che era stato da molti rimito.

Le radici di questa Vicia presentano dei tubercoli radicali, come del resto quasi tutte le leguminose di Lampedusa.

Ho visto esemplari di Vicia triftora uguali a quelli di Lampedusa ed a quelli autentici di Tenore, anche di Algeria, del Cairo e di Grecia.

Cosa sia la Vicia o Cracca calcarata delle flore francesi, collocata da. Grenier e Godron nella rubrica a fiori piccoli, pallidi ed a legumi $2-4$ spermi, non so; certo non è la $V$. triflora Ten. In quanto alla $V$. calcarala Moris (non Desf.), essa va considerata come sinonimo di $V$. triflora Ten., come lo fa Bertoloni.

133. - Vicia Ieucantha Biv. Ervum parviflorem Somm. Piante ined.

Molto frequente ed abbondante, specialmente nelle siepi di fichi d'India, dore cresce alta e rigogliosa; Mrz. fi. e fr. giov., Apr. fr'.! Grazie alle piante in buono stato raccolte quest'anno ho potuto rettificare la mia prima determinazione, fatta sopra gli esemplari secchi ed incompleti raccolti nell'Aprile del 1873.

134. - Eryum Lens L.; Somm. Piante ined.

Nel coltivato presso il Porto; 20 Apr. fi. e fr.

Per le piccole dimensioni del legume $(8-9 \times 5-6 \mathrm{~mm}$.) e del 
seme (4 mm.), che è anche meno compresso che nel tipo, corrisponde all' $E$. dispermum Roxb. $=E$. Lens var. minor Ten. $=$ Lens esculenta 3 subsphxerusperma Godr. in Gr.Godr. Fl. de Fr. I p. $476=$ Lens disperma Rony Fi. de F1". V p. 205 = Vicia Lens $x$ typica b. disperma Fiori Fl. an. II p. 122.

135. - Ceratonia Siliqua L.; Guss. Notiz. p. 81, 86 et Syn. II p. 646 ; Calc. p. 30, 32 et 35; Solli p. 473; Lojac. Esc. p. 12, 19 et Fl. Sic. I, pars II p. 161; Car. in Parl. X p. 102.

Trovansi ancora in discreto numero le piante di Carubbo, specialmente nei valloni più protetti, ma sono in generale di piccole dimensioni. Soltanto in alcuni orti se ne vede qualche pianta meno meschina; Mrz. e Apr. fr. giov.! - Gussone (non oltrepassano l'altezza di 10 a 12 piedi ma benchè poco alti si caricano di frutti), Calcara (circa 300 piante selvatiche sparse qua e lí per l' isola), Solla (fr.), Lojacono (pochi alberi o al'busti qua e lá nella parte meridionale).

\section{XXII. - ROSACEAE.}

136. - Rubus ulmifolius Schott. R. Pruticoses Guss. Suppl. Prodr. p. 157. R. Dalmaticus Guss. Syn. I p. 568 ; Solla p. 465 (in nota).R. Dalmaticus b. rotundifolius Calc. p. 26; Lojac. Esc. p. 19. R. ulmifolius var. rolundifolius Lojac. Fl. Sic. I, pars II p. 169 (in nota).

Presso la chiesa della Madonna in un orto, una sola pianta; Mrz. fi.! - Gussone (satis rarus), Calcara (alla Madonna), Zodda (Cala Madonna, foglie).

Questo rogo, giả detto raro da Gussone, si è mantenuto, almeno dal tempo di Calcara in poi, sernpre nel medesimo luogo, alla Madonna. La pianta che ho vista, vecchia, rigogliosa e lungamente scandente (lunga 3-4 m.), mi fu assicurato essere la sola esistente adesso nell' isola.

137. - Poterium muricatum Spach; Solla p. 474.

Trovato solamente da Solla (fi.).

$$
\text { XXIII. - Lythraceae. }
$$

138. - Lythrum Graefferi Ten., Solla 1) 175; : Lojac. Esc. p. 19. L. aculangulem Lojac. Fl. Sic. I, pars II p. 229. 
Intorno a qualche pozzanghera, raro; Mrz. foglie! - Solla (ii.), Lojacono.

139. - Lythrum Hyssopifolia L.; Ross p. 347.

Nei luoghi che più a lungo mantennero l'umidita, consociato a Elatine e Bulliarda: Mrz. foglie, Apr. fi. e fr.! - Ross, Zodda (fi. e fr. giov.).

I numerosi esemplari che ho raccolti, come pure quelli di Zodda, sono assai piccoli (al massimo 6-7 $\mathrm{cm}$.), decombenti, ed hanno calici a $8-10$ denti.

\section{XXIV. - Crassulaceae.}

140. - Tillaea muscosa L.: Guss. Syn. I p. 167; Calc. p. 23 ; Lojac. I'l. Sic. I, pars II p. 213; Car. in Parl. Fl. It. IX p. 92.

Abbastanza frequente $n$ ei terreni aridi ed in alcuni luoghi abbondante fra le microfite, anche sull'isola dei Conigli; Mrz. fi. e fr.: - Gussone, Zodda (fi. e fr.).

141. - Bulliarda Vaillantii (Willd.) DC.; Guss. Syn. I p. 211 ; Calc. p. 23 ; Lojac. Fl. sic. I, lar's II 1). 21.1; Carr. in Parl. Fl. It. IX p. 91.

L' ho trovata sempre associata alla Elatine negli incavi della roccia dove l'acqua si raccoglie in piccole pozzanghere che in Marzo non et'ano ancora prosciugate. Tali pozzanghere trovansi qua e lá sparse per l' isola; Mrz. fi. - - Gussone, Zodda (fr.).

142. - Umbilicus pendulinus DC.; Solla p. 475.

Solla solo (fi. e fr.).

143. - Umbilicus horizontalis DC. Cotyledon horizontalis Guss. Syn. I p. 514 ; Calc. p. 26 ; Lojac. Esc. p. 19 ; Car. in Parl. FI. It. IX p. 16.

Frequente sulle rupi; Mrz. primi fiori e frutti secchi dell'anno avanti, Apr. fr.! - Gussone, Calcara, Lojacono.

144. - Sedum stellatum L.; Solla p. 475.

Solla solo (fi.).

145. - Sedum Cepaea I. S. galioides Solla p. 495.

Solla solo.

146. - Sedum dasyphyllum L.; Car. in Parl. Fl. It. IX p. 67. S. glanduliferm Guss. Syn. I p. 517 ; Lojac. Esc. p. 19. S. glanduliferum et $S$. Neapolilanum Calc. p. 26. 
Frequente sulle rupi, anche nell' Isola dei Conigli; Mrz. foglie, Apr. fr. ! - Gussone, Calcara, Lojacono, Zodda (fi.).

Calcara, che tiene distinto il S. Neapolitanum Ten. dal S. gitanduliferim Guss., indica a torto con due ** che il prime fu raccolto anche da Gussone. Questi per Lampedusa indica soltanto il S. glanduliferum, e non conosce il $S$. Neapolilamum altro che di Pantelleria. Io considero l'uno e l'altro come varieti del S. dasyphyllum. Tutte le piante che ho vedute di Lampedusa appartengono alla val'. glanduliferum (Guss.).

147. - Sedum caespitosum (Cav.) DO.; Solla p. 475.

Raro, 30 Apr. fr.! - Solla (fi.), Zodda (ad Est dell'abitato raro, $f r$.$) .$

148. - Sedum litoreum Guss.; Ross p. 347 ; Lojac. FI. Sic. I pars II p. 214.

Rupi e terreni aridi, frequente, anche nell' Isola dei Conigli; Mr\%. foglie, Apr. fi. e fi.! - Ross. Lojacono, nella Flora Sicula, indica il Sedum liloreum di Lampedusa come raccolto da sé, e cita la sua Fl. Lamp. p. 18. Peró in "Una escurs. botan: in Lamp. " a p. 18 non vi è alcun Sedzm, e a p. 19 sono citati soli i S. glanduliferum e S. Nicaeense.

L' ho trovato per lo più fra le microfite, ridotto ad esigue dimensioni $(2-3 \mathrm{~cm}$.), e con pochi fiori, talvolta uno solo o due. Ma vi sono tutti i passaggi alle forme più alte e ramificate. Gussone ha detto, erl altri hanno ripetuto, che per l'abito questa specie somiglia al $S$. saxatile. Sul secco, quando non è più manifesto il colore dei petali, e suno cascate per la maggior parte le foglie, mi pare che somigli maggrormerte al Sedum mubens. Da esso però si distingue bene, anche in quello stato, oltre che per la mancanza di pubescenza glandolosa, per la forma dei follicoli che sono più grossi alla base ed ottusi all'apice, e per essere caduco lo stilo. A perfetta maturitá, i follicoli essendo quasi triangolari, il loro orlo interno è orizzontale nella sua metá superiore, mentre l'orlo esterno è ascendente.

149. - Sedum Hispanicum L.; Solla p. 475.

Indicato solamente da Solla (fi.).

150. - Sedum Nicaeense All.; Calc. p. 26 ; Lojac. Esc. p. 19. S. allissimem Guss. Suppl. Prodr. p. 134; Solla p. 475 ; Lojac. Fl. Sic. I, pars II p. 221 ; Car, in Parl. Fl. It. IX p. 47. S. ru- 
fescens Guss. Syn. I p. 521 (confr. vol. II Add. et Emend. p. \$26).

Sulle rupi e nel terreno arido frequente; Mrz. e Apr. sole foglie! - Gussone, Calcara, Solla (sfiorito), I。ojacono.

\section{XXV. - Mesemrryanthemaceae.}

151. - Mesembryanthemum nodiflorum L.; Guss. Syn. I p. 554 ; Calc. p. 26 ; Solla p. 475 ; Lojac. Esc. p. 19.

Molto comune nei terreni aridi e sulle rupi presso il mare; anche nell' isola dei Conigli; Mrz. foglie, Apr. fi.! - Gussone, Calcara, Solla (fi.), Lojacono, Zodda (fi.):

152. - Mesembryanthemum crystallinum L.; Guss. Syn. I p. 554; Calc. p. 26 ; Solla p. 475 ; Lojac. Esc. p. 19 et Fl. Sic. I, pars II p. 241 ; Fiori e Paol. Fl. an. I p. 326. M. glaciale Guss. Suppl. Prodr. p. 155.

Non comune; luoghi incolti presso il Porto e isola dei Conigli; Mrz. foglie, 19 Apr. fi.! - Gussone (sotto al Capo di Ponente e sotto al Castello), Solla (foglie), Lojacono (luoghi coltivati).

- Mesembryanthemum acinaciforme L. Solla p. 475.

É citato dubitativamente (in fiore) da Solla, probabilmente perchè non persuaso della sua naturalizzazione. Altri non lo citano. Io non l' ho visto.

\section{XXVi. - Cactéceae.}

153. - Opuntia Ficus Indica Mill.; Guss. Notiz. p. 85 et Syn. I p. 549 ; Calc. p. 26 et 34 ; Solla p. 475 ; Lojac. Esc. p. 19. Cactus Opuntia Guss. Suppl. Prodr. p. 153.

Adoperato per far siepi, trovasi in grandissima quantità nelle parti coltivate dell' isola; Mrz. e Apr. senza fi. nè fr.! - Gussone, Calcara (alla Madonna, alla Casina, resti della piantagione fatta dai Maltesi), Solla (fi.), Lojacono.

\section{XXViI. - Myrtaceae.}

- Punica Granatum L. ; Guss. Notiz. p. 97 ; Sanvisente p. 61.

Gussone ha trovato nel Vallone della Madonna un Melagrano, avanzo di antiche colture, e Sanvisente riporta questa indica- 
zione. Calcara non ne fa menzione. Ora il Melagrano si coltiva negli orti con altri alberi da frutto, ma in piccola quantità; non l' ho visto inselvatichito.

154. - Myrtus communis L.; Guss. Suppl. Prodr. I p. 153 et Syn. I p. 550 ; Lojac. FI. Sic. I, pars II p. 225.

Gussone solo.

Io ne ho veduto una sola pianta, grossa e vecchia, in un orto; Mrz. foglie! Mi fu assicurato che vi era stata piantata, e che questa specie non esisteva spontanea in Lampedusa. Gussone essendo il solo che la cita, se ne potrebbe dedurre che fosse stata distrutta dopo il suo tempo. Peró è da notare che Calcara non la riporta (non si sa se intenzionalmente o per dimenticanza) e che nessun esemplare di Lampedusa esiste nell' Erbario di Gussone, come me ne ha informato il prof. Delpino. Inoltre Gussone, parlando della macchia di Lampedusa in "Notizie », non fa alcuna menzione del Mirto. Tutto ció lascia qualche dubbio (non ostante i versi dell' Ariosto !) sull'attendibilità dell' indicazione di Gussone nel Suppl. al Prodr. e nella Synopsis.

\section{XXVIII. - UMBELLIFERAE.}

155. - Eryngium dichotomum Desf.; Guss. Syn. I p. 303; Calc. p. 24; Solla p. 475, 467 et 468; Lojac. FI. Sic. I, pars II p. 261 ; Car. in Parl. Fl. It. VIII p. 211 ; Fiori e Paol. Fl. an. II p. 149. Eryngium sp. Lojac. Esc. p. 19.

Comune nei terreni aridi; Mrz. foglie, Apr. fi. ! - Gussone, Solla (piccolo e prostrato sulla costa Nord, eretto ed alto $20-30 \mathrm{~cm}$. sulla costa Sud, fi.), Lojacono (questi mi scrive che la specie indeterminata della sua «Esc. » era l'E. dichotomum), Zodda (fi.).

Nei terreni piu aridi trovasi spesso ridotto quasi, ed anche interamente, acaule.

156. - Smyrnium Olusatrum L.; Guss. Syn. I p. 314; Calc. p. 24; Solla p. 475; Lojาc. Esc. p. 20 et Fl. Sic. I, pars II p. 273; Car. in Parl. Fl. It. VIII p. 479.

Molto comune specialmente nella parte coltivata dell'isola; rigoglioso fra i fichi d'India e nei terreni più freschi; Mrz. foglie, Apr. fr.! - Gussone, Solla (fi. e fr., ed in pieno frutto sullo scoglio dei Conigli), Lojacono, Zodda (fi. e fr.). 
157. -- Bupleurum glaucum Rob, et Cast. in DC.; Guss. Syn. I p. 309 ; Calc. p. 24; Solla p. 475 ; Lojac. Esc. p. 19 et Fl. Sic. I, pars II p. 269. B. semicompositum Car. in Parl. FI. It. VIII p. 412.

Molto comune nei luoghi aridi; Mrz: foglie, Apr. fi. e fr.! Gussone, Calcara, Solla (fi. e fr.), Lojacono, Zodda (fi.).

Trovasi per lo più ridotto a piccolissime dimensioni $(1-3 \mathrm{~cm}$.), però se ne incontrano esemplari alti $20 \mathrm{~cm}$. e piú; alcuni hanno le brattee leggermente seghettate al margine, altri le hanno liscie (forma leve Le Grand).

158. - Bupleurum subovatum Link; Car. in Parl. Fl. It. VIII p. 391 ; Lojac. Fl. Sic. I, par's II p. 268; B. protractum Solla p. 475 .

Frequente nel coltivato; Mrz. fi., Apr. fr.! - Solla (fr.).

$\mathrm{L}$ ' ho veduto per lo più nano $(10-5 \mathrm{~cm}$. e talvolta meno) e poco ramificato.

159. - Ptychotis ammoides (L.) Kioch. P. verticillata Guss. Syn. I p. 321 ; Calc. p. 24 ; Lojac. Esc. p. 19. Seseli verticillatum Guss. Suppl. Prodr. p. 79. Apium Ammios Car. in Parl. Fl. It. VIII p. 436.

Gussone, Lojacono.

160. - Scandix Pecten-Veneris L.; Solla p. 475; Lojac. Esc. p. 20; Car. in Parl. Fl. It. VIII p. 377.

Comunissima in tutta l' isola, tanto nel coltivato quanto lontano da esso; Mrz. fi. e fr., Apr. fr.! - Solla (fr.), Lojacono, Zodda (fr.).

161. - Seseli Bocconei Guss.; Guss. Syn. I p. 322; Calc. p. 24; Lojac. Esc. p. 19 et Fl. Sic. I, par's II p. 279; Car. in Parl. Fl. It. VIII p. 313; Fiori e Paol. Fl. an. II, p. 168.

Gussone e Lojacono.

162. - Foeniculum vulgare Mill. F. piperahem Guss. Syn. I, p. 324; Calc. p. 24; Lojac. Esc. p. 19. Foeniculum sp. Solla p. 475. F. capillaceum $\beta$ piperitum Car. in Parl. Fl. It. VIII 1. 310. Neum piperalum Guss. Suppl. Prodr. p. 79.

Qua e lá; Mrz. e Apr. foglie sole! - Gussone, Lojacono, Solla (foglie), Zodda (foglie).

Ho trovato tanto il tipo capillacerm quanto la var. piperitum. Anche fra le piante raccolte da Zodda vi sono le due forme. 
163. - Magydaris pastinacea (Iam.) l'anletti; Fiori e Panol. Fl. an. II p. 205. MI. tomentosa Guss. Syn. I p. 345 ; Calc. p. 24 ; Ross p. 347; Lojac. Esc. p. 19 et Fl. Sic. I, pars II p. 286; Car. in Parl. Fl. It. VIII p. 233. Athamantha panacisfolia Guss. Suppl. Prodr. p. 70.

Qua e li piuttosto rara, frequente invece nel Vallone dell'Imbriacola; Mrr. foglie, Apr. fi. e fr.! - Gussone, Ross (Iungo le vie e margini dei campi), Lojacono (rara), Zodda (Imbriacola, in aridis saxosis, fi.).

164. - Crithmum maritimum L.; Guss. Syn. I p. 326 ; Calc. p. 24; Solla p. 465 (in nota); Lojac. Esc. p. 19 et Fl. Sic. I, pars II p. 280; Car. in Parl. Fl. It. VIII p. 241. Cachyys maritima Guss. Notiz. 86 et Suppl. Prodr. p. 82.

Sulle rupi marine; raccolto a Cala Pisana il $30 \mathrm{Apr}$. sole foglie! - Gussone, Calcara, Lojacono. Solla dice di averlo cercato invano, e Zodda di averlo veduto soltanto nei dirupi inaccessibili. $L$ ' ho ricevuto in boccio, raccolto questo Agosto dalla guardia campestre Martorana.

Gussone (Synopsis) dice che gli abitanti di Lampedusa ne mettono le foglie sottaceto dopo averle fatte lessare nel ranno.

165. - Ferula nodiflora L.; Guss. Syn. I p. 353; Calc. p. 24; Lojac. Esc. 19 et Fl. Sic. I, pars II p. 289. F. communis Car. in. Parl. Fl. It. VIII p. 299.

Qua e là in varî luoghi ed isola dei Conigli; Mrz. primi fi., Apr. fi.! - Gussone, Lojacono, Zodda (fi. e fr. giov.).

Gussone osserva che gli esemplari di Lampedusa hanno le lacinie delle foglie metá più corte del normale, i frutti perfettamente orbicolari e i fusti alti da 2 a 5 piedi. Sull'isola dei Conigli l' ho trovata assai rigogliosa ed alta. Non ne ho veduto i frutti, ma la brevità delle lacinie foliari ha colpito anche me.

166. - Tordylium Apulum L.; Guss. Syn. I p. 319 ; Calc. p. 21 ; Solla p. 475 ; Lojac. Esc. p. 19 ; Car. in Parl. Fl. It. VIII p. 246. Comunissimo in tutta l'isola, tanto nelle parti coltivate quanto anche lontano da esse; Mrz. fi., Apr. fi. e fr.! - Gussone, Solla (fr.), Lojacono, Zodda (fi. e fr.).

Sono frequenti gli esemplari di dimensioni ridotte $(7-15 \mathrm{~cm}$.$) ,$ ma si trova anche normalmente sviluppato nei luoghi meno. aridi. 
167. - Bifora testiculata (L.) DC. B. flosculosa Guss. Syn. I p. 342 ; Calc. p. 24 ; Lojac. Esc. p. 20.

Gussone, Juojacono.

168. - Daucus Siculus Tin.; Guss. Syn. I p. 334 ; Calc. p. 24 ; Solla p. 475; Lojac. Esc. p. 20 et Fl. Sic. I, pars II p. 298. $D$. Gingidium Car. in Parl. Fl. It. VIII p. 549.

Rupi marine qua e lit, anche nell'isola dei Conigli; Mrz. foglie, Apr. fi. ! - Gussone, Solia (fi. e fr.). .

Calcara ha dimenticato $l^{\prime}$ * per indicare che era stato trovato da Gussone. Questa specie è dimenticata nella Florula del Sanrisente. Lojacono nella Flora Sicula non si cita come raccoglitore. Peró dice di averla distribuita nelle Pl. Sic. rar. n. 217, probabilmente avuta da un corrispondente.

Caruel ha riferito la pianta di Lampedusa raccolta dall'Ajuti e da me, che io ritengo essere il $D$. Siculus, alla sua specie complessiva D. Gingidium.

169. - Daucus rupestris Guss.; Guss. Syṇ. I p. 335 ; Calc. p. 24 ; Ross p. 347 ; Solla p. 468 et 475 ; Lojac. Esc. p. 20 et Fl. Sic. I, pars Il p. 300 (per Lampione solo); Nyman Consp. p. 279. D. australis Guss. Suppl. Prodr. p. 74 ; Bert. Fl. It. III p. 171. D. Gingidium $\beta$ breviaculeatus Car. in Parl. Fl. It. VIII p. 549. D. Carola var. rupester Fiori e Paol. Fl. an. II p. 187.

Gussone, Ross (molto lrequente nella macchia), Solla (il quale nota le grandi differenze di statura e di ramificazione secoudo il luogo dore cresce, fi. e fr.).

Questa specie è indicata pure dell' isolotto di Lampione da Gussone. La Flora analitica dice per errore «Lampione nelle Eolie» invece di dire nelle Pelagie. Anche Bertoloni sbaglia nel citare la localita di questa pianta, dicendo «habui ex Egadibus » mentre doveva dire "ex Pelagiis".

170. - Daucus Lopadusanus Tineo Plant. rar. Sicil. fasc. 3 p. 38 ; Calc. p. 24 et 31 ; Lojac. Esc. p. 19 et Fl. Sic. I, pars II p. 298; Car. in Parl. Fl. It. VIII p. 551 ; Nyman Consp. p. 280. D. Carola rar. Lopadusanus Fiori e Paol. Fl. an. II p. 187.

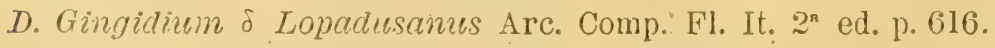
Luoghi aridi, abbondante; raccolto sull' isola dei Conigli e verso Capo Ponente, Capo Rupestre e Cala Francese; Mrz. foglie, Apr. fi. e fr.! - Calcara (Cala Grande), Zodda (Cala Malucco, 
Cala Pisana, Cala Francese in rupibus maritimis et saxosis, fi.). Lojacono lo ha distribuito di Lampedusa nelle PI. It. sel. n. IS (sull' etichetta è scritto D. Lopadresanus e nella nota stampata di quella centuria è scritto Siculues), e nella Flora Sicula dice di averlo raccolto presso al Porto, mentre in "Una Escur's. » mette il segno $\bigcirc$ che significa non averlo egli trovato. Questa specie manca nella Florula del Sanvisente.

$\dot{\mathrm{E}}$ degno di nota che chi ha raccolto il $D$. mupestris, non ha raccolto il $D$. Lopadusanus e ricerersa. Ciò fa nascere il dubbio che sia stata data una diversa interpretazione alla medesima pianta, il che non sarebbe aflatto strano in un genere cosi critico. I miei esemplari di $D$. Lopadusanus presentano le medesime grandi rariazioni di statura notate dal solla per il $D$. mupestris.

171. - Torilis nodosa (L.) Gaertn. ; Guss. Syn. I p. 338; Calc. p. 21; Solla p. 475; Lojac. Esc. p. 20. Caucalis nodosa Car. in Parl. Fl. It. VIII p. 563.

Comunissima dovunque nell' isola e nell' isolotto dei Conigli; Mrz. fi. e fr. riov., Apr. fi. e fr.! - Gussone, Solla (frr.), Lojacono, Zodda (fi. e fr.).

Negli esemplari più alti, il peduncolo delle ombrelle inferiori oltrepassa talvolta $1 \mathrm{~cm}$.

172. - Thapsia Garganica L.; Guss. Syn. I p. 359 ; Calc. p. 24 ; Solla p. 475 ; Lojac. Esc. 20; Car. in Parl. Fl. It. VIII p. 511.

Comune nei terreni aridi e sull isola dei Conigli; Mrz. primi bocci, A pr. fi: e fr. ! - Gussone, Solla (fi. e fr.; sullo scoglio dei Conigli in pieno frutto), Lojacono, Zodda (fi. e fr. giov.).

\section{XXIX. - RUBiaceaE.}

173. - Sherardia arvensis $I_{.}$; Guss. Syn. I p. 182; Cálc. p. 23 ; Lojac. Esc. p. 20 ; Tanf. in Parl. Fl. It. VII p. 72.

Nolto comune dovunque nell' isola; Mrz. fi., Apr. fi. e fr.! Gussone, Lojacono, Zodda (fi. e fr.).

174. - Crucianella rupestris Guss. Suppl. ad Fl. Sic. Prodr. p. 44 ; Bert. Fl. It. II p. 144 ; Guss. Syn. I p. 192 ; Calc. p. 23 et 31 ; Ross. p. 347 ; Lojac. Esc. p. 20 et Fl. Sic. II, pars I p. 28; Tanf. in Parl. Fl. It. VII p. 99; Arc. Comp. Fl. It. 2a ed. p. 625; 


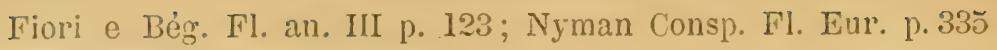
(pro subsp. C. marilimae).

Abbondante nelle fessure deile rupi in molti lunghi; Mrz. foglie, Apr. fi.! - Gussone (nelle rupi al Nord ed in quelle del Vallone della Croce e del Muro, Synops.) (in rupibus calcareis maritimis, non vero in arenosis, Suppl. ad Prodr.), Calcara (Vallone della Madonna e della Croce), Ross (fessure delle roccie in varie vallate della costa Nord di faccia alla Colonia), Lojacono (nelle rupi qua e li, massime a Capo Grecale e Cala Galera dove è abbastanza copiosa), Zodda (Capo Grecale e Cala Galera aả rupes, fiori). - Distribuita da Lojacono nelle Pl. It. sel. col n. 11 .

175. - Rubia peregrina L.; Guss. Syn. I p. 193; Calc. p. 23 ; Lojac. Esc. p. 20 et Fl. Sic. II, par's I p. 12; Tanf. in Parl. Fl. It. VII p. 17; Fiori e Bẻg. Fl. an. III p. 103.

Qua e là non comune; Mrz. foglie! - Gussone, Calcara (Vallone dell' Imbriacola), Lojacono.

Tanto Calcara quanto Lojacono "Una Escur's. » indicano per Lampedusa la var. angustifolia, mentre Gussone indica il tipo. Nella Flora Sic. Lojacono non si cita più come raccoglitore, ed attribuisce a Lampedusa il tipo anzichè la varieta. La pianta che ho raccolta, stando alla descrizione di Gussone Syn., appartiene al tipo.

176. - Galium cinereum All. G. pallidum Guss. Syn. I p. 184; Lojac. FI. Sic. II, pars I p. 16. G. corrudaefolium Tanf. in Parl. Fl. It. VII p. 31.

Gussone solo. Calcara ha dimenticato di citare questa specie nella Florula di Lampedusa.

Seguo l'esempio di De Candolle, Nyman (Consp.), Béguinot (Fl. an.) e di altri, unendo il G. pallidum Presl al G. cinereum All., rammentando peró che Gussone e Lojacono protestano contro tale riunione.

177. - Galium saccharatum All.; Guss. Syn. I p. 189; Calc. p. 23 ; Solla p. 475 ; Lojac. Esc. p. 20. G. Vaillantia Tanf. in Parl. Fl. It. VII p. 61 .

Comunissimo; Mrz. fi. e fr., Apr. fi. e fr.! - Gussone, Solla (fr.), Lojacono, Zodda (fi. e fr.).

Nei luoghi piu freschi, specialmente sotto le Opuntia si trova 
abbondante la forma b. éfuszen Bẻg. in Fiori e Bèg. Fl. an., raccolta pure dia Zodda.

178. - Galium Aparine L, ; Guss. Syn. I p. 183; Tanf. in Parl. Fi. It. VII p. 58.

Qua e la nelle siepi e negli orti; Mrz. primi fi., Apr. fi. e fr.! - Gussone, Zodda (fi. e fr.). Calcara lia dimenticato questa pianta raccolta da Gussone.

179. - Callipeltis muralis (I..) MIoris; Tanf. in Parl. Fl. It. VII p. 71. Galium merale Guss. Syn. I p. 100; Calc. p. 23; Lojac. Esc. p. 20.

Comune sotto le siepi, sulle rupi, fra le microfite; Mrza fi. e fr., Apr. fr.! - Gussone, Lojacono, Zodda (fi. e fr.).

180. - Vaillantia muralis L.; Lojac. Esc. p. 20 et Fl. Sic. II, pars I p. 10. Valantia muralis Guss. Syn. II p. 639 ; Calc. p. 30 et 31. Galium vexans Tanf. in Parl. FI. It. VII p. 69.

Ancora più comune della specie precedente, anche sull'isola dei Conichli; Mrz. fi. e fr., Apr. fr. ed ancora alcuni fi.! - Gussone, Lojacono, Zodda (fi. e fr.).

\section{XXX. - Caprifoliaceae:}

181. - Lonicera implexa Ait.; Solla p. 475; Tanf. in Parl. FI. It. VII P. 1:2.2.

Raccolta nel 1873 nella macchia del Vallone dell' Imbriacola. Quest'anno ne ho visto solo poche piante nei residui di macchia di Cala Grecale; $13 \mathrm{Mrz}$. foglie, $30 \mathrm{Apr}$. in boccio! Solla (fi.). Riceruta dalla guardia Martorana fiorita in Maggio.

- Lonicera Etrusce Santi; Tanf. in Parl. Fl. It. VII p. 125; Fiori e Bẻg. Fl. an. III p. 128.

Tanfani nella Flora Italiana cita quesia pianta come raccolta a Lampedusa da Calcara; e Bẻguinot nella Flora analitica riporta questa indicazione. Effettivamente ho riscontrato che trovasi nell' Erbario Centrale un esemplare di Lonicera Elrusca con un cartellino che dice: "Lonicera implexa; Lampedusa». Vi è poi l'annotazione: «avuta da Calcara Luglio 1846 ».

Io dubito che sia avvenuta qualche confusione per te seguenti ragioni: $1^{\circ}$ La sconcordanza fra il nome di implexa scritto sull'etichetta e dell' esemplare che è di Etrusca. $2^{\circ}$ Calcara non 
cita alcuna Lonicera nella Florula di Lampedusa. $3^{\circ}$ Altri raccoglitori non hanno veduto a Lampedusa altra Lonicera che la implexa.

\section{XXXI. - VAlerianaceaE.}

182. - Valerianella microcarpa Lois; Tanf. in Parl. Fl. It. VII p. 181. V. mixta Guss. Syn. I p. 29; Calc. p. 22. Fedia microcarpa Guss. Suppl. Prodr. p. 6.

Trovata solo da Gussone.

183. - Fedia Cornucopiae (L) Gaertn.; Guss. Syn. I. p. 27 ; Calc. p. 22; Solla p. 475 ; Lojac. Esc. p. 20 ; Tanf. in Parl. Fl. It. VII p. 161.

Trovata una sola volta a Sanguedolce; Mrz. fi. e fr. giov.! Gussone, Solla ( $f^{2}$.), Lojacono.

184. - Centranthus Calcitrapa (L.) Dufr.; Lcjac. Esc. p. 20; Tonf. in Parl. Fl. It. VII p. 159.

Trovato soltanto da Lojacono.

$$
\text { XXXII. - DipsaCEAE. }
$$

185. - Knautia hybrida Coult.; Tanf. in Parl. Fl. It. VII p. 205. K. integrifolia Guss. Syn. I p. 170; Calc. 23; Lojac. Essc. p. 20. Scaliosa integrifolia Guss. Suppl. Prodr. p. 37.

Trovata da Gussone soltanto.

186. - Scabiosa Cretica L.; Guss. Syn. I p. 177; Calc. p. 23; Lojac. Esc. p. 20; Tanf. in Parl. Fl. It. VII p. 242; Fiori e Bég. Fl. an. III p. 153.

Gussone, Lojacono.

XXXil. - COMPositae.

187. - Conyza ambigua DC.; Calc. p. 29.

Calcara solo.

188. - Bellis annua L.; Guss. Syn. II p. 508 ; Calc. p. 29. Trovata soltanto da Gussone. Sembra strano che egli abbia potuto riconoscere, in Agosto, le traccie di questa effimera specie invernale e primaverile. 
189. - Senecio vulgaris L.; Somm. Piante ined.

Comune, specialmente dal lato del Porto; Mrz. fi. e fr., Apr. fi. e fr. !

190. - Senecio pygmaeus DC.

Nei terreni arili fra Cala Croce e Cala Madonna e nelle ricinanze del Porto, con altre microfite; Mr\% fi. e fr.!

La pianta di Lampedusa corrisponde perfettamente alle descrizioni, alla figura di Lojacono Fl. Sic. II, par's I tab. VIII ed agli esemplari di S. pygmaeus raccolti a Gozo (Malta) da Duthie e da me stesso. Fiori Fl. an. include questa specie nel $S$. leucanthemifolius; peró in Lampedusa, dove quest'ultimo è cosi comune e cosi variabile, non ho veduto che presentasse alcuna forma di passaggio al S. pygmaeus. Bastano a distinguere la specie di De Candolle anche da Iontano, la piccolezza delle sue calatidi, e l'assenza di fiori ligulati. Il S. leucanthemifolius, quando si riduce nano, ha un fusticino semplice e dritto ed il capolino, anche quando è solitario, di grandezza normale; il S. pygmaers invece è ramoso diffuso, il che, unito alla piccolezza dei suoi capolini, gli dá un aspetto del tutto diverso. Ha peró in comune col S. leucanthemifolius l'odore muschiato.

191. - Senecio leucanthemifolius Poir. S. vernus et S. cressifoliues Guss. Syn. II p. 473 et 474 ; Calc. p. 29 ; Lojac. Esc. p. 20. S. incrassatus et S. crassifolius Lojac. Fl. Sic. II, par's I p. 65 et 66. S. crassifolius Solla p. 475. S. leucanthemifolius var. vermes Arc. Comp. $2^{a}$ ed. p. 668; Fiori e Bẻg. Fl. an. III p. 211.

Molto comune e abbondante non solo sulle rupi marine, ma anche nell' interno dell' isola, e nell' isola dei Conigli; Mrz. fi. e fr., Apr. fi. e fr.! - Gussone, Solla (fi.), Lojacono (distribuito in Pl. It. sel. n. 80 col nome di S. crassifolizes), Zodda (fi. e fr.).

Serguo l'esempio di Bertoloni e di Moris, tenendo uniti i $S$. crassifolius Willd., incrassalus Guss. e vernus Biv. Le forme sotto le quali il Senecio lencanthemifolius cosi inteso si presenta a Lampedusa sono molto varie, ma vi sono tutti i passagrogi dalle une alle altre. Tutte hanno le foglie più o meno carnose ed un forte odore di muschio, che conservasi ancora marcatissimo negli esemplari raccolti 33 anni fa. Specialmente sulle rupi marine si trova nano, ridotto ad un fusto semplice, alto pochi centimetri (talvolta non più di $2-3 \mathrm{~cm}$.) con una sola 
calatide. Peró anche in questi esemplari nani la calatide si mantiene grande come negli esemplari più alti, ed i fiori del raggio sono sempre ligulati.

192. - Senecio Cineraria DC. S. Cineraria var. bicolor Fiori e Bẻz. Fl. an. III p. 215. Cineraria bicolor Guss. Syn. II p. 480 ; Calc. p. 29; Lojac. F'l. Sic. II, par's I 1\% 80.

Gussolle solo.

193. - Pinardia coronaria (L.) Less.; Solla p. 476; Lojac. Esc. p. 20. Chrysanthemum coronarium Guss. Syn. II p. 484; Calc. p. 29 .

Comune nelle parti coltivate; Mr\%. fi., Apr. fi. e fr.! - Guissone, Calcara, Solla (fi.), Lojacono, Zodda (fi. e fr.).

Gli esemplari di Zodua appartengono alla forma bicolor MIicheletti, colle linguette d'un giallo piủ intenso alla base, ciò che vedesi ancora sul secco. Io peró, in quest'anno, benchè cercassi di questa forma bicolor che avevo vista cosi abbondante i giorni avanti a Linosa, non ho potuto redere in Lampedusa altro che la forma concolore.

194. - Matricaria Chamomila L.; Sanvisente L'isola di Lampedusa p. 65.

Presso il coltirato, $19 \mathrm{Apr}$. fi. e fr.! - Indicata gia dal Sanvisente «all'antica Casina, alla Madonna».

195. - Cotula aurea L. Matricaria aurea Ross Eine bot. Exc. p. 317 et Buil. Herb. Boiss. VII n. 4 p. 347 ; Lojac. Esc. p. 20 et Fl. Sic. II, pars I p. 77; Fiori e Bẻg. Fl. an. III p. 236.

Abbondante presso il Porto, lungo le vie e nei luoghi ruderali; ma trovasi pure in molte altre parti dell' isola, ed anche nana $(2-3 \mathrm{~cm}$. e con un solo capolino) frale microfite nei terreni aridi; Mrz. fi. e fr., Apr. fi. e fr.! - Ne ho ricevuto dal sig. Conti degli esemplari in pieno fiore ed in parte gia fruttificati, raccolti il 6 Dicembre 1873. Ross, Lojacono, Zodda (fi. e fr.). - Distribuita da Ross nel suo Herb. Sic. 11. 35̆, e da Lojacono nelle Pl. It. sel. n. 85 sotto il nome di Malricaria aurea.

196. - Anthemis secundiramea Biv. A. intemelia Lojac. Esc. p. 20. A. Lopadusana et A. secundiramea c. Urvilleana Lojac. Fl. Sic. II, pars I 85 et 86 . A. secundiramea $\beta$ intermedia Fiori e Bẻg. Fl. an. III p. 255. Matricaria secundiramea Somm. Piante ined. 
Comune nei !noghi aridi, anche sull' isola dei Conigli; Mrz. foglie, Apr. fi.! - Lojacono.

Mi trovo costretto a riunire alla $A$. secuncimamea Biv. la A. Lopadresana di Lojacono (della quale l'autore dice di non arer visto né $\mathrm{i}$ fori, quantunque la sua figura la rappresenti in pieno fiore, nè ghi achenî), perchè fra i numerosi esemplari da me raccolti in Lampedusa, ve ne sono alcuni ai quali conviene la descrizione della nuova specie, mentre altri concordano con la A. sacuntivamea, e fra grli uni e gli altri vi sono tutti i passaggi che dimostrano trattarsi soltanto di variazioni dorute alla natura del luogo dove sono cresciute. Nel suo primo lavoro sopra Lampedusa, Lojacono citara una sola specie di Anthemis, la intermedia Guss., la quale in verita diflicilmente può separar'si specificamente dalla A. secundiramea Biv. Secondo Fiori (in literis) la A. Lopadusana Lojac. è una forma della A. secundiramea, intermedia fra il tipo e la var. intemedia.

Gli esemplari che ho raccolti nel 1873 variano molto per il portamento e la maggiore o minore ramificazione, per la glabrescenza più o meno pronunziata e per l'ampiezza delle foglie ora pennate ed ora bipennate. Hanno in generale il peduncolo assai breve, e con tendenza poco manifesta ad ingrossare.

197. - Anthemis aryensis L. A. areensis et A. incrassata Solla p. 475 et 476.

Questa specie è indicata soltanto da Solla (fi.), sotto le sue due forme a peduncoli cilindrici e a peduncoli ingrossati, forme che a mio parere non si possono tenere distinte. Solla invece non cita la $A$. secundircmea tanto comune in Lampedusa, il che fa nascere qualche dubbio sulla esattezza della sua determinazione.

198. - Anthemis fuscata Brot.; Solla p. 476.

Indicata soltanto da Solla (fi.).

199. - Artemisia arborescens L.; Guss. Notiz. 87 et Syn. II p. 456 ; Calc. p. 29 et 31 ; Lojac. Esc. p. 20 et Fl. Sic. II, pars I p. 72.

Sulle rupi a Cala Galera, dove è frequente, Aria Rossa, Cala Vilgia; Mrz. foglie, Apr. fi.! - Gussone (che la dice rara), Calcara, Lojacono, Zodda (in boccio).

200. - Inula viscosa (L.) Ait.; Guss. Notiz. 86 et Syn. p. 503; Calc. p. 29; Lojac. Esc. p. 20. 
Nei luoghi ruderali, intorno al Porto; Mrz. e Apr. foglie sole! - Gussone (comune tra i frutici bassi).

201. - Inula graveolens (L.) Desf.; Guss. Syn. II p. 504 ; Calc. p. 29 ; Lojac. Esc. p. 20.

Gussone, Lojacono.

202, - Inula crithmoides L.; Guss. Notiz. p. 86 et Syn. II p. 503; Calc. p. 29 ; Lojac. Esc. p. 20.

Qua e lá, generalmente vicino al mare, Valle Pollicino, Grotta della Tabaccara, Cala Pisana e isola dei Conigli; Mrz. e Apr. sole foglie! - Gussone, Lojacono.

203. - Jasonia glutinosa (L.) (1)C. saltem pro p.) Guss.; Guss. Notiz. 86,87 et Syn. II p. 451; Calc. p. 29 et 31 ; Lojac. Esc. p. 20; Nyman Consp. Fl. Eur. p. 395; Fiori e Bég. Fl. an. III p. 291. Orsinia camphorata Bert. Fl. It. IX p. 100 ; Lojac. FI. Sic. II, pars I p. 100. Inula saxatiits Arc. Comp. FI. It. $2^{a}$ ed. p. 693.

Luoghi aridi sassosi molto comune, ed isola dei Conigli ; Mrz.e Apr. foglie e resti d' infiorescenza dell'anno precedente! - Gussone (colli marini aridi), Calcara, Lojacono, Zodda (sole foglie). Questa pianta a fioritura estiva è stata distribuita da Ross Herb. Sic. 11. 346 (Jasonia camphorata) e da Lojacono Pl. It. sel. n. 179, raccolta in fiore in estate dai loro corrispondenti.

204. - Asteriscus aquaticus ([..) Moench; Lojac. Esc. p. 20. Buphthalmum aquaticuin Guss. Notiz. p. 86 et Syn. II p. 505; Calc. p. 29 ; Lojac. FI. Sic. II, pars I p. 95 ; Fiori e Bég. Fl. an. III p. 296.

Comunissimo ed abbondante nei luoghi aridi, ed anche nell'isola dei Conigli; Mrz. piante secche dell'anno avanti, Apr. (i. e fr.! - Gussone (ricopre le piủ aride pianure in riposo), Calcara, Lojacono, Zodda (fi.).

Trovasi ridotto spesso ad umilissime dimensioni.

205. - Asteriscus spinosus (L.) Gren. et Godr. Pallenis spinose Solla p. 476.

Raro; valie Pollicino, Aria Rossa, isola dei Conigli; Mrz. primi fiori ! - Solla (fi.), Zodda (ne ha visto un solo esemplare a Cala Galera, fi.).

206. - Calendula arvensis L.; Ross p. 317. C. ceratosperma Lojac. Esc. p. 21 ?; Nyman Consp. Fl. Eur. Suppl. II p. 178. C. ceratocarpa Lojac. Fl. Sic. II, pars I p. 116. 
Comune nel coltivato; Mrz. fi. e fr., Apr. fi. e fr.! - Ross, Lojacono?, Zodda (fi. e fr.).

La gran maggioranza della $C$. arvensis di Lampedusa appartiene alla var. b. floribus croceis di Gussone; ma si trovano anche esemplari con fiori zolfini.

Nella Flora anal., Fiori riferisce la C.ceralosperma di Lojacono alla $C$. fugida Raf., peró dubitativamente.

207. - Calendula parvifiora Raf. C. ollicinalis ò parviflore Fiori e Bẻg. Fl. an. III p. 297.

Frequente nei campi incolti ; Mrz. fi. e fr.! - Zodda (fi. e fr.).

Gli achenî marginali rostrati presentano ben marcato il largo margine scarioso espanso e denticolato all'orlo, caratteristico delia specie. I fiori sono crocei e rare volte \%olfini.

208. - Calendula micrantha Tineo et Guss.; Calc. p. 29; Ross p. 347 ; Lojac. Esc. p. 21?

Campi incolti fra il Porto e Cala Francese, ivi abbondante; Mrz. fi. e fr.! - Calcara, Ross (terreno incolto vicino al Castello), Isojacono (?) il quale in «Una Esc. » ha dei dubbî sulla determinazione e in «Fl. Sic. " pare la riferisea alla C. ceralocarpa.

Nolla Flora analitica, Fiori suppone che la C. micrantha di Lojacono possa essere invece la C. Eyyptiuca Desf., e perció indica quest'ultima specie per" Lampedusa soltanto dubitativamente.

209. - Calendula Fegyptiaca Desf. C. ofricinalis $\gamma$. Eyyntiace Fiori, in Fiori e Bég. Fl. an. III p. 297.

Comune nelle parti coltirate dell' isola; Mrz. fi. e fr.! - Zodda (fi. e fr.).

Questa specie, a Lampedusa come a Linosa, ha sempre le ligole crocee, ed i fiori del disco porporini. Il croceo però col tempo in erbario sbiadisce.

210. - Calendula fulgida Raf.; Guss. Syn. II p. 523; Calc. p. 29; Lojac. Fl. Sic. II, pars I p. 117. C. Crislayalli Lojac. Esc. p. 21? C. slellata Nym. Consp. FI. Eur. Suppl. II p. 178. C. officinalis * fulgida Fiori e Bẻg. Fil. an. III p. 298.

Gussone, Lojacono (?).

Nella Flora analitica, Fiori anzichè a questa specie, riferisce la C. Cristagalli di Lojacono alla C. parvillora Raf. Nella Flora Sicula Lojacono non fa più menzione della sua C. Cristagalli di. Lampedusa, né cita la C. parviflora per quest' isola. 
- Calendula Siculı Lojac. Fl. Sic. II, pars I p. $11 \%$.

Nella Flora Sicula come in "Una Escurs. », Lojacono indrea pel' Lampedusa tre specie di Calendula; per due peró cambia la sua primitiva determinazione. Ció mostra che non solo i varî raccoglitori hanno interpretato dirersamente le Calendule osservate in Lampedusa, ma che anche uno stesso autore ha avuto, ad epoche diverse, opinioni differenti. Credo quindi più prudente non ammettere per Lampedusa una sesta specie, senza peró sapere indicare a quale delle cinque precedenti debba riferirsi la C. Sicula di Lojacono: Lojacono stesso non fa sapere nella Flora Sicula sotto quale nome l'avesse citata in «Una Esc. ».

211. - Phagnalon saxatile (L.) Cass.; Lojac. Esc. p. 20. Comy a saxatilis Guss. Syn. II p. 500; Calc. p. 20.

Frequente sulle rupi; Mrz. fi. e fr. giov., Apr. fi. e fr.! Gussone, Calcara, Lojacono, Zodda ( $\mathrm{fr}$.).

La pianta di Lampedusa ha le squame involucrali esterne un poco dilatate in alto e meno acute che nel tipo, e per. questo carattere si avvicina alla varietá intermedium (Lag.). Nei luoghi più protetti trovasi con cauli alti fino $\% / 2$ metro.

212. - Phagnalon rupestre (L.) DC.; Lojac. FI. Sic. II, par's I p. 54. Ph. Tenorii Solla p. 476; Lojac. Esc. p. 20. Conyza Tenorii Guss. Syn. II p. 500 ; Calc. p. 29. Ph. rupestre $\propto$ T'enomii et $\beta$ Graecum Fiori e Bẻg. Fl. an. III p. 284.

Rupi e luorhi sassosi aridi, frequente anche più del precedente, ed isola dei Conigli; Mrz. bocci, Apr. fi.efr.! - Gussone, Solla (fi.), Lojacono, Zodda (fr.).

Gussone 1. c. dice che a Lampedusa ha raccolto una varietá di questa specie caratterizzala da: «anthodii foliolis exterioribus acutiusculis subsquarrosis», varieta che Fiori (Fl. anal.) ritiene essere il Ph. Graecum di Boiss. Nessuno dei molti esemplari da me raccolti né di quelli di Zodda presenta questo carattere; alcuni sono nani, alti $3-6 \mathrm{~cm}$. Lojacono pure pare abbia raccolto il tipo, poichè in Fl. Sic. menziona come raccoglitore della rarietá Gussone solo.

213. - Filago Germanica L. F. myramidata var. spathulata Guss. Syn. II p. 462. F. pyramidata Calc. p. 29 ; Lojac. Esc. p. 20. F. Germanica var. prostrata Fiori e Bég. Fl. an. III p. 274. 
Comune nei luoghi aridi; Mrz. bocci, $A$ pr.' fr.! - Gussone, Lojacono, Zodda (fi. e fr.).

Le piante raccolte da me, come quelle di Zodda, appartengono alla var. mrostrate (Parl.).

214. - Filago Gussonei Lojac. FI. Sic. II, pars I p. 110. Evax tomeifolia Guss. Syn. II p. 460 ; Calc. p. 29; Solla p. 476 ? F. Cossyrensis Lojac. Esc. p. 21 ed in exsiccata, non Tin. nec Lojac. Fl. Sic. F. Germanica \& Gressonei Fiori, in Fiori e Bẻg. Fl. an. III p. 274.

Comune nelle terre aride fra le microfite e nell'isola dei Conigli; Mrz. fi., Apr. fi. e fr.! - Gussone (luoghi aridi sterili calcarei), Calcara (3 Giugno 1840 secondo l'etichetta), Solla? (fi.), Lojacono, Zodda (fi. e fr.).

Lojacono arendo verificato che la pianta raccolta da Calcara in Lampedusa il 3 Giugno 1840 ed etichettata "Evax tenuifolia Guss. ", non era una Evax ma una Filago, credette da principio (Una Esc. Lamp. p. 21) che fosse la Filago Cossyrensis Tineo, e sotto questo nome la distribui nelle sue exsiccata n. 78. Ma poi, essendosi persuaso che non era la specie di Tineo, nella Flora Sic. la descrisse col nome di Filago Gussonei. Il prof. Lojacono avendomi mandato un esemplare della pianta ritenuta da Calcara per Evax tenuifolic, ho potuto verificare che era identico a quelli raccolti dall'Ajuti e da me, come è identico a quelli raccolti da Lojacono a Lampedusa nell'A prile 1884 e distribuiti nelle sue exsiccata col nome di Filago Cossyrensis. Rimanendomi il dubbio che la pianta raccolta da Gussone nel 1828, su cui fondó la sua specie Evax tenufolia, fosse altra cosa, pregai il prof. Delpino di mandarmi un esemplare autentico di Gussone. L' ispezione di questo esemplare mi ha reso certo dell' identitá della Evax tenuifolia Gussone e della Filago Gussonei Lojacono. L'errore di genere in cui è caduto Gussone si spiega col fatto che nei suoi esemplari, raccolti con frutti maturi in Agosto, i pappi molto decidui sono tutti caduti e glì achenî appaiono nudi.

\section{5. - Filago arvensis L.; Solla p. 476.}

Indicata solamente da Solla (fi. e fr.).

Non avendo veduto gli esemplari, non so a quale forma di questa specie debba riferirsi la pianta di Lampedusa; molto probabilmente ad una di quelle esistenti in Sicilia. 
216. - Filago Callica L.; Solla p. 470.

Indicata da Solla solamente (la var. tenuifolia [Presl], fi. e fr.). 217. - Evax pygmaea (L.) Pers.; Lojac. Esc. P. 20. Evax sp. Solla p. 470 .

Comunissima dovunque nei terreni aridie nell' isola dei Conigrli; Mrz. fi., Apr. fi. e fr': - Solla (fi.), Lojacono, Zodda (fi. e fi'.).

Nentre la forma, comunissima in tutta l' isola, ha cauli semplici, o poco e brevemente ramosi, in un punto, non lontano dal Porto, ho racculto molti esemplari tutti appartenenti ad una forma assai diverșa, a foglie piủ strette, a capolini piủ picccli, a caule per lo più molto ramoso alla base, ed a rami rarie volte più lunghi dei capolini, gracili e prostrati per terra. Negli altri caratteri rion dif'eriscono dall' E. pygmaea, per cui ritengo che siano una semplice forma di questa specie (fi. e fr. $20 \mathrm{Apr}$.!). A questa forma forse va riferita la Evax sp. di Solla.

218. - Carlina Ianata L.; Calc. p. 20; Lojac. Esc. p. 21.

Qua e li nei luoghi aridi; Mrz. foglie radicali, Apr. non ancora in fiore! - Calcara, Lojacono, Zodda (sole foglie). Ne ho ricevute da Lampedusa alla fine di Giugno delle piante in fiore.

219. - Carlina involucrata Poir.; Guss. Syn. II p. 434; Calc. p. 29. C. corymbosa $\gamma$ involucrata Fiori, in Fiori e Bẻg. Fl. an. III p. 312 (et $C$. conymbosa o globosa Fiori Fl. an. III p. 313 quoad plantam Lopadusanam?).

Cala Galera, Capo Rupestre, luoghi aridi, ed isola dei Conigli ; Mrz. e Apr. foglie griovani e piante secche dell' anno precedente ! - Gussone.

Nella Flora analitica p. 313, Fiori riferisce la C. involucrata di Gussone alla C. corymbosa $\gamma$ globosa Arc. Ma Fiori non avendo visto gli esemplari raccolti da Gussone a Lampedusa, è permesso supporre che essi appartengano alla rera $C$. involucrata da me raccolta in Lampedusa.

220. - Carlina Sicula Ten.; Lojac. Esc. p. 21; Fiori e Bẻg. Fi. all. III p. 31?.

Solo Lojacono (comunissima).

Lojacono, non so per quale ragione, dice che è questa la pianta chiamata da Calcara C. involucrata. Calcara non ha fatto altro che trascrivere il nome di Gussone, poichè egli stesso non ha raccolto questa specie (contrassegnata con un solo*). 
221. - Microlonchus Salmanticus (L.) DC.

L' ho ricevuto quest' anno raccolto in fiore ed in frutto nel mese di Giugno dalla guardia campestre Nartorana.

222. - Centaurea Melitensis L.; Guss. Syn. II p. 516 ; Calc. p. 29 ; Solla p. 476 ; Lojac. Esc. p. 22 et Fl. Sic. II, pars I p. 142.

Molto comune nei luoghi incolti aridi; Mrz. primi capolini alla base dei fusti, Apr. fi. e fr.! - Gussone, Calcara, Solla (bocci), Lojacono, Zodda (bocci). L'ho avuta dalla guardia Martorana, raccolta in fiore e frutto in Giugnn.

223. - Centaurea Nicaeensis All.; Fiori e Bẻg. Fl. an. III p. 346. C. fuscata Lojac. Esc. p. 22.

Solo Lojacono (un unico esemplare nei luoghi aperti dei fruticeti di Ponente).

224. - Carthamus lanatus L. Kentrophyllum lanatum Guss. Syn. Il p. 430 ; Calc. p. 29 ; Lojac. Esc. p. 22.

Terranova, Taccio Vecchio; Mrz. foglie giovani e steli dell'anno avanti! - Gussone, Lojacono. I' ho ricevuto quest'anno raccolto in fiore in Giugno dalla guardia Martorana.

Gli esemplari mandatini da Martorana sono robusti, molto ramificati, a capolini ovato-lanceolati piu stretti del solito, ed a foglie dell' involucro lunghe fino a $4 \mathrm{~cm}$., oltrepassanti assai i fiori. Sembrano corrispondere alla descrizione del Trentrophylhm turbinatum Gasparr. nella Synopsis di Gussone II p. 430.

225. - Carduus argyroa Biv.; Guss. Syn. II p. 440 ; Calc. p. 29 ; Arc. Comp. $2^{a}$ ed. p. 721 ; Lojac. Fl. Sic. II, pars I p. 167. C. argyroa var. ramosissimus Lojac. Esc. p. 21. C. argyroa a. typicus Fiori e Bẻg. Fl. an. IIl p. 360.

Frequente in varie parti dell' isola, rigoglioso in Val Pollicino e sull isolotto dei Conigli; Mrz. fi. e fr., Apr. fi. e fr. ! - Gussone, Lojacono, Zolda (ii. e fr.).

Lojacono nel suo primo lavoro riferisce la pianta di Lampedusa ad una var. ramosissimus che non descrive. Nella Filora Sicula non fa piủ menzione di questa varietà.

226. - Carduus pycnocephalus L.; Solla 1). 476; Lojac. Fl. Sic. II pars I p. 167. C. pycnocephahes var. Gussoneanus Lojac. Esc. 1). 21?

Presso il Porto nei ruderati, e Cala Pisana; Mrz. foglie, Apr. fi.! - Solla (fi.), Lojacono (luoghi incolti e viottoli), Zodda 
(presso l'abitato e presso il camerone dei cuatti, fi. e fro.). Ross l'ha distribuito nel Herb. Sic. col n. 404 raccolto in fi. e fr. in Apr. 1904 da un corrispondente, ed io l'ho ricevuto in fi. e fr. raccolto in Giugno dalla guardia Martorana.

I miei esemplari come quelli del Herb. Sic., quelli di Martorana ell uno di quelli di Zodda, appartengono al C. pycnocephalus tipico.

Zordda inoltre ha raccolto varî esemplari appartenenti ad una forma robusta che differisce dal tipo per avere i capolini affastellati, il fusto alato fino sotto i capolini, e le foglie più profondamente divise ed armate, come le ali del fusto, di spine piu robuste. Suppongo che possa essere questa forma che Lojacono in « Una Esc. » aveva chiamata var. Gussoneanus, senza però darne la descrizione. Nella Flora Sicula Lojacono non fa più menzione di questa varietá, altro che in nota a p. 170. Degli esemplari di Lampedusa del C. pycnocephalus dice "forma robusta spinosior», il che si potrebbe applicare alla forma raccolta da Zodda.

227. - Carduus brevisquanus (Fiori, in Fiori e Bigg. Fl. an. III p. 359 pro var. C. pycnocephali).

Zodda solo (presso il Porto, in incultis saxosis, î. e fr.).

Il dott. Zodda ha trovato, tanto a Lampedusa quanto a Linosa, questo Carcuris, che mi pare meritevole di essere considerato come specie distinta del C. pycnocephalus. Esso sembra formare il passaggio fra il C. pycnocephalus e il C. marmoratus. A quest'ultimo somiglia per le squame dell' involucro, ma ne differisce per non avere le foglie involucrali oltrepassanti i capolini nè munite di spine robuste.

Questa specie è chiamata C. Arabicus da Gussone, da Lojacono e dagli autori italiani in genere, ma secondo Fiori non è il C. Arabicus Jacq. Non si puó dare a questa specie il nome di C. peregrinus Ten. perchè non è certo che ad essa si applichi, e del resto era già stato dato ad altro Carduus da Retzius. E neppure gli si puó dare il nome di C. Panormilanus perché è nome nudo e d'incerto significato. Bisogna dunque prendere il nome di Fiori dando alla sua varietá il valore di specie.

Inoltre il dott. Zodda ha raccolto un esemplare che non appartiene ad alcuna delle forme sopra citate. Sembra intermedio. 
fra la forma Gussoneanus ed il C. brevisquamus e potrebbe esserne un-ibrido.

Come si vede, vi è a Lampedusa tutto un ciclo di forme i cui punti estremi sono il C. pycnocephalus tipico e il C. marmoratus, che meriterebbero di essere ulteriormente studiate con abbondante materiale.

228. - Carduus marmoratus Boiss. et Heldr.; Fiori e Bẻg. FI. an. III p. 360. C. pycnocephales var. $\gamma$ Arc. Comp. $1^{2}$ ed. p. 402. 6. pseutlosyriacus Lojac. Esc. p. 22 et FI. Sic. II, pars I p. 170; Nyman Consp. Suppl. II p. 182. C. pycnocephalus $\gamma$ Lopadesanus Arc. Comp. $2^{\mathrm{a}}$ ed. p. 722.

Terreni incolti alla Cala Pisana ed in varî luoghi presso il Porto; Apr. fi. e fr.! - Lojacono (nelle vie e luoghi incolti presso il paese, presso il mare a Porto Vecchio; distribuito poi nelle Pl. It. sel. n. 91 col nome di C. pseudosyriaceus sull' etichetta, e di C. Lopadusanus sulla nota stampata).

Arcangeli aveva ritenuto che la pianta di Lampedusa fosse una varietá del C. pycnocephalus, mentre Lojacono l'aveva considerata come una specie nuova, e descritta col nome di C.pseudosyriacus. Fiori ritiene invece che sia identica alla specie descritta da Boissier e Heldreich col nome di C. marmoratus, e. indicata fino allora di un sol luogo di Grecia, ma che esiste anche a Malta. Vi sono difatti nell' Erbario Centrale di Firenze degli esemplari di quest' isola, avuti da Todaro e Calcara col nome di C. pycnocephalus, che sono conformi a quelli di Lampedusa. Anche Lojacono dice di avere visto la stessa specie di Malta. Io. poi in quest' anno l' ho trovata abbondante nelle isole Maltesi.

229. - Carduus corymbosus Ten.; Solla p. 476.

Indicato solamente da Solla (fi.).

Non mi sembra improbabile che Solla abbia dato questo nome ad una delle specie precedenti.

230. - Notobasis Syriaca (L.) Cass.; Lojac. Esc. p. 22. Cnicus Syriacus Arc. Comp. $2^{\mathrm{a}}$ ed. p. 723. Cirsium Syriacum Fiori e Bẻg. Fl. an. III p. 364.

Qua e là vicino al paese; Apr. fi. e fr. giov.! - Lojacono. (luoghi incolti presso il paese), Zodda (presso l'abitato, in boccio). Ricevuta quest'anno raccolta in frutto in Giugno dalla guardia Martorana. 
Lungo la via che conduce alla Cala Pisana ne ho trovato una forma assai aberrante che se fosse costante meriterebbe di essere designata col nome di var. conferta, cosi caratterizzata: Planta obscure virens et humilior (pedalis); capitula in racemum abbreviatum congesta; folia involucrantia breviora.

231. - Cynara Cardunculus L. C. horrida Guss. Syn. II p. 436 ; Calc. p. 29 ; Lojac. Esc. p. 22 et Fl. Sic. II, pars I p. 164. C. Cardunculus a typica Fiori e Bẻg. Fl. an. III p. 380.

In luoghi aridissimi tra Taccio Vecchio e Albero del Sole, ivi abbondante; Mrz. foglie! - Gussone, Zodda (all'Aria Rossa, bocci).

- Cynara Scolymus L. ; Sanvisente l'isola di Lamp. p. 64.

Sanvisente nella sua Florula cita questa pianta che si coltiva negli orti, ma non è inselvatichita.

232. - Silyhum Marianum (L.) Gaertn. Carcleeses Marianies Sanvisente L'Isola di Lampedusa p. 65.

In varî luoghi vicino alle case; Mrz. foglie! - Sanvisente era il solo che avesse citato questa specie "al Ponente ", il che è assai strano pensando che il suo lavoro sembra calcato su quello di Calcara. Io ne avevo ricevuto una giovane rosetta di foglie fin dal 1873 raccolta dal Conti in inverno.

233. - Echinops spinosus L.; Tineo Plant. rar. Sic. p. 48; Calc. p. 29 et 31 ; Solla p. 470 ; Lojac. Esc. p. 21 , Malpighia anno I p. 289 et Fl. Sic. II, pars I p. 175; Arc. Comp. $2^{a}$ ed. p. 730 ; Nicotra, Gli Echinops italiani, Bull. Soc. bot. it. 1901 p. 237; Fiori e Bẻg. Fl. an. III p. 307. E. viscosus Guss. Syn. II p. 526 ; Ross p. 347 ; Arc. Comp. $1^{a}$ ed. p. 410.

Sulle rupi marine della costa Nord a Taccio Vecchio e a Capo Rupestre; Mrz. foglie, Apr. bocci! - Gussone (sulla Costa del Nord e presso al Castello), Calcara (Punta Rupestre), Ross (macchia presso Capo Ponente e sulla Costa Nord presso la Guardia del Prete), Solla (fi.), Lojacono (luoghi petrosi aperti dei fruticeti a Punta Ponente, rarissimo), Zodda (Capo Grecale, ad sepes raro, fi.). L' ho ricevuto quest'anno raccolto in fiore in Giugno dalla guardia Martorana. Dal segno! messo da Nicotra (loc. cit.) à Tineo, si potrebbe credere che Nicotra avesse veduto esemplari di Echinops spinosus raccolti a Lampedusa da questo botanico. Ma Tineo non fu mai in Lampedusa ed egli stesso Plant. rar. Sic. indica come raccoglitore per Lampedusa il solo Calcara. 
Lojacono ha rilevato nella Malpighia, come fosse errato il nome di E.viscosues dato da Gussone alla pianta di Lampedusa. Il Ross é stato indotto in errore dalla Synopsis di Gussone come egli ha poi riconosciuto (vedi Nicotra loc. cit. p. 236). Fiori (Fl. an. III p. 308) nota egli pure come sia probabilmente errata l'indicazione dell' $E$. viscosus per Lampedusa.

234. - Scolymus maculatus L.; Lojac. Esc. p. 22; Fiori e Bẻgr. Fl. an. III p. 383.

Lojacono solo. Pertanto nella Flora Sicula non menziona piu Lampedusa.

235. - Hyoseris radiata L.; Guss. Syn. Il p. 416; Calc. p. 28 ; Solla p. 476 ; Lojac. Esc. p. 23. H. lucilla Guss. Syn. II p. 417; Calc. p. 29 ; Lojac. Fl. Sic. II, par's I p. 186. H. radiata $\beta$ Baetica Fiori Fl. an. III p. 389.

Comunissima in tutta l'isola e nell' isolotto dei Conigli; Mr'z. fi. e fra, Apr. fi. e fr.:! - Gussone, Solla (fi.), Lojacono, Zodda (fi. e fr.).

Quantunque Gussone stesso citi per Lampedusa le Hyoseris radiala e lucida come specie diverse, non posso tenerle separate. Gussone stesso le dice distinte da "characteribus satis infirmis », e Boissier Flora Or. IiI p. 719 ritiene che la vera $H$. lucida di Linneo si trovi solamente in Egitto, e che la H. lucida di Gussone sia una semplice forma della $H$. radiata. - A Lampedusa si trovano forme diversissime congiunte fra di loro da passaggi insensibili. Specialmente sulle rupi marine questa pianta è piccola ed ha foglie carnosette, con lobi arrotondati interi, talvolta remoti e talvolta imbricati, corrispondendo in questo secondo caso perfettamente alla $H$. lucida b. imbricata Guss. ( $\beta$ Baetica Fiori Fl. an.). In luoghi piu freschi ed in terreno meno arido se ne trovano piante alte, a foglie sottili, con lobi acuti e discosti (a typica Fiori Fl. an.).

236. - Hyoseris scabra L.; Guss. Syn. II p. 417; Calc. p. 29; Lojac. Esc. p. 23 ; Arc. Comp. $2^{a}$ ed. p. 732; Fiori e Bèg. Fl. all. III p. 389 .

Frequente nei terreni aridi e nell' isola dei Conigli; Mrz. fi. e fr., Apr. fr.! - Gussone, Lojacono, Zodda (fi. e fr.).

237. - Hedypnois polymorpha I) H. Heblaeformis Guss. Syn. II p. 419 ; Calc. p. 29 ; Lojac. Esc. p. 23. H. Cretica Lojac. Esc. p. 23. 
Comune nei campi e nei luoghi incolti; Mrz. fi. e fr., Apr. fi. e fr.! - Gussone, Lojacono, Zodda (fi. e fr.).

Se ne trovano esemplari che corrispondono perfettamente alla var. tubxefurmis per i peduncoli eccessivamente rigonfi (con capolini ora glabri ora scabri), ed altri che corrispondono al tipo (H. Mrauritanica in Guss. Syn.); altri ancora sono intermedî fra le due forme.

238. - Seriola Etnensis L. ; Guss. Syn. II p. 421 ; Calc. p. 29 ; Solla p. 476 ; Lojac. Esc. p. 22.

Molto comune in tutta l' isola e sull' isolotto dei Conigli; Mrz. primi fi., A pr. fi. e fr.! - Gussone, Solla (fi.), Lojacono, Zodda (fi. e fr.).

Se ne trovano forme irsutissime ed altre glabrescenti; spesso è nana nei terreni più aridi.

239. - Cichorium Intybus L.; Lojac. Esc. p. 23. C. Inlybus var. pumilum Fiori e Bẻg. Fl. an. III p. 386. C. salvatica (sic) Sanvisente, L' Isola di Lampedusa p. 64.

In rarî luoghi; Mrz. foglie, Apr. fi.! - Sanvisente, Lojacono, Zodda (fi.).

Ho trovato tanto la forma tipica quanto la var. pumilum.

- Cichorium Endivia L.; Sanvisente, L'Isola di Lampedusa p. 64.

Questa specie che Sanvisente cita come facente parte della florula di Lampedusa, si coltiva negli orti ma non pare che rinasca subspontanea.

240. - Thrincia tuberosa (L.) DC. ; Somm. Piante ined.

Comunissima nei luoghi aridi incolti; Mrz. fi. e fr., Apr. tì. e fr.! - Zodda (fi. e fir.).

241. - Picris spinulosa Bert.; Lojac. Esc. p. 23. P. hieracioides $\beta$ spinulosa Fiori e Bẻg. Fl. an. III p. 403.

Lojacono solo (Cala Pisana, rarissima).

242. - Urospermum picroides (L.) Desf.; Guss. Syn. II p. 386; Calc. p. 28; Solla p. 476; Lojac. Esc. p. 23.

Frequente nelle parti coltivate; Mrz. fi., Apr. fi. e fr.! Gussone, Calcara, Solla (fi. e fr.), Lojacono.

243. - Tragopogon Cupani Guss.

Ricevuto quest'anno raccolto in frutto in Giugno dalla guardia Martorana. 
- Lactuca sativa L.; Sanvisente, L'Isola di Lampedusa

p. 64 .

Questa pianta citata nella Florula del Sanvisente, trovasi soltanto collivata negli orti.

244. - Sonchus tenerrimus L.; Guss. Syn. II p. 392 ; Calc. p. 28 ; Lojac. Esc. p. 23.

Presso il Porto, verso Caia Francese, e sull'isola dei Conigli; Mrz. fi., גpr. fi. e fr.! - Gussone, Lojacono, Zodda (fr.).

245. - Sonchus levis Bartal. S. oleraceus Guss. Syn. II p. 391 ; Calc. p. 28; Solla p. 470 ; Lojac. Esc. p. 23.

Comunissimo nel coltivato, e anche lontano da questo; Mrz. fi. e fr., Apr. fi. e fro ! - Gussone, Solla (fi.), Lojacono, Zodda (fi.).

246. - Sonchus asper Hill.; Calc. p. 28.

Calcara solo.

E probabile che questa citazione di Calcara vada riferita alla specie seguente.

247. - Sonchus glaucescens Jord.; Lojac. Fl. Sic. II, par's I p. 206. S. decomes? Lojac. Esc. p. 23. S. oleraceus var. $\beta$ asper forma e. glaucescens Fiori Fl. an. III p. 418.

Scogliere marine rerso Ponente e sull' isola dei Conigli dove è abbondante; Mrz. î. e fr., Apr. fi. e fr'! - Lojacono (Capo di Ponente, luoghi pietrosi, calcarei, erbosi fra i fruticeti, rarissimo e sporadico).

Questa bella specie, di abito singolarissimo, è bene distinta dal S. asper per i suoi capolini più crrandi, di un giallo dorato, affastellati in un denso corimbo, per l'eccessivo turgore di tutte le sue parti, il che ne rende la disseccazione una ardua impresa (i suoi fusti hanno fin piü di $15 \mathrm{~mm}$. di diametro) e per. gli achen̂i più largamente ellittici, cinti da un margine membra. naceo ben distinto, largo più del mezzo diametro dell'achenio.

248. - Sonchus maritimus L.; Lojac. Esc. p. 23. S. arvensis $\gamma$ marilimus Fiori e Bẻg. Fl. an. III p. 419.

Solo Lojacono (arene della spiagrgia sotto il Castello).

Lojacono pertanto non indica Lampedusa per questa specie nella Fl. Sic.

249. - Picridium vulgare Jesf.; Lojac. Esc. p. 23. Sonchus picrioides Guss. Syn. II p. 393 ; Calc. p. 28. P. vulgare b. crassifolium Lnjac. Fl. Sic. II, par's I p. 207. 
Comune nei luoghi incolti aridi e rupestri ; Mrz. fi. e fr., Apr. fi. e fr.! - Gussone, Calcara, Lojacono.

250. - Picridium intermedium C. H. Schultz; Solla p. 476.

Verso Cala Francese, in terreno arido; Mrz. fi. e fr.! - Solla (fi. e fir.).

Questa specie non è citata di Sicilia da Gussone, ma bensi dagli autori posteriori. A Lampedusa ne ho veduto soltanto la var. gracile Schultz quale è descritta in Willk. et Lge. Fl. Hisp. II p. 233 ed in Lojacono Fl. Sic. II, pars I p. 208.

251. - Picridium Tingitanum Desf: Arc. Comp. $2^{\mathrm{a}}$ ed. p. 746. Isolotto dei Conigli, fra i frutici sulle scogliere marine, abbondante; Mrz. fi.!

Qui il $P$. Tingitanum cresce rigoglioso come in generale tutte le piante su quell' isolotto. Ha fusti alti fino a $35 \mathrm{~cm}$., ramosi e fogliosi anche in alto, capolini grandi, foglie larghe, largamente abbraccianti e poco profondamente incise, quasi del tutto prive di papille; si avvicina quindi piủ all' a genuinum Willk. ed anche al P. Gaditanum Willk. (in W. et L. Fl. Hisp.).

Questa specie era stata citata di Lampedusa da Arcangeli per sbaglio, dovendosi leggere nel Compendio Linosa invece di Lampedusa. Ora peró è constatata la sua presenza anche in questa isola, o per lo meno sull isolotto dei Conigli che le appartiene.

\section{2. - Crepis foetida L.}

Ricevuta da Lampedusa quest'anno, raccolta in fiore e in frutto in Giugno dalia guardia campestre Martorana; esemplari robusti e molto ramificati.

253. - Crepis bulbosa (L.) Froel. Hieracium bulbosum Guss. Syn. II p. 403; Calc. p. 28; Lojac. Esc. p. 23. Etheorhiza butcosa Solla p. 476 ; Lojac. Fl. Sic. II, pars I p. 214.

Frequente nei luoghi coltivati ed incolti e nell' isolotto dei Conigli; Mrz. fi., Apı. fr'.! - Gussone, Solla (fi.), Lojacono.

254. - Andryala sinuata L.; Solla p. 476.

Indicata soltanto da Solla (fi. e fr.).

\section{XXXiV. - Campanulaceae.}

255. - Campanula Erinus L.; Guss. Syn. I p. 250; Calc. p. 24 ; Solla p. 470 ; Lojac. Esc. p. 23 et FI. Sic. II, pars I p. 231 ; 'Tant. in Parl. Fl. It. VIII p. 112. 
Sulle rupi e sui terreni aridi, comune; Mrz. foglie, Apr. fi. e fr.! - Gussone, Calcara, Solla (frr.), Lojacono, Zodda (fi. e fr $r^{\circ}$ ).

\section{XXXV. - CucurbitaceaE.}

256. - Bryonia acuta Desf.; Guss. Notiz. p. 87 et Syn. If p. 621 ; Calc. p. 30 et 31 ; Nym. Consp. Fl. Eur. p. 246 ; Ross p. 347 ; Solla p. 474 ; Lojac. Esc. p. 19 et Fl. Sic. I, pars II p. 238 ; B. acuta \& typica Fiori e Bẻg. Fl. an. III p. 159.

Frequente sui muri e nelle siepi fra i fichi d'India; Mrz.fi., Apr. fi. e fr. ! - Gussone (siepi e macerie), Calcara, Ross (il quale l' ha poi distribuita nel Herb. Sic. n. 431, raccolta da un corrispondente, Apr.-Magg. 1903 fi. e fr.), Solla (fi. e fr.), Lojacono (distribuita in PI. It. sel. n. 100), Zodda (fi. e fr.).

257. - Ecballium Elaterium (L.) Rich.; Guss. Syn. II p. 620; Calc. p. 30; Lojac. Esc. p. 19 et Fl. Sic. I, pars II p. 239.

Verso Cala Malucco, raro; 14 Mrz. fi.! - Gussone, Lojacono.

\section{XXXVI. - ERICACEAE.}

258. - Arbutus Unedo L.; Guss. Notiz. p. 86 et Syn. I p. 464; Calc. p. 26 et 32 ; Solla p. 465 (in nota); Lojac. Esc. p. 23; Car. in Parl. Fl. It. VIII p. 723.

Vallone dell' Imbriacola; 30 Apr. 1873 sole foglie! - Gussone (il quale in «Notizie ecc. » dice che trovasi sparso per. la macchia) e Calcara (il quale, p. 1 e «Rapporto ecc.» p. 6, dice che trovasi in gran numero nel burrone dell' Imbriacola « unde nomen »). Lojacono e Solla dicono di avere ricercato invano questa pianta la quale pure in antico dovera essere comune in Lampedusa, poichè da essa ha derivato il suo nome il Vallone dell'Imbriacola. Quando vi siamo stati l'Ajuti ed io, nel 1873, lo abbiamo ancora raccolto in quel vallone. Ora non vi esiste piu. Mi è stato assicurato quest'anno che se ne trova ancora qualche pianta in luoghi inaccessibili all'estremo Ponente, cioé nella parte più lontana dall' abitato.

259. - Erica multiflora L.; Guss. Notiz. p. 86, 87 et Suppl. Prod. p. 113; Solla p. 476 ; Car. in Parl. Fl. It. VIII p. 702. E. pedencularis Guss. I p. 447; Calc. 25, 31 et 32; Lojac. Esc. p. 23. 
Ora molto rara, verso Cala Francese, poche informi ceppaie, Mrz. foglie; a Cala Sottile, Apr. fr.! - Gussone (a Cala Pisana), Calcara, Solla, Lojacono (rara).

\section{XXXVII. - OLEACEAE.}

260. - Olea Europaea L.; Guss. Notiz. p. 85, 86, 97 et Syn. I p. 11 ; Calc. p. 22,32 et 35 ; Solla p. 470 ; Lojac. Esc. p. 12 et 23; Car. in Pirl. I'l. It. VIII p. 156.

Le piante di oleastro che ancora esistono sono ridotte per lo più a meschini arbusti erinacei, talvolta quasi pulvinari. Qua e lá peró, in luoghi piú protetti, se ne vede ancora qualche pianta con portamento normale; Mrz. e Apr. foglie! - Gussone (forma dense boscaglie insiemie a Plitllyrea media, Pistacia Lentiscus ecc.; nei luoghi elevati per l'azione del vento forma densi ed umili cespugli, mentre nelle valli cresce più alto; $\mathrm{i}$ suoi rami e le foglie giovani sono in Linosa e Lampedusa quasi l'unico cibo dei bovi e delle capre), Calcara (dovanque), Solla (coltivato), Lojacono, Zodda (foglie).

Calcara valutara a 2000 circa gli alberetti d'ulivo salvatico che sarebbero stati adatti all' innesto. Lojacono non ne crede raccomandabile la coltura in Lampedusa. All'epoca della sua visita in Lampedusa (1828) Gussone vi trovò 46 ulivi innestati da antichi coloni.

261. - Phillyrea variabilis Timb.; Car. in Parl. VIII p. 161. Ph. media Guss. Notiz. p. 83, 85, 86 et Syn. I p. 10; Calc. 22, 32 et 34 ; Solla p. 476 ; Lojac. Esc. p. 23. Ph. stricta Guss. Syn. II Addend. et emend. p. 775 ; Calc. p. 22; Lojac. Esc. p. 23.

Ora trovansene soltanto qua e la piante isolate e destinate esse pure a morire fra breve; Mrz. e Apr. bocci! - Gussone (uno dei costituenti della densa boscaglia), Calcara (in tutta l' isola), Solla, Lojacono (una delle essenze dei fruticeti), Zodda (foglie). Sanvisente cita soltanto la Ph. media.

Ho visto e raccolto tanto la forma media quanto la latifolia, con foglie ora intere ed ora seghettate. Gussone nell'Addenda, Calcara e Lojacono indicano la Ph. stricta, forma che io non ho vista. Gussone nella Synopsis osserva specialmente come a Lampedusa e a Linosa se ne trovino molte varietá. - Gussone 
(Notizie) dice che le capre ne mangiano avidamente i frutti, e che i bovi e le capre si cibano dei rami teneri e delle foglie di questa pianta come dell' Oleastro e della Periploca.

\section{XXXVII. - ASCLEPIADACEAE.}

262. - Periploca levigata Ait.; Car. in Parl. Fl. It. VI p. 717; Fiori e Bẻg. Fl. an. II p. 345. P. angrestifolia La Billardière Ic. plant. Syr. rar. dec. 2a Tab. VII p. 13-14; Guss. Notiz. \$3, 85, 86, 87 et Syu. I p. 286- 7 ; Bert. Fl. It. III p. 2-3; Calc. 24, 31 et 32 ; Ross p. 347 ; Solla p. 476 ; Lojac. Esc. p. 24.

Ancora frequente, specialmente sui muri e nelle siepi, ma spesso deformata dal dente degli ovini; trovasi anche nell'isola dei Conigli; Mrz. fi. e fr. giov., Apr. fi. e fr. ! - La Billadière (?), ${ }^{4}$ Gussone (rupi calcaree al mezzogiorno dell' isola; uno dei costituenti delle dense boscaglie), Calcara (dorunque), Ross, Solla (fi.), Lojacono, Zodda (fi., fr. giov. e f'r. maturi).

Secondo Gussone perde le foglie nei grandi calori estivi; gli abitanti di Lampedusa la chiamano «albero della seta » e raccolgono le chiome dei suoi semi per farne dei guanciali; le capre, le pecore ed $i$ bovi, quando sono secche le altre piante, costretti dalla fame, ne mangiano le foglie, le quali da principio infiammano ed esulcerano la loro bocca, e cagionano ematuria, ma in seguito, assuefatti, non ne risentono alcun danno, anzi grli abitanti affermano che con tal cibo si accresce la quantità e si migliora la qualità del latte. Cosi Gussone; a me peró fu detto che l'ematuria delle capre e delle pecore, che si verificava talvolta, era dovuta ad altra pianta.

263. - Stapelia Europaea Guss. Notiz. in Acta Soc. Borb., p. 87-91; Guss. Syn. I p. 289; Calc. p. 24 et 30 ; Bert. Fl. It. III p. 4; Nym. Consp. Fl. Eur. p. 497 ; Solla p. 470 ; Lojac. Esc. p. 24 ; Arc. Comp. $2^{a}$ ediz. p. 365 ; Fiori e Bẻg. Fl. an. II p. 347. Apteranthes Gussoneana Mikan Eine von $\mathrm{D}^{\mathrm{r}}$ Gussone auf Eur. Bod. entdeckte Slapelia; Battandier et Trabut Flore de l'Algé-

'C' è nell'erbario Webb un esemplare di Periploca angustifolia con cartellino dell' erbario La Billardière, sul quale è seritto « nora species », il che farebbe supporre che sia scritto (lello stesso La Billadière. Però non è indicata la provenionza. 
rie I p. 587. Boucerosia Gussoneana Ross p. 347 ; Car. in Parl: Fl. It. VI p. 725 .

Nei terreni più aridi e sassosi, frequente in varie parti dell'isola; Mrz. fi., Apr. fi. e fr.! - Gussone (nelle fessure delle rocce calcaree ripiene di terra, sulle pendici meridionali dell' isola), Calcara (luoghi sassosi, Punta Rupestre e Capo Rupestre), Ross (sparsa nella macchia fra le pietre, abbondantemente fiorita ed in parte fruttificata in Aprile), Solla (fi.), Lojacono (in due o tre luoghi fra $\mathrm{i}$ sassi nel suolo argilloso), Zodda (fi.). Il dott. Ross nel suo «Herbarium Siculum» col n. 263 sotto il nome di Caralluma europaea N. E. Br. ha distribuito questa pianta raccolta in fiori e in frutti da un suo corrispondente nel Giugno del 1900.

Questa strana specie dall'aspetto cactaceo esotico, che trovasi soltanto sulla costa settentrionale d'Africa ed in pochi punti della Spagna meridionale, ed è l'unico rappresentante del genere nel nostro emisfero, è certo la più interessante delle inquiline di Lampedusa.

Gussone (Notiz. p. 83) dice che gli abitanti di Lampedusa l'applicano sulla fronte contro le cefalee.

XXXIX. - Gentianaceae.

264. - Chlora perfoliata L.; Solla p. 476; Car. in Parl. Fl. It. VI p. 733. C. intermedia Guss. Syn. I p. 445; Calc. p. 25 ; Solla p. 476; Lojac. Esc. p. 24.

Gussone, Calcara, Solla (fi.), Lojacono.

Questi autori indicano tutti la forma intermedia e Solla inoltre anche il tipo.

265. - Erythraea Centaurium (L.) Pers.; Guss. Syn. I p. 281; Calc. p. 24 ; Lojac. p. 24. E. grandifora Guss. Suppl. Prodr. p. 63.

Gussone, Lojacono e Zodda (Cala Croce in aridis, fi.). - Nel Supplemento al Prodromo Gussone citava Lampedusa per la E. grandiflora e non per la Centaurium, donde mi pare certo che sono gli stessi esemplari che nella Synopsis attribuisce alla E. Centaurium.

266. -- Erythraea pulchella (Sw.) Horn.; Solla p. 476; E. ramosissima, Guss. Syn. I p. 282; Calc. p. 24 ; Lojac. p. 24 ; Car. in Parl. Fl. It. VI p. 736. 
Verso Capo Ponente e Capo Rupestre; 28 e 30 Apr. fi. e fr.! Gussone, Calcara (Valtone dell' Imbriacola), Solla (fi.), Lojacono.

I miei esemplari appartengono in parte alla microflora, taluni non oltrepassando $1 \mathrm{~cm}$. d'altezza.

267. - Erythraea spicata (L.) Pers.; Guss. Syn. I p. 283; Calc. p. 24; Lojac. Esc. p. 24 ; Car. in Parl. Fl. It. VI p. 740. Gussone (nella Salina sotto al Castello), Lojacono.

\section{XL. - Convolvulaceae.}

268. - Convolvulus lineatus L.; Ross p. 318 ; Solla p. 476 et 465 (in nota); Lojac. Esc. p. 24; Car. in Parl. Fl. It. VI p. 801; Fiori e Bég. Fl. als. II p. 385.

Frequente nei terreni aridi; Mrz. foglie, Apr. fi.! - Ross, Solla (fi.), Lojacono, Zodda (fi.).

Lojacono osserva che si trova in esemplari nani. Tali sono per lo più anche i miei.

269. - Convolvulus althaeoides L.; Solla p. 476 ; Car. in Parl. Fl. It. VI p. 817. C. Italicus Calc. p. 24; Lojac. Esc. p. 24.

Rupi, muri e siepi, frequente; Mrz. foglie, Apr. fi.! - Calcara, Solla (fi.), Lojacono, Zodda (fi.). Calcara cita a torto Gussone per questa pianta, poichè nella Synopsis non viene fatta menzione di Lampedusa.

Tutte le piante che ho viste appartengono alla forma a pelurie patente e foglie poco divise, generalmente distinta col nome di Italicus, ed alla quale riferiscono pure i loro esemplari Calcara e Lojacono.

270. - Convolvulus arvensis L.; Guss. Syn. I p. 241; Calc. p. 24; Lojac. Esc. p. 24; Car. in Parl. Fl. It. VI p. 813.

Molto comune nella parte coltivata dell' isola; Mrz. foglie, Apr. fi. ! - Gussone (var. b.), Calcara, Lojacono, Zodda (bocci).

Gussone riferisce la pianta da esso raccolta a Lampedusa ad una forma a foglie strette, mentre Lojacono vi ha raccolto piante a foglie grandissime. Le mie non sono notevoli né per larghezza né per strettezza.

271. - Convolvulus tricolor L.; Car. in Parl Fl. It. VI p. 810; Fiori e Bég. Fl. an. II p. 386.

Nei campi presso il Porto; $20 \mathrm{Apr}$. fi. e frr.! 
272. - Convolvulus Siculus L.; Guss. Syn. I p. 244 ; Calc. p. 24; Lojac. Esc. p. 24; Car. in Parl. Fl. It. VI p. 808; Fiori e Bẻg. Fl. an. II p. 385.

Frequente; piccolo e prostrato nei luoghi aridi diventa alto e subscandente nei luoghi piu fertili e nelle siepi; Mrz. fi., Apr. fi. e fr. ! - Gussone, Calcara, Lojacono, Zodda (fi. e fr.). Questa specie manca nella Florula del Sanvisente.

273. - Cuscuta Epithymum (L.) Murr.; Car. in Parl. Fl. It. V1 p. 823. C. alba Calc. p. 24.

Sul Cistus complicalus $30 \mathrm{Apr}$. fi. ! - Calcara, Zodda (Carallo Bianco e Cala Uccello, sulla Scandix e sopra diverse papilionacee, fi.).

274. - Cuscuta Palaestina Boiss.; Somm. Piante incd.

A Capo Ponente sul Thymus capitatus 28 Apr. fi.! - Zodda (Capo Grecale sull' Hupericum Egyptiacuin, fi.).

275. - Cuscuta Europaea L.; Solla p. 476.

Solla solo (ii.).

276. - Cuscuta planiflora Ten. ?; Lojac. Esc. p. 24.

Lojacono cita questa specie solo dubitativamente, per cui è possibile che si tratti di una delle specie precedenti.

E evidente che le Cuscute di Lampedusa vanno ulteriormente studiate. In Marzo ne ho raccolte senza fiori e quindi indeterminabili.

\section{XLi. - Boraginaceae.}

277. - Echium parvifilorum IIoench. E. calycinzmiz Guss. Syn. I 1. 233 ; Calc. p. 23; Lojac. Esc. 1. 24.

Piuttosto frequente nei luoghi incolti; Mrz. fi.! - Gussone, Lojacono, Zodda (fi. e fr:.).

278. - Echium arenarium Guss.; Guss. Syn. I p. 233 ; Calc. p. 23; Lojac. Esc. p. 24; Car. in Pall. Fl. It. VI p. 935 ; Fiori e Bẻg. Fl. an. II p. 366. E. arenarium b. Guss. Syn. I p. 233; Lojac. Esc. p. 24. E. arenarium var. macranthum Fiori in Fl. an. II p. 366. E. Delileanum Lojac. Fl. Sic. II, par's II (nondum editum).

Gussone (il tipo sotto al Castello, la varietá b. nei campi coltivati), Lojacono (il tipo e la varietii). 
La varieti macranthum Fiori (= var. b. caule robustiore, corollis majoribus Guss.) è detta da Lojacono intermedia fra l'E. arenarium e l'E. maritimum. Gussone stesso nel Prodromo Fl. Sic. l'areva riferita come varieti all' E. marilimum anzichè all'arenarium. Io pure per varî esemplari di Linosa sono stato incerto se doressi riferirli all' E. marilimum o all'arenarizm. A Lampedusa peró tutti gli Echium che ho raccolti mi sono sembrati indubbiamente appartenenti al maritimum ossia confusum.

279. - Echium confusum De Coincy; Fiori e Bẻg. Fl. an. II p. 366. E. maritimum auct., non Willd.; Calc. p. 23; Ross p. 348 ; Solla p. 477 ; Lojac. p. 24 ; Car. in Parl. Fl. It. VI p. 936.

Comune nei luoghi colti ed incolti, e nell'isola dei Conigli; Mrz. fi. e fr. giov., Apr. fi. e fr.! - Calcara, Ross, Solla (fi.), Lojacono, Zodda (fi. e fr.).

Anche gli esemplari di Zodda mi sembrano appartenere all'E. confusum piuttostochè all'E. arenarium macrunthum.

280. - Borago officinalis L.; Solla p. 477.

Qua e lá nelle parti coltivate non comune; Mrz. fi.! - Solla (fi.), Zodda (fi. e fro.).

\section{1. - Anchusa Italica Retz.}

Raccolta e mandatami in fiore in Giugno quest'anno dalia guardia Martorana.

282. - Heliotropium Europaeum L.; Guss. Syn. I p. 212 ; Calc. p. 23 ; Lojac. Esc. p. 24 ; Car. in Parl. Fl. It. VI p. 832.

Gussone, Calcara, Lojacono.

\section{XLII. - SOLANACEAE.}

283. - Hyosciamus albus L.; Guss. Notiz. p. 83 et Syn. I p. 268 ; Calc. p. 24 et 31 ; Car. in Parl. Fl. It. VI p. 670.

Qua e lá nei luoghi ruderali, non comune; Mrz. e Apr. fi. ! Gussone, Calcara (alla Madonna), Zodda (in ruderatis ubique, fi. e fr. giov.).

Gussone dice che gli abitanti di Lampedusa fanno uso delle foglie di questa pianta, pestate insieme a foglie di Mandragora, come suppuratiro.

284. - Lycium Europaeum L.; Guss. Syn. I p. 273; Calc. p. 24 et 32 ; Lojac. Esc. p. 24; Car. in Parl. Fl. It. VI p. 702. 
Nelle siepi e tra le Opunzie; Mrz. e Apr. foglie! - Gussone, Calcara (alla Salina), Lojacono, Zodda (alla Salina, ad sepes, fi.). Gli esemplari miei sarebbero da riferirsi piuttosto alla forma a typicum Fiori in Fl. an. e quelli del dott. Zodda alla forma $\beta$ remulosum Dun., Fiori in Fl. an.

- Nicotiana glauca Grah.

Vicino alla cappella della Madonna vi sono alcuni alberetti di questa specie nati spontaneamente, a quanto assicurano. Ad ogni modo anche se la prima pianta vi fu messa dall'uomo, adesso rinasce da sè; Mrz. fr.!

285. - Solanum Sodomaeum L.; Guss. Syn. I p. 271 ; Calc. p. 24; Solla p. 477 ; Lojac. Esc. p. 24 ; Car. in Parl. Fl. It. VI p. 689. S. Sodomaenm a Mediterraneum Fiori e Bẻg. Fl. an. II p. 401.

Comune nella parte cóltivata dell' isola; Mrz. fi., Apr. fi. e fr. giov. : - Gussone (alla Cala della Madonna e alla Croce), Calcara, Solla (fi.), Lojacono, Zodda (fi.). Raccolto e mandato dal Conti in fiore in Novembre 1873.

Nella Flora analitica è indicata per Lampedusa e Linosa la forma glabra ( $\propto$ Mediterraneum Dun.); peró tanto gli esemplari miei quanto quelli di Zodda appartengono alla forma stellatopelosa ( $\beta$ Hermanniae Dun.).

286. - Solanum nigrum L.; Lojac. Esc. p. 24. S. moschahum Guss. Syn, I p. 272.

Qua e lả non comune; Mrz. e Apr. fi,! - Gussone, Lojacono, Zodda (fi. e fr.).

287. - Mandragora autumnalis Bert.; Car. in I'arl. Fl. It. VI p. 699. M. oflicinarem Guss. Syn. I p. 269 ; Calc. p. 24 ; Lojac. Esc. p. 24.

Gussone e Lojacono.

Secondo Gussone (Syn. I p. 268) le foglie della Mandragora, pestate assieme a quelle del Giusquiamo, sono adoperate dagli abitanti di Lampedusa come suppurativo.

Xliit. - Plantaginageae.

288. - Plantago Psyllium L.; Guss. Syn. I p. 201; Calc. p. 23; Solla p. 477; Lojac. Esc. p. 23 ; Fiori e Bẻg. Fl. an. III p. 101 (in nota). 
Comune; Mrz. fi. e frr., Apr. fi. e fr.! - Gussone, Solla (fr'), Lojacono (che gli dá il nome di var. prostrata $=P$. Afra L.), Zodda (fi. e fr.).

Gli esemplari che ho raccolti sono in parte umili e prostrati (f. divaricata [Zucc.] Béguinot in Fl. an. III p. 101) come sembra, dal nome di var. prostrata, che lo fossero quelli raccolti dal Lojacono. Anche gli esemplari di Zodda presentano la forma a rami eretti e quella a rami divaricati.

Nicotra (Naturalista sicil. X p. 67-8 e Commentario diagnostico delle piante vascolari siciliane p. 106-7) dice (e Bẻguinot Fl. anal. III p. 101 in nota riporta) che forse trovasi in Lampedusa ed in Linosa la $P$. Psylliem var. Zwierleinii Nicotra, ma la indicazione di questo habitat è incerta. La diagnosi della varieta Zwierleinii trovasi nei due lavori sopracitati di Nicotra.

\section{9. - Plantago albicans $L$.}

In contrada di Cavallo Bianco andando a Cala Malucco, in terreno arido; Mrz. fi. e fr.!

290. - Plantago Coronopus I, ; Guss. Sy'n. I p. 200 ; Calc. 1) 23 ; Lojac. Esc. p. 23. P. commutata Guss. Syn. I p. 200 ; Calc. p. 23; Lojac. Esc. p. 23.

Comune nelle terre aride; Mrz. e Apr. fi.! - Gussone, Lojacono, Zodda (fi. e fr.).

Non ho veduto e raccolto aitro che la var. commulata, mentre Gussone e Lojacono indicano anche il tipo. Zodda, oltre alla var. commulata, ha raccolto pure la var. pusilla Moris (in Fiori e Bẻg. Fl. an.) con scapi ridotti fino a $1 \mathrm{~cm}$, spighe di pochi tiori, raccorciate, talvolta subsferiche, foglie intere o quasi. Non avendo semi maturi non si puó sapere se questi siano alati come nella varietà microcephala Somm. Fl. Giglio.

291. - Plantago Serraria L.; Solla p. 477, et 465 (in nota). Solla solo (fr.).

\section{XliV. - Scrofulariaceae.}

292. - Verbascum sinuatum L.; (iuss. Syn. I p. 203; Calc. p. 24 ; Lojac. Esc. p. 25 ; Car. in Pari. Fl. It. VI p. 586 . Verbascum sp. Solla p. 477 ? 
Qua e li non comune; Mrz. foglie! - Gussone, Lojacono, Solla (se pure a questo si riferisce il suo Verbascum sp. trovato con sole foglie), Zodda (foglie).

293. - Scrofularia peregrina L.; Guss. Syn. II p. 127; Calc. p. 27; Solla p. 477; Lojac. Esc. p. 25; Car. in Parl. Fl. It. VI p. 554 .

Qua e lå specialmente nei luoghi più freschi; Mrz. fi., Apr. fi. e fr. ! - Gussone, Solla (bocci), Lojacono, Zodda (fr.).

294. - Antirrhinum Orontium L.; Lojac. Esc. p. 24.

Lojacono solo.

295. - Linaria reflexa (L.) Desf.; Ross p. 348 ; Solla p. 477; Car. in Parl. Fl. It. VI p. 624. L. reflexa var. pumila Lojac. Esc. p. 25; Fiori e Bẻg. Fl. an. II p. 422.

Molto comune specialmente nei terreni aridi lontano dal coltivato; Mrz. fi. e fr., Apr. fi. e fr.! - Ross (sempre con fiori azzurri), Solla (fi. e fr.), Lojacono (il quale l' ha distribuita nelle Pl. It. sel. n. 56), Zodda (fi. e fr.).

Se la caratteristica principale della var. pumila è la colorazione violetta della corolla (Lojacono loc. cit. dice che il tipo in Sicilia ha sempre corolle bianche) e la piccolezza delle foglie, tutta la $L$. reflexa che ho veduta di Lampedusa appartiene a questa varietá. I fiori difatti sono sempre di un violaceo più o meno intenso con palato giallo, e le foglie sono sempre piccole. Peró i suoi fusti raggiungono talvolta $30 \mathrm{~cm}$. ed i suoi fiori fino a $23 \mathrm{~mm}$. di lunghezza, e le foglie sono spesso quasi orbicolari. Anche Ross ha notato che a Lampedusa le corolle erano sempre colorate.

\section{6. - Veronica arvensis L.}

Luogo fresco nella Valle Pollicino, vista in quel luogo solo; Mrz. fi. e fr'.!

\section{Xlv. - Orobanchaceae.}

297. - Orobanche sangúinea Presl; Car. in Parl. Fl. It. VI p. 369 ; Fiori e Bèg. Fl. an. II p. 478. O. crinata Guss. Syn. II p. 136 ; Calc. p. 27 ; Lojac. Esc. p. 25 . Orobanche sp. Solla p. 477 ? Sulle radici del Lolus cytisoides ver'so Capo Ponente, non frequente; 28 Apr. fi.! - Gussone, Lojacono (sulle radici del Lotus 
cytisoides). Riferisco qui a caso la Orobanche sp. di Solla (fi.), che egli non potè determinare perchè gli esemplari raccolti si guastarono. Zodda (fra l'Imbriacola e Pozzo del Monaco, sul Lotus cytisoides, fi.).

298. - Orobanche minor Sutt. Orobanche sp. Lojac. Esc. 25?

Zodda (verso Capo Ponente e all'Imbriacola, fi. e fr.). - Riferisco a caso a questa specie quella indeterminata di Lojacono.

Gli esemplari raccolti da Zodda appartengono alla var. litorea (Guss.) Bẻg. in Fl. an. II p. 484.

299. - Kopsia Muteli (F. Schultz) Bẻug. Plelipaea Muteli Lojac. Esc. p. 25.

Lojacono, Zodda (Cala Galera e Cavallo Bianco, fi. e fr.).

Gli esemplari di Cala Galera appartengono ad una forma alta $20 \mathrm{~cm}$. e ramificata; quelli di Cavallo Bianco ad una forma piủ piccola, alta $4-6 \mathrm{~cm}$., non ramificata e, secondo che dice l' etichetta, a fiori bianchi.

\section{Xlvi. - Labiatae.}

300. - Thymus capitatus (L.) Hoffm. et Link; Guss. Syn. II p. 95 ; Calc. p. 27 et 31 ; Lojac. Esc. p. 24; Car. in Parl. Fl. It. VI p. 101 ; Fiori e Bẻg. Fl. an. III p. 65. Satureja capitata Guss. Notiz. p. 86 et 87.

Ancora comune nei luoghi aridi rocciosi, ma generalmente ridotto a miseri sterpi; Mrz. e Apr. foglie ! - Gussone, Calcara, Lojacono (forma a solo dei fruticeti caratteristici nella parte orientale dell' isola), Zodda (foglie).

301. - Satureja fasciculata Raf.; Guss. Syn. II p. 93; Calc. p. 27; Car. in Parl. Fl. It. VI p. 121. S. Graeca ל fasciculata Fiori e Bẻg. Fl. an. III p. 58. Micromeria fasciculata Lojac. Esc. p. 24.

Valle Imbriacola nei luoghi rocciosi, ivi frequente, ma in area ristretta e non vista altrove; Mrz. fi.! - Gussone, Lojacono.

302. - Satureja microphylla (Urv.) Guss.; Guss. Syn. II p. 92; Calc. p. 27; Solla p. 477; Car. in Parl. Fl. It. VI p. 124; Fiori e. Bẻg. Fl. an. III p. 58. Micromeria microphylla Lojac. Esc. p. 24.

Qua e là nei luoghi aridi sassosi; Mrz. fi., Apr. fi. e fr.!Gussone (la var. b.), Solla (fi. e fr.), Lojacono, Zodda (fi. e fr.). 
Tutto quanto ho visto di Lampedusa corrisponde alla var. b. di Gussone, caratterizzata per le foglie superiori porporascenti come la parte superiore del fusto che inoltre è pubescente, e per' i peli del calice patenti.

303. - Saluia clandestina L.; Solla p. 477; Lojac. Esc. p. 24; S. verbenaca \& vulgaris Car. in Parl. Fl. It. VI p. 257.

Molto comune nei luoghi erbosi e nei campi incolti; Mrz. fi., Apr. fr.! - Solla (sfiorita), Lojacono. L' ho riceruta dal Conti che la raccolse fiorita in Norembre.

Caruel nella Flora Italiana riferisce gli esemplari dell'Ajuti alla $S$. verbenaca * vulgaris, mentre avrebbe dovuto riferirli alla var. $\beta$ australis. Ció è derivato dal non averne il Caruel veduto $\mathrm{i}$ fiori.

304. - Rosmarinus of̂́icinalis I. : (inss. Notiz. p. 83, 89, 87 et Syn. I p. 20; Calc. p. 22, 31, 32 et 34 ; Solla p. 465 (in nota); Lojac. Esc. p. 24 ; Car. in Parl. Fl. It. VI p. 281.

A Capo Rupestre; 30 Apr. sole foglie ! - Gussone (var. c. angustifolius), Calcara (Punta Rupestre), Lojacono (forma dei luoghi sterilissimi a foglie angustissime).

Solla dice di averlo cercato invano. Neanche io l'ho più reduto quest'anno; ma mi fu assicurato che esistera ancora in contrada di Terranova e verso Capo Ponente. Ad ogni modo è diventato raro assai.

I miei esemplari appartengono ad una forma a foglie strette come notano Gussone, Calcara e Lojacono; questa è del resto la forma più comune sotto cui presentasi anche altrove il Rosmarinus selvatico. Gussone (Noti\%. p. 83) dice che è adoperato dagli abitanti di Lampedusa come pianta medicinale.

- Nepeta Cataria L.; Sanvisente L'Is. di Lamp. p. 63.

Non tengo conto di questa specie nella numerazione, dubitando che si tratti di un equiroco, poichè Sanvisente dice che si trova in Lampedusa « da per'ogni dove » mentre nessun altro ve l' ha trovata.

305. - Sideritis Romana L.; Guss. Syn. II p. 66; Solla p. 477; Lojac. Esc. 1. 24 ; Car. in Parl. Fl. It. VI p. 65. S. Romana et S. appruximala Calc. p. 27.

Comunissima specialmente nei terreni aridi; Mrz. fi. e fr., Apr. fr. e ancora qualche fi.:-Gussone, Calcara, Solla (fr.), Lojacono, Zodda (fi. e fr.). 
La Siderilis approximata Gasp. in Guss. citata da Calcara, secondo Caruel loc. cit., non è neanche una varieti della S. Romana. Alcuni dei miei esemplari e quelli di Zodda hanno verticilli multiflori che cominciano fino dalla base del caule, carattere attribuito da Gussone alla S. approximata. Abbondano nei terreni aridi gli individui nani.

306. - Marrubium vulgare L.; Guss. Syn. II p. 80 ; Calc. p. 27; Solla p. 477; Car. in Parl. Fl. It. VI p. 70. M. A pulum Guss. Syn. II p. 81 ; Calc. p. 27 ; Lojac. Esc. p. 24. M. vulgare var. Apulum Ross p. 348.

Qua e lá vicino all'abitato e nei campi incolti; Mrz. foglie, Apr. fi. e fi: : - Gussone, Calcara, Ross, Solla (fi. e fir.), Lojacono, Zodda (fi.).

Nonostante che Gussone stesso indichi le due specie vulgare e Apulum per Lampeilusa, non posso tenerle distinte, poichè mi sembrano semplici variazioni senza importanza.

307. - Lamium amplexicaule L.; Lojac. Esc. p. 24; Car: in Parl. Fl. It. VI p. 213.

Comune nella parte coltirata dell' isola; Mrz. fi., Apr. fi. e fr.. Lojacono (forma dalle corolle lungamente exserte), Zodda ( $\mathrm{fr}^{\mathrm{r}}$ ). Specialmente in Aprile ho trovato freruenti i fiori casmogami, con corolle lungamente sporgenti dal calice.

308. - Prasium majus L.; Guss. Notiz. p. 87 et Syn. II p. 107 ; Calc. p. 27 et 31 ; Solla p. 477 ; Lojac. Esc. p. 24 ; Car. in Parl. Fl. It. VI p. 229.

Ancora frequente nelle siepi, muri, roccie, anche nell' isola dei Conioli ; Mrz. fi., Apr. fr.! - Gussone, Calcara, Solla (fi.), Lojacono, Zodda (fi.).

Gussone dice che perde le foglie dopo la fruttificazione. Nei miei esemplari fruttificati le foglie sono ancora fresche; ma Gussone visitó Lampedusa nell Agosto ed i miei esemplari fruttificati sono di Aprile.

\section{9. - Ajuga Iva L.}

Solo Zodda (Cala Cruce in arvis, fi. e fr.).

Le piante raccolte da Zodda sono da riferirsi alla var. pinnatifida (Forsk.).

310. - Teucrium fruticans L.; Guss. Notiz. p. 83; Calc. p. 27, 30 et 32 ; Solla p. 477 ; Lojac. p. 24 ; Cal. in Parl. Fl. It. VI p. 299. 
Ancora frequente nei luoghi rupestri e nei residui di macchia, ma generalmente mutilato; Mrz. e Apr. fi.! - Gussone, Calcar'a, Solla (fi.), Lojacono, Zodda (fi.).

Gussone dimentica di citare Lampedusa nella Synopsis. Nelle « Notizie ecc. » p. 83 dice che gli abitanti di Lampedusa lo adoprano come stomachico.

311. - Teucrium Creticum L.; Guss. Syn. II p. 57 ; Calc. p. 27 ; Solla p. 465 (in nota); Lojac. Esc. p. 24; Car. in Parl. Fl. It. VI p. 300; Fiori e Bẻg. Fl. an. III p. 11. T. rosmarinifolium Nym. Consp. Fl. Eur. p. 564 .

Questa pianta è stata trovata a Lampedusa solamente da Gussone. Nella Flora italiana di Parlatore è citata come raccolta da Pasquale, probabilmente perchè da questi fu mandato a Firenze un esemplare dell' erbario Gussoneano.

È questa l'unica localitá non solo italiana ma europea di questa specie orientale.

312. - Teucrium flavum L.; Calc. p. 27; Solla p. 477 ; Lojac. p. 24 ; Car. in Parl. Fl. It. VI p. 288.

Raro, trovato in due soli luoghi; Mrz. foglie! - Calcara, Solla (fi.), Zodda (foglie).

\section{XLVII. - GLobUlariaceae.}

313. - Globularia Alypum L.; Guss. Notiz. p. 83, 86, 87 et Syn. I p. 168; Calc. p. 23, 30 et 31 ; Lojac. Esc. p. 25 ; Car. in Parl. Fl. It. VI p. 41 ; Fiori e Bẻg. Fl. an. III p. 92.

Qua e là nei luoghi sassosi aridi ; Apr. foglie sole! - Gussone, Calcara, Lojacono (rara).

Considerata dagli abitanti di Lampedusa come medicinale, secondo Gussone.

\section{XLViII. - Primulaceae.}

314. - Asterolinum Linum stellatum (L.) Duby. Lysimachia Linum stellalum Guss. Syn. I p. 238; Calc. p. 23.

Gussone solo.

315. - Anagallis arvensis L.; Calc. p. 23; Solla p. 477; Lojac. p. 24. 
Comunissima in tutta l'isola, tanto nelle parti coltivate quanto Iontano da queste, e nell'isola dei Conigli; Mrz. fi. e fr., Apr. fi. e fr. ! - Calcara, Solla (fi. e fr.), Lojacono, Zorda (li. e fr.). Calcara con due * * indica che fu raccolta anche da Gussone. Ció peró non risulta dalla Synopsis.

Pare che non esista in Lampedusa altro che la valietá a corolla azzurra. Per quanto ne facessi ricerca non ho potuto vederne una pianta con corolla rossa. Anche gli esemplari raccolti nel 1873 e quelli di Zodda appartengono alla var. comuleu.

\section{Xlit. - Plumbaginaceae.}

316. - Statice monopetala L.; Guss. Syn. I p. 374; Bert. Fl. It. III p. 533; Calc. p. 25; Lojac. Essc. p. 23; Mori in Parl. Fl. It. VIII p. 572; Fiori e Bér. Fl. an. II p. 328.

Gussone solo (Scoglio dei Conigli e nel lido che gli resta dirimpetto).

317. - Statice echioides L.; Guss. Syn. I p. 373; Bert. Fl. It. III p. 525 ; Calc. p. 25 ; Lojac. Esc. p. 23 ; Martelli Rir. Crit. Stat. It. p. 20 ; Mori in Parl. Fl. It. VIII p. 590 ; Fiori e Bẻg. Fl. an. II p. 328.

Presso il Porto; Aprile bocci!- - Gussone (nel lido presso il Porto, e nella Salina), Calcara. Nella Flora Italiana é citato come raccoglitore Todaro ii quale non fu mai a Lampedusa, ma distribui esemplari raccolti da Calcara.

318. - Statice sinuata L.; Guss. Syn. I p. 374 ; Calc. p. 25; Lojac. Esc. p. 23; Mori in Parl. Fl. It. VIII p. 573; Fiori e Bẻg. Fl. an. II p. 328.

Gussone solo.

319. - Statice psiloclada Boiss.; Mori in Parl. Fl. It. VIII p. 578 saltem pro parte. S. albida et S. belliclifolia Guss. Syn. I p. 369. S. psiloclada var. gracilis Fiori in Fl. an. II p. 330. S. bellidifolia Bert. FI. It. III p. 524 ; Lojac. Esc. p. 23. S. albida Calc. p. 24; Lojac. Esc. p. 23. S. bellidifolia f. albida pro parte Martelli Riv. crit. Stat. It. p. 12. S. psiloclada $\varepsilon$ albida Fiori in Fl. an. II p. 330.

Molto comune in tutta la zona piủ prossima al mare dore forma dei densissimi pulvinuli alti $2-3$ decimetri; Mr'z. foglie, 
Apr. primi fi.! - Gussone, Calcara, Lojacono. L' ho ricevuta in fiol'e dal Conti e dal Nartorana. L' hanno distribuita Ross n. 483 e Lojacono n. ${ }^{1} 125$ e 126, coi nomi di psiloclada rar. gracilis e albidla. Mori in Parl. Fl. It. cita la $S$. psiloclada come raccolta in Lampione da Pasquale e da Todaro, i quali né l'uno nè l'altro visitarono quest' isola.

Mi sono trorato obbligato a riunire le S. albida e bellidifolia di Gussone senza neppure poterle distinguere come varietá, perchè questo autore deve essere incorso in qualche errore nelle descrizioni e negli habitat di queste due specie o forme. Difatti quello che dice della bellidifolia "planta saepe in cœespitem semiglobosum diametro $1-1 \%$ pedali congesta.... in Lampedusa vulgarissima " non lascia alcun dubbio che egli chiamasse bellidifoliu la sola specie di Statice che sia comunissima in Lampedusa, la sola trovata da Lojacono. Ma ad essa attribuisce rami inferiori sterili mentre nella specie comune in Lampedusa i rami sono tutti fertili. Della S. albida invece, che egli indica di Lampione soltanto, dice «ramis sterilibus nullis». Da ció è derivato anche la diversa interpretazione data dagli autori posteriori ai nomi Gussoneani. Ad ogni modo credo che, anzichè di due specie distinte, si tratti di forme poco diverse, come gia opinara Bertoloni, il quale della albida Guss. facera una var. $\beta$ della bellicifolia.

320. - Statice intermedia Guss.; Guss. Syn. I p. 369; Calc. p. 25; Lojac. Esc. p. 23. S. globulariaefolia $\beta$ Bert. FI. It. III p. 519. S. bellidifolia $\beta$ albida pro parte Martelli Riv. Crit. Stat. It. p. 12 S. psiloclack Mori in Parl. Fl. It. VIII p. 578 pro parte. S. psiloclara .6 intermedia Fiori in Fl. an. II p. 330.

Gussone solo (luoghi inondati salsi alla Salina).

Bertoloni scrive di avere ricevuto questa specie da Gussone di Lampelusa, di Erusa (Farignana), di Phoenicusa e d'Icesia (due delle Lipari), mentre Gussone nella Synopsis la indica soltanto di Lampedusa. Questo è una riprova della confusione che esiste in questo difficilissimo genere.

321. - Statice virgata Willd. S. Smithii b. major Guss. Syn. I p. 370; Calc. p. 25; Lojac. Esc. p. 23. S. oleaefolic Bert. Fl. It. III 1. 517 .

Solo Gussone. 
322. - Statice dubia Andrews ex Guss.; Calc. p. 25 ; Lojac. Esc. p. 23.

Calcara solo (var. glabra).

323. - Statice minuta L.; Guss. Syn. I p. 373.

Indicata so!o da Gussone; Calcara dimentica di riportare la citazione della Synopsis.

Le tre ultime specie che sono soggette alle più varie interpretazioni, andrebbero forse con rantaggio riunite sotto il nome di S. minuta; ed alla S. psiloclada, nella quale ho gia fuso due delle specie di Gussone, andrebbe forse riunita la S. intermedia.

\section{L. - Polygonaceae.}

324. - Polygonum aviculare L.; Guss. Syn. I p. 453; Calc. p. 25 ; Lojac. Esc. p. 25.

Gussone e Lojacono. Calcara ha dimenticato di apporre un* a questa specie, per indicare che era stata raccolta da Gussone. 325. - Polygonum maritimum L. Guss. Notiz. p. S3 et Syn. I p. 452 ; Calc. p. 25 ; Lojac. Esc. 25.

Gussone (isolotto dei Conigli), Lojacono.

Gussone (Notizie ecc.) dice che gli abitanti di Lampedusa ne fanno uso come diuretico.

326. - Emex spinosa (L.) Campd.; Guss. Syn. I p. 436 ; Calc. p. 25; Lojac. Esc. p. 25; Fiori e Paol. Fl. an. I p. 297. Rumex spinosus Guss. Suppl. Prodr. p. 110.

Qua e lá nelle vicinanze del Porto; Mrz. foglie, Apr. fr.! Gussone, Lojacono, Zodda (fi. e fr.)

\section{7. - Rumex pulcher L.}

Alle cave presso il paese e a Terranova in luoghi umidi, raro; Mrz. foglie e fr. secchi dell' anno precedente!

328. - Rumex bucephalophorus L.; Guss. Syn. I p. 432 ; Calc. p. 25 et 32 ; Solla p. 477 ; Lojac. Esc. p. 25.

Qua e lá nei terreni incolti, non frequente; Mrz. fi., Apr. fi. e fr.! - Gussone, Solla (fr.), Lojacono, Zodda (fi. e fr.). 


\section{Li. - Cirenopodiaceae.}

329. - Obione portulacoides (L.) Moq. Tand.; Lojac. Esc. p. 25. Atriplex portulacoides Guss. Notiz. p. 86 et Syn. II 1. 588; Calc. p. 30.

Gussone solo (la var. c. angustifolia).

330. - Atriplex roseum L.; Lojac. Esc. p. 25.

Solo Lojacono (al Porto).

331. - Atriplex Halimus L.; Guss. Syn. II p. 587 ; Calc. p. 30 ; Solla p. 477 ; Fiori e Paol. Fl. an. I p. 307.

Nelle vicinanze del Porto in riva al mare, dei grossi tronchi legnosi con poche foglie, e sull' isola dei Conigli abbondante e rigoglioso; Mrz. e Apr. sole foglie! - Gussone, Calcara, Solla (foglie).

Gussone loc. cit. fa delle osservazioni sulla forma a forlie larghe, argentee ed a fiori disposti in glomeruli densi e piccoli, formanti spighe brevi, sotto cui trovasi in Lampedusa.

332. - Bota maritina L.; Guss. Syn. I p. 298 ; Calc. p. 24.

Presso il Porto sotto il Castello; 27 Apr. fi. e fr. ! - Gussone.

333. - Beta macrocarpa Guss.; Guss. Syn. I p. 298 ; Calc. p. 24. B. vulgaris L. x macrocarpa Fiori e Paol. Fl. an. I p. 308.

Insieme alla precedente; $27 \mathrm{Apr}^{\circ}$. fi. e fr. ! - Gussone e Calcara.

Sanvisente (p. 58), oltre alle Beta marilima e B. macrocarpa, cita anche la $B$. vulgaris, col quale nome intende probabilmente una razza coltivata.

334. - Chenopodium murale L.; Guss. Syn. I p. 294; Calc. p. 24 ; Solla p. 477 ; Lojac. Esc. p. 25.

Comune nelle parti coltivate come pure lontano da esse; Mrz. fi., Apr. fi. e fr.. - Gussone, Calcara, Solla (fr.), Lojacono, Zodda (fi.).

Gussone, Calcara e Lojacono riferiscono la pianta di Lampedusa alla var. b. pruinosum Guss., alla quale corrispondono pure i miei esemplari e più o meno anche quelli di Zodda.

335. - Arthrocnemum glaucum (Del.) Ung. Stbg. Salicornia macrostachya Guss. Syn. I p. 7 ; Calc. p. 22 ; Lojac. Esc. p. 25. Gussone, Calcara (Salina).

Gussone indica per Lampedusa tanto il tipo quanto una varietà che chiama b. glaucescens, mumilis (alla Salina sotto il 
Castello), e dice che gli abitanti di Lampedusa mangriano questa pianta in insalata.

336. - Salsola longifolia Forsk.; Solla p. 47 a t 4tic (in nota); Fiori e Paol. Fl. an. I p.318. S. oppositifolia Guss. Notiz. 86, 87 et Syn. I p. 301.

L' ho vista soltanto nell' isola dei Conigli; Mrz. foglie! - Gussone (rupi e pendici marine al Sud e sullo scoglio dei Conigli), Solla (fi.). Calcara ha dimenticato di citare questa specie.

337. - Salsola Tragus L.; Guss. Syn. I p. 299 ; Calc. p. 24 et $3 \mathbf{i}$; Lojac. Esc. p. 25 .

Gussone, Calcara.

338. - Salsola Soda L.; Calc. p. 24 et 34 ; Lojac. Esc. p. 25.

Solo Calcara, il quale ne raccomanda la coltura.

339. - Suaeda fruticosa (L.) Forsk. Chenopodium fruticosım Guss. Notiz. p. 86 et Syn. I p. 297; Calc. p. 24; Lojac. Esc. p. 25.

In riva al mare nelle ricinanze del Porto e abbondante nell'isola dei Conigli; Mrz. foglie, Apr. fi. ! - Gussone, Calcara, Zodda (Capo Ponente, in r'upibus, fi.).

Gussone e Calcara riferiscono la pianta di Lampedusa alla varieti b. confertiflora alla quale corrispondono anche i miei esemplari.

\section{LiI. - URTICACEAE.}

340. - Theligonum Cynocrambe L.; Guss. Syn. II p. 600 ; Calc. p. 30 ; Lojac. Esc. p. 26 ; Parl. Fl. It. IV p. 310.

Sulle rupi frequente; Mrz. fi. e fr., Apr. fr.! - Gussone, Calcara, Lojacono, Zodda (fr.).

341. - Urtica urens L.; Somm. Piante ined.

Frequente presso l'abitato; Mrz. fi. e fr., Apr. fi. e fr.!

342. - Urtica membranacea Poir.; Guss. Syn. II 1. 579; Calc. p. 29; Lojac. Esc. p. 26. U. Lusitanica a membranacea Solla p. 478. U. neglecta Guss. Syn. II p. 579 ; Lojac. Esc. p. 20. U. membranacea var. neglecta Parl. Fl. It. IV p. 320.

Nolto comune vicino all'abitato, ma anche lontano da questo, come nell' isolotto dei Conigli; Mrz. fi. e fr., Apr. fi. e fr.! Gussone, Solla (fr.), Lojacono, Zodda (fi. e fr.). 
Ho raccolto tanto il tipo quanto la var. neglecta. Anche fra le piante di Zodda trovansi le due forme.

343. - Urtica pilulifera L. ; Parl. FI. It. IV p. 331. U. Balearica Guss. Syn. II p. 581 ; Lojac. Esc. p. 26.

Gussone e Lojacono. Calcara dimentica il segno * per indicare che è stata trovata da Gussone.

344. - Parietaria officinalis L.; Parl. Fl. It. IV p. 335 ; Ross p. 348. P. diffusa Guss. Notiz. 83 et Syn. II p. 640 ; Calc. p. 30; Lojac. Esc. p. 26.

Comunissima sotto le siepi, sulle rupi e sui muri, anche nell' isola dei Conigli ; Mrz. fi., Apr. fi. e fr.! - Gussone, Calcara, Ross, Lojacono, Zodda (fi. e $\mathrm{fr}^{\circ}$.).

Calcara riferisce la pianta da lui raccolta alla var. Sicula, e Ross alla rar. mamiflora. Io ho notato negli orti e nei luoghi più freschi e fertili la forma erecta, sulle rupi la forma Judaica, e fra l'una e l'altra tutte le forme di transizione. Sull' isola dei Conigli la forma Judaica.

Gussone (Notizie) dice che gli abitanti di Lampedusa applicano sulle contusioni questa pianta schiacciata insieme al Rosmarinus.

345. - Parietaria Cretica I.; Guss. Syn. II p. 642 ; Calc. p. 30 ; Solla p. 478; Parl. Fl. It. IV p. 338 ; Fiori e Paol. Fl. an. I p. 280. Gussone, Calcara, Solla (fi. e fr.).

346. - Ficus Carica L.; Guss. Notiz. p. 85 et 97 ; Calc. p. 30 et 35 ; Solla p. 478 ; Lojac. Esc. p. 12 et 26.

Calcara, Solla e Lojacono citano il fico soltanto come pianta coltivata ed jo non l'ho visto allo stato spontaneo, benchè mi abbiano detto che si trovava anche selvatico. Calcara ne raccomanda la coltura e dice che a tempo suo ve ne erano alcune piante nel Vallone della Madonna. Lojacono invece ritiene che farebbe cattiva prova. Gussone (Notizie) ne trovo 12 alberi piantati da antichi coloni; nella Synopsis non fa menzione di Lampedusa.

\section{LiII. - BalanophoraceaE.}

347. - Cynomorium coccineum L.; Guss. Syn. II p. 561; Calc. p. 29: Parl. Fl. It. IV p. 383.

Gussone solo. 


\section{LIV. - THYMELAEACEAE.}

348. - Passerina hirsuta L.; Guss. Syn. I p. 450 ; Calc. p. 25 , 31 et 32 ; Solla p. 478.

Frequente nei terreni aridi e rocciosi in vicinanza del mare; Mrz. fi., Apr. fr.! - Gussone, Calcara, Solla, Zodda (fi. e fro.).

349. - Daphne Gnidium L.; (russ. Notiz. 1) 86 et Syn. I p. 4.19 ; Calc. p. 25 et 32 ; Solla p. 465 (in nota).

Gussone, Calcara. Solla dice di averla cercata invano.

\section{LV. - LaUraceae.}

350. - Laurus nobilis L.; Guss. Notiz. p. 86 et Syn. I p. 459; Calc. p. 25; Solla p. 465 (in nota).

Gussone (Vallone dello Scoglio), Calcara, il quale nella Florula lo cita come raccolto anche da lui, ma poi al capitolo boschi non ne parla piu. Credero che questa specie fosse stata distrutta dagli uomini poichè nessuno l'aveva piủ citata, e Solla, dopo arerla cercata invano, aveva scritto che gli abitanti ai quali ne chiese nou saperano neppure più della sua esistenza nell' isola. Quest'anno però $\mathrm{mi}$ fu detto che esisteva ancora in un vallone che per questo si chiamava Vallone dell' Alloro. Non so peró quanto sia attendibile questa informazione.

\section{I.VI. - Santalaceae.}

351. - Thesium humile Tahl; Ross p. 348; Solla p. 478; Lojac. Esc. p. 25; Fiori e Paol. Fl. an. I p. 285.

Nei campi incolti, Vallone dell' Imbriacola; $30 \mathrm{Apr}$. f1.! Ross (in un campo in riposo), Solla (fr.), Lojacono.

\section{LViI. - Callitrichaceae.}

352. - Callitriche pedunculata DC.; (iuss. Syn. I p. 9; (alc. p. 22; Parl. Fl. It. IV p. 420. C. palustris \& pedunculata Fiori e Paol. Fl. an. II p. 294.

Gussone solo. 


\section{LVIII. - EuphorbiaceaE.}

353. - Euphorbia Peplis L.; Guss. Syn. I p. 532 ; Calc. p. 26; Lojac. Esc. p. 25; Parl. Fl. It. IV p. 446.

Gussone, Calcara, Lojacono.

- Euphorbia Chamaesyce L.; Solla, p. 465 (in nota).

Solia la cita come raccolta in Lampedusa dall' Ajuti e da me. Dubito però che si tratti di un errore, poichè fra le mie piante non esiste, e neppure trovasi neğli erbarî di Firenze dore sono conservate le piante dell'Ajuti.

354. - Euphorbia helioscopia L.; Guss. Syn. I p. 540 ; Calc. 1) 26: Lojac. Lsc. 1) 25: Parl. Fl. It. IN 1. 491.

Comune specialmente nella parte coltivata dell' isola; Mr'z. fi. e fr., Apr. fr. : - Gussone, Lojacono.

355. - Euphorbia Peplus L.; Guss. Syn. I p. 533; Calc. p. 26; Lojac. Esc. p. 25 ; Parl. Fl. It. IV p. 498. E. peploides Solla p. 478.

In tutta l' isola e sull' isolotto dei Conigli; Mrz. fi. e fr., Apr. fr.! - Gussone, Lojacono, Solla (fi. e fr.), Zodda (fr.).

Il tipo trovasi qua e là, non comune, piu particolarmente nel coltirato; la var. peploides Gouan invece è molto comune fra le microfite, specialmente nei terreni aridi. Sull' isolotto dei Conigli trovasi la varietà.

356. - Euphcrbia exigua L.; Guss. Syn. I p. 535 ; Calc. p 26; Lojac. Esc. p. 25; Parl. Fl. It. IV p. 504. E. exigua var. retusa Solla p. 478.

Quasi dorunque nei terreni aridi fra le microfite, e nell' isola dei Conigli ; Mrz. fi. e fr., Apr. fr. ! - Gussone, Solla (fi. e fr.), Lojacono, Zodda (fi. e fir.).

Tutta l'E. exigua di Lampedusa che ho veduta, appartione alla var. retusa (Cav.). Essa varia del resto per la statura (da 1-2 a $15 \mathrm{~cm}$.) e per le foglie ora strette, allungate e quasi affatto lineari, ora cuneate, ora accorciate e largamente obovato-cuneate, sempre peró retuse. Spesso tutta la pianta è colorata in rosso.

357. - Euphorbia pinea I..; Guss. Notiz. 1. 83 et Syn. I 1. 53!); Calc. p. 26; Solla p. 478; Lojac. Fisc. p. 25; Parl. Fl. It. IV p. 521. 
Comunissima ed abbondante in tutta l'isola e nell'isola dei Conigli ; Mrz. fi. e fr., Apr. fi. e fr.! - Gussone (var. foliis glaucescentibus), Calcara, Solla (fr.), Lojacono, Zodda (fi. e fr.).

Sulle rupi marine e in generale vicino al mare si trova una forma più bassa, più ramificata, a foglie più conferte, spesso rosseggianti, molto diversa dalla forma alta, verde e lassa che trovasi più lontano dal mare; ma fra le due vi sono tutti i passaggi.

358. - Euphorbia Paralios L.; Guss. Syn. I p. 537 ; Calc. p. 20; Lojac. p. 25 ; Parl. Fl. It. IV p. 526.

Nella spiaggia arenosa detta Arena Bianca; Mrz. primi fi. ! Gussone (var. b. palens, sotto al Castello, alla Cala della Madonna e della Croce), Calcara, Lojacono.

La varietà b. di Gussone non mi sembra distinguibile dal tipo.

\section{9. - Euphorbia Terracina L.}

Zodda solo (Cala Francese, in saxosis, fi. e fr.).

360. - Euphorbia dendroides L.; Guss. Notiz. p. 86 et Syn. I p. 536 ; Calc. p. 26, 32 et 34 ; Solla p. 478; Lojac. Esc. p. 25 ; Parl. Fl. It. IV p. 553.

E ancora frequente in varie parti dell' isola, ma non abbondante dovunque come era una volta; trovasi anche nell'isola dei Conigli; Mrz. fi., Apr. fr.!-Gussone, Calcara, Solla (fr.), Lojacono (nei fruticeti di cui è una delle essenze principali).

Gussone dice che in estate, mancando le erbe, i conigli sono obbligati a cibarsi delle corteccie di questa Euphorbia e della E. Pinea. È chiamata dagli isolani Camarrone.

361. - Mercurialis annua I.. ; Calc., p. 30 et 32 ; Solla p. 478 ; Lojac. Esc. p. 26.

Molto comune nei lnoghi coltirati, come lontano da essi; Mrz. fi. e fr., Apr. fr.! - Calcara (?), Solla (fr.), Lojacono, Zodda $(\mathrm{fr}$.$) . Calcara mette un* che indicherebbe essere stata raccolta$ da Gussone e non da lui, mentre la Synopsis non la cita. 


\title{
MONOCOTYLEDONEAE.
}

\author{
LIX. - ORCHIDACEAE.
}

\section{2. - Ophrys arachnites (L.) Lam.}

Qua e lá sporadica e rara, nei luoghi dore prima fu la macchia. Trovata a Cala Galera, all Imbriacola e a Sanguedolce, sempre in pochi esemplari; Mrz. fi.!

363. - Ophrys Speculum Link; Lojac. Esc. p. 26; Fiori e Paol. Fl. an. p. 237.

Sporadica qua e lí in molti luoghi dove prima fu la macchia, ma che ora sono scoperti. L' ho trovata quasi in ognuna delle gite, ma mai copiosa; Mrz. fi., Apr. frutii con fiori secchi ! Lojacono (luoghi ombrosi nei fruticeti di Ponente), Zodda (Cala Galera, Cala Uccello, Imbriacola, Sanguedolce, Aria Rossa e verso Ponente, haud rara in dumetis, fi. e fr. giov.).

\section{4. - Ophrys lutea Cav.; Solla p. 478?}

Solo Solla il quale non è certo della determinazione, avendola trorata sfiorita. E quindi specie dubbia; potrebbe difatti trattarsi della specie precedente.

\section{LX. - IRIDACEAE.}

365. - Romulea Columnae Seb. et Mauri. R. Bulbocodium Guss. Syn. I p. 33 ; Calc. p. 22; Parl. Fl. It. III p. 243. Ixia Bulbocodium Guss. Suppl. Prodr. p. 7. Trichonema Bulbocodium Solla p. 478.

Nelle terre aride fra le microfite, comunissima in tutta l'isola, salvo nelle parti coltivate; trovasi pure nell' isolotto dei Conigli; Mrz. fi. e fr., Apr. fi. ! - Gussone, Solla (fr.).

Non esito a riferire alla $R$. Columnae la pianta creduta da Gussone R. Bulbocodium, perché Gussone stesso era ilcerto della determinazione avendo visto la pianta secca. E Solla che non vide $\mathrm{i}$ fiori sari stato tratto in errore dalla autoriti di Gussone. Se vi fosse la $R$. Bulbocodium in Lampedusa, non avrei potuto mancare di vederla in Marzo; la $R$. Colummae 
invece s'incontra ad ogni passo. Essa varia in Lampedusa per il colore dei.fiori; talora si trova coi tre tepali interni violetti e gli esterni rerdognoli di fuori, talora con tutti i tepali più o meno intensamente violetti. Più rari sono i fiori di un bianco verdognolo appena sfumato di violetto. Non ho osservato alcuna forma che si arricinasse alla $R$. ramifora.

366. - Gladiolus dubius Guss.; Somm. Piante ined.

Qua e lá molto meno comune del seguente; Marz. foglie, Apr. fr. !

La grande capsula (fino $2 \%$ non lasciano dubbio sulla determinazione di questa specie. Non l' ho veduta in fiore; ma quest'anno ne ho ricevute di nuovo, dalla guardia Nartorana, delle capsule mature raccolte in fine di Maggio.

367. -- Gladiolus segetum Ker-Gawl.; Solla p. 478; Lojac. Esc. p. 26; Fiori e Paol. Fl. an. I p. 228.

Comune nei seminati, alcuni campi ne sono addirittura infestati; Mrz. fi.! - Solla (fi.), Lojacono, Zodda (fi.). Me ne sono state mandate capsule mature in fine di Maggio.

E detto dai contadini «spatolilla».

368. - Gynandriris Sisyrinchium (L.) Parl.: Solla 1). 478; Lojac. Esc. p. 26. Iris Sisyrinchium Fiori e Paol. Fl. an. I p. 226.

Nei luoghi aridi comune; Mrz. foglie, Apr. fi. e fr.! - Solla (fi.), Lojacono, Zodda (fi.).

\section{9. - Iris Germanica L.}

Zodda solo (fra Imbriacola e Poggio Monaco, in dumetis umbrosis, fi.).

\section{0. - Iris Florentina $L$.}

Zodda (fra Imbriacola e Poggio Monaco in dumetis umbrosis, fi.). Mi è stata mandata pure dalla guardia Martorana.

Zodda nota espressamente sull' etichetta che queste due I'is, le quali crescerano nello stesso luogo, si trovavano allo stato selratico. Si tratta pero probabilmente di un inselratichimento o di un avanzo di coltura posteriore al 1884, anno in cui Lampedusa fu visitata da Lojacono, Ross, Zwierlein e Solla, nessuno dei quali ve le trovó, come non ve le avevano trovate nè Gussone, nè Calcara. 


\section{LXI. - Amaryllidaceae.}

371. - Pancratium maritimum L.; Guss. Syn. I p. 383; Calc. 1. 25; Lojac. Esc. 1). 20 ; Parl. Fl. It. III 1) 101.

Nelle arene marine vicino al Porto, nel luogo detto Arena Bianca, ivi abbondante, ma in area molto ristretta; Mrz. foglie! - Gussone, Calcara (alla Salina), Lojacono.

372. - Agave Americana L.; Solla p. 478.

Qua e là in vicinanza del Porto dove appena si può dire inselvatichita; Mrz. foglie e steli secchi! - Solla (in principio di fioritura).

\section{LXII. - ASPARAGACEAE.}

\section{3. - Asparagus albus $\mathrm{L}$.}

In Valle Pollicino, molto raro; Mrz. messe giov.! - Zodda (Capo Ponente, in dumetis rarissimus, foglie).

374. - Asparagus acutifolius L.; Calc. p. 25.

Nelle siepi di fichi d'India; 19 Apr. giov. foglie! - Calcara, Zodda (Sanguedolce in dumetis, foglie).

375. - Asparagus aphyllus L.; Somm. Piante ined.

Comunissimo nelie siepi e tra i frutici, anche sull' isola dei Conigli; Mrz. e Apr. foglie sole!

E strano che non sia stata segnalata da altri questa specie che avevo giả raccolta nel 1873 , e che quest'anno era tanto abbondante che la mia guida ogni giorno ne portava a casa un grosso mazzo di giovani messe che mi fornivano un' eccellente pietanza.

Varia molto per la grossezza e lunghezza dei cladodî che ora. sono tutti non più lunghi di 4-5 mm., ora raggiungono $15-18 \mathrm{~mm}$.

376. - Asparagus stipularis Forsk.; Parl. Fl. It. III p. 25. A. horridus Guss. Syn. I p. 418 ; Calc. p. 25; Lojac. Esc. p. 26. A. aphyllus is stipularis Fiori e Paol. Fl. an. I p. 209.

Gussone (sotto il Capo di Ponente), Lojacono (presso il Porto, rarissimo).

Solla (p. 467 e 478) indica un Asparagus sp. (sole foglie) che sarà da riferirsi ad uno dei quattro sopra citati. 
377. - Smilax aspera L.; Calc. p. 30 et 32 ; Solla p. 478?; Lojac. Esc. p. 26. S. Mfaurilanica Guss. Syn. II p. 629 ; Calc. p. 30 ; Lojac. Esc. p. 26.

Gussone, Calcara, Solla (foglie), Lojacono.

Gussone e Lojacono indicano, oltre al tipo, anche la var. Mauritanica (Desf.).

\section{LXIII. - LILIACEAE.}

378. - Ornithogalum Narbonense $I_{1 .}$; Guss. Syn. I p. 404 ; Calc. p. 25; Solla p. 478 ; Lojac. Esc. p. 26 ; Parl. Fl. It. II p. 448.

Raccolto il $28 \mathrm{Apr}$. in li.! - Gussone, Solla (fi.), Lojacono (luoghi arenosi coltivati, Vallone Imbriacola), Zodda (Cala Croce, in vineis, bocci).

379. - Urginea maritima (L.) Baker. Scille marilima Guss. Notiz. p. 85 et Syn. I p. 407 ; Calc. p. 25 et 38 ; Solla p. 467 et 478 ; Lojac. Esc. p. 26. Urginea Scilla Parl. Fl. It. II p. 455.

Comunissima in tutte le parti non coltivate dell' isola e nell' isolotto dei Conigli; Mrz. e Apr. foglie ! - Gussone, Calcara, Solla (sole foglie), Lojacono.

Questa Scilla e l'Asfodelo sono adesso le piante più appariscenti nelle parti non coltivate dell' isola.

Gussone dice che le pecore ne mangiano le foglie secche, e che le testuggini in estate ne rodono avidamente $\mathrm{i}$ bulbi, benchè caustici. Io pure ho visto le capre a mangiare tutte le punte ingiallite delle foglie, fermandosi lá dove la foglia era ancor verde.

330. - Scilla autumnalis L.; Guss. Syn. I p. 408; Calc. p. 25 Parl. Fl. It. II p. 469.

Frequente nelle terre aride; Mrz. foglie! - Gussone. L' ho ricevuta raccolta il 28 Ottobre 1873 in fiori dal Conti.

- Scilla sp. Lojac. Esc. p. 26.

Lojacono ha trovato a Punta Grecale una Scilla in uno stato indeterminabile, che sembragli poter essere la S. intermedia.

381. - Muscari comosum (L.) Mill.; Guss. Syn. I p. 410 ; Calc. p. 25; Lojac. Esc. p. 26. Leopoldia comosa Parl. Fl. It. II p. 496.

Frequente nei campi di grano; Mrz. fi.! - Gussone, Lojacono. 
382. - Allium roseum L.; Guss. Syn. I p. 388; Calc. p. 25 ; Solla p. 478; Lojac. Esc. p. 26 ; Parl. Fl. It. II p. 524.

Comunissimo in tutta l' isola, tanto nel coltivato quanto nelle parti incolte; Apr. fi. e fr.! - Gussone, Solla (fi.), Lojacono, Zodda (fi. e capsule giov.).

Gli esemplari che ho raccolti appartengono in parte alla forma speciale ai terreni molto aridi che ho chiamata var. humile (Flora del Figlio); nessuno è bulbillifero. Zodda invece ha trovato, oltre al tipo, la forma a ombrella bulbillifera, ed anche la forma a fiori bianchi (majale Cyr.) (a Cala Francese fiori e bulbilli).

383. - Allium subhirsutum L.; Lojac. Esc. p. 26.

Lojacono solo.

384. - Allium vernale Tineo.

Zodda solo (Sanguedolce in saxosis, $\mathrm{fr}^{\circ}$.).

Insieme alla forma tipica, Zodda ha raccolto un esemplare mostruoso, con ombrelle composte, cioè aventi alcuni raggi alla loro volta ombrelliferi. Zodda (in schedula) nota inoltre che aveva il perianzio lutescente. La stessa forma mostruosa ho trovata a Linosa.

385. - Allium Chamaemoly L.; Guss. Syn. I p. 391 ; Calc. 1). 25: Parl. Fl. It. II p. 531.

Gussone solo, il quale peró nel Suppl. al Prodr. non lo aveva citato di Lampedusa.

386. - Allium tenuiflorum Ten.; Guss. Syn. I p. 396 ; Calc. p. 25; Parl. Fl. It. II p. 548 .

Gussone, Calcara (Vallone dell' Imbriacola).

387. - Allium sphaerocephalum L.: Guss. Syn. I p. 393; ralc. p. 25; Parl. Fl. It. II p. 505.

Gussone solo.

388. - Allium margaritaceum Sibth. et Sm.; Guss. Syn. I 1). 301: Calc. 1. 25: Parl. Fl. It. II 1). 569.

Solo Gussone (al Muro Vecchio).

389. - Allium Ampeloprasum L. var. commutatum (Guss. Fl. Inarimensis pro specie). A. rotundum Guss. Syn. I p. 392, non L. (monente ipso Gussone Fl. Inar. p. 339); Calc. p. 25. A. commudatum Parl. Fl. It. II p. 574; Solla p. 465 (in nota).

Haec varietas recedit a typo: spatha longius cuspidata, um- 
bella fere globosa nec ovata, staminibus longius exsertis, capsulis tepala excedentibus, bulbo minus prolifero.

Valle Pollicino, Vallone dell' Imbriacola, Cala Francese, dintorni del Porto, isola dei Conigli ; Mrz. foglie, Apr. fi. e fr.! Gussone (nell' isola dei Conigli), Zodda (all'Aria Rossa, fi. e fr.).

Riferisco la mia pianta di Lampedusa e dei Conigli all' 1 . commutatum (A.rotundum della Synopsis), per la località, o perché combina colla descrizione di Gussone, salvo che ha le foglie perfettamente liscie negli orli e nella carena. In quanto alla scabridità dei tepali sugli orli e nel dorso, essa varia nei miei esemplari di Lampedusa che ora la mostrano ed ora no. I fiori sono di un rosa più o meno carico.

Oltre alla var. commulatum, l'A. Ampeloprasum presenta a Lampedusa un'altra forma che propongo di chiamare:

\section{Var. hemisphaericum.}

A varietate praecedente differt: pedunculis minus numerosis, omnibus erectis, ascendentibus, vel horizontaliter patentibus (quapropter umbella hemisphaerica nec globosa apparet) et capsulis minoribus. Specimina plurima gracilia et humilia (20$30 \mathrm{~cm}$.), sed majora (ultra $\% \mathrm{~m}$.) non desunt.

Verso Capo Ponente; 28 Apr. fi. e fr.!

Questa forma presenta i caratteri della var. commutatum, salvo che ha l'ombrella meno fornita e mancante di peduncoli reflessi, ed ha le capsule più corte dei tepali.

Fiori nella Flora analitica d' Ital. I p. 195, considera l' A. commulatum Guss. quale varietà dell'A. rotundum L., unendolo all'A. rubellum Presi (non M. B.), sotto il nome di rar. Preslianum. A me sembra più affine all' A. Ampeloprasum, e per ciỏ 'lo riferisco come varietá a questa specie dalla quale in veritá differisce pel' caratteri di ben poco valore. Con l'A. Ampeloprasum meglio combina anche per la sua stazione marittima e meridionale. In quanto alla identificazione coll' $A$. mebellum Presl che fa il Fiori, osserveró che la descrizione di Presł si attaglierebbe alla mia varieta hemisphaericum per la forma dell'ombrella da esso detta emisferica; inoltre nei miei esemplari più piccoli e gracili di questa rarietá, lo scapo è anche meno folioso e più lungamente nudo che nel tipo, come Presl dice del suo A. rubellum; ma questo autore attribuisce alla sua specie uno scapo angoloso, 
il che non combina più colla pianta di Lampedusa. Per questo credo sia meglio lasciare l'A. vubellum di Presl-fra le specie dubbie, dove giả la relegarono Gussone e Parlatore.

- Allium sativum L.; Sanvisente p. 59.

Sanvisente cita nella Flor'ula di Lampedusa questa pianta che però vi si trova soltanto coltivata negli orti.

390. - Asphodelus ramosus L. : Guss. Notiz. p. S5 et Syn. I p. 413 ; Calc. p. 25 et 32 ; Solla p. 467 et 478 , Lojac. Esc. p. 26. A. microcarpus Parl. Fl. It. II p. 599.

Comunissimo in tutte le parti incolte dell' isola, trovasi anche nell' isola dei Conigli; Mrz. fi. e fr., Apr. fr.! - Gussone, Solla (sfiorito), Lojacono, Zodda (it. e fr:).

Quest'anno la maggior parte degli Asfodeli non areva fatto fiori. Vi è a Lampedusa la credenza che gli anni in cui l'Asfodelo fiorisce poco, siano anni cattivi per il grano.

Gussone dice che in estate, quando manca altro cibo, le pecore mangiano le foglie secche dell'Asfodelo, ma che dopo le prime pioggie queste diventano amare e vengono rifiutate dalle bestie.

\section{LiNiV. - Colchicaceae.}

391. - Colchicum Bertolonii Stev.; Somm. Piante ined.

Frequente nelle terre aride ed in alcuni luoghi, come ad esempio fra Cala Croce e Cala Madonna, abbondantissimo; foglie erolute e capsule quasi mature in Marzo! - L'arevo ricevuto nel 1873 dal Conti, raccolto in fiore l'11 Novembre.

La pianta fruttifera di Lampedusa ha le foglie evolute lunghe funo a $25 \mathrm{~cm}$., e larghe fino a $1 \mathrm{~cm}$., scabre o quasi liscie ai margini, mai cigliate. Appartiene dunque alla rar. b. Crepani Parl. Fl. It. III p. $190=$ C. Cupani Guss. ex parte $=$ C. montanum $\gamma$ Cupani Fiori e Paol. Fl. an. I p. 177. Al momento dell'antesi, negli esemplari Conti, le foglie sono poco piủ o poco meno lunghe dei fiori, e larghe al massimo $5 \mathrm{~mm}$. Negli esemplari presi quest'anno a Lampedusa e fioriti in autunno a Firenze invece, le foglie al momento dell'antesi hanno raggiunto il doppio della lunghezza dei fiori, mantenendosi strette. Questi esemplari presentano quindi la forma chiamata C. Valery da Tineo (C. montanum $\gamma$ Cupani b. Valery Fl. an.). 


\section{LXV. - Juncaginaceae.}

392. - Triglochin Barrolieri Lois.; Guss. Syrn. I p. 439; Calc. j). 25; Parl. Fl. It. III p. 616.

Gussone solo (alle Saline).

\section{LXVI. - JUNCACEAE.}

393. - Juncus bufonius L.; Guss. Syn. I p. 424; Calc. p. 25; Lojac. Esc. p. 26. J. insulanus Guss. Suppl. Prodr. p. 107. J. hybridees Guss. Syn. I p. 425 ; Calc. p. 25 ; Lojac. Esc. p. 26. J. bufonizes c. hybridues Parl. Fl. It. II p. $3 \breve{4} 4$.

Comunissimo ; Mrz. fi. e fr., Apr. fr.! - Gussone (il tipo senza localita, la var. hybridus alla Salina), Lojacono, Zodda ( $\mathrm{fr}^{\circ}$.).

La forma tipica è rara; l'ho trovata bene sviluppata, alta fino a $25 \mathrm{~cm}$., soltanto in pochi luoghi rimasti ancora umidi. Più frequente è la var. hybridus. Gussone e Lojacono indicano l'una e l'altra. Na abbondantissima è la forma nana, chiamata da Grisebach var. pumilio. ${ }^{1}$ Essa forma dei cespuglietti compatti, talvolta rosseggianti, quasi acauli, con steli spesso meno alti di un centimetro, lungamente oltrepassati dalle foglie. Cresce in quantità fra le micloite nelle terre che sono prosciugate ed aride in Aprile ed anche in Marzo, ma che dovettero per' qualche tempo in inverno mantenersi umide.

\section{LXVII. - NAJADACEAE.}

394. - Posidonia Oceanica (L.) Del. Caulinia Oceanica Calc. p. 29.

Depositata in alti strati sulla spiaggia in varie Cale; Mrz. foglie! - Calcara.

395. - Cymodocea nodosa (Ucria) Asch Phiceagrostis major Calc. p. 29.

Calcara solo.

\section{LXVIII. - ARACEAE.}

396. - Ambrosinia Bassii J.; Guss. Syn. II p. 504 ; Calc. p. 30 ; Lojac. Esc. p. 26; Parl. Fl. It. Il p. 232.

Gussone, Lojacono.

'Cfr. Somsrar, Aggiunt alla Fl, di Capraia in N. Giorn. bot. it.' 1893, p. 134, e Sommer, Flora del Giglio, p. 87. 
397. - Arisarum vulgare Targ. Tozz.; Guss. Syn. II p. 595; Calc. p. 30 et 32 ; Solla p. 4\%8; Lojac. Esc. p. 26.

Dovunque nelle parti coltivate ed incolte, e nell'isola dei $\mathrm{Co}$ nigli ; Mrz. fi., Apr. fr..! - Gussone, Solla (fr.), Lojacono, Conti (fi. in Novembre).

\section{LXIX. - CYPERACEAE.}

398. - Schoenus nigricans L.: Solla p. 478.

Solo Solla (fr.).

399. - Carex Halleriana Asso.; Somm. Piante ined.

Nella macchia verso Cala Galera, rara; 28 Apr. fr.!

$$
\text { LXX. - Graminaceae. }
$$

400. - Phalaris brachystachys Link; Lojac. p. 26.

Lojacono solo (nei seminati).

401. - Phalaris minor Retz.; Solla p. 479.

Qua e là nel coltivato; Mrz. fi., Apr. fi. e fí.! - Solla (fi. e fr.), Zodda (fi.).

402. - Anhhoxanthum odoratum L.; Fuss. Syn. I p. I57; Calc. p. 23 ; Parl. Fl. It. I p. 59.

Gussone solo.

403. - Cynodon Dactylon (L.) Pers.; Guss. Syn. I p. 110; Calc. p. 22.

Nelle arene marine a Cala Francese; $30 \mathrm{Apr}$. foglie sole! Gussone, Zodda (alla Salina, in arenosis, foglie).

404. - Andropogon hirtus L.; Guss. Syn. I p. 163; (alc. p. 23 : Parl. Fl. It. I p. 112.

Gussone solo (il quale non lo aveva citato nel Supplemento al Prodromo).

405. - Andropogon pubescens Vis.; Somm. Piante ine\}.

Luoghi rocciosi, Cala Galera e in un altro luogo, raro; Mrz. bocci, Apr. fi.!

406. - Arundo Donax L.

In varie Cale, dove probabilmente fu introdotta, ma ora si è fatta spontanea. Nasce p. es. perfettamente selvatica nella spiaggia arenosa detta Arena Bianca; Mrz. foglie! 
407. - Ampelodesmos tenax (Tahl) Link: Parl. Fl. It. I p. 464. Amonido Ampelodesmos Guss. Syn. I p. 138; Calc. p. 23 ; Solla p. 465 (in nota).

Gussone solo.

408. - Sporobolus pungens (Schreb.) Kunth; Parl. Fl. It. I p. 196. Agrostis pungens Guss. Syn. I p. 134; Calc. p. 23; Lojac. Esc. p. 26.

Gussone solo (sotto al Castello). Lojacono dice di averlo cercato invano, ma dimentica il segno $\bigcirc$ per indicare che non fu da esso raccolto.

409. - Triplachne nitens (Guss.) Link; Solla 11. 479; Parl. Fl. It. I p. 178; Fiori e Paol. Fl. an. I p. 62. Agrostis nitens Guss. Syn. I p. 135 ; Calc. p. 23.

Capo Rupestre; $30 \mathrm{Apr}$. fr.! - Gussone (Capo di Ponente), Solla (fi.), Zodda (alla Salina, in arenosis, fi.).

410. - Gastridium lendigerum (L.) (raud.; (russ. Syn. I p. 132; Calc. p. 23; Parl. Fl. It. I p. 176. Milium lendigerm Guss. Suppi. Prodr. p. 14.

Raro; 28 Apr. fi. e fr.! - Gussone.

411. - Polypogon Monspeliensis (L.) Dest.; Guss. Syn. I p. 126; Calc. p. 22; Lojac. Esc. p. 27; Parl. Fl. It. I p. 199.

Gussone (var. b. panicula minore, spiciformi, ecc.), Lojacono.

412. - Polypogon maritimus Willd.; (russ. Syn. I p. 120 ; Calc. p. 22 ; Solla p. 479 ; Lojac. Esc. p. 27 ; Parl. Fl. It. I p. 201.

In varî luoghi; Apr. fr.! - Gussone (alla Salina), Solla (fi.), Lojacono, Zodda (fi. e fr.).

Gussone indica di Lampedusa tanto il tipo di questa specie quanto la sua var. b. aculiflorus. I miei esemplari non appartengono al tipo, ma tutti presentano caratteri al tempo stesso della var. acutiflorus e della var. subspathaceus (Req.), cioè : glume acute non dilatate, e pannocchia, benchè maturissima, quasi interamente rinchiusa entro la guaina dell'ultima foglia. Gli esemplari di Zodda sono uguali ai miei.

413. - Lagurus ovatus L.; Guss. Syn. I p. 127 ; Calc. p. 23; Solla p. 479 ; Lojac. Esc. p. 27 ; Parl. Fl. It. I p. 205.

Comune, allche nell' isola dei Conigli; Mrz. in principio di fioritura, Apr. fi.! - Gussone, Solla (fi.), Lojacono, Zodda (fi.).

Gussone istituisce per la pianta di Lampedusa una varietå 
b. nanus, caratterizrata da statura umile e pannocchia subsferica. Ho notato che le piante di Lampedusa presentano in generale questa forma microflorata, la quale peró non mi sembra meritare di essere distinta rieppure come varieti.

414. - Stipa tortilis Desf.; Guss. Syn. I p. 129; Calc. p. 23 ; Lojac. Esc. p. 26; Parl. Fl. It. I p. 169.

Gussone, Lojacono, Zodda (Cala Galera, in sterilibus satis rara, fr.).

415. - Milium coerulescens Desf.; ( illss. Syn. I p. 130; Calc. 1. 23 ; Parl. Fl. It. I p. 158.

Gussone solo.

416. - Milium multislorum Cav.; Guss. Syn. I p. 130; Calc. 1). 23; Parl. Fl. It. I p. 160.

Solo Gussone, il quale (Syn. I p. I31) nota che gli esemplari di Lampedusa sono suffrutescenti, alti $2-4$ piedi, hanno i rami inferiori della pannocchia verticillati e generalmente sterili, e crede che possa essere forse l'Urachne frutescens Link. Nel vol. II Addenda et emend. p. 786-7, conferma questo suo dubbio.

417. - Aira Cupaniana Guss.; Guss. Syn. I p. 149 ; Calc. p. 23 ; Ross p. 348 ; Solla p. 479 ; Lojac. Esc. p. 11 et 27 ; Parl. Fl. It. I p. 253. A. capillaris Guss. Suppl. Prodr. p. 16.

Gussone, Ross (costa Sud), Solla (fr.), Lojacono (luoghi ombrosi dei fruticeti, rara a Cala Galera e Capo Ponente).

418. - Avena sterilis L.; Somm. Piante ined.

Raccolta in frutto il 20 Aprile!

419. - Avena fatua L.; Guss. Syn. I p. 155; Calc. p. 23; Lojac. Esc, p. 27.

Gussone, Lojacono.

420. - Avena barbata Brot.; Solla 1. 479. A. atherantha Calc. p. 23. A. hirsula et $A$. atherantha Lojac. Esc. p. 27.

Dovunque, anche nell' isola dei Conigli; Mirz. fi. e fr'., Apr. fr. : - Calcara, Solla (fi. e fr'.), Lojacono, Zodda (fr.).

Lojacono distingue col nome di A. hirsuta var. racemosa, degli esemplari stentati di questa specie.

421. - Trisetum aureum Ten.; Parl. Fl. It. I p. 267. Avena condensata Guss. Sy'n. I p. 152 ; Calc. p. 23.

Gussone solo. 
422. - Melica minuta L.; Guss. Syn. I p. 142 ; Calc. p. 23 ; Solla p. 479; Lojac. Esc. p. 27 ; Parl. Fl. It. I p. 305̆. M. pyramidalis Guss. Suppl. Prodr. p. 17.

Luoghi rupestri e fra i residui della macchia, nei valloni piủ freschi, piuttosto rara; Mrz. fi., Apr. fi. o fr.!-Gussone (inter fruticeta, non satis obvia), Calcara (Cala della Madonna), Solla (fr.), Lojacono, Zodda (Cala Uccello, fi. e fir.).

Gussone avverte che per errore nel suo Suppiemento al Prodromo aveva riferito la localita. di Lampedusa alla Melica pyramidalis Bert. Le piante che ho raccolte hanno le foglie quasi interamente convolute, ma la linguetta troncata, con due appendici ai lati.

423. - Scleropoa maritima (L.) Parl.; Ross p. 318.

Ross (nell' insenatura occidentale del Porto), Zodda (alla Salina, in arenosis maritimis, fi. e f $1^{\circ}$ ).

424. - Scleropoa rigida (L.) Griseb.; Solla p. 479 ; Parl. Fl. It. I p. 474. Poa rigida Guss. Suppl. Prodr. p. 2'. Selerochloa rigida Guss. Syn. I p. 94 ; Calc. p. 2 i.

Comune; Mrz. piante secche dell'anno precedente, Apr. fi. e fr. : - Gussone, Solla ( $\mathrm{fr}$.), Zodda (fi. e fr.). Calcara indica la localita di Salina, non citata da Gussone, quantunque con un* attribuisca il ritrovamento della specie a Gussone solo.

Gussone riferisce la pianta di Lampedusa alla sua var. glaucescens; ma i miei esemplari, in parte assai grandi ed a pannocchia ramificata e lassa, non corrispondono a questa varietá, e neppure alla forma di Linosa chiamata da Lojacono S. Zivierleinii. Più si avvicinano a quelle forme gli esemplari di Zodda.

425. - Poa annua L.; Guss. Syn. I p. 96; Calc. p. 22; Parl. Fl. It. I p. 349.

Comune, anche nell' isola dei Conigli; Mrz. fi. e fr.! - Gussone, Zodda (fr.). Non si sa da dove Calcara abbia preso l'indicazione di «Salina » poichè non trovasi in Gussone, e Calcara non ha raccolto questa specie, poichè è segnata con un solo*.

426. - Sphenopus divaricatus (fouan) Roichb.: (ills.s. Syn. I p. 95 ; Calc. p. 22 ; Lnjac. Esc. p. 28 ; Parl. Fl. It. I 1) 377. Poa divaricata Guss. Suppl. Prodr. p. 21.

Luoghi inondati d'inverno, in vicinanza del Porto; 19 e 27 Apr. fi. e fr.! - Gussone (alla Salina), Lojacono, Zordda (alla 
Salina, in arenosis abunde cum Spergularia rubra et Plantagine commutata; a Cala Malucco in saxosis rara, fi. e fr.).

427. - Eluropus repens (Desf.) Parl. : Ross. p. 348 ; Lojac. Esc. p. 26 ; Parl. Fl. It. I p. 463. Daclylis repens Guss. Notiz. p. 87 et Syn. I p. 91 ; Calc. p. 22 et 31. Aluropus litoralis $\beta$ repens Fiori e Paol. Fl. an. I p. 84.

Luoghi inondati d' inverno, in vicinanza del Porto; $27 \mathrm{Apr}$. foglie sole! - Gussone (alla Salina sotto il Castello), Calcara, Ross (sulla spiaggia occidentale del Porto), Lojacono (sulle arene della Salina).

428. - Dactylis giomerata L.; ( + uss. Syn. I p. 90 ; Calc. p. 22; Lojac. Esc. p. 27; Parl. Fl. It. I p. 459. D. Hispanica Guss. Suppl. Prodr. p. 22.

Capo Ponente; $28 \mathrm{Apr}$. fi. e fr.! - Gussone (var. Hispanica), Lojacono, Zodda (Capo Ponente, in dumetis haud communis, bocci).

I miei esemplari appartengono alla forma Hispanica spiccatissima, come pure quelli di Zodda, i quali inoltre sono nani.

429. - Lamarckia aurea (L.) Moench; Solla p. 479; Lojac. Esc. p. 27; Parl. Fl. It. I p. 334. Cynosurus aureus Guss. Suppl. Prodr. p. 20. Chrysurus cynosuroides Guss. Syn. I p. 107; Calc. p. 22.

Qua e lá, più specialmente vicino all'abitato; Mrz. fi., Apr. fr. ! - Gussone, Solla (fi.), Lojacono, Zodda (fr.).

430. - Chrysurus echinatus Pal. de Beauv. Cynosirnes echinaters Guss. Syn. I p. 108; Calc. p. 22; Parl. Fl. It. I p. 337.

Gussone solo.

431. - Koeleria phleoides (Vill.) Pers.; Ross p. 318; Solla p. 479 ; Lojac. Esc. p. 28.

Comune, raccolta in luoghi diversi; Apr. fi. e fr.! - Ross, Solla (fi. e fr.), Lojacono, Zodda (fi. e fri.).

Fra i miei esemplari, come fra quelli di Zodda, se ne trovano colle spighette pubescenti ed altri colle spighette glabre.

432. - Koeleria villosa Pers.; Lojac. Esc. p. 28.

Lojacono solo.

433. - Koeleria hispida (Savi) DC.; Solla p. 479.

Solla solo (fi. e fr.).

434. - Vulpia Ligustica (All.) Link; Solla p. 479.

Solla solo (fi. e fr.). 
435. - Vulpla ciliata (Pers.) Link; Lojac. Esc. p. 27.

Lojacono (gia disseccata in Aprile), Zodda (Cavallo Bianco, in herbosis saxosis, fi. e fr.).

436. - Bromus tectorum L.; Cruss. Syn. I p. 78 ; Calc. p. 22 :

Lojac. Esc. p. 27 ; Parl. Fl. It. I p. 409.

Gussone, Lojacono.

437. - Bromus Madritensis I.; Guss. Syn. I p. 78; Calc. p. 22 :

Solla p. 479 ; Lojac. Esc. p. 27 ; Parl. Fl. It. I p. 406.

Comune nell' isola, e nell' isolotto dei Conigli; Mrz. fi., Apr. fr. ! - Gussone, Solla (fr.), Lojacono, Zodda (fi. e fr.).

Trovasi a Lampedusa tanto la forma a spighette glabre quanto quella con spighette pubescenti-irte detta da Gussone b. ciliakes.

438. - Bromus maximus Desf.

Qua e là nei luoghi erbosi delle parti coltivate; Mrz. fi. e fr. giov.!

Le piante che ho vedute e raccolte si avvicinano alla var. Gussonei (Parl.).

439. - Eromus fasciculatus Presl ; (inss. Syn. I p. 81; Calc. p. 22; Solla p. 479; Parl. Fl. It. I p. 411.

Qua e li; Mrz. fi., Apr. fr.! - Gussone, Solla (fr.), Zodda (fi. e fro.).

L'ho trovato tanto con le spighetie pubescenti, quanto con le spighette glabre.

440. - Serrafalcus mollis (L.) Parl.; Solla p. 479; Lojac. Esc. p. 27; Parl. Fl. It. I p. 396. Bromus mollis Guss. Syn. I p. 74 ; Calc. p. 22.

Comunissimo in molte parti dell'isola, e nell'isolotto dei Conigli; Mrz. fi. e fr., Apr.fi. e fr. ! - Gussone, Solla (fi. e fr.), Lojacono, Zodda (fi. e fr.).

Nei terreni aridi abbonda nano fra le microfite, ridotto fino a $2-3 \mathrm{~cm}$; ma, in luoghi più fertili, trovasi anche alto e con pannocchia ramificata.

441. - Sorrafalcus intermedius (Guss.) Parl.?; Solla 1\% 479.

Osservato solamente da Solla (fi. e fr.), il quale peró ha dei dubîi su questa determinazione.

442. - Lolium perenne L.; Guss. Syn. I p. 58; Calc. p. 22; Lojac. Esc. p. 28; Parl. Fl. It. I p. 530. 
Verso Capo Ponente; 28 Apr. fi. ! - Gussone (la var. a. vulgare, l'ara), Lojacono, Zodda (presso la Vigna del Sindaco, in incultis vulgatum, fi.).

443. - Lolium Siculum Parl.; Somm. Piante ined.

Presso il Porto; $30 \mathrm{Apr}$. fi.! - Zodda (Cavallo Bianco, in herbosis incultis, fi.).

444. - Lolium rigidum Gaud.; Solla p. 479.

Frequente nel coltivato; Apr. fi.! - Solla (fi. e fr.), Zodda (Cavallo Bianco, in aridis, fi.).

445. - Catapodium loliaceum (IIuds.) Link; (miss. Syn. I p. 69; Calc. p. 22 ; Solla p. 479 ; Lojac. Esc. p. 27. Trilicum loliaceum Guss. Suppl. Prodr. p. 36.

Comunissimo nei terreni aridi con altre microfite, e nell'isola dei Conigli; Mrz. fi.! - Gussone, Solla (fi. e fr.), Lojacono, Zodda (fi.).

- Castellia tuberculosa (= Calapodium tuberculosum Moris (1841), Castellia tuberculata Tin. (1846).

Questa specie è stata distribuita da Todaro con etichette su cui è scritto «Lampedusa", e quindi trovasi in molti erbarî con quella falsa indicazione. Da ció forse è derivato l'errore che notasi nella Fl. Or. di Boissier V p. 635, nella Fl. d'Algérie di Cosson e Durieu p. 189, nella Fl. d'Alger et catal. des pl. d'Algérie di Battandier et Trabut p. 100 e nel Catal. raisonné des pl. vasc. de la Tunisie di Bonnet et Barratte p. 482, opere tutte nelle quali è indicata la Castellia tuberculosa per Lampedusa, mentre non è mai stata trovata in questa isola. Gli esemplari distribuiti da Todaro sembrano coltivati, e sono probabilmente nati nell orto botanico di Palermo da semi di Sardegna.

446. - Brachypodium distachyum (L.) Pal. de Beauv.; Guss. Syn. I p. 73 ; Calc. p. 22 ; Solla p. 479; Lojac. Esc. p. 27; Parl. Fl. It. I p. 492. Festuca distachya Guss. Notiz. p. 86 et Suppl. Prodr. p. 25.

Comune; Mrz. fi., Apr. fr.! - Gussone (quasi sola graminacea a formare il pascolo primarerile), Calcara, Solla (fr.), Lojacono, Zodda (fi. $\theta$ fr.).

Gussone (Notizie) dice che cresce in quantita, ma rimane pigmeo a causa della sterilitá del terreno. Io pure l'ho trovato frequentemente nano, quasi acaule, alto $1-2 \mathrm{~cm}$. Ma ne ho raccolto pure piante alte fino a $75 \mathrm{~cm}$., con fuglie ondulate ai mar- 
gini, con 3-5 spighette e con culmi ingrossati sotto l' inserzione delle spighette, che corrispondono alle varieta c. pentastachyum e d. undulatum di Gussone. Anche Lojacono indica per il Vallone dell' Imbriacola lá var. pentastachyım.

447. - Triticum villosum (L.) M. Bieb.; Solla p. 479.

Solla solo ( $\mathrm{fr}$.).

448. - Eggilops ovata L.; Solla p. 479 ; Lnjac. Esc. p. 28.

Solla (fr.), Lojacono.

449. - Hordeum murinum L.; Guss. Syn. I p. 63; (aic. p. 22 :

Solla p. 479; Lojac. p. 28 ; Parl. Fl. It. I p. 520.

Comune presso l'abitato, ma anche lontano da questo, enell'isola dei Conigli ; Mrz. fi., Apr. fi. e fr.! - Gussone (abbastanza raro), Solla (fr.), Lojacono, Zodda (fi. e fr.).

Solla indica il tipo e la var. leporinum (Linli) $=$ var. majus Gren. et Godr. Tutti gli esemplari che ho esaminati appartengono a questa varietà.

460. - Lepturus incuryatus (L.) Trin.; Ross p. 348 ; Lojac. Esc. p. 28; Parl. Fl. It. I p. 537. Rottboellia incuretata Guss. Syn. I p. 57 ; Calc. p. 22.

In varî luoghi in viçinanza del mare; Mrz. primi fi., Apr. 1i. ! - Gussone, Ross, Lojacono, Zodda (fi.).

Ross dice che i suoi esemplari erano nani, alti pochi $\mathrm{cm}$. Fra i. miei, ve ne sono degli altissimi $(40 \mathrm{~cm}$.), come anche dei nani.

451. - Lepturus filiformis Trin. L. incurvalus var. filiformis Ross p. 348.

Ross solo (macchia vicino al mare, piante nane).

452. - Lepturus cylindricus (Willd.) Trin.; Parl. Fl. It. I p. 53\%. Rollboellice selnelale Guss. Suppl. Prodr. p. 3:3. R. cylinarica Guss. Syn. I. p. 56 ; Calc. p. 22 ; Lojac. Esc. p. 28.

Gussone, Lojacono.

G YMNOSPERMAE.

LXXI. - CoNIFERaE.

453. - Pinus Halepensis Mill.; Guss. Notiz. p. 86 et Syn. II p. 614 ; Calc. p. 30 et 32 ; Solla p. 465 (in nota) et 466; Lojac. Esc. [. 20 ; Parl. Fl. It. IV p. 11. 
Cala Greca; 29 Apr. strobili giov.! - Gussone (non oltrepassa l'altezza di 10 a 12 piedi), Calcara, Lojacono.

Questa specie, che ai tempi di Gussone pare non fosse rara, lo era già al tempo della mia prima visita, e adesso è del tutto scomparsa.

454. - Juniperus Phoenicea L.; Guss. Syn. II p. 634; Calc. p. 30 et 32 ; Ross p. 348 ; Solla p. 478; Lojac. Esc. p. 26; Parl. Fl. It. IV p. 91. J. Lycia Guss. Notiz. p. 86.

Adesso isolato e raro in alcuni valloni rocciosi; Mrz. foglie, Apr. fr. giov. e fr. maturi! - Gussone (costituente della macchia alta), Calcara, Ross (nella macchia), Solla (fr.), Lojacono, Zodda (fr. giov. e fr. maturi dell' anno precedente).

\section{P'TERIDOPHYTAE.}

\section{LXXII. - LyCOPODIACEAE.}

455. - Selaginella denticulata (L.) Spring; Lojac. Esc. p. 28. Lojacono solo (luoghi ombrosi nei fruticeti).

\section{LXXIII. - FILICES.}

456. - Grammitis leptophylla (L.) Swartz; Calc. p. 30 ; Lojac. Esc. p. 28.

Valle Pollicino sulle r'upi ombrose; Mrz. con. spore! - Lojacono. Calcara con un * indica come raccoglitore Gussone; ma questa specie non è citata per Lampedusa nella Synopsis, donde si dovrebbe concludere che fu raccolta da Calcara il quale avrebbe dovuto citarla senza :.

457. - Aspleniun Adiantum nigrum L. : (alc. p. 30 ; Lojac. Esc. p. 28.

Calcara solo (la forma detta da Gussone a. cuneifolium). 458. - Adiantum Capillus Veneris L.: Guss. Synl. II 1).660; Calc. p. 30 ; Lojac. Esc. p. 28.

Trovato soltanto in uno stillicidio nella grotta della Tabaccara in riva al mare, non lontano dall' isola dei Conigli; 10 Mrz. con spore! - Gussone, Calcara, Lojacono. 


\section{Musci.}

(Cl. marchese A. Bottini determinavit).

ACROCARPI.

\section{1. - Phascum rectum With.}

Vallone Pollicino, fra Cala Galera e Cala Greca, e verso Cala Malucco; 10, 13 e 14 Mrz. fr.!

\section{2. - Eucladium verticillatum (L.) Br. Eur.}

Stillicidî nella Grotta Tabaccara, in luogo dove cresceva il Capelvenere; $10 \mathrm{Mrz}$. ster.!

\section{3. - Fissidens incurvus Starke.}

Regione di Terranova; 13 Mrz. fr.!

4. - Fissidens tamarindifolius (Don, Turn.) Brid. $\alpha$.

Non lontano dal Porto, nella regione detta Cavallo Bíanco; 8 Mrz. fr.!

La forma tipica è rara in Italia, o almeno è passata inosservata. Sono frequenti le forme di passaggio al $F$. incurvus del quale, in sostanza, non è che una varietà rimarchevole. Si conosceva di varie localitá isolate e saltuarie della penisola.

\section{5. - Fissidens pusillus Wils.}

Vallone Pollicino; $10 \mathrm{Mrz}$. fr. e pianta $\delta^{\top}$ gemmiforme a! piede della pianta fertile, nascente dalle radici di questa!

\section{6. - Pottia minutula (Schleich.) Br. Eur.}

Verso Cala Francese; 8 Mrz. fr. !

\section{7. - Pottia Starkeana (Hedw.) C. Müll.}

Comunissima sui terreni più aridi; raccolta anche nell'isola dei Conigli; 8, 10, 12 e $13 \mathrm{Mr}^{2} \mathrm{z}$. fr. !

\section{8. - Pottia mutica Vent.}

Da Cala Francese a Capo Grecale, e fra Cala Greca e Cala Galera; 9 e 13 Mrz. fr.!

Specie sporadica e rara, nota soltanto di Vestfalia, Trento, Lugano, Livorno, Argentaro, Pianosa, Malta. 
9. - Trichostomum crispulum Bruch var. brevifollum $\mathrm{Br}$. Eur.

Vallone Imbriacola; $12 \mathrm{Mr} z$. ster.!

Var. viridulum (Bruch) Braithw.

Questa varietà trovata da Zodda solo (frr.).

10. - Trichostomum mutabile Bruch.

Aria Rossa e vicino al Porto; 8 e 12 Mrz. fr.! - Zodda (fr.).

Var. densum Br. Eur.

Fra Cala Francese e Capo Grecale; 9 Mrz. fr.!

11. - Trichosiomum nitidum (Lindb.) Schimp. a obtusuni Boulay.

Isola dei Conigli; $10 \mathrm{Mrz}$. ster.! - Zodda (ster.).

12. - Trichostomum viridîllavum De Not.

Verso Aria Rossa; 12 Mrz. ster.!

E una semplice forma, piuttosto rara, del Trichostomum $f a-$ vovirens Bruch.

13. - Timmiella Barbula (Schwägr.) Limpr.

Vallone Pollicino; $10 \mathrm{Mrz}$. fr.!

14. - Earbusa unguiculata (Huds.) Hedw. var. apiculata (Hedw.) Br. Eur.

Sotto i fichi d'India; 8 Mrz. ster.!

15. - Aloina aloides (Koch) Kindb.

Non lontano dal Porto; 9 Mrz. fr. !

16. - Aloina ambigua (Br. Eur.) Limpr.

Verso Cala Greca e verso Cala Francese; 8 e 13 Mrz. fr.!

17. - Tortula muralis (L.) Hedw.

Miolto comune sulle rocce; 10, 12 e 13 Mrz. fr.! - Zodda (fr.).

Var. incana $\mathrm{Br}$. Eur.

Vallone Pollicino e Vallone della Madonna; 10 e 11 Mrz. fr.! -Zodda (fr.).

18. - Tortuia marginata (Br. Eur.) Spruce.

Comune sulle rocce; 8,10 e 11 Mrz. fr. e ster.!

19. - Entosthodon curvisetus (Schwägr.) C. Müll.

Nolto frequente quasi in tutta l' isola; $8,9,10,11,12,13$ e 14 Mrz. fr'. !

20. - Entosthodon pallescens Juratzka in Unger et liotschỵ, die Insel Cypern (1865) p. 170.

Vallone Pollicino e Vallone della Madonna;10 e 13 Mrz. fr.! 
Bella specie mediterranea scoperta a Cipro, e nota in Italia soltanto di Roma, Sorrento, Capri e Sicilia (quest'ultima localitá non ancora pubblicata).

21. - Funaria Mediterranea Lindb. $\alpha$

Vallone Pollicino; 10 Mrz. fr.!

22. - Funaria convexa Spruce. Operculum planum, sed interdum plano-mamillatum.

Regione detta di Cavallo Bianco e fra Cala Francese e Capo Grecale; 8 e 9 . Mrz. fr.!

23. - Bryum capillare L.

Vallone Pollicino; 10 Mrz. ster.!

24. - Bryum torquescens Br. Eur.

Fra Cala Greca e Cala Galera; 13 Mrz. fr.!

25. - Bryum Duriaei Schimp. mss. in Herb. Dur. - Bescher. Cat. d. Mousses d'Algeérie (1882) p. 24, n. 6.

Vallone Pollicino e regione di Terranova; 10 e 12 Mrz. fr.!

Il Bryum Duriaei, che in veritá potrebbe considerarsi come una forma robusta di $B$. murale vegetante sulla terra è nuovo per l'Europa.

26. - Bryum murale Wils.

Non lontano dal Porto; 8 Mrz. ster.!

27. - Bryum atropurpureum (haud Wahlenb.) Br. Eur.

Frequente; 9, 12 e 13 Mrz. fr. e ster.!

Var. dolioloides Solms-Laub.

Fra Cala Galera e Cala Greca; 13 Mrz. fr. !

28. - Bryum argenteum L. var. lanatum (P. B.) Br. Eur.

Sotto i fichi d'India; 8 Mrz. fr.!

\section{Pleurocarpi.}

29. - Eurhynchium circinatum (Brid.) Br. Eur.

Frequente, raccolto nel Vallone Pollicino ed in regione di Terranova; 10 e 14 Mrz. ster.!

Forma attenuatum Boulay Muscin. (1884) p. 115.

Regione detta dell'Imbriacola; 12 Mrz. ster.!

30. - Rhynchostegiella tenella (Dicks.) Limpr.

Vallone della Madonna; 13 Mrz. fr.! 


\section{Hepaticae.}

(Cl. Caro Massalongo determinavit).

\section{1. - Fossombronia caespitiformis De Not.}

Comunissima in molte parti dell' isola, raccolta in ogni gita; Mrz. fr.! - Zodda (ster.).

32. - Petalophyllum Ralfsii (Dicks.) Gottsche.

Luoghi ombreggiati, specialmente sotto i fichi d' India, in diversi punti non lontano dal Porto, in direzione di Cala Francese e Cala Malucco; 8, 11 e 14 Mrz. ster.!

E la quarta localitá italiana nota per questa rara epatica che fino a poco tempo fa era conosciuta soltanto della Gran Brettagna e dell'Algeria. '

\section{3. - Sphaerocarpus Michelii Bell.}

Luoghi ombreggiati sotto i muri, qua e lá; 9 e 13 Mrz. fr. giov.!

\section{4. - Lunularia vulgaris Mich.}

Luoghi ombrosi nel Vallone Pollicino e nella Cala Greca; 10 e 13 Mrz. ster. cum scyphis!

35. - Riccia insularis Levier.

In molti luoghi, specialmente lungo i muri e sotto i fichi d' India; 8, 9, 13 e 14 Mrz. !

36. - Riccia lamellosa Raddi.

In varî luoghi; 8, 9 e $13 \mathrm{Mr} z$. fr.!

37. - Riccia nigrella DC.

Verso Cala Francese; 8 Mrz. fr.!

38. - Anthoceros dichotomus Raddi.

Vallone Pollicino, luoghi ombrosi; 10 Mrz. ster.!

1 Vedi Massalongo, Bull. Soc. bot. it., 1902, p. 37; Sommer, ibid., p. 54 ө p. 73; TH. Herzog, Beitr. z. Kenntn. der Laub-und Lebermoosflora von Sardinien, Zürich-Oberstrass, 1905, p. 16. 


\section{I CHEN ES.}

(Cl. A. Jatta determinavit).

1. - Collema pulposum Ach. Syn. p. 311; G. Albo I primi lich. di Lin. e Lamp."

Sulle rupi e sulla terra muscosa frequente; anche sull' isolotto dei Conigli; Mrz.! - Zodda (sulla terra).

2. - Cladonia pyxidata L. var. neglecta Fllk. Clad. p. 49.

Sul terreno all' Inbriacola; Mrz.!

3. - Cladonia rangiferina Hoffm. Fl. G. II p. 114; Solla Phytobiol. Beob. p. 480.

Solla.

4. - Cladonia furcata Hoffm. var. racemosa Flk. Clarl. p. 152; Solla p. 480.

Solla.

5. - Cladonia endiviaefolia (Dcks.) Fr. L. E. p. 212; Solla p. 480.

Sul terreno all' Imbriacola; Mrz.! - Solla.

6. - Ramalina Duriaei DNrs. Fram. lich. p. 216.

In abbondanza sulla scorza di alcuni alberi da frutto; Mrrz.!

- Var. dilacerata Mass. I. I p. 175.

Insieme al tipo; Mrz.!

7. - Roccella tinctoria DC. Fl. Fr. II p. 334: Albo l. c.

Zodda (sulla roccia, fra Cala Pisana e Cala Grecale).

1 I licheni raccolti a Lampedusa ed a Linosa dal dott. Zodda nel 1905 hanno formato oggetto di una memoria del prof. Albo, e sono pubblicati nel Bullettino della Soc. bot. it., Adunanza di maggio 1907, col titolo: «I primi licheni di Linosa e di Lampedusa ». Questo lavoro del prof. Albo non figura nella mia bibliografia, essendo pubblicato posteriormente.

Devesi osservare che il titolo dell'Albo non è esatto, poichè qualche lichene tanto di Lampedusa che di Linosa era stato già notato da Ross e da Solla, opere cit., dal JATta nel suo Lich. Ital. meridionalis Man. V, N. Gior. bot. it. XVIII, 1886, p. 78 e nella Mon. Lichenum It. meridionalis, Trani, 1889 e dallo STIzenberger nella Lichenaea africana, S.t Gallen, 1891, pag. 234 e seg. 
8. - Roccella phycopsis Ach. Univ. p. 440; Albo l. c.

Sulle rupi dell' isolotto dei Conigli ; Mrz.! - Zodda (sulla roccia).

9. - Roccella pygmaea Mtg. Alg. p. 266; Albo l. c. Zodda (sulle rocce fra Cala Pisana e Cala Grecale).

Specie nuuova per la Sicilia (Albo l. c.).

10. - Physcia parietina (L.) DNrs. Parm. p. 23.

Sulle scorze, frequente; Mrz.!

- Var. dealbata Müll. Hrb.

Insieme al tipo; Mrz.!

- Var. ectanea (Schaer.) Nyl. Syn. p. 411; Solla p. 480. Solla (sulle rupi).

- Var. aureola Fr.; Jatta Syll. p. 140; Albo l. c. Zodda (sui tronchi di Morus alba).

11. - Diphratora spadicea (Fw.) Jatta Syll. p. 204. Ricasolia spadicea Bgl.; Solla p. 480.

Solla.

12. - Diphratora olivacea (Bgl.) Jatta Syll. p. 264; Albo l. c. Zodda (sulla roccia).

13. - Diphratora polycycla Anzi Com. cr. II p. 9 ; Albo l. c. Zodda (sulla roccia).

Specie nuova per la Sicilia (Albo l. c.).

14. - Diphratora candicans (Frr.) Jatta Syll. p. 263; Albo l.c. Zodda (sulla roccia).

15. - Caloplaca murorum (Hoffm.) Fr. Th. Scand. p. 170. Placodium murorem Solla p. 480.

Solla.

16. - Caloplaca Callopisma (Ach.) Jatta Syll. p. 239 ; Albo 1. c. Zodda (sulla roccia).

- Var. centroleuca Mass. Bl. p. 58; Jatta 1. c.; Albo l. c. Zodda (sulla roccia).

Varietá non per anco indicata di Sicilia (Albo I. c.).

17. - Caloplaca ochracea (Schaer.) Jatta Syll. p. 251; Albo 1. c. Xanthocarpiae sp. Mass. : $\mathrm{ch}$. cr. p. 77.

Zodda (sulla roccia).

18. - Bacidia atrogrisea (Hep.) Krrb. Prg. p. 133.

Sulle scorze; Mrz.!

19. - Platygrapha rimata (Fw.) Nyl. Prod. p. 162.

Sulle scorze; Mrz.! 
Questa specie, nuova per l'Italia, era nota fino adesso soltanto dell'Algeria e del Portogallo.

20. - Urceolaria scruposa (Ach.) Jatta Syll. p. 287; Albo l. c. Zodda (sulla terra):

21. - Lecanora (Hymenelia) lithophraga Mass. Sym. p. 28; Albo l.c.

Zodda (sulla roccia).

22. - Lecidea (Biatora) chondrodes Mass. Sym. p. 28; Albo l. c. Zodda (sulla roccia).

Specie nuova per la Sicilia (Albo l. c.).

23. - Dirina repanda (Fr.) Nyl. Pr. p. 97; Albo l. c.

Zodda (sulla roccia).

Specie nuova per la Sicilia (Albo 1. c.).

24. - Dirina Ceratoniae ( $F r$.) DNrs. G. B. It. II p. 188.

Sui tronchi di Ceratonia Siliqua; Mrz.!

25. - Opegrapha herpetica Ach. v. fuscata Schr. En. p. 156. Sulle scorze; Mrz.!

26. - Opegrapha Mougeothii Mass. Mem. P. 103; Albo 1. c. Opegrapha Chevallievi Leigth.

Zodda (sulla roccia).

Specie nuova per la Sicilia (Albo l. c.).

27. - Opegrapha (Lecanactis) grumulosa Duf. var. platycarpa Nyl. Pr. p. 152; Albo l. c.

Zodda (sulla roccia).

La specie era nota dell' Italia meridionale, della Sicilia e di Malta, ma la varietá non era stata segnalata in Italia (Albo l. c.).

28. - Arthonia melanophthalma (Duf.) Nyl. Prod. p. 166.

Sulle scorze; Mrz.!

29. - Endopyrenium haepaticum (Ach.) Nyl. Pyr. p. 15.

Sulla terra, fra i muschi e le epatiche; Mrz.!

30. - Sagedia byssophila Kĩrb. Prg. p. 3ð̌. S. persicina Albol. c. Zodda (sulla roccia).

- Var. chlorotica (Mass.). Mass. Ricc. p. 159. S. persicina v. chlorotica Albo l. c.

Zodda (sulla roccia).

31. - Verrucaria rupestris Schral. Spic. p. 109; Albo I. c.

Zodda (sulla roccia). 


\section{A L G A E. 1}

(Cl. A. Borzì determinavit).

1. - Amphiroa rigida Lamour.; Piccone Risult. algol. della crociera del «Violante» in Ann. Mus. Civ. St. Nat. Genova volume XX (1884) p. 130. ${ }^{2}$

D'Albertis, Settembre 1876.

2. - Bryopsis disticha J. Ag.; Solla Auf. einer Excurs. n. d. Pelagischen Inseln gesammelte Meeresalgen (vedi bibliogr.) p. 4.

Solla, A prile 1884 (Cala Galera. Miolto frequente, attaccata alla roccia e nuotante presso la superficie).

\section{3. - Calothrix confervicola $\mathrm{Ag}$.}

Aderente a varie alghe marine; Mrz.!

4. - Ceramium flabelligerum J. Ag.

Sulle rupi della costa S. presso il porto; Mrz.!

5. - Chroococcus turgidus Näg. Einz. Alg. p. 46.

Sulle pareti umide degli stihiicidî; Mrz.!

6. - Cladophora glomerata Kuetz. Phyc. germ. p. 212.

Nelle fosse di acqua piovana; Mrz.!

7. - Cladophora prolifera Kuetz.; Picc. l. c. p. 110.

D'Albertis.

8. - Cladostephus verticillatus Ag.; Picc. 1. c. p. 117.

D'Albertis.

1 Oltre alle alghe marine raccolte dal capitano D'Albertis e dal prof. Solla, ed alle pochissime riportate da Riccobono e da me nel 1906, ne cito due, comunicatemi dal prof. Borzi, che furono raccolte da Calcara nel 1846 e sono conservate nell'Erbario Palermitano. - 'Trabucco (L'isola di Lampedusa p. 10) dà il nome di alcune alghe marine che sono tutts tolte dal lavoro di Solla.

Le alghe terrestri sono state tutte, meno il Phyllosiphon, raccolte da Riccobono e da me, e døterminate dal prof. A. Borzi.

2 Le alghe raccolte a Lampedusa dal Cap. D'Albertis si trovano citate da Piccone in 3 lavori: in D'Albertis "Crociera del Violante» estratto dagli Ann. del Mus. Civ. di Genova vol. XI a p. 223 in nota (1878) - nelle Memorie Sc. fis. mat. nat. dei Lincei ser. III vol. IV col titolo di « Catalogo delle alghe raccolte durante le crociere del cutter Violante» (1879) - e negli Annali del Mus. Civico di Genova vol. XX (1884). In questi tre elenchi trovansi alcune lievi differenze. Ho scelto la versione degli Annali del 1884, perchè posteriore alle altre. 


$$
\text { LAMPEDUSA - ALGAE }
$$

9. - Corallina officinalis L.; Solla p. 3.

Solla (la var. Mediterranea [Arsch.], isolotto dei Conigli dal lato E., a piccola profonditá, e Cala Galera, frequente).

10. - Cyanocystis versicolor Bor'zi Note Morl. e Biol. Fic. II p. 314.

Aderente ai fili di Rhizoclonium hieroglyphicum; Mrz.!

11. - Cystoseira barbata Ag.

Calcara 1846 (nell' Erbar. Palerm.).

12. - Cystoseira sp.: Solla p. 3.

Solla (Cala Galera e qua e lå sulle coste di S., di E. e di O., a poca profonditå).

13. - Dasycladus clavaeformis Ag.; Solla p. 4.

Solla (raro negli incavi delle rupi dell'isolotto dei Conigli, lato N., vicino alla superficie).

14. - Dictyota dichotoma Lamour.; Picc. 1. c. p. 119 ; Solla p. 3. D'Albertis, Solla (la forma implexa [Lamour.], Cala Galera, frequente).

15. - Dictyota Fasciola Lamour.; Picc. 1. c. p. 1ï; Solla p. 3. Sulle rupi della costa S. presso il porto; Mrz.! - D'Albertis, Solla (Cala Galera con D. dichotoma).

16. - Dictyota linearis Ag.; Picc. 1. c. p. 118.

D'Albertis.

17. - Enteromorpha compressa Grev.?; Solla p. 4.

Solla (rigettata sulla spiaggia della costa $\mathrm{S}$.).

18. - Gelidium crinale Ag.; Solla p. 2.

Solla (isola dei Conigli, lato N., in acqua piuttosto profonda, raro).

19. - Gloeothece palea Rabenh. Fl. europ. Alg. II p. 61.

Sulle pareti umide sottoposte agli stillicidî; Mrz.!

20. - Gracilaria dura Ag.; Picc. l. c. p. 132.

D'Albertis.

21. - Halimeda Tuna Lamour.; Solla p. 4.

Cala Galera, Mrz.! - Solla (piuttosto rara sulle rupi dell'isolotto dei Conigli, vicino alla superficie).

22. - Halyseris polypodioides Ag.; Picc. I. c. p. 120. Dictyopteris polypodioides Solla p. 3.

D’Albertis, Solla (Cala Galera e vicino alla Lanterna sulla costa S., esemplari piccoli, fruttificati).

23. - Hapalidium Phyllactidium IRuetz. ; Picc. l. c. p. 130.

D'Albertis (sulla Cladophora prolifera). 
24. - Hydroclathrus sinuosus Zan.; Solla p. 4.

Solla (nel Porto, sulle pietre a diversa profonditå, ed anche rigettato a riva, ma non comune).

25. - Hypnaea musciformis Lamour.; Solla p. 2.

Solla (Cala Galera, a quasi un braccio di profonditá, isolata ma non rara, senza frutti).

26. - Jania adhaerens Lamour.; Picc. 1. c. p. 131. J. gracilis Picc. in D'Albertis Croc. Violante p. 233.

D'Albertis (sopra alghe maggiori).

27. - Jania rubens Lamour.

Sulle rupi della costa S. presso il porto; Mrz.!

28. - Laurencia obtusa Lamour.; Solla p. 3.

Solla (Cala Galera, e sulla costa S. presso la Lanterna, piuttosto frequente).

29. - Lyngby a majuscula Hrv. in Hook. Brit. Fl.; Solla p. 4. Solla (Cala Galera negli incavi della roccia, alla superficie).

30. - Melobesia pustulata Lamour.; Solla p. 3.

Solla (scogli dell'isolotto dei Conigli, e qua e là sulla costa S., a diverse profondità).

31. - Melobesia verrucosa Lamoul'; Picc. Risult. algol. p. 130.

D'Albertis (sulle foglie della Posidonia).

32. - Microcoleus vaginatus Gomt. Ess. class. p. 93.

Sulla terra umida piuttosto comune in Lampedusa e sull' isolotto dei Conigli; Mrz.!

33. - Nostoc insulare Borzi nor. spec.

N. thallis minutis, raro ad millimetrum crassis, globoso-pyriformibus aut ovoideis, solidis, olivaceo-fuscis, peridermate firmo tenacissimo, densissime aggregatis; trichomatibus haud vaginatis, torulosis, articulis sphaerico-depressis, ad 6-8 micr. latis, heterocystis globosis articulis vegetativis paulo majoribus denique in sporis transmutatis.

Sulla terra argillosa umida, in Lampedusa e sull'isolotto dei Conigli ; Mrz. !

Fra le moltissime forme o specie di Nostoc questa si distin. gue a prima vista perchè costituisce, sul suolo argilloso umido bagnato dall'acqua piovana dell'inverno, un sottile strato olivaceo scuro, il quale, per azione della umidità, piglia l'aspetto di una crosta minutamente tubercoloso-granulosa, molto resistente e compatta. Questa apparisce costituita da una infiniti di minu- 
tissime granulazioni solide e cosi strettamente ravricinate tra di loro che a fatica si riesce ad isolarle. Questo carattere mi pare a prima giunta molto singolare e degno di considerazione. Inoltre la notevole durezza dei singoli talli, le loro esigue dimensioni, la stazione costantemente terrestre, mi hanno persuaso di considerare questa forma come affatto distinta dal $N$. coeruleum, col quale presenta le maggiori affinitá.

Quanto mai caratteristica è presso questa specie la formazione delle spore, le quali procedono dalla trasformazione degli eterocisti, o almeno dalla più grande parte di questi; fenomeno che trova riscontro solamente nel $N$. commune, mentre presso le altre specie di Nostoc, le spore derivano dagli articoli vegetativi. Non è qui il luogo di descrivere nei suoi minuti particolari e discutere le modalità di tale processo tanto nel N. commune come nel $N$. insulare. Ricorderó solo, per la storia, che la trasformazione degli eterocisti del Nostoc commune in spore è stata per la prima volta segnalata dal Brand (V. Ber.d. deutsch. Botan. Gesell. 1901, pag. 152) e le mie osservazioni, tanto nella detta specie, quanto nel $N$. insulare, concordano perfettamente con quelle di quest'autore. Solo è da aggiungere che tale fenomeno va considerato come perfettamente normale, ed è la sola forma di propagazione per germi immobili ibernanti che si conosca nel $N$. commune. Lo stesso deve dirsi per il $N$. insulare.

34. - Nostoc muscorum Ag. Disp. Alg. Suec. p. 44.

Sulla terra muscosa umida; Mrz. !

35. - Oscillaria brevis Kuetz. Phyc. gener. p. 186.

Sul fondo delle pozze d'acqua piovana; Mrz.!

36. - Oscillaria limosa Ag. Disp. Alg. Suec. p. 35.

Non molto frequente sulla terra umida; Mrz.:

37. - Oscillaria tenuis Ag. Alg. Decad. p. 25.

Sul fondo delle fosse ripiene d'acqua piovana; Mrz.!

38. - Padina Pavonia Gaill.; Solla p. 3.

Solla (nel Porto a varie profondità, molto frequente).

39. - Peysonnelia rubra J. Ag.; Picc. 1. c. p. 129.

D'Albertis.

40. - Phyllosiphon Arisari İ̈̈hn; Ross Eine bot. Exc. (vedi bibliogr.) p. 349 .

Ross (parassita sulle foglie dell' Arisamum vulgare). 
41. - Pleonosporiuın Borreri Näg.; Solla p. 2.

Solla (Cala Galera, negli incavi delle rupi presso la superficie, piuttosto frequente).

42. - Polysiphonia fruticulosa Spreng.; Solla p. 3.

Solla (costa 0. della Cala Galera, vicino alla superficie, frequente).

43. - Rhizoclonium hieroglyphicum Kíuetz. Phyc. gen. p. 205 .

Comune nelle fosse ripiene d'acqua piovana, dove ricuopre spesso di un fitto strato pannoso i tappeti di Elatine e di Bulliarda; Mrz.!

44. - Rhodymenia Palmetta Grev.; Picc. 1. c. p. 127.

D'Albertis (dragata a $60 \mathrm{~m}$. di profonditá).

45. - Rhodymenia 11. sp. ? Picc. 1. c p. 127. Rhodophyllis bifida Picc. in D'Albertis Croc. Violante p. 233, non Ktz.

D'Albertis (frammenti ritirati colla draga e ritenuti nel suo primo lavoro per la Rhodophyllis bifida, ma poi giudicati appartenenti a specie non descritta di Rhodymenia).

46. - Rytiphlaea pinastroides Ag.; Solla p. 3.

Solla (Cala Galera, a un braccio di profondità).

47. - Rytiphlaea tinctoria Ag.; Picc. Risult. algol. p. 136 ; Solla p. 3.

D'Albertis, Solla (con la precedente, rara).

48. - Sargassum linifolium Ag.; Solla p. 3.

Calcara 1846 (nell' Erb. Palerm.), Solla (piuttosto frequente sulla costa S., esemplari molto piccoli).

49. - Scenedesmus quadricauda Bréb. Alg. Fal. p. 66.

Sul terreno umido, frammista ad altre alghe; Mrz.!

50. - Scytonema ocellatum Lyngbye Hydr. dan. p. 97.

Molto raro in terreni acquitrinosi, argilloso-calcarei, presso Cala Galera; Mrz.!

51. - Spelaeopogon Sommieri Borzi nov. spec.

Strato late expanso intricato, aerugineo, filis primariis torulosis, in fasciculos repentes dense coalitis, 8-10 micr. crassis, secundariis paulo tenuioribus, spar'sis et irregulariter et laxe intricatis, continuis, articulis cylindraceis 5-8 micr. latis, membrana achroa tenuissima, heterocystis ovato-globosis aureo-fuscis, ad 6-9 micr., hormocystis lineari-oblongis, leviter torulosis; 8-16articulatis, rectis membrana crassiuscula olivaceo-fuscesscente. 
Sulle marne argillose calcaree acquitrinose, in luoghi ombrosi, fra i muschi e rizomi di Adiantum Capillus Veneris nella grotta 'Tabaccara sulla costa meridionale; Mrz.!

Il genere Spelaeopogon venne da me costituito pochi anni fa per distinguere alcune forme di Stigonemacee prive quasi del tutto di quelle caratteristiche ramificazioni che contraddistinguono questo gruppo di Mixoficee, ma che alle stesse Stigonemacee si riferiscono per la struttura dei filamenti. Questi sono infatti formati da porzioni di tricomi a cellule sferico-depresse, quindi torulose, e da porzioni ad articoli cilindrici continui. Peró nel genere Spelaeopogon lievissima è la differenza fra lo spessore delle dette due porzioni; nè quelle cilindriche si alternano con quelle torulose, come accade normalmente negli Hapalosiphon; queste ultime possono solamente divenir torulose per dar luogo alla formazione di organi di propagazione, cioè le ormocisti. Le porzioni torulose stanno aggregate in densi fascetti, che nello S. Sommieri, come in un'altra specie, rinvenuta presso Catania, lo $S$. Cavarae, crescono repenti e abbandonati sul substrato, mentre nello S. lucifugus, essi sono eretti e formanti dei densi cespuglietti. Notevole è inoltre in questo genere il meccanismo di ampliamento del tallo, il quale si effettua per spontanea frammentazione dei filamenti, senza l'intervento di eterocisti; anzi in qualche specie questi mancano o sono rarissimi. Spesso la separazione dei tricomi è determinata dalla presenza di un setto di materia gelatinosa densa lucida che si frappone fra due articoli consecutivi. Quando la rottura del filamento, al punto dove si è formato tale setto, non avviene rapidamente, continuando i filamenti a crescere in lunghezza, prendono origine delle disposizioni che possono indurre la convinzione che il tallo si accresca anche per mezzo di vere ramificazioni.

Il genere Spelaeopogon è un importante acquisto per la sistematica delle Mixoficee. Esso segna il passaggio fra le Stigonemacee tipiche e le Scitonemacee. Piủ importante è ancora dal punto di vista biologico, poichè le sue specie sono caratterizzate da un habitat particolare, crescendo sulle pareti di grotte, in punti per lo più pochissimo rischiarati, sopra un sulsstrato di natura calcarea argillosa. 
Lo stesso S. Sommieri, quantunque non rinvenuto sulle pareti di una vera grotta, mostra di preferire localitá molto ombreggiate, e i suoi filamenti si stendono tra gli steli dei muschi e rivestono le parti superficiali dei rizomi del Capelvenere, rimanendo in gran parte nascosti e sottratti alla luce.

Il genere Spelaeopogon non è stato finora raccolto al di fuori della Sicilia. Lo S. Sommieri, oltre che a Lampedusa, cresce pure all'Isola di Gozo (Malta) sulle pareti esposte agli stilli. cidî dove è stato raccolto, sebbene in piccolissima quantitả, dallo stesso. Sommier lo scorso anno.

52. - Sphacelaria filicina Ag.; Picc. 1. c. p. 117. S. scoparia Picc. in D'Albertis Croc. Viol. p. 233.

D'Albertis.

53. - Sphaerococcus coronopifolius Ag.; Picc. Risult. algol. p. 131.

D'Albertis.

54. - Spyridia filamentosa Hrv. in Hook. Brit. Fl.; Solla p. 2.

Solla (Cala Galera, ad un braccio di profonditá, rara, associata qua e lì alla Laurencia).

55. - Ulothrix flaccida Kuetz. Sp. Alg. p. 349.

Comune sulla terra umida e nelle pozze d'acqua piovana in Lampedusa e sull'isolotto dei Conigli ; Mrz. !

56. - Ulva Lactuca (L. p. p.) Jol. Alg. mar. Cherb. p. 38; Solla p. 4.

Solla (nel Porto, e rigettata sulla costa S. E.).

57. - Vaucheria dichotoma Ag. Syn. Alg. Scand. p. 47.

Comunissima sulla terra umida, nei luoghi inondati durante l'inverno in Lampedusa e sull'isolotto dei Conigli; Mrz.!

58. - Vaucheria sessilis DC. Fl. franç. II p. 63.

Piuttosto rara sulla terra umida; Mrz.!

59. - Vidalia volubilis J. Ag.; Picc. l. c. p. 137.

D'Albertis (esemplari che giungono ad arere fino a $20 \mathrm{~cm}$. di lunghezza).

60. - Zy gnema cruciatum Ag.; Syst. Alg. p. 77.

Comunissimo sulla terra nel fondo di pozze ripiene d'acqua piovana stagnante sull' isolotto dei Conigli ; Mrz. ! 


\section{LINOSA}

\section{NOME E STORIA.}

Linosa fu dagli scrittori di varie epoche chiamata Ethusa, Larenusia, Lenusa, Lalenusa, Nemousa, Limosa. ' Ethusa è il nome più antico datole da Plinio e da Tolomeo. Secondo Müller (in Ptolomaei Geogr. vol. I pars II p. 661), viene da $\mathbf{\Lambda} i$ fovox (ardente) nome che, dice Müller, ben si conviene a quest'isola che è tutta vulcanica. Strabone non ne fa menzione.

Se la storia poco ci dice di Lampedusa, di Linosa tace quasi del tutto. Non ebbe questa isoletta la sorte di essere cantata, come Lampedusa, nè da un Ariosto né da un Wieland; non

1 Sono stati applicati a Linosa anche i nomi di Algusa ed Egusa. Il nome di Egusa peró viene generalmente dato ad una delle Egadi (Favignana). Plinio pare facesse già questa confusione, poichè nẹl Libro III della sua Historia naturalis dice «Ethusa quam alii Egusam scripserunt 》. Qualcuno dei suoi commentatori però (p. es. Harduinus, Torino 1831) crede che Plinio designasse invece Linosa col nome di Caene, e che da questo sia derivato il suo nome più recente di Limoza o Limosa (a caeno seu limo). Anche Fra Leandro Alberti (Isole appartenenti all'Italia, in Descrittione di tutta l'Italia \& Isolo pertinenti ad essa, Venetia 1581 p. 69) ripete con Plinio che Ethusa ed Egussa sono due nomi dati ad una stessa isola; ma dicendo che è situata vicino a Trapani, mostra di crederla una delle Egadi.

Il nome di Ethusa trovasi usato ancora in altro senso. Vediamo p. es. che Coronelli (Isola di Rodi ecc., Venezia 1688) chiama così una isola nei pressi di Rodi «ivi ruotolata dal terremoto a gala »; e Nigro (Geogr. comment., Basileae 1557) c'insegna che il nome di Ethusa fu dato anche a Costantinopoli!

Della confusione fra Linosa ed altre isole si trovano le prove in molte antiche opere. Fra le tante che si potrebbero citare rammen- 
ebbe una Madonna miracolosa che attirasse su di essa l'attenzione degli scrittori di cose sacre, in modo che varî autori i quali dedicarono intere pagine a Lampedusa, di Linosa appena citano il nome. ${ }^{1}$

Traccie di abitazioni, monete, ruderi diversi e numerose cisterne (136 secondo Calcara) ${ }^{2}$ dimostrano chiaramente che fu abitata al tempo dei Romani, e probabilmente più tardi dagli Arabi.' Ma i pochi autori che ne fanno menzione nei quattro secoli passati la dicono deserta. Deserta la trovó il capitano Smyth, al principio del secolo passato, e neppure vi rinvenne alcun grande mammifero; per questo vi porto alcune capre ed alcuni conigli. Deserta pure la trovó Gussone nel 1828; ma vide che le capre lasciate dallo Smyth si erano moltiplicate tanto che ne valutó il numero a circa 200 ; ed i conigli vi erano gia « in tale abbondanza che non poteva darsi un passo senza vederne fuggire moltissimi ». Ancora priva di abitanti era Linosa nel 1844 quando la visitó il tenente di vascello Sanvisente, Governatore di Lampedusa.

La sua colonizzazione rimonta al 1845 quando, per ordine del Re Ferdinando di Borbone, vi sbarcarono 30 individui provenienti

terò solo ancora l'edizione di Basilea del 1545 della Geographia univers. di Tolomeo, dove troviamo citate (p. 66) come cose diverse, $\Theta$ rappresentate poi molto distanti fra loro sulla Tabula Aphricae II «Larunesiae insulae duae » ed « Ethusa ». Però tre anni dopo, nella edizione di Mattioli (Venezia 1548) della stessa opera, vi è una «Africa Minor, nova tabula » di Jacopo Gastaldo, sulla quale Linosa e Lampedusa trovansi segnate in posizioni molto prossime al vero, coi nomi di Limosa e Lampedola, e non trovansi più le Larunosiae. Del resto nei portolani, da quello di Pietro Vesconte del 1311 fino a quelli della fine del secolo XVI, troviamo Linosa segnata molto corrottamente, generalmente col nome di Limosa, nuova prova di quanto fosse rimasta arretrata la geografia scolastica.

${ }^{1}$ Nigro, è vero (Geogr. Comment. Basileae 1557 p. 363), identificando Limosa con l'antica Gaudos, dice che secondo Callimaco era quella l'isola di Calipso. Lo stesso Nigro però aggiunge « quod Straboni non placet ».

2 Pare che tutte queste cisterne fossero in stato inservibile quando il Re Ferdinando ordinò la colonizzazione di Linosa, poichè Calcara dice che l'acqua vi doveva essere portata in botti dal continente. Adesso invece molte sono di nuovo in uso. 
da Girgenti, fra cui un deputato di sanitá, un medico e un prete, e vi vennero fabbricati un magazzino, una chiesa ed una casa. Nel 1846, quando Calcara visitò Linosa, il numero dei suoi abitanti era di 85, e nel 1847 Sanvisente scriveva che erano 116. Poco progredita trovai la popolazione dell' isola all'epoca della mia prima visita nel 1873. Gli unici edifizî in muratura erano ancora la chiesa, il magazzino e la casa abitata dal dott. Buonadonna, capo della colonia e rappresentante del Governo, che cumulara gli ufficî di capitano del porto, di prosindaco, di notaro, di medico e di farmacista! I coloni abitavano miseramente da trogloditi entro grotte scavate nel tufo. Lo stesso stato di cose vi trovò il capitano d'Albertis nel 1876. Dopo d'allora però, col concorso del Governo che forni gratuitamente la pietra calcarea portata da Lampedusa per fare la calcina, e il legname, sono state costruite delle basse casupole di un piano e di poche stanze ognuna, ed ora le grotte non servono più che di stalla per le bestie e di magazzini. Le abitazioni sono raggruppate in un piccolo villaggio vicino allo scalo vecchio, a mezzogiorno dell'isola. Dal lato opposto, a N. E. é stato costruito un fanale. Gli isolani, in numero oggi di circa 250, sono tutti agricoltori. Alcuni di essi si dedicano inoltre alla pesca, ma poco. Quelli immigrati dopo la prima colonizzazione provengono in parte, come i primi, da Girgenti, ed in parte da Pantelleria e da Ustica, in maggior numero da quest'ultima isola.

\section{DESCRIZIONE, GEOLOGIA, CLIMA, PRODOTTI.}

E situata Linosa fra $35^{\circ} 51^{\prime}, 2$ e $35^{\circ} 52^{\prime}, 5$ Lat. N., e fra $0^{\circ} 23^{\prime}, 7$ e $0^{\circ} 25^{\prime}, 7$ Long. E. di Roma. Il faro sulla costa N. E. è a $35^{\circ} 52^{\prime} 13^{\prime \prime}$ Lat. N. e $0^{\circ} 25^{\prime} 25^{\prime \prime}$ Long. E. ỏi Roma. É distante $42 \mathrm{Km}$. da Lampedusa, 122 da Pantelleria, 120 da Gozo (Malta), 161 dal punto più vicino della Sicilia (Marina di Palme), 163 dal punto più vicino della Tunisia (Capo Mehediah). E di forma quasi circolare. Il suo massimo diametro, da Punta Levante a Punta Ponente, è di Km. 3,5 ed il minimo, da Sud a Nord, di $2 \mathrm{Km}$. Il suo cir- 
cuito è di $11 \mathrm{Km}$. La sua cima più alta, il Monte Vulcano, raggiunge $195^{\mathrm{m}}$. La sua superficie è di Kmq. 5,4302.'

$$
*^{*} *
$$

Linosa è, sotto tutti i rapporti, differente da Lampedusa. In Lampedusa la roccia è tutta calcarea, sedimentare, ed essendo in gran parte denudata, é di un bianco che acceca. In Linosa il suolo

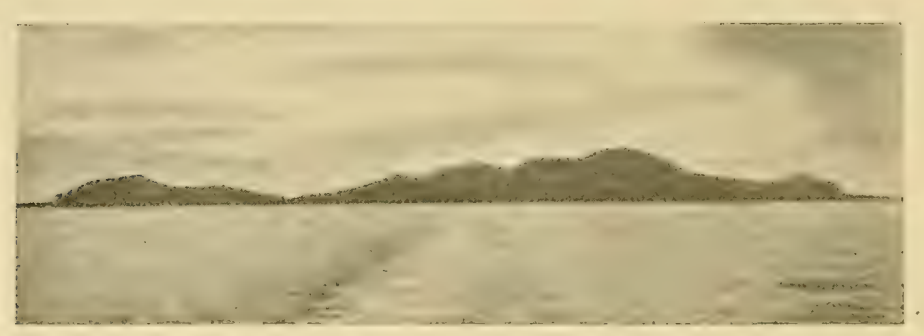

Linosa veduta dal lato di mezzogiorno. (Da una mia fotografla)

è interamente vulcanico e di colore scuro, composto come è di basalti antichi ai quali sono sovrapposte colate di nerissima lava, scorie, tufi vulcanici, bombe, pomici, lapilli, sabbie e ceneri, materiali talvolta rossastri o giallastri, ma per lo più neri o nerastri. Inoltre, mentre Lampedusa è pianeggiante, Linosa è tutta montuosa per i coni più o meno rovinati degli apparati vulcanici che le hamno dato origine traversando le antiche assise basaltiche ed eruttando le lave ed i materiali detritici sovrapposti a quelle assise. Pianeggianti sono soltanto il fondo dei crateri (di cui uno solo, al centro dall' isola, ha una discreta estensione), alcune distese di terra sabbiosa fra la base dei monti e il mare, le antiche assise basaltiche alla periferia dell' isola, ed alcune colate di lava, queste peró a superficie scoriacea irregolare; frastagliata ed irta di punte in modo da rendervi assai faticoso il cammino. In alcuni punti si vedono interi fianchi dei

1 Atrilio Mori, L'area delle minori isole italiane, in Rivista geogr. ital., anno III, fasc. X, dicembre 1896. Allo stesso prof. Mori devo le precise indicazioni geografiche che sopra. 
coni di deiezione formati da frane di materiale incoerente, affatto privi di vegetazione (come lo mostra la fotografia della Montagna di Ponente), ed in altri punti si vedono scogli di basalte sui quali non cresce altro che la varietá ectanea della

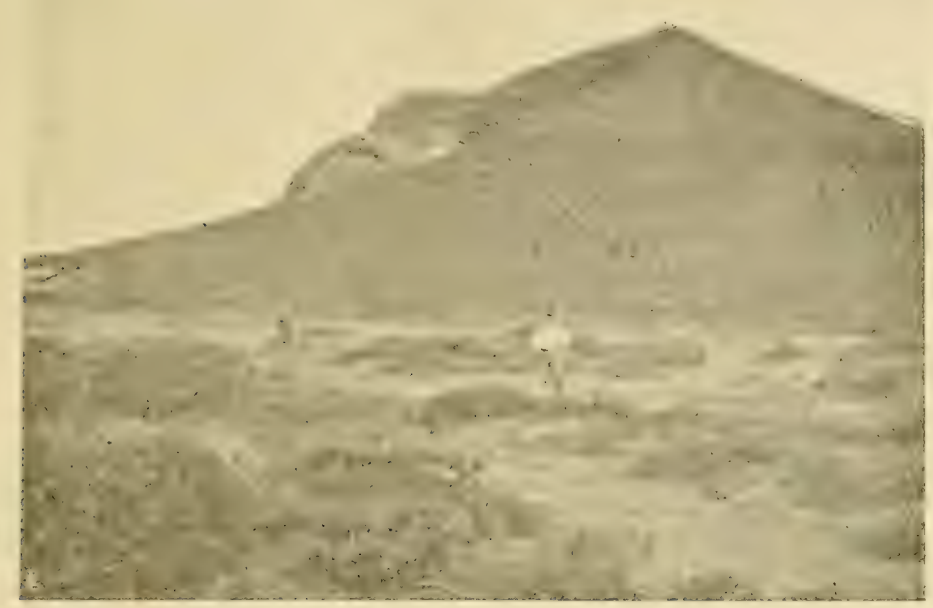

Montagna e Vulcano di Ponente.

(Da una mia fotografia)

Physcia parietinc, la quale con il suo tallo arancione fa un bel contrasto con la roccia nera cui è attaccata. La maggior parte dell'isola però in primavera è verdeggiante, ma di un verde attraverso al quale traspare il colore scuro del suolo.

L'attivita vulcanica è adesso interamente cessata in Linosa, e a differenza di Pantelleria non vi si trovano nè sorgenti termali né fumarole. Non evvi neppure alcun ricordo storico di ertuzioni in quest' isola.

$$
* *
$$

Primo a dare notizie sulla geologia di Linosa fu Gussone, seguito poi da Calcara e da Speciale. Più estesi ed accurati studî sulla sua struttura geologica ed origine troviamo nelle memorie del prof. Trabucco, il quale potè anche determinare alcuni fos- 
sili trovati nei suoi tufi. Trabucco arriva alle seguenti conclusioni sulla storia geologica e dinamica dell' isola:'

«La base dell'isola è costituita da basalte amigdaloide, eruttato sott'acqua da bocca sconosciuta.

«Successivamente sopra questa base si innalzarono e cominciarono ad emergere dai flutti alcuni coni vulcanici, i quali, nelle fasi successive di attivitá, vomitarono delle lave e dei materiali detritici che, mentre andavano a mano a mano innalzando i loro apparati, discendevano anche sott'acqua, costituendo il banco di basalte porfirico e gli strati tufacei che ricoprono i basalti amigdaloidi. Intanto i materiali detritici, nel formare i tufi impigliarono i fossili che ora contengono.

«Più tardi un bradisismo ascendente fece emergere i basalti amigdaloidi e porfirici e portò la panchina tufacea fossilifera fino a circa $30 \mathrm{~m}$. sul livello del mare.

« L' isola dovette, verosimilmente, emergere dalle onde durante il quaternario antico e deve la sua origine agli spiragli apertisi sulla linea di frattura che corre tra la costa Est della Sicilia ed il litorale di Tunisi, per l'innalzamento post-pliocenico, sorgendo essa sulla linea che separa i bassifondi dal mare profondo.

«Questa conclusione è avvalorata dai seguenti fatti:

« $1 .^{\circ}$ l'etá dei fossili che abbondano nei tufi stratificati della base orientale del Monte di Ponente;

« $2 .^{\circ}$ la natura delle rocce di cui l' isola è costituita;

«3. da una parte la mancanza di tracce di elementi eruttivi nei calcari pliocenici della limitrofa isola di Lampedusa e dall'altra la mancanza di ricordi storici di fenomeni vulcanici dell' isola.

* Più difficile riesce di stabilire il periodo in cui cessò l'attivitả vulcanica. $\mathrm{Na}$, anche qui, la mancanza di ricordi storici di eruzioni, fumarole, sorgenti termali, ecc. provano che la medesima cessò da tempo storico remotissimo.»

1 Recentemente il Washington (The Titaniferous Basalls of the Western Mediterranean (Quart. Journ. of the Geol. Soc., 1907, vol. 63, n. 249) dà l'analisi (p. 74-75) di alcune camptoniti, basalti titaniferi di Linosa, ch'egli avvicina a quelli di Pantelleria, di Monte Ferru in Sardegna e di Catalogna. 
Da questo risulta dunque certo che Linosa non è mai stata congiunta con altre terre emerse, ciò che è importante notare per la storia della sua flora.

Sul clima di Linosa non abbiamo alcun dato preciso. Pare peró che le pioggie vi siano più abbondanti che a Lampedusa, e che la temperatura vi si innalzi di più a causa del colore scuro del suolo e del riparo parziale dai venti dovuto alla natura montuosa dell' isola. ${ }^{1}$ Grande vi è la siccitả in estate, e giå Gussone notò che per questa causa la Periploca, i Rhus e l'Olea vi perderano le foglie in quella stagione, e che allora tale era la mancanza di vegetazione erbacea, che i conigli (non essendovi allora i fichi d"India) erano costretti a cibarsi della corteccia delle Euforbie.

Non vi è in Linosa alcuna acqua superficiale. Scavando dei pozzi nei punti più bassi, anche al centro dell' isola, si raggiunge, al livello del mare, uno strato di acqua salata quanto quella marina. In conseguenza gli abitanti non hanno per gli usi domestici e per abbeverare i loro animali, altra acqua che quella delle cisterne.

La terra vegetale formata da sabbia e ceneri vulcaniche e dal polviscolo risultante dal disfacimento delle roccie, dei tufi e specialmente del materiale detritico di cui è composta l'isola, il tutto misto ai prodotti della decomposizione delle piante, è di colore scuro ed è incoerente e suddivisa in modo da sem-

1 Il riscaldamento maggiore del suolo a Linosa farebbe supporre che le fioriture vi fossero più precoci che a Lampedusa, come asserisce Solla il quale dice che ciò si rileva anche dalle sue annotazioni fenologiche. Tuttavia lo spoglio di queste annotazioni mi ha mostrato che la grande maggioranza delle specie in comune alle due isole fu da Solla trovata allo stesso stato di fioritura o fruttificazione a Linosa $\theta$ a Lampedusa. Il fatto che ne trovò alcune in uno stadio un poco più avanzato a Linosa si spiega sufficientemente per esservi egli stato una decade più tardi. Io invece, che in quest'anno visitai Lampedusa dopo Linosa, trovai una piccola sproporzione in favore di Lampedusa. Da ciò risulta che, almeno nella prima primavera, il riscaldamento maggiore del suolo non ha avuto un effetto molto sensibile sulle fioriture. 
brare polvere finissima. Si ha una prova di quanto sia sciolta, vedendo in erbario le lunghe e sottili fibre radicali che se ne sono potute facilmente estrarre senza romperle. Ciò permette di distinguere a prima vista le piante raccolte in Linosa da quelle provenienti da Lampedusa dove le radici non si possono estrarre dalla terra compatta e coerente; senza mutilarle. Per la sua

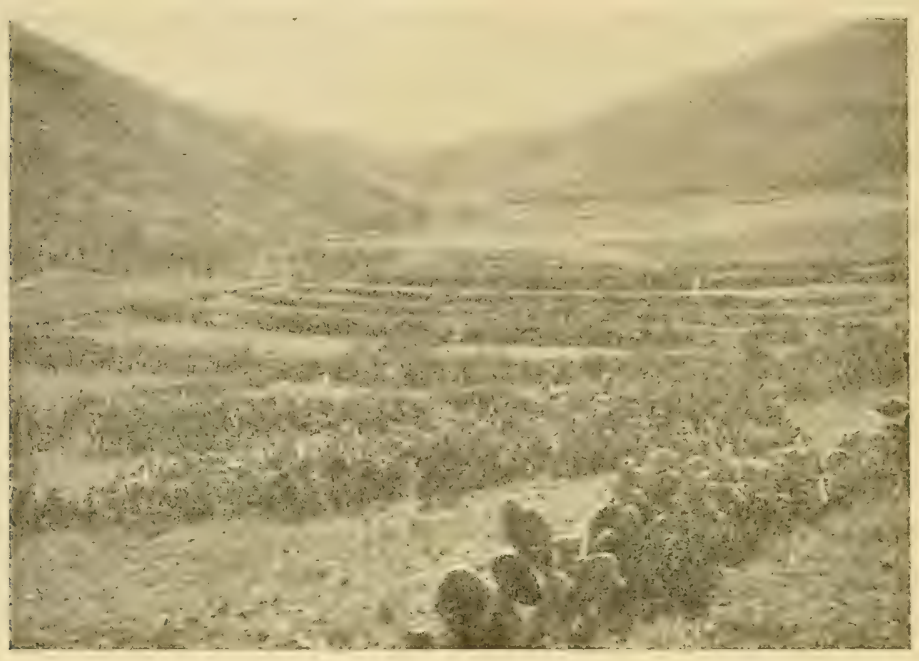

Fondo del cratere nel centro dell'isola, con siepi di fichi d'India. (Da una mia fotografia)

composizione, per la conformazione del suolo che meno si presta al dilavamento, come per le pioggie più frequenti e le esposizioni diverse, la terra a Linosa é più fertile che a Lampedusa.

Le parti pianeggianti dell' isola sono per lo più coltivate. Oltre ai fichi d'India, che vi crescono abbondanti e rigogliosi, raggiungendo notevoli aitezze (fin 3 e $4 \mathrm{~m}$.), ed alla vite di cui vi sono ora circa 20,000 piante, ' si coltivano le patate, varie leguminose, fra le quali meritano speciale menzione le lenti perchè si esportano e godono fama speciale in Sicilia, l'orzo usato principalmente per foraggio, pochissimo grano, il lino per uso domestico, pochi ortaggi e pochi alberi da frutto (principal-

\footnotetext{
${ }^{1}$ Secondo Lo Re, nel 1884 ve ne erano poco più di 10,000. In quest'anno mi scrivono che furono fatti 40 ettolitri di vino.
} 
mente il Fico). Il Carubbo, che cresce spontaneo a Linosa, vi viene anche coltivato. Il prodotto principale dell'isola, per l'espor'tazione, è la Soda, che si ottiene bruciando le piante secche del Mesembryanthemum crystallinum (non Salsola Soda come dice Lo Re), chiamato dagli isolani Erba Cristallina o Erba Soda. Questa specie, introdotta a quanto pare dopo i tempi di Gussone, vi cresce ora spontanea in grande quantita, ed inoltre ri si coltiva, quantunque fino dal 1884 Lo. Re ne sconsigliasse la coltura a causa dell'impoverimento del terreno che ne risulta, impoverimento del quale sono consapevoli anche gli isolani. I campicelli sono tutti circondati da muri a secco o da siepi di Opunzie e di Lycium. Le siepi di fichi d'India in alcuni punti sono cosi ravvicinate, che da una certa distanza si direbbe che è la sola pianta coltivata. Queste Opunzie sono una grande risorsa per gli isolani, non soltanto perchè ne mangiano i frutti, ma anche perchè le loro pale forniscono un buon cibo alle bestie nella stagione in cui tutte le erbe sono disseccate.

Gli isolani tengono un discreto numero di ovini e di vaccine che si esportano a Lampedusa, dove la mancanza di pascolo non permette di tenerne una quantita sufficiente al consumo." Col latte che non consumano in paese, fanno dei formaggi che in parte si esportano. Nelle stagioni fresche, quando la pesca è stata fruttuosa, approfittano della cor'sa settimanale del piroscafo per mandare del pesce in Sicilia.

\section{FAUNA.}

Della fauna di Linosa ha dato poche notizie Calcara, il quale descrive una Helix nuova trovata in quest' isola, la H. Linusae, che egli dice esser la sola specie di mollusco terrestre che si rinviene in grande abbondanza in Linosa. Però vi si trovano anche chiocciole di altre qualitá, poichè gli isolani vi distinguono dei castroni e dei babbaluci. Trabucco dá egli pure alcuni elenchi di animali, dai quali peró risulta che, come per la flora, ha confuso

1 Quest'anno mi fu detto che le vaccine in Linosa erano circa 300 , le pecore 250 e le capre 300. 
Lampedusa con Linosa. Linosa è stata visitata a varie riprese dal zoologo prof. E. H. Giglioli; ma egli non ne ha fatto argomento di una pubblicazione speciale.

Le capre selvatiche, discendenti da quelle importate dal capitano Smyth, che Gussone valutava a 200 nel 1828, all'epoca della visita di Calcara erano giá ridotte a un centinaio, ed ora sono da un pezzo interamente distrutte. Anche i conigli, tanto abbondanti al tempo di Gussone, sono ora del tutto scomparsi. Sono frequenti invece nella macchia il gatti inselvatichiti. Di altri mammiferi, che mi consti, vi sono soltanto topi nelle case e in campagna, ed una specie di pipistrello.

Molte sono le specie di uccelli che nelle loro migrazioni fanno una sosta in Linosa, e poche quelle che vi nidificano. Calcara då un breve elenco delle specie osservate. Io ho veduto Alcedo Ispida, Tadorna cornuta e Ciconia alba presi nell' isola. Le rondini, le tortore, i colombi vi si fermano al passo, ma non nidificano. Le quaglie, i passeri ed alcuni altri piccoli uccelli vi fanno il nido. Vi si cacciano le lodole, i tordi, varie anatre, le beccaccie, i beccaccini.

Fra i rettili abbondano la comune lucertola (Podarcis muralis) ed il Gongylus ocellatus, rappresentati l'uno come l'altro da razze che, per adattamento alla roccia nera ed alle terre scure su cui vivono, hanno assunto una colorazione scura. Questa colorazione è cosi marcata, che differenzia in modo da colpire anche il profano, il Gongylus di Linosa da quello di Lampedusa. Trovansi inoltre, $\mathrm{ma}$ in assai minore quantitá, Plalydactylus Mauritanicus ed Hemidactylus verruculatus. Mancano del tutto, a quanto mi assicurarono i coloni, le serpi. Le testuggini vi esistono, ma molto rare. Mancano affatto gli anfibi, non essendovi neppure alcun rospo di cui vi è una specie in Lampedusa.

Fra i coleotteri da me riportati da Linosa vi è una Tentyria ${ }^{4}$

1 Tentyria Sommieri F. Baudi, "Catalogo dei Tenebrioniti della fauna europea e circummediterranea appartenenti alle collezioni del Museo Civico di Genova ", Ann. Mus. Civ., Ser. I, Vol. VI, 1874, p. 99 e Naturalista Siciliano, 1896, p. 103. Tutti i coleotteri conosciuti delle Pelagie sono citati da Ragusa in "Catalogo ragionato dei coleotteri di Sicilia 》nel Naturalista Siciliano, catalogo incominciato nel 1883, e tutt'ora in corso di stampa. 
non conosciuta di altro luogo, e che ivi è comunissima sul suolo arenoso. Fra le formiche da me raccolte, il prof. Emery ha riconosciuto le seguenti due specie: Messor barbarus capitatus Latr. v. sancta For. e Tapinoma erraticum nigerrimum.

Il mare intorno a Linosa è ricco di pesci, ma meno, a quanto pare a causa della sua maggiore profondità, di quello di Lampedusa, la cui ricchezza in pesci gode di una fama speciale. Sulle scogliere marine abbondano le patelle, fra le quali è facile riconóscere più d'una specie.

\section{FLORA.}

Il rivestimento vegetale in oggi è molto più ricco a Linosa che a Lampedusa. La macchia ha già sofferto per l'azione dell'uomo e degli animali domestici, e specialmente hanno diminuito le piante di alto fusto. Ma tuttavia la macchia esiste ancora nella maggior parte dell' isola. Suoi elementi costitutivi sono: Pistacia Lentiscus, la specie più comune e di cui esistono ancora degli alberetti alti più di due metri che in qualche punto formano quasi bosco, Euphorbia dendroides che abbonda e spicca fra le altre piante e sulla roccia nera per il verde chiaro delle sue fronde cupoliformi, Lycium Europaeum comune nella macchia ed inoltre adoprato dai coloni per fare delle siepi alte, fitte ed impenetrabili, Periploca angustifolia, Rhus dioica, Ceratonia Siliqua, Prasium majus (più specialmente fra le rupi), Ruta bracteosa, Olea Europaea (notevolmente diminuita in questi ultimi anni), Juniperus Phoenicea adesso raro. Gussone cita ancora la Phillyrea, ma oggi è quasi del tutto scomparsa, forse perchè è la più appetita dal bestiame.

Queste piante, quando crescono nella macchia, hanno il Ioro portamento naturale; ma spesso si trovano isolate, ed allora i loro rami inferiori si adagiano a terra ed esse si presentano come densi cespugli cupoliformi, certo per opera della brucatura degli ovini non meno che per effetto del vento. Alcune di queste piante isolate, quasi prive di foglie, e nelle quali affiorano soltanto rami nudi e spine in modo da prendere un 
aspetto quasi erinaceo, sono invase dai licheni e sembrano morenti.

Poche sono le specie esclusive della zona più prossima al mare, di continuo spruzzata dall'acqua salata, come Statice virgata, Crithmurin maritimum, Silene sedoides, Bellium minu-

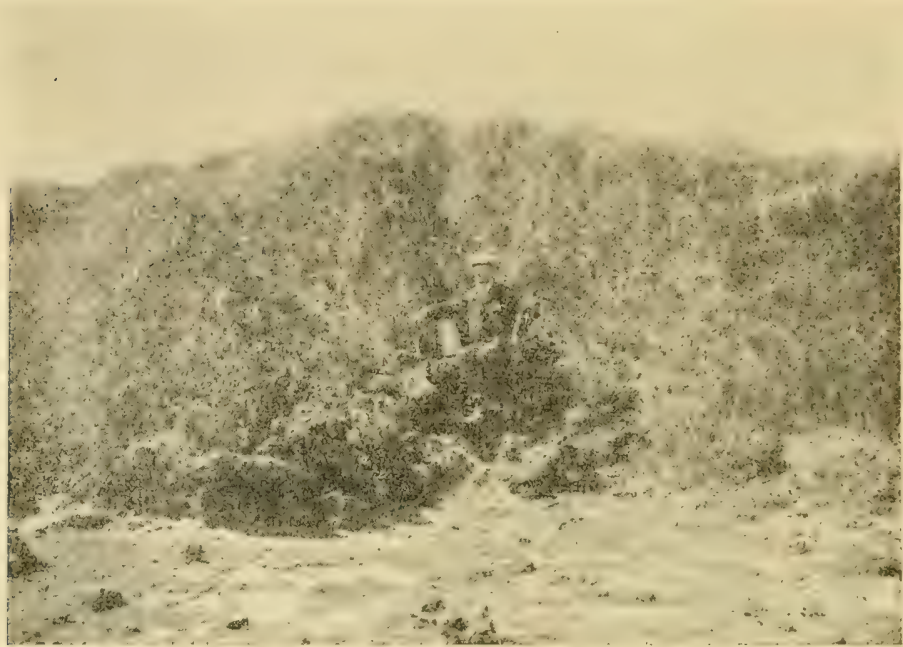

Lycium Europaeum.

(Da una mia fotografia)

tum, Frankenia intermedia e pulverulenta, Mesembryanthemum nodiflorum. Nei luoghi pianeggianti, sabbiosi o rupestri, che in gran parte dell' isola succedono alla zona prettamente mariua, fra i frutici bassi e rari abbondano le microfite e le piante prostrate al suolo quali Plantago Coronopus e Psyllium, Ophioglossum Lusitanicum, Rumex bucephalophoris, Trifolium suffocatum, Sagina maritima, Catapodium loliaceum, Tillaea muscosa, Euphorbia peploides, Echium arenarium, Trigonella maritima, Medicago litoralis, ecc. Ivi spesso il terreno è coperto di epatiche, fra le quali più comuni sono le Fossombronia, le Riccia, la Tessellina pyramidata, la Targionia hypophylla, l'Anthoceros levis, e di molte specie di muschi arenicoli, fra i quali primeggiano la piccola Pottia intermedia che spesso forma da sola dei fitti tappeti riccamente fruttificati, 
anche nei luoghi vicinissimi al mare, la IVeisia viridula essa pure gregaria e formante spesso da sola delle estese colonie, il Trichostomm flavovirens, le Tortula. In alcuni punti vedesi rosseggiare il terreno non per fiori, ma per la colorazione delle foglie del Rumex bucephalophorus e della Tillaea muscosa.

Se abbonda il terreno sabbioso, le vere arene marine sono rare, di piccola estensione e generalmente prive di vegetazione. Solo sulla piccola spiagria detta "Arena bianca », formata in parte da detriti di conchiglie marine e quindi il solo lembo di terreno contenente del carbonato di calce libero che esista in Linosa, si trovano tre piante prettamente psammofile: Euphorbia Paralios, Polygonum maritimum e Pancratium maritimum (non ho ritrovato l'Euphorbia Peplis indicata da Gussone).

Le rupi ombrose e quindi più umide che si trovano negli anfratti delle correnti di lava vicino al mare, e più specialmente quelle delle pendici volte a Nord nell'interno dell' isola, sono rivestite di Parietaria, di Vaillantia, di Callipeltis; vi crescono Grammitis leptophylla, Polypodium vulgare, Asplenium obovatum, Cotyledon horizontalis e pendulinus, Campanuia Erinus, Centranthus Calcitrapa, Sedum litoreum, ecc., e vi prosperano varie epatiche come la Clevea Rousseliana, le Fossombronia, la Lunularia, la Targionia, la Corsinia e molti muschi, fra i quali specialmente abbondanti Entosthodon curvisetus, Fissidens tamarindifolius, Eurhynchium circinatum, varie Funaria, e varî Bryıım.

Nei luoghi più freschi, e sotto la macchia più folta, albergano Geranium Robertianum, Arenaria serpyllifolia, Aslerolinum stellatum, Cardamine hirsuta, Succowia Balearica, Galium spurium, Torilis nodosa, Erodium Chium, Vicia lathyroides e V. leucantha. Nei luoghi rupestri e nella macchia più rada s'incontra Asphodelus tenuifolius e si adagia al suolo la elegante Fumaria bicolor che rifugge dai coltivati prediletti dalle altre specie del genere.

Fra le rupi rivestite di licheni, nei luoghi più denudati ed aridi nascono Thymus capilatues, Satureja microphylla, Notholaena vellea, Picridium Tingitanum.

Nei luoghi coltivati, specialmente nei campi in maggese, lungo i viottoli ed in generale lá dove più si è fatta sentire l' influenza 
dell'uomo, crescono Brassica fruticulosa e Tournefortii, Echium confusum, Calenduta Egyptiaca, Astragalus Baeticus, Solanum Sodomaeum, Fumaria media, Naiva microcarpa, Lavatera Cretica, la vistosa Pinardia Coronaria concolore e discolore, i Trifolicm, ed altre comuni piante arvensi ed antropocore. Parimenti nei campi coltivati si trovano la elegante $\mathrm{Am}$ berboa Lippii ed il bel Lupinus pilosus.

Del resto poco distinte fra loro sono le stazioni, e molte le piante comuni nell' isola che crescono quasi dovunque, dal mare fino alla cima dei monti, sotto la macchia come nei luoghi aprici, quali: Anagallis arvensis, Arisarum vulgare, Stellaria media, Polycarpon tetraphyllum, Geranium molle, Sonchus lenerrimus, Crepis bulbosa, Medicago litoralis, e più specialmente nei luoghi aprici: Silene neglecla, Euphorbia Terracina, Avena barbata, Stipa tortilis, Mesembryanthemum crystallinum, Lotus edulis, la forma nana del Brachypodium distachyum. Dovunque, in alto come in basso, spicca sulle rupi nere il verde glauco delle foglie del Sedum litoreum; e la Scilla maritima, che è sparsa per tutta l' isola, costituisce colle sue larghe foglie una caratteristica del paesaggio. Cosi pure la microflora, di cui ho detto che domina nei terreni sabbiosi piani del basso (salvo nel coltivato), ritrovasi con elementi poco variati più o meno dovunque fino sulla cima dei monti, e vediamo per esempio che l'Ophioglossum Lusitanicum è comune fra i minuti lapilli della cima del Monte Rosso (m. 186) quanto vicino al mare, e che il Trifolium suffocatum forma dei fitti tappeti verdi tanto in alto quanto in basso. Fra quelle microfite trovansi, ridotte nane per le condizioni speciali di vita alle quali sono soggette, anche alcune piante in cui non si suole osservare tale nanismo, come Senecio vulgaris, Silene neglecta, Antirrhinum Orontium, Urtica membranacea, Mercurialis annua. Alcune delle specie che sono rappresentate da individui nani nella microflora, trovansi invece lussureggianti in altri luoghi dove si mantiene più a lungo l'umiditá.

In questi cenni sulia distribuzione delle piante in Linosa, ho riferito quanto ho osserrato quest'anno nel mese di Marzo; ma ben diverso deve presentarsi l'aspetto floristico dell' isola in primavera inoltrata ed in estate. In Agosto ce lo descrive Gussone, 
il quale dice di non avervi potuto riconoscere più di 50 specie di piante, e di avervi trovato spogliati di foglie alcuni frutici per loro natura sempre verdi.

\section{LE PIANTE RACCOLTE A LINOSA.}

Mentre Gussone si trattenne quasi un mese a Lampedusa, visitó Linosa soltanto da mattina a sera il 30 Agosto. A p. 87 delle sue «Notizie» dice di avervi raccolto 50 fanerogame; ma dallo spoglio della «Synopsis » risulta che egli indica per quest' isola, dove fino ai suoi tempi non era stato altro botanico che lui, 88 fanerogame. ${ }^{1}$

Calcara pare vi soggiornasse due o tre giorni. Dal suo «Rapporto » sappiamo che vi giunse l' 8 Giugno; e tutte le etichette sue di Linosa che ho vedute, portano le date del 9 o del 10 Giugno. Egli avverte a p. 20 della sua descrizione dell'isola di Linosa, che nella Florula contrassegna con un * le specie raccolte da Gussone solo, con due ** quelle raccolte da lui stesso e da Gussone, e che tutte le specie senza asterisco sono state raccolte dal solo Calcara. Ne risulterebbe che Calcara non vi raccolse in tutto più di 38 piante, ritrovando 5 sole di quelle raccolte da Gussone, aggiungendone quindi 33 nuove, e portando così il totale a 101. E da notare pertanto che delle 33 specie alle quali non ha messo *, alcune figurano già nella Synopsis, e che Calcara ha dimenticato di riportare nella sua Florula alcune delle specie citate da Gussone. Da ciò risulta che Calcara attribuisce a Gussone soltanto 68 specie, mentre, come ho detto, dallo spoglio della Synopsis si rileva che Gussone ne cita 88. Come si vede, il contributo portato da Calcara alla conoscenza della flora di Linosa è ben meschino, tanto più se si considera che

1 Ho osservato (p. 54 in nota) a proposito delle piante indicate da Gussone nella Synopsis per Lampedusa, che forse alcune non erano state raccolte da lui ma dal barone Porcari. Potrebbe darsi che questo stesso raccoglitore avesse mandato a Gussone anche delle pianto di Linosa e che questa sia la causa della discrepanza fra le indicazioni della « Synopsis» e delle « Notizio». 
egli visitó quest' isola in epoca piủ propizia di Gussone e che avera seco un aiuto. Se il numero di piante da esso aggiunte a quelle di Gussone, benchè esiguo, è pure maggiore di quello che esso aggiunse alla flora di Lampedusa, ció si spiega colla brevità del soggriorno di Gussonie in Linosa.

Ross, nel suo lavoro, cita soltanto 38 delle piante vascolari da esso raccolte in compagnia dei barone Zwierlein, dal 16 al 20 Aprile 1884, ed inoltre due licheni e un' alga.

Lojacono in «Una escursione ecc. » cita 39 specie di Linosa, per la metá circa diverse da quelle indicate da Ross, che dice essergli state tutte comunicate dal barone Zwierlein. Non sempre pero ha indicato come raccoglitore lo Zwierlein. Anzi, in qualche caso cita Calcara, per cui non si puó essere certi che tutte quelle 39 specie siano state veramente raccolte da Zwierlein. Qualche modificazione alle sue prime indicazioni del resto si trova nella Flora Sicula di Lojacono.

Solla fece in Linosa un soggiorno più lungo (dal 21 al 30 Aprile 1884). Egli enumera 125 piante vascolari raccoite da lui stesso, oltre a 12 licheni e 18 alghe.

Zodda e Sturniolo, dal 9 al 16 Aprile 1905, raccolsero in Linosa 130 piante vascolari, oltre alle crittogame cellulari.

Durante il nostro soggiorno dal 21 al 25 Aprile 1873, Ajuti ed io averamo raccolto in Linosa 160 specie vascolari.

In quest'anno (1906) con Riccobono, dal $1^{\circ}$ all' 8 Narzo, abbiamo raccolto 190 specie vascolari, 47 briofite, 12 licheni e 23 alghe.

Il parroco di Linosa, Don Luigi Sangermano, mi ha poi fatto alcuni incii di piante raccolte quest'anno dopo la nostra visita, fra le quaii ho trovato 4 specie non ancora raccolte da altri.

Cosi il totale delle specie vascolari conosciute di Linosa viene portato a 294. '

Per Linosa posso ripetere quello che ho detto per Lampedusa, che cioè alcune specie figurano certamente nella mia Florula

${ }^{2}$ Le specie di Linosa da me studiate, cioè quelle di Zodđa, di Sangermano, e quelle raccolte da me stesso, ammontano a 237. Sono quindi 57 le specie citate in questa Florula da me non viste, e che possono essere state determinate con criterî diversi dai miei. 
sotto due nomi, e che quindi alcuni nomi dovranno passare in sinonimia. Na la diminuzione nel numero di specie che ne risulterá deve esser certo più che compensata dalle specie ancora sfuggite alle ricerche.

Per le citazioni degli autori, nella seguente Florula, ho seguito lo stesso sistema che in quella di Lampedusa (v. p. 57-58). La frequenza e la stazione sono riportate quali io stesso le ho notate quest'anno sul posto.

Apr.! significa raccolto dall'Ajuti e da me nel 1873, fra il 21 e il 25 Aprile. Mrz.! significa raccolto in quest'anno (1906) da Riccobono e da me fra il l.o e l's Marzo. Zodda significa raccolto da questo botanico. in compagnia di Sturniolo fra il 9 e il 16 Aprile 1905 e veduto da me nell'erbario Martelli. Menziono gli altri raccoglitori secondo le indicazioni degli scritti citati per le singole specie; noto però come per le ragioni esposte sopra (p. 157-88), si rimanga talvolta incerti riguardo a Calcara, a Zwierlein ed anche a Gussone.

Dopo Gussone si sottintende 31 Agosto 1828; dopo Calcara 9-10 Giugno 1840; dopo Ross e Zwierlein 16-20 Aprile 1884; dopo Solla 21-30 Aprile 1884. 



\section{FLORULA DI LINOSA}

DICOTYLEDONEAE.

\section{I. - Randnculaceae.}

1. - Clematis cirrhosa L.; Guss. Fl. Sic. Syn. II p. 36 ; Lojac. Fl. Sic. I p. 28.

Qua e lả nella macchia tra i frutici, sulle rupi e sui muri; Marzo frutti, Aprile foglie! - Gussone (il quale nella Synopsis osserva che a Linosa come a Lampedusa perde le foglie in estate), Zodda (foglie), Sangermano (Dicembre fi. e fr. giov.). Calcara ha dimenticato di citare questa pianta per Linosa.

Foglie per la maggior parte intere o trilobe; alcune peró, verso l'estremità dei rami, sono ternate come a Lampedusa.

\section{II. - Papaveraceae.}

2. - Papaver hybridum L.; Calc. Descr. Linosa p. 22 ; Solla Phyt. Beob. p. 472.

Nel coltivato; Apr. fr.! - Calcara lo dice raccolto in Linosa da Gussone; questi peró nella Synopsis l' indica soltanto di Lampedusa. Solla (fro).

3. - Papaver Rhoeas L.; Guss. Syn. II p. 8.

Indicato per Linosa soltanto da Gussone. Calcara ha dimenticato di citarlo.

4. - Papaver setigerum DC. ; Guss. Syn. II p. 8 ; Calc. p. 22. Gussone solo.

5. - Glaucium flavum Crantz. Glarecium luteım Guss. Syn. II p. 5 ; Calc. p. 22.

Gussone. Zodda lo indica fia le piante viste e non raccolte. 
- Glaucium conniculatum (L.) Curt.; Fiori e Paol. Fl. an. I p. 484. G. phoenicerm Lojac. Fl. Sic. I p. 57 .

Lojacono, nella Flora Sicula, cita questa pianta di Linosa sulla autoritá di Calcara; ma questi nella Florula di Linosa non ne fa menzione, e non se ne trovano esemplari negli erbarî di Palermo. Fiori e Paoletti evidentemente non hanno fatto altro che riportare la citazione di Lojacono. Credo quindi di dovere escludere il Glaucium comiculatum da questa Florula.

- Iypecoum procumbens L.; Nicotra Le Fumariacee Italiane p. 22.

Nicotra indica questa pianta come trovata a Linosa dal barone Zwierlein. Siccome peró non la menzionano per quest'isola nè Lojacono che vide le piante raccolte a Linosa dallo Zwierlein, nè Ross che fu di questi compagno, ritengo l'indicazione del Nicotra come dubbia, tanto più che la sua citazione non essendo seguita da un! ció significa che non ne ha reduto esemplari. Per questo non l'ammetto nel novero delle piante di Linosa.

\section{III. - FUMARIACEAE.}

- Fumaria parviflora Lam.; Nicotra Le Fum. It. p. 71.

Nicotra, come raccoglitori di questa specie in Lampedusa e Linosa, cita Lojacono, Zwierlein, Ajuti e Sommier. Ma siccome i due ultimi hanno raccolto questa specie soltanto in Lampedusa, siccome Lojacono non è stato a Linosa, e siccome le piante di Zwierlein furono determinate dal Lojacono il quale non cita Linosa, credo di non dovere per ora includere la $F$. parviflora nella flora di quest' isola.

\section{6. - Fumaria bicolor Somm.}

Abbondante in alcuni punti della macchia e sulle rupi, lontano dai luoghi coltivati; Mrz. fi. e fr. !

Questa specie, in Linosa, si scosta alquanto dal tipo per avere i pedicelli un poco meno lunghi e sottili, talvolta un poco incurvati, la corolla un poco meno gracile ed il calcare per lo più sensibilmente rigonfio; e per questi caratteri si avvicina alla $F$. flabellata. Potrebbe designarsi col nome di: $F$. bicolor forma Linosana. 
Forse alla $F$. bicolor, frequente in Linosa, anzichè alla flabellata che vi è rara, andrebbero riferite le citazioni di Ross, Nicotra e Lojacono.

7. - Fumaria flabellata Gasparr.; Ross Eine bot. Exc. p. 3.16 ; Nicotra Le Fum. It. p. 60 et Altri ragguagli s. Fum. It. p. 8 (in nota). $F$ : pallidiflora Lojac. Una Esc. p. 15?

Nella macchia, rara; Mrz. fi. e fr.! - Ross, Zwierlein? (in Lojacono). Lo stesso Lojacono dice che la pianta raccolta da Zwierlein e da esso chiamata $F$. pallidiflora, è stata determinata per flabellata dall'Ascherson. Nella sua Flora Sic. poi (p.61), cita la $F$. pallicliflora come affine alla sua nuova specie $F$. nemorosa, ma della localitá di Linosa non riparla.

Come ho detto sopra, pare che a Linosa vi siano dei passaggi fra la $F$. bicolor e la $F$. flabellata. In questa peró i sepali si mostrano sempre molto piủ grandi, ed i frutti pure sono più grandi.

- Fumaria capreolata L.; Nicotra Le Fum. It. p. 45. F. pallidiflora Lojac. Esc. p. 15? (nisi species sit antecedens).

Trovo questa specie indicata di Linosa in due varietá, flavescens e speciosa, soltanto nella monografia del Nicotra, ed attribuite ambedue allo Zwierlein. Siccome peró Lojacono, il quale studiò la collezione Zwierlein, indica soltanto Lampedusa per la $F$. speciosa e siccome la F. pallidiflora che egli indica di Linosa sulla fede di Zwierlein è stata dall'Ascherson riferita alla $F$. flabellata, mi pare che l'indicazione di Nicotra il quale non ha visto gli esemplari, sia molto dubbia, e per questo escludo per ora questa specie dalla flora di Linosa.

8. - Fumaria media Lois. F. Gussonei Ross p. 346 ; Nicotra Le Fum. It. p. 53 et Altri ragg. s. Fum. It. p. 7.

Comune nel coltivato; Mrz. fi. e fr., Apr. fi. e fr.! - Ross, Zodda (fi. e fr.), Sangermano (Dic. fi.).

Qui, come in Lampedusa, ho trovato più frequente in Aprile la forma piu scandente, con racemi tutti oltrepassati dalle cime dei rami, con fiori più pallidi, corrispondente alla var. confusa '(Jord.); ed in Marzo invece, ho trovato piủ frequente la var. Gressonei (Boiss.), spesso con fusto corto, quasi semplice ed eretto (forma vernalis). Alla var. Gussonei appartengono pure gli esemplari raccolti in Dicembre da Sangermano. 
- Fumaria agraria Lagasca; Nicotra Le Fum. It. p. 58.

Nicotra cita questa pianta di Linosa sulla fede di Lojacono (Una Esc. p. 15 sub $F$. microsepala); ma l'indicazione di Lojacono si riferisce a Lampedusa e non a Linosa, come ha osservato più tardi lo stesso Nicotra in Altri ragguagli ecc. p. 9.

\section{IV. - CRUCIFERAE.}

9. - Matthiola incana (L.) R. Br.; Car. in Parl. Fl. It. IX p. 796.

Presso l'abitato; 24 Apr. fi. e fr.!

10. - Cardamine hirsuta L.

Nella macchia alle falde del Monte Vulcano, rara; 2 Mrz. fi. e fr.! 11. - Brassica fruticulosa Cyr.; Car. in Parl. Fl. It. IX p. 995.

Abbondante nel coltivato; Mrz. fi. e fr., Apr. fi. e fr.! Zodda.(fi.), Sangermano (Dic. fi. e fr. giov.).

Viene mangiata lessa dagli isolani che la chiamano Rapuddi.

12. - Brassica Tournefortii (iouan; Ross 1. 346; Car. in Parl. Fl. It. IX p. 990 ; Fiori e Paol. Fl. an. I p. 447.

Molto frequente nel coltivato ed anche lontano da questo; Mrz. fi. e fr., Apr. fi. e fr.! - Ross, Zodda (fi. e fr.).

13. - Brassica adpressa (Moench) Boiss.; Car. in Parl. Fl. It. IX p. 1008. Sinapis incana Guss. Syn. II p. 203 ; Calc. p. 22. Trovata soltanto da Gussone.

\section{4. - Eruca sativa Lam.}

Rinasce spontanea vicino ai luoghi dove è stata coltivata; Mrz. fi.!

- Car'richtera annua (L.) Prantl; Fiori e Paol. Fl. all. I p. 442. Carrichtera Vellae Lojac. Esc. p. 16.

Lojacono l' indica di Linosa in Una Esc. bot. ecc., ma nella Flora Sic. la cita soltanto per Lampedusa, per cui rimane as. sai dubbio che egli l'abbia realmente ricevuta di Linosa dal barone Zwierlein, tanto più che non è citata dal dott. Ross, compagno dello Zwierlein. Perció l'escludo dalla numerazione. Fiori e Paoletti ripetono l' indicazione di Lojacono.

15. - Succowia Balearica (L.) Nedic.; Ross p. 346; Solla p. 472 ; Lojac. Esc. p. 16 et Fl. Sic. I p. 91 ; Car. in Parl. Fl. It. IX p. 1015; Fiori e Paol. Fl. an. I p. 441. 
Nella macchia, abbondante; Mrz. fi., Apr. fi. e fr.! - Ross, Solla (fi. e fr.), Zwierlein (in Lojacono), Zodda (fi. e fr.).

16. - Rapistrum rugosum (L.) Ber.s.; Somm. Piante ined. di Lamp. e Lin.

Abbondante nei luoghi coltivati ed incolti; $24 \mathrm{Apr}$. fi. e fr. giovani !

Come gli esemplari di Lampedusa, anche quelli di Linosa appartengono alla forma lejocarpum e si avvicinano alla var. $H i$ spanicum (Boiss. et Reut.) Fiori Fl. an.

17. - Alyssum maritimum (L.) Lam.; Guss. Syn. II p. 165; Calc. p. 22. Koniga maritima Car. in Parl. Fl. It. IX p. 755.

Abbastanza abbondante sui Monte di Levante, ma non visto altrove; Mrz. fi. e fr., Apr. fi. e fr.! - Gussone.

18. - Biscutella lyrata L.; Solla p. 472.

Indicata soltanto da Solla (fi. e fr.).

\section{V. - Capparidaceae.}

19. - Capparis rupestris Smith; Guss. Syn. II p. 3 ; Calc. p. 22. Sulle rupi, rara; Mrz. e Apr. foglie! - Gussone, Calcara.

\section{Vi. - Cistaceae.}

20. - Cistus Monspeliensis L.; Guss. Syn. II p. 13; Calc. p. 22 ; Parl. Fl. It. $\mathrm{V}$ p. 589.

Gussone lo indica di Linosa nella Synopsis; Calcara e Parlatore riportano questa indicazione, ma nessuno dopo Gussone ve l' ha ritrovato, per cui è permesso dubitare di un errore. Ad ogni modo, anche se vi esisteva al tempo di Gussone, doveva essere raro, ed è probabile che oggi sia distrutto. Gli isolani ai quali ne mostrai degli esemplari, mi dissero che non si trovava in Linosa.

\section{Vit. - Frankeniaceae.}

21. - Frankenia levis L.; Guss. Syn. I p. 428 ; Calc. p. 21 ; Lojac. Fl. Sic. I p. 146; Car. in Parl. Fl. It. IX p. 237-38 (pro parte). 
Gussone (var. b. caulibus villosiusculis subvelutinis, calycibus. ad angulos sparse strigoso-pilosis). Calcara e Lojacono l' indicano soltanto sulla fede di Gussone.

22. - Frankenia intermedia DC. F. hirsitc Solla 1) 473. F. levis $\beta$ cinerascens Car. in Parl. Fl. It. IX p. 237-38.

Abbondante sulle rupi e nel terreno arenoso in vicinanza del mare; Mrz. foglie, Apr. fi.! - Solla (fi.).

Nella Flora Italiana (Caruel in Parl.), la localitá di Linosa per la $F$. levis va riferita, per quanto riguarda gli esemplari dell'Ajuti, alla var. cinerascens. Fra le piante osservate e raccolte, ho notato una certa diversità nella pelurie, ma non tale da poterle dividere in $F$. levis tipica e $F$. hirsuta L. E probabile che a Linosa come a Lampedusa le citazioni di $F$. levis siano da riferirsi a $F$. intermedia.

23. - Frankenia pulverulenta L.; Fuss. Syn. I 1. 429 ; Cale. 1. 21; Solla p. 473; Car. in Parl. Fl. It. IX p. 235.

Sulle rupi marine, meno frequente della precedente; Mrz. e Apr. fi..: - Gussone, Solla (fi.), Calcara. Questi ha dimenticato di indicare che era stata raccolta anche da Gussone, come risulta dalla Synopsis.

Sono frequenti gli esemplari esigui (1-2 cm.) e pauciflori, appartenenti alla microflora.

Solla (p. 470) dice delle Frankenia in genere, che nei luoghi più bassi e vicini al mare formano come un feltro che ricuopre il terreno. Ciò però si verifica soltanto in pochi luoghi.

VIII. - CaryophylLaCeaE.

24. - Silene Gallica L.; Solla p. 473; Lojac. Fl. Sic. I p. 151 ?

Campi incolti dal lato di Levante e presso il paese, non comune; Mrz. fi. e fr. ! - Solla (fr.), Zodda (costa di Levante in arvis, fi. e fr.).

Lojacono, Flora Sic., indica come raccolta a Linosa da Zwierlein una S. Gallica var. decumbens. Questa indicazione pertanto non si trova in Una Esc. Ivi per Linosa sono citate soltanto S. nocturna (una forma), e S. neglecta var. diffresa; è dunque probabile che nella Flora Sic. abbia a torto ritenuto per forma della Gallica quella che prima aveva con ragione. riportata alla $S$. neglecta. 
25. - Silene nocturna L.; Lojac. Esc. p. 17 ; Tanf. in Parl. Fl. It. IX p. 365 .

Qua e lá, non molto frequente, più che altro nelle parti coltivate; Mrz. fi. e fr., Apr. fr.! - Zwierlein (in Lojacono, una forma a grossi calici).

26. - Silene neglecta Ten. s. neglectr var. diffusa Lojac. Esc. p. 17 (1884); Ross Sulla Silene neglecta, Naturalista Sicil. XI n. ${ }^{1}$ 6, 7, 8 cum tabula (1892). Silene sp. Ross Eine bot. Excurs. p. 346. S. reflexa Tanf. in Parl. Fl. It. IX p. 362 ; Fiori e Paol. Fl. an. I p. 367. S. reflexa f. divaricata Lojac. Add. et emend. ad Fl. Sic., Malpighia XX p. 184.

Comunissima dovunque nel coltivato ed anche nelle parti incolte dell' isola; Mrz. fi. e fr., Apr. fi. e fr.! - Ross, Zwierlein (in Lojacono), Zodda (fi. e fr.). Lojacono, come ho detto a proposito della S. Gallica, a torto non cita piú la S. neglecta (ossia reflexa) per Linosa nella sua Flora Sicula, però ne fa menzione nelle sue Addenda et emend., dandole questa volta il nome di S. reflexa forma divaricata.

La eccellente monografia del sig. Ross, accompagnata da figura, mi dispensa dall' entrare in maggiori particolari intorno a questa specie. ${ }^{2}$ Aggriungeró soltanto che essa, a Linosa, fornisce un elemento alla microflora, trovandosene in gran copia esemplari nani, ma eretti, nelle arene vulcaniche, ridotti uniflori e alti da uno a pochi centimetri, mentre d'altra parte se ne trovano esemplari alti fino $50 \mathrm{~cm}$. e ramosissimi fino dalla base, in modo da formare dei grossi cesti. Queste forme vigorose, per varî caratteri si avvicinano più alla var. erecta che alla var. diffusa. Ho spesso osservato in Linosa degli esemplari con fiori aperti in pieno giorno, carattere che si riscontra, secondo Ross, soltanto nella var. erecta o in forme di transizione fra le due varietá.

Non si puó negare che la $S$. neglecta sembri un intermediario fra le $S$. Gallica e nocturna, e che in alcuni casi si rimanga in dubbio sulla sua determinazione. Il fatto peró che essa si trova a Linosa in quantita molto maggiore di quelle due specie, parla

- Dalla Silene neglecta ho già parlato io puro diffusamente nella « Florula del Giglio ». 
contro l'ipotesi di una origine ibrida alla quale è pure contrario Ross.

Il dott. Terracciano mi scrive che nell' Erbario Palermitano vi è una Silene con una etichetta su cui è scritto, di pugno di Tineo: « Silene neglecta Ten. $\beta$ glandulosa b. b. divaricata nobis. Linosa 9 Giugno 1846 ». Questa frase di Tineo corrisponde perfettamente alla var. diffusa di Lojacono e di Ross, e dimostra che anche Calcara raccolse questa forma della $S$. neglecta in Linosa, quantunque non l'abbia citata nella Florula di quest'isola. Tineo, come si sa, non fece altro che determinare le piante di Calcara.

27. - Silene sericea Guss. an All.?; Calc. p. 22; Tanf. in Parl. FI. It. IX p. 371.

Indicata soltanto da Calcara come var. angustifolia.

28. - Silene apetala Willd.; Tanf. in Parl. Fl. It. IX p: 372; Arc. Comp. Fl. It. $2^{\text {a }}$ ed. p. 309; Fiori e Paol. Fl. an. I p. 366 ; Lanza Note sulla flora di Sic. in Boll. R. Orto bot. di Palermo IV 1905 p. 31.

Ajuti ed io l'abbiamo trovata in pochi esemplari nel terreno arenoso il 24 Aprile in frutto! - Il dott. Lanza ha recentemente riconosciuto questa specie fra le piante raccolte da Calcara in Linosa, e rimasta indeterminata nell' erbario di Palermo. Il cartellino che l'accompagna porta scritto di pugno di Tineo « Silene sp. Linosa, Giugno 1846 ». Gli esemplari raccolti da Calcara hanno i fiori avvicinati in gruppetti all'apice dei rami e devono quindi riferirsi alla var. congesta Godr. (Rohrbach, Monogr. Sil.), alla quale si arvicinano anche i nostri.

É l'unica località nota per questa specie in Italia. In Europa era stata trovata solamente in Spagna.

29. - Silene sedoides Poir.; Calc. p. 22; Tanf. in Parl. Fl. It. IX p. 401.

Frequente sulle rupi prossime al mare; Mrz. primi fi., Apr. fi. e fr.! - Calcara, Zodda (fi. e fr'.).

Tanfani 1.c. cita come raccoglitore Tineo il quale peró non fu mai a Linosa nè a Lampedusa, ma determinò e distribui le piante raccolte da Calcara.

30. - Silene inflata Smith; Guss. Syn. I p. 486; Solla p. 473. S. Behen b. angrestifolia Guss. Suppl. Prodr. p. 124. S. Cucubalus Tanf. in Parl. Fl. It. IX p. 345. Silene sp. Solla p. 473? 
Sulla costa di Tramontana rara, 4 Mrz. foglie! - Gussone, Solla (fi.). Calcara ha dimenticato di citare questa specie. Perde le foglie in estate secondo Gussone.

Solla, oltre alla $S$. inflata, cita una Silene sp. che dice affine alla marilima, e che sara forse una varieta delia S. inflata.

Il dott. 'Terracciano ini avverte che nell' Erbario di Palermo trovasi un esemplare di Silene con etichetta su cui sta scritto «S. anmulata Linosa » dello scritto di Todaro, senza data nè nome di raccoglitore. Na dietro questa semplice indicazione non credo di dover ammettere la presenza in Linosa della Silene Cretica L.. (= S. anmulata Thore), essendochè Terracciano non conferma l'identità della specie, e Lojacono, il quale ha spogliato tutto l'Erbario di Palermo, non ne fa menzione per quest' isola.

\section{1. - Silene Behen L.}

Sulla costa di Levante e verso il Faro; Mrz. fi., Apr. fr.!

I pochi esemplari che abbiamo raccolti con Riccobono in Marzo, appartengono ad una forma ubertosa, con foglie fiorali inferiori lunghe $5 \mathrm{~cm}$. e larghe 2 ; quelle inferiori del fusto raggiungono $7 \mathrm{~cm}$. di lunghezza e $2 \%$ di larghezza.

\section{2. - Cerastium glomeratum Thuill.}

In varî luoghi, specialmente nella macchia in alto, come sul M. Rosso, M. Vulcano e M. di Ponente; Mrz. fi. e fr. !

Trovasi in Linosa tanto la forma apetala quanto quella con petali quasi il doppio del calice. S' incontra tanto nano quanto ben sviluppato. Gli esemplari nani di solito appartengono alla forma apetala.

\section{3. - Cerastium semidecandrum L.}

Frequente tra le microfite in terreno arenoso, specialmente in alto, come sul M. Rosso e sul M. Vulcano; Mrz. fi. e fr.! Zodda (M. Vulcano fi. e fr.).

Trovasi raramente alto fino a $\$-9 \mathrm{~cm}$. e multifloro; per lo più è nano, talvolta alto appena $1 \mathrm{~cm}$. e unilloro.

34. - Stellaria media (L.) Cyr.; Guss. Syn. I p. 494; Calc. p. 22 ; Solla p. 473 ; Tanf. in Parl. FI. It. IX p. 525.

Comunissima tanto nel coltivato quanto nella macchia; Mrz. fi. e fr., Apr. fi. e fr.! - Gussone (var. a. e var. b.), Solla (fi. e fr.), Zodda (fi. e fr.), Sangermano (Dic. fi. e fr.). 
É più comune in Linosa la forma apetala (Ucria), ma non mancano le forme con petali uguaglianti la metá del calice, o lunghi quasi quanto esso. Alla f. apetala appartengono gli esemplari raccolti in Dicembre da Sangermano.

35. - Arenaria serpyllifolia L.; Solla p. 473; Tanf. in Parl. Fl. It. IX p. 549. Alsine leptoclados Calc. p. 22. Arenaria leptoclados Lojac. Fl. Sic. I p. 175.

In varî luoghi, abbastanza frequente specialmente nella macchia; Mrz. fi. e fr., A pr. fi. e fr.! - Calcara, Solla (fi.), Zodda (fi. e $f^{2}$.).

Tutta l'A. serpyllifolia che ho veduta di Linosa appartiene ad una forma glandoloso-vischiosa della var. leptoclados, corrispondente alla $A$. serpyllifolia $\beta$ tenuior Koch b. minutiflora (Loscos) Fiori e Paol. Fl. an. I p. 345. Spesso è ridotta nana (1 cm.) ed uniflora.

36. - Alsine procumbens (Vahl.) Fenzl ; (russ. Syn. I p. 497; Calc. p. 22 ; Tanf. in Parl. Fl. It. IX p. 608. Arenaria procumbens Guss. Suppl. Prodr. p. 128.

Solo Gussone.

37. - Sagina apetala L.; Solla p. 473; Tanf. in Parl. Fl. It. IX p. 570.

Frequente, specialmente sotto la macchia fra le microfite; Mrz. fi. e fr. ! - Solla (fi.).

La pianta di Linosa come quella di Lampedusa è di solito (ma non sempre) glabra, senza ciglia alla base delle foglie, ed ha i sepali appressati alla capsula; corrisponde quindi alla S. Lamyi F. Schultz in Rouy et Fouc. Fl. Fr.

38. - Sagina maritima Don; Tanf. in Parl. Fl. It. IX p. 568.

Abbondante sulle rupi e nelle spianate arenose vicino al mare, formando talvolta da sola dei fitti e bassi tappeti; Mrz. fi. e fr., Apr. fi. e fr.!

39. - Spergula pentandra L.; Somm. Piante ined. S. arvensis Tanf. in Parl. Fl. It. IX p. 612.

Luoghi incolti verso il centro dell'isola e sotto il M. di Ponente: Mrz. fi. e fr., Apr. fr.! - Zodda (presso l'abitato, in arenis rulcanicis, $\mathrm{fl}^{\mathrm{P}}$.).

Tanfani 1. c. indica a torto la spergula arvensis di Linosa, poichè gli esemplari dell'Ajuti conservati al Museo Fiorentino, appartengono, come i miei, alla S. pentandra. 
LINOSA - CARYOPHYLL., PORTUL., PARONYCH.

40. - Spergularia rubra (L.) Pers.; Tanf. in Parl. Fl. It. IX p. 617. Arenaria rubra Guss. Suppl. Prodr. p. 129. Alsine rubra Guss. Syn. I p. 501 ; Calc. p. 22. Lepigonum rubrum Lojac. Esc. p. 18.

Qua e lả nei luoghi coltivati e incolti; Mrz. fi. e fr., Apr. fi. e fr. ! - Gussone, Zwierlein (sec. Lojacono).

Trorasi sotto due forme assai diverse: una prostrata, a internodî raccorciati, a fiori brevemente peduncolati e ravvicinati in cime brevi, corrisponde \pm alla var. campestris Fenzl in Ledeb. Fl. Ross; l'altra eretta, a internodî e foglie allungate, a cime lasse oltrepassanti lungamente le foglie, a fiori più grandi ed a peduncoli inferiori $2-3$ volte più lunghi dei calici, corrisponde press' a poco alla var. pinguis Fenzl I. c. Non mancano i passaggi fra l'una e l'altra.

41. - Spergularia media (L.) Per's. Alsine heterosperma Guss. Syn. I p. 501 ; Calc. p. 22. S. salina Lojac. Fl. Sic. I p. 184; Tanf. in Parl. Fl. It. IX p. 621.

Vicino al màre presso lo Scalo e presso il Faro, rara; Mrz. fi. e primi fr.! - Gussone, Calcara il quale dimentica l'* per indicare che era stata gia trovata in Linosa da Gussone.

Nelle poche capsule mature che ho trovate, i semi sono per la maggior parte alati; ma le piante sono troppo poco sviluppate per riconoscere se appartengono alla stessa forma di quelle di Lampedusa.

\section{ix. - Portulacaceae.}

\section{2. - Portulaca oleracea L.}

Ricevuta dal parroco di Linosa, Sangermano, raccolta in frutto, senza indicazione di data.

\section{X. - Paronychiaceae.}

43. - Herniaria cinerea DC. ; Güss. Syn. I p. 292; Calc. p. 21. Gussone. Zodda scrive di averla veduta a Linosa, ma non raccolta.

44, - Loeflingia Hispanica L. ; Calc. p. 20 ; Bert. Fl. It. VII p. 611 ; Tanf. in Parl. Fl. It. IX p. 629; Fiori e Paol. Fl. an. I p. 336. 
Trovata da Calcara solo. La citazione di Bertoloni «da Tineo » si riferisce evidentemente ad esemplari raccolti da Calcara e distribuiti da Tineo. Esemplari raccolti a Linosa da Calcara, il 10 Luglio 1846, trovansi nell' Erbario di Palermo. Esemplari di Linosa distribuiti da Tineo si trovano anche al Museo di Firenze.

45. - Polycarpon tetraphyllum (L.) L. ; cruss. Syn. I p. 166 ; Calc. p. 21 ; Solla p. 475 ; Tanf. in Parl. Fl. It. IX p. 625.

Nolto comune nelle parti coltivate, come pure lontano da esse; Mrz. fi. e fr., Apr. fi. e fr.! - Gussone, Calcara (il quale dimentica di mettere il segno * per indicare essere stato raccolto anche da Gussone), Solla (fi.), Zodda (fi. e fr.).

Il Polycarpon di Linosa, al pari di quelli di Lampedusa, si avvicina spesso alla varieta alsinefolium. Trovasi anche nano.

\section{XI. - Malvaceae.}

46. - Malva sylvestris L. Mr. silvestris B erecta Fiori e Paol. Fl. an. II p. 267.

Zodda (ad vias, fi.).

L' indicazione della Flora analitica mi era sembrata dubbia perchè non avero trovato la $M$. sylvestris indicata di Linosa da alcun racogglitore. Ora peró.la sua presenza in quest' isola è accertata da Zoddạ. L'esemplare di Zodda nella collezione Martelli è troppo giovane per poterlo riferire con sicurezza alla varietá erecta.

47. - Maiva microcarpa Desf.; Solla p. 473. M. flexiesa var. canescens Calc. p. 23. M. flexuosa Lojac. Esc. p. 18. M. parviflora Ross p. 346.

Molto comune lungo i viottoli e nelle parti coltivate; Mrz. fi. e. fr. giov., Apr. fi. e fr.! - Calcara, Ross, Solla (fi. e fr.), Zwierlein (sec. Lojacono), Zodda (fi. e fr.).

Le piante che ho raccolte a Linosa differiscono da quelle di Lampedusa per essere più spesso erette, per avere i fiori in generale meno numerosi all'ascella delle foglie, i peduncoli più allungati, i calici fruttiferi più patenti e talvolta un poco colorati, i carpelli notevolmente più piccoli (poco più di $1 \mathrm{~mm}$. di diametro) e meno rugosi. Hanno ugualmente i peli a fascetti e 
la radice annua. Solla riferisce le piante da lui trovate a Linosa alla M. microcarpa, e quelle di Lampedusa alla M. parviflora. Non ostante le differenze suaccennate, non credo di dovere ritenere la pianta di Linosa come specificamente distinta da quella di Lampedusa. Ad ogni modo si avvicina più di quella di Lampedusa alla 1 . parviflora.

48. - Lavatera Cretica L. L. sylvestris Calc. p. 23 ; Lojac. Fl. Sic. I p. 198.

Presso l'abitato, non rara; Mrz. fi. e fr. giov.! - Calcara, Zodda (fi.). Secondo Lojacono Flor. Sic. sarebbe stata raccolta anche da Zwierlein; peró in Una Esc., non ne aveva fatto menzione.

\section{9. - Lavatera arborea L.}

Presso l'abitato; 24 Apr. fi. e fr.! - Zodda (presso l'abitato, ad sepes valde rara, fí.).

La stazione presso l'abitato, e la raritå di questa bella specie, frequentemente coltivata come pianta ornamentale anche da pescatori e contadini, fa dubitare che sia soltanto sfuggita dalle colture e non veramente inselvatichita.

\section{XiI. - Geraniaceae.}

50. - Geranium molle L.; Guss. Syn. II p. 216; Calc. p. 23; Ross p. 346 ; Solla p. 473; Parl. Fl. It. V p. 183.

Dovunque comunissimo, tanto nelle parti coltivate quanto nella macchia; Mrz. fi. e fr., Apr. fi. e fr.! - Gussone, Ross, Solla (fi.), Zodda (fi. e fr.), Sangermano (Dic. fi.).

51. - Ceranium rotundifolium L.; Solla p. 473.

Qua e lá nella macchia, piuttosto raro; Mrrz. fi., Apr. fi. e fr.! - Solla (fi.), Sangermano (Dic. fi.).

\section{2. - Geranium Robertianum L.}

Frequente nei luoghi ombrosi; Mrz. fi. e fr. giov.! - Zodda (fi. e ir.).

In tutte le piante di Linosa da me vedute i carpelli sono glabri come in quelle di Lampedusa.

53. - Erodium cicutarium (L.) L' Hẻrit.; Calc. p. 23 ; Solla p. 473 ; Lojac. Esc. p. 18.

Comunissimo in tutta l'isola, specialmente nelle sue parti in. 
colte; Mrz. fi. e fr., Apr. fi. e fr.! - Calcara, Solla (fi. e fr.), Zwierlein (sec. Lojacono), Zodda (fi. e fr.).

L'E. cicutarium si presenta a Linosa sotto aspetti assai diversi per la statura che giunge fino a $40 \mathrm{~cm}$., per la divisione delle foglie, per la lunghezza dei pedicelli, per la radice or'a sottile ed ora formante un lungo fittone legnoso in apparenza perennante, in modo che si potrebbe riferire a direrse delle innumerevoli forme che sono state distinte in questa specie, in generale peró a quelle del ciclo pimpinellifolium (nel senso di Rouy et Fouc. Fl. Fr.). E frequente fra le microfite una forma acaule, nana (talvolta alta solo $2-3 \mathrm{~cm}$.), con peduncoli radicali ed uni-biflori, che corrisponde alla var. praecox (Willd.) DC.

Nel 1873 Ajuti ed io abbiamo raccolto alcuni esemplari di una forma anomala di questa specie, che ho ritrovata pure fra le piante raccolte da Zodda (fi. fr.). Essa è notevole per avere nella maggior parte delle ombrelle i fiori tanto breremente pedicellati che formano un capolino compatto in cima al peduncolo. Questa anomalia sembra dovuta a infezione fungina o a puntura d'insetti. É strano però che sia stata trovata identica a 32 anni di distanza, da noi e da Zodda. In quest'anno non l'ho veduta.

54. - Erodium Chium (Burm.) Willd.; Somm. Piante ined.

Non raro nelle radure della macchia; Mrz. fi. e fr., Apr. fi. e fr. ! - Zodda (fi. e fr.).

Fra le piante di Linosa ve ne sono con foglie larghe 4 e lunghe $5 \mathrm{~cm}$. (var. platyphyllum Rouy et Fouc. Fl. Fr. IV p. 119), ed altre con foglie assai più piccole (le inferiori da 1 a $1 \%$ più divise e meno distintamente cordate alla base (var. microphyllum Rouy et Fouc. 1.c. p. 120). Tanto l'una quanto l'altra forma presentano esemplari microflorati, alti $5-6 \mathrm{~cm}$., con peduncoli uni-biflori. Le forme precoci ridotte hanno petali lunghi il doppio del calice. Nessuna delle forme di Linosa ha peli glandolosi; tutte peró in alto, specialmente sulla faccia inferiore delle foglie giovani, hanno delle glandolette sessili.

55, - Erodium laciniatum (Cavan.) Willd. val. grandiflorum mihi.

Petalis calyce duplo longioribus.

Nelle radure della macchia, alla base del $\mathrm{m}$. Vulcano, sopra la Pozzolana di Levante; 3 Mrz. fi. e fr.! 
Le piante da me raccolte appartengono ad una forma precoce ridotta (alta $5-10 \mathrm{~cm}$.), a fusto eretto poco ramoso, ed a fiori noteroli per la loro grandezza, essendo i petali lunghi il doppio del calice (8-9 mm.). Hanno le prime foglie radicali superficiảlmente lobate, con lobi crenulati, le successive pennatofesse o pennatopartite, e le cauline profondamente pennatopartite, con partizioni acute ed acutamente dentate, munite sulle due faccie di peli corti ed applicati. I pedicelli sono glabri o pubescenti per peli non glandolosi. Il rostro è lungo 40-45 $\mathrm{mm}$.

56. - Erodium angulatum Pomel Nouv. matér. pour la Fl. Atlantique, 1875, p. 341 var. Linosae mihi.

Radice annua, caulibus ascendentibus hispidulis $25 \mathrm{~cm}$. usque altis, foliis pilis brevibus adpressis plus minus pubescentibus glandulisque sessilibus abunde conspersis ambitu ovato-oblongis basi cordatis pinnatifidis vel pinnatipartitis, partitionibus obtusis obtuse crenato-lobatis, stipulis mediocribus praeter supremas abbreviatas orato-triangularibus acutiusculis, pedunculis subquinquefloris (variant $1-7$ flori) cum pedicellis et calycibus pubescentibus interdum glanduloso-pubescentibus, sepalis $4-5 \mathrm{~mm}$. longis mucrone brevissimo apice setigero terminatis, petalis violaceis calycem aequantibus vel parum excedentibus, filamentis glabris, valvarum foveolis parvis eglandulosis plica concentrica angusta auctis, earum cauda $30-35 \mathrm{~mm}$. longa jam a basi intus longe pilosa et contorta.

Species fere intermedia inter $E$. malacoidem W. et $E$. laciniatum IV. Propius accedit ad E. malacoidem a quo primo intuitu foliorum partitione distinguitur; ab eo praeterea differt fovea valvarum eglandulosa minore, plica concentrica minus conspicua et rostro longiore. Ab E. laciniato differt foliis glanduloso-punctatis non dimorphis, superioribus minus profunde partitis eorum partitionibus lobulisque obtusis, valvarum foveis plica concentrica donatis, earum cauda breviore basi crassiore et jam a basi spiraliter contorta et pilis longis barbata, sepalorum mucrone breviore, pedunculis et pedicellis densius pubescentibus, stipulis minus latis. Forma E. laciniati quae in Linosa adest praeterea magnitudine florum eximie differt.

Frequente nelle radure della macchia, sulle rupi ed anche nei luoghi arenosi ; 2, 3, 4, 5 e $6 \mathrm{Mrz}$. fi. e fr., 23 e $24 \mathrm{Apr}$. fr.! 
Fra le microfite trovasi, come avviene per molti Erodium, anche ridotto unilloro e alto $2-3 \mathrm{~cm}$. In questi esemplari nani, le foglie sono grandemente ridotte, ma i fiori e i frutti sono di dimensioni normali.

Questo Erodium, quantunque in Linosa varî alquanto per la statura, la pubescenza, la dimensione e la divisione più o meno profonda delle foglie, pure non presenta alcuna forma di passaggio alle due specie affini che si trovano nell'isola.

Per distinguerla da queste basterebbe un solo carpello. Difatti nell' $E$. laciniaurm (come nel Chium) la fossetta del carpello è superficiale e priva affatto di piega concentrica, e la sua codetta in basso è sottile, diritta e poco pelosa, mentre nella nostra pianta (come nell' E. malacoides) la codetta fin dalla sua base è meno sottile, munita all' interno di lunghi peli ed avvolta in spira. Dal carpello del malacoides quello dell'angulatım differisce per la fossetta senza glandole, più piccola, circondata da una cresta meno rilevata, e munita di una piega concentrica meno cospicua, ed inoltre per la codetta più lunga. I carpelli della nostra pianta, anzichè con quelli di queste due specie, potrebbero più facilmente confondersi con quelli dell' E. cicularium ai quali molto assomigliano.

Riferisco la pianta di Linosa come varietá all' $E$. angulatum di Pomel per la descrizione (troppo breve) che ne danno questo autore e Battandier e Trabut Fl. d'Algẻrie I, p. 128, e per lopinione dei sigg. Battandier e Bonnet ai quali ho mandato la pianta di Linosa con preghiera di confrontarla negli erbarî di Algeri e di Parigi coi tipi di Pomel. La var. Linosae, a quanto pare, differisce dal tipo per la gracilitå di tutta la pianta, per la pubescenza minore, e per la divisione più profonda delle foglie, presentando del resto tutte le principali caratteristiche della specie.

Avevo dubitato un momento che la pianta di Linosa fosse l'E. Subtrilobum di Jordan. Ma questo dubbio svani leggendo la descrizione originale di Jordan (Pugill. plant. nov. praesertim Gallicar. 1852 p. 42) dove è detto che nell' E. subtrilobum soltanto le foglie radicali sono profondamente lobate, e che il becco del frutto è lungo non piủ di $20-22 \mathrm{~mm}$. Del resto il sig. Bonnet che ha avuto la compiacenza di confrontare la mia pianta di 
Linosa col tipo di Jordan negli Erbarî di Parigi, mi dice che è del tutto diversa da questo.

É probabile che l'E. anguclatum, indicato fin ora soltanto di Algeria, si trovi pure in Tunisia. Fra gli exsiccata di Kralik dell'Erbario Centrale di Firenze ve n'è uno (Pl. Tunetanae n. 19 ad muros umbrosos Gabes) che, per quanto lo stato infelice dell'esemplare permette di gindicare, dere riferirsi a questa specie.

Sospetto pure che $I^{\circ} E$. angulatum esista in Spagna e vi sia stato confuso coll'E. subtrilobum Jord:

\section{XIII. - LinaCEAE.}

57. - Linum angustifolium Huds.?; Solla p. 473.

Solla solo (fi.).

Solla cita questa specie dubitativamente. Io ho raccolto soltanto degli esemplari subspontanei di Linum usitatissimum che vien coltivato qualche volta dai contadini.

\section{XIV. - ZyGOPHYLLACEAE.}

58. - Tribulus terrestris L.; Somm. Piant. ined.

Nel coltivato; Mrz. frutti secchi dell'anno avanti, Apr. fi. e fr.!

$$
\text { XV. - Rutaceae. }
$$

59. - Ruta bracteosa DC.; Solla p. 473; Lojac. Esc. p. 18 ; Parl. Fl. It. V p. 356.

Frequente nella macchia e sulle rupi; Mrz. fi., Apr. fi. e fr.! Solla (fi. e.fr.), Zwierlein (sec. Lojacono).

\section{XVI. - ANacardiaceae.}

60. - Pistacia Lentiscus L.; Guss. Notiz. p. 86 et Syn. II p. 627 ; Calc. p. 23 et 24; Ross p. 345 ; Solla p. 473 ; Parl. Fl. It. V p. 379 ; Lojac. Fl. Sic. I pars II p. 15.

Comunissima in tutta l' isola dove è l'elemento costituente principale della macchia; Mrz. foglie, Apr. fi. ! - Gussone, Calcara, Ross, Solla (fi. e fr.). 
Se ne trovano ancora sul M. Vulcano, in luogo riparato, delle piante annose ed alte, che formano quasi bosco. Gli esemplari isolati prendono aspetto cupoliforme per effetto dei venti e degli animali che ne mangiano le foglie.

61. - Rhus dioica Brouss.; Calc. p. 21 ; Parl. Fl. It. V p. 391; Lojac. Fl. Sic. I pars II p. 14. R. oxyacantha Fiori e Bẻg. Fl. an. II p. 226.

Sui colli nella macchia, abbastanza frequente; Mrz. fr., Apr. fr.! - Calcara (var. pubernela), Zodda (frr.). Lojacono Fl. Sic. cita Solla fra quelli che hanno raccolto in Linosa il $R$. dioica. Solla indica invece $R$. pentaphylla.

62. - Rhus pentaphylla Desf.; Guss. Srrı. I p. 362 ; Calc. p. 21 ; Solla p. 473 ; Parl. Fl. It. V p. 393; Fiori e Bẻg. Fl. an. II p. 226. R. Thezera Guss. Notiz. p. 78 et Suppl. Prodr. p. 85.

Gussone (il quale nell'Agosto lo trovò spoglio di foglie per la grande siccitá), Solla (fr.).

$\dot{\mathrm{E}}$ da notare come chi dice di avere raccolto in Linosa il Rhus pentapluylla non indica il $R$. dioica, e viceversa. Ció induce a credere che a Linosa esista una sola di queste specie. Tanto nel 1873 quanto in quest'anno ho visto e raccolto in copia il $R$. dioica benissimo caratterizzato, con foglie tutte trifoliolate, e non ho veduto alcuna pianta di $R$. pentainhylla, nè forme di passaggio a questa specie. Non farebbe meraviglia che Gussone fosse stato indotto in èrrore indicando il $R$. pentaphylla, perchè trovó i Rhus di Linosa completamente privi di foglie, e quindi della caratteristica specifica principale. E non farebbe neppure meraviglia che Solla, nel determinare le sue piante, fosse stato indotto in errore dall'autorità di Gussone. Se cosi fosse, si dovrebbe cancellare dalla Fl. di Linosa il $R$. pentaphylla.

\section{XVII. - Papilionaceae.}

63. - Anagyris foetida L.; Guss. Syn. I p. 460 ; Calc. p. 22; Tanf. in Parl. Fl. It. X p. 10r.

Verso il centro dell' isola raro; 3 Mrz. fi., Apr. fr.! - Gussone. 64. - Lupinus angustifolius L.; Solla p. 474; Tanf. in Parl. Fl. It. I p. 109.

Terreni incolti; 24 Apr. fr.! - Solla (fi. e fr.). 
Per le foglioline piegate in due, per la larghezza dei legumi (8-9 mm.), per le dimensioni dei semi (4 per $5 \mathrm{~mm}$.) e per essere questi giallo-grigiastri con reticolatura rosso scura, i miei esemplari appartengono alla var. linifolizes (Roth) $\beta$ reticulatus. (Desv.) quale è descritto in Rouy et Fouc. Fl. de France IV p. 194 ed in Fiori e Bẻg. Fl. an. II p. 10. Hanno le radici abbondantemente provviste di tubercoli radicali.

65. - Lupinus Termis Forsk.

Qua e lá non raro nei campi incolti; Mrz. fi. e fr. giov.!

Questo Lupino, che viene coltivato, sembra adesso essersi naturalizzato nell' isola.

66. - Lupinus pilosus Murr.; Solla p. 475; Lojac. Fl. Sic. I par's II p. 34; Fiori e Bẻg. Fl. an. II p. 11. L. varius Tanf. in Parl. Fl. It. X p. 113.

Abbondante nei campi in riposo di cui è un ornamento; Mrz. fi. e fr. giov., Apr. fi. e fr.! - Solla (fi. e fr.), Zodda (fi.).

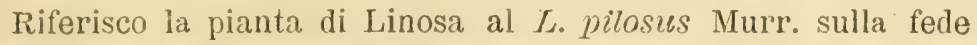
di Solla, come hanno fatto Lojacono e Fiori; ma senza frutti maturi come sono gli esemplari miei e quelli di Zodda, non saprei distinguerli dal $L$. Cosentini Guss. (che del resto ne è considerato come un puro sinonimo da Tanfani in Parl.). - Anche questa specie è provvista di tubercoli radicali.

67. - Ononis mollis Savi; Somm. Piante ined.

Frequente nell' isola, specialmente nelle radure della macchia; Mrz. bocci, Apr. fr. ed ancora qualche fi.! - Zodda (fi. e fr.).

Frequenti sono qui, come a Lampedusa, le forme nane; alte anche soli $2 \mathrm{~cm}$., e con pochissimi fiori.

68. - Ononis serrata Forsk.; Solla p. 474.

In varî luoghi, piủ che altro nei campi abbandonati; Mrz. fi., Apr. fi. e fr.! - Solla (fi. e fr.) ; Zodda (fi. e fr.).

69. - Ononis diffusa 'Ten.; Lojac. Esc. p. 18 et Fl. Sic. I par's II p. 43. O. serrata $\beta$ major Ross p. 347.

Più frequente della precedente; Mrz. foglie, Apr. fi. e fr.! Ross, Zwierlein (sec. Lojacono), Zodda (fi. e fr.).

70. - Trigonella Monspeliaca L.; Solla p. 174; Lojac. Fl. Sic.

I pars II p. 71 .

Non comune, verso il Faro e alle falde del M. Vulcano; Mrz. fi. e fr. giov. ! - Solla (fi. e fr.), Zwierlein (sec. Lojacono). 
71. - Trigonella maritima Delile; Ross p. 347. T. maritima $\beta$ derra Solla p. 474 .

Sulle rupi e nei luoghi arenosi vicini al mare, molto frequente; Mrz. fi., Apr. fi.e e fr.! - Ross, Solla (fi.), Zodda (fi. e fr.).

Solla riferisce la pianta di Linosa alla var. dirra Vis., e quella di Lampedusa al tipo. Fra i miei esemplari di Linosa e quelli di Lampedusa non so scorgere alcuna differenza.

72. - Medicago secundiflora Dur.; Somm. Piante ined.

Luoghi arenosi aridi, salendo al Monte Rosso dal versante meridionale, ivi abbastanza abbondante; 23 Apr. fi. e fr.!

Questa specie, comune sulle coste settentrionali d'Africa, non era nota per l' Europa quando la trovammo in Linosa nel 1873. E stata segnalata di poi, sempre in piccole isolette, nel mezzo. giorno della Francia, e all' isolotto di Port' Ercole presso il Monte Argentaro.

\section{3. - Medicago Helix Willd. ?}

Campi incolti non lungi dal paese, Mrz. fi.!

Non sono sicuro della determinazione, per la mancanza di frutti; ma è certamente una delle specie raggruppate da Fiori Fl. an. sotto la denominazione complessiva di $M$. obscura.

\section{4. - Medicago tribuloides Desr.}

Zodda solo (in incultis fi.e fr.).

Le piante raccolte da Zodda sono da riferirsi piuttosto alla forma crassispina. (Viv.) Fiori Fl. an. II p. 36.

75. - Medicago litoralis Rhode; Ross p. 347 ; Lojac. Esc. p. 19 et Fl. Sic. I pars II p. 59. M. striata Solla p. 474.

Comunissima in tutta l' isola, tanto nelle sue parti coltivate quanto lontano da esse; Mrr. fi. e fr. giov., Apr. fi. e fr.! Ross, Solla (fi.), Zwierlein (sec. Lojacono), Zodda (fi. e fr.).

Lojacono, nella Flora Sicula, cita di Linosa una M. striata var. major dell' Erbario di Palermo, senza dire da chi fu raccolta. - In quanto alla $M$. striata del Solla, credo di dorerla riferire alla $M$. litoralis sensu lato. - Il dott. Ross attribuisce la sua $M$. litoralis di Linosa alla var. brevisela DC.

Le piante che ho raccolte presentano forme molto varie e pelurie molto differenti. Alcune hanno legumi maturi più larghi che lunghi (diametro $4 \mathrm{~mm}$.), con aculei lunghi 1 e $2 \mathrm{~mm}$., che potrebbero riferirsi alla var. arenaria (Ten.). Altre hanno 
legumi con gli stessi caratteri salvo che la loro altezza è uguale alla loro larghezza. Altre ancora hanno frutti assai piccoli, $3 \mathrm{~mm}$. di diametro, con lunghezza che varia da 3 a $5 \mathrm{~mm}$. e spine brevissime o anche ridotte a semplici tubercoli. Queste varie forme oscillano fra le varietà depressa Urb., cylindracea (DC.) e presilla (Viv.), avvicinandosi più spesso a quest'ultima. Gli esemplari di Zodda in parte si avvicinano alla var. cylindracea (DC.), in parte alla var. tricycla (DC.).

Tutte queste forme presentano fra di loro tanti passaggi, che riesce impossibile tenerle separate le une dalle altre. Sono in parte destrorse ed in parte sinistrorse.

76. - Medicago denticulata Willd.; Solla p. 474.

Solla (fi. e fr.), Zodda (presso l'abitato, in aridis, fi. e fr.).

Gli esemplari di Zodda appartengono alla var. lappacea (Desr.). Solla non ha indicato a quale varietá appartenessero i suoi.

77. - Medicago minima Grufb. in L.; Solla p. 474.

In diversi luoghi del piano e dei colli ; Mrz. fi. e fr. giov., Apr. fi. e fr. ! - Solla (fi.), Zodda (fi. e fr..).

Le piante di Linosa hanno per lo più spine lunghe almeno quanto il diametro del giro medio, ed appartengono quindi alla varietá longiseta $\mathrm{DC}$., che del resto è la sola indicata di Sicilia (Guss., Lojac.); ed essendo grigio-tomentosa, va riferita alla forma mollissima (Roth.). Si trova peró in Linosa anche una forma con spine un poco più corte e legumi più piccoli, che si avvicina alla var. canescens Ser. in DC.

78. - Melilotus Neapolitanus Ten.; Solla p. 474.

Solla solo (fi.).

79. - Melilotus elegans Salzm.; Somm. Piante ined.

Frequente; fi. e fr. giov.; Apr.!

80. - Melilotus sulcatus Desf.; Somm. Piante ined.

In varî luoghi; Mrz. foglie, Apr. fi. e fr. giov.! - Zodda (fi. e fr.).

I miei esemplari hanno il racemo piuttosto lasso, corto e brevemente peduncolato, come quelli di Lampedusa. Alcuni di quelli di Zodda sono conformi ai miei; altri che hanno racemo piủ compatto, peduncolo lungo quanto il racemo, e foglioline più larghe, presentano press' a poco la forma compactus (Salzm.). 


\section{1. - Trifolium suffocatum $\mathrm{L}$.}

Abbondante, forma spesso dei fitti e bassi tappeti verdi, tanto in riva al mare quanto in alto; Mrz. fi.! - Zodda (f $1^{\circ}$.).

82. - Trifolium glomeratum L.; Gibelli e Belli Rir. crit. Trif. It. Sez. micrantheum Mem. R. Acc. Sc. Torino Ser. II Ton. XLI, estratto p. 56 ; Somm. Piante ined.

Nei luoghi erbosi, piuttosto raro; 23 Apr. fi. e fr.! - Zodda (M. Calcarella, in dumetis, fi. e fr.).

\section{3. - Trifolium arvense L.; Solla p. 474.}

Abbastanza comune; Mrz. fi., Apr. fi. e fr.! - Solla (fi.), Zodda (fi. e fr.).

Per i denti del calice almeno tre volte più lunghi del tubo e oltrepassanti di molto la corolla, la pianta di Linosa si avricina alla rar. longisetum (Boiss. et Bal.; Gib. e Belli Riv. crit. Trif. It. Sez. Lagopus p. 27).

84. - Trifolium scabrum L.; Guss. Syn. II p. 327 ; Calc. p. 23; Solla p. 474; Gibelli e Belli Riv. Crit. Trif. It. Sez. Lagopees p. 49.

Comune in varie stazioni; Mrz. primi fi., Apr. fi. e fr.! Gussone, Solla (fr.), Zodda (fi. e fr.).

Piante per lo più di piccole dimensioni ed appartenenti alla microflora.

85. - Trifolium Ligusticum Balb.; Guss. Syn. II p. 329 ; Calc. p. 23.

Gussone solo.

86. - Trifolium Cherleri L.; Guss. Syn. II p. 332 ; Calc. p. 23. Ġussone solo.

87. - Trifolium stellatum L.; Solla p. 474.

In molti luoghi, tanto nel coltivato quanto nelia macchia; Mrz. primi fi.! - Solla (fr.), Zodda (fi. e fr.).

Sono frequenti in Marzo gli esemplari nani e pauciflori.

88. - Trifolium tomentosum L.; Solla p. 474 ; Gibelli e Belli Riv. Crit. Trif. It. Sez. Galeraia p. 21.

In varî luoghi, tanto nel piano' quanto in alto; Mrz. fi. e fr., Apr. fi. e fr.! - Solla (fi.), Zodda (fi. e fr.).

89. - Trifolium nigrescens Viv.; Guss. Syn. II p. 339 ; Calc. p. 23 ; Lojac. Fl. Sic. I pars II p. 81.

Gussone solo. 
90. - Trifolium agrarium Poll.; Solla p. 474.

Comune; Mrz. primi fi., Apr. fi. e fr. ! - Solla (fi. e fr.), Zodda (fi. e fir.).

I miei esemplari del 1873 appartengono ad una forma nana che, secondo Gibelli e Belli i quali li hanno esaminati, corrisponde al T. agrarium b. minus Iroch (Gib. e Belli Malpighia III p. 207). Peró ne ho visto quest'anno esemplari perfettamente sviluppati in tutte le loro parti, e alti fino a $35 \mathrm{~cm}$.

91. - Lotus angustissimus L.; Guss: Syrr. II p. 351; Calc. p. 23 ; Lojac. Fl. Sic. I pal's II p. 109.

Gussone solo.

92. - Lotus pusillus Viv.; Solla p. 474.

Abbondante in varî luoghi nel suolo arenoso, in alto e in basso; Mrz. fi. e fr. giov., Apr. fi. e fr.! - Solla (fr.), Zodda (fi. e fi.).

La maggior parte degli esemplari che ho raccolti tanto nel 1873 quanto in quest'anno, come quelli di Zodda, appartengono alla forma distinta col nome di $\beta$ major da Boiss. Fl. Or. II p. 173, per le dimensioni maggiori (altezza fino a $30 \mathrm{~cm}$.) ed i peduncoli bi-triflori. Gli esemplari tutti hanno tubercoli radicali abbondanti, come del resto la maggior parte delle leguminose di Linosa.

93. - Lotus cytisoides L.; Guss. Syn. II p. 356 ; Calc. p. 23. Gussone solo.

94. - Lotus Creticus L.; Solla p. 474.

Solla solo (fi.).

Solla indica per Linosa il L. Creticus, e per Lampedusa il L. cytisoides, donde bisogna concludere che ha trovato nelle due isole forme diverse. Tuttaria le differenze fra $L$. Creticus e L. cytisoides presentano cosi poca fissitá e sono interpretate dai varî autori in modo tanto diverso, che è permesso sospettare che Gussone e Solla abbiano dato nomi diversi alla medesima pianta, e che quindi i miei n. ${ }^{i} 93$ e.94 ne formino uno solo.

95. - Lotus peregrinus L.; Somm. Piante ined.

Ne ho raccolto un solo esemplare il 23 Apr. con fi. e fr.!

Questa specie orientale non era stata ancora indicata per l' Italia. Il L. Dioscoridis All. di Liguria le è stato erroneamente riferito da Reichenbach e da Nyman Consp. 184, secondo 
Burnat Fl. Alp. Mar. II p. 151 (vedi anche Fiori e Bẻg. Fl. an. II p. 73). Lojacono Fl. Sic. I pars II p. 113 mostra di avere avuto il presentimento che questo Lotı.s si dovesse trorare in Sicilia.

96. - Lotus edulis L.; Guss. Syn. II p. 350 ; Calc. p. 23; Solla p. 474 .

Molto comune specialmente nelle parti coltivate; MIrz. fi. e fr., Apr. fi. e fr.! - Gussone, Solla (fr.), Zodda (fi. e fr.).

Gli isolani lo conoscono col nome di Cannavuci e ne mangiano i semi.

97. - Astragalus Baeticus L.; Solla p. 474. A. Crelicus Lojac. Esc. p. 19 (lapsu calami ut ipse clarus Lojacono mihi scripsit).

Comune e rigoglioso nei luoghi coltivati; Mrz. fi. e fr., Apr. fr.! - Solla (fr.), Zwierlein (sec. Lojacono), Zodda (fr.).

98. - Hippocrepis multisiliquosa L.; Ross p. 347; Solla p. 474.

Molto frequente nelle parti coltivate; Mrz. fi. e fr. giov., Apr. fi. e fr. ! - Ross, Solla (fr.), Zodda (fi. e frr.).

99. - Lathyrus Clymenum L.; Solla p. 474. L. tenuifolizes Guss. Syn. II p. 278; Calc. p. 23.

Qua e lá nella macchia; Mrz. fi., Apr. fi. e fr.! - Gussone, Solla (fi. e fri.).

I miei esemplari hanno foglie strette come quelli di Lampedusa, ed appartengono quindi alla forma temeifolizes (Desf.).

100. - Lathyrus sphaericus Retz.; Somm. Piante ined.

Varî luoghi, nella macchia e nel coltivato; Mrz. fi., Apr. fi.fr. !

Per lo più con le parti vegetative molto sviluppate, avendo foglioline larghe fino ad $1 \mathrm{~cm}$.

101. - Lathyrus setifolius L.; Solla p. 474.

Solla solo (fi.).

102. - Vicia sativa L. V. macrocarpa Solla p. 474.

Comune nel coltivato e nella macchia; Mrz. fi., Apr. fi. e fr.! Solla (fr.), Zodda (fi. e fr.).

Solla indica di Linosa la $V$. macrocarpa. Nè i miei numerosi esemplari, nè quelli di Zodda appartengono a questa varietà.

\section{3. - Vicia lathyroides L.}

Nella macchia sui fianchi N. O. del M. Vulcano, ivi abbastanza frequente, ma non vista altrove; $2 \mathrm{Mrz}$. fi. e fr. giov. ! 
104. - Vicia atropurpurea Desf.; Solla 1. 47.4 ; Lojac. Esc. p. 19.

In diversi luoghi, macchia e campi incolti; Mrz. fi., Apr. fi. e fr.! - Solla (fi. e fr.), Zwierlein (sec. Lojacono), Zodda (fi. e fr.).

105. - Vicia dasycarpa Ten. V. varia Solla p. 474.

Solla (fi. e fr.), Zodda presso l'abitato (fi. e fr.).

106. - Vicia pseudocracca Bert.; Guss. Syn. II p. 295: Calc. p. 23.

Varî luoghi, nei campi incolti e nella macchia; Mrz. fi. e fr. giov., Apr. fi. e fr.! - Gussone.

107. - Vicia leucantha Biv.

Nella macchia e fra i fichi d'India alle falde N. O. del M. Vulcano, ivi abbondante, ma non vista altrove; 2 Mrz. fi. e fr. giov.!

108. - Eryum Lens L.; Somm. Piante ined.

Nel coltivato; Mrz. foglie, Apr. fi. e fr.! - Zodda (ad vias, fi. e fr.).

I miei esemplari appartengono alla medesima forma da noi trovata a Lampedusa, e che corrisponde alla var. dispermum Roxb. = subsphaerospermum Godr. Quelii di Zodda invece, che questi dice subspontanei, si avvicinano al tipo.

Le lenti si coltivano su larga scala a Linosa, dove costituiscono un articolo d'esportazione.

109. - Ceratonia Siliqua L.; Guss. Syn. II p. 646; Calc. p. 25 ; Lojac. Fl. Sic. I pars II p. 161; Car.in Parl. Fl. It. X p. 102.

Qua e là ; Mrz. e Apr. foglie ! - Gussone. Calcara nella Florula di Linosa dimentica di citare il Carubbo, ma lo menziona al Cap. IX p. 25.

Il Carubbo si trova nell isola tanto selvatico nella macchia, quanto coltivato. Anche le piante coltivate, per effetto del vento, alzano poco il tronco e distendono lungamente i loro rami fino sul suolo, prendendo un aspetto cupoliforme.

\section{XVIII. - ROSACEAE.}

\section{0. - Rubus uImifolius Schott.}

Verso il centro dell'isola, al piede della Montagna Rossa, poche piante; 4 Mrz. fi.!

La medesima forma a fiori rosei che a Lampedusa. 


\section{XIX. - Crassulacéae.}

111. - Tillaea muscosa L.; Car. in Parl. Fl. It. IX p. 92.

Abbondante specialmente nel piano arenoso, dove fa talora rosseggiare il terreno, ma trovasi anche in alto; Mrz. fi. e fr., Apr. fr.!

112. - Umbilicus pendulinus DC. Cotyledon Umbilicus Veneris Car. in Parl. Fl. It. IX p. 15.

Sulle rupi frequente; Mrz. fi. e frutti secchi dell'anno avanti, Apr. fi. e fr.! - Zodda (fr.).

L'U. pendulinus di Linosa presenta delle forme di transizione all' $U$. horizontalis, cioé forme con corolle più piccole, pedicelli più corti delle brattee e fiori orizzontali. I frutti però diventano penduli, ciò che non avviene nell' $U$. horizontalis. Il dott. Bẻguinot ha egli pure osservato, nelle isole Ponziane, delle forme di passaggio fra gli $U$. horizontalis e pendulinus, alle quali ha dato il nome di U. horizontalis f. intermedius (La Veget. delle is. Ponziane e Napol. p. 371). Boissier nella Fl. Or. ha descritto col nome di $U$. intermedius una specie orientale che dice pure essere intermedia fra gli $U$. horizontalis e pendulinus; ma i caratteri che Boissier assegna a questa specie non convengono alle piante di Linosa.

113. - Umbilicus horizontalis DC.; Solla p. 475. Cotyledton 7orizontalis Lojac. Esc. p. 19; Car. in Parl. Fl. It. IX p. 16.

Sulle rupi, non raro; Mrz. frutti secchi dell'anno avanti, Apr. fi. e fr.! - Solla (sfiorito), Zwierlein (sec. Lojacono).

114. - Sedum dasyphyllum $L$.

Rupi sulla cima della Montagna Rossa; $4 \mathrm{Mr}$. foglie!

La pianta di Linosa appartiene alla varietá glanduliferum (Guss.), come quella di Lampedusa.

115. - Sedum rubens L. Crassula mbens Calc. p. 21.

Indicata soltanto da Calcara. Non mi sembra improbabile che Calcara abbia confuso questa specie col Sectum litoreum che egli non cita, benchè sia tanto comune in Linosa.

116. - Sedum litoreum Guss.; Ross p. 347; Lojac. Fl. Sic. I pars II p. 214.

Comunissimo sulle rupi e specialmente nei loro crepacci, o alla loro base, spiccante col verde chiaro delle sue foglie sul 
colore scuro della roccia, in tutta l'isola dal mare alla cima dei monti; Mrz. foglie, Apr. fi. e fr.! - Ross, Zodda (fi. e fr.). Lojacono nella Fl. Sic. cita questa specie come raccolta da Zwierlein in Linosa, e si riferisce alla sua Fl. Lamp, intendendo citare, suppongo, "Una Esc. bot. Lamp. », dove peró non ne è fatta menzione.

I miei esemplari sono in parte microflorati come quelli di Lampedusa.

117. - Sedum Hispanicum L.; Solla p. 475.

Indicato solamente da Solla (sfiorito).

Mi sembra probabile che l'indicazione di Solla debba riferirsi alla specie precedente, comunissima, e da lui non citata. Lo stesso dicasi del S. Hispanicum di Lampedusa.

118. - Sedum Nicaeense All. : Calc. p. 22. S. altissimum Guss. Suppl. Prodr. p. 134 ; Car. in Parl. Fl. It. IX p. 47 ; Lojac. FI. Sic. I pars II p. 221. S. mefescens Guss. Syn. I p. 521 (cfr. vol. II Addenda et emend. p. 826).

Trovato soltanto da Gussone.

\section{XX. - Mesembryanthemaceae.}

119. - Mesembryanthemum nodiflorum L.: Guss. Syn. I p. 554 ; Calc. p. 22 ; Solla p. 475; Lojac. Fl. Sic. I, pars II p. 242.

Rupi e luoghi arenosi vicino al mare, comune; Mrz. foglie, Apr. fi. e fr.! - Gussone, Solla (fi.). Dalla Florula di Calcara non si rileva se egli pure abbia raccolto questa pianta, poichè ha dimenticato di contrassernarla con un asterisco per indicare che era stata trovata da Gussone. La citazione di Lojacono peró farebbe credere che si trovano esemplari di Calcara nell' Erbario Palermitano.

120. - Mesembryanthemum crystallinum L. ; Solla 1. 470 et 475 ; Lojac. Fl. Sic. I pars II p. 241.

Molto comune ed abbondante nella zona piủ vicina al mare, ma trovasi anche nell'interno dell'isola e fino sulla cima dei monti; Mrz. foglie, Apr. fi.! - Solla (in tutti i luoghi bassi cuopre il terreno di un fitto tappeto, fi.).

Questa pianta, detta dagli ișolani Erba Cristallina o Erba Soda, che oltre ad essere molto abbondante allo stato spontaneo viene 
anche coltivata, non è citata nè da Gussone nè da Calcara, il che fa supporre che sia stata introdotta dopo il loro tempo. La soda che si ricava tanto dalla pianta spontanea quanto da quella coltivata, costituisce uno dei principali articoli d'esportazione di Linosa. Questa esportazione si faceva giả nel 1884, come sappiamo da Solla (p. 470 in nota) e da Lo Re.

\section{XXI. - CACTACEAE.}

121. - Opuntia Ficus Indica Mill.; Calc. p. 25; Solla p. 470 et 475

In tutte le parti coltivate dell'isola; Mrz. e Apr. senza fi. e senza fr.! - Solla (fi.). Calcara non la cita altro che per raccomandarne la coltura, onde si puó concludere che a tempo suo non vi era ancora stata introdotta.

Il fico d'India trovasi ora in grandissima quantità e di grandi dimensioni in Linosa, in modo da costituire il tratto più saliente del paesaggio botanico in tutte le parti coltivate dell'isola. Esso forma delle siepi utilissime perchè, oltre ad essere efficace difesa per i campi contro gli animali, fornisce cibo all'uomo coi suoi frutti, e alle bestie con le sue pale. Se ne trovano anche dei veri boschetti. L'ho visto qualche volta rinascere da sè, lontano dai lưoghi ove è stato messo dall'uomo, ma raramente. Come giả notó Solla (p. 470 in nota), col sugo dei suoi frutti cotti e spremuti si fa, in Linosa, una specie di mosto, ritenuto buono contro i raffreddori, e che serve anche nella confezione di dolci. La varietá coltivata a Iinosa, come quella di Lampedusa, è quasi senza spine.

\section{MYRTACEAE.}

- Punica Granatum L.; Solla p. 174.

Il Melagrano è citato da Solla nel suo elenco di specie osservate in Linosa, ma soltanto come pianta coltivata. Lo trovó in fiore.

XXII. - UMBELliferaE.

122. - Scandix Pecten Veneris L.

Zodda solo (in arvis, fr.). 
123. - Foeniculum vulgare Mill. F. piperatem Guss. Syn. I p. 324 ; Calc. p. 21. F. capillaceum Car. in Parl. Fl. It. VIII p. 310. Merm piperatum Guss. Suppl. Prodr. p. 79.

Centro dell' isola alle falde del M. Vulcano; 5 Mrz. foglie! Gussone, Zodda (costa di Levante, foglie).

Tanto le piante da me vedute quanto quelle raccolte da Zodda sono da riferirsi al tipo capillacerm Gilib. piuttosto che alla varietà piperitum (DC.).

124. - Crithmum maritimum L.; Guss. Syn. I p. 326 ; Calc. p. 21 ; Solla p. 475 ; Lojac. Fl. Sic. I pars II p. 280 ; Cal' in Parl. FI. It. VIII p. 241. Cachrys maritima Guss. Suppl. Prodr. p. 82.

Rupi marine presso lo Scalo; $4 \mathrm{Mrz}$ foglie ! - Gussone, Solla (foglie), Calcara. Questi ha dimenticato di indicare che era stato trovato anche da Gussone.

125. - Ferula nodiflora L.; Guss. Syn. I p. 353 ; Calc. p. 21 ; Lojac. Fl. Sic. I pars II p. 289. F. communis Car. in Parl. Fl. It. VIII p. 299.

Nella macchia della Montagna Rossa; Mrz. foglie! - Gussone.

I segmenti delle foglie non sono raccorciati come nella $\mathrm{Fe}$ rula di Lampedusa.

126. - Daucus Gingidium L. sensu lato.

Raro; Mrz. e Apr. foglie!

Riferisco le piante che ho raccolte con sole foglie tanto nel 1873 quanto in quest'anno a questa entiti complessiva, perchè in quello stato é impossibile giudicare a quale delle specie in cui è stato frazionato il D. Gingidium esse appartengano. Caruel nell' Erbario Centrale ha collocato gli esemplari Linosani di Ajuti fra i D. Gingidium; peró non menziona Linosa nella Flora Italiana.

127. - Daucus Carota L. sensu lato.

Luoghi coltivati verso il centro dell'isola; Mrz. fi.!

Riferisco questa pianta, che non ho vista in frutto, al Daucus Carota sensu lato, avvertendo che potrebbe darsi che fosse quella forma che da Gussone fu chiarnata Daucus foliosus. Gli esemplari che ho raccolti hanno ombrelle prolifere, carattere teratologico di cui Gussone fa menzione soltanto per questa sua specie, e somigliano assai ad un esemplare autoptico di Gussone di $D$. foliosus proveniente da Stromboli, che si conserva nell' Erbario Centrale di Firenze. 
128. - Torilis nodosa (L.) Gaert.; Guss. Syn. I p. 338 ; Calc. p. 21 ; Solla p. 475. Caucalis nodosa Car. in Parl. Fl. It. VIII p. 563. Frequente nella macchia ed anche in vicinanza dei luoghi coltivati ; Mrz. fi. e fr. giov., A pr. fi. e fr.! - Gussone, Solla (fr.), Zodda (fi. e fr.).

Trovasi a Linosa tanto il tipo a ombrelle sessili o quasi, quanto la forma peduncularis Ten. con ombrelle inferiori portate da peduncoli lunghi fino a $11 / 2 \mathrm{~cm}$.

\section{XXIII. - RUBIACEAE.}

129. - Sherardia aryensis L.; Guss. Syn. I p. 182; Calc. p. 21; Solla p. 475 ; Tanf. in Parl. Fl. It. VII p. 72.

Luoghi coltivati e incolti, molto frequente; Mrz. fi., Apr. fi. e fr.! - Gussone, Solla (fi.), Zodda (fi. e fr.).

130. - Rubia peregrina L.; Tanf. in Parl. Fl. It. VII p. 17.

Nella macchia qua e lá, non comune; Apr. fi.! - Zodda (M. Vulcano, in dumetis, fi.).

Tanfani, 1. c., riferisce l' esemplare dell'Ajuti che trovasi nell'Erbario Centrale, alla rar. lucida. Na è appunto uno di quegli esemplari dai quali risulta che neanche come varietá si puó distinguere la $R$. lucida L., poiché ha le foglie inferiori largamente ovate, appena più lunghe che larghe, e le superiori dello stesso ramo strettamente lineari e allungate. Del resto ho altri esemplari di Linosa con foglie tutte strette e lunghe $(40 \times 3 \mathrm{~mm}$.). e scaberrime agli orli e sulla carena, corrispondenti alla forma angustifolia, ed altri ancora con tutte le foglie lanceolate $(30 \times 12)$ liscie sulla carena e poco scabre agli orli, che corrisponderebbero alla $R$. peregrina var. b. in Gussone Syn.

131. - Galium saccharatum All. G. Vaillantia Tanf. in Parl. FI. It. VII p. 61.

Qua e lá, non raro nelle parti coltivate; Mrz. fi. e fr. giov. Apl'. fir. :

Non ho veduto in Linosa la forma effusum Bég., che è frequente in Lampedusa.

132. - Galium Aparine L.; Guss. Syn. I p. 188; Tanf. in Parl. Fl. It. VII p. 58.

Piuttosto raro: Mrz. fi. e fr.! - Gussone. 
133. - Galium spurium L. G. Aparine var. spurium Tanf. in Parl. Fl. It. VII p. 58; Fiori e Bẻg. Fl. an. III p. 115.

Sotto le Opunzie e nella macchia, molto frequente; Mrz. fi. e fr., Apr. fr.! - Zodda (fr.).

Tutto il G. spurium che ho visto di Linosa appartiene alla var. Vaillantii (DC.) Gr. Godr. con frutti ispidi ( $G$. Aparine $\delta$ Vaillantii e non $\gamma$ spurium come indica la Flora analitica). Anche la pianta nata nell'Orto botanico di Firenze dai semi di Linosa aveva i frutti ispidi, come risulta dalla descrizione di Parlatore Fl. It. VII p. 58-59.

134. - Callipeltis muralis (I.) MIoris; Tanf. in Parl. Fl. It. VII p. 71 .

Sulle rupi e sotto la macchia, frequente fra le microfite; Mrz. fi. e fr., Apr. fr.! - Zodda (M. Vulcano e Mr. Calcarella, fr.). Una parte degli esemplari di C. muralis di Linosa appartiene ad una forma che mi sembra meritare di essere distinta come

- Forma calvescens fructibus omnino glabris, etiam apice pilis destitutis; tota planta glabra, folia angusta, nervo medio valido.

Non ho mai visto questa forma altrove, e tutti gli autori sono concordi nel dire che gli achenî della Callipeltis muralis, se anche talvolta sono glabri sul dorso, non mancano mai di un ciuffo di peli allapice. Trovasi in Linosa anche il tipo con gli achenî pelosi e muniti di lunghi peli all'apice, e trovansi pure forme intermedie.

135. - Vaillantia muralis L. Valantir manalis Guss. Syn. II p. 639 ; Calc. p. 23. Vaillantia muralis e V. intricala Lojac. Fl. Sic. II pars I p. 10 et 11 . Galium vexans Tanf. in Parl. Fl. It. VII p. 66.

Comunissima fra le microfite in tutta l'isola, tanto sulle rupi quanto nei luoghi arenosi ; Mrz. fi. e fr., Apr. fi. e fr.! - Gussone, Zwierlein (sec. Lojacono), Zodda (fr.).

La forma piú comune in Linosa è quella descritta da Lojacono col nome di $V$. intricata, che ha frutti piccoli, cauli diffusi, procumbenti, molto ramificati e quindi intricati, sottili, ad internodî inferiori allungati, e che nel portamento somiglia alla Callipeltis muralis. E questa una forma molto frequente fra le microfite nelle zolle erbose della macchia littoranea anche nell' Italia 
centrale, e non si può distinguere neanche come varietá dal tipo più robusto, più eretto, meno ramificato, a verticilli più ravvicinati ed a frutti più grossi, al quale è collegata da tutte le forme intermedie varianti a seconda del luogo dove la pianta è cresciuta. Non so cosa sia la V. tenella glabra alla quale Lojacono dice che la sua nuova specie è affine.

Sulla cima della Montagna Rossa abbondava fra i lapilli una forma estremamente nana, a fusti semplici.

\section{XXIV. - VALERIANACEAE.}

136. - Valerianella puberula (Bert. in Guss.) DC. ; Solla p. 475.

Abbondante fra i lapilli sulla cima della Muntagna Rossa; $4 \mathrm{Mrz}$. primi fi.! - Solla (fr.), Zodda (vetta di M. Vulcano, fr.). - Fedia Comucopiae (L.) Gaert.; Tanf. in Parl. Fl. It. VII p. 161.

Questa specie è citata per sbağlio dal Tanfani 1. c. come raccolta da Calcara. Essa non figura nella Florula di Linosa di questo autore, come dice Tanfani, a p. 160, bensi in quella di Lampedusa.

137. - Centranthus Calcitrapa (L.) Dufr.; Solla p. 475; Lojac. Esc. p. 20; Tanf. in Parl. Fl. It. VII p. 159.

Molto abbondante sulle rupi e nella macchia; Mrz. fi., Apr. fi. e fr. ! - Solla (fi. e fr.), Zwierlein (sec. Lojacono), Zodda (M. Vulcano, M. Calcarella e Montagna Rossa, in dumetis abunde, fr.).

XXV. - Compositae.

138. - Conyza ambigua DC.

Ho ricevuto questa specie dal parroco di Linosa, Sangermano, raccolta probabilmente in Aprile, con fiori e frutti.

139. - Bellium minutum L.; Arc. Comp. Fl. It. $2^{\mathrm{a}}$ ed. p. 666; Fiori e Bẻg. Fl. an. III p. 226. B. Sommierii Parl. in Arc. Comp. Fl. It. $1^{a}$ ed. p. 342 ; Lojac. Fl. Sic. II pars I p. 57.

Sulle rupi prossime al mare a Capo Ponente, ivi abbondante; 6 Mrz. rosette di foglie, 24 Apr. fi. e fr.! - Zodda (Capo Ponente, ad scopulos, fi. e fr.). 
Boissier (Fl. Or. III p. 173) indica questa specie di pochi punti dell'Arcipelago (rreco e dell'isola di Rodi, e dice di arerne risto soltanto due o tre esemplari. Nyman (Consp. Fl. Eur. p. 389), la dice «planta rarissima ed aggiunge dubitativamente la localitá di Creta. Parlatore, quando vide gli esemplari numerosi raccolti a Linosa dall'Ajuti e da me, credendola specie non descritta (allora non trovavasi negli erbarî di Firenze il $B$. minutum L.), le appose sull'etichetta il nome di Bellium Sommierii. Come tale fu pubblicata dall'Arcangeli nella prima edizione del Compendio. Nella seconda edizione peró viene riportata all' antica specie Linneana. I numerosi esemplari (più di 100) che tutt'ora conservo della specie di Linosa, raccolti nel 1873, differiscono dalla descrizione del B. minutum nella Flora Orientalis per: la pubescenza non papillosa; per gli scapi, o meglio peduncoli, non appena, ma due a tre volte più lunghi delle foglie; per le foglioline dell'involucro non $7-8$, nè ovato-oblunghe cucullate ed ottusissime, ma in due o tre serie ben distinte (quelle della serie interna più piccole, glabre e quasi interamente scariose) in numero che giunge fino a 20, ellittico-lanceolate acute; finalmente per essere brevemente caulescenti, e quindi per non essere le foglie tutte radicali (il che è evidente soltanto negli esemplari più sviluppati). Variano in altezza i miei esemplari, da 2 a $6 \mathrm{~cm}$.; le foglie maggiori hanno $2 \mathrm{~cm}$. di lunghezza e $5 \mathrm{~mm}$. di larghezza; i capolini maggiori $5-6 \mathrm{~mm}$. di diametro. Gli esempläri più sviluppati sono fogliati fino all'altezza di oltre $1 \mathrm{~cm}$. Gli achenî, dopo 33 anni si sono mantenuti di un verde gaio. I numerosi esemplari raccolti da Zodda sono di dimensioni assai piccole (1-3 cm.) ed hanno le foglie tutte radicali o quasi radicali. Vi sono adesso nell'Erbario Centrale degli esemplari di Bellium minutem di Kassos (fra le isole di Creta e di Rodi) raccolti dal D. Forsyth Najor il 29 Aprile 1886, che combinano con la pianta di Linosa, solamente sono piú piccoli dei miei, hanno peduncoli piu corti, e le foglie in apparenza tutte radicali.

140. - Senecio vulgaris L.; Ross p. 347; Lojac. Esc. p. 20. Comune presso al coltivato ed anche nella macchia; Mrz. fi. e fr., Apr. fi. e fr.! - Ross, Zwierlein (sec. Lojacono), Sangermano (Dic. fi. e fr.).

Sotto la macchia, fra le microfite, trovasi spesso ridotto a 2$3 \mathrm{~cm}$. di altezza e ad una sola calatide. 
141. - Senecio Cineraria DC.; Solla p. 475. S. bicolor Ross p. 347. S. Cineraria var. bicolor Fiori é Bẻg. Fl. an. III p. 215. Cineraria bicolor Guss. Syn. II p. 480 ; Calc. p. 23 ; Lojac. Esc. p. 20 et Fl. Sic. II pars I p. 70.

Fra lo scalo e la Cala della Pozzolana sulle rupi presso il mare; Mrz. foglie, Apr. primi fi.:-Gussone, Calcara, Ross (vicino al mare, nelle arene vulcaniche della costa Sud), Solla (bocci), Zwierlein (sec. Lojacono).

Non saprei decidere se le piante che ho vedute e raccolte a Linosa siano da ascriversi al tipo Cineraria o alla varietá $b i$ color. Mi è sembrato che vi fosse una forma sola, ed è certo quella che da Gussone, Calcara e Ross è stata chiamata bicolor e da Solla Cineraria.

142. - Pinardia coronaria (L.) Less.; Solla p. 476.

Abbondante nei luoghi coltivati; Mrz. fi., Apr. fi. e fr.! Solla (fi. e fr.), Zodda (fi. e fr.).

La forma più frequente è quella con ligole concolori; ma in alcuni campi, invece di questa forma normale, si trova quella a linguette gialle soltanto alla base, e scolorite nel resto (f. bicolor Micheletti). Queste forme sono gregarie e non si trovano miste.

143. - Anthemis secundiramea Biv. A. intermedia Solla p. 476.

S Solla solo (fi.).

\section{4. - Artemisia arborescens L.}

Sulle rupi a Ponente dello Scalo, rara; Mrz. foglie!

145. - Ambrosia maritima L.; Somm. Piante ined.

Qua e lả nei luoghi arenosi, anche lontano dal mare; Mrz. e Apr. foglie! - Zodda (foglie).

146. - Asteriscus spinosus (L.) Gren. et Godr. Pallenis spinosa Solla p. 476.

Solla solo (fi. e fr.).

147. - Calendula arvensis L.; Solla p. 476.

In diversi luoghi; Mrrz. fi. e fr.! - Solla (fr.).

Questa specie è molto meno frequente a Linosa della seguente. Sul Monte Vulcano, e specialmente abbondante sulla Montagna Rossa fra i minuti lapilli, se ne trova una forma ridotta in tutte le sue parti. Tutta la $C$. arvensis da me vista a Linosa aveva fiori crocei. 
148. - Calendula $\mathbb{E g y p t i a c a}$ Desf'. C. olficinalis $\gamma$-Egyptiaca Fiori in Fiori e Bẻg. Fl. an. III p. 297. C. micrantha Ross p. 347 ?

Molto comune, specialmente nei luoghi coltivati, ma anche Iontano da essi; Mrz. fi. e fr., Apr. fi. e fr.! - Sangermano (Dic. fi. e primi $\mathrm{fr}_{\text {. }}$.

A questa specie, cosi abbondante in Linosa, penso debba attribuirsi la $C$. micrantha di Ross. La C. Egyptiaca differisce dalla $C$. micrantha specialmente per avere i fiori del disco porporini come la $C$. bicolor, e per essere papillosa, irta e vischiosa. Questa specie era nota soltanto della Tunisia, dell'Egitto, della Grecia, della Palestina e della Mesopotamia.

\section{9. - Phagnalon saxatile (L.) Cass.}

A Lerante dello Scalo, sulle rupi, piuttosto raro; Mrz. fi. ! Solla (bocci).

Le piante di Linosa sono più tipiche di quelle di Lampedusa, non presentando la dilatazione in alto delle squame involucrali esterne che si osserva nella maggior parte degli esemplari di Lampedusa.

150. - Filago Germanica L. F. spathulata var. mostrate Ross p. 347. F. Germanica var. prostrata Fiori e Bẻg. Fl. an. III p. 274.

Solo Ross (nelle arene vulcaniche la var. prostrata [Parl.]).

151. - Filago Gallica L. $F$. temifolic Calc. p. 23. $F$. Gallica var. tenuifolic Ross p. 347; Solla p. 476. $F$. Cossyrensis Tin. ined. in Herb. R. Hort. Bot. Panorm.; Lojac. Fl. Sic. II pars I p. 110, non Lojac. Esc. p. 21, nec in exsiccatis (monente ipso Lojacono Fl. Sic.).

Molto frequente nel piano in suolo arenoso, ma trovasi anche in alto; Mrz. primi fi., Apr. fi. e fr.! - Calcara, Ross, Zwierlein (sec. Lojacono), Solla (fi. e fr.), Zodda (fi. e fr.).

Io nel 1873, come Calcara, Ross, Solla e Zodda, avevo raccolto soltanto la var. tenuifolia (Presl). Quest'anno peró, oltre alla varietà lenuifolia, abbondante e ben caratterizzata, ho raccolto più rara una forma che sembra diversa per essere più tomentosa, più prostrato-ramificata, e per avere foglie più corte e un poco più slargate in alto. Ma questa seconda forma non essendo ancora fiorita, non posso pronunziarmi sul suo valore. Mi pare certo che sia quella la forma chiamata da Tineo Cossyrensis e figurata da Lojacono FI. Sic. II pars I tab. XX. 
152. - Centaurea Melitensis L. ; Calcara 11. 23 ; Lojac. F1. Sic. II pars I p. 142.

Colli aridi; 23 Apr. fi. e fr.! - Calcara.

153. - Amberboa Lippii (L.) DC.; Ross 1). 347 : Lojac. Fl. Sic. II pars I p. 152. A. Lippii var. subdiscolor Lojac. Esc. p. 22. Centaurea Lippii Arc. Comp. Fl. It. $2^{a}$ ed. p. 717; Fiori e Bég. Fl. an. III p. 323 .

Abbastanza abbondante in alcuni campi vicino al paese, non trovasi lontano dal coltivato; Mrz. fi.! - Ross (sulle pendici del M. Rosso sulla costa orientale, a Ponente del paese), Zwierlein (sec. Lojacono), Zodda (costa di Levante e alla Pozzolana, fi. e fr.).

Lojacono, nel suo primo lavoro, dice che la pianta di Linosa è abbastanza diversa da quella di Spagna per costituire una varietá di cui dả la diagnosi. Ma nella Flora Sicula riconosce l'insussistenza di questa varietà. L'A. Lippii è stata distribuita da Ross Herb. Sic. n. 467 e da Lojacono Pl. It. Sel. n. 90, ricevuta da corrispondenti di Linosa, ed in parte nata da semi nell'orto botanico di Palermo. É l'unica localitá italiana dove trovisi questa specie, nota inoltre delle Canarie, della Spagna meridionale, dell'Africa settentrionale e dell'Arabia petrea.

154. - Carduus pycnocephalus L.; Solla p. 476.

Vicino all'abitato, lungo le vie, non frequente; 4 Mrz. bocci ! Solla (fi.).

Gli esemplari che ho raccolti in Linosa, per quanto si puó giudicare in quello stato giovanile, appartengono alla forma raccolta a Lampedusa da Zodda, e distinta dal tipo per essere più robusta, per avere i capolini affastellati, il fusto alato fino sotto ai capolini, le foglie piủ profondamente divise ed armate come le ali del fusto di spine più robuste (forma Gussoneanus Lojac. ?).

155. - Carduus brevisquamus (Fiori in Fiori e Bẻg. Fl. an. pro var. C. pycnocephali) Somm. Fl. Lamp. p. 112."

${ }^{1}$ Dopo avere veduto in quest'anno (1907) il Carduus marmoratus a Malta in quantità sterminata, ed in tutti gli stadî di sviluppo, mi sono persuaso che il $C$. brevisquamus gli si avvicina molto piu che al $C$. pycnosephalus, e forse andrebbe considerato come una sua semplice forma o varietà. 
Vicino all'abitato; Mrz. foglie! - Zodda (ad vias, fi. e fr.).

Le foglie det C. brevisquamus si distinguono da quelle del C. pycnocephalus per essere assai meno profondamente dirise, più debolmente spinose e molto meno grigio-tomentose di sotto.

156. - Carduus marmoratus Boiss. et Heldr.

Ho ricevuto questa specie da Sangermano, parroco di Linosa, raccolta in fiore e frutto alla fine di Aprile.

Gli esemplari che ho sott'occhio sono identici a quelli che ho raccolti a Lampedusa.

157. - Onopordon Sibthorpianum Boiss. et Heldr.; Fiori e Bég. Fl. an. III p. 382; Lojac. Fl. Sic. II pars I p. 163. O. corymbosim Lojac. Esc. p. 21.

Presso alle grotte e verso il centro dell'isola; Mrz. foglie, Apr. fi. e fr. ! - Zwierlein (sec. Lojacono), Zodda (presso l'abitato, ad vias, raro, bocci).

È l'unica localitá italiana dove sia stata trovata con certezza questa specie propria del bacino mediterraneo orientale e meridionale, poichè la localitá di Malta, indicata nella Flora analitica, è dubbia.

158. - Rhagadiolus stellatus DC.; Solla p. 476.

Solla solo (fi. e fr.).

159. - Hyoseris radiata L.; Guss. Syn. II p. 416; Calc. p. 23. Gussone solo.

160. - Hyoseris scabra $L$.

In diversi luoghi; Mrz. fi. e fr. giov.! - Zodda (fi. e fr.).

161. - Hedypnois poiymorpha DC. H. polymorpha $\delta$ erecte Solla p. 476.

In diversi luoghi; Mrz. fi. e fr. giov, !- - Solla (fr.).

Ho trovato soltanto la forma a peduncoli poco ingrossati ed a squame involucrali glabre (H. Mauritanica in Guss. Syn.).

162. - Seriola Etnensis L. ; Guss. Syn. II p. 421; Calc. p. 23; Solla p. 476.

Gussone, Solla (fi.).

163. - Cichorium Intybus L. C. Intybues $\beta$ glabratum Fiori e Bẻo. Fl. an. III p. 385.

Raccolto il $23 \mathrm{Apr}$. in fiore!

Come lo nota Fiori Fl. an., gli esemplari raccolti dall' Ajuti e da me appartengono alla varietá glabratum (Presl). 
164. - Urospermum picroides (L.) Desf.; fruss. Syn. II p. 386; Calc. p. 23 ; Solla p. 476 .

Frequente specialmente nelle parti coltivate; Mrz. fi. e fr., Apr. fi. e fr.! - Gussone, Calcara, Solla (fr.).

Trovasi, a seconda dei luoghi, rigogliosamente sviluppato o nano.

165. - Sonchus tenerrimus L.; Guss. Syn. II p. 392 ; Calc. p. 23; Solla p. 476.

IIolto comune nelle parti coltivate, e specialmente rigoglioso nella macchia; Mrz. fi. e fr., Apr. fi. e fr. ! - Gussone, Solla (fi.).

Viene mangiato dagli isolani che lo chiamano Cardedda.

166. - Sonchus levis Bartal.; Somm. Piante ined.

Varû luoghi; A pr. fi. e fr. ! - Zodda (M. Calcarella in dumetis, fi. e fr.).

167. - Picridium vulgare Desf.

Zodda solo. (in aridis, raro, fi. e fr.).

168. - Picridium Tingitanum (L.) Desf.; Ross p. 31\%. P. Tingitanum $\beta$ minus Lojac. Esc. p. 23. $P$. vulgare b. crassifolium Lojac. Fl. Sic. II pars I p. 207? Reichardia Tingilana $\beta$ Orientalis Fiori e Bẻg. Fl. an. III p. 425.

Luoghi rocciosi nelle radure della macchia, in varî punti del. l' isola, specialmente abbondante sulla Montagna Rossa; Mrz. fi. e fr., Apr. fr. e ultimi fi. ! - Ross, Zwierlein (sec. Lojacono), Zodda (fi. e fr.).

Nella Flora Sicula Lojacono non cita più per Linosa il $P i$ cridium Tingitanum, come aveva fatto in Una escurs. ecc., ma invece vi indica il $P$. vulgare var. crassifolium Willk., il che farebbe supporre che si è ricreduto per la primitiva determinazione degli esemplari di Zwierlein, quantunque non lo dica esplicitamente. Anche i miei esemplari del 1873 non sono molto tipici, tanto che ero stato io pure incer to se dovessi riferirli al $P$. Tingitanum o al P. vulgare. É questa la forma per la quale Fiori ha creato la sua var. Orientalis. Quest' anno peró, in stagione meno inoltrata, ne ho raccolto degli esemplari in pieno fiore assai rigogliosi, con foglie larghe e capolini grandi, cinti da squame largamente marginate, che molto si arvicinano alla forma da me trovata a Lampedusa nell' isolotto dei Conigli. Ho notato del resto che anche in questa stagione era assai varia- 
bile per le dimensioni di tutta la pianta, che é più o meno prostrato-ascendente, e per la divisione delle foglie.

169. - Crepis bulbosa (L.) Froel. Hieracium bulbosum Guss. Syn. II p. 403 ; Calc. p. 23 ; Lojac. Esc. p. 23. Etheorhiza bulbosa Solla p. 476; Lojac. Fl. Sic. II pars I p. 214.

Comunissima nel piano, dove in qualche luogo occupa quasi da sola il terreno, e da dove sale fino sulla cima dei monti; Mrz. fi., Apr. fi. e fr.! - Gussone, Solla (fi.), Zwierlein (sec. Lojacono), Zodda (fi. e fr.).

170. - Andryala sinuata L.; Ross p. 347. A. sinuata $\gamma$ Cossy. rensis Solla p. 476. A. undulata Lojac. Esc. p. 23. A. integrifolia $\gamma$ undulata Fiori e Bég. Fl. an. III p. 426.

Molto comune nel suolo arenoso; Mrz. foglie e qualche boccio, Apr. fi. e fr.! - Ross, Solla (fi. e fr.), Zwierlein (sec. Lojacono, il quale non la riporta nella Flora Sic.), Zodda (fi. e fr.).

La pianta di Linosa è spesso ramificata fino dalla base, ed ha l'inflorescenza pannocchiato-racemosa e glandolosa. Le foglie sono più o meno profondamente sinuato-dentate. Essa quindi combina colla var. undtelata (Presl) e non puó confondersi colla var. Cossyrensis (Guss.).

\section{XXVi. - CaMpanUlaceae.}

171. - Campanula Erinus L.; Solla p. 476 ; Tanf. in Parl. Fl. It. VIII p. 112.

Sulle rupi, frequente; Mrz. foglie, Apr. fi. e fr.! - Solla (fr.), Zodda (fi. e fr.).

I miei esemplari per la maggior parte sono ridotti nani.

XXVII. - CucurbitaceaE.

172. - Ecballium Elaterium (L.) Rich. Momordica Elaterium Solla p. 474.

Solla solo (fi. e fr.).

\section{XXVIII. - ERICACEAE.}

173. - Arbutus Unedo L.; Guss. Syn. I p. 464; Calc. p. 22; Car. in Parl. Fl. It. VIII p. 723. 
Gussone nella Synopsis cita questa pianta per Linosa, ed è seguito in questo da Calcara e da Caruel nella Fl. It. Tuttavia, da quanto Gussone scrive a p. 86 in Notizie sulle isole Linosa, Lampione e Lampedusa, parrebbe che avesse osservato il Corbezzolc soltanto in Lampedusa. Nessun altro l' indica di Linosa, e gli isolani che pure lo conoscono col nome di Imbriacola e sanno che esiste a Lampedusa, assicurano che non si trova in Linosa. Per questo mi sembra certo che adesso non vi esiste, e dubbio che vi fosse anche al tempo di Gussone.

\section{XXIX. - Oleaceae.}

174. - Olea Europaea L.; Guss. Notiz. p. 78, 86, et Syn. I p. 11 ; Calc. p. 20, 24 et 25; Tanf. in Parl. Fl. It. VIII p. 156. O. Oleaster Solla p. 476.

Nella macchia; Mrz. foglie, Apr. bocci! - Gussone, Calcara, Solla (bocci), Sangermano (Dic. fr.).

Nelle parti dell' isola più vicine all' abitato gli Oleastri sono ridotti a pochi, e questi pochi sono mutilati dagli animali bovini e ovini che ne mangiano le foglie, riducendoli ad umili cespugli di forma cupolare, quasi erinacea per l'affiorimento dei rami spinosi. Però in alcune parti esistono ancora in discreto numero, in modo da formare quasi bosco. Si trova tanto la forma con foglie piccole ed arrotondate che quella a foglie grandi ed allungate, quest'ultima proveniente forse da antichi innesti. - Calcara scrive che a tempo suo vi erano nell' isola circa 200 Oleastri alti e ben vegeti, suscettibili di innesto.

Gussone in Notizie ecc. dice che ha trovato l'Olivastro privo di foglie nell'Agosto, per la grande siccitá. Nella Synopsis dice che le sue foglie giorani ed i rami teneri formano quasi l'unico cibo delle capre selvatiche, che allora erano gli unici grossi animali in Linosa.

175. - Phillyrea variabilis Timb.; Tanf. in Parl. Fl. It. VIII p. 161. Ph. media Guss. Notiz. p. 86 et Syn. I' p. 10.

Trovata da me una volta sola sui fianchi del M. Vulcano; Mrz. foglie! - Gussone. Calcara ha dimenticato di riportarla.

La pianta che ho vista a Linosa appartiene alla varietả media (L.), indicata pure da Gussone. La Phillyrea è probabil- 
mente destinata a scomparire totalmente da Linosa coll'aumentare degli animali domestici. L'unico esemplare che ne ho visto era un misero cespuglio cupoliforme, ricoperto da licheni, e sembrava destinato a prossima morte.

\section{XXX. - Asclepiadaceae.}

176. - Periploca levigata Ait.; Car. in Parl, Fl. It. VI p. 717; Fiori e Bẻg. Fl. an. II p. 345; Lojac. Fl. Sic. II pars II p. 55. P. angrestifolia Guss. Notiz. p. 78 et Syn. I p. 286 ; Calc. p. 21; Ross p. 347 ; Solla p. 476 ; Lojac. Esc. p. 24.

Comune nella macchia e fra i fichi d'India, se ne trovano anche arbusti isolati, morenti e invasi dai licheni; Mrz. fi. e fr. giov. ed altri quasi maturi, Apr. fi. e fr.! - Gussone, Ross, Solla (sfioriti), Zwierlein (sec. Lojacono).

Ho trovato la Periploca in pieno fiore in Marzo, mentre portava dei frutti a tutti gli stadî di sviluppo, il che prova che la sua fioritura ha una lunga durata.

Le osservazioni di Gussone per la Periploca di Lampedusa si applicano in parte anche a quella di Linosa (vedi mia Fl, di Lamp. p. 121).

\section{- Stapelia Europaea Guss.}

Nyman Syll. Fl. Eur. nel 1854 ha indicato erroneamente questa specie per Linosa. Tale errore si trova ripetuto posteriormente da varî autori quali Willkomm e Lange Prodr. Fl. Hisp., Bunnet e Barratte Catal. plant. Tunisie, e Nyman Conspect. Fl. Eur.

\section{XXXi. - Gentianaceae.}

177. - Erythraea Centaurium (L.) Per's. ; (russ. Syn. I 1. 281 ; Calc. p. 21. E. grandiftora Guss. Suppl. Prodr. p. 63. Gussone solo.

178. - Erythraea pulchella (Sw.) Horn.; Solla p. 476. Solla solo (fi.).

XXXII. - Convolvulaceae.

179. - Convolvulus althaeoides L.; Somm. Piante ined.

Sui muri a secco vicino al paese; 6 Mrz. e Apr. sole foglie! 
I miei esemplari di Linosa appartengono alla medesima forma di quelli di Lampedusa, cioè alla forma a peluria patente, ed a foglie poco divise, che è generalmente distinta col nome di Italicus.

180. - Convolvulus Siculus L.; Ross p. 348 ; Car. in Parl. Fl. It. VI p. 808 ; Fiori e Bẻg. Fl. an. Il p. 385.

Qua e là nella macchia e nelle siepi; Mrz. fi., Apr.fi. e fr. ! Ross.

181. - Cuscuta Epithymum (L.) Murr.; Car. in Parl. Fl. It. VI p. 823; Lojac. Fl. Sic. II pars II p. 100.

Sulla Succowia Balearica, sulla Plantago Psylizum e su di alcune composte, non rara; Mrz. fi., Apr. fi.! - Zodda (sulla Succowia ed altre piante, fi.).

\section{XXXIII. - BORAginaceaE.}

182. - Echium parviflorum Moench. E. calycinemin Ross p. 348. Ross solo (arene vulcaniche presso il mare).

183. - Echium arenarium Guss.; Ross p. 348; Car. in Parl. Fl. It. VI p. 935 ; Fiori e Bẻg. Fl. an. II p. 366. E. Delileanum Lojacono Fl. Sic. II pars II p. 79-80 et Appendice p. 406.

Comune in basso, nel suolo arenoso; Mrz. fi., Apr. fi. e fr.! Ross, Zodda (fi. e fr.).

Nei luoghi arenosi più vicini al mare, fra le microfite, l'E. arenarium è piccolo e prostrato ed ha corolle piccole. Peró nei campi e luoghi dove il terreno è piú fertile si trovano forme assai diverse che sembrano passaggi all'E. confusum, e lasciano spesso nell' incertezza. Credo che debbano riferirsi alla var. b. Guss. = var. macranthum Fiori Fl. an. $=E$. Delileanum Lojac.

184. - Echium confusum De Coincy. E. maritimnem Auct. non Willd.; Calc. p. 21 ; Solla 1. $47 \%$.

Molto comune, specialmente nelle parti coltivate, ma anche lontano da queste; Mrz. fi. e fr., Apr. fi. e fr. ! - Calcara, Solla (fi.), Sangermano (Dic. fi.).

Oltre alla forma che ritengo tipica, e che è molto comune in Linosa, ne ho notata e raccolta un'altra, meno frequente, che propongo di chiamare:

- var. bracteatum mihi.

Differt a tipo: floribus minoribus, bracteis inferioribus foliis 
similibus flores remolos longe superantibus, bracteis superioribus calyces et flores excedentibus racemum juvenem comantibus; planta elata, flaccidior.

Nelle parti coltirate; Mr'z. fi.!

- Echium vulgave L.; Car. in Parl. Fl. It. VI p. 928.

Certamente per errore Caruel, nella Flora Italiana, cita questa pianta di Linosa come raccolta dall'Ajuti, poichè negli Erbarî dell' Istituto botanico di Firenze vi sono, di Linosa, soltanto le forme di Echium arenarium e confusum che vi ho rac. colte coll'Ajuti e che trovansi nel mio erbario.

185. - Heliotropium Europaeum I..; russ. Syll. I p. 212; Calc. p. 21; Solla p. 477; Car. in Parl. Fl. It. VI p. 832.

Gussone, Solla (fi.).

186. - Heliotropium Eichwaldi Steud.; Car. in Parl. Fl. It. VI p. 835. H. Europaeum var. Eichwaldi Fiori Fl. an. II p. 383. II. dolosim Lojac. Fl. Sic. II pars p. 90.

Qua e lá nel coltivato; Apr. fi. ! - Il parroco di Linosa, Sangermano, mi ha mandato questa specie raccolta in epoca più inoltrata in fi. e fr.

Quando Gussone citó l'H. Europaeum di Linosa, non aveva ancora descritto il suo $H$. macrocarpem (Fl. Inarim.) $=H$. Eichwalcli Steud. Può quindi darsi che la sua citazione, ed in conseguenza quella di Solla debbano riferirsi al H. Eichwaldi anzichè al H. Europaeum.

Le mie piante di Linosa corrispondono perfettamente all' $H$. macrocarpum Guss. di Ischia.- Ho riferito la specie di Gussone come sinonimo alla specie piủ antica di Steudel sull'autoritá di Caruel e di Fiori. Lojacono peró, nel fascicolo della Flora Sicula testè pubblicato, ritiene che $H$. macrocarpum Guss. $H$. Eichwaldi Steud. e H. dolosum De Not. siano tre specie diver'se.

XXXIV. - Solanaceae.

187. - Hyosciamus albus L.; Solla p. 477; Car. in Parl. Fl. It. VI p. 670.

Presso l'abitato, 24 Apr. fi. ! - Solla (fi.).

188. - Lycium Europaeum L.; Guss. Syn. I p. 273 ; Calc. p. 21 : Cai. in Parl. Fl. It. VI p. 702; Lojac. Fl. Sic. II pars II p. 103. L. Arabicum Solla.p. 477 (ex FI. an.). L. Europaeum $\beta$ ramu- 
losum Fiori Fl. an. II p. 398. L. intricatum Lojac. Fl. Sic. II pars II p. 103?

Molto comune; Mrz. foglie, Apr. fi. ! - Gussone, Solla (fi.), Zodda (fi.).

Questa pianta che è comune nella macchia e trovasi anche ad arbusti isolati, spesso cupoliformi per effetto del vento e degli animali, forma in molti luoghi delle folte ed impenetrabili siepi, alte fino più di tre metri, di un assai bell'effetto. Visto da lontano, questo Lycium, colle sue foglie fini di un verde molto chiaro, ha un aspetto quasi vaporoso, di modo che sulle fotografie, i suoi cespugli in distanza sembrano delle nuvolette chiare a contorni sfumati (vedi la vignetta a p. 184).

Sembra che vi sia in Linosa tanto il tipo quanto la forma ramiulosum Dun.; ma confesso di aver fatto poca attenzione alle forme sotto cui questa pianta, ivi cosi comune, si presentava. Ne ho raccolto un ramo giovane, forse un pollone, assai diverso dagli altri, avendo esso foglie larghe fino a 11 e lunghe $45 \mathrm{~mm}$. e la scorza ricoperta di una fitta lanugine bianca che si estende anche sulle spine. Gli altri esemplari invece hanno i rami glabri e le foglie assai più piccole. Gli autori in generale dicono che il L. Europaeum ha la scorza glabra; ma Willk. e Lange Fl. Hisp. II p. 532, osservano che presenta talvolta rami pubescenti. Lojacono, 1. c., sospetta che il Lycium di Linosa, come pure quello di Lampedusa, siano il L. intricatum Boiss.

189. - Solanum Sodomaeum L.; Solla p. 477 ; Car. in Parl. Fl. It. VI p. 689. S. Sodomaeum $\propto$ Mediterraneum Fiori e Bég". Fl. an. II p. 401.

Molto comune specialmente lungo i viottoli presso alle abitazioni, ma trovasi qua e lá anche nelle parti incolte dell' isola; Mrz. fi., Apr. fi. e fr. ! - Solla (fi. e fr.).

Gli esemplari da me raccolti appartengono alla var. Hermanni Dun. e non alla var. Mediterraneum indicata dalla Flora analitica per Linosa.

190. - Solanum nigrum L. ; Solla p. 477.

Qua e là nelle parti coltivate; Mrz. fi. e fr., Apr.fi. e fr.! Solla (fi. e fr.), Sangermano (Dic. fi).

Tutte le piante che ho viste avevano le bacche nere. 


\section{AxyV. - Plantaginaceae.}

191. - Plantago Psyllium L.; Solla p. 477.

Comunissima in tutta l'isola; Mrz. fi., Apr. fr.! - Solla (fr.), Zodda (fi. e fr.), Sangermano (Dic. fi.).

Variabilissima a seconda della stazione. Nei luoghi arenosi meno fertili trovasi ridotta ad un fusticino semplice, alto talvolta un solo centimetro. Da quelle forme nane si passa per tutte le gradazioni alle forme erette, alte e ramificate, ed alle forme prostrate con l'asse primario accorciato, e coi rami inferiori molto allungati e distesi sul suolo quasi a modo di rosetta (b. divaricala [Zuccagni] Bẻguinot Fl. an. III p. 101), che sono forse individui perennanti, sopravvissuti dall'autunno dell'anno precedente.

Nicotra (Naturalista Siciliano X p. 67-8 e Commentario diagn. p. 106-7) dice, e Béguinot riporta (FI. an. III p. 101 in nota) che trovasi forse in Linosa e Lampedusa la var. Zwierleinii Nicot. di questa specie. Ma Nicotra stesso non è certo che le piante avute dal barone Zwierlein siano di questa provenienza. Dalla descrizione, del resto, non sembra improbabile che la var. Zuvierleinii sia da identificarsi con la forma divaricala (Zucc.).

192. - Plantago Coronopus L.

Comunissima in tutta l'isola; Mrz. fi., Apr. fi. e fr.! - Zodda (fi. e fr.).

Nei luoghi arenosi, specialmente in basso vicino al mare, abbonda la forma nana a foglie intere ed a spighe ovali o globose (v. musilla Moris), dalla quale si passa per gradi alla varietá commutata Guss. normalmente sviluppata. Non ho visto il tipo Coronopus in Linosa.

193. - Plantago Serraria L.; Solla p. 477.

Solla solo ( $\mathrm{fr}$.).

\section{XXXVI. - ScrofulariaceaE.}

194. - Scrofularia peregrina L.; Solla p. 477; Car. in Parl. Fl. It. VI p. 554.

Frequente, specialmente nella macchia e setto i fichi d'India; Mrz. fi. e fr. giov., Apr. fr.! - Solla (fi. e fr.), Zodda (fi. e fr.). 
195. - Antirrhinum Orontium L.; Solla p. 47\%; Car. in Parl. Fl. It. VI p. 655.

In varî luoghi abbastanza frequente; Mrz. fi. e fr., Apr. fi. e fi. ! - Solla (fi. e fr.).

Trovasi talvolta nella macchia ridotto nano $(3-4 \mathrm{~cm}$.) ed unifloro.

196. - Linaria cirrhosa (L.) Dum.-Cours.; Guss. Syn. II p. 118; Calc. p. 22; Car. in Parl. Fl. It. VI p. 613; Fiori e Bég. Fl. an. II p. 420 ; Lojac. Fl. Sic. II pars II p. 137.

Solo Gussone.

197. - Linaria pseudolaxiflora Lojac. Esc. p. 25 et Fl. Sic. II pars II p. 132; Fiori Fl. an. appendice p. 169. L. virgata forma albiflora Ascherson in Ross p. 348; Fiori Fl. an., appendice all'iconografia, fig. 2917, p. 483. L. laxiflora v. pseudolaxiflora Fiori e Bẻg. Fl. an. II p. 423.

Verso la cima del M. Vulcano e sul M. di. Ponente; sulle rocce e fra i lapilli, abbondante; 5 e $6 \mathrm{Mr} z$. fi. ! - Ross (nella rena vulcanica a Levante del paese), Zwierlein (sec. Lojacono), Zodda (vetta del M. Vulcano, in rupestribus haud communis, fi. e fr.).

Ascherson che studió le piante raccolte da Ross, ritenne che questa Linaria fosse una forma della $L$. virgata (Poir.) Desf., specie comune sulle vicine coste d'Africa, ma non ancora trovata in Europa. Lojacono invece la giudicó specie nuova, e come tale la descrisse. Il confronto dei numerosi esemplari raccolti da

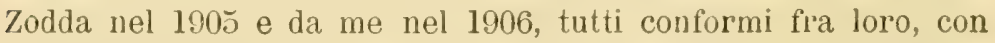
la figura della Flora Atlantica (II, tab. 135) e con gli esemplari d'Africa dell' Erbario Centrale, mostrano differenze tali da indurre anche me a ritenerla specie diversa.

La $L$. virgata quale è figurata da Desfontaines, e quale presentasi in generale negli esemplari d'Africa da me visti, ha cauli quasi eretti, alti fino a $2 \mathrm{dm}$., foglie larghe fino $5-6 \mathrm{~mm}$., fiori numerosi, lunghi con lo sperone $20 \mathrm{~mm}$., disposti in spiga densa, mentre tutte le piante di Linosa hanno cauli prostrati, lunghi raramente fino ad $1 \mathrm{dm}$., foglie larghe tutt'al più $3 \mathrm{~mm}$. e fiori lunghi (con lo sprone) al massimo $15-16 \mathrm{~mm}$., per lo più solitarî, raramente due in cima al caule. Queste differenze, è vero, sono più che altro di sviluppo e di portamento, cosicchè si potrebbe supporre che la Linaria di Linosa fosse soltanto un caso 
di eccessiro microfitismo della $L$. virgatu. Le differenze però, benchè di sviluppo e di portamento, sono tali, e sembrano tanto costanti, che ritengo trattarsi di forma fissata la quale, essendo inoltre geograficamente isolata, merita di esser considerata come specie distinta, tanto piü che sembra essere direrso anche il colore della corolla. Questa difatti è detta azzurra nella pianta d'Africa, mentre nella pianta di Linosa, come ho notato sul vivo, lo sperone e il labbro superiore sono bianchi leggerissimamente sfumati di violetto, la fauce è gialla, ed il labbro inferiore biancastro è percorso da due linee gialle. Le antere sono biancastre.

\section{XXXVII. - OROBaNCHACEAE.}

198. - Kopsia lavandulacea (Reichb.) Caruel.

Zodda solo (costa di Levante, fi. e fir.).

\section{XXXVIII. - LaBiatae.}

199. - Thymus capitatus (L.) Hoffm. et Link; Guss. Syn. II p. 95 ; Calc. p. 22 ; Car. in Parl. Fl. It. VI p. 101 ; Fiori e Bẻg. Fl. an. III p. 65 ; Lojac. Fl. Sic. II pars II p. 202.

Sulle rupi, MI. Vulcano e.M. di Levante dove è abbondante; Mrr. e Apr. foglie! - Gussone.

200. - Satureja microphylla (Urv.) Guss.; Car. in Parl. Fl. It. VI p. 124; Fiori e Bẻg. Fl. an. III p. 58.

Sulle rupi apriche qua e lá, sopra diversi monti dell' isola; Mrz. fi., Apr. fi. e fr.! - Zodda (fi. e fr.).

Le piante di Linosa, a fusti raccorciato-prostrati, pubescenti, a calici coperti di peli patenti, a foglie porporascenti, appartengono, come quelle di Lampedusa, alla rarietá b. di Gussone Syn. II p. 91.

201. - Sideritis Romana L.; Guss. Syn. II p. 66.

Gussone solo. Calcara ha dimenticato di citare questa specie nella sua Florula di Linosa.

202. - Marrubium vulgare L. ; Solla p. 177; I ojac. Esc. 1). 24; Car. in Parl. Fl. It. VI p. 70. M. vulgare var. Apulum Ross p. 348. 
Presso alle abitazioni e nei campi incolti, non comune: Mrz. foglie, Apr. fi. e fr. ! - Ross, Solla (fi. e fr.), Zwierlein (sec. Lojacono), Zodda (fi.).

203. - Stachys arvensis L.; Ross p. 348; Solla p. 477; Lojac. Esc. p. '24. S. arvensis var'. colorata Lojac. Fl. Sic. II pars II p. 236 .

Nel coltivato, non molto comune; Mrz. fi. e fr.! - Ross, Solla (fi.), Zwierlein (sec. Lojacono).

204. - Lamium amplexicaule L.; Solla p. 477; Car. in Parl. Fl. It. VI p. 213.

Comune, specialmente nel coltivato; Mrz. fi., Apr. fi. e fr.! Solla (fi.), Sangermano (Dic. fi.).

Quasi in tutte le piante da me osservate, come in quelle di Sangermano fiorite in Dicembre, vi erano fiori casmogami frammisti ai cleistogami.

205. - Prasium majus L.; Guss. Syn. II p. 107 ; Calc. p. 22; Solla p. 477 ; Car. in Parl. Fl. It. VI p. 229.

Sulle rocce e nella macchia, comune; Mrz. e Apr. fi.! Gussone, Solla (fi.), Zodda (fi. e fr.).

Oltre al tipo trovasi a Linosa, ma meno frequente, la varietà Liparitanum Mandr. in Tod. ex Lojac. (conf. Bẻguinot in Fl. an. III p. 15), con foglie e caule pubescenti, talvolta anche densamente pubescenti.

Secondo Gussone, qui e a Lampedusa il Prasium perde le foglie dopo la fruttificazione.

\section{XXXIX. - PRIMUlaceaE.}

206. - Asterolinum Linum-stellatum (L.) Duby; Somm. Piante ined.

Frequente fra le microfite sotto la macchia; Mrz. fi. e fr., Apr. fr. ! - Zodda (fi. e fr.).

207. - Anagallis aryensis L.; Somm. Piante ined.

Comunissima in tutta I' isola, specialmente nelle sue parti coltivate; Mrz. fi. e fr., Apr. fi. e fr. ! - Zodda (fi. e fr.), Sangermano (Dic. fi., fr.).

Quest'anno ho notato espressamente che tutte le piante da me vedute appartenevano alla var. coerulea, e che non avevo potuto 
trovare neanche un esemplare a fiori rossi; per quanto ne facessi ricerca. Alla varietá coemulea appartengono pure gli esemplari di Zodda, quelli raccolti in Dicembre da Sangermano, e quelli che avevo raccolti nel 1873. Sembra dunque che la var. phoenicea manchi a Linosa.

\section{Xl. - Plumbaginaceae.}

- Statice psiloctada Boiss.; Mori in Parl. Fl. It. VIII p. 578. S. psiloclada var. gracilis Fiori in Fiori e Bég. Fl. an. II p. 330. Non ammetto l' esistenza di questa specie a Linosa, perchè nella Flora Italiana Linosa è citata sulla fede di Lojacono (sub bellidifolia), come risulta dalla bibliografia, mentre Lojacono (in Una Escurș. ecc.) cita la S. bellidifolia soltanto di Lampedusa, e non indica alcuna specie di questo genere per Linosa. La citazione della Flora analitica è probabilmente desunta dalla Flora Italiana.

208. - Statice virgata Willd.; Mori in Parl. Fl. It. VIII p. 582. S. Smilhii Calc. p. 21; Lojac. Fl. Sic. II pars II p. 23. S. minuta var. virgata Martelli Riv. crit. Stat. It. p. 15.

Sulle rupi marịne, abbondante presso lo Scalo e a Capo Ponente; Mrz. foglie e fiori secchi dell'autunno precedente, Apr. bocci ! - Calcara.

Le piante vive che abbiamo prese a Linosa, hanno fiorito in questa estate nell' Orto botanico di Palermo, e per effetto del trapiantamento in terreno più ricco, hanno allungato lo scapo fino a $80 \mathrm{~cm}$. ed hanno prodotto spighette molto distanti fra loro (le inferiori distanti $1 \mathrm{~cm}$.). Per questi caratteri apparterrebbero alla var. remotispicula (Lacaita) Fiori Fl. an. II p. 331, la quale appare quindi essere soltanto una variazione dovuta al luogo dove cresce, poichè le piante fiorite a Linosa raggiungono al massimo $40 \mathrm{~cm}$. d'altezza ed hanno spighette ravvicinate fra di loro (distanti non più di $2-3 \mathrm{~mm}$.).

XLi. - Polygonaceae.

209. - Polygonum aviculare L.; Guss. Sy11. I p. 153; Calc. p. 22. P. dissitiflorim Solla p. 477. 
Nei campi e lungo le vie, non comune; 24 Apr. fi.! - Gussone, Solla (fi.), Zodda (foglie).

I miei esemplari e quello di Zodda appartengono ad una forma eretta come quelli del Solla, stando al nome che questi ha loro dato.

\section{0. - Polygonum maritimum L.}

Presso lo Scalo sulla spiagrgia detta Arena Bianca, insieme alla Euphorbia Paralios, raro; $4 \mathrm{Mrz}$. foglie! - Zodda (presso lo Scalo nelle arene marine, fi. e $\mathrm{fr}$.).

211. - Emex spinosa (L.) Campd.; Solla p. 477 ; Fiori e Paol. Fl. an. I p. 297.

Qua e lá, abbastanza frequente nei campi incolti e lungo i viottoli; Mrz. fi. e fr., Apr. fi. e fr.! - Solla (fi. e fr.), Zodda (fi. e fr.).

212. - Rumex bucephalophorus L.; Guss. Syn. I p. 432 ; Calc. p. 22; Solla p. 477 .

Comunissimo in tutta l'isola, dai luoghi arenosi vicino al mare fino alla cima dei monti, formando spesso estesi tappeti d'un bel rosso; Mrz. fi. e fr., Apr. fi. e fr.! - Gussone, Solla (fr.), Zodda (fi. e fr.).

Trovasi in gran quantitá nano fra le microfite; in vicinanza del mare ha generalmente le foglie assai carnose, e tutta la pianta assume una colorazione rosso intenso. Viene mangiato in insalata dagli isolani che lo chiamano Carceitola.

XLiI. - AMarantaceae.

213. - Amarantus graecizans L. ex Fiori e Paol. Fl. an.

Ho ricevuto questa specie da Sangermano, parroco di Linosa, raccolta in Dicembre in fiore e. frutto.

\section{XLiII. - ChenopodiaceaE.}

214. - Atriplex Halimus L.; Calc. p. 23.

Solo Calcara.

215. - Beta maritima L. ; Guss. Syn. I p. 298 ; Calc. p. 21. Nel suolo arenoso specialmente nelle parti coltivate; Mrz. fi.e fr., Apr. fi. e fr. ! - Gussone, Zodda (fi. e fr.). 
216. - Beta macrocarpa Guss.; Solla p. 477.

Solla solo.(ii. e fr.).

\section{7. - Beta Cycla L.}

Zodda solo (fi.).

L'esemplare raccolto da Zodda appartiene alla forma b. sulcata (Gasp.) Fiori Fl. an. I p. 308.

218. - Chenopodium murale L.; Calc. p. 21; Ross 1) 318 ; Lojac. Esc. p. 25 et Fl. Sic. II pars II p. $28 \%$.

Comune presso l'abitato, ma trovasi anche distante dalle colture; Mrz. fi. e fr., Apr. fi. e fr.! - Calcara, Ross, Zwierlein (sec. Lojacono), Zodda (fi. e fr.).

Calcara riferisce i suoi esemplari alla var. b. pruinosum Guss., alla quale corrispondono (più o meno caratterizzati) anche i miei e quelli di Zodda.

219. - Chenopodium opulifolium Schraul.; Somm. Piante ined.

Nel coltivato, 24 Apr. foglie! — Zodda (foglie), Sangermano (Dic. fi.).

220. - Salsola Tragus L.; Guss. Syn. I p. 299; Calc. p. 21.

Luoghi arenosi presso il mare, non comune; Apr. foglie! Gussone, Zodda (foglie), Sangermano (fr.).

221. - Salsola Soda L.; Somm. Piante ined.

Luoghi arenosi marini in vicinanza dello Scalo; $22 \mathrm{Apr}$., sole foglie!

XLIV. - URTICACEAE.

222. - Theligonum Cynocrambe L.; russ. Syn. II p. 600 ; Calc. p. 23; Solla p. 478 ; Parl. FI. It. IV p. 310.

Sulle rupi; 25 Apr. foglie! - Gussone, Solla (fr.).

223. - Urtica urens $\mathrm{L}$.

Frequente nel coltivato; Mrz. fi. e fr.! - Zodda (fi. e fr.).

224. - Urtica membranacea Poir.; Guss. Syn. II p. 579 ; Calc. p. 23; Parl. Fl. It. IV p. 319.

Molto comune presso le abitazioni ed anche lontano da queste nella macchia, fino sulla cima dei monti; Mrz. fi. e fr., Apr. fi. e fr. ! - Gussone, Sangermano (Dic. fi.).

Ho raccolto anche alcuni esemplari della var. neglecta Guss. La $U$. membranacea trovasi spesso fra le microfite sotto la macchia, ridotta a piccolissime dimensioni $(2-3 \mathrm{~cm}$.). 


\section{5. - Urtica pilulifera L.}

Non vista altrove che lungo la via, verso il centro dell'isola; $4 \mathrm{Mr} \%$ fi. e fr.!

226. - Urtica dioica L.; Solla p. 478.

Solla solo (fi. e fr.).

227. - Parietaria officinalis L.; Ross p. 348. P. diffusa Calc. p. 23. P. diffusa var. microphylla Lojac. Fl. Sic. II pars II p. 351.

Molto comune in tutta l'isola; Mrz. fi. e fr., Apr. fi. e.fr.! Calcara, Ross (var. ramiflora), Zodda (fi. e fr.).

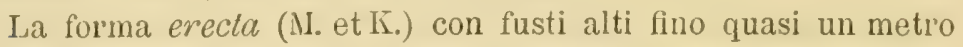
e foglie larghe fino a 5 e lunghe $10 \mathrm{~cm}$. nei luoghi ombrosi, e la f. Judaica (L.) sulle rupi, con infinite forme intermedie.

228. - Parietaria Cretica L.; Guss. Syn. II p. 642; Calc. p. 23 ; Ross p. 348; Solla p. 478; Parl. Fl. It. IV p. 338 ; Lojac. Esc. p. 26; Fiori e Paol. Fl. an. I p. 280. P. Cretica var. thymoides Lojac. Fl. Sic. II pars II p. 353.

Comune sulle rupi e nelle loro fessure, specialmente vicino al mare; Mrz. fi. e fr., Apr. fi. e fr.! - Gussone, Ross (fra i ciottoli vulcanici nel cratere del Monte Vulcano), Solla (fr.), Zwierlein (sec. Lojacono), Zodda (fi. e fr.).

\section{9. - Parietaria Lusitanica L.}

Sulle rupi ombrose frequente; Mrz. fi. e fr.!

- Ficus Carica L.; Calc. p. 25; Solla p. 478.

Citato nelle Florule di Calcara e di. Solla ma soltanto come pianta coltivata. Mi fu assicurato dalla gente dell'isola che non trovasi spontaneo.

\section{XLV. - Euphorbiaceae.}

230. - Euphorbia Peplis L.; Guss. Syn. I p. 532; Calc. p. 22. Gussone solo.

231. - Euphorbia helioscopia L.; (iuss. Syır. I p. 510 ; Calc. p. 22.

Gussone solo.

232. - Euphorbia Peplus L. ; Guss. Syn. I p. 533 ; Calc. p. 22; Parl. Fl. It. IV p. 498.

Molto comune; Mrz. fi. e fr., Apr. fi. ! - Gussone.

Gussone indica per Linosa il tipo e non la var. peploides (Gouan). 
Io invece, tanto quest'anno quanto nel 1873 , ho trovato soltanto la varietà e non il tipo. Abbonda nana ed a foglie densamente imbricate tra le microfite nei luoghi bassi arenosí. Sotto la macchia cresce piủ alta, con foglie più lasse e di un verde più gaio. Alcuni di questi esemplari, che hanno glandole verdognole anzichè rosse, presentano un passaggio al tipo, senza raggiungerlo.

233. - Euphorbia pinea L.; Guss. Notiz. 1. 86 et Syn. I p. 53:39; Calc. p. 22.

Comunissima nella zona marina, tanto sulle roccie quanto nei luoghi arenosi. 'Trovasi pure lontano dal mare, ma piủ rara; Mrz. fi. e fr., Aprile fi. e fr. ! - Gussone, Zodda (fi. e fr.). Calcara dimentica il segno * per indicare che fu raccolta da Gussone.

Qui come a Lampedusa, le piante della zona prettamente marina presentano un aspetto assai diverso da quello delle piante cresciute più lontane dal mare.

Secondo Gussone i conigli nell'estate erano costretti a cibarsi della corteccia di questa Euphorbia e di quella della E. dendroides. In oggi peró, conigli non vi sono più, e se vi fossero troverebbero probabilmente pascolo sufficiente nelle pale dei fichi d'India.

234. - Euphorbia Paralios L. ; (iuss. Syn. I p. 537 ; Lojac. Fl. Sic. II pars II p. 342.

Vicino allo Scalo, sulla piccola spiaggia arenosa contenente molti detriti di conchiglie, e detta per questo Arena Bianca; ivi in discreta quantitá, ma non vista altrove; Mrz. foglie! Gussone, Zodda (in arenosis maritimis foglie). Calcara ha dimenticato di citare questa specie nella sua Florula di Linosa.

235. - Euphorbia Terracina L.; Guss. Syn. I p. 535; Calc. p. 22; Solla p. 478; Parl. Fl. It. IV p. 537.

Comunissima vicino al mare ed anche nell'interno; Mrz. fi. e fr., Apr. fr.! - Gussone, solla (fr.).

236. - Euphorbia dendroides L.; (iuss. Notiz. 1). S6 et Syn. I p. 536 ; Calc. p. 22 ; Ross p. 345 ; Solla p. 478 ; Parl. Fl. It. IV p. 553.

Comunissima in tutta l' isola; Mrz. fi. e fr', Apr. fr.! - Gussone, Calcara, Ross (con Pistacia Lentiscus è l'elemento predominante della macchia), Solla (fr.), Zodda (fr.). Calcara ha dimenticato di segnare che era stata raccolta da Gussone. 
Questa pianta è una caratteristica del paesaggio di Linosa, per la sua abbondanza e per la vivacita della tinta delle sue foglie e dei suoi fusti, di primo d'un verde chiaro e gaio, e rosseggianti coll' inoltrar'si della stagione. Secondo Gussone i conigli, costretti dalla fame, mancando in estate le erbe, ne rosicavano la corteccia.

237. - Mercurialis annua L.; Solla p. 478.

Comune nel coltivato ed anche nella macchia; Mrz. fi. e fr., Apr. fr.! - Solla (fr.), Sangermano (Dic. fi.).

Nella macchia si vede talvolta nana, alta $1-2 \mathrm{~cm}$. Ho trovato. anche la var. ambigz a, ma più rara.

- Ricinus commumis L.; Solla p. 470 et 478 .

Indicato da Solla (fi.) il quale ne vide un solo esemplare. Non menziona che fosse coltivato, ma è permesso supporlo. II capitano Smyth scrive di avere gettato semi di Ricino e di altre piante nella allora deserta isola di Linosa, al principio del secolo passato. Oggi non vi si trova nè coltivato nè spontaneo.

\section{MONOCOTYLEDONEAE.}

\section{XLVI. - IRIDACEAE.}

238. - Romulea Columnae Seb. et Maur.? Romulea sp. Somm. Piante ined.

Abbondante sul M. di Ponente e raccolta anche altrove; Mrz. fr. giov., Apr. fr. secchi !

Rimango in dubbio sulla determinazione di questa Romulea di cui non ho visto i fiori. Dalla $R$. Columnae di Lampedusa differisce alquanto per arere foglie un poco più sottili e capsule un poco più piccole.

239. - Gynandriris Sisyrinchium (L.) Parl.

Zodda solo (in aridis ubique, fi.).

- Iris Germanica L.

Trovasi nel centro dell' isola, in luogo dove fu piantata ma dove, a quanto mi assicurarono, si è moltiplicata da sè, e vive ora senza l'intervento dell'uomo. 


\section{XLVII. - AMARYLLIDACEAE.}

240. - Pancratium maritimum L.; Solla p. 478.

Nelle arene marine, raro; 24 Apr. foglie! - Solla (foglie).

241. - Agave Americana L.

Vicino all'abitato; Mrz. foglie!

Non potrei assicurare che si sia reaimente inselvatichita a Linosa.

XLVIII. - Asparagaceae.

242. - Asparagus acutifolius L.; Lojac. Esc. p. 26.

Zwierlein solo (sec. Lojacono).

243. - Asparagus aphyllus L.; Ross p. 348. A. stipularis Somm. Piante ined.

Qua e lá nella macchia, non raro; Mrz. e Apr. foglie! Ross.

Qui come a Lampedusa si trovano forme assai diverse per la grossezza e lunghezza dei cladodî. La serie di forme che ho. raccolte quest'anno mi ha persuaso che gli esemplari a cladodî lunghi e robusti raccolti nel 1873 e da me ritenuti per $A$. stipularis, non erano altro che un estremo di variazione dell' $A$. aphinllues.

\section{XLix. - Liliaceae.}

244. - Urginea maritima (L.) Baker. Scilla maritima Guss. Syn. 1 p. 407; Calc. p. 21; Solla p. 478. Urginea Scilla Parl. Fl. It. II 1). 455 .

Frequente, trovasi fino sulla cima dei monti; Mrz. e Apr. foglie! - Gussone, Calcara, Solla (foglie).

245. - Allium roseum L.; Solla p. 478.

Solla solo (fi, e. fr.).

246. - Allium subhirsutum L.

Zodda solo (MI. Calcarella, in collibus aridis, fi. e fr.).

Gli esemplari raccolti da Zodda hanno le foglie quasi glabre, e quindi si avvicinano alla forma glaberrimum Noris. 
247. - Allium vernale Tin.; Somm. Piante ined.

Molto comune specialmente nei luoghi rupestri; Mrz. fi., Apr. fiori secchi e capsule mature! - Zodda (fi.).

In Marzo, quest'anno, ho visto una grande quantitá di giovani piante di Allium in boccio o. con sole foglie, di cui non posso dire se fossero $A$. vernale o $A$. subhirsutum, due specie del resto molto affini. Ho poi trovato un esemplare di $A$. vernale rigoglioso, con foglie larghe fino ad $1 \% \mathrm{~cm}$. e con ombrella prolifera, identico a quello trovato a Lampedusa da Zodda (v. Flora Lamp. p. 146).

248. - Allium Ampeloprasum L.

Zodda solo (fi. e fr.).

Gli esemplari raccolti da Zodda appartengono alla var. commictatum (Guss.) che trovasi anche a Lampedusa.

249. - Asphodelus tenuifolius Cav.; Ross p. 318; Lojac. Esc. p. 26. A. fistulosus var. macrior Calc. p. 21. A. fistulosus $\beta$ tenuifolius Arc. Comp. Fl. It. $2^{a}$ ed. p. 140; Fiori e Paol. Fl. an. I p. 204.

Fra le piante della macchia in suolo roccioso, M. di Ponente e M. di Levante, in questi due luoghi abbondante; Mrz. fi. e fr., Apr. fr. ed ultimi fi.! - Calcara, Ross (fessure delle rocce del Monte di Ponente), Zwierlein (luoghi sassosi sec. Lojacono).

La pianta di Linosa corrisponde perfettamente all' $A$. tenuifolius di Spagna, di Grecia e d'Africa, come giustamente osserva Lojacono il quale si è accertato che a questa specie andava riferito $l^{\prime} A$. fishulosus var. macrior di Calcara.

E l'unica localitả conosciuta per questa specie in Italia.

$$
\text { L. - NAJADACEaE. }
$$

250. - Posidonia Oceanica (L.) Del.

Rigettata in abbondanza sulle spiaggie; Mrz. foglie!

\section{Li. - Araceate.}

251. - Ambrosinia Bassii L. ; Cuss. Syn. II p. 594 ; Calc. p. 23 ; Parl. Fl. It. II p. 232.

Gussone solo. 
252. - Arisarum vulgare Targ. Toz\%; Guss. Syn. II p. 595; Calc. p. 23 ; Solla p. 478.

Comunissimo dovunque nell' isola; 4 Mrz. fi. e fr., A pr. fr.! Gussone, Solla (fr.), Zodda (fi.), Sangermano (Dic. fi.).

Dai tuberi si partono dei lunghi rizomi i quali alla loro volta emettono delle lunghe radici. Questi rizomi e queste radici, a Linosa, si estraggono facilmente dal terreno che dovunque é arenoso e sciolto.

253. - Arum Italicum Nill.; Solla p. 478.

Verso il centro dell' isola lungo la via, raro e da me non visto altrove; Mrz. foglie! - Solla (il quale non era certo della determinazione, fr.), Zodda (alla Pozzolana, inter scopulos, fi.).

\section{LiI. - GraminaceaE.}

254. - Phalaris minor Retz.; Solla p. 479.

Solla (fi. e fro), Zodda (in arvis, fi.).

255. - Cynodion Dactylon (L.) Pers.; Guss. Syn. I p. 110: Calc. p. 21.

Solo Gussone, il quale peró, nel Supplemento al Prodromo p. 20, non l'avera citato di Linosa.

256. - Andropogon hirtus L.; Solla p. 479.

Solla solo (fi.).

E possibile che Solla abbia dato questo nome, preso nel senso più lato, alla specie seguente. Se cosi fosse, I'A. hirtus sensu stricto andrebbe cancellato dalla Florula di Linosa.

257. - Andropogon pubescens Vis.

Rupi del M. Pozzolana di Ponente, raro; 3 Mrz. bocci ! - Zodda (fra M. Vulcano e M. di Levante, in praeruptis collium, fi.).

258. - Triplachne nitens (Guss.) Link; Lojac. Esc. p. 26. Agrostis nilens Calc. p. 21.

Nelle arene marine; 22 e 24 Apr. fi. e fr.! - Calcara, ZwierIein (sec. Lojacono), Zodda (Montagna di Levante, in arenis vulcanicis abunde, fi. e fr.).

259. - Polypogon maritimus Willd.: Sonm. Piante ined.

Luoghi aridi; 25 Apr. fi. e fr.!

I miei esemplari, per la forma acuta delle glume, e per avere la pannocchia in parte arvolta dalla guaina della foglia supe- 
riore, sono intermedî fra le varietả acutiflom Guss. e subspathacers (Req.).

260. - Lagurus ovatus L.; Solla p. 479.

Solla solo (fi.).

261. - Stipa tortilis Desf.; Guss. Syn. I p. 129; Calc. p. 21; Solla p. 479.

Molto comune tanto nel piano quanto in alto; Mrz. fi. e fr.s Apr. fr.! - Gussone, Solla (fi. e fr.), Zodda (fr.). Calcara ha dimenticato il segno * per indicare che era stata raccolta da Gussone.

Trorasi spesso nana.

\section{2. - Milium multiflorum Car.}

Zodda solo (Scoglio di Ponente, in dumetis, fi. e fr.).

263. - Aira Cupaniana Guss.; Guss. Syn. I p. 149; Calc. p. 21 ; Solla p. 479; Parl. Fl. It. I p. 253. A. ${ }^{*}$ Capillaris Guss. Suppl. Prods. p. 16.

Gussone, Solla (var. incerla Cesati con spighette biaristate, fr.), Zodda (M. Vulcano, fr.).

La pianta raccolta a Linosa da Zodda è secondo ogni probabilitá la stessa ivi raccolta da Gussone e chiamata nel Suppl. al Prodr. Aira capillaris, e nella Synopsis A. Cupaniana; ed è certamente quella riferita da Solla alla $A$. Cupaniana var. incerta Ces., poichè anche negli esemplari di Zodda le spighette hanno per la maggior parte due fiori aristati. Tuttavia la pianta di Linosa differisce alquanto dalla descrizione di A. Cupaniana in Gussone per avere i pedicelli delle spighette più lunghi di queste, e per essere le spighette biflore.

264. - Aira Tenorei Guss.; Guss. Syn. I p. 148; Calc. p. 21. Fiorinia pulchella Parl. Fl. It. I p. 234.

Gussone solo.

265. - Avena barbata Brot.; Solla p. 479.

Molto comune in tutta l' isola; Mrz. fi. e fr., Apr. fr. ! - Solla (fi. e fr.), Zodda (fr.).

Trovasi tanto di dimensioni normali quanto nana $(5-15 \mathrm{~cm}$.) e pauciflora, talvolta con una spighetta sola. Sembra che, indipendentemente dalla statura, vi siano due forme distinte per le dimensioni delle spighette. Nell'una la gluma maggiore misura circa $15 \mathrm{~mm}$. e la glumetta inferiore con la resta $30 \mathrm{~mm}$. 
Nell'altra la gluma maggiore raggiunge $30 \mathrm{~mm}$. e la glumetta inferiore con la resta $50 \mathrm{~mm}$. Le dimensioni delle spighette non sono sempre in relazione con le dimensioni delle piante, trovandosi anche esemplari alti $50 \mathrm{~cm}$. con spighette piccole, ed esemplari nani con spighette grandi.

266. - Trisetum aureum Ten. T. condensaticn Lojac. Esc. p. 27.

Zwierlein solo (sec. Lojacono).

267. - Melica minuta L.

Sulle rupi tra gli arbusti verso Capo Ponente e verso lo Scalo; Mrz. foglie! - Zodda (Capo Ponente, in dumetis rara, fi. e fr.).

268. - Scleropoa rigida (L.) Griseb. Poa rigida Guss. Suppl. Prodr. p. 22. Sclerochloa rigida Guss. Syn. I p. 94 ; Calc. p. 21. S. Zwierleinii Lojac. Esc. p. 27.

Arene e rupi, frequente; Mrz. fi., Apr. fi. e fr.! - Gussone (var. glaucescens), Zwierlein (nei luoghi el'bosi assieme alla Castellia tuberculosa sec. Lojacono), Zodda (M. Vulcano, M. Calcarella e M. Rosso, fi. e fr.). Calcara ha dimenticato l'* a indicare che fu raccolta da Gussone, e non si sa quindi se egli pure l'abbia trovata.

Tutti gli esemplari di Linosa che ho visti appartengono ad una forma stentata, benchè non sempre nana, ad infiorescenza depauperata, stretta, poco o punto ramificata. Questa è certamente la forma descritta da Lojacono col nome di S. Zwierleinii (riferita anche nella Flora analitica alla S. rigida). Gussone indica di Linosa la var. glaucescens; però la sua descrizione di questa varietá non si attaglia ai nniei esemplari i quali non sono sempre nani, e non hanno la pannocchia conferta e rigida, nè il culmo violaceo. Forme depauperate come questa di Linosa trovansi del resto frequentemente anche altrove che a Linosa, insieme alle forme tipiche.

269. - Poa annua L.; Guss. Syn. I p. 96 ; Calc. p. 21 ; Parl. Fl. It. I p. 349.

Molto comune dorunque, nel piano come in alto; Mrz. fi. e fr.! - Gussone.

270. - Dactylis glomerata L.; Guss. Syn. I p. 90 ; Calc. p. 21. D. Hispanica Guss. Suppl. Prodr. p. 22.

Gussone solo (rar. Hispanica [Roth.]). 
271. - Lamarckia aurea (L.) Moench; Solla p. 479; Parl. Fl. It. I p. 334. Cynosurees aureus Guss. Suppl. Prodr. p. 20. Chrysurus cynosuroides Guss. Syn. I p. 107; Calc. p. 21.

Assai frequente; Mrz. fi. e fr., Apr. fr.! - Gussone, Solla (fi. e fr.), Zodda ( $(\mathrm{r} r$.$) . Calcara ha dimenticato di indicare con un *$ che era stata trovata da Gussone, quindi in questo come in altri casi simili, non si sa se egli stesso l'abbia raccolta.

272. - Chrysurus echinatus Pal. de Beauv. Cynosumes echinatees Guss. Syn. I p. 108 ; Calc. p. 21 ; Parl. Fl. It. I p. 337.

Gussone solo.

273. - Koeleria phleoides (Vill.) Pers.; Calc. p. 21 ; Ross p. 348 ; Solla p. 479.

Comune nei luoghi arenosi ; Mrz. fi., Apr. fi. e fr.! - Calcara, Ross (nelle arene vulcaniche, molto grande e rigogliosa), Solla (fi. e fr.), Zodda (fi. e fr.), Sangermano (fi. e frr.).

Parte dei miei esemplari ha le spighette glabre e parte le ha pubescenti. Le stesse due forme trovansi fra le piante raccolte da Zodda e da Sangermano. L' ho trovata bene sviluppata, con pannocchia lobata lunga fino $12 \mathrm{~cm}$., come pure spesso l'ho trovata nana.

274. - Avellinia Michelii Parl.; Solla p. 479; Lojac. Esc. p. 27.

In luoghi arenosi vicino al mare; Apr. fi. e fr.! - Solla (fi. e fr.), Zwierlein (sec. Lojacono), Zodda (M. Vulcano, in aridis collium, fi. e fr.).

275. - Vulpia uniglumis (Sol.) Reichb.; Somm. Piante ined.

Luoghi aridi ; Apr. fr. ! - Zodda (M. Vulcano e costa di Levante, in dumetis, fi.).

276. - Vulpia myurus (L.?) Gmel.

Zodda solo (presso l'abitato, in arvis, fi. e fr.).

277. - Vulpia ciliata (Pers.) Link; Somm. Piante ined.

Qua e lá; Mrz. fi., Apr. fr.! - Zodda (fi. e fr.).

Spesso nana, non più alta di $1-2 \mathrm{~cm}$.

278. - Bromus tectorum L.; Guss. Syn. I p. 78 ; Calc. p. 20 ; Parl. Fl. It. I p. 409.

Gussone solo.

279. - Bromus Madritensis L.; ( Guss. Syn. I p. 78; (alc. 1). 21 ; Parl. Fl. It. I p. 406. 
Qua e lid specialmente nelle parti coltivate; Mr'. fi. e fr., Apr. fr.: - Gussone, Zodda (fi.).

Ho veduto soltanto la forma a spighette glabre.

280. - Bromus maximus Desf.

Zodda solo (costa di Levante, in cultis, fi.).

Le piante di Linosa appartengono alla stessa forma di quelle di Lampedusa.

281. - Bromus fasciculatus Presl; Calc. p. 21 ; Lojac. Esc. p. 27.

Frequente nel piano e sui colli; Apr. fi. e fr.! - Calcara, Zwierlein (sec. Lojacono), Zodda (fi.).

Parte dei miei esemplari ha spighette glabre, parte le ha pubescenti. Tutti quelli di Zodda le hanno glabre.

282. - Lolium rigidum Gaud.; Somm. Piante ined.

Raro; 24 Apr. fi. !

283. - Catapodium loliaceum (IIuds.) Link; Solla p. 479.

Comunissimo, specialmente nei luoghi arenosi prossimi al mare, con altre piante appressate al suolo come Medicago litoralis e Plantago Coronopes; Mrz. fi. e fr., Apr. fi. e fr.! Solla (fi. e fr.), Zodda (fi. e fr'.).

284. - Catapodium Siculum (Jacq.) Link; Solla p. 479.

Solla solo (fi. e fr.).

285. - Castellia tuberculosa (Moris sub Catapodio). C. tubercielata Tineo Plant. rar. Sic. fasc. $2^{\circ}$ p. 18; Calc. p. 20 ; Ross p. 348 ; Solla p. 479 ; Lojac. Esc. p. 28; Parl. Fl. It. I p. 480. Triticum tuberculosum Bert. Fl. It. VII p. 620. Festuca tuberculosa Cosson et Durieu de Maisonneuve Flore d'Algérie in Explor. sc. Alg. p. 189. Desmazierea tuberculosa Bonnet et Barr. Catal. rais. des plant. vasc. de la Tunisie p. 482 ; Battandier et Trabut Flore d'Alger et Catal. des pl. d'Algérie p. 100. Catapodium tuberculosum Fiori e Paol. Fl. an. I p. 96.

Abbondante nella macchia, dove l' ho raccolta in molti luoghi dal 22 al 24 Apr. fr.! - Calcara (nelle arene vulcaniche, sec. Tineo loc. cit.), Ross (nella parte orientale dell' isola), Solla (fi. e fr.), Zwierlein (nelle arene vulcaniche, sec. Lojacono), Zodda (Montagna Rossa, in dumetis, fi. e fr.). - Il dott. Ross l'ha distribuita nel suo Herbarium Siculum, n. 397, col nome di Fesluca tuberculosa, raccolta a Linosa da qualche suo corrispondente nel Maggio del 1903. 
Gli esemplari più ubertosi, tanto fra i miei, quanto fra quelli di Zodda, giungono ad avere $80 \mathrm{~cm}$. d'altezza, pannocchia con rami allungati e spighette con fino a 12-14 fiori.

Pianta con distribuzione geografica assai strana. Nota per molto tempo soltanto di Linosa e dei dintorni di Cagliari in Sardegna, è stata poi trovata in una isoletta della Grecia, in un punto dell'Algeria occidentale e nelle isole Canarie. L'indicazione di Lampedusa, come ho detto a p. 136, è erronea.

286. - Brachypodium distachyum (L.) Pal. de Beauv.; Guss. Syn. I p. 73 ; Ross p. 348 ; Parl. Fl. It. I p. 492. Festuca distachya Guss. Suppl. Prodr. p. 25.

Molto comune dovunque nell' isola; Mr'z. fi. e fr.! - Gussone (è quasi l'unica graminacea che formi pascoli primaverili), Ross, Zodda (fr.). Calcara ha dimenticato questa pianta indicata da Gussone.

Si trova sotto forme molto diverse, da quella nana (alta talvolta non più di $1-2 \mathrm{~cm}$.), rigida e ad una sola spighetta (a. monostachya Guss.) dei luoghi aridi, a quelle di uno sviluppo normale. Notevole è una forma che distinguo col nome di:

- Var. paradoxum mihi.

Planta elata $(35-45 \mathrm{~cm}$.) gracilis, culmis numerosis a basi ramosis geniculatis, foliis elongatis flaccidis obscure virentibus, panicula 4-6stachya, spiculis vix compressis aristis ante anthesin in penicillum conniventibus. Habitus $B$. sylvatici.

Nelle siepi e fra i fichi d'India, fra il Paese e lo Scalo; 4 Mrz. fi.!

Questa forma sembra intermedia fra il B. distachyım e il B. sylvaticum. Dal primo differisce per le caratteristiche sopra enumerate, e dal secondo per la radice annua, per le guaine e le spighette glabre, per la pannocchia più rigida e non flessuosa.

287. - Hordeum murinum L.; Solla p. 479.

Molto comune, specialmente nelle parti coltivate e lungo i viottoli; Mrz. e Apr. fi.! - Solla (fr.)., Zodda (fi. e fr.).

Gli esemplari che ho raccolti appartengono alla var. majus Gren. et Godr. $=H$. leporinum Link.

288. - Lepturus incurvatus (L.) Trin.; Solla p. 479; Parl. Fl. It. I p. 537. Rottboellia increvvata Guss. Syn. I p. 57; Calc. p. 20. 
Frequente in vicinanza del mare; Mrz. foglie, Apr. fi.! Gussone (il quale peró nel Suppl. al Prodr. non lo aveva citato di Linosa), Solla (fi.).

GYMNOSPERMAE.

$$
\text { LiIi. - Coniferae. }
$$

289. - Juniperus Phoenicea L. ; Cruss. Syn. II p. 634 ; Calc. p. 23 et 24; Ross p. 348 ; Solla p. 478 ; Pạl. Fl. It. IV p. 91; Lojac. Fl. Sic. II pars II p. 402. J. Lycia Guss. Notiz. p. 86.

Nella macchia, raro; Mrž e Apr. fr.! - Gussone, Ross, Solla (fr.). Calcara ha dimenticato il segno $*$ a indicare che era stato raccolto da Gussone. Non si sa quindi se egli pure l'abbia raccolto.

PTERIDOPHYTAE.

\section{LIV. - Filices.}

\section{0. - Ophioglossum Lusitanicum L.}

Molto comune nel piano, trovasi anche sulle cime dei monti; Mrz. spore! - Sangermano (Dic. spore).

E particolarmente abbondante nei terreni arenosi non lontani dal mare fra le microfite, insieme alla Tessellina, alle Riccia ed altre epatiche.

291. - Notholaena vellea (Ait.) R. Br.; Somm. Piante inerl.

Sulle rupi del M. di Ponente e del M. Vulcano, rara; Mrz. e Apr. spore !

292. - Polypodium vulgare L.; Solla p. 479.

Sulle rupi ombreggiate in varî punti dell' isola; Mrz. e Apr. spore! - Solla (per lo più secco), Zorda (spore).

Tutto il $P$. vulgare che ho visto di Linosa appartiene alla forma serratum Willd. Trovasi del resto tanto con fronde bene sviluppate quanto nano. 
293. - Grammitis Ieptophylla (L.) Swartz ; Solla p. 479.

Sulle rupi ombrose, tanto nelle buche delle colate di lava vicino al mare, quanto nelle parti più centrali dell' isola, non rara; Mrz. e Apr. spore! - Solla (interamente secca), Zodda (spore).

294. - Asplenium obovatum Viv.; Solla p. 479; Lojac. Esc. p. 28. A. lanceolatum var. obovatum Ross p. 348.

Sulle rupi in varî punti dell' isola; Mrz. e Apr. spore ! Ross, Solla, Zwierlein (sec. Lojacono), Zodda (spore).

Trovasi con le fronde bene sviluppate, bipennatosette, lunghe fino oltre $25 \mathrm{~cm}$. e larghe $8-9 \mathrm{~cm}$., e trovasi anche ridotto a piccolissime dimensioni (1-3 cm.) con foglie semplicemente pennatosette, che quasi somigliano all' $A$. Trichomanes.

B R Y O P Y T A E.

\section{Musci.}

(C1. marchese A. Bottini determinavit).

ACROCARPI.

1. - Phascum rectum With; Zodda Briofite Sicule, Contribuzione prima, in Malpighia XX (1906) p. 94.'

Zodda (sulle rupi del monte Vulcano, fr.).

2. - Gymnostomum calcareum Bryol. Germ. var. muticum Boulay.

Sulle rupi, alla Grotta dei Colombi ; 2 Mrz. ster.!

3. - Weisia viridula (L.) Hedw.

Zona sabbiosa piana vicino al mare; I Mrz. fr.!

Var. arenicola Limpr. Peristomii dentes magni in linea divisurali conspicua saepe pertusi, vel apice fissi, vel bipartiti.

Abbondante nella zona piana sabbiosa vicino al mare, ove

1 Questo lavoro, che contione alcune briofite raccolto a Linosa dallo stesso Zodda nel 1905, e da Solla nel 1884, manca nella mia bibliografia, essendo posteriore ad essa. 
forma talvolta da sola dei tappeti, e trovasi spesso mista ad epatiche; 1 Milrz. fr.!

Varietá fin ora sfuggita ai briologi italiani.

4. - Fissidens tamarindifolius (I)on. T'ur'11.) Brit. Formae typica et non typicae.

Sparsa più 0 meno per tutta l'isola, dalla zona marina fino in alto, sulle rupi e nella macchia; 1 e 6 Mrz. ster. e fr.!

Vedansi le osservazioni a questa specie nella Florula di Lampedusa, a p. 159 .

\section{5. - Fissidens pusillus Wils.}

Luoghi ombrosi alla Grotta dei Colombi e sul monte Vulcano; 2 Mrrz. fr.!

6. - Pottia intermedia (Turn.) Fürnr.

Questo è il più comune dei muschi nella zona piana di terreno sabbioso vicino al mare, dove abbonda e forma spesso degli estesi e fitti tappeti riccamente fruttificati; 1 e 6 Mrz. fr.!

7. - Pottia Wilsoni (Hook.) Br. Eur.

Sulle rupi alla Grotta dei Colombi e sul m. Vulcano; 2 Mrz. fr.! Trovata nell'Arcipelago Toscano, in Sardegna, in Corsica e a Nizza.

8. - Pottia Starksana (Hedw.) C. Müll.

Nella zona marina sulla costa Nord; 4 Mrz. fr.!

9. - Pterygoneurum lamellatum (Lindb.) Jur.

Monte di Ponente sopra la Pozzolana; $6 \mathrm{Mr}$. fr. perfetto!

Sporadico inell Europa nordica e media. Rarissimo in Italia (Trento, Cuneo, Modena).

10. - Didymodon tophaceus (Brid.) Jul. f. acutifolius Boulay.

Abbondante sulle rupi alla Grotta dei Colombi; 2 Mrz. fr. giov.!

\section{1. - Trichostomum crispulum Bruch?}

Zodda (esemplari imperfetti, in herb. Martelli).

12. - Trichostomum mutabile Bruch var. densum I3r. Eur.

Alla Pozzolana e a Capo Ponente; 3 e 6 Mrz. ster. e fr.!

13. - Trichostomum nitidum (Lindb.) Schimp. var. medium Boulay.

Zodda (monte di Ponente, in herb. Martelli, ster.).

Var. obtusum Boulay.

Monte Vulcano; 2 Mrz. ster.! 
14. - Trichostomum flavovirens Bruch.

Abbondante nei luoghi sabbiosi piani, non lontano dal mare; 3 e 6 Mrz. ster. e fr.!

Var. nitidocostatum Bott. I primi muschi delle isole Eolie Bull. Soc. Bot. it. 1903; Zodda loc. cit. p. 92.

Zodda (sulle rupi, ster.).

15. - Leptobarbula Berica (De Not.) Schimp.

Monte Vulcano, qua e là; 2 Mrz. fr.!

Specie sporadica e rar'a in Italia e fuori.

16. - Tortella tortuosa (L.) Iimpl'. ral' fragilifolia Jur.

Luoghi pianeggianti della zona marina, dove forma dei tappeti; 1 Mrz. ster.!

17. - Barbula revoluta (Schrad.) Brid.

Grotta dei Colombi, rara; 2 Mrz. ster.!

18. - Barbula convoluta $\mathrm{Hdw}$.

Luoghi arenosi vicino al mare, insieme a specie di Riccia; 1 Mr'z. ster.!

19. - Tortula atrovirens (Smith) Lindb. Desmatodon atrovirens Zodda 1. c. p. 93.

Frequente, raccolta sul monte di Ponente, e sui terreni pianeggianti della zona marina; 1 e 6 Mrz. fr.! - Zodda (sulle rupi, ster.).

20. - Tortula muralis (L.) Hedw.; Zodda 1. c. p. 93.

Sulle rupi molto comune in molte parti dell' isola; 2 e $6 \mathrm{Mrz}$. ster. e fr.! - Solla (alla bocca di una cisterna, sec. Zodda, anteridî e fr.), Zodda (in herb. Martelli).

21. - Tortula aestiva P. de B.; Zodda 1. c. p. 92.

Solla ( $m$. Bandiera sul tufo vulcanico, sec. Zodda, anteridî e fr.).

22. - Tortula marginata (Br. Eur.) Spruce; Zodda 1. c. p. 92.

Frequente sulle rupi; raccolta alla Grotta dei Colombi e sul monte Vulcano; 2 e $3 \mathrm{Mrz}$. fr. ! -- Solla (alla bocca di una cisterna, sec. Zodda, fr.).

23. - Tortula Solmsii (Schimp.) Vent. et Bott.!

Alla Pozzolana di Ponente, ivi abbondante; 3 Mrz. fr. giov.!

Bellissima e rara specie, nota soltanto di Algarvia di Madera, dell'isola di San Pietro in Sardegna, dell'isola di Salina, di Messina, Malta e Pantelleria. 
24. - Grimmia pulvinata Smith var. minor Boulay; Zodda l. c. p. 93.

Zodda (sulle rupi, ster.).

25. - Grimmia Lisae De Not.

Sulle rupi alla Grotta dei Colombi; 2 Mrz. fr.! - Zodda (in herb. Martelli, fr.)

26. - Entosthodon curvisetus (Schwgrr.) C. Müll.

Frequente sulle rupi nei luoghi piu ombrosi, misto a varie epatiche, in diverse parti dell' isola; 2 e 6 Mrz. fr.!

27. - Entosthodon pallescens Jur.

Sulle rupi alla Grotta dei Colombi, raro; 2 Mrz. fr.!

Vedansi le osservazioni a questa specie nella Flor. Lampedusa p. 161.

28. - Funaria Mediteranea Lindb. var. patula Br. Eur.

Alla Grotta dei Colombi ; 2 Mrz. fr.!

29. - Funaria dentata Crome.

In varî punti dell' isola; 2-4 Mr'z. fr. !

30. - Funaria hygrometrica (L.) Sibth.; Zodda 1. c. p. 92. Luoghi arenosi piani, vicino al mare; I Mrz. fr.! - Solla (alla bocca di una cisterna, sec. Zodda, fr.).

Var. calvescens (Schwaegr.) Br. Eur.; Zodda 1. c. p. 92.

Don Luigi Sangermano (fr.), Solla (col tipo alla bocca di una cisterna, sec. Zodda, fr.).

31. - Funarla convexa Spruce.

Zodda (monte Bandiera, in berb. Martelli, fr.).

32. - Bryum torquescens Br. Eur.

Monte Vulcano; 2 Mrz. fr. e fi. \& !

Forma orthophyllum Bottini Flor. briol. dell'Arcip. toscano p. 184.

Sulla costa Nord, raro; 4 Mrz.! fr. e fi. \&!

33. - Bryum Donianum Grev.

Pozzolana di Ponente, raro; 3 Mrz. ster !

34. - Bryum caplllare L.

Monte Vulcano, raro; $5 \mathrm{Mrz}$. fr. e fi。 o !

Var. flaccidum Br. Eur. Folia usque ad $4 \mathrm{~mm}$. longa!

Sulla costa Nord, raro; 4 Mrz. ster.!

35. - Bryum caespiticium L. var. Kunzei (Hpp. et IIornsch.); Zodda 1. c. p. 91.

Secondo Zodda (sulle rupi, con anteridî). 
36. - Bryum murale Wils.; Zodda 1. c. p. 91.

Solla (alla bocca di una cisterna sec. Zodda, con anteridî).

37. - Bryum atropurpureuin Br. Eur. non Wahl.

Varî luoghi, ma non abbondante, nei terreni arenosi vicino al mare e sul m. Vulcano; 1 e 2 Mrz. fr.!

Var. dolioloides Solms Laub.

Verso il centro dell'isola, alla Grotta dei Colombi ; 2 Mrz. fr.!

38. - Bryum Canariense Brid.

Qua e lá, raccolto sul M. Vulcano, ed in luoghi arenosi presso

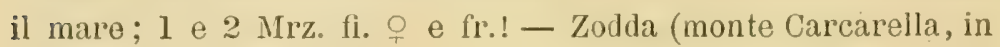
Herb. Martelli, fi. ㅇ).

E la forma dioica e meno sviluppata di un tipo che comprende altra forma robusta, sovente (non sempre) ermafrodita, cioé il nostro $\mathrm{Br}$. provinciale. Era giả noto di Rapallo, di Toscana, di Corsica e di Bari.

\section{PleURocarpi.}

39. - Eurhynchium circinatum Brid. Br. Eur.; Zodda 1. c. p. 91.

Molto comune in tutta l'isola, specialmente nei luoghi piu freschi; 2-6 Mrz. ster. e fr. ! - Zodda (in herb. Martelli, ster.). Forma attenuatum Boulay.

M. Vulcano; 2 Mrz. ster.!

Var. nov. myosuroideum. Tenellum: formis minoribus Eurhynchii myosuroidis facie persimile. Surculi erecti, dendroides ramosi, ramis et ramulis in unum sensum curvatis, apice attenuatis haud circinatis. Folia ramulina minus dense imbricata, longius acuminata, parte superiore patentia vel subpatula. Bottini.

Punta di Levante; 3 Mrz. fi. ठౌ!

Varietå nuova, molto spiccata e singolare.

40. - Rhynchostegium tenellum (Dicks.) Br. Eur.

Alla Pozzolana: 3 Mrz. fr.! 


\section{Hepaticae.}

(Cl. Caro Massalongo determinavit).

\section{1. - Gongylanthus ericetorum (Raddi) Nees.}

Luoghi sabbiosi bassi, sotto il monte di Ponente; $3 \mathrm{Mrz}$. ster. ! 42. - Fossombronia angulosa Raddi.

Comunissima in tutta l'isola, specialmente nei luoghi pianeggianti sabbiosi, con altre epatiche, nei luoghi dove crescono l'Ophioglossum Lusitanicum ed altre microfite; Mrz. ster. e fr.!

\section{3. - Fossombronia caespitiformis De Not.}

Alla Pozzolana di Ponente e alla Grotta dei Colombi ; Mrz. fr.!

44. - Fossombronia pusilla Du Mort.

Abbondante in varie parti dell'isola, specialmente nei luoghi bassi sabbiosi ; Mrz. fr.!

45. - Fossombronia cristata Lindb.

Alla base del monte di Ponente, nel suolo sabbioso; $6 \mathrm{Mrz}$. fr. giov. !

46. - Lunularia cruciata (L.) Du Mort. Lemularia vulgaris Zodda Briof. Sic. I in Malpigh. XX p. 94.

Frequente specialmente nei luoghi ombrosi piu freschi; Mrz. piante gremmifere! - Solla (sul tufo di monte Bandiera, sec. Zodda), Zodda (ster, in herb. Martelli).

47. - Reboulia hemisphaerica (L.) Raldi; Zoddia I. c. p. 94. Solla (sul tufo di m. Bandiera, sec. Zodda).

48. - Cievea Rousseliana (Mont.) Leitg.

Bella ed abbondante sulle rupi esposte a N., nel luogo detto Grotta dei Colombi, dove crescono pure rigogliose la Targionia, la Corsinia, l'Entosthodon curviseles ed altre briofite: trovata pure in piccola quantiti sul monte di Ponente; 2 e 6 Mrz. fr. !

Questa interessante Marcanziåcea, scoperta in Algeria dal Roussel, si conosce inoltre soltanto di Palermo, dove fu trovata per la prima volta in Europa dal D. ${ }^{\mathrm{r}}$ Lanza, di Acireale, dove l'ha raccolta il D. Zodda, e di Corfù.

49. - Targionia hypophylla L. ex Micheli; Zodda I. c. p. 94.

Comunissima sulle rupi ombrose dei monti, trovasi anche nel piano; fr. Mrz.! - Solla (tufi del monte Bandiera, sec. Zodda). 
50. - Corsinia marchantioides Raddi.

Comune nei luoghi ombrosi freschi, particolarmente abbondante sulle rupi ombrose del luogo detto Grotta dei Colombi; Mrz. fr.!

51. - Tessellina pyramidata Du Mort.

Abbondante, mista alle Riccia ed altre epatiche nei luoghi piani sabbiosi; l'ho trovata pure sul monte Vulcano; Mrz.!

52. - Riccia glauca L.

Monte di Pollente; $6 \mathrm{Mrz}$.

53. - Riccia insularis Levier.

Abbondante nei luoghi bassi sabbiosi, insieme ad altre epatiche, in terreno da microflora; Mrz.!

54. - Riccia nigrella DC.

Luoghi pianeggianti sabbiosi, rara; $1^{\circ}$ Mrz.!

55. - Anthoceros levis L.

Frequente insieme ad altre epatiche, specialmente nei luoghi bassi sabbiosi, dove crescono l' Ophioglossum Lusitanicum ed altre microfite; Mrz. ster. e fr. immat.!

\section{LICHENES.}

(Cl. A. Jatta determinavit).

1. - Usnea Soleirolii Duf. Bryopogon arenarium Fr. in Ross p. 349.

Ross (colla Roccella phycopsis, ma meno comune).

2. - Evernia Prunastri Ach. Univ. p. 442; Jatta Syll. p. 60; Albo, I primi licheni di Linosa e di Lampedusa p. 43. '

Zodda (sui tronchi).

3. - Ramalina Arabum Nyl.; Jatta Syll. p. 62; Albo l. c. p. 43. Alectoria sarmentosa var. crinalis Fr. in Solla Phytobiol. Beob. p. 480 ?

Abbondante sulle rocce; Mrz., Apr.! - Solla?, Zodda (sulle rupi).

1 Vedi la nota a p. 163 nella Florula di Lampedusa. 
La Alectoria sarmentosa Ach. var. crinalis Fr. essendo un lichene che viene sulle conifere d'alto fusto nelle selve dell'Italia settentrionale, e non potendo quindi trovarsi a Linosa, è probabile che la citazione di Solla debba riferirsi alla Ramalina Arabum Nyl., che, per la forma esterna del tallo, puó confondersi con essa.

4. - Ramalina Îraxinea Ach. Unir. p. 602; Jatta Syll. p. 64; Albo p. 44.

Zodda (Albo l. c. dice « ad rupes»; ma è specie che cresce sui tronchi).

5. - Ramalina digitellata Nyl. in Bot. Soc. Brotel. VI p. 211 ; Albo p. 42 et 44 .

Sulla terra nella macchia; Mrz.! - Zodda (sulle rupi).

Questa specie, nuova per l'Italia, era finora nota soltanto delle coste del Portogallo e dell'isola di San Thomè.

6. - Ramaina Duriaei De Not.; Jatta Syll. p. 66. R. pollinaria Ach. var. Duriaei De Not, in Solla p. 480 ; Albo p. 44.

Invade il tronco e i rami degli arbusti morenti; Mrz.! Solla, Zodda (sul legno; Albo 1.c. dice certo erroneamete che cresce anche sulle rupi, probabilmente per aver confuso la sta. zione di questa specie con quella della specie seguente).

7. - Ramalina Bourgaeana (Mtg.) Nyl.; Jatti Syll. 1). 67. R. maciformis Del. in Solla P. 480 ; Albo p. 42 et 44 .

Negli anfratti delle rupi; Mrz.! - Solla, Zodda (Albo 1. cit. dice per errore sul legno, poichè questa specie è essenzialınente sassicola).

Specie nuova per la Sicilia (Albo 1. cit.).

8. - Cladonia pungens IIrb.; Jatta Syll. p. 93; Albo p. 44.

Sulla terra nella macchia; Mrz.! - Zodda (sulla terra).

9. - Cladonia muricata Del. in Dub. Bot. Gall. p. 662; Jatta Syll. p. 93 ; Albo p. 44.

Zodda (sulla terra).

10. - Cladonia pyxidata Fr.

Monte Vulcano, sulla terra; Mrz.!

11. - Roccella tinctoria DC.; Solla p. 480; Jatta Syll. p. 71 ; Albo p. 44.

Abbondante sulle rupi; Mrz., Apr.! - Solla, Zodda (sulle rupi). 
12. -- Roccella phycopsis Ach.; Ross, Eine bot. Excurs. p. 348; Jatta Syll, p. 71.

Sulle rupi; Mrz.! - Ross (molto comune sulla lara nuda).

13. - Roccella pygmaea Mtg. Alg. p. 266; Jatta Syll. p. 71; Albo p. 42 et 44 .

Zodda (sulle rupi).

Specie nuova per la Sicilia (Albo I. cit.).

14. - Parmelia perlata L. var. ciliata Schaer. Imbricaria perlata var. ciiialu Schaer. in Solla p. 480.

Solla.

15. - Physcia stellaris (Ach.) Fr. var. leptalea (. $\mathrm{ch}$.) Th. Fr. Scand. p. 140 ; Jatta Syll. p. 141. Parmelia stellaris var. adscendens ** leptalea Albo p. 44.

Zodda (sulle rupi).

16. - Physcia setosa (Ach.) Nyl.; Jatta Syll. p. 142. Parmelia setosa Albo p. 42 et 44 .

Zodda (sulle rupi).

Specie nuova per la Sicilia (Albo l. c.).

17. - Xanthoria parietina (L.) Fr. Ph!lscia parielsace De Not. in Solla p. 480 .

Il tipo raccolto da Solla solo.

Var. ectanea Nyl.; Jatta Syll. p. 149; Aibo p. 44.

Abbondante sulle rocce anche le più nude su cui non cresce altra pianta: Mrz.! - Zodda (sulle rupi).

Col suo tallo di un arancione intenso, che fa un bel contrasto col colore nero delle rocee vulcaniche a cui aderisce, questa varieta, che trovasi in tutte le parti dell'isola, forma un tratto caratteristico del paesagrgio botanico.

Var. aureola Fr.; Solla p. 480; Jatta Syll. p. 149 ; Albo p. 44. Sulle scorze; Mrz.! - Solla, Zodda (sulle rupi).

18. - Theloschistes villosus (Dub.) Norm.; Plyscia villosa Dub. in Solla p. 480.

Solla.

19. - Lecanora crassa Ach. val. caespitosa Schaer. Spic. p. 432 ; Jatta Syl!. p. 175 ; Albo p. 44.

Zodda (sulla terra).

20. - Lecanora dispersa (Pers.) Krb.; Jatta Syll. p. 186; Albo p. 44.

Zodda (sulle rupi). 
21. - Lecanora albella Ach. Univ. p. 369 ; Jatta Syll. p. 194; Albo p. 44.

Zodda (sul legno).

22. - Lecanora Hageni Ach. var. lithophila ( $/$ 'llr.) Krb. Prg. p. 80 ; Jatta Syll. p. 196 ; Albo p. 44.

Zodda (sulle rupi).

23. - Lecanora pallescens Schaer. var. Parella Fr. L. E. p. 133; Jatta Syll. p. 209; Albo p. 44. Ochrolechia pallescens var. Parella (Fr.) Krb. in Solla p. 480.

Solla, Zodda (sulle rupi).

24. - Lecanora calcarea smrf. val. viridescens (Mass.) Kr'b. Prg. p. 95 ; Jatta Syll. p. 214 ; Albo p. 45.

Zodda (sulle rupi).

25. - Caloplaca aurantiaca I.gthf. var. salicina (Schrad.) Mass. Blast. p. 77 ; Jatta Syll. p. 248; Albo p. 45.

Zodda (sui tronchi).

26. - Caloplaca cerina (Ehr') 'Th. Fr. var. Ehrarti Krb. Syst.

p. 127 ; Jatta Syll. p. 253 ; Albo p. 45.

Zodda (su varî tronchi).

27. - Caloplaca subsimilis Th. Fr. Scand. 1. 189; Jatta Syll.

p. 257 ; Albo p. 45.

Zodda (sulle rupi).

28. - Rinodina exigua Mass. $R$. exigua var. pyrina Solla p. 480 .

Solla.

29. - Rinodina atrocinerea (Dcks.) Irrb. Syst. 10. 125; Jatta Syll. p. 273 ; Albo p. 42 et 45.

Zodda (sulle rupi).

Specie nuova per la Sicilia (Albo 1. c.).

30. - Dirina Ceratoniae Fr.; Solla p. 480.

Solla.

31. - Lecidea contigua Hoffm.; Solla p. 480.

Solla.

32. - Lecidea sabuletorum Flk. Berl. May. 1s0S 1). 309 ; Jatta Syll. p. 348 ; Albo p. 45.

Sulle rocce; Mrz.! - Zodda (sulle rupi).

33. - Lecidea viridans FW. in Fl. 1828 p. 697; Jatta Syll. p. 348 ; Albo p. 43 et 45 . 
Zodda (sulle rupi).

Specie nuova per la Sicilia (Albo l. c.).

34. - Buellia canescens (Dclis.) 1)e Not. Fram. p. 197; Jatta Syll. p. 385 ; Albo p. 45. Diploïcia epigaea var. efrigurata Solla p. 480.

Frequente sulle rocce; Mrz.! - Solla, Zodda (sulle rupi).

35. - Buellia Ieptoclinis (Fw.) Ir rb. Syst. p. 225; Jatta Syll. p. 389 ; Albo p. 45.

Zodda (sulle rupi).

36. - Buellia spuria Krrb. var. lactea Mass. Sch. cr. p. 153; Jatta Syll. p. 390 ; Albo p. 46.

Zodda (sulle rupi).

37. - Diplotomma porphyricum Arnd.

Sulle rocce; Mrz.!

38. - Diplotomma alboatrum (Hoffm.) Krb. var. corticola Schaer. En. p. 122; Jatta Syll. p. 425; Albo p. 46.

Zodda (sul legno).

\section{ALGAE. 1}

(Cl. A. Borzì determinavit).

1. - Amphiroa cryptarthrodia Zanard. corall. p. 21. Mrz. 1906 !

2. - Amphiroa rigida Lamour. polyp. flex. p. 297. Mrz.!

3. - Aphanocapsa membranacea Rabh. Fl. Eur. Alg. aqu. dulc. II p. 49.

Sulla spiaggia; Mrz.!

4. - Brachytrichia maculans Gomt. in Johs.

Sugli scogli marini; Mrz.!

Oss. - Tutti gli esemplari osservati erano contraddistinti da fronde piane, crostacee, aderenti fortemente al substrato e co-

1 Trabucco ( $\mathrm{L}^{\prime}$ is. di Linosa ecc. p. 8) dà un piccolo elenco di alghe marine tolte dal lavoro di Solla; ma avendo sbagliato nota, come per gli insetti, attribuisce a Linosa le alghe di Lampedusa. 
stituenti delle macchie tenuissime di color nericcio, a contorno sinuoso irregolare. Per tali caratteri l'alga va riferita senza dubbio alla specie descritta dal Gomont, nel lavoro succitato, col nome di $B$. maculans. Peró io dubito che questa forma possa considerarsi come distinta dalla $B$. Balani Born. et Fl., specie abbastanza diffusa sugli scogli e sui gusci delle conchiglie sulle coste dell'Atlantico e nel IIediterraneo, poichè in quest' ultima forma, anche le fronde allo stadio giovanile, assumono un aspetto crostiforme e aderiscono al substrato, mentre più tardi, com'è noto, divengono spesse, carnose e nostociformi. Checchè ne sia, importante è il fatto che la $B$. maculans è stata finora rinvenuta nei mari del Siam in Asia. Tale specie deve essere anche abbastanza comune sulle coste della Sicilia. Il sig. Antonio Riccobono ne raccoglieva dei saggi sulle rupi marittime dello Scoglio dei Ciclopi, presso Catania, nella primavera del 1903.

5. - Bryopsis plumosa Ag.; Solla Auf einer Excurs. n. d. pelag. Inseln gesammelte Meeresalgen p. 7 .

Solla (sulle pietre alla superficie).

6. - Calothrix crustacea Thr: Notes Alg. I, p. 13-16, tab. IV. Sui gusci delle patelle; Mrz.!

7. - Ceramium ciliatum Ducl.; Solla p. 5.

Solla (sulla costa meridionale, alla superficie, non ráro).

8. - Ceramium filabelligerum J. Ag. advers. p. 27. Mr'z. !

9. - Cladophora albida Kuetz. ph. germ. p. 240.

Nirz. !

10. - Cystoseira abrotanifolia Ag. sp. alg. p. 63.

Mrz.!

11. - Cystoseira amentacea Bory; Solla p. 6.

Solla (sugli scogli di lava nelle piccole insenature delia costa occidentale, vicino alla superficie, non rara).

12. - Cystoseira concatenata Mont. fl. alg. p. 15.

Nir.!

13. - Cystoseira discors Ag. sp. alg. p. 62 .

Mr $\%$ !

14. - Dasycladus clavaeformis Ag. sp. alg. p. 16.

MI'z.!

15. - Dictyota fasciola Lamour. in Journ. d. bot. 1809; Solla p. 6. 
Mrz.! - Solla (costa meridionale, non rara).

16. - Enteromorpha compressa Grev.; Solla p. 6.

Solla (sulla costa O. sotto Vulcano di Ponente, e piu raro sulla costa S., nuotante alla superficie con Ulva, Lacheca).

17. - Enteromorpha intestinalis Link sp. in Fl. ph. Berol.p. J.

Mrz.; stato gioranile!

18. - Gastroclonium Salicornia Kuetz.; Solla p. 5.

Solla (sugli scogli della costa meridionale, ad un braccio di profonditi, raro).

19. - Gelidium capillaceum Kuet\%. ; Solla p. 5.

Solla (sulla costa meridionale, vicino alla superficie, frequente).

20. - Gigartina acicularis Lamour. ess. p. 48.

Mr\%.!

21. - Halimeda Tuna Lamour. exp. m. p. 27.

Mrz.!

22. - Haliseris polypodioides Ag. sp. p. 142 .

Mrz. !

Var. microphylla Vin. not. alg. p. 42.

Mrz.!

23. - Hydroclathrus sinuosus Zan.; Solla p. 0.

Solla (sugli scogli della punta S. O., ad una discreta proionditá, non frequente).

24. - Jania rubens Lamour. polyp. (lex. p. 272.

Mr'z.!

25. - Laurencia obtusa Lamour. ess. p. 42; Solla p. 6 .

Mrz. - Solla (allo Scalo nuoro sulla costa di ponente, non lontano dalla superficie, non rara).

26. - Laurencia papiliosa Grev. alg. brit. 1830.

Mrz.!

27. - Melobesia sp. Solla p. 6 .

Solla (sulle Cystoseira).

28. - Nodularia Harveyana Thr. in Anu. Sc. nat. VI sẻr. I p. 378.

Sulla spiaggia; Miz.!

29. - Padina Pavonia Gaillon. dict. d'H. N. LIII p. 371; Solla p. 6 .

Mrz. : - Solla (sugli scogli della costa meridionale, a diverse profonditá, non rara). 
30. - Phyllosiphon Arisari lïlhu; Ross Eine bot. Exc. p. 319.

Ross (parassita sulle foglie dell'Arisarum vulgare).

31. - Polysiphonia opaca Zan.; Solla p. 6.

Solla (sulla costa meridionale, ad un braccio di profonditá, meno rara della $P$. sertularioilles).

32. - Polysiphonia sertularioides J. Ag.; Solla p. 6.

Solla (sugli scogli della costa meridionale, ad un braccio di profonditi, piuttosto rara).

33. - Porphyra leucosticta Thur. in Le Jol.; Solla p. 5.

Solla (sugli scogli della costa meridionale, alla superficie).

34. - Sphacelaria scoparia Lyngb. hydr. dan. p. 104.

MIr\%.!

35. - Ulya Lactuca L. sp. pl. II p. 1163; Solla p. 6.

Mrz.! - Solla (scogli della costa meridionale, vicino alla superficie).

36. - Valonia utricularis Ag.; Solla p. 7.

Solla (sulla costa meridionale, alla profondita di un braccio, rara).

37. - Zoddaea viridis Borzi Nuova Notarisia Serie XVII Gennaio 1906.

Zodda sulle rupi vulcaniche umide.

Genere nuovo di cloroficee, scoperto a Linosa nell'Aprile del 1905 dal dott. Zodda, e dal prof. Borzi dedicato al suo scuopritore.

\section{EUNGI.1}

(Cl. P. A. Saccardo determinavit).

\section{1. - Melaspilea opegraphoides $\mathrm{Nyl}$.}

Sui rami di Periploca levigata; Mrz.!

\section{2. - Stropharia merdaria $F \mathrm{r}$.}

Sul Monte Vulcano; Mrz.!

3. - Uromyces Pisi (Pers.) De Bary.

St. pycnid.: Ecidiolum exanthemalicum Ung.

Sulla Euphorbia Terracina; Mrz.!

1 Mi fu detto dagli abitanti di Linosa che d'inverno si trovavano dei funghi mangerecci, e che funghi velenosi non vi erano. 


\section{LAMPIONE}

Questa isoletta, dove non mi fu possibile andare neppure quest'anno a causa del tempo burrascoso, ma che dall'alto di Capo Ponente ho vista disegnarsi come una sottile lineetta all'orizzonte, ci è descritta dallo Smyth (p. xxxv e 289) e da Gussone (Notizie p. 80-81). Calcara non potè visitarla «sendo essa per la sua piccolezza inabitata, e reso a un tempo consapevole che le regole sanitarie ne proibiscono l'approdo ». Sanvisente ne dice solo poche parole, perchè neppure lui poté andarvi «onde non assoggettare a quarantena le altre die isole ».

Lampione (Lat. N. $35^{\circ} 33^{\prime}$, Long. O. di Roma $0^{\circ} 8^{\prime}$ ) è situato a Ponente di Lampedusa, e ne è distante Km. 17,5. É la piú piccola delle Pelagie, avendo incirca un diametro massimo, da N. a S., di $250^{\mathrm{m}}$, un diametro minimo da E. a O. di $100^{\mathrm{m}}$, un perimetro di $700^{\mathrm{m}}$ ed un'area di Kmq. 0,03. ${ }^{1}$

In passato venne chiamato Scola, Schola o Scolla, nomi coi quali lo vediamo segnato presso a Lampedusa sulla maggior parte degli antichi portolani. Secondo Gussone gli furono dati pure i nomi di Fanale e di Scoglio de' Scolari. D'Avezac (Iles de l'Afrique p. 119), contrariamente all'opinione generale, crede che il nome di Ethusa si applicasse a Lampione anzichè a Linosa.

Ha forma quasi triangolare. La sua superficie è un piano inclinato che dal lato maggiore del triangolo che guarda Ponente, e dove raggiunge le altezze di 33 e $36 \mathrm{~m}$., va gradatamente abbassandosi verso Levante dove forma una punta bassa, molto

\footnotetext{
1 Indicazioni favoritemi dal prof. Attilio Mori dell' Istituto geografico militare.
} 
frequentata, al dire del Cap. Smyth, dalle foche, e che si prolunga sutt'acqua in bassi fondi. Meno che verso questa punta, la costa è formata da dirupi quasi verticali. Vi si trovano traccie di abitazioni antiche cuoprenti uno spazio di 60 piedi, nelle quali lo Smyth trovo ancora una volta ben conservata con intonaco colorato, e resti di pavimento tessellato in marmo, di epoca romana.

La formazione geologica di Lampione è identica a quella di Lampedusa; gli strati e banchi delle due isole sono perfettamente corrispondenti, ed il canale che le separa non oltrepassa la profonditá massima di $85 \mathrm{~m} .{ }^{1}$ cosicchè si deve supporre che fossero una volta unite. Le corrosioni del mare devono lentamente ma continuamente diminairne la superficie, la quale probabilmente anche in tempi storici, quando Lampione fu abitato, era notevolmente magggiore di oggi, poichè male si spiegherebbe che in un isolotto cosi piccolo ssi costruissero edifizî a volta con pavimento di marmo.

Il prof. Giglioli che ha visitato Lampione due volte, nel Iuglio 1882 e nell'agosto 1890, mi dice di avervi trovato abbondante un falco, Ilypotriorchis Eleonorae (Gené) ed un palmipede, il Puffinus Kuhlii (Boie), e, cosa strana, la comune lucertola, Podarcis miralis (Laur.), che manca a Lampedusa.

Gussone, il solo botanico che abbia visitato Lampione, vi stette poche ore il. 15 agosto 1828. Egli scrive (Notizie p. 87) che le piante fanerogame di Lampione non oltrepassano le 20 specie. Nella sua Synopsis non ho trovato indicato che le seguenti $11:^{2}$

Capparis rupestris Gussone Syn. II p. 3.

Lavatera arborea Gussone Notiz. p. 87 et Syn. If p. 229.

Mesembryanthemum nodiflorum Syn. I p. 554 .

1 Nediterranean, Sardinia to Malta including Sicily; compiled from the latest British and foreign government Surveys. London, $19 \mathrm{Ag}$. 1880, published under the superintendence of Cap. F. J. Evans.

2 Nella Flora analitica, vol. II p. 330, Fiori cita la Statice Panormitana 'I'od. per Lampione, come raccolta da Pasquale. Questi peró non fu mai a Lampiono, e soltanto distribui alcune delle piante raccolte da Gussone o conservate a Napoli. Nell'erbario centralo di Firenze non trovasi alcun esomplare corrispondente alla citazione del Fiori. 
Daucus rupestris Syn. I p. 335.

Statice alkida Syn. I p. 369.'

Arthrocnemum glaucum Syn. I p. 7 (sub Saliconnia macrostachya).

Obione portulacoides Syn. II p. 588 (sub Alriplice).

Atriplex Halimus Syn. II p. 587. 2

Allium Ampeloprasum rar. commutatum Syll. I p. 392 (sub A. rotundo). ${ }^{3}$

Dactylis glomerata var. Hispanica Syn. I p. 90.

Lepturus incurvatus Syn. I p. 57 (sub Rottboellia).

E certo che anche il numero di 20 assegnato da Gussone a Lampione, è molto inferiore al numero di fanerogame che questo isolotto alberga, poichè nel mese di agosto, epoca della visita di Gussone, di non poche piante invernali e primaverili non esistono più traccie riconoscibił. Il prof. Giglioli che vi fu pure nella stagione estiva, mi dice che ví trovó la vegetazione interamente bruciata dal calore e dall'ariditá. Sull'isolotto dei Conigli presso Lampedusa, che ha una superficie di poco superiore a quella di Lampione (Kmq. 0,04), in meno di un'ora di erborazione in marzo, abbiamo raccolto 75 fanerogame.

${ }^{1}$ Specie indicata da Gussone per Lampione soltanto, e che io unisco alla S. bellidifolia sotto il nome di $S$. psiloclada Boiss. Vedasi quello che ho detto in proposito nella Florula di Lampedusa, a p. 134.

\& La medesima forma che a Lampedusa.

${ }^{3}$ Come ho detto nella Florula di Lampedusa a p. 146, Gussone nella Florula Inarimensis ha riferito all' $A$. commulatum tutto ciò che egli nella Syn. Fl. Sic. avera chiamato $A$. rotundum. 


\section{Aggiunte alla Florula di Lampedusa}

Gli invii di piante fattimi dalla guardia campestre di Lampedusa Martorana, dopo che era giá stampata la mia Florula, la pubblicazione recente di un volume della Flora Sicula e delle «Addenda et emendanda " di Lojacono, ed alcune citazioni di piante Lopadusane che mi erano sfuggite, mi obbligano a pubblicare queste "Aggiunte 》. Importante era specialmente che venissero premesse alla seguente tabella le 6 specie di cui viene ad arricchirsi la Florula di Lampedusa, onde giustificare la loro citazione nella tabella stessa.

Il totale delle specie vascolari di Lampedusa viene cosi portato a 464 .

Dopo 158 si inserisca:

1) - Ammi majus I. A. intermedicm Lojacono Add. et em. ad Fl. Sic. in Malpighia XX p. 294.

Lojacono loc. cit. dice che Calcara ha raccolto a Lampedusa I'A. intermedium DC., che io considero come semplice varieta dell'A. majes. Calcara nella sua Florula non ne aveva fatto menzione.

A 187 Conyza ambigua DC. si aggiunga:

Questa specie che fin'ora era stata indicata di Lampedusa da Calcara soltanto, mi è stata mandata in Gennaio 1907 da Martorana, in fiore e in frutto.

Dopo 221 si inserisca :

\section{2) - Centaurea acaulis L.}

Me ne è stato mandato da Martorana un esemplare in boccio, raccolto il 10 di Marzo 1907.

Questa bella specie non era ancor'a stata indicata per l'Europa. Essa è comune in tutta la Tunisia dove dalle sue radici si estrae un colore giallo adoprato dai tintori. Trovasi pure in Algeria (Bonnet et Barratte Catal. rais. d. pl. de la Tunisie 
p. 246 e 509), - Nartorana, rispondendo alle mie domande, mi informa che cresce in campi di grano, dove peró è rara assai, e che ha fatto la sua comparsa a Lampedusa soltanto da una diecina di anni. Si tratterebbe dunque di una specie di nuova introduzione, destinata forse a sparire, forse invece ad estendersi ed a conquistare definitivamente questo territorio.

\section{Dopo 261 si inserisca:}

- Fraxinus Ormus L.; Tanf. in Parl. Fl. It. VIII p. 166.

Tanfani, nella Flora Italiana, cita questa specie di Lampedusa sulla autoritá di Bertoloni. Ció deriva da un errore di lettura o di trascrizione, poichè nè Bertoloni nè altri fa menzione alcuna di Fraxinus per Lampedusa.

A 269 Convolvetlus althaeoides si aggiunga:

Lojacono Fl. Sic. II pars. II p. 95 istituisce una varietả glaberrimus del Convolvulus: Italicus, che dice crescere anche a Lampedusa (non dice da chi raccolta). Fra i miei esemplari del C. althaeoides, che come ho detto appartengono tutti alla forma Italicus, non trovasi la vạietá descritta da Lojacono.

\section{Dopo 279 si inserisca:}

- Echium vulgare Car. in Parl. Fl. It. VI p. 928.

Caruel, nella Flora Italiana, cita questa specie come raccolta in Lampedusa dall' Ajuti. Ma deve essere incorso in errore come per Linosa, poichè tutti gli esemplari di Echium raccolti a Lampedusa dall'Ajuti, che si conservano nell' Erbario Centrale, appartengono all'E. confusum De Coincy.

\section{Dopo 282 si inserisca:}

\section{3) - Heliotropium Eichwaldi Steud.}

L'ho ricevuto da Martorana nel Gennaio del 1907, in fiore e in frutto.

Questi esemplari di Lampedusa sono conformi a quelli di Linosa.

A 284 Lycium Europaeım si aggiunga:

Lojacono Fi. Sic. If pars II p. 103 crede che il Lycium di Lampedusa, come quelli di Linosa e di Pantelleria, possa riferirsi 
al L. intricatum Boiss. Tuttavia cita per Lampedusa anche il L. Europaeim, ma dubitativamente.

Dopo 302 si inserisca:

- Satureja Acinos Scheele; Car. in Parl. Fl. It. VI p. 142. Questa specie è indicata da Caruel nella Flora Italiana come raccolta in Lampedusa da Calcara. Mi era sfuggita perchè non è citata da aitri. Ho verificato che se ne trova effetfivamente un piccolo esemplare nell' Erbario Centrale di Firenze. Sull'etichetta originariamente era scritto soltanto « Lampedusa, Calcara »«avuta da Calcara in Luglio 1848 ». Questa specie fu mandata evidentemente indeterminata, poichè il nome Calamintha arvensí è scritto posteriormente di pugno di Caruel. Sembra strano che Tineo, il quale studiò le piante di Calcara, non l'avesse determinata e che quindi non figuri nella Florula di Calcara. Il professore Borzi m'informa che negli erbarî di Palermo non trorasi alcun rappresentante di questa specie di Lampedusa. Si puó dunque avere fondati dubbî sulla provenienza di questo esemplare. Tali dubbî crescono pensando che questa specie non è indicata di Tunisia, e che Gussone la cita soltanto di localitá elevate della Sicilia dove anzi, secondo Lojacono Fl. Sic., non esiste affatto ed è rappresentata soltanto nelle regioni montuose da specie vicarianti. Per questo la escludo dalla numerazione.

A 319 Statice psilocladx si aggiunga:

Lojacono Fl. Sic. II pars II p. 18, oltre alla S. psiloclada var. albicla, cita per Lampedusa una sua nuova varietả spathrılaefolia di questa specie.

A 329 obione portulacoides si aggiunga:

Lojacono Fl. Sic. II pars II p. 279 riferisce dubitativamente alla Obione glauca Moq. Tand. la pianta di Lampedusa che Gussone aveva chiamata Obione porlulacoides. In conseguenza peró Lojacono avrebbe dovuto sopprimere la citazione di Lampedusa per quest'ultima specie.

A 331 Ariplex Halimus si agriunga:

Lojacono Fl. Sic. II pars II p. 280 cita per Lampedusa, oltre alla $A$. Halimus, anche la $A$. halimoides Tineo $=A$. Halimus L. var. verruculosa Guss., senza dire da chi fu raccolta. 
Dopo 333 si inserisca:

\section{4) - Chenopodium olidum Curt.}

Ricevuto da Martorana in Gennaio 1907, sole foglie.

A 337 Salsola Tragus si aggiunga:

Ricevuta in fore in Gennaio 1907 da Nartorana. Lojacono Fl. Sic. II pars II p. 271-2 cita per Lampedusa le forme glabra e hirta della Salsola controversa Tod., la quale rientra nella S. Tragis L. sensu lato.

\section{A 345 Parietaria Cretica si aggiunga:}

Lojacono Fl. Sic. II pars II p. 353 descrive una varietả thymoides della $P$. Cretica, caratterizziata da «foliis minutissimis》 che dice trovarsi anche a Lampedusa ed a Linosa. In "Una Escurs. ecc. " non citara affatto la $P$. Cretica per Lampedusa.

Dopo 344 si inserisca:

5) - Parietaria Lusitanica L.; Lojacono Fl. Sic. II pars II p. 353.

Lojacono nella Flora Sicula cita questa specie per Lampedusa, come da lui trovata nel Vallone dell' Imbriacola. In "Una Escurs. ecc. » aveva citato soltanto la $P$. diffiesa.

Dopo 361 si inserisca:

6) - Chrozophora tinctoria (L.) A: de Juss.

Ricevuta da Martorana in fiore e in frutto in Gennaio 190\%.

A. 361 Mercurialis annua si aggiunga:

Di questa specie ho ricevuto da Martorana la var: ambigua (L.) raccolta in fiore e in frutto in Gennaio 1907.

A 392 Triglochin Barrelieri si aggiunga:

Questa specie che non era più stata raccolta a Lampedusa dal tempo-di Gussone in poi, mi è stata mandata da Martorana il quale la raccolse in abbondanza, in fiori e frutti, il 10 Marzo 1907. 


\section{PROSPETTO}

DELLE:

\section{FLORULE DI LAMPEDUSA E DI LINOSA}

con indicazione delle specie di queste isole che trovansl

IN

Pantelleria, Malta, Sicilia e Tunisia

\section{AVVERTENZE.}

Nel seguente prospetto sono riportate tutte le specie delle due precedenti Florule, comprese le aggiunte (p. 271-274). Nelle due prime colonne é indicata con una linea la presenza delle singole specie a Lampedusa e a Linosa. Nelle colonne seguenti $\grave{e}$ indicato se quelle specie si trovano rispettivamente a Pantelleria, nelle Isole Maltesi, in Sicilia ed in Tunisia.

Le fonti consultate per* Pantelleria sono indicate nella mia nota sulla Florula di quell' isola."

Per la flora dell' Isole Maltesi ho consultato i seguenti lavori:

Zerapha Stefano, Florae Melitensis thesaurus, sive plantarum enumeratio, quae in Melitae Gaulosque insulis aut indigenae aut vulgatissimae oscurvunt, etc. Malta, fasciculus I, 1827. Fasciculus alter 1831.

J. C. Grech Delicata, Flora Melitensis. Malta, 1853.

Gulia Gavino, Stirps Compositarum florulae Melitensis. Bull. Soc. bot. de France. Paris, 1869, p. 253-54.

Gulia Gavino, Mreltese lotany. In varî Numeri del giornale di Milta «II Barth».

J. F. Duthie, Notes on the Flora of Malta and Gozo. Journal of Botany, 1872.

J. F. Duthie, On the botany of the Maltese islands in. 1874. Journal of Botany, 1874 e 1875.

J. Daveau, Excursion à Malte et en Cyrénä̈que. Bullet. Soc. botan. Fr., 1876.

1. S. Sommen, Materiali per una Flonla di Pantelleria. Bull. Soc. bot. it., 1907, p. 48.60. 
E. Armitage, Appunti sulla flora dellisola di Malta. Nuovo Giorn. bot. it., 1889, p. 495.

A. p. 449 vi è l'elenco delle specie da aggiungere alla Flora Melitensis di Grech Delicata.

Caruana Gatto Alfredo, Dello stato presente delle nostre cognizioni sulla vegetazione Maltese. Atti Congr. bot. Genova, 1893.

L. Nicotra, Le Fumariacee italiane.

F. Parlatore, Flora Italiana.

Fiori, Paoletti e Béguinot, Flora analitica d' Italia.

Nyman, Conspectus Florae Europaeae.

Alle piante indicate in questi lavori ne ho aggiunto alcune altre che non sono pubblicate, ma che furono trovate da me stesso, o la cui presenza nelle Isole Maltesi mi è stata attestata dal distinto botanico Conte Caruana GatTo che da molti anni esplora quelle isole.

Per segnare nella quinta colonna le specie delle isole Pelagie che si trovano in Sicilia, ho consultato Gussone, Synopsis Florae Siculae; LoJacono, Flora Sicula (i due volumi, in quattro parti, fin'ora comparsi); Parlatore, Flora Italiana; Fiori, Flora analitica; Nicotra, Le Fumariacee Italiane.

-Per la Tunisia ho consultato: ED. Bonnet et G. Barratte, Catalogue raisonné des plantes vasculaires de la Tunisie, ed alle specie citate in quel lavoro ne ho aggiunto alcune altre che il prof. Ed. Bonnet del Muséum di Parigi ha avuto la somma cortesia di indicarmi come pubblicate in lavori più recenti (di Murbeck e altri) o da esso ricevute dalla Tunisia e non ancora pubblicate.

Nella colonna dedicata alla Tunisia ho poi indicato con tre punti, invece della linea, le piante che non sono note della Tunisia, ma che sono state trovate in Algeria; e con due soli punti quelle trovate in altri punti dell'Africa settentrionale, servendomi specialmente di BatTander et Trabut (Flore de l'Algérie e Flore analyl. et synopt. de l'Algérie et de la Trunisie), di J. BaLL (Spicilegium florae Maroccanae in Journ. Linn. Soc. Vol. XVI), di BoIssier (Flora Orientalis) e delle indicazioni epistolari del sig. BonNET. 


\section{PROSPETTO}

\begin{tabular}{|c|c|c|c|c|c|c|}
\hline DTCOTYLEDONEA & 琴 & $\stackrel{g}{\underline{3}}$ & 吾 & 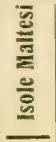 & 咅 & \\
\hline 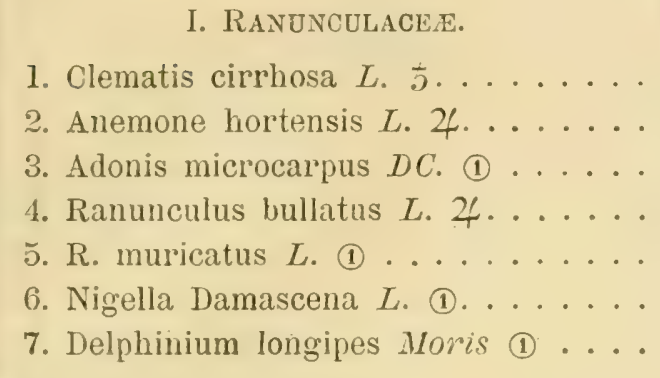 & $\begin{array}{l}- \\
- \\
- \\
- \\
- \\
-\end{array}$ & - & $\begin{array}{l}- \\
- \\
- \\
-\end{array}$ & $\begin{array}{l}- \\
- \\
- \\
- \\
- \\
-\end{array}$ & $\begin{array}{l}- \\
- \\
- \\
- \\
- \\
-\end{array}$ & - \\
\hline $\begin{array}{ll}\text { Ranuncel. 7. } & \text { Lamp. } 51 ; 42 ; \text { (1) } 4 . \\
& \text { Lin. } 51 .\end{array}$ & 7 & 1 & 5 & 7 & 7 & 5 \\
\hline 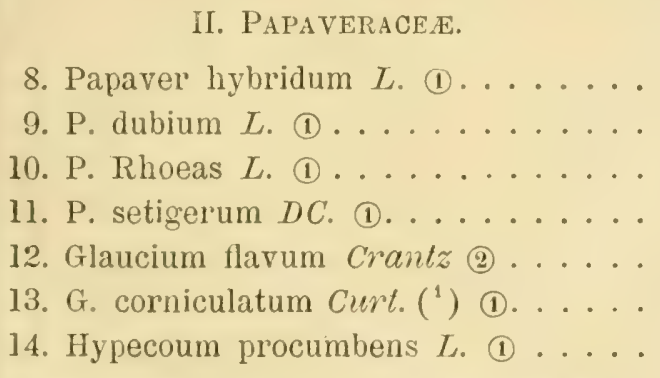 & $\begin{array}{l}- \\
- \\
- \\
- \\
- \\
-\end{array}$ & $\begin{array}{l}- \\
-\end{array}$ & $\begin{array}{l}- \\
- \\
- \\
- \\
-\end{array}$ & $\begin{array}{l}- \\
- \\
- \\
-\end{array}$ & $\begin{array}{l}- \\
- \\
- \\
- \\
-\end{array}$ & - \\
\hline $\begin{array}{l}\text { Lamp. (2) e (1) } 7 . \\
\text { Lill. (2) e (1) } 4 .\end{array}$ & 17 & 4 & 6 & 0 & 7 & 7 \\
\hline
\end{tabular}

* I punti nell'ultima colonna significano, come ho detto nelle Arveltenze al Prospetto, che la specie trovasi nell'Africa boreale, ma non in Tunisia. 
III. FuMARIACEE.

15. Fumaria densiflora $D C$. (1)......

16. F. parriflona Lam. (1) . . . . . . .

17. F. bicolor Somm. (1) . . . . . . .

18. F. flabellata Guspurm. (1)........ - - - - -

19. F. media Lois. (1)........... $-{ }_{-}--$

20. F. agraria Lagasce (1)........ - - $1-|-|-$

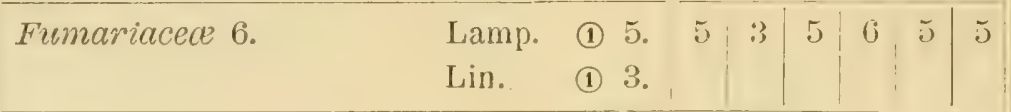

\section{CRUCIFERE.}

21. Natthiola incana $R$. $B r \cdot 2$

22. Cardamine hirsuta $I$. (1).......

23. Sisymbrium officinale $L$. (1) . . . . . -

24. Brassica fruticulosa Cyr. $\left.{ }^{2}\right)$ 4...

25. B. Tournefortii Gouan (1).......

20. B. alpressa Boiss. (1).........

27. B. Sinapistrum Boiss. (1) . . . . . .

28. Eruca sativa Lam. (1) . . . . . . . .

29. Diplotaxis erucoides $D C$. (1) ...... -

30. D. scaposa DC. $\left({ }^{3}\right)$ (1) . . . . . . -

31. Carrichtera annua Prantl (1) ...... -

32. Succowia Balearica Med. (1)...... $---|-|-$

33. Rapistrum rugosum Berg. (1)...... - - -

34. Cakile maritima scop. (1)....... - $\quad--$

35. Alyssum maritimum Lam. 4..... - - - - -

36. Capsella Bursa-Pastoris Moench (1) . - - - - - - -

37. Hutchinsia procumbens Desv. (1) ... - - $\quad-\quad-$

38. Biscutella lyrata $L$. (1) ........

\begin{tabular}{llllll|lll|lll} 
Cruciferce 18. Lamp. \& & 2 ; (1) 14. & 16 & 10 & 8 & 15 & 17 & 16
\end{tabular} Lin. 43 ; (1) 7 .

V. CAPPARIDACEE.

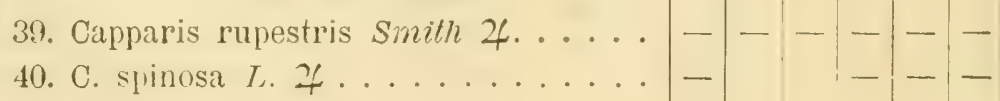




\begin{tabular}{|c|c|c|c|c|c|c|}
\hline VI. RESEDACEA. & 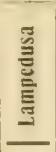 & 总 & 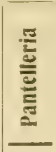 & 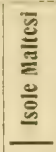 & 意 & $\begin{array}{l}\text { 愛 } \\
\text { 咅 }\end{array}$ \\
\hline 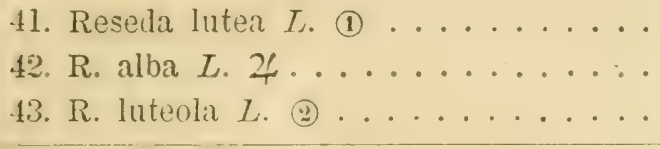 & - & & - & - & - & $\begin{array}{l}- \\
- \\
-\end{array}$ \\
\hline 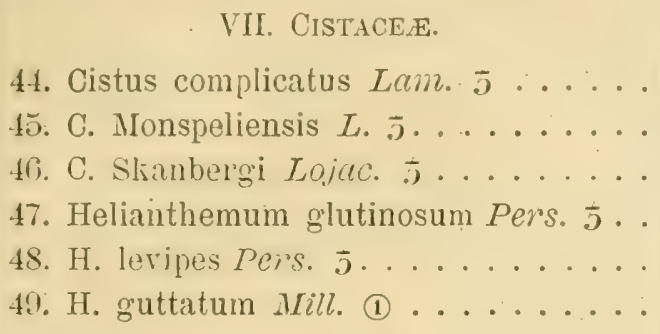 & $\begin{array}{l}- \\
- \\
- \\
- \\
-\end{array}$ & - & - & - & $\begin{array}{l}- \\
-\end{array}$ & - \\
\hline $\begin{array}{ll}\text { Cistacece } 6 . & \text { Lamp. } \\
& \text { Lin. } 5 \text {; (1) } 1 . \\
& 51 .\end{array}$ & 6 & 1 & 3 & 2 & 4 & 4 \\
\hline 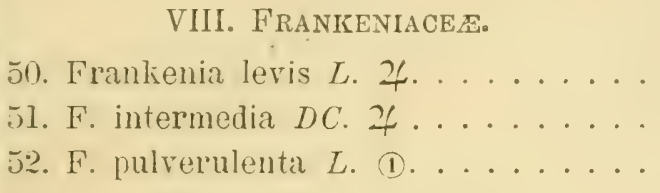 & $\begin{array}{l}- \\
- \\
-\end{array}$ & $\begin{array}{l}- \\
-- \\
-\end{array}$ & - & - & $\mid-$ & - \\
\hline 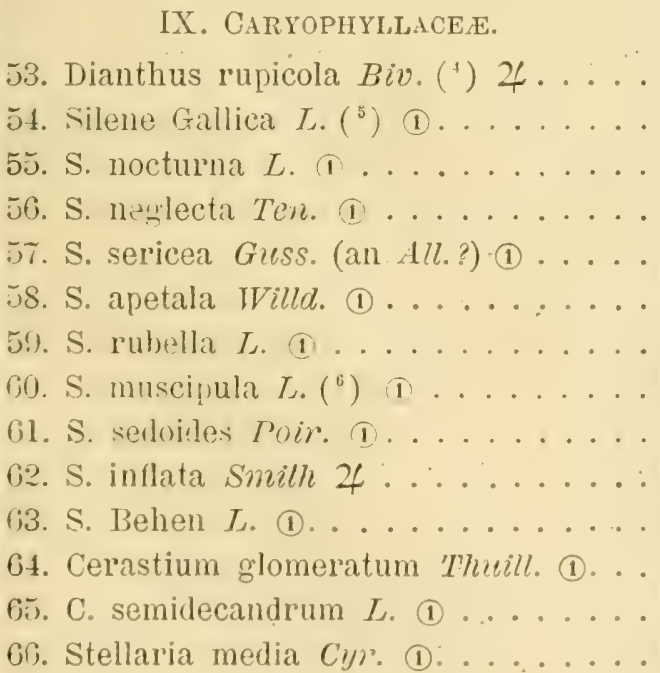 & $\begin{array}{l}- \\
- \\
- \\
- \\
-\end{array}$ & $\begin{array}{l}- \\
- \\
- \\
- \\
-\end{array}$ & - & - & $\begin{array}{l}- \\
- \\
- \\
-\end{array}$ & $\begin{array}{l}- \\
- \\
\cdots \\
- \\
- \\
- \\
- \\
- \\
- \\
- \\
-\end{array}$ \\
\hline
\end{tabular}


67. Arenaria serpyllifolia $L$. (1) .....

68. Alsine procumbens Fenzl 4 . . . .

69. Sagina apetala $L$. (1)

70. S. maritima Dom (1).......... - - - - -

71. Spergula pentandra $L \cdot\left({ }^{7}\right)$ (1) . . .

72. Spergularia rubra Pers. (1)..... - - - - -

73. S. diandra Boiss. (1).........

74. S. media Pers. (2) ........... - - - - -

\begin{tabular}{lllll|l|l|l|l|} 
Caryophyll. 22. Lamp. 24 2; (2) e (1) 13. & 15 & 18 & 13 & 13 & 20 & 19
\end{tabular} Lin. 42 ; (2) e (1) 16 .

\section{Portulacaceae.}

75. Portulaca oleracea $L$. (1) . . . . . .

Xi. Paronychiaceae.

76. Herniaria cinerea $D C .4 \ldots . . .--$

77. Loeflingia Hispanica $L$. (1)...... -

78. Polycarpon tetraphyllum $L$. (1).... $|--|-|-|-$

XiI. Elatinace.e.

79. Elatine Hydropiper $L$. (1

XIII. HYPERICACEE.

80. Hypericum Egyptiacum L. J..... -

XIV. MalvaceE.

81. Malva Cretica Cav. (1) .........

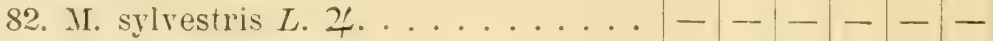

83. II. microcarpa Desf. (1)........ - - - - -

84. Lavatera Cretica L. (2)......... - - - - - -

85. L. arborea L. . ............. - - - -

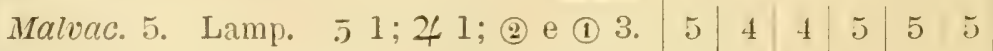
Lin. $51 ; 21$; (2) e (1) 2 . 


\section{GERANIACE}

86. Geranium molle $L$. (1)

87. G. rotundifulium $L$.

88. G. Robertianum L. (1)

89. Erodium cicutarium L'Hẻrit. (1). . . .

90. E. moschatum LiHérit. (1) ......

91. E. Chium Willd. (1) ..........

92. E. laciniatum Villı. (1)........

93. E. angulatum Pomel (1) .......

94. E. malacoides Wille. (1)

$\begin{array}{ll}\text { Geraniacece 9. } & \text { Lamp. (1) } 7 . \\ \text { Lin. } & \text { (1) } 7 .\end{array}$

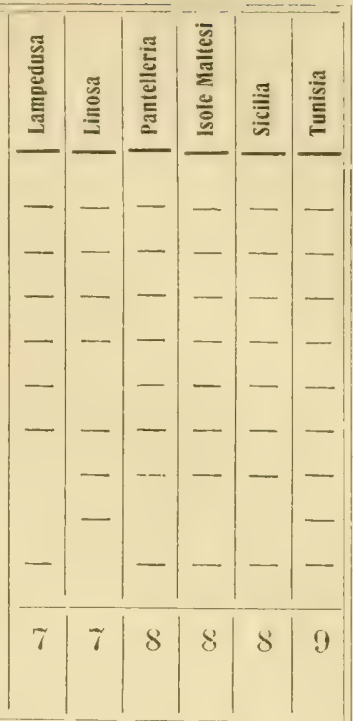

XVI. LINACEE.

95. Linum Gallicum L. (1) ......... -

96. L. strictum $L$. (1) ............ . . .
97. L. angustifolium Huds. (1) ......

XVII. Oxalidace正.

98. Oxalis cernua Thunbg. 4 ......

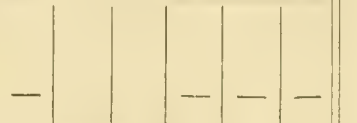

XVIII. ZYGOPHYLLACEE.

99. Tribulus terrestris $L$. (1). . . . . .

XIX. RUTACE.E.

100. Ruta bracteosa $D C . \bar{j} \ldots \ldots \ldots$

XX. AnaCardiaceE.

101. Pistacia Lentiscus

102. Rhus dioica Brouss. 5 . . . . . .

103. R. pentaphylla Desf. 5 .......

XXI. RhannaCEE.

104. Rhamnus Alaternus $L .5 \ldots \ldots$ - 
XXII. PAPILIONACEe.

105. Anagyris foetida $L . \overline{5}$

106. Spartium junceum $L$. J.

107. Lupinus angustifolius $L$. (1)

108. L. Termis Forsh. $\left({ }^{8}\right)$ (1)

109. L. pilosus ML(Gr). (1).

110. Ononis Sieberi Bess. (1)

111. O. ornithopodioides $L$. (1)

112. O. mollis Savi

113. O. serrata Forsh. (1)

114. O. difrusa Ten. (1)

115. Trigonella Monspeliaca $L$. (1)

116. T. maritima Del. (1)

117. Medicago secundiflora Dur. (1)

118. M. orbicularis $A l l$.

119. M. Helix Wille:

120. M. tribuloides Des?

121. M. litoralis Rhode (1)

122. M. denticulata Willd.

123. M. minima Gruf. (1)

124. Melilotus Neapolitanus Ten. (1) :.

125. .I. parviflorus Desf. (1) . . . . . . .

126. M. elegans Salzm. (1)

127. M. sulcatus Desf.

128. Trifolium suffocatum $L$. (1)

129. T. glomeratum L. (1)

130. T. arvense $L$. (1)

131. T. scabrum L. (1).

132. T. Ligusticum Balb. (1) . . . . . .

133. T. Cherleri $L$. (1).

134. T. stellatum $L$. (1)

135. 'T. resupinatum $I$.

136. T. tomentosum $L$. (1)

137. T. nigrescens Viv. (1)

138. T. agrarium Poll. (1).

139. Anthyllis tetraphylla $L$. (1)

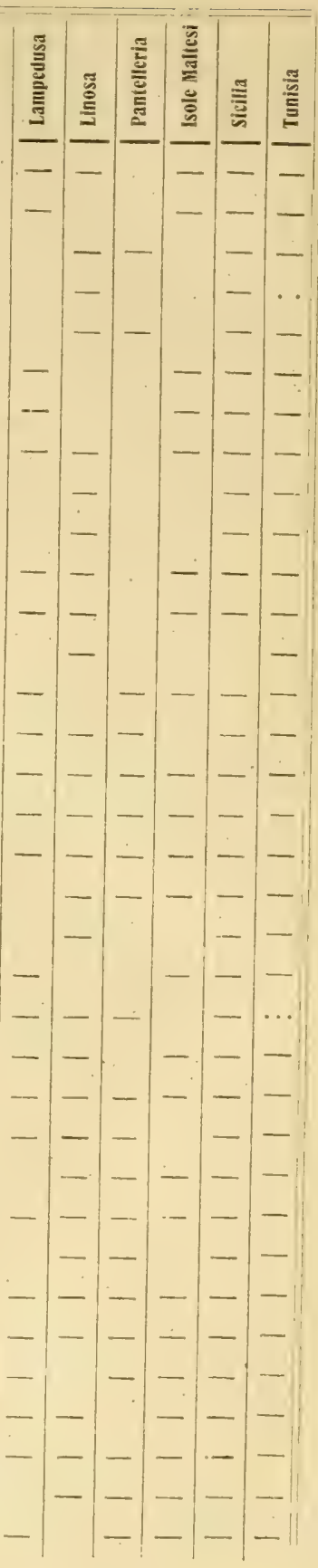


140. Lotus angustissimus $L$. (1).... 141. L. pusillus Viv. (1) ........... 142. I. cytisoides T. 7 . 143. L. Creticus L. $₹$. 144. L. peregrinus $L$. D

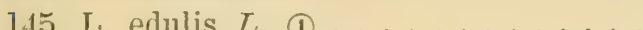
146. I. ornithopolioides $L \cdot$ (1) . . . . . . . .

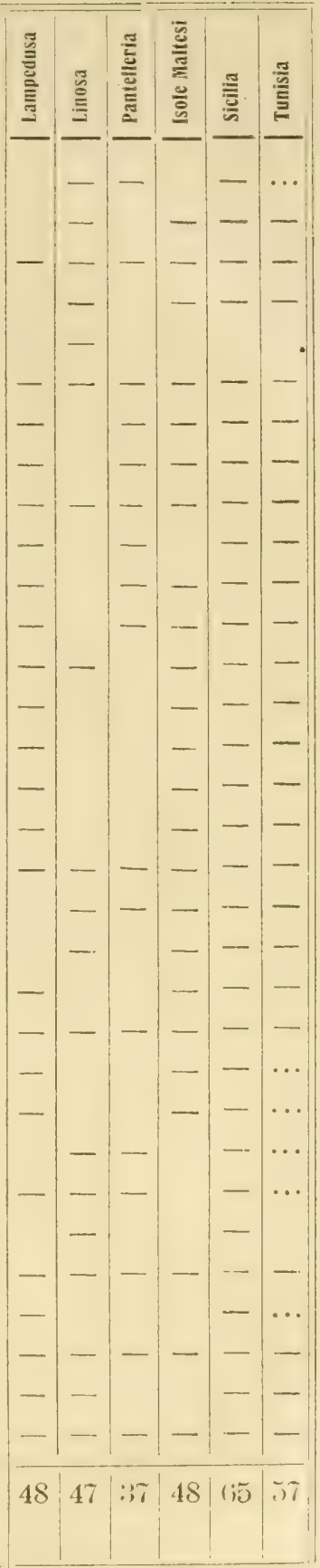

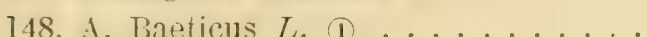

149. Biserrula Pelecinus $L$. (1)...... 150. Scorpiurus subvillosus $L$. (1) . . . . 151. Coronilla scorpioides Koch (1).... 152. Hippocrepis multisiliquosa $L$. (1). . . 153. H. ciliata Willd. (1). . . . . . . . . 154. I. unisiliquosa $L$. I. . . . . . . . 155. Hedysarum capitatum Desf. (1)... 156. Lathyrus Ochrus $D C$. (1) ...... 157. L. Clymenum $L$. (1) . . . . . . . . 158. L. sphaericus Retz. (1). . . . . . . 159. L. setifolius $i$. (1) . . . . . . . 160. L. Cicera L. (1) . . . . . . . . . . 161. Vicia sativa $L$. (1) . . . . . . 102. V. maculata Presl 1 ........ 163. V. peregrina L. (1) . . . . . . . 164. V. lathyroides $I$. I ........ 165. V. atropurpurea Desf. (1) . . . . . 166. V. dasycarpa Ten. $\left({ }^{9}\right)$ (1) . . . . . 167. V. pseudocracca Bert. (1) ....... 168. Y. triflora Teir. (") I ....... 169. V. leucantha Biv. (1) ......... 170. Ervum Lens $L$. (1) . . . ....... 171. Ceratonia Siliqua $L . \bar{J}$

Papilion. 67. Lamp. 5 $3 ; 2$ 1; (1) 44 . Lin. $52 ; 22$; (1) 43 . 
XXIII. ROSACEE.

172. Rubus ulmifolius scholt $5 \ldots .$.

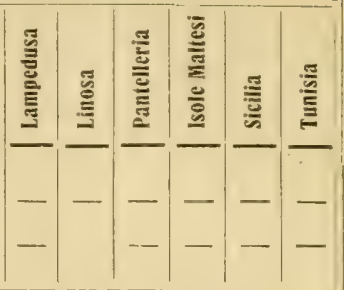

XXIV. LYTHRACEE.

'174. Lythrum Graefferi Ten. 4...... -

175. L. IIyssopifolia L. (1. . . . . . . -

\section{Crassulace.e.}

176. Tillaea muscosa

$L$. (1)

177. Bulliarda Vaillantii $D C$. (1)..... -

178. Umbilicus pendulinus $D C .4 \ldots$.

179. U. horizontalis $D C$. $4 \ldots \ldots \ldots$. .

180. Sedum stellatum $L .\left(^{18}\right)$ (1) . . . .

181. S. Cepaea L. (1)............ -

182. S. dasyphyllum $L .4 \ldots \ldots \ldots$

183. S. caespitosum DC. (1)........ -

184. S. rubens $L$. (1) . . . . . . . .

185. S. litoreum Guss. (1)........ - -

186. S. Hispanicum $L$. (1)......... - - $\quad-$

187. S. Nicaeense $A l l .24 \ldots \ldots . .$.

\begin{tabular}{lll|l|l|l|l|l|l|l|} 
Crassulacece 12. & Lamp. $\quad 4$ 4; (1) 7. & 11 & 8 & 6 & 8 & 12 & 8
\end{tabular} Lin. $\quad 44$; (1) 4 .

XXVI. Mesembryanthemace.

188. Mesembryanthem. nodiflorum $L$. (1) . - - - - -

189. M. crystallinum L. (1........ $|--|-$

XXVII. CACTACEE.

190. Opuntia Ficus-Indica Mill. 5.....

XXVII. Myrtaceae.

191. Myrtus communis $L . \overline{.} \ldots \ldots \ldots,-|-|-$ 
XXIX. UMBELLIFERæ.

192. Eryngium dichotomum Desf. 4 . . 193. Smyrnium Olusatrum $L$. (2) . . . . 194. Buplenrum glaucum DC. (1) . . . . - 195. B. suboratum Link (1. . . . . . . 196. Immi majus $L$. I.......... 197. Ptychotis ammoides Koch (1)..... 198. Scandix Pecten-Veneris L. (1). . . . 199. Seseli Bocconei Guss. 4 . 200. Foeniculum vulgare Mill. 4 . . . . 201. Magydaris pastinacea Paolelli 4 . . 202. Crithmum maritimum L. 4 . . . . 203. Ferula noliflora $L$. Ұ. . . . . . . 204. Tordylium Apulum $L$. (1) . . . . . . 205. Bifora testiculata $D C$. (1) ...... 206. Daucus Gingidium L. $\left({ }^{12}\right)$ (2) . . . . 207. I) (arota L. . . . . . . . . 208. D. Siculus Tin. (") (2) . . . . . . 209. D. rupestris Gues. (2) . . . . . . . . 210. D. Lopratusanus Tin. (2). . . . . . 211. Torilis nodosa Guertn. (1)....... 212. Thapsia (iarganica L. 4 .

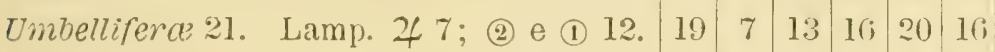
. Lin. 24 3; (2) e (1) 4 .

XXX: RUBIACE

21\%. Sherardia arvensis $L$. (1) . . . . . 211. Crucianella rupestris Gers. $\left({ }^{14}\right) 4$. 21.). Rubia peregrina $L .4$. . . . . . 216. Galium cinereum All. $\left({ }^{15}\right) 4 \ldots$. 217. 'i. saccharatum All. ( .......

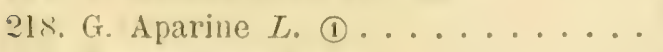
219. (r. spurium
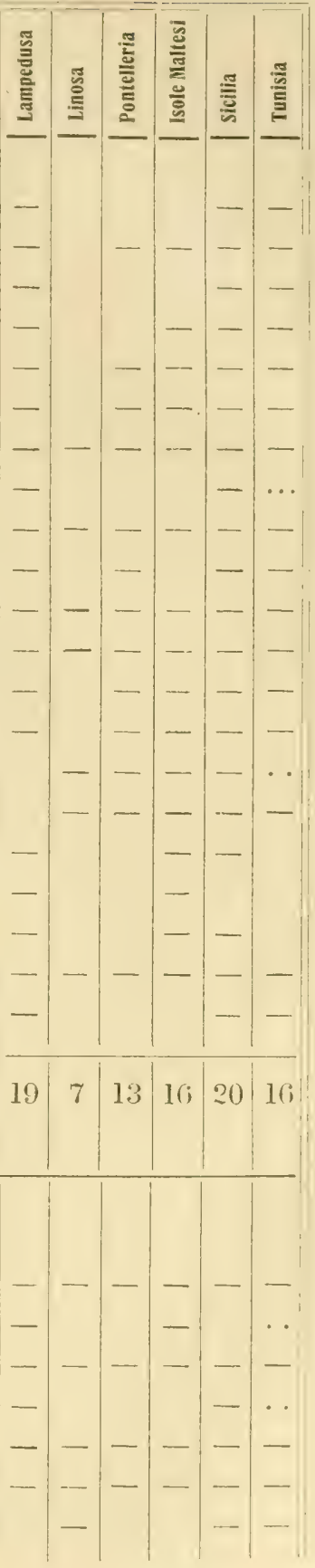


\begin{tabular}{|c|c|c|c|c|c|c|}
\hline & 言 & 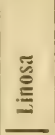 & 莺 & 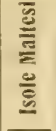 & $\frac{g}{\bar{z}}$ & 吾 \\
\hline $\begin{array}{l}\text { 220. Callipeltis muralis Moris (1) . . . . } \\
\text { 221. Vaillantia muralis } L \text {. (1). . . . . }\end{array}$ & - & - & - & - & - & - \\
\hline $\begin{array}{ll}\text { Lamp. } \& 3 & 3 \text {; (1) } 5 . \\
\text { Lin. } & \text { \& } 1 ; \text { (1) } 6 .\end{array}$ & 8 & 7 & $1 ;$ & 17 & 8 & 7 \\
\hline $\begin{array}{l}\text { XXXI. CAPRIFoliaCE.E. } \\
\text { 22. Lonicela implexa Ait. .) . }\end{array}$ & - & & - & - & - & $1-$ \\
\hline $\begin{array}{l}\text { XXXII. VALERIANACEE. } \\
\text { 223. Valerianella microcarpa Lois. (1) . } \\
\text { 224. V. puberula } D C \text {. (1) . . . . . . . } \\
\text { 225. Fedia Cornucopiae Gaertin. (1) . . . } \\
\text { 226. Centranthus Calcitrapa Dufr. (1) . }\end{array}$ & - & - & $\begin{array}{l}- \\
- \\
-\end{array}$ & $\begin{array}{l}- \\
- \\
-\end{array}$ & $\begin{array}{l}- \\
- \\
-\end{array}$ & - \\
\hline $\begin{array}{l}\text { XXXIII. Dipsace E. } \\
\text { 227. Knautia hybrila Conlt. } \\
\text { 228. Scabiosa Cretica L. } 4 \text {. . }\end{array}$ & - & & & & $\mid-$ & - \\
\hline 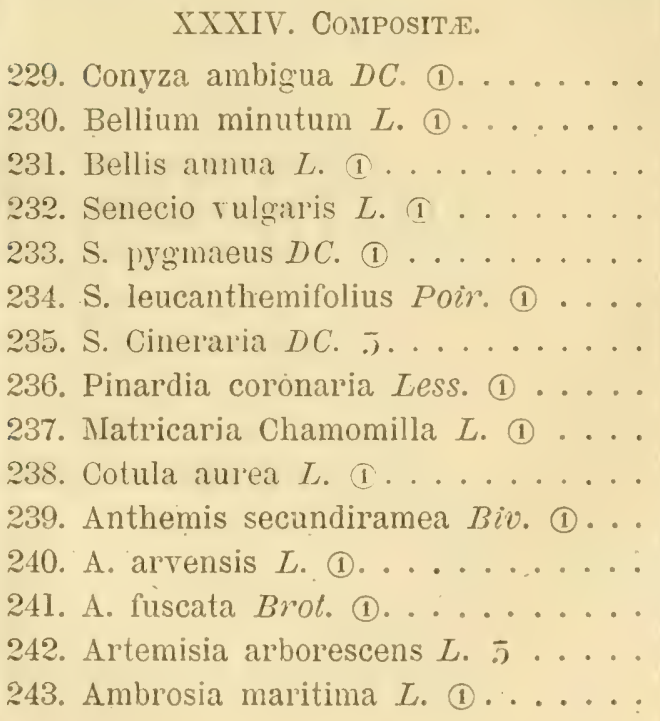 & $\begin{array}{l}- \\
- \\
- \\
- \\
- \\
- \\
- \\
- \\
- \\
- \\
- \\
-\end{array}$ & - & $\begin{array}{l}- \\
- \\
-\end{array}$ & $\begin{array}{l}- \\
- \\
- \\
- \\
- \\
- \\
- \\
- \\
- \\
-\end{array}$ & $\left\{\begin{array}{l}- \\
- \\
- \\
- \\
- \\
- \\
-\end{array}\right.$ & $\begin{array}{l}- \\
- \\
- \\
\cdots \\
- \\
- \\
- \\
- \\
-\end{array}$ \\
\hline
\end{tabular}


241. Inula viscosa Ait. 4 . . . . . . . 245. I. graveolens Desf. (1). . . . . . 246. I. crithmoiles L. $\mathcal{\Psi}$. 247. Jasonia glutinosa $D C$. 4 2.18. Asteriscus aquaticus Moench (1). : 249. A. spinosus $G r$. et Godr. 4 .... 250. Calendula arvensis $L$. (1) ....... 251. C. parviflora Raf. (1) . . . . . 252. C. micrantha Tin. (1). 2.53. C. Aegyptiaca Desf. (1. 251. C. fulgilla Raf: 255. Phagnalon saxatile Cass. 4 .... 256. P. rupestre $D C .24$. . . . . . . 257. Filago Germanica L. (1). . . . . . . 258. F. Gnssonei Lojic. (1) . . . . . . . 259. F. arrensis $L$. (1 . . . . . . . 260. F. Gallica L. (1)............ 261. Evax pygmaea Pers. (1)....... 2(12. Carlina lanata $L$. (1).......... 203. C. involucrata Poir. 4 ....... 26.t. C. Sicula Ten. 2 .

265. Microlonchus Salmanticus $D C$. (1) $\therefore$ 26if. Centaurea acaulis $L$. (1). . 26\%. C. Melitensis $L$. (1) . 26is. C. Nicaeensis All. (1).......... 269. Carthamus lanatus $i$. (1)....... 27). Amberboa Liprii $D C$. (1).

271. Carduus argyroa Biv. $\left({ }^{16}\right)$ (1).... 272. C. pycnocephalus $L$. (1) ........ 27:3. C. brevisquamus (Fioni) (1) . . . . 274. C. marmoratus Boiss. et Heldr. (1) . - 275. C. corymbosus T'en. (1) ........ 276. Notobasis Syriaca Cass. (1)...... 27\%. Cynara Cardunculus $L .4 \ldots .$.

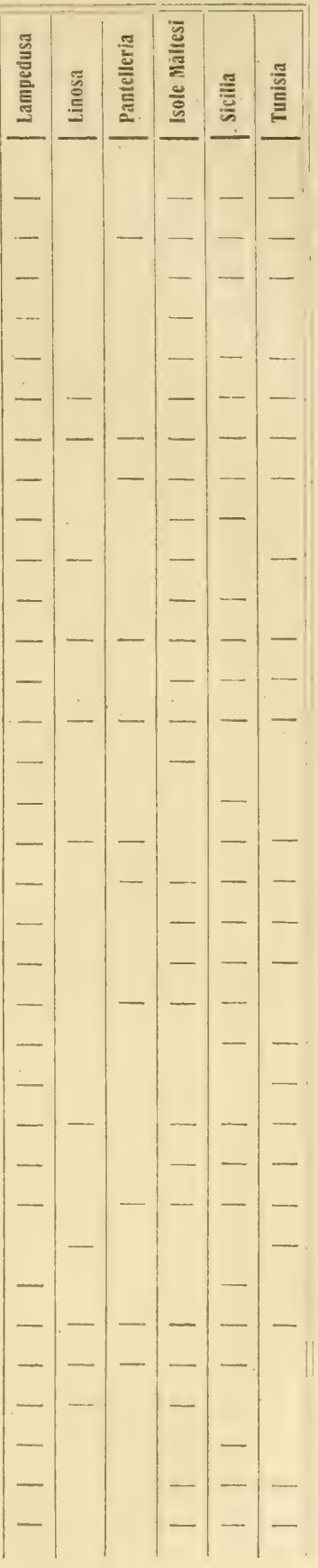


278. Onopordon Sibthorpianum B. et H. (2) 279. Silybum Marianum Gaert. (2) .... 280. Echinops spinosus L. 2 281. Scolymus maculatus $I$ 282. Rhagadiolus stellatus $D C$. (1) ... 283. Inyoseris rarliata $L$. 7 284. H. scabra L. (1) 285. Hedypnois polymorpha $D C$. (1) 286. Seriola Etnensis $L$. (1) 287. Cichorium Intybus $L . \mathcal{Z}$ 288. Thrincra tuberosa I) 289. Picris spinulosa Ber 290. Urospermum picroides Desf. (1) . . 291. Tragonogon Cupani Guss. (1)..... 292. Sonchus tenerrimus $L$. $\mathcal{Z}$ 293. S. levis Bartal. (1) 294. S. asper Hill. (1) 295. S. glaucescens Jord. 296. S. maritimus $L$. 24 297. Picridium vulgare Desf. $\not{Z}$ 298. P. intermedium Schuılz 299. P. 'Tingitanum Lesf. (1) 300. Crepis foetida $L_{0}\left({ }^{17}\right)$ 301. C. bulbosa Froel. Z4 302. Andryala sinuata $L$. (1) .......

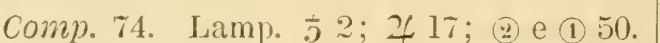
Lin. . 2; यै \%; (2) e (1) 21 .

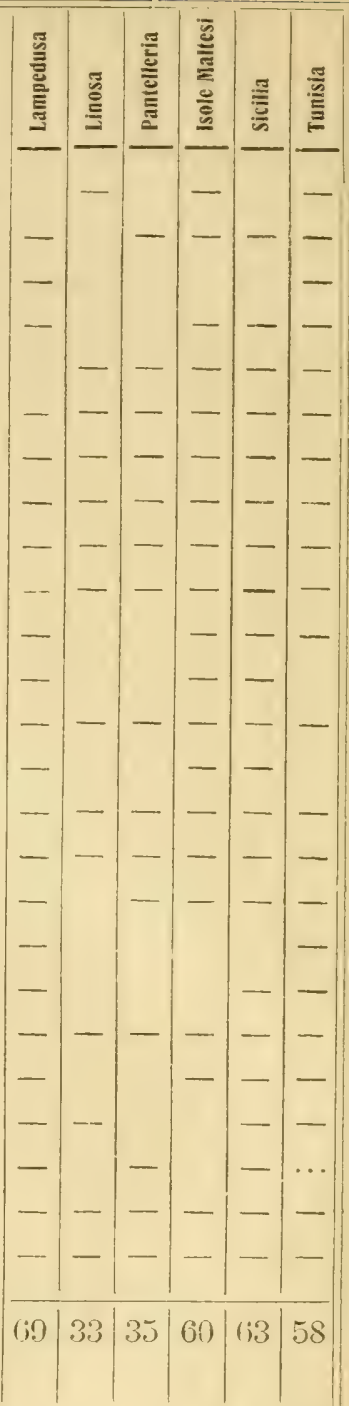

XXXV. CAMPANULACEE.

303. Campanula Erinus $L$. (1

XXXVI. CUCURBITACEE.

304. Bryonia acuta Desf. 2 305. Echallion Elaterium Rich. 4 ... 


\begin{tabular}{|c|c|c|c|c|c|c|}
\hline $\mathrm{XXX}$ & 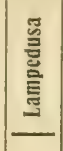 & 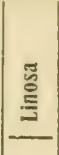 & 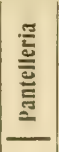 & 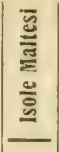 & 墨 & 总 \\
\hline $\begin{array}{l}L .5 \\
L .5\end{array} \ldots \ldots \ldots$ & - & - & - & - & - & - \\
\hline 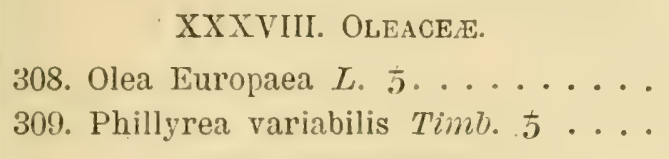 & $|-|$ & - & - & - & $\mid-$ & - \\
\hline $\begin{array}{l}\text { XXX } \\
\text { 310. Periploca } \\
\text { 311. Stapelia }\end{array}$ & - & - & - & - & - & - \\
\hline $\begin{array}{l}\text { XL. Gentianace E. } \\
\text { 312. Chlora perfoliata L. (1) . . . . . . } \\
\text { 313. Erythraea Centaurium Pers. (1) . . } \\
\text { 31.4. E. pulchella Horn. (1) . . . . . . . } \\
\text { 315. E. spicata Pers. (1). . . . . . . . }\end{array}$ & $\begin{array}{l}- \\
- \\
- \\
-\end{array}$ & - & - & $\begin{array}{l}- \\
- \\
-\end{array}$ & $\begin{array}{l}- \\
- \\
- \\
-\end{array}$ & - \\
\hline 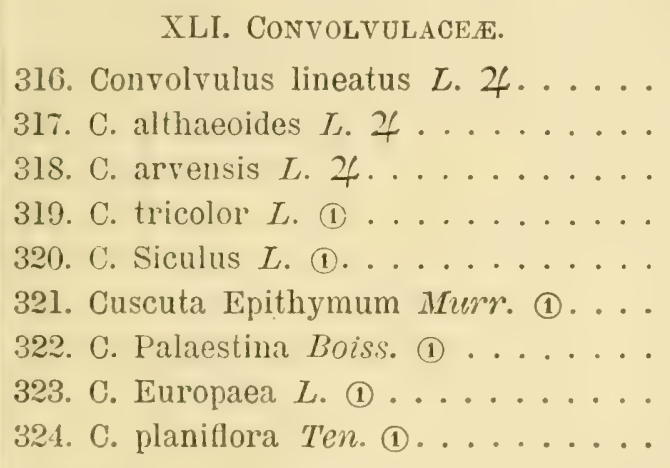 & $\begin{array}{l}- \\
- \\
- \\
- \\
- \\
- \\
- \\
- \\
-\end{array}$ & - & $\begin{array}{l}- \\
- \\
-\end{array}$ & $\begin{array}{l}- \\
- \\
- \\
- \\
- \\
- \\
-\end{array}$ & $\begin{array}{l}- \\
- \\
- \\
- \\
- \\
-\end{array}$ & $\begin{array}{l}- \\
- \\
- \\
- \\
- \\
- \\
\cdots \\
-\end{array}$ \\
\hline $\begin{array}{lll}\text { Lamp. } & 43 \text {; (1) } 6 . \\
\text { Lin. } & 41 \text {; } 1 \text { (1) } 2 .\end{array}$ & 9 & 3 & 5 & $\tau$ & 8 & 8 \\
\hline $\begin{array}{l}\text { viflorum Moench (1) . . } \\
\text { n Gizss. (2) . . . . . . } \\
\text { De Coincy (2) . . . }\end{array}$ & $\begin{array}{l}- \\
- \\
-\end{array}$ & - & - & $\begin{array}{l}- \\
- \\
-\end{array}$ & $\begin{array}{l}- \\
- \\
-\end{array}$ & - \\
\hline
\end{tabular}


328. Borago officinalis $L$. (1) . . . . : 329. Anchusa Italica Retz. (2) 330. Heliotropium Europaeum L. (1) ... 331. H. Eichwaldi Stererl. (1) . . . . . . . Boraginacece 7.

Lamp. (2) e (1) 7 .
Lin. (2) e (1) 5 .

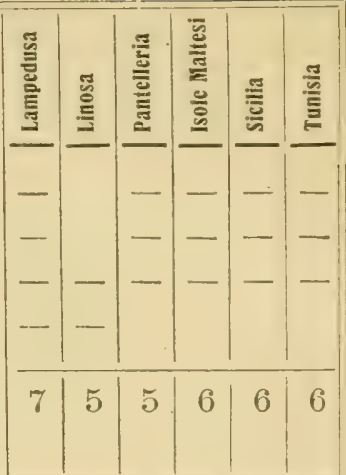
XLIII. SOLANACE E.

332. Hyosciamus albus $L$. (2) 333. Lycium Europaeum L. . . . . . . . 334. Solanum Sodomaeum L. 5 . . . . 335. S. nigrum $L$. (1). . . . . . . . 336. Mandragora autumnalis Bert. 4 . . Solanac. 5. Lamp. $52 ; 2$ 1; (2) e (1) 2 . Lin. $\quad 52 ; \quad$ (2) $\mathrm{e}(1) 2$

XliV. Plantaginace

337. Plantago Psyllium $L$. (1) ....... - - - - 338. P. albicans $L .4 \ldots \ldots \ldots \ldots$ 339. P. Coronopus L. (1). 340. P. Serraria

XLV. Scrofulariage正.

341. Verbascum sinuatum $L$. (2). . . . . 342. Scrofularia peregrina L. (1). . . . . 343. Antirrhinum Orontium $L$. (1). . . . 344. Linaria cirrhosa Dum.-Cours $\left({ }^{18}\right)$ (1) 345. L. reflexa Desf. (1).......... . 346. L. pseudolaxiflora Lojac. (1) . . . . 347. Veronica arvensis $L$. (1). . . . . Scrofulariacea 7. Lamp. (2) e (1) 5 . Lin. (1) 4 . 
XLVI. OROBANCHACEE.

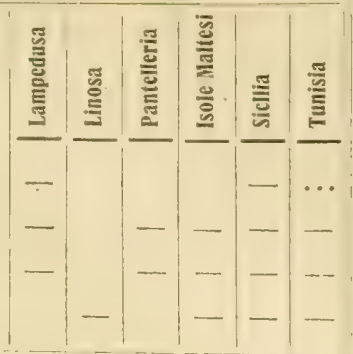

XLVII. LABIATE.

352. Thymus capitatus Hoffm. et $L \hbar$. j. 353. Satureja fasciculata Raf. 4 . . . . 354. S. microphylla Guss. $\left({ }^{19}\right) 4 \ldots \ldots$ 355. Salvia clandestina L. 4 . . . . . 356. Rosmarinus officinalis $L$. . .... 357. Sideritis Romana $L_{\text {. }}$ ( ) . . . . . . . . 358. Narrubium vulgare $L .4 \ldots \ldots$. . 359. Stachys arvensis $L$. (1) . . . . . . 360. Lamium amplexicaule $L$. (1) ..... 361. Prasium majus $L$. .). . . . . . . 362. Ajuga Ira Schreb. \& ....... 363. Teucrium fruticans $L$. . . . . . . 361. Т. Creticum L. 5 . . . . . . . 36.5. T. flavum L. 5. . . . . . . . . Labiutce 14. Lamp. $5 ; 245$; (1) 2 . lill. 5 $2 ; \% 2:$ (1) 3 .

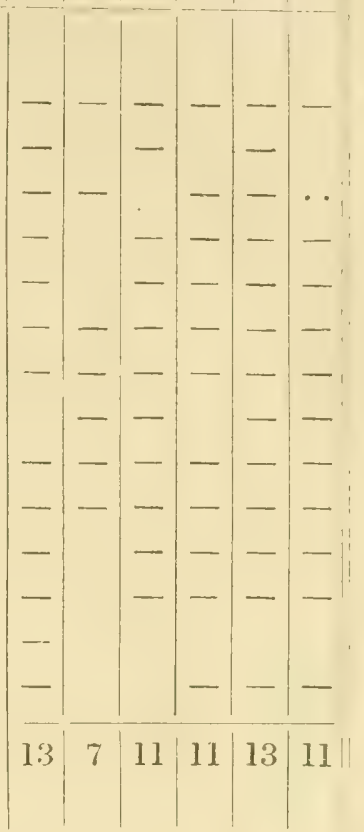

XLVIII. Globulariace.

366. Globularia Alypum L. 5 .

XLIX. PRIMUlace \&.

367. Asterolinum Linum-stellat. Duby (1) 368. Anagallis arvensis L. (1) .......

L. Plumbaginaceas.

369. Statice monopetala $L .5 \ldots \ldots \ldots$ 370. S. echioides $L$. (1) . . . . . . . -

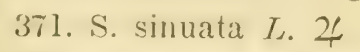
372. S. psiloclada Boiss. 4 . 
373. Statice intermedia Guss. 4

374. S. virgata Willd. 4

375. S. dubia Andrews 2

376. S. minuta $L .\left({ }^{20}\right) 4$

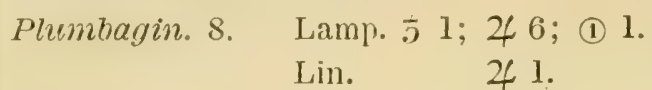

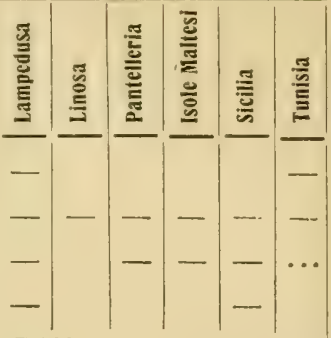

\section{Li. PolygonaceE.}

377. Polygonum aviculare $L$. (1). . . .

378. P. maritimum L. $\psi$.

379. Emex spinosa Campd. (1)

380. Rumex pulcher $L$. (2) . .

381. R. bucephalophorus L. (1) ......,-----

\section{LiI. Amarantaceat.}

382. Amarantus graecizans $L$. (1)....

\section{ChenopodiaceE.}

383. Obione portulacoides Moq. 5..... -

384. Atriplex roseum $L$. (1) ........ -

385. A. Halimus $L$. 5 . . . . . . . .

386. Beta maritima L. 4 . .

387. B. macrocarpa Guss. (1)

388. B. Cicla $L$. (2)

389. Chenopodium olidum Curt. (1) ....

390. C. murale L. (1) . . . . . . . . .

391. C. opulifolium Schrad. (1).......

392. Arthrocnemum glauc. Ung. Sternb. 5

393. Salsola longifolia Forsh. 5......

394. S. Tragus $L$. (1) . . . . . . . .

395. S. Soda $L$. (1) . . . . . . . . . .

396. Suaeda fruticosa Forsk. . .....

Chenop. 14. Lamp. 市 5; 4 1; (1) 6 .

Lin. $51 ; 41$; (2) e (1) 6 .
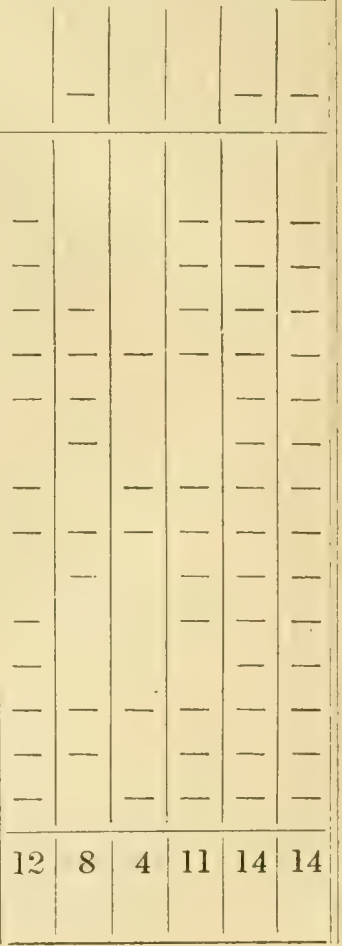


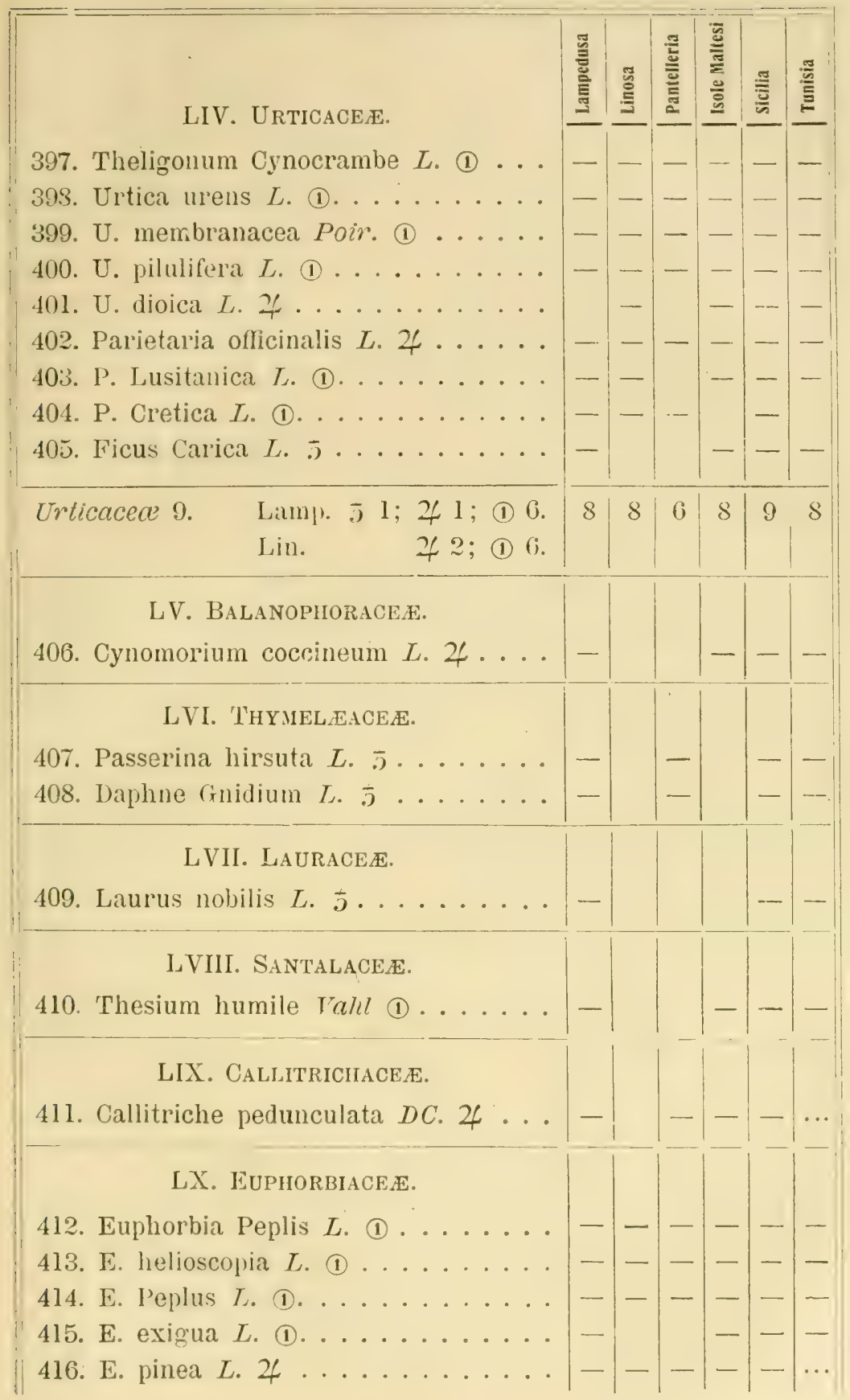




\begin{tabular}{|c|c|c|c|c|c|c|}
\hline & 焉 & $\begin{array}{l}\text { 总 } \\
\text { E. } \\
\end{array}$ & 丞 & 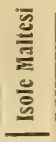 & 咅 & \\
\hline 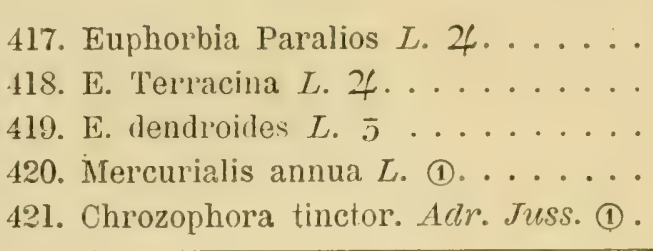 & $\begin{array}{l}- \\
- \\
- \\
-\end{array}$ & $\begin{array}{l}- \\
- \\
-\end{array}$ & - & $\begin{array}{l}- \\
- \\
- \\
-\end{array}$ & $\begin{array}{l}- \\
- \\
- \\
- \\
-\end{array}$ & - \\
\hline $\begin{array}{ll}\text { Euphorb. 10. } & \text { Lamp. } 51 ; \not 43 ; \text { (1) } 6 . \\
& \text { Lin. } 51 ; \not 33 ; \text { (1) } 4 .\end{array}$ & 10 & 8 & 7 & 10 & 10 & \\
\hline $\begin{array}{l}\text { MONOCOTYLEDONEA } \\
\text { LXI. ORCHIDACEE. } \\
\text { 422. Ophrys arachnites Lam. } 4 \ldots \ldots \\
\text { 423. O. Speculum Link } 4 \ldots \ldots \ldots \\
\text { 424. O. lutea Cav. } 4 \ldots \ldots \ldots \ldots\end{array}$ & - & & & - & $\begin{array}{l}- \\
- \\
-\end{array}$ & - \\
\hline $\begin{array}{l}\text { LXII. IRIDACE. } \\
\text { 425. Romulea Columnae Seb. Maur. } 4 \text {. } \\
\text { 426. Gladiolus dubius Guss. } 4 \ldots \ldots \ldots \\
\text { 427. G. segetum Ker.-Gawl. } 4 \ldots \ldots \\
\text { 428. Gynandriris Sisyrinchium Parl. } 4 \text {. } \\
\text { 420. Iris Germanica L. } 4 \ldots \ldots \ldots \ldots \\
\text { 430. I. Florentina L. } 4 \ldots \ldots \ldots \ldots\end{array}$ & $\begin{array}{l}- \\
- \\
- \\
- \\
- \\
-\end{array}$ & - & - & $\begin{array}{l}- \\
- \\
- \\
-\end{array}$ & $\begin{array}{l}- \\
- \\
- \\
- \\
-\end{array}$ & $\begin{array}{l}- \\
- \\
- \\
\cdots\end{array}$ \\
\hline $\begin{array}{l}\text { Lamp. } \& 6 . \\
\text { Lin. } ¥ 2 .\end{array}$ & 6 & 2 & 1 & 4 & 6 & 4 \\
\hline 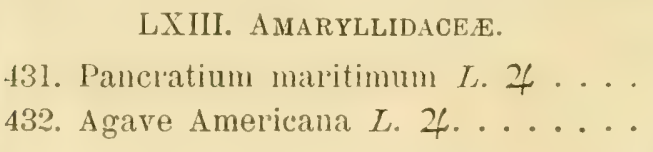 & - & - & - & - & - & - \\
\hline 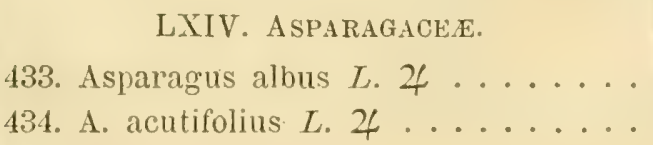 & - & - & - & - & & \\
\hline
\end{tabular}


435. Asparagus aphyllus $L .4 \ldots \ldots$. .

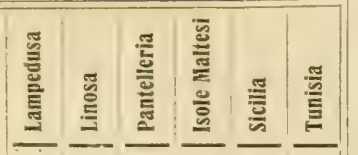

436. A. stipularis Forsh 4.

437. Smilax aspera $L .5$

\section{LILIACE E.}

438. Ornithogalum Narbonense $L$. 4. . . -

439. Urginea maritima Baker 4 . . . . .

440. Scilla autumnalis L. 24........ -

411. Nuscari comosum Mill. 4 ...... -

442. Allium roseum L. 24........

443. A. subhirsutum L. 4 .

44. A. vernale $T i n .24$.

445. A. Chamaemoly L. 4

440. A. tenuiflorum Ten. ( $\left.{ }^{21}\right)$ 4 . . . .

447. A. sphaerocephalum $L .4 \ldots \ldots$

448. A. margaritaceum Sibth. et Sm. 4.

449. A. Ampeloprasum L. 4. . . . . . .

450. Asphodelus tenuifolius Cav. (1) ...

451. A. ramosus $L .4 \ldots \ldots \ldots \ldots$. . .

\begin{tabular}{|c|c|c|c|c|c|c|c|}
\hline Liliacece 14. & $\begin{array}{l}\text { Lamp. \& } 13 . \\
\text { Lin. \& } 5 \text {; (1) } 1 .\end{array}$ & 13 & (j) & 8 & 10 & 13 & 13 \\
\hline
\end{tabular}

LXVI. COLCHICACE\&.

452. Colchicum Bertolonii Stev. $\psi$.

LXVII. JUNCAgINACEÆ.

453. Triglochin Barrelieri Lois. 4.... -

LXVIII. JUNCACE屟.

454. Juncus bufonius $L$. (1)........ -

LAXIX. NAJADACEE.

45.). Posidonia Oceanica Del. 4 .....

450. Cymodocea nodosa Asch. 4 
LXX. ARACE E.

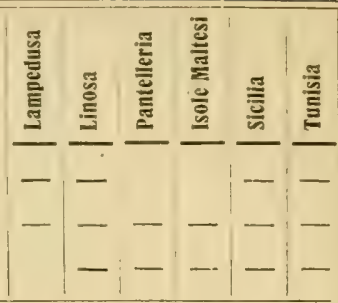

LXXI. CYPERACEE.

460. Schoenus nigricans $L$. 4 . . . . . -

461. Carex Halleriana Asso 4.

\section{GraminaceE.}

462. Phalaris brachystachys Link (1)... -

463. P. minor Retz. (1) ... . . . . .

464. Anthoxanthum odoratum L. 4 . .

465. Cynodon Dactylon Pers. 4.....

466. Andropogon hirtus $L .4 \ldots \ldots$. .

467. A. pubescens Vis. 4. . . . . . .

468. Arundo Donax L. 4........

469. Ampelodesmos tenax $\operatorname{Link} 4 \ldots$

470. Sporobolus pungens Kunth 4 ...

471. Triplachne nitens $\operatorname{Link}$ (1)......

472. Gastridium lendigerum Gaud. (1) ..

473. Polypogon Monspeliensis Desf. (1)..

474. P. maritimus Willd. (1) .......

475. Lagurus ovatus $L$. (1) . . . . . .

476. Stipa tortilis Desf. (1) .........

477. Milium coerulescens Desf. 4 ....

478. M. multiflorum Cav. 4 .......

479. Aira Cupaniana Guss: (1).......

480. A. Tenorei Guss. (1).........

481. Avena sterilis $L$. (1) . . . . . . -

482. A. fatua $L$. (1). . . . . . . . . -

483. A. barbata Brot. (1)......... - - - - -

484. Trisetum aureum Ten. (1)...... ----

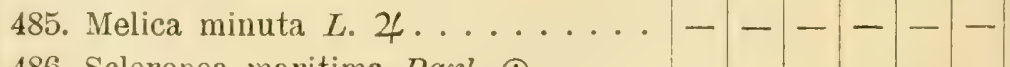

486. Scleropoa maritima Parl. (1)..... - $\quad$ - -

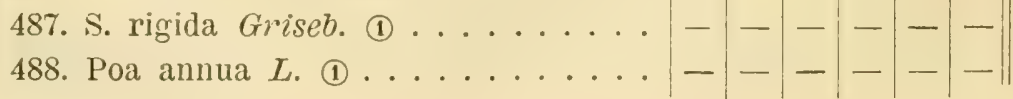


489. Sphenopus divaricatus Reichb. (1) . 490. Aluropus repens Parl. \& ..... 491. Dactylis glomerata $L .4 \ldots . .$. 492. Lamarckia aurea Moench (1).... 493. Chrysurus echinatus Pal. de B. (1). 494. Koeleria phleoides Pers. (1)...... 495. K. villosa Pers. (1) 496. K. hispida $D C$. (1) 497. Avellinia Nichelii Parl. (1)..... 198. Vulpia uniglumis Reichb. (1) ..... 499. V. Ligustica Link (1) 500. V. myurus Gmel. (1)......... 501. V. ciliata Link (1) 502. Bromus tectorum 503. B. Madritensis 504. B. maximus Desf. (1). 505. B. fasciculatus Prest 506. Serrafalcus mollis Part. 507. S. intermedius Parl. 508. Lolium perenne 509. I. Siculum Par\%. 510. L. rigidum Gaud. 511. Catapodium loliaceum Link (1).... 512. C. Siculum Link (1) .......... 513. Castellia tuberculosa Tineo (1).... 514. Brachypodium distachyum P. de B. (1) 515. Triticum villosum M. Bieb. (1).... 516. Aegilops ovata $L$. (1) ........ 517. Hordeum murinum L. (1)....... 518. Lepturus incurvatus Trin. (1) .... 519. L. filiformis Trin. (1) . . . . . . . 5:0. I. cylindricus Trin. (1) ....... Graminacee 59. Lamp. 413 ; (1) 40. Lin. \& 6 ; (1) 29 .

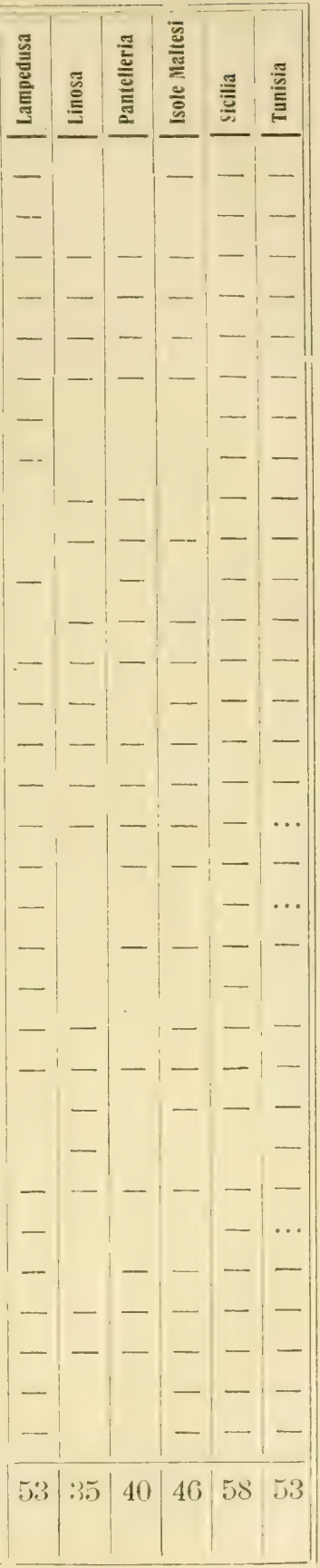




\begin{tabular}{|c|c|c|c|c|c|c|}
\hline$G Y \square$ GOSDER & 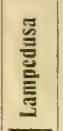 & 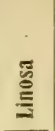 & 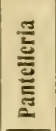 & 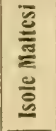 & 竧 & 总 \\
\hline $\begin{array}{l}\text { LXXIII. CONIFERæ. } \\
\text { 521. Pinus Halepensis Mill. } .5 \ldots \ldots \text {. } \\
\text { 522. Juniperus Phoenicea } L .5 \ldots \ldots \ldots\end{array}$ & - & - & - & - & - & - \\
\hline $\begin{array}{c}\text { PTERIDOPHYTAE } \\
\text { LXXIV. LYCOPODIACEE. } \\
\text { 523. Selaginella denticulata Spring. } 4 \text {. }\end{array}$ & $1 \div$ & & - & $1-$ & $1-$ & - \\
\hline 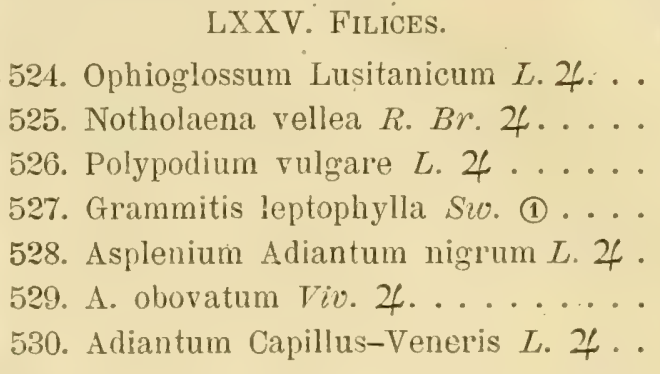 & - & $\begin{array}{l}- \\
- \\
- \\
-\end{array}$ & $\begin{array}{l}- \\
- \\
- \\
- \\
- \\
-\end{array}$ & - & $\begin{array}{l}- \\
- \\
- \\
- \\
-\end{array}$ & $\begin{array}{l}- \\
- \\
- \\
- \\
\cdots \\
-\end{array}$ \\
\hline $\begin{array}{l}\text { Lamp. } 42 \text {; (1) } 1 . \\
\text { Lin. } 44 \text {; (1) } 1 .\end{array}$ & 3 & 5 & 7 & $\mid 2$ & 17 & 6 \\
\hline
\end{tabular}

NO'TE AL PROSPETTO.

(1) Glaucium corniculatum Curt. - Questa specie non è indicata della Sicilia stessa, ma trovasi nella vicina isola di Maretimo.

${ }^{2}$ ) Brassica fruticulosa Cyr. - Secondo Cosson, come pure secondo Battandier a 'Trabut, benchè in Algeria non si trovi il tipo, vi se ne trovano delle varieti.

(3) Diplotaxis scaposa DC. - La presenza di questa specie non è indicata nei lavori sull'Africa settentrionale; ma io $l^{\prime}$ ho ricevuta dai dintorni di Tunisi. 
Di Maita invece è indicata da Duthie, Gulia, Caruel in Parl. (Fl. it.) e Fiori (Fl. anal.), ma a torto, poichè gli esemplari distribuiticon quel nome da Duthie, sui quali si fondano quelle indicazioni, appartengono alla Diplotaxis viminea DC.

(4) Dianthus rupicola Biv. $=D$. Bisignani Ten. - Citato da Battandier e Trabut per l'Algeria nella Flore d'Algérie (e riportato dal Fiori nella Fl. analitica sulla loro autorità), e poi messo in dubbio nella $2^{a}$ appendice p. v, nella Fl. aualyt. et syn. do l'Alg. et de la Tunisie degli stessi autori non è più citato affatto per l'Algeria. Per la Tunisia sola è citato il D. Bisignani Ten. var. Hermaeensis. Il D. Hermaeensis Coss. essendo da Bonnet et Barratte considerato come specio autonoma distinta, non metto alcun segno al D. rupicola nella mia ultima colonna.

${ }^{(5)}$ Silene Gallica L. - È indicata per le Isole Maltesi da Gulia, ma come me ne informa il conte Caruana Gatto, vi è fondata ragione per ritenere che sia un equivoco. Quindi non la segno nella relativa colonna.

${ }^{6}{ }^{6}$ Silene muscipula L. - Gussone (Syn.) non la cita, e Lojacono (F1. Sic.) dice espressamente che manca in Sicilia; ma Tanfani (in Parl. Fl. It.) dice che vi è stata raccolta presso Catania.

$\left({ }^{7}\right)$ Spergula pentandra L. - Indicata per la Sicilia soltanto da Lojacono (Fl. Sic.). E possibile che questa specie, indicata qua e là per molte parti d'Italia, por la Corsica e per la Sardegna, sia meno rara anche in Sicilia di quanto parrebbe, e che sia stata confusa con la $S$. arvensis L., come lo furono gli esemplari di Linosa da Tanfani (in Parl. Fl. It.).

(8) Lupinus Termis Forsk. - Questa specio è stata indicata sulla costa d'Africa soltanto dell' Egitto.

$\left.{ }^{9}\right)$ Secondo le indicazioni degli autori sembrerebbe che nell'Africa boreale vi fosse la Vicia villosa Roth., e mancasse la $V$. dasycarpa Ten. Sarebbe perú forse stato miglior consiglio consideraro l'una e l'altra come due varietà della specie complessiva $V$. varia Host.

(10) Vicia triflora Ten. - Segno questa specie per l'Algeria, quantunque non sia menzionata nei lavori citati, perchè io stesso l'ho vista di quella provonienza, come ho detto a p. 90.

(11) Sedum stellatum L. - Non l' ammetto per Malta, quantunque sia citato da Gulia, perchè il conte Caruana Gatto ritione che sia una indicazione erronea.

(12) Daucus Gingidium L. - Ė indicato della costa d'Africa solo per il Marocco. Ma della distribuzione geografica dei Daucus è difficilo parlare a causa del modo cosi diverso nel quale sono state intese le specie.

$\left.{ }^{13}\right)$ Dausus Siculus Tin. - Essendo questa specie stata citata solo dubitativamente per l'Algeria da Battandier e Trabut nella Fl. d'Algérie e poi soppressa nella loro Fl. analyt. et synoptique del 1904, nou la segno neppure con punti nella mia ultima colonna. 
(14) Crucianella rupestris Guss. - Indicata pør l'Africa settentrionale soltanto dell' Egitto (Parl. Fl. It. VII p. 99); ma è specie molto affine (da Boissier considerata come puro sinonimo) alla C. maritima L. comune in 'Tunisia e nel resto della costa N. dell' Africa.

(15) Galium cinereum All. - Sulla costa d'Africa è indicato soltanto della Cirenaica é della Tripolitania.

(10) Carduus argyroa Biv. - Fiori (Fl. an.) lo indica per l'Africa bor. occ., ritenendo che il $C$. myriacanthus indicato per l'Africa boreale, sia sinonimo del $C$. confertus Moris che egli considera alla sua volta come varietá del $C$. argyroa. Ma secondo Bonnet, come secondo Battandier a Trabut, il Carduus indicato per l'Africa boreale col nome di $C$. myriacanthus non è la specie di Salzmann, come credeva il Fiori, ma va riferito al C. Balansae Boiss. ot Reut. Non segno dunque il $C$. argyroa, neppure con dei punti, nell'ultima colonna. (17) Crepis foetida L. - In Sicilia questa specio è rappresentata dalla var. glandulosa (Guss.), che è la forma sotto cui trovasi pure a Lampedusa.

(18) Linaria cirhosa Dum.-Cours. - È indicata da Boissier per l'Egitto. Secondo la Flora Orientalis troverebbesi anche in altre parti dell'Africa boreale ed in Sicilia; ma non vedo questa indicazione confermata da altri autori, e per ciò mon segno questa specio nella colonna della Sicilia.

(19) Satureja microplyylla Guss. - Non è indicata nè di Tunisia nè d'Algeria, ma è detta comune al Marocco.

$\left.{ }^{(20}\right)$ Statice minuta L. - Questa specie che Battandier e Trabut ammettevano nella Fl. d'Algérie, dichiarando però di non averla vista, nella Fl, analyt. et syn. del 1904 è da loro definitivamente soppressa.

(21) Allium tenuiflorum Ten. - Della costa boreale d'Africa è indicato soltanto per il Marocco. In Tunisia come in Algeria trovasi I'affine $A$. pallens. 


\begin{tabular}{|c|c|c|c|c|c|c|c|}
\hline \multirow{7}{*}{$\begin{array}{l}\mathbb{J} \\
\stackrel{\mathscr{W}}{0} \\
\stackrel{\Xi}{\Xi}\end{array}$} & Sp. perenni legnose & 92 & 0 & - & $\overrightarrow{s i}$ & 0 & $\overrightarrow{G i}$ \\
\hline & Sp. perenni erbacee & 우 & $\overrightarrow{\mathrm{A}}$ & 0 & $\overrightarrow{0}$ & $\vec{H}$ & 13 \\
\hline & Specie bienni & 은 & 0 & 0 & $\stackrel{\circ}{-1}$ & 0 & 으 \\
\hline & Specie annue & $\underset{\sim}{\vec{\theta}}$ & 8 & 0 & $\stackrel{-1}{\leftrightarrows}$ & $\neg$ & $\stackrel{20}{S}$ \\
\hline & Totale delle specie & $\begin{array}{c}1-5 \\
\text { and } \\
a 1\end{array}$ & 25 & -1 & $\stackrel{\circ}{a}$ & 10 & $\overrightarrow{\text { ते }}$ \\
\hline & Generi & 莺 & ळे & -1 & $\underline{0}$ & 10 & $\stackrel{\infty}{-\infty}$ \\
\hline & Famiglie & $\stackrel{10}{9}$ & 5 & -1 & 19 & -1 & 20 \\
\hline \multirow{7}{*}{ 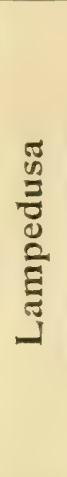 } & Sp. perenni legnose & $\stackrel{19}{71}$ & $T$ & a & $\stackrel{\infty}{\rightarrow+}$ & 0 & क्ञ \\
\hline & Sp. perenni erbacee & 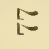 & ? & 0 & $\stackrel{\mathscr{N}}{\sim}$ & $\infty 2$ & 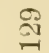 \\
\hline & Specie bienni & 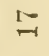 & 0 & 0 & $\stackrel{-1}{=}$ & 0 & 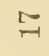 \\
\hline & Specie annue & \&D & F & 0 & क्षे. & -1 & 尺 \\
\hline & Totale delle specie & जे & के & or & क्षे & +1 & है \\
\hline & Generi & 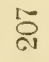 & 品 & $\approx$ & $\stackrel{\infty}{\approx}$ & +1 & จิ \\
\hline & Famiglie & $\begin{array}{l}\infty \\
10\end{array}$ & 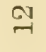 & - & $F$ & $\infty$ & $\stackrel{9}{2}$ \\
\hline \multirow{7}{*}{ 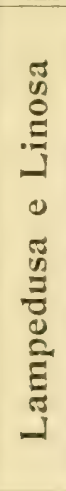 } & Sp. perenni legnose & 5 & -1 & बi & 88 & 0 & 8 \\
\hline & Sp. perenni erbacee & $\vec{\infty}$ & 8 & 0 & $\vec{m}$ & $\leftarrow$ & 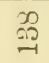 \\
\hline & Specie bienni & $\vec{a}$ & 0 & 0 & $\vec{a}$ & 0 & $\vec{a}$ \\
\hline & Specie annue & 영 & $\stackrel{\infty}{\rightarrow}$ & 0 & .ें & -1 & $\overrightarrow{\lambda 1}$ \\
\hline & Totale delle specie & $\vec{\Im}$ & \& & c & 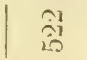 & $\infty$ & i⿱ \\
\hline & Generi & âं & 3) & $\sim v$ & $\begin{array}{l}10 \\
2 \\
2 i\end{array}$ & 10 & $\overrightarrow{\tilde{a}}$ \\
\hline & Famiglie & 8 & $\stackrel{11}{11}$ & -1 & $i^{2}$ & 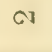 & ${ }^{10}$ \\
\hline & & 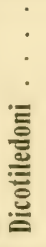 & 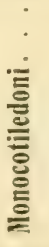 & 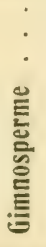 & 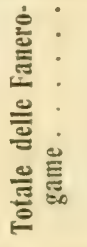 & 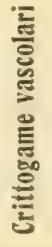 & 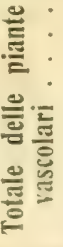 \\
\hline
\end{tabular}




\section{PANTELLERIA}

In attesa del giorno in cui verra scritta una Florula particolareggiata di Pantelleria, credo fare cosa utile pubblicando questo elenco delle 155 piante mancanti alle Pelagie che, aggiunte alle 313 della terza colonna del precedente Prospetto comuni a Pantelleria ed alle Pelagie, rappresentano la Florula vascolare di Pantelleria quale è nota oggi (468 specie). Ho indicato altrove le fonti alle quali ho attinto.' Sono contrassegnati con $+\mathrm{i}$ generi che mancano nelle Pelagie, e quindi non si trovano nel Prospetto.

\section{Piante di Pantelleria non comprese nel precedente Prospetto.}

1. Ranunculus Sardous Crtz. (1)

2. IR. parviftorus L. (1)

3. Papaver obtusifolium Desf. (1)

4. Matthiola sinucuta R. Br. (2)

5. Mr. tricuspidata R. Br. (1)

6. $\dagger$ Lepidium graminifolium L. 2

7. Sisymbrium Thatianum Gay (1)

8. † Raphanus Raphanistr. L. (1)

9. $\dagger$ Bunias Errucago L. (1)

10. Cistus villosus L. $\overline{5}$

11. C. Creticus L. 方

12. C. salvifolius L. $\frac{1}{5}$

13. Dianthus prolifer L. (1)

14. D. velutinus Guss, (1)

15. Cerastium brachypetal. Desf. (1)

16. Alsine tenuifolia Crtz. (1)

17. Spergula arvensis L. (1)

18. † Corrigiola litoratis I. (1)

19. † Paronychia cchinata Lam. (1)

20. $P$. argentea Lam. 2

21. Hypericum perforatum L. 2

22. $H$. perfoliatum L. 2

23. Malva Niccensis All. (1)
24. Geranium columbinum L. (1)

25. G. lucidum L. (1)

26. Erodium Botrys Bert. (1)

27. E. Romanum L'Hérit. 2

28. † Radiola linoides L. (1).

29. Oxalis comiculata L. (1)

30. + Calycotome villosa Link .j

31. † Genista aspalathoides Lam. $\frac{1}{5}$

32. G. candicans $I_{\text {d. }} 5$

33. †Cytisus triflorus L'Hérit. j)

34. Lupinus luteus. L. (1)

35. L. hirsutus L. (1)

36. Medicago marina L. 2

37. M. elegans Jacq. (1)

38. M. spharocarpa Bert. (1)

39. †Hymenocarpus circin. Savi (1)

40. Trifolium subterraneum L. (1)

41. T. Bocconei Savi (1)

42. $T$. angustifolium L. (1)

43. T. lappacerm L. (1)

44. T. repens L. '2

45. †Dorycnium hirsutum Ser. 方

46. Lotus parviflorus Desf. (1)

1 Sommer, Materiali per una Florula di Pantelleria. Bull. Soc. bot. it., 1907, p. 48. Ivi, per diversa interpretazione di alcune specie, avevo indicato un totale di 471, che secondo la mia valutazione attuale si riduce a 468 . 
47. Lotus hispidus Dosf. (1)

13. † Ornithapus ebracteat. Brot. (1)

4!). O. compressus L. (1)

5). $\dagger$ Pisum elatius M. Bieb. (1)

51. Lathyrus Aphaca I. (1)

52. Ervum pubescens DC. (1)

5i). E. parviflorum Bert. (1)

51. E. nigricans MI. Biob. (1)

55. $\dagger$ Amygdalus communis L. 5

56. † Rosa sempervirens L. 5

57. Eryngium naritimum T. 2

59. + Eehinophora spinosa L. 2

59. † Pimpinella lutea Desf. 2

60. Daucus gummifer Lam. (2)

61. Torilis purpurea Guss. (1)

62. † Asperula arvensis L. (1)

63. Galium divaricatum Lam. (1)

61. † Chrysanthemum Myconis L. (1)

65. C. hybridum Guss. (1)

66. C. segetum L. (1)

(i7. † Diotis candidissima Desf. 2 65. † 11elichrysum saxatile Moris 2 (i). Onupordon horridum Viv. ${ }^{1}$ (2) 70. + Galactites toment. Mönch (2)

71. Scolymus Hispanicus I. (2)

72. S. grandiflorus Desf. 2

7:. + Tolpis umbellata Bert. (1)

74. T. quadriaristata Biv. =

75. † Hypocheris glabra L. (1)

76. Urosperm. Dalechamp. Dsf. 2

77. † Chondrilla juncea L. (2)

78. Crepis vesicaria L. (1)

79. Andryala Cossyrensis Guss. (1)

80. Campanula dichotoma L. (1)

81. + Cucumis Colocynthis L. (1)

S.2. Erica arborea L. .5

S3. Erythrea maritima Pers. (1)

8.t. Echium plantagineum L. (1)

85). + Myosotis collina Hoffm. (1)

85. + Datura Metel L. (1)

S7. Plantago Lagopus L. 2

88. P. Bellardi All. (1)
89. Serofularia pinnatif. Guss. 2

90. Antirrtinum majus L. 2

91. A. tortuosum Pers. 2

92. Linaria commutata Bernh. (1)

93. Veronica Cymbalaria Bod. (1)

94. V. Panomitana Tin. (1)

95. Orobanche crenata Forsk. (1)

96. +Lavandula Stochas L. .)

97. † Mentha Pulegirm I. 2

98. Satureja Groca L. 方

99. S. Clinoporlium Caruel 2

100. S. Nepeta Scheele 2

101. † Ballota nigra L. 2

102. +Verbena officinalis I. (1)

103. + Acanthus mollis L. 2

104. + Samolus Valerandi L. 2

105. Statice densiflora Guss. 2

106. S. Cossyrensis Guss. 2

107. † Plumbago Europeca L. F

108. Polygonum Convolvulus L. (1)

109. P. Bellardi All. (1)

110. $P$. equisetiforme $\mathrm{S}$. et $\mathrm{S} .2$

111. Chenopodium album L. (1)

112. + Cytinus Hypocistis L。 2

113. Enphortia Chamaesyce L. (1)

114. E. serrata L: 2

115. †Quercus Ilex L. $\frac{1}{5}$

116. † Serapias longipetala Poll. 2

117. S. cordigera L. 2

118. + Tinea intacta Boiss. 2

119. Ophrys Scolopax Cav. 2

120. Romulea ramiflora Ten. 2

121. Narcissus serotinus L. 2

122. †Tamnus communis L. 2

123. + Caruelia Arabica Parl. 2

124. Ornithogal. tenuifol. Guss. 2

125. Asphodelus fistulosus L. 2

126. Juncus capitatus IVeig. (1)

127. † Cyperus levigatus L. 2

128. C. esculentus L. 2

129. C. rotundus I. 2

130. Carex vulpina L. 2

1 Un Onopordon vi è di certo in Pantelleria, ma è dubbio se sia l'O. horridum Viv. o il Sibthorpianum. Non avendo, nel Prospetto, assegnato a quest' isola l' $O$. Sibthorpianum, le assegno qui l' O. horridum. 
131. Carex muricata L. $z$

132. C. distachya Desf. 2

133. † Setaria viridis P. de B. (1)

134. S. verticillata P. de B. (1)

135. † Digitaria sanguinal. Scop. (1)

136. † Dactyloctenium Eigypt. W. (1)

137. Andropogon distachyus L. 2

138. Aira capillaris Host. (1)

139. A. intermedia Guss. (1)

140. Melica citiata L. 2

141. Poa bulbosa I. if

142. † Eragrostis poceoides P. B. (1)

143. E. megastachya Link (1)
144. † Briza maxima L. (1)

145. Chrysurus eleg. R. et Sch. (1)

146. $\dagger$ Gaudinia fragilis P. de B. (1)

147. Brachypod. pinnat. P. B. ₹

148. Pinus Pinaster Ait. 方

149. † Isoëtes Duricei Bory 2

150. + Ceterach officinar. Willd. 2

151. Asplenium Trichomanes L. 2 152. A. marinum L. 2

153. + Scolopendrium officin. Sm. 2 154. $\dagger$ Pteris aquilina L. 2

155. $\dagger$ Cheilanthes odora Swartz 2

In Pantelleria sono rappresentate sei famiglie di piante vascolari mancanti alle Pelagie, e cioè le Verbenacee, Acantacee, Citinacee, Cupulifere, Dioscoreacee, Isoëtacee. Aggiungendo queste 6 famiglie alle 62 che quest' isola ha in comune con le Pelagie, abbiamo per Pantelleria un totale di 68 famiglie.

I generi di piante vascolari di Pantelleria che mancano alle Pelagie sono 53 (quelli segnati con una $\dagger$ nell' elenco qui sopra). Aggiunti ai $223^{1}$ che Pantelleria ha in comune con le Pelagie, portano il totale dei generi oggi conosciuti di Pantelleria a 276.

Escludendo 15 specie, 12 generi e 3 famiglie di crittogame vascolari, abbiamo per Pantelleria 453 specie, 264 generi e 65 famiglie di fanerogame. Dunque in quest'isola, per le fanerogame il numero medio di generi per famiglia è 4,06, quello di specie per famiglia è 6,97 e quello di specie per genere è 1,72 .

Se cerchiamo il rapporto in Pantelleria fra le dicotiledoni (unendovi le gimnosperme) e le monocotiledoni, troviamo che le prime (362) stanno alle seconde (91) come 3,97 sta a 1.

Le piante vascolari annue in Pantelleria sono 270 (di cui 194 trovansi nelle Pelagie); le bienni sono 19 (di cui 13 Pelagie); le perenni erbacee sono 134 (di cui 76 Pelagie); le perenni legnose 45 (di cui 30 Pelagie) - totale 468 . Le piante annue costituiscono dunque il $57,69 \%$ della flora vascolare di Pantelleria.

1 Di questi 223 generi 10 sono rappresentati in Pantelleria soltanto da specie che mancano nelle. Pelagie. 


\section{CONSIDERAZIONI SULLA FLORA DELLE ISOLE PELGGIE}

Avanti di confrontare le flore di Lampedusa e di Linosa fra loro, e con quelle delle terre più vicine, devo dichiarare che fosso tenere conto soltanto delle sprecie quali le ho registrate nelle due florule e nel precedente prospetto, cioè in un senso in generale assai largo. ${ }^{1}$ Sarebbe certo molto importante il prendere in esame le specie critiche, le sottospecie, microspecie o varietá che chiamar si vogliáno, che potrebbero forse rivelare degli inizîi di differenziazioni locali, dei micro- o neo-endemismi, o potrebbero mostrare, nelle forme delle Pelagie, delle affinità maggiori sia con quelle dell'Africa, sia con quelle della Sicilia, e quindi indicarne la provenienza o dimostrare che la loro differenziazione è proceduta parallelamente con le une o con le altre Ma, oltre che ciò allargherebbe troppo il campo di queste considerazioni, tali forme subordinate sono troppo controverse, e sono dai varì autori intese in modo troppo differente perchè, nella maggior parte dei casi, vi si possano trovare elementi di confronto sicuri, senza avere sott'occhio abbondanti materiali dai varî territorî che si vogliono confrontare. Per questo stimo più prudente non basare su di esse dei ragionamenti che peccherebbero per la loro base, e limitarmi alle specie sulle quali gli autori sono per lo più d'accordo, e che nelle varie flore di cui dero servirmi sono state generalmente intese nel medesimo modo.

1 Confesso però che, per quanto abbia cercato di attenermi al senso Linneano della specie, in non pochi casi ho dovuto scostarmene e, per mancanza di elementi di giudizio, ammettere come specie dello forme forse subordinate. 


\section{Confronto fra la flora delle isole Pelagie e quelle delle terre più vicine.}

Prima di esaminare partitamente le florule di Lampedusa e di Linosa, consideriamole nel loro complesso. Anzitutto constatiamo la mancanza nelle Pelagie di endemismi primarî, ossia di endemismi di specie intese nel modo che ho detto sopra. Difatti, dellè due sole specie del mio prospetto non conosciute di altro luogo, il Cistus Skanbergi e la Linaria pseudolaxiflora, la prima è un ibrido, e la seconda fu da altri giudicata varietá della $L$. virgata, specie Africana. ${ }^{1}$ Esaminiamo dunque le piante delle Pelagie riguardo alla loro presenza nelle terre più vicine, incominciando con la Sicilia e le coste mediterranee d'Africa. Non tengo conto delle crittogame cellulari per le quali i dati di confronto sono per ora insufficienti.

Su di un totale di 530 specie, le Pelagie ne hanno 471, cioé $89 \%$ in comune tanto con la Sicilia quanto coll'Africa boreale. Delle rimanenti 59 specie, 25 mancano nell'Africa boreale ma trovansi in Sicilia, 22 mancano in Sicilia ma trovansi nell'Africa boreale, e 12 sole, cioé poco piủ di $2 \%$, mancano tanto in Sicilia quanto in Africa.

Specie delle Pelagie che mancano tanto in Sicilia quanto nell'Airica boreale.

Lin. . . Fumaria bicolor Somm. . . . . II. e P.

Lamp. . . Cistus complicatus Lam.

Lamp. . . Cistus Skanbergi Lojac.

1 Grisebacir, La végétation du Globe (traduzione di Tchihatchef จol. I, p. 506 e 552), cita come endemico di Lampedusa il Daucus Lopadusanus; ma questa specie, del resto assai critica, è stata indicata di Malta da varî autori, e di Sicilia (a Trapani) da Lojacono.

Fra le crittogame abbiamo due specie nuove di alghe terrestri; ma appartengono ad una categoria di piaute che sono state raccolte e studiate troppo poco perchè si possa ritenere che non si trovino altrove.

2 Lamp. avanti il nome della specie significa che è di Lampedusa, e Lin. che è di Linosa. La lettera MI dopo il nome significa che trovasi a Malta, e la lettera $\mathrm{P}$ che trovasi a Pantelleria. 
Lin.. . . Lotus peregrinus L.

Lamp. . . Daucus rimpestris Guss. . . . . M.

Lin.. . . Bellium minutum L.

Lamp. . . Jasonia glutinosa Guss. . . . . M.

Lamp. . . Filago Gussonei Lojac. . . . . . M.

Lamp. e Lin. Cardues mamorates Boiss. et Heldr. M.

Lamp. e Lin. Ileliotropium Eichwaldi Steud.

Lin.. . . Linaria psendolaxiflora Lojac.

Lamp. . . Teucrium Creticum L.

Questo numero gia tanto esiguo di piante Pelagie che non si trovano nè in Africa nè in Sicilia, andrebbe ancora ridotto volendosi attenere strettamente al senso Linneano della specie.' Ammettendo l'autonomia di queste 12 specie, vediamo che 5 di esse si trovano nelle isole Maltesi, le altre. salvo il Cistus Shanbergi e la Linaria pseudolaxiflora, si trovono in parti piu orientali. Tutte, meno l'Heliolropium Eichualdi, mancano nell'Italia continentale. Una sola si trova in Pantelleria.

Specie delle Pelagie che mancano nell'dirica boreale ma si trovano in Sicilia.

Lamp. . . Anemone hortensis I. . . . . . M. e P.

Lamp. . . Dianthus mupicola Biv.

Lin.. . . Ticia delsycarpa Ten.

Lamp. e Lin. Serlen litorem Guss. . . . . . . . M.

Lamp. e Lin. Sedum Hispanicum L.

Isamp. . . . Inancess Sicules Tin. . . . . . . .I.

1 Se ne dovrebbero togliere, per le ragioni dette alla pagina precedente, il Cistus Slanbergi e la Linaria psendolaxiflora. Il Daucus rupestris è specie dubbia e ad ogni modo molto afime a specie diffuse nel bacino mediterraneo. La Fumaric bicolor, specie di un genere critico, e recentemente descritta, $\dot{e}$ forse stata indicata di Sicilia e di Africa sotto altri nomi. Cosi pure la Filago Gussonei in Africa, e forse anche in Sicilia, puó essere stata confusa con la $F$. mostralc Parl. Il Carduzs marmoratus si puó supporre che sia stato indicato in Africa e in Sicilia sotto il nome C. Arabiers a l'Heliotropium Eichualdi col nome di II. Europaeum. Cosi si ridurrebbero a 5 sole lo specie delle Polagie che non si trovano nè in Sicilia ne in Africa. 
Lamp. . . Daucus Lopadusanus Tin. . . . . . M.

Lamp. . . Scabiosa Cretica L.

Lamp. . . Senecio pygmreus DC. . . . . . M.

Lamp. . . Calenchula micrantha Tin. . . . . . M.

Lamp. . . Calendula fulgidu Ral. . . . . . II.

Lamp. . . Filago arvensis L.

Lamp. . . Carlina Sicula Ten. . . . . . M. e P.

Lamp. . . Carcuus argyroa Biv.

Lamp. e Lin. Carduses brevisquamus (Fiori) . . . M. e P.

Lamp. . . Carduus corymbosus Ten.

Lamp. . . Picris spinulosa Bert. . . . . . M.

Lamp. . . Tragopogon Cupani Guss. . . . . M.

Lamp. . . Salıveja fasciculala Raf. . . . . . P.

Lamp. . . Statice minuta L.

Lamp. e Lin. Parietaria Cretica L. . . . . . . P.

Lamp. . . Ophrys arachnites Lam.

Lamp. . . Gladiolus duthius cruss. . . . . . . II.

Lamp. e Lin. Trisetum aureum Ten. . . . . . . M. e P.

Lamp. . . Lolium Siculum Parl.

Di queste 25 specie, 13 si trovano nelle isole Maltesi; le altre hanno per lo più un' area geografica che si estende tanto a Ponente quanto a Levante. Sei si trovano a Pantelleria. Tutte meno quattro (Daucus Lopadusanus, Senecio pygmaeus, Calenduza fulgida, Parietaria Cretica) trovansi nell'Italia continentale. Alcune di queste 25 specie si potrebbero eliminare considerandole come microspecie forse indicate d'Africa sotto un nome più complessivo, e cosi si diminuirebbe alquanto il numero delle specie Siculo-Pelagie mancanti alla costa d'Africa. Se peró invece di prendere in considerazione tutta la costa settentrionale dell'Africa ci limitiamo alla Tunisia (aggiungendo all' elenco qui sopra le 32 specie che si trovano in Sicilia e sono segnate soltanto con dei punti nell'ultima colomna del mio prospetto ${ }^{1}$ ), troviamo che è molto più grande, cioè 57, il numero di specie Siculo-Pelagie mancanti in quella parte dell'Africa, quantunque

1 Le specie segnate con dei punti nell'ultima colonna del prospetto sono $37 ;$ ma 5 di esse mancano in Sicilia. 
sia piủ ricina e più estesa della Sicilia, e la sua flora sia abbastanza conosciuta.

Specie delle Pelagie che mancano in Sicilia ma trovansi nell'Airica boreale.

Lamp. . . Diplotaxis scaposa DC.

Lin. . . . Silene apelala Willd.

Lamp. e Lin. Silene Behen I. . . . . . . . . . M.

Lamp. . . Hypericum Egyptiacrom L. . . . . M.

Lin.. . . Erodium angutatum Pomel

Lin.. . . Medicago secundiftora Dur.

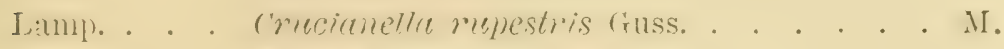

Lamp. . . Colnele anerea L. . . . . . . . . M.

Lamp. e Lin. Calendeta Egyptiaca Boiss. . . . . . M.

Lamp. . . Centaurea acaulis L.

Lin.. . . Amberboa Lippii DC.

Lin.. . . Onopordon Siblhorpianzm Boiss. . . . M.

Lamp. .. . Echinops spinosus L.

Lamp. . . Sonchus glaucescens Jord.

Lamp. . . Bryonia acula Desf.

Lamp. . . Stapelia Europaea Guss.

Lamp. . . Cuscuta Europaea L.

Lin.. . . Linaria cirrhosa Dum. . . . . . . P.

Lamp. . . Slatice echioides L.

Lamp. . . Statice intermedia Guss.

Lin... . Asphodelus tenuifolius Cav.

Lin. . . . Castellia tuberculosa Tin.

L'area geografica di queste 22 specie Afro-Pelagie mancanti in Sicilia $\dot{e}$, in generale, un poco meno estesa verso Oriente di quella delle Siculo-Pelagie mancanti in Africa. Sei sole si trovano nello isole Maltesi, e cinque sole nell' Italia continentale. Una sola si trova in Pantelleria, il che sembra strano, considerando la vicinanza di quest'isola alla Tunisia.

Un esame critico di queste 22 specie potrebbe diminuirne un poco il numero. Na considerandole come specie autonome, vediamo che 5 di esse (Silene Behen, Hypericum Egyptiacum, Crucianella rupestris, Cuscuta Europaea e Linaria cirrhosa) 
non sono indicate di Tunisia, e quindi il numero di specie Tuneto-Pelagie che non si trovano in Sicilia é di sole 17. Trovansi nelle Pelagie ed in Tunisia i generi Cotula, Castellia e Stapelia mancanti in Sicilia; ma la Colula aurea e la Castellia tuberculosa possono riferirsi ai generi Malricaria e Catapodium, cosicchè rimane il solo genere Stapelia assolutamente estraneo alla flora sicula.

Se ora confrontiamo statisticamente la flora delle isole Pelagie con quella delle isole Maltesi, troviamo che nella prima vi sono 131 specie mancanti alla seconda. Nanca dunque, nelle isole Maltesi, un numero di piante Pelagie molto maggiore di quello che manca alla Sicilia o alla Tunisia, il che non deve fare meraviglia pensando quanto sia più piccola l'area delle isole Maltesi e quindi minore il numero delle ioro piante. (Il numero delle specie rascolari conosciute delle isole Maltesi é di circa 800 , quello della Sicilia di circa $2586,{ }^{1}$ e quello della Tunisia di circa $1947^{2}$ ). Si potrebbe supporre che questa differenza fosse dovuta in parte anche al fatto che, se Lampedusa ha terreni della stessa natura di quelli delle isole Maltesi, in queste mancano affatto i materiali vulcanici di cui è interamente composta Linosa. Tale supposizione però non sarebbe confermata dai fatti, come vedremo confrontando separatamente ognuna delle Pelagie con le isole Maltesi.

Notiamo ancora che delle 59 specie Pelagie non appartenenti alla flora Afro-Sicula, 24 appartengono alla flora Maltese.

Passando poi a fare il confronto con Pantelleria, troviamo che in quest'isola mancano 217 specie delle Pelagie, numero

1 Cifra data dallo Ziccardi (in Guss. Synops. Fl. Sic., II, p. 693) ed accettata da Nicotra (Elementi statistici della Fl. Siciliana).

2 Questa cifra data da Doumet-Adanson in Bonnet et Barratte, Catalogue rais. des plantes vasc. de la Tunisie, Paris, 1896, p. xum, comprende le varieta ben caratterizzate, da molti considerate come specie. Bonnet, in Géogr. bot. de la Tunisie, dava la cifra di 1915. 
ancora assai maggiore di quello mancante alle isole Maltesi. Dobbiamo cercarne la ragione principale nel numero di piante molto più piccolo in Pantelleria (Malta 800, Pantelleria.471), inferioritá dovuta, in parte almeno, all' essere l'area di Pantelleria assai più piccola di quella delle isole Maltesi. Vedremo più oltre come non sarebbe giustificato l'attribuire la mancanza in Pantelleria di tante specie delle Pelagie al non trovarsi in quest' isola i terreni calcarei di cui è costituita Lampedusa. Delle 59 specie Pelagie mancanti alla flora Afro-Sicula, soltanto 8 si trovano in Pantelleria, e di queste, 5 si trovano nelle isole Maltesi.

Da questi confronti risulta che le isole Pelagie hanno una flora eminentemente Afro-Sicula. La zona inferiore della Sicilia e la vicina costa d'Africa hanno un gran numero di piante in comune, ed è appunto fra queste che le Pelagie hanno reclutato la grandissima maggioranza della loro flora. Ció era prevedibile, visto la loro posizione intermedia fra la Sicilia e l'Africa. A priori però si sarebbe creduto di trovare, fra le specie non comuni alla Sicilia e all' Africa boreale, un predominio mareato delle Africane, perchè una delle Pelagie è più lontana dalla costa di Sicilia che da quella d'Africa, perché entrambi sono sensibilmente più a Sud dell'estrema punta meridionale della Sicilia mentre i loro paralleli attraversano la 'Tunisia e l'Algreria per ben 20 gradi di longitudine, e finalmente perchè Lampedusa è considerata come geologicamente appartenente all'Africa. Invece non è cosí, ed abbiamo visto che vi era un leggiero predominio, nelle Pelagie, degli elementi Siciliani sugli Africaui, predominio che diveniva assai mareato se, invece di prendere in considerazione tutta l'Africa boreale, si limitava il confronto alla Tunisia: Difatti le specie Siciliane (496) entrano per $94 \%$, e le Tunisine (456) soltanto per $86 \%$ nella costituzione della flora delle Pelagie. 
E naturale che in isole cosi riarse durante molti mesi dell'anno, si debbano trorare specialmente piante dotate di adattamenti per resistere alla prolungata siccita. Non mi dilungherò nel rilevare i varî e ben noti adattamenti xerofili di cui presentano esempî le piante delle Pelagie. Solo insisteró su di uno che nelle Pelagie assume un carattere di generalita, cioè sull'arresto della vita nella stagione calda. Questo è ottenuto nel modo più perfetto colla breviti del periodo vegetativo e colla sospensione della vita nel seme delle piante annue, pèr cui in tal modo anche qualche pianta idrofila puó stidare il prosciugamento completo e prolungato del terreno. E vediamo difatti che nelle Pelagie le piante annue costituiscono il $61 \%$ della flora vascolare, mentre nell' Arcipelago Toscano raggiungono soltanto il $41 \%{ }^{\circ}{ }^{1}$ Possiamo dunque dire che la forte proporzione delle piante annue è una caratteristica della flora delle isole Pelagie, ed è in relazione con le loro condizioni ecologiche. "

1 Le specie annue, come è indicato nel riassunto del prospetto a p. 301, sono 321 nelle Pelagie. Nell'Arcipelago Toscano (Bull. Soc. bot. ital., anno 1903, p. 192) le annue e bienni sono 648, e deducendone 71 bienni, rimangono 577 annue, sopra un totale di 1411 specie vascolari.

${ }^{2}$ Questa spiegazione della prevalenza delle piante annue nelle Pelagie, sembra accordarsi male col fatto che dello 11 specie conosciute di Lampione, due sole sono annue. Ciò però si spiega pensando che Gussone, il quale vi ha raccolto queste 11 specie, fu in Lampione di Agosto, allorquando delle piante annue per lo più nou rimane traccia visibile. Non è del resto improbabile che la proporzione di piante annue vi sia realmente minore che in Linosa ed in Lampedusa, perchè quello scoglio esposto in ogni sua parte alla furia dei venti ed allo spruzzo delle onde salate, non presenta quei ripari necessarî per molte delle piante annue, tenere e delicate, come li presenta Lampedusa e più ancora Linosa. Inoltre, il vento che sotfia quasi di continuo deve portar via i semi ed ostacolare quindi grandemente la riproduzione delle piante annue. Gia Hooker ha notato che le piante annue, per mantenersi, avevano bisogno di aree di una certa estensione, e che per questo erano in minor numero nelle piccole isole. 
Ad un risultato analogo giungono molte piante perenni erbacee con la morte delle loro parti aeree in estate, e con il lungo letargo dei loro bulbi, tuberi o rizomi sotterranei. Ed anche di queste è piuttosto forte la proporzione nelle Pelagie.

Un altro modo di ottenere una sospensione, parziale almeno, della vita in estate, che si riscontra in varie specie legnose delle Pelagie, è la perdita delle foglie in quella stagione, come osservasi anche altrove nel mezzogiorno d'Italia, ad esempio nella Euphorbia dendroides, nell'Anagyris foetida, nel Thymmes capitatus, nella Thapsia Garganica, e come fu notata da Gussone in Linosa e Lampedusa anche in altre piante che egli in Sicilia e nel mezzogiorno d'Italia aveva viste sempre verdi, quali Clemalis cimohosa, Olea, Periploca, Rhus. Qui dunque, come in certe parti asciutte della zona torrida, vediamo prodursi per effetto dell' alidore estivo, lo stesso fenomeno della caduta delle foglie che in altri climi avviene d'inverno per elfetto del freddo.

La frequenza del nanismo nelle specie annue si puó ancora citare come una caratteristica della flora delle Pelarie. Tale nanismo, che è congiunto ad una vita vegetativa più breve della normale, è anch'esso l'espressione di condizioni ecologiche speciali, ma forse si doviebbe chiamare in molti casi una mostruosita, o una anomalia come puó esserlo la cadiuta delle foglie in aicune delle specie legnose," piuttosto che un adattamento fissato, poichè forse molte delle piante cosi ridotte non abboniscono i semi, o producono semi che germogliando in altre condizioni darebbero origine a piante normali. Questa è del resto una questione intorno alla quale non vi sono suflicienti osservazioni, e che va meglio studiata sperimentalmente.

Possiamo notare ancora, come caratteristica della flora delle Pelagie confrontata con quella della zona marittima della regione mediterranea in generale, la scarsita delle psammofile marine esclusive, cosi abbondanti nelle arene marine sulle coste del Me-

1 Gussone non ha detto come avveniva la caduta dello foglie da lui osservata nelle piante sempreverdi. Ultimamente il sig. J. Schiller (Esterr. bot. Zeitschr., 1907, n.6) ha descritto il modo anomalo, per rottura del picciolo, nel quale avviene sul littorale Adriatico la caduta delle foglie in alcune piante legnose sempreverải (fra cui l'Olea E'uropaea), per effetto di un vento forte ed asciutto. 
diterraneo, il che denota la piccolissima estensione di. questa stazione nelle Pelagie. Nancano ugualmente quasi tutte le idrofite, potendosi designare come tali soltanto l'Elatine Hydropiper, la Bulliarda Vaillantii e la Callitriche pedunculata, tre piante annue che si derono dire piuttosto anfibie che vere idrofite, poichè compiono l'ultima fase della loro vita in terreno quasi asciutto, e che hanno inoltre la preziosa facolta di conservare poi la vita latente nel seme durante la lunga estate, quando sono interamente prosciugate tutte le acque superficiali. Molto scarse sono pure le piante idrofile, ossia amiche dei luoghi umidi, potendosi contare come tali solo i due Lythrum, l'Anthemis fuscata, il Sonches maritimus, l'Arthrocnemum glaucum, il Triglochin Barrelieri, il Juncus bufonius, l'Eluropus repens e l'Adiantum Capillus Teneris. Idrofite e idrofile trovansi esclusivamente a Lampedusa. Le piante delle summentovate stazioni, oltre ad essere rappresentate nelle Pelagie da un numero piccolo di specie, vi si trovano in generale anche in numero scarsissimo di individui. ' Le alofite sono pievalentemente rupestri, come le Statice, il Crithmum, la Silene sedoides, il Bellium minutum, la suaeda fruticosa, la Salsola longifolia. Abbondanti sono le antropocore.

$$
\text { *** }
$$

Se esaminiamo la tlora delle Pelagie riguardo alla proporzione in cui vi sono rappresentati i gruppi sistematici, troviamo che le differenze con la flora delle terre piu vicine non sono meno marcate che nella proporzione dei gruppi biologici. Difatti le famiglie, seriate secondo la loro ricchezza di specie, salvo le tre piủ ricche, non si seguono, nelle Pelagie, nello stesso ordine che in Sicilia ed in Tunisia, nei quali due paesi, come si vede dal seguente specchietto, le sette famiglie più ricche occupano gli stessi posti.

1 Abbondanti sono soltanto l'Elatine e la. Bulliarda che hanno trovato negli incavi delle roccio impermeabili di Lampedusa dei piccoli serbatoî di acqua che si prosciugano solamente a primavera inoltrata, ed il Juncus bufonius che si trova in Lampedusa sotto la forma pumilio, nana ed a periodo vegetativo breve, adattata a terreni che si prosciugano presto. 
Famiglie principali ordinate secondo la loro ricchezza di specie.

\section{Pelagie.}

Totale delle specic: 530.

1. Composte... 74

2. Papilionacee . 67

3. Graminacee . 59

4. Cariofillacee . 22

כ. Ombrellifere. 21

6. Crocifere... 18

\%. Labiate....

"Chenopodiac. 14

» Gigliacee...

8. Crassulacee. . 12

9. Euforbiacee - 10

10. Geraniacee..

» Rubiacee...

„Convolvulac. . 9

》Urticacee...

11. Plumbaginac.

12. Ranunculacee

» Papaveracee.

»Borraginacee. 7

»Scrofulariacee

» Felci......

13. Fumariacee.

\Cistacee... 6

Irilacee....

14. Malracee...

„Solanacee. .. I

» Poligonacee.

\Asparagacee.

15. Valerianacee.

» Genzianacee./

» Plantaginacee (
Sicilia. ${ }^{1}$

'Totale delle specie: 2586 .

1. Composte... 315

2. Papilionacee . 285

3. Graminacee . 275

4. Crocifere ... 131

5. Ombrellifere . 113

6. Labiate ... I0S

7. Cariofillacee. 81

8. Rosacee.... 1 \%3

\$Scrofulariac. .

). Gigliacee ... (6)

10. Orchidacee . . 60

11. Ranunculace 59

1:. Ciperacee. . . 18

13. Borraginacee. 46

14. Rubiacee ... 39

15. Cistacee... (38

》Euforbiacee . 38

16. Chenopodiacee 33

17. Malvacee . . . :8

18. Poligonacee . 26

19. Geraniacee. .

„Crassulacee. . . .

» Plumbaginac.

\Giuncacee ...

2). Iridacee... . 21

21. Iipsatcee ...
» Convolvulac.

»Solanacee... 1 s

"Plantaginacee

»Cupulifere ..

22. Felci..... 18

23. Paronichiacee ।

Orobancacee.
Tunisia."

Totale delle specie: 1917.

1. Composte .. 247

2. Papilionacee . 223

3. Graminacee . 202

4. Crocifere ... 97

๖. Ombrellifere . 92

6. I abiate ... 77

7. Cariofillacee . 61

8. Scrofulariac. . )

» Gigliace ... 47

?. Borraginacee. 44

11). Chenopodiacee 43

11. Ciperacee... 36

1:. Rubiacee ... 35

13. Ranunculacee 34

14. Orchislacee. . 27

15. Geraniacee.. 26

16. Cistacee... 25

"Paronichiacee

17. Rosacee... 23

18. Plumbaginac. 20

19. Poligonacee - 18

»Felci......

20. Malvacee... 17

》Giuncacee...

21. Crassulacee. • 15

"Convolvulac. .

22. Fumariacee.

» Plantaginacee 14

» Naiadacee. . .

23. Valerianacee.

> Primulacee. 1

orobancacee. 1 . 13

$\gg$ Iridacee...

1 L. Sicotra, Elementi slatistici vella filore Siciliana in Nuov, Giorn, hot. it., XVI, 1884, p, 347.

2 LD. Boxiet, Giograplic botanique de la Tunisic. Extr. du Journal de botanique, IA et $\mathrm{x}$,

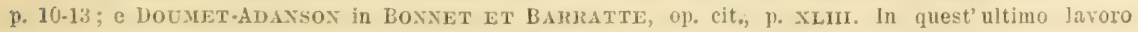
sono dimenticate le Poligonace. 
Notevole $\dot{e}$, come si rede, nelle Pelagie in confronto della Sicilia e della Tunisia, la scarsita delle Rosacee, Ciperacee, Giuncacee, Orchidacee, Scrofulariacee, Borraginacee, Ranunculacee, Cistacee, Malvacee, Paronichiacee, ed invece la ricchezza delle Crassulacee, Chenopodiacee, Geraniacee, Fumariacee, Euforbiacee, Plumbaginacee, Papaveracee, Felci, Convolvulacee, Urticacee.

In molti casi la scarsitá e la ricchezza di specie vanno d'accordo con una corrispondente scarsita e ricchezza di individui.

Riguardo all'ordine in cui si seguono le famiglie nello specchietto qui sopra, sembra che le Pelagie presentino deile divergenze, ossia differenze di livello, un poco più accentuate con la Sicilia che con la Tunisia. ${ }^{1}$ Si potrebbe a prima vista credere che cio derivasse dalla esistenza, nella flora Siciliana, di molti elementi della zona montana. Na a tale interpretazione contraddice il fatto che le divergenze sono minori fra la Sicilia e la Tunisia che fra le Pelagie e questi due paesi. Questa concordanza fra loro delle flore di due terre separate da buon tratto di mare, maggiore che fra ognuna di esse e quella di isole a quelle terre interposte, si deve attribuire al fatto che, in una medesima regione botanica, più è piccola l'area di cui si studia la flora, più primeggia l'influenza dei fattori ecologici locali, cancellando o mascherando le leggi generali della distribuzione dei vegetali in quella regione; più è grande invece l'area contemplata, più ci si arricina a questa legge di distribuzione.

Importante è il notare che le differenze di livello, o divergenze, fra le famiglie delle Pelagie e quelle delle altre due colonne, se per lo piü sono diverse di grado, pure in tutti i casi si verificano nello stesso senso; in altri termini le famiglie che nelle Pelagie sono relativamente più ricche che in Sicilia, sono anche piú ricche che in Tunisia, e quelle più povere che in Sicilia sono anche più povere che in Tunisia.

1 Le somiglianze e le differenze nella composizione di diverse flore, anche dal punto di vista puramente sistematico e statistico, si possono intendere e valutare in modi diversi e quiudi sfuggono ad un calcolo matematico esatto. Per questo mi sono limitato qui al confronto dell'importanza che hanno le famiglie principali, desunta dal posto (o livello) che occupano quando sono messe in serie per ricchezza di specie. 
Se facciamo questo medesimo confronto fra le famiglie seriate per ricchezza di specie nelle Pelagie e nell'Arcipelago Toscano, altro distretto floristico ben conosciuto, mediterraneo ed insulare come le Pelagie, ma situato ben sette gradi più a Nord e meno distante da terre continentali, vediamo che le differenze di livello delle famiglie poco difleriscono in entità da quelle che corrono fra le Pelagie e la Sicilia e la Tunisia; ed anche qui, nella gran maggioranza dei casi, si verificano nella stessa direzione, cioé sono in generale le stesse famíglie che si distinguono per ricchezza o povertả rispetto all'Arcipelago 'Toscano, come rispetto alla Sicilia e alla Tunisia." Dunque le medesime cause che fanno divergere sotto questo rapporto la flora delle Pelagie da quelle della Sicilia e della Tunisia, la fanno divergere in modo press'a poco uguale dalla flora di altri distretti floristici anche più lontani, ma della medesima zona e della medesima regione.

Si puó ancora osservare che nessuna delle famiglie che in Tunisia contano più di 9 specie, manca nelle Pelagie; e che delle famiglie Siciliane mancanti nelle Pelagie, una sola, quella delle Cupulifere, conta in Sicilia più di 9 specie.

In quanto alla proporzione fra le dicotiledoni (includendori le gimnosperme) e le monocotiledoni, essa è : ${ }^{2}$

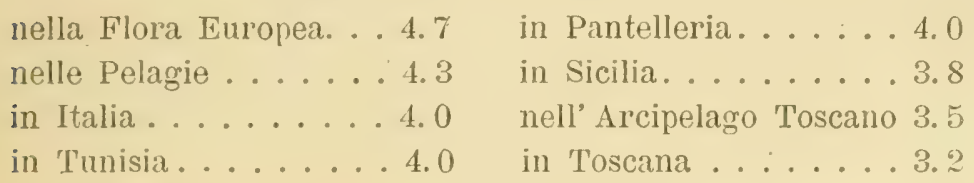

1 Non riporto qui, per brevita, la seriazione delle famiglie nell'Arcipelago Toscano. Si puo desumere dalla mia Flora dell'Arcipelago Toscano (N. Giorn. bot. it., Vol. X, 1903).

2 Por la. Sicilia, l'Italia, l'Europa e li Toscana riporto le cifre date da Nicotra, Elementi statistici della Flora Siciliana, paragr. IV, p. 312. Per la Tunisia ho rilevato il rapporto dai dati di DoumetAdanson in Bonnet at Barratte, Catal. $l$. Plantes vase. de la Tunisie, p. xurII; per Pantelleria dal mio Prospetto nel presente lavoro, con le Aggiunte a p. 302-4; per l'Arcipelago 'T'oscano da Sommer, Prospetto della Flora dell'Arcipelago 'Toscano in N. Giorn. bot. ital., 1903, p. 141. 
Le Pelagie sono dunque proporzionalmente più ricche di dico. tiledoni, e piú povere di monocotiledoni della Sicilia, della Tunisia, di Pantelleria, dell'Italia, delia Toscana e dell'Arcipelago Toscano; sono invece piủ ricche di monocotiledoni e più povere di dicotiledoni dell' insieme d'Europa. La differenza minore, a questo riguardo, la presentano con la Tunisia, con Pantelleria, e con l'insieme della flora Italiana; la differenza maggiore con la 'Toscana.

$$
\text { *** }
$$

Molti autori si sono occupati dei rapporti numerici fra specie, greneri e famiglie nelle flore di paesi diversi, cercando quale relazione vi fosse fra questi rapporti e le condizioni ecologiche dei paesi di cui studiavano statisticamente le flore. Per. questo ho esposto nel seguente quadro, quanto sia in media il numero. di generi e di specie per famiglia, e quanto il numero di specie per genere nelle Pelagie ed in alcune altre parti della regione mediterranea, e cioè in tutta l' Italia, in Sicilia, in Tunisia, in Pantelleria, in Toscana, nell'Arcipelago Toscano, in due delle isole di questo Arcipelago (quelle che per numero di specie si avvicinano di più alla flora delle Pelagie, cioé Capraia e Pianosa), e finalmente nelle cinque isolette che troransi lungo la costa della Liguria e che esse pure, nel loro insieme, hanno un numero di specie che si arricina a quello delle Pelagie. Ho disposto queste varie flore secondo il numero delle loro specie, incominciando con la piu ricca. Mi sono limitato alle fanerogame, come hanno fatto in generale gli autori che si sono occupati dell'argomento. 


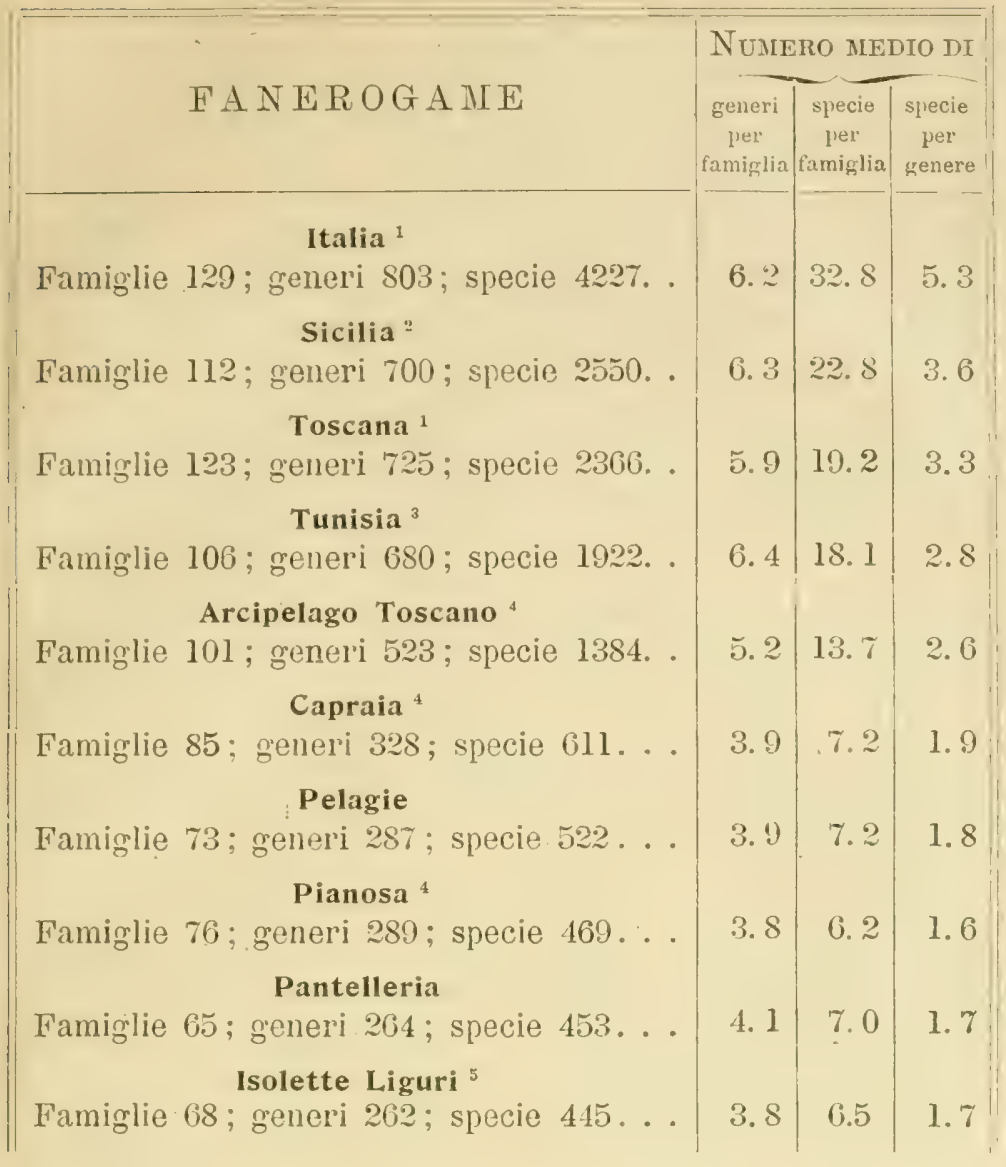

1 Le cifre pex l' Italia e per la Toscana sono prese da CARUEL, Stutistica botanica della T'oscanc, p. 39.e 48. Le proporzioni fra ordini, generi e specie sono calcolate sopra queste cifre. Per l'Italia differiscono un poco dai rapporti indicati da Caruel stesso a p. 50 (specialmente il rapporto fra specie e famiglie, per il quale Caruel di la cifia di 34,1 , mentre dalle cifre di Bertoloni, sulle quali si basa Caruel, risulta di 32,767). Dall'epoca in cui fu scritto il lavoro del Caruel ad oggi, i molto progredita la conoscenza delle thore di Toscana e d'Italia; ma credo che non saranno per questo molto cambiati i numeri medî di specie per famiglia e per genere, e di generi per famiglia.

2 Il numero di famiglie, generi e specie di Sicilia e preso da Nucotin (Elementi stafist. $d$. Fl. Sicil., \& $1,2,3$, p. 339, 3t1), il quale, per le specie, ammette il numero dato

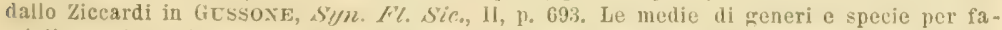
miglie, e di specie per genere che do qui sopra, sono calcolate sopra queste eifre, e differiscono leggermente da quelle indicate dallo stesso Nicotra il quale per i suoi calcoli sembra essersi servito del numero di specie un poco differente indicato per la Sicilia da De Candolle.

3 Da BoxגET ET BARIATTE, op. cit.

4 Da SOMYIER, op, cit.

5 Da A. BÉglixot, the regetazione relle Isole Liguri, Gallinra, liergeigi, Palmaria, T'ino e T'inetto, in Anaali del IIuseo Civico di Genova, Vol. Xlll (1907), 1\%,39 (463). 
Come si vede da questi esempîscelti in parti del bacino mediterraneo abbastanza lontane fra loro, più è picculo il numero di specie di una flora, più piccolo è il numero medio di specie per genere. Una piccola irregolarita insignificante presenta a questo riguardo soltanto Pianosa. Meno costante, benché in generale riconoscibile, è la diminuzione delle specie e dei generi per famiglia col diminuire del numero di specie prese in esame. Si vede pure che le noteroli differenze ecologiche fra le Pelagie, le due isole Toscane ed il gruppo delle Liguri, quattro distretti che poco differiscono per numero di specie, non portano sensibile variazione nelle proporzioni fra specie, generi e famiglie. Cio mi sembra una prova che questi rapporti nei varî distretti o dominî appartenenti ad una medesima regione, siano l'espressione matematica di un calcolo di probabilita basato sul numero di specie, di generi e di famiglie esistenti nella regione, calcolo pochissimo disturbató dall'azione dei fattori ecologici proprî ai distretti o ai dominî presi in esame; e mi pare quindi che poco abbia a valersi di questi rapporti l'ecologia botanica.'

1 Nella flora di uno scoglio sul quale crescesse una sola specie, $\nabla i$ sarebbero un genere ed una specie per famiglia, ed una specie per genere. Se vi crescessero due specie, secondo ogni probabiliti esse appartorfebbero a due generi e a due famiglie. Con ogni specie addizionale aumenterebuero le probabilita che questa specie appartenesse ad una famiglia e ad un genere già rappresentati e quindi che aumentasse il numero medio di specie per famiglia e per genere. Sull' isolotto di Lampione, p. es. ( 11 specie appartenenti a $\delta$ famiglie e a 11 generi. Prendendo in esame altre isolette, si vedrebbe che man mano aumenta il numero di specie, diminuisce la proporzione del numero di famiglie e di generi. Il calcolo che ho fatto per diverse piccole flovule lo ha quasi invariabilmente confermato.

$\mathrm{Vi}$ è chi ha detto che il numero medio di specie per genere diminuiva man mano si andava da Sud a Nord e dal piano verso la cima dei monti, oppure che diminuiva per effetto della steriliti del suolo. E stata pure formulata la legge che «il numero medio di specie per genere aumenta coll'aumentare della diversità nelle condizioni ecologiche dei territorî presi in esame ». Vi è poi chi ha ritenuto che un piccolo numero di specie per genere fosse una caratteristica delle flore insulari, specialmente delle piccole isole, $\theta$ questo è stato da molti ripetuto come un assioma. La maggior 


\section{Modificazioni nella flora delle Pelagie.}

All'azione diretta dell'nomo, ed a quella degli animali da esso introdotti, ho accennato a p. 47 e 183. Anche nel breve spazio di tempo che ci separa dalla visita di Gussone, possiamo constatare nella flora delle Pelagie dei profondi mutamenti dovuti all'uomo, non solo per la distruzione o grande diminuzione di alcune specie legnose, ma anche per l'introduzione di varie piante. L'introduzione volontaria del Fico d'India ha grandemente cambiato il paesaggio botanico tanto di Lampedusa che di Linosa; quella del Mesembryanthemum crystallinum. (introdotto per l'estrazione della soda) ha influito sul paesaggio botanico di Linosa. L'introduzione involontaria dell' Oxalis cernuca ha fornito a Lampedusa uno dei suoi elementi floristici più cospicui e piú diffusi. Molte sono le specie erbacee citate da Gussone e da Calcara che, come si puó vedere nelle mie florule, non furono più trovate da altri; ed è lecito supporre che varie di esse sono definitivamente scomparse insieme alla macchia alla quale erano

parte degli esempî portati in appoggio confermano questi varî modi di vedere, che del resto non sono contrarî gli uni agli altri. Mi pare tuttavia che sia stata disconosciuta la vera causa della diminuzione del numero di specie per genere, che risiede unicamento nel minor numero di specie prese in esame. Difatti sterilità e uniformiti di condizioni ecologiche fanno impoverire le flore, e quindi la differenza nel numero di specie per genere non è da ricercarsi nei fattori ecologici, ma nel numero diverso di specie nelle flore confrontate. Cosi se è piccolo il numero di specie per genere nelle isole, specialmente nelle minori, è perchè piccolo è il numero di specie che albergano. De Candolle, nella sua Géograptie botanique raisonnée ha complicato il problema introducendo nei confronti le aree delle diverse flore, e rendendo cosi meno evidente la relazione fra il numero di specie delle varie flore $\theta$ il rapporto fra generi $\theta$ specie. Secondo me, volendo formulare una legge, bisogna dire che "il numero medio di specie per genere è direttamente proporzionale al numero di specie prese in esame, indipendentemente, o quasi, dalle condizioni ecologiche del territorio da cui provengono 》. Questa legge andrebbe documentata con gran numero di esempî, o ne andrebbero ricercato le cause; ma non è questo il luogo per dare all'argomento lo svolgimento che merita. 
consociate. " Ed inversamente molte sono le piante specialmente arvensi ed antropocore ora comuni, che non furono notate né da Gussone nè da Calcara, e di cui una parte almeno si deve ritenere introdotta dopo il loro tempo. Qualcuna delle specie annue osservate da un solo raccoglitore puó darsi che fosse eflimera, e rappresenti un tentativo infruttuoso d'immigrazione.

Quale parte abbiano avuto gli agenti naturali nelle modificazioni avvenute nella flora dall'epoca di Gussone in poi, è impossibile stabilire. Ma qualche parte probabilmente è da attribuirsi anche ad essi, specialmente in unione con l'azione dell'uomo il quale, dissodando terreni, prepara una stazione propizia agli immigranti per qualunque via essi vengano.

Sull' azione dell' uomo avanti il tempo di Gussone non possiamo fare altro che delle congetture basate sopra quello che sappiamo degli ultimi tre quarti di secolo, e di quello che ci insegnano altri paesi ${ }^{2}$ ma certo sará stata grande, specialmente a Lampedusa; e ad essa possiamo per esempio attribuire la mancanza fin dallora nelle Pelagie del leccio così caratteristico della regione, ma troppo ricercato dall'uomo per il suo legno.

\section{Confronto fra le florule di Lampedusa e di Linosa.}

Non ostante le grandi differenze nel rilievo e nella natura del loro suolo, essendo Lampedusa piana e calcarea, con terreno quasi tutto compatto, Linosa invece montuosa e vulcanica, con abbondanza di terreni sciolti, la differenza nella ric-

1 Calcara, Descr. di Lampedusa, p. 33, parla di tronchi d'alberi rivestiti di muschi e licheni. Se realmente fosse cosi, bisognerebbe dire che con gli alberi sono scomparsi tutti i muschi arboricoli, poichè non ne ho trovato neanche uno, nè a Lampedusa nè a Linosa. Ma della esattezza di Calcara è lecito dubitare, specialmente trattandosi di crittogame.

2 IVarming, ad esempio, in Den Danske Planteverdens Historie efter istiden, valuta a circa 150 le fanerogame la cui introduzione in Danimarca si puó attribuire all'uomo nel corso del solo secolo 190; ○ dice che forse più della metà delle 1400 fanerogame della Dani- 
chezza delle loro flore fanerogamiche è press'a poco quale ci si sarebbe potuto aspettare per la diversiti delie loro aree se avessero avuto condizioni di suolo eguali. Ció desumo dal confronto con due isole dell'Ar'cipelago 'Toscano, Giamnutri e Pianosa, distanti l'una dall'altra poco piu che Linosa da Lampedusa, ed arenti aree che stanno press'a pcco nello stesso rapporto (cioè di circa uno a quattro). ${ }^{1}$ Benchè Giannutri e Pianosa sieno ugualmente pianegrianti ed interamente composte dello stesso calcare cavernoso, il rapporto numerico delle loro specie è press'a poco Io stesso di quello tra Linosa e Lampedusa. ${ }^{2}$ Si deve dunque riconoscere che in Linosa ed in Lampedusa le grandi differenze moifologiche, fisiche e chimiche del suolo influiscono poco sul numero delle specie fanerogame. Molto invece sembra che influiscano sul numero delle specie crittogame. Infatti già nelle crittogame vascolari vediamo invertite le proporzioni, e Linosa con 5 specie superare Lampedusa che ne ha 4 sole, nonostante che Lampedusa abbia un'area quasi quattro volte magriore di Linosa. Questa disproporzione in favore della piccola Linosa si accentua ancora più nelle briofite, tanto nei muschi (Lampedusa ne ha 30 e Linosa 40) quanto nelle epatiche (Lampedusa 8, Linosa 15). La proporzione si mantiene in favore di Linosa

marca sono immigrate in questo modo nei tempi storici. - La scomparsa dall' isola di S. Elena della maggior parte della flora indigena, e la sua sostituzione da piante quasi tutte inglesi, importate involontariamente dall'uomo, ci offromo un bell'esempio di quanto possa il fattore antropico in breve volgere di tempo.

1 Lampedusa ha kmq. 20.20, Linosa kmq. 5.43. - Pianosa ha kmq. 10.25, Giamnutri ne ho. 2.31. Linosa è dunque, per rapporto a Lampedusa, un poco meno piccola che Giannutri per xapporto a Pianosa.

2 La flora fanerogamica di Linosa che conta 289 specie, sta a quella di Lampedusa che ne conta 460, come 62.83 sta a 100. Quella di Giannutri con 275 specie sta a quella di Pianosa con 469 specie, come 58.63 sta a 100 (v. Nuov. Giorn. bot. ital., X, 1903, p. 190 e Bull. Soc. bot. it., 1903, p. 228). Vi è dunque una piccola differenza in favore di Linosa nel numero delle specie, come vi è nel rapporto delle aree.

Riconosco però che tali confronti fra isole di areo diverse, ed in condizioni assai differenti per clima e per distanza da altre terre, hanno un valore molto rolativo. Confronti fra altre isolo appartenenti alla Sicilia non lio potuto fare per mancanza di dati statistici. 
anche nei licheni (Linosa 38, Lampedusa 31). La ricchezza di crittogame relativamente molto maggiore in Linosa non si manifesta soltanto nel numero piủ grande di specie, ma anche nella abbondanza di individui, tanto che nel visitare successiva. mente le due isole si è colpiti dalla prevalenza del rivestimento crittogamico in Linosa.

Il paragone con le isole dell'Arcipelago Toscano ci mostra pure che a paritả di superficie le Pelagie sono più povere di specie vascolari delle isole Toscane. Difatti Lampedusa con kmq. 20.20 ne ha solo 464, mentre Capraia con limq. 19.53 ne ha 627 e il Giglio con kimq. 21.21 ne ha 700. Anche la flora di Pianosa, con 478 specie vascolari, supera un poco quella di Lampedusa, quantunque abbia un' area di circa metá. Linosa con limq. 5.43 ha 294 specie, mentre con delle superficie metả più piccole, Gorgona (kmq. 2.23) ne ha 465 e Giannutri (kmq. 2.31) ne ha 280. ${ }^{1}$ La povertá relativa delle Pelagie è dunque ugualmente accentuata in Linosa e in Lampedusa, e si osserva tanto nelle crittogame vascolari quanto nelle fanerogame. Oramai la flora vascolare delle Pelagie è abbastanza bene conosciuta per ritenere che ulteriori ricerche non potranno modificare sensibilmente questa disproporzione. Confronti intorno alla ricchezza in crittogame cellulari sarebbero prematuri, le isole dell'Arcipelago Toscano essendo a quel riguardo molto meglio esplorate delle Pelagie. Da quello che sappiamo peró, in quanto a briofite sembrerebbe che Linosa non fosse inferiore alle isole Toscane, e Lampedusa invece fosse assai più povera.

La povertà della flora vascolare nelle Pelagie deve certo attribuirsi, in gran parte almeno, alla brevitả del periodo vegetativo in causa della prolungata siccitá dell'estate, alla mancanza di acque superficiali, alla grande uniformitá del terreno in ognuna delle due isole e quindi alla pochissima varietá di stazioni. Puó forse dipendere in parte anche dalla più grande distanza da terre più estese, e quindi dalle difficoltà maggiori

${ }^{1}$ La flora di Giannutri, che dal Prospetto della Fl. dell'Arcipelago Toscano risultava di 187 fanerogame e 191 piante vascolari, è stata per ulteriori esplorazioni portata a 275 fanerogame e a 280 piante vascolari (v. Bull. Soc. bot. it., 1903, p. 228). 
per la immigrazione di molti semi. A questo fa riscontro il fatto gia da altri osservato, che le isole sono più povere di un'area uguale sul continente più vicino. Suppongo che ció sia perché la conquista del terreno passo a passo è più efficace di quella longinqua, contro la quale meglio si difeudono le associazioni, anche se porere, quando hanno preso piede in un'isola, e che diventa tanto meno efficace quanto maggiore è l'isolamento ossia la distanza dai centri di diflusione.

$$
\text { *** }
$$

Vediamo ora quanto e come differiscano nella loro composizione le florule di Lampedusa e di Linosa.

Delle 522 fanerogame che si trovano in Lampedusa e in Linosa, le due isole ne hanno in comune 227 , cioè $43,5 \%$. Pianosa e Giannutri, con una flora complessiva di 535 specie, ne hanno in comune 209 , ossia $39,1 \%$. Abbiamo veduto dianzi che proporzionatamente alle loro aree le florule di Lampedusa e di Linosa differiscono, in ricchezza, press' a poco quanto quelle di due altre isole uguali fra loro per la costituzione del suolo prese ad esempio nell'Areipelago Toscano. Ora vediamo che nella loro composizione le flore delle due Pelagie differiscono sensibilmente meno l'una dall'altra delle stesse due isole Toscane. Troviamo dunque il contrario di quanto avremmo potuto presumere considerando la similarita di configurazione e di composizione del suolo di Giannutri e di Pianosa. Ne dobbiamo trarre la conclusione che nelle Pelagie, configurazione e natura del suolo sono fattori che hanno avuto meno influenza di altri nel determinare tanto la ricchezza della flora fanerogamica quanto la diversitá (statisticamente parlando) della sua composizione.

Diversamente anche in questo si comportano le crittogame. Difatti, delle loro 8 crittogame vascolari, le due isole ne hanno una sola in comune; nelle briofite le specie comuni alle due isole (23) formano soltanto il $33 \%$ del totale (70), e nei licheni la proporzione delle specie comuni alle due isole è ancora minore. Ció puó dipendere in parte, per le crittogame cellulari, da una investigazione meno accurata; ma le differenze con la proporzione delle fanerogame sembrano troppo grandi per renire cancellate da una piủ completa esplorazione. 
Da quanto precede si puó trarre la conclusione che le crittogame ci presentano un reattivo assai più sicuro delle fanerogame per rivelare le differenze edafiche in distretti di ugual clima.

Analizzando ora sommariamente le differenze fra le florule rascolari di Lampedusa e di Linosa, notiamo anzitutto che è più marcata in Linosa la prevalenza delle specie annue, le quali formano il $66,3 \%$ della sua flora, mentre in Lampedusa raggiungono soltanto il $58,2 \%$ Questa maggriore prevalenza è abbastanza marcata perchè si debba riconoscere in essa l'espressione di condizioni ecologiche generali dell'isola. In quanto all'azione dell'uomo che si è esercitata molto meno in Linosa che in Lampedusa, più lungamente abitata e più estesamente coltivata, essa non potrebbe aver agito che nel senso. opposto, poiché tende a favorire l'introduzione e la moltiplicazione delle piante annue. ${ }^{1}$

Il maggior numero delle specie annue in Linosa mi sembra che si possa spiegare con la natura fisica del suolo, indipendentemente dalla sua natura chimica. Le roccie nere di Linosa devono riscaldarsi più dei calcari bianchi di Lampedusa, e le sabbie scure che ricuoprono gran parte dell'isola vulcanica devono prosciugarsi profondamente più presto dei terreni compatti dell'isola sedimentaria. Quindi in Linosa deve farsi sentire anche più che in Lampedusa la necessità di adattamenti per sopportare il calore e l'aridita della lunga estate. Ed ottimi fra questi, come abbiamo detto a p. 312, sono la brevità del periodo vegretativo ed il mantenimento della vita allo stato latente nel seme di una pianta annua. Specialmente adattato a Linosa deve essere questo modo di difesa contro l'estate infuocata se, come pare, le pioggie invernali vi sono più abbondanti che in Lampedusa, aumentando la differenza fra le stagioni e favorendo le piante annue a breve periodo vegetativo, prive di altri adattamenti xerofili. E naturale quindi che abbia preso dimora in Linosa un numero maggiore di tali piante.

1 Vedi De Candolle, Géogr. bot. raisonnée, p. 991; Flahault, La distribution géograph. des végét. dans un coin du Languedoc, Montpellier, 1893, p. 80, e Sommer, Una cima vergine nelle Alpi Apuane, in N. Giorn. bot. it., 1894, p. 15 e 18. 
Il terreno sciolto di Linosa pare invece meno propizio di quello compatto di Lampedusa alle specie aventi serbatoî sotterranei di sostanze nutritrici, poichè vi è minore la proporzione di piante fornite di bulbi, tuberi o rizomi carnosi. Minore è pure in Linosa la proporzione di piante alofite. Maggiore invece vi è la proporzione delle specie psammofile. Mancano del tutto a Linosa non solo le vere idrofite, ma anche le piante amiche dei luoghi umidi, non trovandovisi neppure una di quelle poche che crescono in Lampedusa e che ho citate a p. 314. La proporzione delle antropocore non è minore in Linosa che in Lampedusa, il che sembra in contradizione con l' estensione maggiore delle colture in Lampedusa, ma va d'accordo col predominio delle specie annue in Linosa, le antropocore essendo in grandissima maggioranza annue.

Se, seguitando la nostra analisi, ordiniamo le famiglie principali secondo la loro ricchezza di specie come abbiamo fatto per la flora delle Pelagie in complesso, troviamo differenze assai marcate fra le due isole.

Famiglie che nell' una o nell'altra delle Pelagie contano almeno 4 specie.

\begin{tabular}{|c|c|}
\hline usa. & Linosa. \\
\hline 1. Composte & 1. Papilionacee ..... \\
\hline 2. Graminacee...... 53 & 2. Graminacee.... \\
\hline 3. Papilionacee . . . . . 48 & 3. Composte \\
\hline 4. Ombrellifere ..... 19 & 4. Cariofillacee. . . . . \\
\hline 5. Crocifere. . . . . . . 16 & 5. Crocifere ....... \\
\hline 6. Cariofillacee. . . . . 15 & 6. Crassulacee. . \\
\hline 7. Labiate ........ & »Chenopodiacee \\
\hline$»$ Gigliacee ........ & Urticacee. \\
\hline 8. Chenopodiacee ..... 12 & $»$ Euforbiacee...... \\
\hline sulacee....... 11 & 7. Geraniacee ..... \\
\hline 10. Euforbiacee. . . . . 10 & » Ombrellifere . . \\
\hline 11. Convolvulacee .... 9 & " Rubiacee ........ \\
\hline 12. Rubiacee ....... & "Labiate. \\
\hline bas & 8. Gigliacee \\
\hline
\end{tabular}


Lampedusa.

13. Ranunculacee. .....

$\gg$ Papaveracee ......

$»$ Geraniacee .......

\Borraginacee.....

》Urticacee .........

14. Cistacee.......

Iridacee........

15. Fumariacee.......

». Malvacee ........

$\gg$ Solanacee........

»Scrofulariacee .....

» Poligonacee.......

»Asparagacee ......

16. Genzianacee. ......

》Plantaginacee .....

17. Felci.........
Linosa.

@. Borraginacee......

》Felci.......... 5

10. Papaveracee......

» Malvacee ........

»Solanacee........ 4

》 Scrofulariacee .....

» Poligonacee.......

11. Fumariacee.......

»Convolvulacee .... 3

» Plantaginacee .....

12. Genzianacee .....

Iridacee............ 2

»Asparagacee ......

13. Ranunculacee.....

Notevole, in confronto di Lampedusa, è la ricchezza in Linosa delle Papilionacee, Cariofillacee, Urticacee, Geraniacee e Felci, e la povertả delle Composte, Ombrellifere, Convolvulacee, Iridacee, Asparagacee, la presenza di una sola Ranunculacea, di una sola Slatice, di una sola Cistacea (ed anche quella dubbia). In Linosa mancano 21 delle famiglie. Pelagie (rappresentate in Lampedusa da 24 generi e 29 specie): Resedacee, Elatinacee, Ipericacee, Ossalidacee, Ramnacee, Litracee, Mirtacee, Caprifogliacee, Dipsacee, Globulariacee, Balanoforacee, Timeleacee, Lauracee, Santalacee, Callitricacee, Orchidacee, Colchicacee, Juncaginacee, Giuncacee, Ciperacee, Licopodiacee. In Lampedusa mancano due delle famiglie Pelagie, le Portulacacee e le Amarantacee, rappresentate in Linosa ognuna da una sola specie.

Delle differenze nel paesaggio botanico nelle due isole, risultante dalle diverse associazioni e dalla abbondanza degli individui di certe specie, ho parlato a p. 47-53 e 183-18\%. Qui osservero soltanto che a tale differenziazione contribuiscono molto l'abbondanza in Lampedusa dei Cistus, delle Statice, di Asphodelus ramosus, Asteriscus aquaticus, Teucrimm fruticans, Lotus cytisoides, Senecio leucanthemifolius, Jasonia glutinosa, Col- 
chicum Bertolonii, Hypericum Ag!pliacum, Crucianella mepestris, Diplotaxis scaposa, Linaria reflexa, Cotula anrea, Oxalis cermea, e la frequenza in Linosa, invece, di alcuni arbusti oramai quasi distrutti in Lampedusa, nonchè l'abbondanza di Silene neglecta, Mesembryanthemum crystallinum, Rumex bucephalophomes, la presenza dei Rhus, dell'Amberboa, dei Lupinus, dell'Asphodelus tenuifolius, degli Erodium laciniatum e angulatcom, e la frequenza delle crittogame.

Abbiamo visto che fra le caratteristiche della flora di Linosa ve ne era una, la prevalenza delle piante annue, che si potera attribuire a condizioni ecologiche speciali dell'isola. La forte proporzione di Papilionacee, di Cariofillacee e di Geraniacee potrebbe dipendere dalle stesse cause, essendovi nella zona marittima della regione mediterranea una grande prevalenza di specie annue in queste tre famiglie, ${ }^{1}$ fra le quali quindi Linosa poteva reclutare maggior numero di specie adattate, per questa ragione, alle sue condizioni ecologiche.

Se confrontiamo lo specchietto delle p. 327-8 con quello a p. 315 , rediamo che per l'ordine in cui si seguono le famiglie, ognuna delle due Pelagie si scosta dalla Sicilia e dalla Tunisia piu delle due isole in complesso, le divergenze (o differenze di livello) mostrandosi fino dalle prime famiglie; e vediamo pure che é Linosa, cioè l'isola più piccola, che più se ne scosta. Ció concorda con quanto abbiamo osservato a p. 316, che più é piccola l'area considerata, meno chiare vi appariscono le leggi di distribuzione della regione, primeggiando i fattori edafici locali. Vediamo pure che l'entità delle divergenze nella seriazione delle famiglie $\dot{e}$ press'a poco uguale fra Linosa e la Sicilia e fra Linosa e la Tunisia; cosi pure Lampedusa, confrontata separatamente con la Sicilia e cor la Tunisia, non mostra sotto questo riguardo piủ aflìnitá con l'una che con l'altra. Nella seriazione delle famiglie non troviamo dunque alcuna ragione per avvicinare piu l'una che l'altra delle Pelagie alla Tunisia o alla Sicilia.

^ Nell'Arcipelago Toscano le Cariofillace contano $71,15 \%$, le $\mathrm{Pa}$ pilionacee $70,91 \%$ e le Geraniacee $94,12 \%$ di specie amnue, mentre nel loro insieme le piante vascolari ne contano soltanto $40,89 \%$. 
In quanto al rapporto fra dicotiledoni (incluse le Gimnosperme) e monocotiledoni, esso è assai diverso nelle due isole. Di fatti in Lampedusa le prime stanno alle seconde come 4,05 sta ad 1, ed in Linosa come 4,67 sta ad 1. Linosa è dunque. notevolmente piủ povera di monocotiledoni. A questa differenza non contribuiscono affatto le graminacee, che invece, in proporzione, sono più numerose in Linosa.

Se cerchiamo la proporzione, nelle fanerogame, fra specie, generi e famiglie, come abbiamo fatto per le due Palagie in complesso, troviamo che sono le seguenti:

FANEROGAME.

\section{Lampedusa}

Famiglie 71 ; generi 268 ; specie 460

\section{Linosa}

Famiglie 53; generi 176 ; specie 289

\begin{tabular}{|c|c|c}
\hline \multicolumn{2}{|c|}{ NUMERO MEDIO DI } \\
\hline $\begin{array}{c}\text { generi per } \\
\text { famighia }\end{array}$ & $\begin{array}{c}\text { specie per } \\
\text { famiglia }\end{array}$ & $\begin{array}{c}\text { specie per } \\
\text { genere }\end{array}$ \\
\hline 3.8 & 6.5 & 1.7 \\
3.3 & 5.5 & 1.6 \\
\hline
\end{tabular}

Confrontando queste cifre con quelle esposte nello specchietto della p. 319, vediamo che confermano quanto ho detto, cioè che quanto più è piccola una flora, tanto minore è il numero di specie per genere e per famiglia.

Facciamo ora il confronto statistico delle flore di Lampedusa e di Linosa separatamente, con quelle delle terre più vicine, come lo abbiamo fatto per la flora delle due Pelagie in complesso.

Lampedusa ha in comune con le isole Maltesi 370 specie vascolari, e Linosa ne ha 235, il che corrisponde per la prima al $79,74 \%$ della sua flora vascolare, e per la seconda al $79,93 \%$. Si può dunque dire che le due Pelagie presentano con le isole Maltesi delle differenze esattamente proporzionali alle loro flore.

Lampedusa ha in comune con Pantelleria 281 specie vascolari, e Linosa ne ha 221 , il che corrisponde per la prima al $60,56 \%$ della sua flora vascolare e per la seconda al $75,17 \%$. Lampedusa 
dunque, nella composizione della sua flora vascolare differisce assai più di Linosa da Pantelleria.

Esaminiamo ancora le 59 specie Pelagie che non sono AfroSicule (p. 306-9). Delle 12 mancanti tanto alla Sicilia quanto all'Africa, 6 si trovano in Linosa, e 8 in Lampedusa. Delle 22 Afro-Pelagie che mancano in Sicilia, 10 si trovano in Linosa, e 14 in Lampedusa. Delle 25 Siculo-Pelagie che mancano in Africa, ve ne sono 6 sole in Linosa, mentre in Lampedusa ve ne sono 24, cioé il quadruplo. Se facciamo il confronto con la Tunisia anzichè con tutta $l^{\prime}$ Africa boreale, troviamo che delle 74 specie Pelagie mancanti in Tunisia 61 si trovano a Lampedusa e 28 sole in Linosa, d'onde risulta che Linosa ha in comune con la Tunisia $90, \mathrm{~L} \%$ delia sua flora vascolare mentre Lampedusa ne ha solo $86,9 \%$. Si vede dunque che il carattere Sículo-Africano, cosi spiccato nella flora delle Pelagie, lo è presso a poco ugualmente nelle due isole, ma che la flora di Linosa si stacca un poco piu di quella di Lampedusa dalla flora della Sicilia ed invece un poco più si avvicina a quella dell'Africa boreale. Troviamo cosi di nuovo l'opposto di quanto avremmo potuto supporre considerando che terreni vulcanici come quelli di Linosa si trovano in Sicilia ma non sulla costa opposta d'Africa, mentre terreni uguali a quello di Lampedusa si trovano in Tunisia, ed inoltre che Lampedusa è più vicina all'Africa, ed è stata forse ad essa unita in epoca geologica non molto remota, mentre Linosa non è stata mai unita ad altre terre emerse. Si vede pure che non è vero quello che fu detto, cioè che floristicamente Lampedusa appartiene all'Africa, ${ }^{1}$ e che anzi statisticamente le affinità floristiche di Lampedusa sono un poco più accentuate con la Sicilia.

Non possiamo terminare i confronti fra Lampedusa e Linosa senza domandarci come e quanto si manifesti nelle loro flore l'influenza della natura chimica del terreno. Quale insegnamento possiamo ricarare in proposito dai confronti statistici fin ora fatti?

2 'Trabucco, I' Tsola di Lampedusa, p. 22. 
Ci si poteva aspettare di redere in qualche modo tradursi in cifre l'azione diversa del suolo esclusivamente vulcanico di Linosa e di quello calcareo di Lampedusa. Mía tali differenze numeriche non si sono palesate chiare. Non abbiamo potuto constatare che la natura del terreno esercitasse alcuna influenza nè sulla ricchezza relativa delle due flore, nè sulla diversitá della loro composizione. Abbiamo trovato che la calcarea Lampedusa non aveva in comune con le isole Maltesi calcaree una proporzione di elementi maggiori della vulcanica Linosa; ed il confronto tanto con tutta l'Africa boreale, quanto con la sua parte piu vicina alle Pelagie, la Tunisia, che ha terreni uguali a quelli di Lampedusa, ci ha mostrato al contrario che la proporzione di elementi Tunisini e Africani era un poco maggiore in Linosa.

Una differenza marcata abbiamo riscontrato nella proporzione di specie annue; ma abbiamo visto che si poteva attribuire alle qualita fisiche del suolo, indipendentemente dalla sua composizione chimica, se non in quanto questa influisce su quella.

Vi è però uno dei confronti statistici che abbiamo fatti, quello di Linosa e Lampedusa con Pantelleria, che, se fosse isolato, sembrerebbe provare come l'uguaglianza mineralogica di Linosa e di Pantelleria abbia avuto per conseguenza una proporzione maggiore di elementi floristici comuni a queste due isole.

Ma se questa fosse la causa, una corrispondente disproporzione avremmo dovuto trovare confrontando le due Pelagie con le isole Maltesi e con la costa d'Africa, mentre abbiamo visto che non è cosi. Vi è poi un altro confronto che ci dá un risultato affatto opposto: Linosa ha in comune con Lampedusa 228 specie, mentre ne ha in comune con Pantelleria soltanto 221. La vulcanica Linosa ha dunque in comune con la calcarea Lampedusa un numero di specie un poco maggiore che con la vulcanica Pantelleria, quantunque la flora di Pantelleria, essendo un poco più numerosa di quella di Lampedusa, avesse anche qualche possibilitá di piủ di possedere elementi floristici in comune con Linosa. Non è dunque alla eguale composizione del suolo che dobbiamo attribuire la somiglianza floristica maggiore fra Pantelleria e Linosa che fra Pantelleria e Lampedusa. Possiamo notare ancora che se fosse la mancanza di calcare che 
impedisce a tante piante di Lampedusa di trovarsi in Pantelleria, esse dovrebbero trovarsi per la massima parte sulla vicina costa calcarea della Tunisia, e dovrebbero invece mancare in Linosa. Vediamo invece che delle 183 specie di Lampedusa mancanti a Pantelleria, 45 mancano anche in 'Tunisia, ed invece 39 si trovano in Linosa.

Da tutto questo risulta che bisogna essere molto cauti nel trarre le deduzioni, quando si adopra il metodo statistico nel confronto delle flore. Ad ogni modo possiamo riconoscere che statisticamente l'influenza della natura chimica del terreno nelle Pelagie rimane mascherata da quella di altri fattori, e deve quindi considerarsi come subordinata a questi.

Ma se la natura chimica del suolo non ha influito in modo rico. noscibile sulla quantità della differenziazione, av rå influito sulla sua qualitả, per cui una analisi accurata dei singoli elementi delle due flore ci dovrebbe mostrare delle differenze attribuibili a questo fattore. Una tale analisi peró dovrebbe essere sussidiata da numerosi confronti e da dati di fatto che in parte ci mancano, poichè le indicazioni sulla relazione fra le piante e la natura chimica del suolo sono incomplete e spesso contradittorie. Bisogna difatti guardarci dal trarre conclusioni da quello che osserviamo in due sole isole, poichè tali conclusioni possono esser contradette da un'al tra flora. Potremmo per esempio credere che l'A sphodehus ramosus fosse esclusivamente calcicolo, vedendo che cuopre Lampedusa, mentre manca interamente a Linosa. Ma se andiamo a Pantelleria lo troviamo abbondante su quei terreni vulcanici quanto sui calcari di Lampedusa. Cosi potrebbe creder'si esclusivamente calcicola l'oxalis cermua quando la si vede una vera peste dei campi a Lampedusa ed a Malta, e si sa che è comune nei terreni calcarei della Tunisia, mentre manca assolutamente a Linosa ed a Pantelleria. Ma trovandola poi ugualmente comune sulle falde del Vesuvio e dell'Etna ed in qualche isolotto interamente vulcanico, si vede che non rifugge dai terreni vulcanici.

Le piante del mio prospetto si possono dividere in tre categorie: 10 piante che si trovano tanto in una almeno delle due isole esclusivamente vulcaniche, Linosa e Pantelleria, quanto nei terreni calcarei di Lampedusa, Malta o Tunisia; $2^{\circ}$ piante che 
mancano nelle due isole vulcaniche; $3^{\circ}$ piante che si trovano soltanto in terreni vulcanici, cioè in Linosa sola o in Linosa e Pantelleria. Nelle prime, che sono di gran lunga le più numerose, dobbiamo riconoscere delle piante che in questi distretti si comportano come indifferenti. Nelle seconde potremmo sospettare delle piante esclusivamente calcofile, nelle terze (che sono pochissime) delle silicicole o calcifughe. Peró se prendiamo delle altre flore di terreni esclusivamente vulcanici, vi troviamo non poche delle piante della seconda categoria. Nella sola flora Vesuviana del Pasquale, ${ }^{1}$ che conta poco più di 650 specie vascolari, troviamo sedici specie che mancano a Linosa ed a Pantelleria mentre si trovano in Lampedusa ed in tutte le altre colonne del mio prospetto; ad Ischia, isola totalmente vulcanica, oltre alla maggior parte di queste sedici specie, ne troviamo altre nove che nel mio prospetto mancano soltanto a Linosa ed a Pantelleria, ${ }^{2}$ ed altre ventidue ne troviamo nelle vulcaniche Eolie. ${ }^{3}$

Nella sopra citata Flora Vesuviana 'e Caprense, a p. 10, Pasquale dá l'elenco di 45 specie vascolari e 3 crittogame cellulari di Capri che considera come caratteristiche dei terreni calcarei di quell' isola e delle quali dice che invano si cercherebbero sul Vesuvio e nei campi Flegrei. Ebbene, 14 di quelle specie vascolari, ed un musco, si trovano in Linosa o in Pantelleria 0 in entrambi quelle isole vulcaniche.

1 J. A. Pasquale, Flora Vesuiviana et Caprensis comparatce. "Atti d. R. Acc. d. Sc. fis. e mat. ». Napoli, 1869.

${ }^{2}$ Gussone, Enumeratio plantarum vascularium in insula Inarime sponte provenientium ecc. Napoli, 1854. (Faccio notare che ivi a p. 416-417 è detto erroneamente che il numero delle specie vascolari d'Ischia è di 960 ; questo numero va corretto, essendovi un grosso errore a p. 416).

3 M. Lojacono, Le isole Eolie e la loro vegetazione. Palermo, 1878. - G. B. Traverso, Elenco delle piante raccolte nelle isole Eolie dal 9 al 13 aprile 1900. «Bollett. Soc. geolog. it. », vol. XIX (1900), p. Lxxir-Lxxiv. - G. Zodda, Una gita alle isole Eolie. "Atti I. R. Acc. Peloritana », vol. XIX, fasc. 1. Si noti che ho confrontato il mio prospetto soltanto con gli elenchi dei sopra citati autori i quali fanno menzione soltanto delle piante raccolte da loro stessi, e che il numero di 22 aumenterebbe certo notevolmente facondo lo spoglio della Synopsis di Gussone. 
Cercando negli elenchi di piante che in altri distretti sono state considerate come calcicole o come silicicole prevalenti o esclusive, si trovano molte altre indicazioni che sono in contradizione con quanto si desumerebbe dal mio prospetto. Ne citeró solo due esempî presi da distretti non lontani, e dove quindi le condizioni climatiche non sono molto diverse. Il dott. Béguinot da, nella sua flora delle isole Ponziane e Napoletane, ${ }^{1}$ un piccolo elenco di «specie piủ fedeli alle roccie silicee ed al terreno da esse derivante e che solo incidentalmente ma senza propagarvisi ed in condizioni del tutto speciali, possono incontrarsi su quello calcareo ». Delle 32 specie che esso cita, le 10 sole che si trovano nel mio prospetto si trovano tutte in qualcuna, se non in tutte le colonne dedicate a terreni calcarei. Alcune anzi sono anche comuni ed abbondanti in Lampedusa, come la Tillaea merscosa, la Spergularia rutha e l'Arbutus Unedo. - Il dott. Zodda nel suo studio sulla flora Messinese ${ }^{2}$ dả un elenco di piante che egli dice «esclusivamente calcicole » in quel distretto. Venti di queste specie si trovano nel mio prospetto, e di queste venti, diciasette si trovano in una delle due isole vulcaniche 0 in tutt'e due. Fra le specie esclusivamente silicicole nel Messinese (p. 17), tre sole appartengono alle Pelarie. Una di esse, Anthoxanthum odoratum, manca per l'appunto soltanto nelle due isole vulcaniche del mio prospetto, mentre trovasi in tutte le altre colonne, ed un' altra, Lamarckia aurea, trovasi in tutte le colonne. Molti altri paragoni si potrebbero fare col mio prospetto, che confermerebbero il fatto, del resto ben noto, che in distretti diversi le medesime specie reagiscono in modo differente alla composizione chimica del suolo, oppure che a questa è stata attribuita una azione che va ricercata altrove. Ció dimostra sempre più quanto sia difficile assegnare il suo giusto valore a questo fattore edafico.

Non bisogna dimenticare, del resto, che nella calcarea Lampedusa non manca la silice, che anzi vi si trova abbondante negli

1 A. Béguinot, La vegetarione delle isole Ponziane e Napoletane, pag. 205. Roma, 1905.

G. ZodDa, Sulla vegetazione del Messinese. Saggio di ecologia botanica, pag. 16. Acireale, 1905. 
strati di marna. E se nei terreni vulcanici di Linosa e di Pantelleria manca il carbonato di calce, non vi manca del tutto la calce la quale, sebbene vi sia in piccola quantitá ed in altre combinazioni, puo venire assimilata dagli or ganismi viventi anche in notevole quantita, come lo prova il guscio calcareo dei molluschi terrestri che vi si trovano, non che quello normalmente sviluppato delle uova di gallina e di altri uccelli.

Tutto quanto abbiamo detto prova ancora una volta che questo capitolo dell: ecologia botanica è ancora molto imperfetto, e richiede ulteriori ricerche sperimentali, ed altri elenchi attendibili di piante di distretti ben delimitati. A tale scopo serviranno specialmente le florule delie isole, nelle quali è da sperare che le crittogame occuperanno il posto importante che spetta loro in questo ordine di ricerche. Probabilmente a chiarire molti punti oscuri, ed a spiegare molte contradizioni apparenti contribuirả lo studio delle microspecie, di quelle entitả morfologricamente poco differenziate che furono dette specie fisiologiche e che sono l'espressione dell' adattamento più o meno fissato di una specie a diversi terreni.

Nelle mie florule di Lampedusa e di Linosa ho accennato ad alcune forme non osservate altrove, che si scostano dal tipo delle specie alle quali le riferisco, e le ho designate come varietc̀ o come forme. Per una sola di esse (Linaria pseudolaxiflora) ho accettato il rango di specie al quale altri l'aveva inalzata. È da notare che esse sono più numerose e marcate a Linosa che a Lampedusa. Queste forme, che si potrebbero considerare come micro- o neo-endemismi, indicherebbero dunque nel distretto vulcanico di Linosa proprietá ecologiche piú adatte a indurre delle differenziazioni. Na non si puó affermare che quelle forme non si trovino pure in Sicilia o nell'Africa boreale, essendo possibile che le lievi differenze che le caratterizzano non sieno state avvertite, o non sieno state giudicate degne di speciale menzione dagli autori che hanno citato in quelle flore le specie alle quali sensu latiore appartengono. 


\section{Origine della flora delle isole Pelagie.}

Ed ora infine possiamo domandarci quale sia l'origine della flora delle Pelagie, cercando di desumerlo dal fin qui esposto. Non $\nabla$ golio ben inteso parlare di origini remote che implicherebbero la ricerca delle origini della flora circummediterranea; mi pongo semplicemente la domanda: da dove, per quale via e con quali mezzi è giunta alle Pelagie la sua popolazione vegetale?

Dobbiamo, per rispondere a questa domanda, consiłerare prima Linosa e Lampedusa separatamente.

Per Linosa di fatti il problema è più semplice. I geologi sono d'accordo nel dirci che essa sorse dal mare dopo la fine del pliocene, cioè in epoca relativamente recente, e che non fu mai congiunta con altre terre emerse. La sua flora è dunque immigrata attraverso al mare, e se ne puó attribuire l'origine soltanto al trasporto di semi a distanza, per mezzo di agenti naturali e dell'uomo. E fra gli agenti naturali principalissimi, anzi quasi unici, si devono riconoscere il vento e gli uccelli. Pochissima parte di fatti vi possono avere avuto le correnti marine, essendo forse il Pancratium maritimum l'unica pianta littoranea con semi muniti di un invoglio adatto al tempo stesso a farli galleggiare e a difenderli lungamente dall'azione dell'acqua marina. In quanto ai venti che iri sofliano impetuosi, è facile comprendere. come essi, data la vicinanza della Sicilia e dell'Africa, portino di continuo da quelle terre in Linosa semi leggieri o muniti di apparecchi per il volo; ed è pure ovvio che in Linosa devono continuamente venire depositati semi ingeriti o trasportati per aderenza dagli nccelli che nelle loro periodiche migrazioni t'ra l'Africa e la Sicilia si fermano a riposare nelle isole Pelagie.

L'azione dell'uomo poi deve essere stata maggiore di quanto si potrebbe supporre a prima vista pensando che Linosa è stata per tanto tempo deserta. Di fatti non solo sappiamo che fu abitata ed estesamente coltivata in antico, e che quindi in quel tempo dovera arere scambî frequenti con altre ter'e, ma anche: durante le epoche in cui non era abitata, fin da quando l'uomo 
incominció a navigare nel Mediterraneo, delle imbarcazioni vi devono avere approdato, volontariamente o involontariamente, un numero infinito di volte, e la grande maggioranza di quelle imbarcazioni doveva provenire dalla Sicilia e dall'Africa. I rilevanti cambiamenti che vi si sono verificati, specialmente per opera dell' uomo, in tre quarti di secolo, dal tempo di Gussone in poi, permettono di arguire delle profonde modificazioni che vi si possono esser prodotte nel corso dei secoli.

La composizione della flora di Linosa che, come abbiamo visto, consta quasi esclusivamente di elementi Africani e Siciliani, è perfettamente d'accordo con questa origine. Delle 6 sole specie di Linosa che non sono nè Sicule nè Africane, la Fumaria bicolor trovasi a Malta ed a Pantelleria, il Carduus marmoratus a Lampedusa ed a Malta, l'Heliotropizem Eichvaldi a Lampedusa, la Linaria pseudolaxiflora si riconnette ad una specie Africana. Il Lotus peregrinus ed il Bellium minutum. sono le due sole specie che si ritrovano soltanto più ad Oriente.

Si puỏ dire di Linosa che è, per rapporto alla flora Afro-Sicula, quello che è l'alto Etna per rapporto alla flora dei monti più antichi della Sicilia e del vicino continente, dai quali è separato da terre basse sulle quali non possono crescere le piante d'alta montagna. L'isola come il monte sono dei neonati che hanno dovuto ricevere la loro flora per trasporto di semi a distanza in tempi relativamente recenti, e che quindi sono privi di endemismi primarî.

Del resto, di immigrazioni di piante per trasporto di semi a distanze anche molto grandi ed attraverso il mare abbiamo tanti e tanti esempî accertati; e tra i più recenti v' è quello di Krakatoa, che dimostra quanto poco tempo basti perchè una terra nuda venga ad albergare una discreta flora.

\section{$\therefore$}

In quanto a Lampedusa, non è escluso dai geologi che essa, dopo la sua emersione, sia stata per un certo tempo congiunta colla costa Tunisina. Il mare fra Lampedusa e la Tunisia non oltrepassa la profonditå di cento metri, e ricuopre delle terre che forse emersero dal mare insieme a Lampedusa ed alla costa 
Tunisina, cioè durante il pliocene. In tal caso l'abbassamento di quelle terre ed il conseguente isolamento di Lampedusa sarebbero avvenuti verso la fine del pliocene o al principio del quaternario. È escluso invece che Lampedusa sia stata unita alla Sicilia o a Malta dalle quali la separano profonditi marine molto maggiori. Si potrebbe quindi attribuire la sua popolazione regetale, in parte almeno, a delle sopravvivenze dell'epoca in cui esisteva la congiunzione colla costa Africana. Ma, oltrechè tale congiunzione è tutt'altro che provata, e che la flora della costa Tunisina poteva allora essere ben diversa dall'attuale, quanto sappiamo della flora di Lampedusa non fornisce alcun argomento in appoggio a questa ipotesi. Abbiamo visto di fatti che, non ostante la maggiore vicinanza, la uguale latitudine, e la presenza di terreni identici, gli elementi Tunisini si trovavano nella flora di Lampedusa in minor numero degli elementi Siciliani. Significativo poi è il fatto che delle due Pelagie, è Linosa, di cui si sa per certo che non fu mai unita alla vicina costa Africana, che possiede in proporzione della sua flora un numero un poco maggiore di elementi Tunisini, e ciò non ostante che sia più discosta dalla Tunisia, ed abbia terreno del tutto diverso. Tutto ci porta dunque a concludere che la flora di Lampedusa è interamente doruta, come quella di Linosa, all'apporto di semi attraverso al mare.

Si potrebbe essere impressionati, a prima visła, dalla presenza in Lampedusa della Stapelia Érropaea, appartenente a un genere cosi caratteristicamente Africano, ${ }^{1}$ e che sembra quindi indicare una più intima connessione con l'Africa, e si potrebbe essere tentati di vedere in essa uno di quei relicta o fossili viventi la cui presenza si è invocata per confermare antiche congiunzioni di isole con altre terre. Ma non vedo alcuna ragione per non ammettere che i semi di questa pianta siano stati trasportati a Lampedusa attraverso al mare da uno degri agenti che hamno popolato di piante Linosa, tanto più che la Slapelia Europaea è comune in 'Tunisia e che i suoi semi muniti di lungo pappo sono appunto fra quelli più facili ad essere traspor-

1 Si noti però cho la Stapelia Éuropaea è stata trovata pure in Spagna. 
tati dal vento. Le spiegazioni geologiche ci seducono perchè una ipotesi ardita è sempre più affascinante di una spiegazione piana e semplice. Ma bisogna andar cauti nel ricorrere alla ipotesi della sopravvivenza di una specie nel suo antico habilat, ipotesi della quale talvolta si é forse abusato non solo per le isole, ma anche per spiegare la presenza di entità e di colonie eterotopiche continentali. Ce lo prova per esempio la presenza in Linosa del Bellium minutum e della Castellia tuberculosa, specie cosi rare e di habital cosi saltuario, le quali tanto bene si presterebbero ad una simile interpretazione, se questa non fosse perentoriamente contraddetta dal fatto che Linosa fu sempre un'isola.

Non bisogna lasciarsi influenzare dalla fisionomia del paesaggio botanico di una terra quando si ricerca l'origine della sua popolazione vegetale. Era naturale che chi conosceva bene la flora Sicula come Gussone, fosse colpito dalla presenza nelle Pelagie anche di poche specie, segnatamente se gregarie, mancanti in Sicilia, e dicesse di alcune che ravvicinavano la vegetazione di Lampedusa a quella di Malta; di altre che la ravvicinavano a quella di Barberia. " $\mathrm{Na}$ con ció non intendeva certo accennare ad antiche congiunzioni con queste terre. La presenza in Lampedusa come in Linosa di alcune piante caratteristiche di altri paesaggi botanici è semplicemente l'indice di condizioni ecologiche esistenti in queste isole, che ne hanno permessso l'attecchire, e favorito la larga diffusione. Del resto tre delle piante più caratteristiche del paesaggio floristico di Lampedusa che mancano a Linosa, Hypericum Egyptiacum, Crucianella rupestris e Jasonia glutinosa, non esistono aftatto in Tunisia, e la Periploca ed i Rhus, citati da Gussone fra le specie che avvicinano le Pelagie alla Barberia, esistono pure a Linosa, anzi, i Rhus soltanto in Linosa. Nulla dunque possiamo trovare che ci induca a credere che Lampedusa non abbia ricevuto la sua flora per la medesima ria di Linosa.

Del resto l'ammettere che tutte le specie ora esistenti in Lampedusa vi sono immigrate per trasporto di semi a distanza, non significa negare che quest'isola sia stata un tempo unita alla Tunisia. Di fatti, anche se tale congiunzione fosse esistita alla

1 Gussone, Notizie, p. 87. 
fine del pliocene, modificandosi il clima, la ílora di Lampedusa avrebbe potuto cambiare completamente, dopo il suo isolamento, per l'apporto continuo di semi, come si é probabilmente cambiata, da quell'epoca in poi la flora Tunisina stessa. Il tempo è un gran fattore del quale è facile di non tenere sufficiente conto, e dalla fine del pliocene in poi ne è passato abbastanza perchè delle modificazioni come quelle che constatiano dopo il tempo di Gussone, venendo a sommarsi ogni secolo, possano avere cambiato interamente la flora di Lampedusa.

$$
*
$$

La composizione della flora delle Pelagie che, bisogna confessarlo, oltre ad esser povera, ha un tipo che si puó chiamare raccogliticcio e volgare, si accorda con la sua supposta genesi arventizia. Essa consta in grandissima maggioranza di piante largamente diffuse nella zona inferiore dalla regione mediterranea, anche nelle sue parti meno meridionali, di quelle piante che si potrebbero dire le vagabonde della regione mediterranea, perchè vi si trovano dappertutto. Delle sue 530 specie 393, vale a dire press'a poco $i$ tre quarti, si trovano nel piccolo Arcipelago Toscano a 7 gradi più a Nord; e delle 137 specie mancanti all'Arcipelago Toscano, 40 si trovano ancora più a Nord, nella sola Liguria. Queste specie largamente sparse nel bacino mediterraneo, oltre ad essere necessariamente fornite di buoni mezzi di diffusione, sono anche quelle che hanno dato prova della maggiore adattabilita ad ambienti diversi, e sono quindi le più idonee alla conquista di nuovi territorî. (Ció puó spiegare anche la difficoltà di trovare nelle Pelagie delle piante che dimostrino spiccata calcofilia o calcofobia.) La similariti nella composizione della flora delle due Pe. lagie, non ostante le grandi diversita geofisiche di queste isole, conferma la supposizione che esse hanno avuto la medesima origine, cioé che sono dovute agli stessi venti, agli stessi uccelli, agli stessi uomini. Con la genesi che attribuisco alla flora delle Pelagie si accorda anche la forte proporzione di specie antropocore, e la mancanza di endemismi primarî. 
Ci si potrebbe domandare ancora quali sono le ragioni che hanno determinato l'attecchire e il moltiplicar'si nelle Pelagie di certune piuttosto che di altre delle molte specie Siculo-Africane di cui ri devono essere giunti i semi. Mi pare che oltre alle condizioni climatiche, che non avranno consentito la vita e la moltiplicazione altro che alle specie della zona bassa, dotate di clima somigliante a quello delle Pelagie, non vi siano altre cause a cui si possa attribuire carattere di generalita. Certo deve avere eser'citato un'azione la natura fisica e chimica del terreno. MIa abbiamo visto che questi fattori edafici non erano tali da controbilanciarne altri. E questi altri fattori ci sfuggono per la loro molteplicità e complessità, come per l'epoca ignota in cui hanno agito. L'elemento cronologico o storico che chiamar si voglia, avanti l'epoca di Gussone, ci sfugge interamente e va quindi relegato fra le cause che per ignoranza dobbiamo chiamare fortuite 0 accidentali, poichè sarebbe vano ricercare perchè sia giunto in un'isola il seme di una pianta prima che quello di un'altra. Eppure il solo fatto di essere giunto primo può avere avuto un'azione determinante, poichè la presa di possesso di un terreno per parte di una pianta o di un'associazione di piante può bastare ad impedire l'introduzione di molte altre specie, quand'anche fossero ugualmente adattate a vivere in quel terreno, ed avessero potuto esserne loro le padrone se vi fossero giunte per prime. Le piante formanti un' associazione fortemente insediata non possono essere sloggiate altro che da specie molto meglio di loro adattate a quel terreno, ed è per questo che l'uomo, distruggendo le aritiche associazioni col lavorare la terra, favorisce l'introduzione di nuovi elementi ai quali offre, nei terreni arati e negli sterri, un campo libero alla competizione.

Il volere sempre trovare nelle condizioni del suolo e del clima le cause dell'accantonamento di certe piante in dati luoghi, mi pare che sarebbe come dire per esempio che le popolazioni diversissime che si trovano accantonate nelle valli del Caucaso vi si sono fissate perchè vi hanno trovato condizioni adattate al loro modo di vivere. Na se la ricerca storica delle vicende e delle migrazioni di quei popoli, che sole ci possono illuminare sulla causa della loro presenza in quelle valli, è molto difficile, asso- 
lutamente impossibile é la ricerca delle modaliti della immigrazione dei semi in un'isola senza storia.

Vi è perỏ un fatto statistico del quale potremmo domandarci la ragione. Perchè le Pelagie, essendo sotto la stessa latitudine della Tunisia e piủ vicine a questa che alla Sicilia, non hanno una maggioranza di piante Tunisine?

Verrebbe fatto, in sulle prime, di attribuirlo al numero di specie tanto maggiore in Sicilia, che avrebbe permesso alle Pelagie di reclutarvi un contingente maggiore. Ma tale spiegazione non regge, perchè la maggiore ricchezza della Sicilia é dovuta a piante di zone e di stazioni che nelle Pelagie non esistono, e che quindi non vi potrebbero allignare. Si potrebbe ancora pensare che fosse perchè il clima insulare delle Pelagie ha maggiore affinitá con quello di un'isola come la Sicilia che con quello di una terra continentale come la Tunisia. Na la causa ne è certo molto più complessa, e vi possono avere avuto parte anche i rapporti umani più frequenti con la Sicilia, la direzione prevalente dei venti e la provenienza degli uccelli di passo nella stagione della maturazione dei semi, come pure possono avere influito alcuni altri di quegli agenti animali che hanno tanti rapporti col mondo vegetale per la diffusione e la fecondazione delle piante, come anche talvolta per la loro distruzione. Probabilmente la ragione principale è che la corrente d'immigrazione è stata prevalentemente da Nord. Bisogna riconoscere pero che la grande maggioranza delle specie Pelagie essendo tanto Africane quanto Siciliane, esse potrebbero essere immigrate nelle Pelagie tanto da Sud quanto da Nord. La ricerca se le probabilitá siano più in un senso che nell'altro implicherebbe la ricerca della origine delle flore sicule e Nord-Africane, ed esorbita quindi dal modesto campo di questo studio. Possiamo dire soltanto che la prevalenza degli elementi Siciliani sopra quelli Tunisini, non ostante la distanza maggiore, è in favore di una maggiore immigrazione da Nord; mentre di alcune specie Afro-Sicule, come Periploca levigala, che raggiungono il loro limite settentrionale in Sicilia, ed ivi sono rare, possiamo ritenere per certo che sieno immigrate da Sud, come da Sud sono necessariamente immigrate le poche specie Africane che non trovansi più a Nord. 


\section{CONSIDERAZIONI SULLA ELORA DELLE ISOLE PELAGIE}

Di una debole, ma pure manifesta immigrazione da Est, ci då la prova il fatto che delle 59 specie Pelagie non Afro-Sicule, 24 si trovano a Malta, e le altre per la maggior parte sono pure orientali.

$$
* * *
$$

Mi auguro che lo studio comparativo di queste due isolette, che ho abbozzato, possa invogliare altri ad intraprenderne uno più esteso e più approfondito su di un maggior numero delle piccole isole del Mediterraneo. Le questioni ecologiche devono attendere molta luce dallo studio comparativo di aree ben circoscritte e spesso con suolo di una unica natura come sono le piccole isole, ed è per questo molto desiderabile vedere moltiplicarsi le florule insulari basate sopra ampio materiale bene raccolto e bene studiato.

Errata - Corrige.

All' ultima riga del penultimo capover'so della pag. 304 invece di: ...... le prime (247) stanno alle seconde (58) come 4,26 sta a 1

si legga:

...... le prime (362) stanno alle seconde (91) come 3,97 sta a 1. 


\section{INDICE DELLE MATERIE}

Prefazione. . . . . . . . . . . . . . Pag. 1

Le isole Pelagie . . . . . . . . . . \$ 5

Botanici che hanno visitato le isole Pelagie. . . \ is

Appunti bibliografici . . . . . . . . . . » 13

Lampedusa - Nome e Storia . . . . . . . . . » 80

Descrizione, prodotti, clima . . . . . . . » 34

Geologia. . . . . . . . . . \4 4

Fauna . . . . . . . . . . 》 41

Flora . . . . . . . . . . . . » 47

Le piante raccolte in Lampedusa. . . . . . . . » 54

Florula di Lampedusa . . . . . . . . . » 59

Linosa - Nome e Storia . . . . . . . . . . » 173

Descrizione, Geologia, Clima, Prodotti. . . . . \ 175

Fauna. . . . . . . . . . . . » 181

Flora. . : . . . . . . . . . . . " 158

Le piante raccolte a Linosa . . . . . . . \ 157

Florula di linosa. . . . . . . . . . . " " 191

Lampione. . . . . . . . . . . . . \265

Aggiunte alla florula di Lampedusa. . . . . . . . . " 271

Prospetto delle Florule di Lampedusa e di Linosa

con indicazione delle specie di queste isole che trovansi in Pantelleria, Malta, Sicilia e Tunisia. - Av-

vertenze . . . . . . . . . . . . \$ 275

Prospetto . . . . . . . . . . . . . . » 27

Note al Prospetto . . . . . . . . . " 294

Riassunto di alcuni dei dati statistici contenuti nel Prospetto . . . . . . . . . . . . " :01

Pantelleria - Piante di Pantelleria non comprese nel precedente Prospetto . . . . . . . . . . » 302

Considerazioni sulfa flora delle isole Peragie. . " 30 ;

Confronto fra la flora delle isole Pelagie e quelle delle terre più vicine. . . . . . . . . . . "

Modificazioni nella flora delle Pelagie. . . . " " 321

Confronto fra le florule di Lampedusa e di Linosa . " "3:2.?

Origine della flora delle isole Pelagie . . . . . " 






